\author{
Andreas Fritsch
}

\title{
Potenzialanalyse von Solarturmkraftwerken mit Flüssigmetallen als Wärmeträgermedium
}
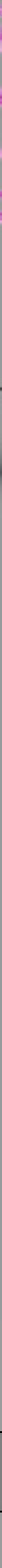



\section{Potenzialanalyse von Solarturmkraftwerken mit Flüssigmetallen als Wärmeträgermedium}

\section{An Analysis of Potential of Solar Tower Power Plants Using Liquid Metals as Heat Transfer Fluid}

Der Fakultät für Maschinenwesen der Rheinisch-Westfälischen Technischen

Hochschule Aachen vorgelegte Dissertation zur Erlangung des akademischen Grades eines Doktors der Ingenieurwissenschaften

VOn

Andreas Fritsch 
Bibliografische Information der Deutschen Nationalbibliothek

Die Deutsche Nationalbibliothek verzeichnet diese Publikation in der Deutschen Nationalbibliografie; detaillierte bibliografische Daten sind im Internet über http://dnb.d-nb.de abrufbar.

(C)Copyright Logos Verlag Berlin GmbH 2018

Alle Rechte vorbehalten.

ISBN 978-3-8325-4724-0

(cC) 6

Logos Verlag Berlin GmbH

Comeniushof, Gubener Str. 47, 10243 Berlin

Tel.: +49 (0)3042851090

Fax: +49 (0)3042851092

INTERNET: https://www.logos-verlag.de 


\section{Vorwort}

Die vorliegende Arbeit entstand am Deutschen Zentrum für Luft- und Raumfahrt in Stuttgart am Institut für Solarforschung in der Abteilung für punktfokussierende Systeme.

Mein besonderer Dank gilt Herrn Professor Bernhard Hoffschmidt für die Betreuung dieser Arbeit und die Übernahme des Hauptberichts. Seine sehr hilfreichen Anregungen haben mich stets unterstützt und voran gebracht. Gleichzeitig gilt mein Dank auch Herrn Professor Thomas Wetzel vom Institut für Kern- und Energietechnik am Karlsruher Institut für Technologie (KIT) für die Übernahme des Zweitgutachters sowie die zahlreichen fachlichen Diskussionen bezüglich Flüssigmetallen.

Außerordentlich dankbar bin ich Ralf Uhlig für die fachliche Betreuung. Seine Offenheit und die konstruktiven Gespräche haben einen wesentlichen Beitrag zum Erfolg dieser Arbeit geleistet. Außerdem danke ich Reiner Buck und allen Kollegen der Solarforschung, die mir stets mit Rat und Tat zur Seite standen und für eine angenehme Arbeitsatmosphäre gesorgt haben. Für die fachliche Unterstützung insbesondere im Bezug auf Flüssigmetalltechnologie danke ich Jonathan Flesch und dem gesamten KALLA-Team.

Cathy Frantz bin ich für die aktive Mitarbeit an ASTRID und die enorme Unterstützung bei der Auslegung der Salzreceiver zu großem Dank verpflichtet. David Trebing danke ich für die vielfältigen und fruchtbaren Diskussionen und Daniel Brenner für die graphische Darstellung der Energieflüsse und Kraftwerkskomponenten in der Einleitung. Schließlich gilt mein allertiefster Dank meiner Familie und meinen Freunden, die mir die Grundlage für diese Arbeit gegeben haben.

Ökologisch sinnvoll erscheint, die fossilen Energieträger durch Solarkraftwerke zu ersetzen. Für einen nachhaltigen Lebensstil muss jedoch vor allem der Bedarf an elektrischer Energie signifikant reduziert werden. Möge diese Arbeit allen an Solarkraftwerken und im Speziellen an Flüssigmetallen Interessierten eine Hilfe für zukünftige Forschung und Entwicklung sein. 

Reich ist, wer viel hat, reicher ist, wer wenig braucht, am reichsten ist, wer viel gibt.

(Gerhard Tersteegen) 



\section{Inhaltsverzeichnis}

Nomenklatur und Verzeichnisse xiii

Abkürzungen ......................... xiii

Formelzeichen . . . . . . . . . . . . . . . . . $\mathrm{xv}$

Indizes . . . . . . . . . . . . . . . . . . . . . . . xvi

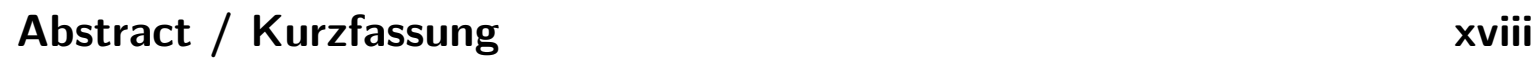

$\begin{array}{ll}1 . \text { Einleitung } & 1\end{array}$

2. Stand der Technik solarthermischer Kraftwerke 17

2.1. Technologien zur Stromerzeugung mit CSP . . . . . . . . 7

2.1.1. Linienfokussierende Systeme . . . . . . . . . . . . 7

2.1.2. Punktfokussierende Systeme . . . . . . . . . . . . . 9

2.2. Komponenten eines Solarturmkraftwerkes . . . . . . . . . 10

2.2.1. Heliostatenfeld . . . . . . . . . . . . . . . 10

2.2.2. Turm . . . . . . . . . . . . . . . . . 11

2.2.3. Receiver . . . . . . . . . . . . . . . . . 11

2.2.4. Energiespeicher . . . . . . . . . . . . . . . . 14

2.2.5. Kraftwerksblock . . . . . . . . . . . . . . . . 14

2.3. Meilensteine der Salzturmtechnologie . . . . . . . . . . 15

2.3.1. Gemasolar (2011) . . . . . . . . . . . . . . . . . . . . . . . . . . . 15

$2.3 .2 . \quad$ Crescent Dunes . . . . . . . . . . . . . . . . 16

3. Eigenschaften und Anwendungen von Flüssigmetallen 19

3.1. Bisherige Anwendungen von Flüssigmetallen . . . . . . . . . 20

3.1.1. Kernreaktortechnik . . . . . . . . . . . . . . 20

3.1.2. Auslassventile in Verbrennungsmotoren . . . . . . . . . 21

3.1.3. CPU-Kühler für PCs . . . . . . . . . . . . . . . . . 21 
3.1.4. Flüssigmetallbatterien . . . . . . . . . . . . . . . . 21

3.2. Flüssigmetalle in Solarkraftwerken . . . . . . . . . . . . . . . . 21

3.2.1. Sandia CRTF (Central Receiver Test Facility)[24] . . . . 22

3.2.2. CRS-Natriumreceiver auf der PSA . . . . . . . . . . 23

3.2.3. Jemalong Solar Thermal Station. . . . . . . . . . . . 27

3.3. Potenzielle Flüssigmetalle in Solarkraftwerken . . . . . . . . . . 29

3.4. Eigenschaften der Flüssigmetalle als WTM . . . . . . . . . . . . 31

3.4.1. Thermohydraulische Eigenschaften . . . . . . . . . . 32

3.4.2. Wärmestromdichtelimit. . . . . . . . . . . . . . . . 33

3.4.3. Receivergeometrie, Druckverlust und Wärmeübergang . . 39

3.4.4. Begleitheizung, Korrosion und Lebensdauer . . . . . . . 48

3.4.5. Einfrier- und Auftauvorgänge im Receiver . . . . . . . . 51

3.4.6. Förderung und Messtechnik . . . . . . . . . . . . . 54

3.4.7. Reaktivität und Toxizität . . . . . . . . . . . . 55

3.4.8. Erfahrung und Sicherheit. . . . . . . . . . . . . . 56

3.4.9. Verfügbarkeit und Kosten . . . . . . . . . . . . 57

4. Kraftwerkskonzepte und -komponenten für Flüssigmetallreceiver 59

4.1. Einteilung der Kraftwerkskonzepte für Flüssigmetallreceiver . 59

4.1.1. Anbindung an eine Dampfturbine . . . . . . . . . . . 59

4.1.2. Anbindung an eine Gasturbine. . . . . . . . . . . . . 61

4.1.3. AMTEC - Alkali Metal Thermal to Electric Converter . 62

4.1.4. LM MHD Generator - Liquid Metal Magnetohydrodynamic Generator . . . . . . . . . . . . . . . . . 63

4.1.5. Flüssigmetalldampfturbine . . . . . . . . . . . . . . . 64

4.2. Thermische Energiespeicher für Flüssigmetallsysteme . . . . . . 64

4.2.1. Modellierung der thermischen Speicherkonzepte . . . . . 65

4.2.2. Ergebnisse und Vergleich mit Literaturdaten . . . . . . . 66

4.3. Elektromagnetische Pumpen (EMP) und Wärmeübertrager . . . 69

4.3.1. Konduktionspumpe . . . . . . . . . . . . . . . . . . . . . 69

4.3.2. Induktionspumpe . . . . . . . . . . . . . . . 70

4.3.3. Wärmeübertrager mit Flüssigmetallen . . . . . . . . 70 
\begin{tabular}{ll} 
5. Receivermodellierung ASTRID & \\
\hline
\end{tabular}

5.1. Analytische Vorauslegung von Rohrreceivern . . . . . . . . . 74

5.1.1. Funktionsumfang und Programmstruktur. . . . . . . . . 74

5.1.2. Berechnung der Receivergeometrie . . . . . . . . . . 82

5.1.3. Thermohydraulische Berechnung . . . . . . . . . 85

5.1.4. Berechnung der Receiverkosten . . . . . . . . . . . . 89

5.2. Thermische Receivermodellierung mit FEM . . . . . . . . 92

5.2.1. Geometrieerstellung und Netzgenerierung . . . . . . . . 93

$5.2 .2 . \quad$ Randbedingungen . . . . . . . . . . . . . . . . . 95

5.2.3. Temperaturregelung und Teillastverhalten . . . . . . . 98

5.2.4. Netzstudie . . . . . . . . . . . . . . . . . . . . 100

5.3. Validierung des ASTRID-Receivermodells. . . . . . . . . . . 102

5.3.1. Validierung mit CFD-Modell. . . . . . . . . . . . . . 102

5.3.2. SOLUGAS-Projekt . . . . . . . . . . . . . 105

5.3.3. SolarTwo-Projekt . . . . . . . . . . . . . . . . . . . 109

6. Konzeptauswahl, Bewertungsmethodik und Spezifikation 117

6.1. Übergeordnete Annahmen . . . . . . . . . . . . . . . . . . 117

6.1.1. Basiskonzept und Leistungsklasse . . . . . . . . . . 118

6.1.2. Kraftwerksblock . . . . . . . . . . . . . . . . 122

6.1.3. Standort und Auslegungszeitpunkt . . . . . . . . . . . 124

6.1.4. Heliostatenfeldauslegung . . . . . . . . . . . . . . . . 124

6.1.5. Receivermodellierung und Wärmeträgermedium . . . . . 126

6.1.6. Leitungssystem und thermischer Energiespeicher. . . . . 127

6.2. Bewertungsmethodik und LCOE-Berechnung . . . . . . . . . 130

6.2.1. Systemmodellierung und Jahresertragsberechnung . . . . 133

6.2.2. Kostenmodelle. . . . . . . . . . . . . . . . . 133

7. Vergleichsstudie Mehrturmsystem (5x140 MW) 141

7.1. Ergebnisse der Komponentenauslegung . . . . . . . . . . . . 141

7.1.1. Heliostatenfeldauslegung . . . . . . . . . . . . . . . 141

7.1.2. Receivermodellierung . . . . . . . . . . . . . . 146

7.2. Ergebnisse der LCOE-Berechnung . . . . . . . . . . . . . . . 149

7.2.1. Solar Salt im Vergleich zu Natrium . . . . . . . . . . . . 149

7.2.2. Sensitivitätsanalyse des Feldwirkungsgrades . . . . . . 152 
7.2.3. Solar Salt im Vergleich zu Blei-Bismut . . . . . . . . . . 154

7.2.4. Kostenanalyse der Wärmeträgermedien . . . . . . . . . . 155

8. Vergleichsstudie Einturmsystem (700 MW-Receiver) 159

8.1. Ergebnisse der Komponentenauslegung . . . . . . . . . . . 159

8.1.1. Heliostatenfeldauslegung . . . . . . . . . . . . . . . 159

$8.1 .2 . \quad$ Receivermodellierung . . . . . . . . . . . . . . . . . 162

8.2. Ergebnisse der LCOE-Berechnung . . . . . . . . . . . . . . 168

8.3. Vergleich von Einturm- u. Mehrturmsystemen . . . . . . . . . . 172

\begin{tabular}{ll}
\hline Zusammenfassung und Ausblick & 173
\end{tabular}

\begin{tabular}{ll}
\hline A. Stoffwerte & 177
\end{tabular}

B. Spannungsuntersuchung des Auftauvorgangs eines mit Natrium befüllten Rohres 181

$\begin{array}{ll}\text { C. Berechnung der Rohrwandstärken } & 183\end{array}$

\begin{tabular}{|ll}
\hline D. Druckverlustberechnung im Receiver & 185
\end{tabular}

\begin{tabular}{ll}
\hline E. Verwendete Nusselt-Korrelationen & 187
\end{tabular}

F. Receivermodell 502 in HFLCAL 189

G. Spezifikationstabellen der Mehrturmsysteme 191

G.1. Übersicht der Konfigurationen . . . . . . . . . . . . . . . . . . . 191

G.2. Konzeptskizzen . . . . . . . . . . . . . . . . . . . . . . 192

G.3. Standort und Auslegungspunkt . . . . . . . . . . . . . . . 198

G.4. Spezifikation Referenzsystem Solar Salt und Rec-only mit Natrium 199

G.5. Spezifikation Refernezsystem Solar Salt und Receiver-only mit LBE . . . . . . . . . . . . . . . . . . . 210

G.6. Spezifikation Referenzsystem Solar Salt und Tower-loop mit Natrium . . . . . . . . . . . . . . . . . . . 213

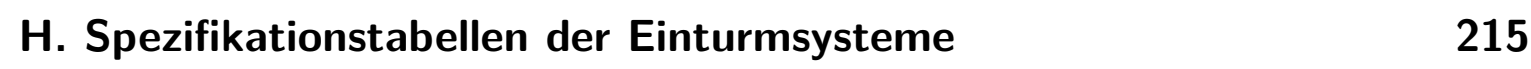

H.1. Übersicht der Konfigurationen . . . . . . . . . . . . . . . 215

H.2. Standort und Design Point . . . . . . . . . . . . . . . . . 216 
H.3. Spezifikation Referenzsystem Solar Salt und Rec-only mit Natrium 217 H.4. Spezifikation Referenzsystem Solar Salt und Tower-loop mit Natrium . . . . . . . . . . . . . . . . . . . . . . . . 229 



\section{Nomenklatur und Verzeichnisse}

\section{Abkürzungen}

ANSYS ANalysis SYStem (FEM-Software)

MatLab MATrix LABoratory (Software von MathWorks zur numerischen Berechnung mithilfe von Matrizen)

AC Wechselstrom (Alternating current)

ALIP Annual Linear Induction Pump

AMTEC Alcali Metal Thermal to Electric Converter

APDL Ansys Parametric Design Language

ASR Advanced Sodium Receiver (Natrium-Receiver Experiment auf der PSA)

ASTRID Advanced Solar Tubular ReceIver Design

CFD Computational Fluid Dynamics (Numerische Strömungsmechanik)

CIFT Contactless Inductive Flow Tomography, Berührungslose Induktive Strömungstomographie

CRS Central Receiver System (Solarturmsystem)

CRTF Central Receiver Test Facility (Teststandort für Turmreceiver von Sandia in Albuquerque, New Mexico)

CSP Concentrated Solar Power (Konzentrierte Solarenergie)

DC Gleichstrom (Direct current)

DCHX Direct Contact Heat Exchanger (Direktkontaktwärmeübertrager)

DNI Direct Normal Irradiation (Direktstrahlung)

DP Design Point (Auslegungszeitpunkt, oft 21.3. um $12 \mathrm{~h}$ )

DSG Direct Steam Generation (Direktdampferzeugung) 
EMP Elektromagnetische Pumpe

EPC Engineering-Procurement-Construction, zu Deutsch: DetailPlanung und Kontrolle, Beschaffungs, Bau- und Montage (Schlüsselfertige Übergabe einer Anlage)

FD Frischdampf

FEM Finite Element Methode

FEMRAY APDL-Makro, welches über SPRAY die Wärmestromdichteverteilung auf der Receiveroberfläche ermittelt und direkt auf das FEM-Modell aufbringt

FLIP Flat Linear Induction Pump

FM Flüssigmetall

HD Hochdruck

HFLCAL Heliostat Field Calculation (Software zur Auslegung und Optimierung von Heliostatenfeldern. Es stehen verschiedene Optimierungsalgoritmen zur Verfügung, die anhand der jährlichen Wärmegestehung die Feldaufstellung optimieren)

IEA International Energy Agency (Internationale Energieagentur)

KIT Karlsruher Institut für Technologie

LBE Lead-Bismuth-Eutectic (Blei-Bismut-Eutektikum)

LCOE Levelized Cost of Electricity

LKA Lorentzkraft-Anemometrie (engl.: LFV = Lorentz Force Velocimetry)

LM Liquid metal

LMFR Liquid Metal Fast Reaktor (Liquid metal cooled nuclear reactor $=$ Flüssigmetallgekühlter Reaktor)

MHD Magnetohydrodynamik, Wechselwirkung von Strömungen elektrisch leitfähiger Fluide mit elektromagnetischen Feldern

$\mathrm{Na} \quad$ Natrium

ND Niederdruck

NREL National Renewable Energy Laboratory

O\&M Betrieb und Instandhaltung (Operation and Maintenance)

PSA Plataforma Solar de Almería (Solartestzentrum in Andalusien, Spanien)

PV Photovoltaik 
SEGS Solar Energy Generation Systems

SOLUGAS SOLar Up-scale GAS Turbine System

SPRAY Solar Power Raytracing Tool, Programm zur Strahlverfolgung und Bestimmung der Wärmestromdichteverteilung

SS Solar Salt

SSPS Small Solar Power Systems

WTM Wärmeträgermedium

ZÜ Zwischenüberhitzung

\section{Formelzeichen}

$\begin{array}{lll}A & {\left[\mathrm{~m}^{2}\right]} & \text { Fläche } \\ C & {[-]} & \text { Konzentrationsfaktor der Solarstrahlung } \\ D & {[\mathrm{~m}]} & \text { Durchmesser } \\ E & {[\mathrm{Wh}]} & \text { Energie } \\ F C R & {[-]} & \text { Annuitätenfaktor (Fixed charge rate) } \\ H & {[\mathrm{~m}]} & \text { Höhe } \\ K & {[€]} & \text { Absolute Kosten } \\ L & {[\mathrm{~m}]} & \text { Länge } \\ N u & {[-]} & \text { Nusselt-Zahl } \\ P e & {[-]} & \text { Peclet-Zahl } \\ P r & {[-]} & \text { Prandtl-Zahl } \\ P & {[\mathrm{~W}]} & \text { Leistung } \\ \operatorname{Re} & {[-]} & \text { Reynolds-Zahl } \\ R \text { f } & {\left[\mathrm{m}^{2} \mathrm{~K} / \mathrm{W}\right]} & \text { Fouling factor, berücksichtigt Verunreinigungen } \\ & & \text { an wärmeübertragenden Flächen } \\ S M & {[-]} & \text { Solarvielfaches (engl.: Solar multiple): Faktor } \\ & & \text { zur Überdimensionierung des Heliostatenfeldes } \\ & & \text { bezüglich der Kraftwerksleistung } \\ T & {\left[{ }^{\circ} \mathrm{C}\right]} & \text { Temperatur } \\ \Delta T & {\left[{ }^{\circ} \mathrm{C}\right]} & \text { Temperaturdifferenz } \\ \Delta p & {[\mathrm{~Pa}]} & \text { Druckverlust } \\ \alpha & {\left[\mathrm{W} / \mathrm{m}^{2} / \mathrm{K}\right]} & \text { Konvektiver Wärmeübergangskoeffizient } \\ \delta & {\left[{ }^{\circ}\right]} & \text { Diffusoröffnungswinkel } \\ & & \end{array}$




\begin{tabular}{|c|c|c|}
\hline$\dot{Q}$ & {$[\mathrm{~W}]$} & Receiverleistung \\
\hline$\dot{V}$ & {$\left[\mathrm{~m}^{3} / \mathrm{s}\right]$} & Volumenstrom \\
\hline$\dot{m}$ & {$[\mathrm{~kg} / \mathrm{s}]$} & Massenstrom \\
\hline$\dot{q}$ & {$\left[\mathrm{~W} / \mathrm{m}^{2}\right]$} & Wärmestromdichte \\
\hline$\epsilon$ & {$[-]$} & Absorptions- bzw. Emissionsgrad \\
\hline$\eta$ & {$[-]$} & Wirkungsgrad \\
\hline$\eta$ & {$[\mathrm{Pa} \mathrm{s}]$} & Dynamische Viskosität \\
\hline$\gamma$ & {$[-]$} & Fit-Parameter \\
\hline$\lambda$ & {$[\mathrm{W} / \mathrm{m} / \mathrm{K}]$} & Wärmeleitfähigkeit \\
\hline$\mu$ & {$[\mathrm{mm}]$} & Rauheit \\
\hline$\nu$ & {$\left[\mathrm{m}^{2} / \mathrm{s}\right]$} & kinematische Viskosität \\
\hline$\rho$ & {$\left[\mathrm{kg} / \mathrm{m}^{3}\right]$} & Dichte \\
\hline$\sigma_{\mathrm{SB}}$ & {$\left[\mathrm{W} / \mathrm{m}^{2} / \mathrm{K}^{4}\right]$} & Stefan-Boltzmann-Konstante: $5.6710^{-8} \mathrm{~W} / \mathrm{m}^{2} / \mathrm{K}^{4}$ \\
\hline$\sigma_{\text {slope }}$ & [rad] & $\begin{array}{l}\text { Winkelfehler der Heliostaten: Abweichung von } \\
\text { idealer Spiegelfläche }\end{array}$ \\
\hline$\sigma_{\text {tracking }}$ & [rad] & Winkelfehler der Heliostaten um x- und y-Achse \\
\hline$\zeta$ & {$[-]$} & Druckverlustbeiwert \\
\hline$b$ & {$[\mathrm{~m}]$} & Breite \\
\hline$c_{p}$ & {$[\mathrm{~J} / \mathrm{K}]$} & Spezifische Wärmekapazität \\
\hline$c$ & {$[\mathrm{~h}]$} & Speicherkapazität \\
\hline$d$ & {$[\mathrm{~m}]$} & Durchmesser \\
\hline$i_{\mathrm{r}}$ & {$[-]$} & Zinssatz (Interest rate) \\
\hline$k$ & {$[€ /]$} & Spezifische Kosten \\
\hline$m$ & {$[\mathrm{~kg}]$} & Masse \\
\hline$n$ & {$[-]$} & Anzahl \\
\hline$p$ & {$[\mathrm{~Pa}]$} & Druck \\
\hline$s$ & {$[\mathrm{~m}]$} & Rohrwandstärke \\
\hline$u$ & {$[\mathrm{~m} / \mathrm{s}]$} & Strömungsgeschwindigkeit \\
\hline$z$ & {$[\mathrm{~m}]$} & Abstand \\
\hline
\end{tabular}




\section{Indizes}

\begin{tabular}{|c|c|}
\hline $\mathbf{a}$ & Absorberrohr außen \\
\hline amb & Umgebung (engl. ambient) \\
\hline ap & Apertur \\
\hline as & Sammler außen \\
\hline assy & Montage \\
\hline aus & Receiverauslass \\
\hline av & Verteiler außen \\
\hline bin & Diskretisierungsabschnitt \\
\hline bulk & Mitteltemperatur (engl. bulk) \\
\hline cav & Kavität bzw. Rückwand bei externen Receiver (engl. cavity) \\
\hline comp & Dehnungskompensator, Metalbalg \\
\hline conv & Konvektion (engl. convection) \\
\hline d & in Rohrdickenrichtung \\
\hline dif & Diffusor \\
\hline drill & Bohrung \\
\hline ein & Receivereinlass \\
\hline el & Elektrisch \\
\hline field & Heliostatenfeld \\
\hline film & Filmtemperatur, (an Flüssigkeitsoberfläche) \\
\hline ges & Gesamt- \\
\hline HX & Wärmeübertrager (engl. heat exchanger) \\
\hline i & Absorberrohr innen \\
\hline inc & Einfallend (engl. incident) \\
\hline inv & Investment \\
\hline ir & Infrarot \\
\hline iso & Dämmung \\
\hline jahr & jährlich \\
\hline 1 & in Rohrlängsrichtung \\
\hline loss & Verlust \\
\hline $\max$ & Maximalwert \\
\hline $\min$ & Minimalwert \\
\hline $\bmod$ & Modul \\
\hline
\end{tabular}




$\begin{array}{ll}\mathbf{o p t} & \text { optisch } \\ \mathbf{p} & \text { Pumpe } \\ \mathbf{p a r} & \text { parasitär } \\ \mathbf{P B} & \text { Kraftwerksblock (engl. power block) } \\ \mathbf{r} & \text { Absorberrohr } \\ \mathbf{r e c} & \text { Receiver } \\ \mathbf{s} & \text { Sammler } \\ \mathbf{s o l} & \text { Solar } \\ \mathbf{s t} & \text { Speicher (engl. storage) } \\ \mathbf{s t r} & \text { Strömungswege } \\ \mathbf{s t r u c t} & \text { Tragwerk, Struktur } \\ \mathbf{s y s} & \text { System } \\ \text { th } & \text { Thermisch } \\ \mathbf{u} & \text { in Rohrumfangsrichtung } \\ \mathbf{v} & \text { Verteiler } \\ \text { wa } & \text { Absorberrohraußenseite } \\ \text { weld } & \text { Schweißnaht } \\ \text { wi } & \text { Absorberrohrinnenseite }\end{array}$




\section{Abstract}

Today solar power plants with central receiver technology often use molten nitrate salts like Hitec or Solar Salt as heat transfer fluid and storage medium. Due to their high heat capacity and the low cost these are well suited for thermal energy storage. Nevertheless, these salts also inherit disadvantages, such as high melting points above $220^{\circ} \mathrm{C}$ demanding high energy for trace heating. The upper temperature limit of Solar Salt is at $565{ }^{\circ} \mathrm{C}$. Therefore, high temperature power conversion cycles with high efficiency are unfeasible. Additionally, its high density results in a high pressure drop in the riser, leading to additional parasitic losses. In plants operating with Solar Salt, freeze events and corrosion problems occur. Moreover, high pressure drops have to be accepted in the absorber tubes to achieve a reasonable heat transfer.

In all the mentioned points liquid metals possess advantages compared to molten salts. Sodium is the most often used liquid metal in research and industry and was already tested at the solar test center PSA in Almería in Spain. The only disadvantage of sodium - its reactivity with water and oxygen - was demonstrated in a sodium fire and the eventual destruction of the test center in summer 1986. During the last 30 years measurement techniques and safety precautions were developed to avoid such accidents.

The present work analyses the properties of liquid metals in detail and compares them with Solar Salt. The custom-built design and simulation tool for tubular receivers ASTRID makes a precise thermohydraulic calculation possible. The assessment of the liquid metal concepts is based on annual yields and LCOE calculations, which are compared to a reference system with Solar Salt. All concepts with liquid metals use electromagnetic pumps. After the solar heating in the receiver the heat is transferred from sodium to Solar Salt in a heat exchanger and then stored in a two-tank storage. Both the reference concept and the liquid metal concepts use the same power block and the same temperatures in storage and turbine.

The results indicate a potential to reduction in LCOE with sodium of up to $16 \%$ compared to the reference system with Solar Salt. 


\section{Kurzfassung}

In aktuellen Solarturmkraftwerken zur Stromerzeugung kommen als Wärmeträgerund Speichermedium häufig flüssige Nitratsalze wie Solar Salt zum Einsatz. Aufgrund ihrer hohen Wärmekapazität und den geringen Kosten eignen sie sich sehr gut zur thermischen Energiespeicherung, jedoch bringen sie auch einige Nachteile mit sich. Der nutzbare Temperaturbereich in der Flüssigphase $\left(220-565{ }^{\circ} \mathrm{C}\right)$ führt einerseits zu hohen Energieverlusten durch Begleitheizung (zu Zeiten von zu schwacher Einstrahlung), andererseits lassen sich dadurch effiziente Hochtemperaturprozesse nicht realisieren. Die hohe Dichte führt außerdem zu einem hohen Druckverlust in der Steigleitung wodurch zusätzliche parasitäre Verluste entstehen. Beim Betrieb mit Solar Salt treten Probleme durch Einfrieren und Korrosion auf. Im Receiver müssen ferner hohe Druckverluste in Kauf genommen werden, um einen akzeptablen Wärmeübergang zu erreichen.

Flüssigmetalle weisen in allen genannten Punkten Vorteile gegenüber den Flüssigsalzen auf. Das in Forschung und Industrie am weitesten verbreitete Flüssigmetall Natrium wurde bereits auf der Solartestplattform PSA in Almería in Spanien getestet. Der einzige Nachteil von Natrium - die Reaktivität mit Wasser und Sauerstoff - manifestierte sich in einem Natriumbrand und der Zerstörung der Anlage im Sommer 1986.

In der vorliegenden Arbeit werden die Eigenschaften von Flüssigmetallen detailliert analysiert und mit Solar Salt verglichen. Das speziell für Rohrreceiver entwickelte Auslegungs- und Simulationsprogramm ASTRID ermöglicht die genaue thermohydraulische Berechnung. Zur Bewertung der Flüssigmetallkonzepte werden die Jahreserträge bzw. die Stromgestehungskosten (LCOE) herangezogen und mit einem Referenzsystem mit Solar Salt verglichen. Alle Konzepte mit Flüssigmetallen verwenden elektromagnetische Pumpen. Nach der solaren Aufheizung im Receiver wird die Wärme in einem Wärmeübertrager auf Flüssigsalz übertragen und in einem 2-Tank-Speicher gespeichert. Sowohl das Referenzkonzept als auch die Flüssigmetallkonzepte wurden alle mit dem gleichen Kraftwerksblock und den gleichen Temperaturniveaus in Speicher und Dampfturbine ausgelegt.

Die Ergebnisse zeigen ein Potenzial zur Reduktion der Stromgestehungskosten mit Natrium um bis zu $16 \%$ gegenüber dem Referenzsystem mit Solar Salt. 


\section{Einleitung}

Seit Beginn der technischen Nutzung des elektrischen Stromes Ende des 19. Jahrhunderts ist dieser nicht mehr aus unserem Leben wegzudenken. Die Stromnetze werden stetig weiter ausgebaut und der Verbrauch steigt kontinuierlich an (siehe Abbildung 1.1). Diese treibende Kraft der Weltwirtschaft basiert nach wie

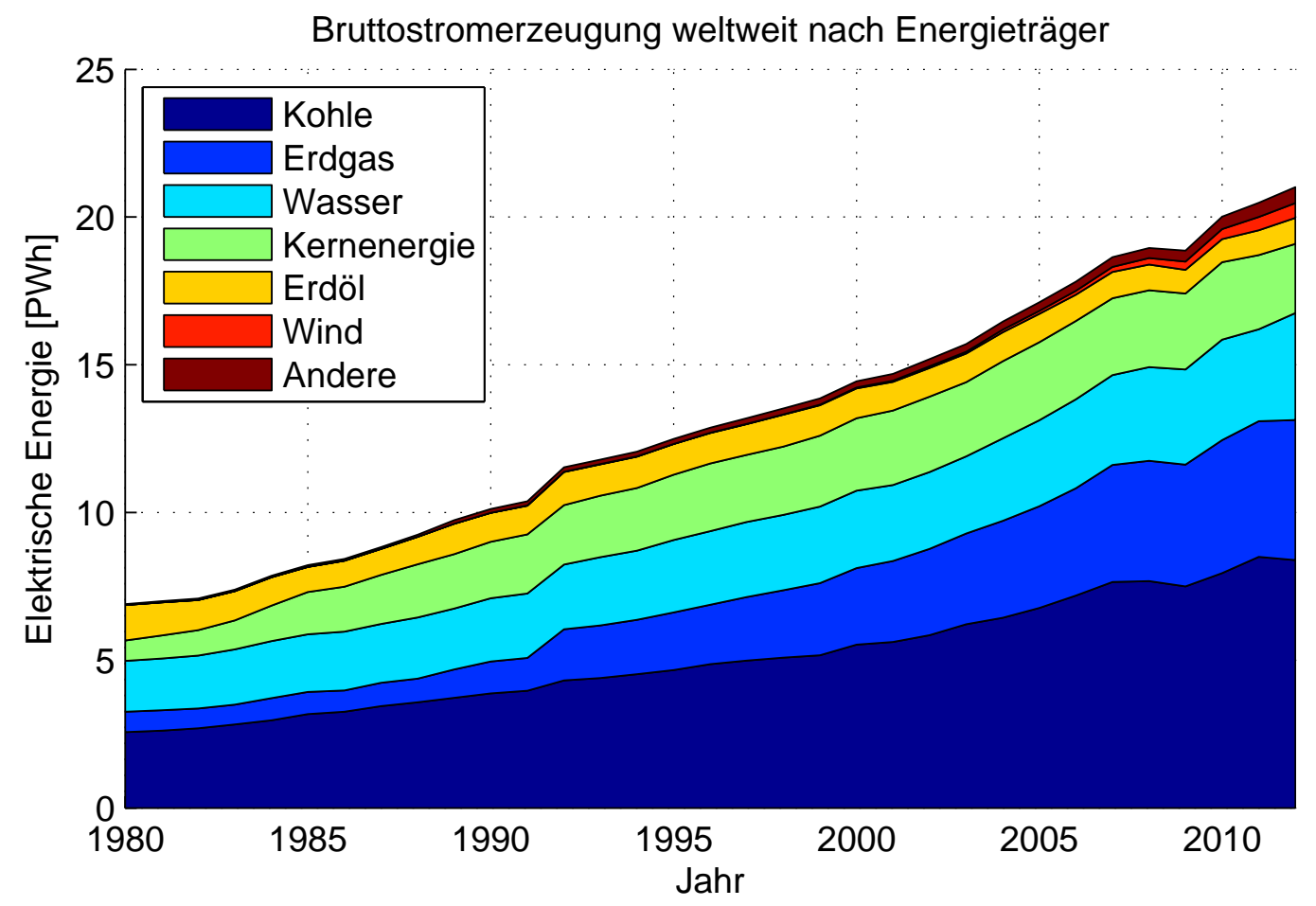

Abbildung 1.1.: Bruttostromerzeugung weltweit seit 1980, aufgeschlüsselt nach Energieträger 1].

vor überwiegend auf fossilen Energieträgern. Das Vorkommen dieser Rohstoffe ist jedoch limitiert. Allein in Deutschland werden über $70 \%$ der elektrischen Energie aus Kohle, Gas und Kernenergie erzeugt (BMWi-Statisik [2] von 2013). Neben der hochgradigen Abhängigkeit von diesen endlichen, fossilen Energieträgern resultieren daraus auch zunehmend Umweltprobleme und politische 
Auseinandersetzungen bis hin zu Kriegen. Aus diesen Gründen rücken die erneuerbaren Energien immer mehr in den Fokus von Politik und Wirtschaft. So hat Deutschland und die Europäische Union beschlossen, den Anteil aller erneuerbarer Energien (Strom, Wärme und Kraftstoffe) am Brutto-Endenergieverbrauch in der Staatengemeinschaft bis zum Jahr 2020 auf $20 \%$ anzuheben [3]. Langfristig muss sowohl die Effizienz gesteigert als auch der Verbrauch reduziert werden, um eine nachhaltige Versorgung zu ermöglichen. Abbildung 1.2 zeigt die Stromgestehungskosten verschiedener Technologien.

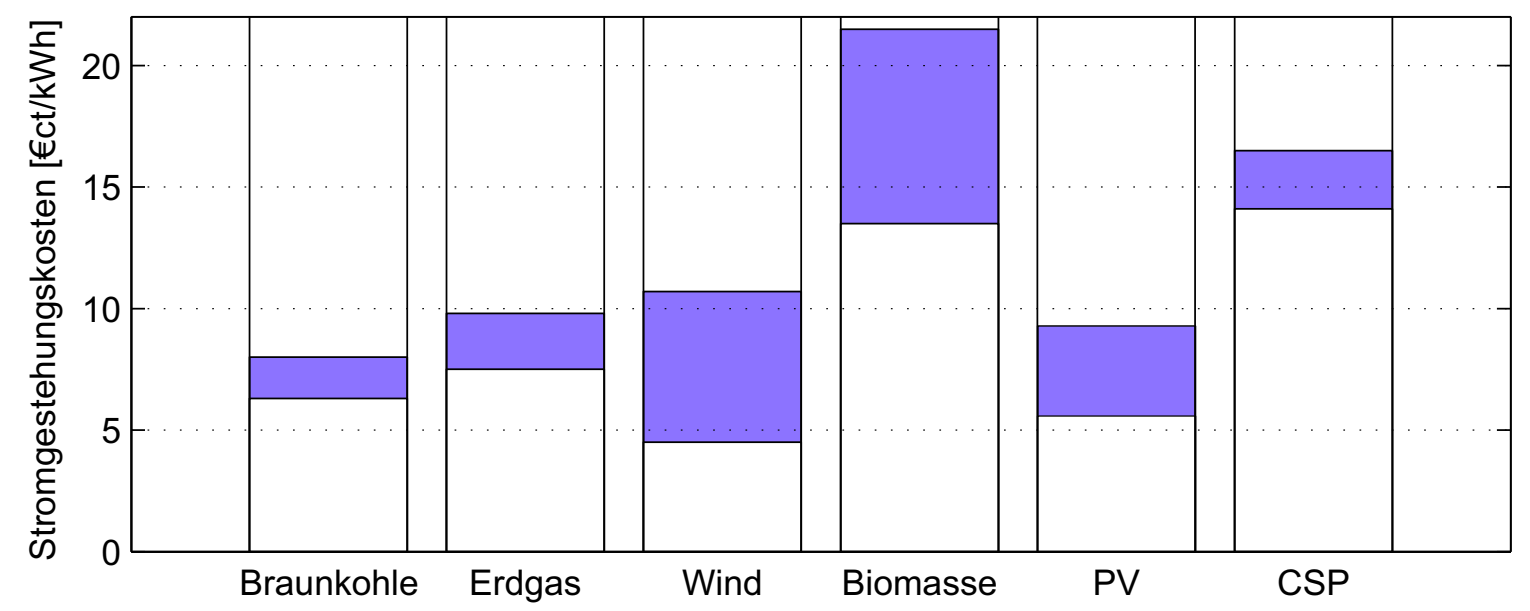

Abbildung 1.2.: Stromgestehungskosten für erneuerbare Energien und konventionelle Kraftwerke an Standorten in Deutschland im Jahr 2013 (außer CSP). Bei PV: solare Globaleinstrahlung von $1000 \mathrm{kWh} / \mathrm{m}^{2} \mathrm{a}$. Bei CSP: sonnenreicher Standort mit hoher solaren Direkteinstrahlung $\left(2500 \mathrm{kWh} / \mathrm{m}^{2} \mathrm{a}\right)$. Daten nach $[4$.

Solarthermische Kraftwerke (CSP) gehören zur Gruppe der erneuerbaren Energien. Ihr größter Vorteil ist die Möglichkeit der zeitlichen Entkopplung von solarer Einstrahlung und Stromerzeugung. Durch einen thermischen Speicher können derartige Kraftwerke auch nachts bzw. je nach Bedarf Strom erzeugen! Der zunehmende Ausbau von Windenergie und Photovoltaik (PV) führt zu stärkeren Fluktuationen in der Stromerzeugung, da diese Technologien jeweils nur bei entsprechenden Wetterbedingungen Strom erzeugen können. Solarthermische Kraftwerke mit Speicher bieten hier die ideale Ergänzung, da sie Grundlastund Regelenergie erzeugen, die die Schwankungen von Windenergie und PV ausgleichen und zur Netzstabilisierung beitragen. Beim Vergleich verschiedener

\footnotetext{
${ }^{1}$ Gemasolar als erstes Solarkraftwerk mit $24 \mathrm{~h}$ kontinuierlicher Stromproduktion $[5]$.
} 
Technologien reicht eine Bewertung rein auf Basis der Stromgestehungskosten (LCOE) folglich nicht aus. Diese Arbeit befasst sich jedoch ausschließlich mit Solarturmsystemen, weshalb ein Vergleich verschiedener Konzepte innerhalb dieser Gruppe anhand der LCOE zulässig ist.

Aktuelle Solarturmkraftwerke nutzen häufig flüssiges Nitratsalz (Solar Salt) als Wärmeträgermedium (WTM). Der große Vorteil dieses Flüssigsalzes ist die hohe Energiedichte, wodurch es sich gleichzeitig als Speichermedium eignet. Jedoch unterliegt dieses Salz auch einigen Beschränkungen, wie beispielsweise dem begrenzten Temperaturbereich, in dem es genutzt werden kann. Das obere Temperaturlimit ist bei Solar Salt auf $565^{\circ} \mathrm{C}$ limitiert, da bei höheren Temperaturen der chemische Zersetzungsprozess beginnt. Flüssigmetalle hingegen sind auch bei hohen Temperaturen chemisch stabil.

Ziel der Forschung im Bereich der CSP ist stets die Reduktion der Stromgestehungskosten. Dies kann einerseits durch geringere Komponentenkosten erreicht werden, oder andererseits durch einen höheren Systemwirkungsgrad. Der Wechsel des WTMs von Flüssigsalz auf Flüssigmetalle setzt an beiden Punkten zur Kostensenkung an.

Flüssigmetalle zeichnen sich im Vergleich zu dem bisher verwendeten Salz durch eine um den Faktor 100-200 höhere Wärmeleitfähigkeit aus. Durch die damit verbundenen sehr hohen Wärmeübergangskoeffizienten kann die Wärme effektiver abgeführt werden. Höhere Wärmestromdichten können zugelassen werden, was bei gleicher Receiverleistung einer Verringerung der Absorberfläche entspricht. Ein vorhergehender Vergleich zwischen Natrium und dem Flüssigsalz Hitec schätzt die Verringerung der Absorberfläche durch Natrium auf $57 \%$ [6]. Die geringere Absorberfläche spiegelt sich nicht nur in den geringeren Receiverkosten wieder, sondern führt auch zu geringeren Wärmeverlusten durch Abstrahlung und Konvektion, wodurch der Receiverwirkungsgrad steigt.

Neben diesen Vorteilen besitzen die Flüssigmetalle aber auch einige Nachteile. Alkalimetalle reagieren bei Kontakt mit Wasser oder Sauerstoff exotherm. Derartige Brände können zu Explosionen führen und sind schwierig zu löschen. Die Frage, ob Flüssigmetalle zu einer gewünschten Kostensenkung von Solarkraftwerken führen hängt sehr stark vom Kraftwerkskonzept ab. Konventionelle Dampfprozesse arbeiten bei Temperaturen bis zu $580{ }^{\circ} \mathrm{C}$. Dampfprozesse mit höheren Temperaturen bringen mit dem aktuellen Stand der Technik kaum 


\section{Einleitung}

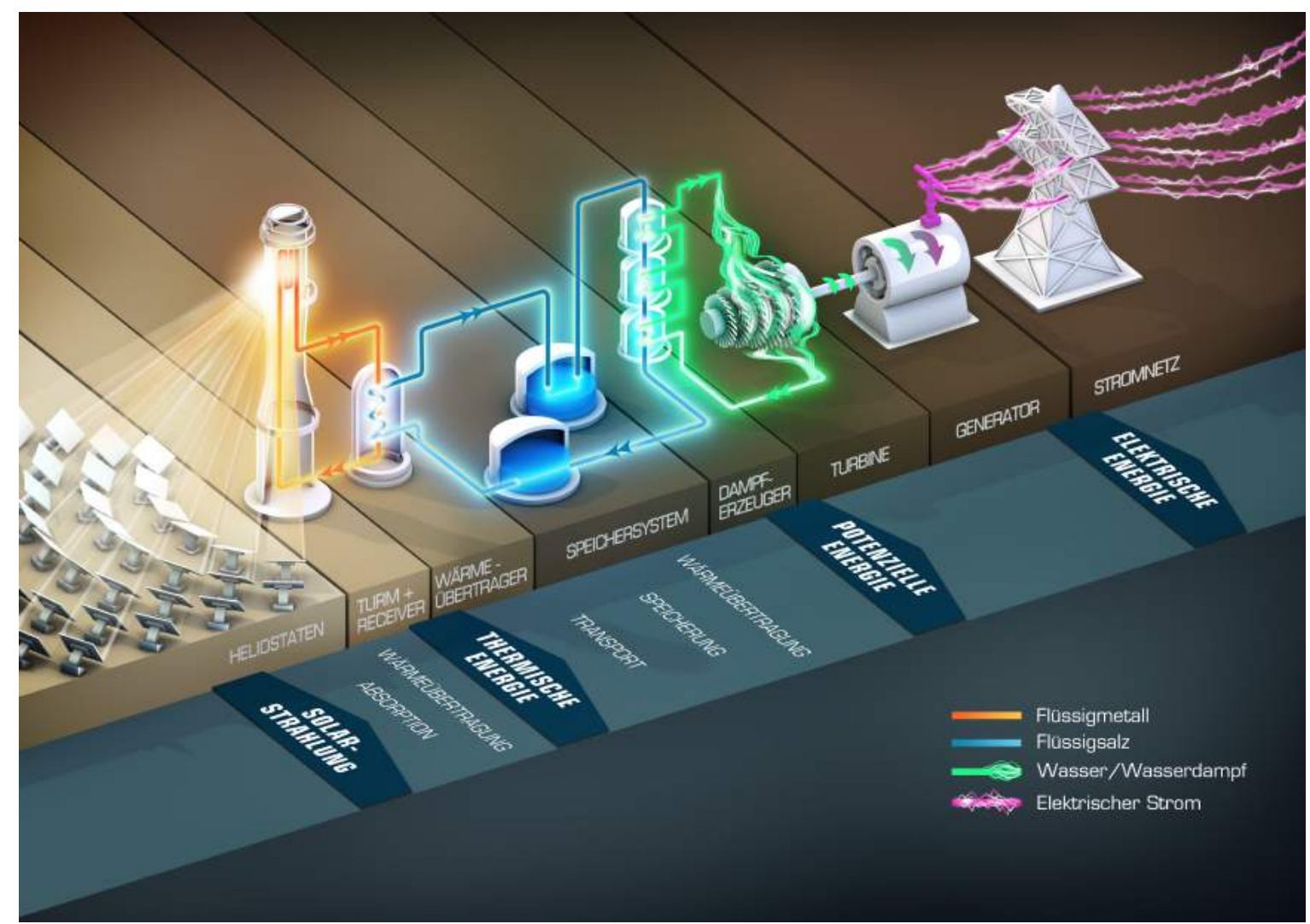

Abbildung 1.3.: Skizze des in dieser Arbeit untersuchten Kraftwerkskonzepts mit allen Komponenten (Heliostatenfeld, Flüssigmetallreceiver, Wärmeübertrager, 2-Tank-Salzspeicher, Dampfturbine und Generator

einen Kostenvorteil [7]. Gasturbinen versprechen höhere Wirkungsgrade, jedoch gibt es bisher keine kommerzielle Anlage mit solarer Wärmequelle. Die meisten in Frage kommenden Flüssigmetalle haben hohe Siedetemperaturen und daher das Potenzial für Hochtemperaturkraftwerksprozesse mit hohen Wirkungsgraden. In thermischen Solarkraftwerken mit derart hohen Temperaturen ist aber die Frage des Kraftwerkskonzepts und der Speichertechnologie noch offen. Daher beschränkt sich die vorliegende Arbeit auf konventionelle Dampfprozesse, was den direkten Vergleich zu Solarkraftwerken mit Flüssigsalztechnologie vereinfacht. Abbildung 1.3 zeigt schematisch das in dieser Arbeit untersuchte Kraftwerkskonzept. Die aus thermodynamischer Sicht hervorragenden Eigenschaften der Flüssigmetalle wurden schon in den 80er Jahren erkannt und in solaren Testanlagen umgesetzt. Das bekannteste Projekt ist das SSPS auf der Plataforma Solar de Almería (PSA). Hier wurden zwei verschiedene Natriumreceiver getestet. Jedoch ging das Kraftwerk bei unsachgemäß ausgeführten Wartungsarbeiten in Flammen auf und brannte ab. 
Seit dieser Zeit wurden Flüssigmetalle in der Solartechnik nicht mehr berücksichtigt. Jedoch wurden vor allem in der Nukleartechnik Flüssigmetalle weiterhin verwendet und erforscht. Mit dem dadurch erlangten Wissenszuwachs, der Erfahrung im Umgang mit Flüssigmetallen und neuer Mess- und Sicherheitstechnik können Fehler wie damals auf der PSA vermieden werden.

In der vorliegenden Arbeit wird das Potenzial von Flüssigmetallen in Solarturmkraftwerken detailliert analysiert und bewertet. Ein parametrisches Modell zur Auslegung und Optimierung von Rohrreceivern wurde speziell dafür entwickelt. Flüssigsalz- und Flüssigmetallsysteme können damit direkt miteinander verglichen werden. 



\section{Stand der Technik solarthermischer Kraftwerke}

\subsection{Technologien zur Stromerzeugung mit CSP}

Die Technologie der CSP umfasst sämtliche Systeme, bei denen das Licht der Sonne durch Spiegelsysteme konzentriert wird. Durch die geometrischen Verhältnisse von Erde und Sonne ergibt sich eine theoretische, maximale Konzentration auf der Erde von $C=46$ 200. Durch unvollständige Reflexion der Spiegel, Oberflächenfehler, Orientierungsfehler sowie der Reflexion und Emission am Absorber sind technisch maximale Konzentrationen zwischen $C=5000-8000$ realisierbar [8]. Nur die direkte Solarstrahlung (DNI) kann konzentriert werden. Daher sind Standorte am Äquator eher ungeeignet für die CSP, da durch die hohe Luftfeuchtigkeit sehr viel diffuses Licht entsteht. Optimale Standorte sind daher die subtropischen Gebiete mit großer Höhe über NN wie Südafrika, Australien, die Sahara, der Westen Nordamerikas oder die Anden. In Chile wird die höchste jährliche direkte Einstrahlung mit über $3800 \mathrm{kWh} / \mathrm{m}^{2}$ gemessen, wohingegen in Deutschland die jährliche Einstrahlung (DNI) stets unter $1000 \mathrm{kWh} / \mathrm{m}^{2}$ beträgt.

\subsubsection{Linienfokussierende Systeme}

Bei linienfokussierenden Systemen wird das Sonnenlicht auf eine Linie konzentriert (2D-Konzentration), in der ein Absorber angebracht ist. Meistens werden sie einachsig der Sonne nachgeführt. Typische technische Konzentrationsfaktoren sind $C=10-100$. Zu den linienfokussierende Systemen zählen Parabolrinnenkraftwerke und Fresnel-Kollektoranlagen 


\subsubsection{Parabolrinne}

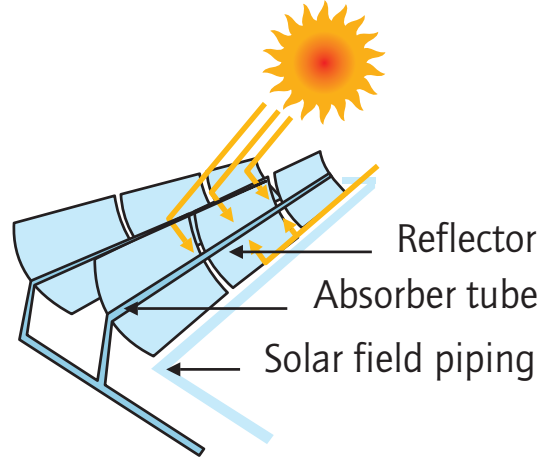

Abbildung 2.1.: Skizze einer Parabolrinne 9

Parabolrinnenkollektoren bestehen aus parabelförmigen Spiegeln, in deren Brennlinie ein Absorberrohr positioniert ist (siehe Skizze). Die Länge solcher Kollektoren variiert je nach Typ zwischen 20-150 m. Die Aperturweite beträgt zwischen 2.5-10 m. Die meisten Parabolrinnen werden nur einachsig der Sonne nachgeführt. Eins der bekanntesten Parabolrinnenkraftwerke ist das SEGS in Kalifornien, Amerika. Die installierte Gesamtleistung aller neun Blöcke beträgt $354 \mathrm{MW}_{\mathrm{el}}$ [10]. Parabolrinnenkraftwerke nehmen aktuell den größten Anteil an solarthermischen Kraftwerken ein. Als WTM kommen bisher Öle, Wasser/Dampf und Flüssigsalze zum Einsatz.

\subsubsection{Fresnel-Kollektoren}

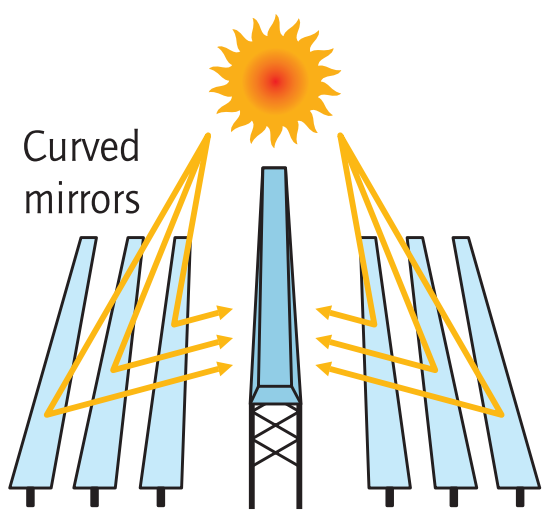

Absorber tube and reconcentrator

Eine weitere linienfokussierende Technologie sind Fresnel-Spiegel-Kollektoren. Nach dem Prinzip einer Fresnellinse werden hier mehrere flache Spiegelsegmente ebenerdig angeordnet. Sie reflektieren das Sonnenlicht auf einen Sekundärspiegel, in dessen Brennlinie sich das Absorberrohr befindet. Die ebenerdig angeordneten Spiegel werden einachsig der Sonne nachgeführt. Ein großer Vorteil gegenüber Parabolrinnenkollektoren ist, dass das Absorberrohr nicht mitbewegt wird und somit keine

Abbildung 2.2.: Skizze eines Fresnelhitze- und druckbeständigen Gelenke erforderkollektors [9] lich sind. Allerdings sind die Verluste durch Verschattung höher als bei der Parabolrinne.

Die bisher größte Fresnel-Kollektoranlage (Puerto Errado) wurde in Murcia, Spanien errichtet und hat eine installierte Leistung von $31.4 \mathrm{MW}_{\mathrm{el}}$. 


\subsubsection{Punktfokussierende Systeme}

Bei punktfokussierenden Systemen wird das Licht auf einen Punkt konzentriert (3D-Konzentration). Typische technische Konzentrationen liegen bei $C=100-$ 1000. Punktfokussierende Systeme werden stets 2-achsig der Sonne nachgeführt.

\subsubsection{Paraboloidkraftwerke}

Paraboloidkraftwerke oder auch Dish-StirlingSysteme besitzen einen Konzentratorspiegel in Form eines Paraboloids. Im Brennpunkt des Paraboloids befindet sich der Absorber. Derartige Systeme sind sehr kompakt und erzeugen sehr hohe Wärmestromdichten auf der Absorberoberfläche. In Odeillo (Frankreich) wurde mit einem Dish-Stirling (Aperturdurchmesser

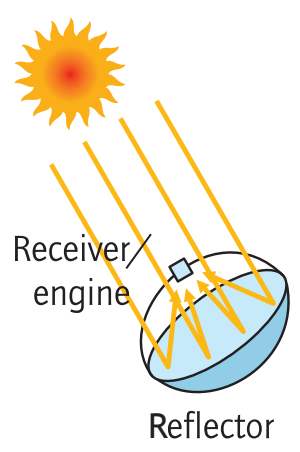
$8.5 \mathrm{~m}$ ) eine Nettoleistung von $9.2 \mathrm{~kW}_{\text {el }}$ erzeugt, was einem Wirkungsgrad von $16 \%$ entspricht. Derartige Kraftwerke eignen sich vor allem für

Abbildung 2.3.: Skizze eines Paraboloidkraftwerks $[9$ die dezentrale Energieversorgung in abgelegenen Regionen. Da eine Speicherung der thermischen Energie schwierig ist, sind Paraboloidkraftwerke derzeit nicht konkurenzfähig zu PV.

\subsubsection{Solarturmkraftwerke}

In einem Solarturmkraftwerk werden hunderte bis tausende Spiegel (sogenannte Heliostate) einzeln der Sonne nachgeführt, sodass das Sonnenlicht auf die Spitze eines Turmes konzentriert wird. Dort befindet sich der Receiver, der die Solarstrahlung absorbiert und an das WTM weitergibt. Große thermische Energiespeicher erlauben die ganztägige Stromproduktion (auch Nachts). Das bisher größte Solarturmkraftwerk (Ivanpah) wurde in der MojaveWüste in Kalifornien, Amerika errichtet und

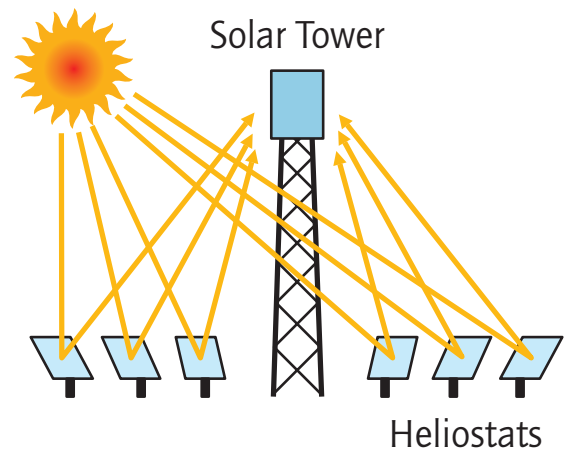

Abbildung 2.4.: Skizze eines Solarturmkraftwerks 9 besteht aus drei Türmen, 173500 Heliostate und hat eine Leistung von $392 \mathrm{MW}_{\mathrm{el}}$. 


\subsection{Komponenten eines Solarturmkraftwerkes}

In dieser Arbeit werden Solarturmkraftwerke behandelt. Die Umformung der solaren Strahlungsenergie der Sonne in elektrische Energie ist mit allen notwendigen Zwischenschritten in Abbildung 1.3 dargestellt. Die jeweils an der Umwandlung beteiligten Komponenten sind ebenfalls ersichtlich. In den folgenden Abschnitten werden diese Komponenten detailliert beschrieben.

\subsubsection{Heliostatenfeld}

Zur Konzentration der Solarstrahlung dienen die sogenannten Heliostate. Sie bestehen aus leicht gekrümmten Spiegelsegmenten und werden einzeln der Sonne nachgeführt, sodass die auftreffende Solarstrahlung stets auf den Receiver konzentriert wird. Dazu muss jeder Heliostat so ausgerichtet werden, dass die Normale der Spiegelfläche der Winkelhalbierenden des Winkels der beiden sich im Mittelpunkt des Spiegels kreuzenden Linien aus Einstrahlrichtung der Sonne und Zielpunkt auf dem Receiver entspricht. Je weiter ein Heliostat vom Turm entfernt ist, desto größer wird dieser Winkel wodurch die effektive Spiegelfläche (projizierte Fläche) abnimmt. Dieser Effekt ist proportional zum Kosinus des Einfallswinkels, weshalb sie auch als Kosinusverluste bezeichnet werden. Außerdem erfährt die reflektierte Strahlung eine atmosphärische Abschwächung zwischen Heliostat und Receiver. Um diese atmosphärischen Verluste zu minimieren wird versucht, die Heliostate möglichst nahe am Turm zu platzieren. Stehen die Heliostate jedoch zu dicht aneinander, kommt es zur gegenseitigen Verschattung und Blockierung der reflektierten Strahlung. Für die Effizienz des Heliostatenfeldes ist die Art der Verteilung der Heliostate von entscheidender Bedeutung und erfordert eine Optimierung. Das Heliostatenfeld ist die teuerste Komponente eines Solarturmkraftwerkes. In der Praxis finden sich sowohl Felder mit vielen kleineren Heliostaten (z.B. je $15 \mathrm{~m}^{2}$ siehe Brightsource, Ivanpah) als auch Felder mit weniger und dafür größeren Heliostaten (z.B. je $120 \mathrm{~m}^{2}$ siehe Sener, Gemasolar). Des Weiteren werden Heliostatenfelder nach ihrer Anordnung klassifiziert. Bei Rundumfeldern sind die Heliostate kreisrund um den Turm $\left(360^{\circ}\right)$ angeordnet, während bei einem Nord- oder Südfeld sich die Heliostaten nur auf einer Seite des Turmes befinden. 


\subsubsection{Turm}

Der Solarturm dient der optimalen Positionierung des Receivers. Der Wirkungsgrad des Heliostatenfeldes hängt stark von der Turmhöhe ab. Vor allem Kosinusverluste, Verschattung und Blockierung der Strahlung nehmen mit der Turmhöhe stark ab. Mit steigender Turmhöhe steigen aber auch die Turmkosten. Solartürme werden sowohl als Gittermast als auch als Stahlbetonbauweise ausgeführt. Tabelle 2.1 zeigt typische Turmhöhen verschiedener Solarturmkraftwerke.

Tabelle 2.1.: Typische Turmhöhen ausgewählter Solarturmkraftwerke

\begin{tabular}{lccccccc}
\hline Name & PS10 & PS20 & $\begin{array}{c}\text { Solar } \\
\text { Two }\end{array}$ & $\begin{array}{c}\text { Gema- } \\
\text { solar }\end{array}$ & Ivanpah & $\begin{array}{c}\text { Crescent } \\
\text { Dunes }\end{array}$ & $\begin{array}{c}\text { Khi Solar } \\
\text { One }\end{array}$ \\
\hline Turmhöhe $[\mathrm{m}]$ & 115 & 165 & 76 & 140 & 140 & 170 & 205 \\
\hline
\end{tabular}

\subsubsection{Receiver}

Im Receiver findet die Umwandlung der Solarstrahlung in Wärme statt. Die absorbierte Wärme wird im Receiver an das WTM übertragen und gelangt über das Leitungssystem in den Energiespeicher bzw. wird direkt zum Kraftwerksblock weitergeleitet. Als WTM kommen Gase, Flüssigkeiten und Feststoffe zum Einsatz. Des Weiteren können Receiver nach der Anordnung des Heliostatenfeldes klassifiziert werden. So gibt es Rundumfeldreceiver für $360^{\circ}$-Heliostatenfelder und Kavitätreceiver in einem Hohlraum mit eingeschränktem Akzeptanzwinkel für Nord- bzw. Südfelder. Je nach geforderter Temperatur und Leistungsklasse gibt es Vor- und Nachteile beider Receivertypen. Bedingt durch den Hohlraum und die damit mögliche Wärmedämmung erreichen Kavitätreceiver tendenziell höhere Wirkungsgrade. Die meist günstigeren Rundumreceiver eigenen sich vor allem für große Leistungen.

Am Receiver treten Reflexionsverluste, Abstrahlungsverluste, Konvektionsverluste sowie parasitäre Verluste durch die Förderung des WTM und der Begleitheizung auf. Im Folgenden werden die Receivertypen Rohrreceiver, volumetrische und direktabsorbierende Receiver näher beschrieben. 


\subsubsection{Rohrreceiver}

Das charakteristische Merkmal von Rohrreceivern sind die Absorberrohre, in denen das WTM strömt. Meistens werden die Rohre aus metallischen Werkstoffen gefertigt. Als WTM kommen Gase und Flüssigkeiten in Frage. Einen Sonderfall stellen DSG-Systeme dar, in denen im Absorberrohr eine Zweiphasenströmung aus flüssigem Wasser und Dampf auftritt. Die konzentrierte Solarstrahlung wird von den Absorberrohren absorbiert und mittels Wärmeleitung durch die Rohrwand geleitet. Im Betrieb treten somit die höchsten Temperaturen auf der Rohraußenseite auf. Um die Wärmeleitung in den Absorberrohren zu verbessern und damit die Übertemperaturen zu reduzieren werden neue Absorbermaterialien erforscht, die einerseits eine hohe Wärmeleitfähigkeit und andererseits eine ausreichende Festigkeit bei hohen Temperaturen haben. Die konvektive Wärmeübertragung an das WTM auf der Rohrinnenseite wird erzwungen, indem das WTM durch die Absorberrohre gepumpt wird und diese damit kühlt. Dieser Wärmeübergang wird durch die Stoffeigenschaften des WTMs und die Hydraulik im Rohr charakterisiert.

Rohrreceiver können sowohl als Rundumreceiver, als auch in einer Kavität Anwendung finden. Die Absorberrohre können beliebig angeordnet werden. Es sind gerade, gebogene oder spiralenförmige Anordnungen möglich. Außerdem können die Rohre in mehreren Schichten, dicht aneinander oder mit Abstand zueinander angeordnet sein. Je nach Konstruktion sind zudem noch Dehnungskompensatoren zum Ausgleich der thermischen Verformungen unter Bestrahlung nötig. Oft werden mehrere Rohre zu einem Modul zusammengefasst. Am Moduleinlass befindet sich ein Verteiler, der die Strömung gleichmäßig auf die Absorberrohre verteilt. Am Austritt wird das WTM im Sammler wieder zusammengeführt und gemischt. Diese meist baugleichen Module können je nach Betriebsstrategie und Temperaturhub in Abhängigkeit von der solaren Einstrahlung parallel oder seriell miteinander verschaltet werden. Auch Rezirkulation und Bypass-Schaltungen sind möglich.

Für Fluidtemperaturen von bis zu $800^{\circ} \mathrm{C}$ werden häufig metallische Rohre aus Nickelbasislegierungen (z.B. Inconel) verwendet. Zur Verringerung der Reflexionsverluste an der metallischen Rohroberfläche werden die Absorberrohre mit 
einer schwarzen, hochabsorbierenden Farbe (Pyromark) beschichtet. In dieser Arbeit werden ausschließlich metallische Rohrreceiver betrachtet.

\subsubsection{Volumetrische Receiver}

In volumetrischen Receivern werden poröse Absorber verwendet, wie beispielsweise ein Drahtgeflecht oder ein Keramikschaum. Die konzentrierte Solarstrahlung dringt in die Absorberstruktur ein und wird somit nicht von einer Fläche, sondern in einem Volumen absorbiert. Das WTM (meist Gase) wird durch diese offenporige Struktur geleitet, wodurch die Wärme übertragen wird. Die Wärmestromdichte ist an der Absorberoberfläche am größten und nimmt beim Eindringen in die poröse Struktur sukzessive ab. Mit der Gas/Luft-Temperatur verhält es sich umgekehrt. Am Einlass ist die Temperatur am niedrigsten und nimmt beim Passieren der porösen Struktur stetig zu. Dies führt zu zwei technologischen Vorteilen: Einerseits erfährt die am stärksten bestrahlte Fläche somit die maximale Kühlung und andererseits kann das WTM theoretisch bis zur maximalen Absorbertemperatur (am Austritt) aufgeheizt werden. Dieser sogenannte „volumetrische Effekt"charakterisiert diesen Receivertyp. Die vergleichsweise geringen Oberflächentemperaturen führen zu niedrigeren thermischen Abstrahlverlusten. Bei hohen Receiveraustrittstemperaturen $>800{ }^{\circ} \mathrm{C}$ werden volumetrische Receiver häufig in Kavitätenbauform ausgeführt. Zur Steigerung des Wärmeübergangs und zur Kombination mit druckbeaufschlagten Wärmekraftprozessen (z.B. Gasturbine) kann ein Receiverfenster aus Quarzglas eingesetzt werden. Durch die hohen Anforderungen an das Fenster sind entsprechende Systeme vergleichsweise teuer. Aktuell gibt es kein kommerzielles Solarturmkraftwerk mit volumetrischem Receiver.

\subsubsection{Direktabsorbierende Receiver}

In direktabsorbierenden Receivern wird das WTM (Feststoffe oder Flüssigkeiten) direkt der Solarstrahlung ausgesetzt. Im Gegensatz zu Rohrreceivern entfällt damit der dort zusätzliche Temperaturgradient durch die Wärmeleitung im Absorberrohr. Dadurch gibt es bezüglich der Bestrahlungsstärke bei direktabsorbierenden Receivern theoretisch keine Grenze. Ein Nachteil ist jedoch, dass 
das WTM im direkten Kontakt mit der Umgebung ist. Die Verunreinigung des WTMs durch Fremdkörper sowie der Austrag des WTM in die Umgebung kann nicht komplett verhindert werden. Direktabsorbierende Receiver sind bisher noch Gegenstand der Forschung und nicht kommerziell verfügbar.

\subsubsection{Energiespeicher}

Im Gegensatz zur Photovoltaik (PV) bieten solarthermische Kraftwerke die Möglichkeit einer vergleichsweise einfachen Speicherung der thermischen Energie (in gedämmten Behältern). Dadurch kann die Stromerzeugung zeitlich von der solaren Einstrahlung entkoppelt werden. Solarthermische Kraftwerke können daher auch Nachts bzw. je nach Bedarf Strom erzeugen. Der zunehmende Ausbau von Windenergie und PV führt zu stärkeren Fluktuationen in der Energiebereitstellung, da diese Technologien jeweils nur bei entsprechenden Wetterbedingungen Strom erzeugen können. Solarthermische Kraftwerke mit Speicher bieten hier die ideale Ergänzung, da sie Grundlast- und Regelenergie erzeugen, die die Schwankungen von Windenergie und PV ausgleicht und zur Netzstabilisierung beiträgt.

Durch den Speicher steigen zwar die Investitionskosten, gleichzeitig erhöht sich aber der Nutzungsgrad der Turbine signifikant, was zu geringeren Stromgestehungskosten führt.

Die am häufigsten eingesetzten Speicher in Solarkraftwerken sind sensible Speicher in 2-Tank Bauweise.

\subsubsection{Kraftwerksblock}

Im Kraftwerksblock findet die Wandlung von thermischer in elektrische Energie statt. Dazu wird in einem Wärmeübertrager die Energie des Speichermediums auf das Arbeitsmedium der Turbine übertragen. Die am häufigsten eingesetzten Arbeitsmittel sind Wasser (Dampfturbine) und Luft (Gasturbine). In der Turbine wird das Arbeitsmittel entspannt, wobei die thermische in potenzielle/kinetische Energie (Rotation der Welle) umgewandelt wird. Mit einem Generator wird 
schließlich die kinetische Energie in elektrische Energie gewandelt. Moderne Dampfturbinen erreichen einen Wirkungsgrad von ca. $40 \%$.

\subsection{Meilensteine der Salzturmtechnologie}

\subsubsection{Gemasolar (2011)}

Gemasolar (Solar Tres) ist das erste kommerzielle und wegweisende Solarturmkraftwerk mit Flüssigsalz (Solar Salt) als Wärmeträger- und speichermedium. Basierend auf dem Forschungsprojekt SolarTwo wurde Gemasolar im Jahre 2009 in Spanien gebaut und ist seit Mai 2011 in Betrieb.

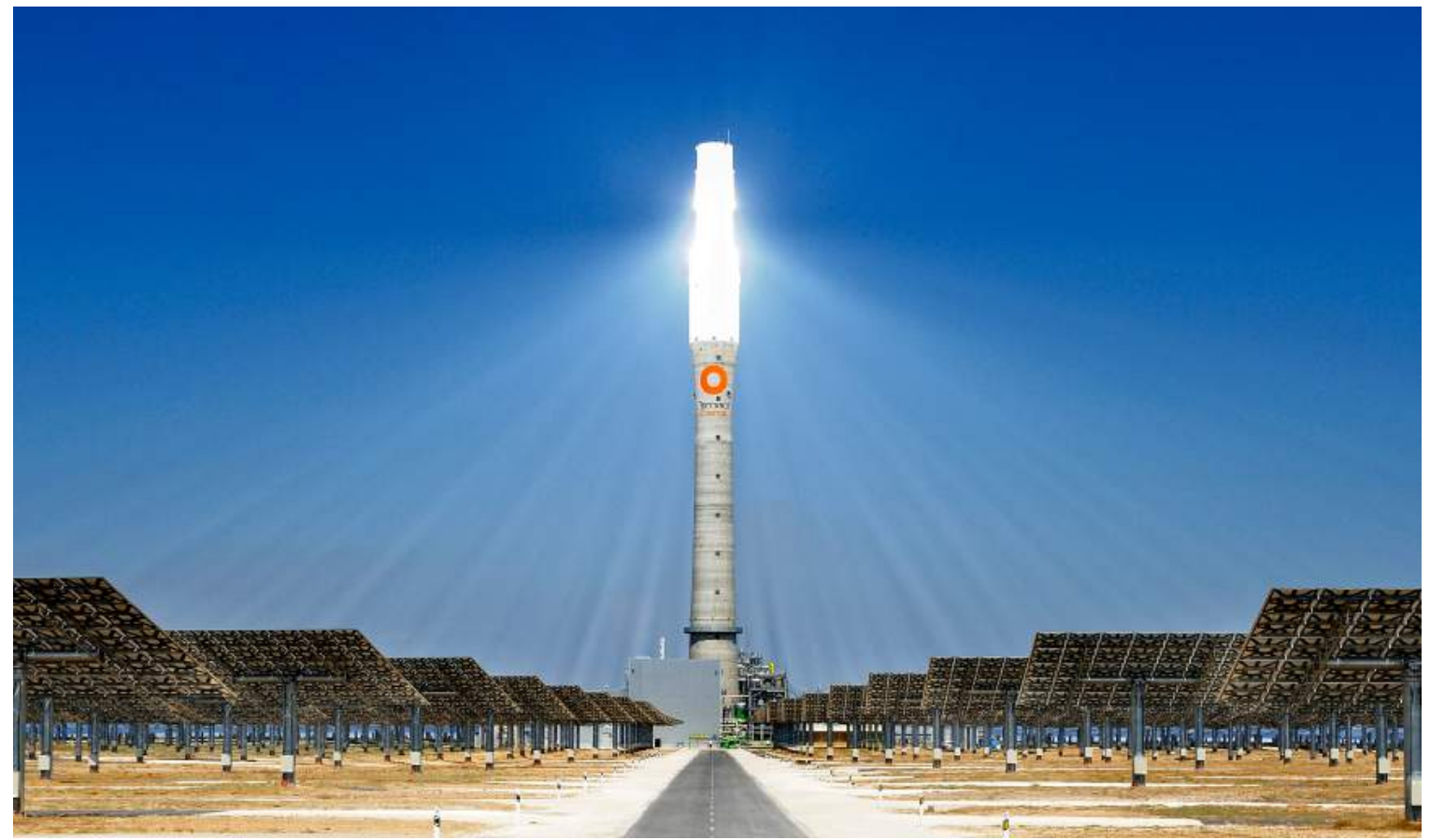

Abbildung 2.5.: Das Solarkraftwerk Gemasolar in Sevilla, Spanien [11].

Das Kraftwerk wurde mit einem hohen Solarvielfachen (Verhältnis der tatsächlichen Spiegelfläche zur für den Nennbetrieb notwendigen Spiegelfläche) von $S M \approx 4.0$ ausgelegt. Dazu besitzt das Kraftwerk einen großen thermischen Energiespeicher, der bei Sonnenschein gefüllt wird und zu Zeiten ohne Sonneneinstrahlung genutzt werden kann. Damit ist das Kraftwerk grundlastfähig bzw. unabhängig von der aktuellen Sonneneinstrahlung. Der Dauerbetrieb 
mit 19.9 MW Turbinenleistung wurde über einen Zeitraum von einer Woche (24 h/Tag) bereits nachgewiesen [12].

Die wichtigsten technischen Daten sind in Tabelle 2.2 zusammengefasst:

Tabelle 2.2.: Technische Daten des Solarkraftwerks Gemasolar 11, 13

\begin{tabular}{ll}
\hline Kraftwerk & Gemasolar \\
\hline Standort & Fuentes de Andalucía, Sevilla \\
Betrieb & seit Mai 2011 \\
Anlagenkonfiguration & Externer Receiver mit 360-Feld \\
Größe des Heliostatenfeldes & $185 \mathrm{ha}$ \\
Reflektierende Gesamtfläche & $304750 \mathrm{~m}^{2}$ \\
Anzahl an Heliostaten & 2650 \\
Aperturfläche eines Heliostaten & $120 \mathrm{~m}^{2}$ \\
Turmhöhe & $140 \mathrm{~m}$ \\
Wärmeträgermedium & Solar Salt \\
Thermische Receiverleistung & $120 \mathrm{MW}$ \\
Einlasstemperatur & $290{ }^{\circ} \mathrm{C}$ \\
Auslasstemperatur & $565{ }^{\circ} \mathrm{C}$ \\
Turbinenleistung & $19.9 \mathrm{MW} \mathrm{(17} \mathrm{MW} \mathrm{netto)}$ \\
Nutzungsgrad & $75 \%$ \\
Jahresenergieertrag & $110 \mathrm{GW} \mathrm{h}$ \\
Thermische Speicherung (2-Tank) & Höhe: $10.5 \mathrm{~m} /$ Durchmesser: $23 \mathrm{~m}$ \\
Speicherkapazität & $15 \mathrm{~h}, 647 \mathrm{MWh}, 8500$ t Salt \\
\hline
\end{tabular}

\subsubsection{Crescent Dunes}

Das aktuell größte Solarturmkraftwerk mit Flüssigsalztechnologie befindet sich in der Wüste von Nevada (Crescent Dunes Solar Energy Project) und wurde im Oktober 2015 mit dem Stromnetz verbunden. Der Durchmesser des Heliostatenfeldes beträgt ca. $2.8 \mathrm{~km}$ mit über 10000 Heliostaten.

Wie auch bei Gemasolar dient ein großer thermischer Speicher zum Ausgleich von Schwankungen der solaren Einstrahlung. Zur Stromerzeugung dient eine konventionelle Dampfturbine von Alstom (125 MW, 115 bar). 


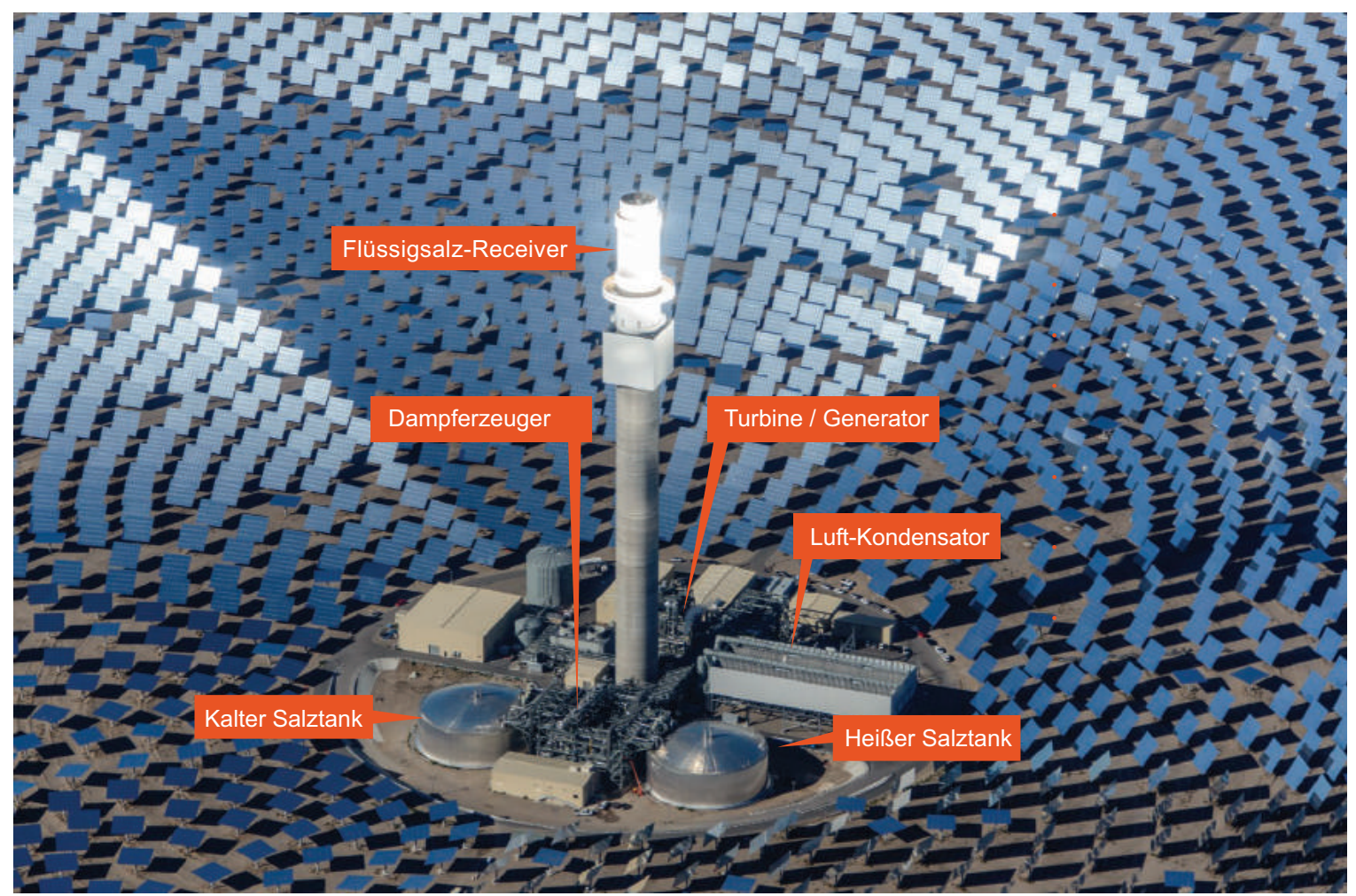

Abbildung 2.6.: Das Solarkraftwerk Crescent Dunes Solar Energy Project nahe Tonopah, Nevada [14.

Tabelle 2.3.: Technische Daten des Solarkraftwerks Crescent Dunes [14, 15

\begin{tabular}{ll}
\hline Kraftwerk & Crescent Dunes \\
\hline Standort & Tonopah, Nevada \\
Betrieb & seit Oktober 2015 \\
Anlagenkonfiguration & Externer Receiver mit 360-Feld \\
Größe des Heliostatenfeldes & ca. 650 ha \\
Reflektierende Gesamtfläche & ca. 1.21 Mio.m ${ }^{2}$ \\
Anzahl an Heliostaten & 10347 \\
Aperturfläche eines Heliostaten & $116.9 \mathrm{~m}^{2}$ \\
Turmhöhe & $195 \mathrm{~m}$ \\
Wärmeträgermedium & Solar Salt \\
Receiverhöhe & $30.5 \mathrm{~m}$ \\
Einlasstemperatur & $288{ }^{\circ} \mathrm{C}$ \\
Auslasstemperatur & $566{ }^{\circ} \mathrm{C}$ \\
Receivergewicht (leer/voll) & $907 \mathrm{t} / 1225 \mathrm{t}$ \\
Turbinenleistung & $125 \mathrm{MW} \mathrm{(110} \mathrm{MW} \mathrm{netto)}$ \\
Jahresenergieertrag & $>500 \mathrm{GW} \mathrm{h}$ \\
Thermische Speicherung (2-Tank) & Höhe: $12.2 \mathrm{~m} /$ Durchmesser: $42.7 \mathrm{~m}$ \\
Speicherkapazität & $10 \mathrm{~h}, 31750 \mathrm{t}$ Salt \\
\hline
\end{tabular}





\section{Eigenschaften und Anwendungen von Flüssigmetallen}

In diesem Kapitel werden die grundlegenden Eigenschaften von Flüssigmetallen detailliert analysiert und diskutiert. Neben den bisherigen Anwendungen und Erfahrungen mit Flüssigmetallen wird auch die aktuelle Entwicklung wiedergegeben. Aus diesen Grundlagen leiten sich die für Solarkraftwerke in Frage kommenden Flüssigmetalle ab. In diesem Kapitel werden alle durch die Verwendung von Flüssigmetallen beeinflussten Komponenten eines Solarturmkraftwerkes (Receiver, Turm, Leitungen, Wärmeübertrager, Pumpen, Betriebsvorgänge wie Einfrier- und Auftauvorgänge sowie Sicherheitstechnik) behandelt und mit den entsprechenden Komponenten für Flüssigsalz verglichen.

Der größte Unterschied von Flüssigmetallen zu anderen Flüssigkeiten besteht in den herausragenden Wärmeübertragungseigenschaften. Grund hierfür ist die niedrige Prandtl-Zahl ( $P r$ stets $<0.05)$. Sie verknüpft das Geschwindigkeitsfeld mit dem Temperaturfeld des Fluids und ist somit ein Maß für das Verhältnis der Dicken von Strömungs- und Temperaturgrenzschicht. Bei Fluiden mit niedrigen Prandtl-Zahlen ist die thermische Grenzschicht dicker als die hydrodynamische Grenzschicht. Somit verteilt sich der Wärmewiderstand über den gesamten Strömungsquerschnitt statt wie bei Fluiden mit höheren Prandtl-Zahlen wie z.B. Luft nur auf den Randbereich [16]. Diese Eigenschaft wird mit den durchgeführten Berechnungen bestätigt und beeinflusst die Komponentenauslegung maßgeblich.

Aus diesem Grund sind Flüssigmetalle schon seit den 60er Jahren Gegenstand der Forschung und werden zur Kühlung von thermisch hochbeanspruchten Flächen verwendet. 


\subsection{Bisherige Anwendungen von Flüssigmetallen}

\subsubsection{Kernreaktortechnik}

Der erste nukleare Versuchsreaktor, der elektrischen Strom erzeugte, nutze eine Legierung aus Natrium und Kalium (NaK) als Kühlmitte円. Die erste kommerzielle Anwendung von flüssigmetallgekühlten Atomreaktoren (LMFR) war der Einsatz in U-Booten. Sowohl die Sowjetunion als auch die USA betrieben erfolgreich zahlreiche U-Boot Klassen mit LMFR seit den 1960er Jahren bis Mitte der 1990 (K-27, Alfa-class, SSN-575). Die meisten dieser Reaktoren nutzten Blei-Bismut als WTM.

Aber auch in der Kraftwerkstechnik gibt es seit den 1950er Jahren viele Versuchsanlagen mit Flüssigmetallen. Die sogenannten schnellen Brüter werden mit flüssigem Natrium gekühlt. In solchen Brutreaktoren dürfen die bei der Kernspaltung entstehenden schnellen Neutronen nicht wie in anderen Reaktortypen zwischen den Brennstäben abgebremst werden. Im Gegensatz zu Leichtwasserreaktoren darf zur Kühlung bei schnellen Brütern kein Wasser eingesetzt werden, da es als Moderator wirkt. Stattdessen wird ein flüssiges Metall (Natrium oder Kalium) verwendet. Der primäre Natriumkühlkreis nimmt die bei der Spaltung eines Kerns entstehende Wärme auf und gibt sie über einen Wärmetauscher an einen Sekundärnatriumkühlkreis weiter. Dieser Sekundärkreislauf produziert in einem Dampferzeuger Frischdampf, der die Dampfturbine antreibt.

Bisher wurden zwölf experimentelle schnelle Brutreaktoren mit einer Leistung von 10-400 $\mathrm{MW}_{\text {th }}$ und sechs kommerzielle Prototypen mit einer elektrischen Leistung von 250-1200 $\mathrm{MW}_{\text {el }}$ gebaut und betrieben. In Summe wurden damit bisher (von 1950-2010) über 350 Reaktorjahre Erfahrung mit LMFRs akkumuliert [17. Der aktuell größte kommerzielle Brutreaktor ist der BN-800 in Belojarsk, Russland mit einer Leistung von $2100 \mathrm{MW}_{\text {th }}\left(864 \mathrm{MW}_{\text {el }}\right)$. Am 11. Dezember 2015 wurde das Kraftwerk erstmals mit der Minimalleistung von $235 \mathrm{MW}$ mit dem Stromnetz synchronisiert [18. Seit 31. Oktober 2016 ist der Atomreaktor im Nennbetrieb [19].

\footnotetext{
${ }^{1}$ Experimental Breeder Reactor I, am 20. Dezember 1951.
} 


\subsubsection{Auslassventile in Verbrennungsmotoren}

Flüssiges Natrium wird auch in der Automobilindustrie zur Kühlung von thermisch hoch beanspruchten Auslassventilen in Verbrennungsmotoren verwendet. Hierzu werden die Ventilschäfte hohl ausgeführt und zum Teil mit Natrium gefüllt. Im Betrieb schmilzt das Natrium und schwappt zwischen der heißen und kalten Seite hin und her. Die Wärme wird dadurch vom heißen Ventilteller effektiv abtransportiert [20]. Bereits im Jahre 1939 verwendete Mercedes-Benz in den Kompressormotoren serienmäßig derartige Ventile [21].

\subsubsection{CPU-Kühler für PCs}

Die Firma Danamics hat bereits im Jahr 2010 einen flüssigmetallgekühlten CPU-Kühler LMX Superleggera auf den Markt gebracht. Als WTM wird die eutektische Mischung von Natrium und Kalium (NaK) verwendet. Zur Förderung des Flüssigmetalls wird eine elektromagnetische Pumpe verwendet. Die Testergebnisse zeigen eine deutlich höhere Kühlleistung als herkömmliche Luftkühler 22.

\subsubsection{Flüssigmetallbatterien}

Flüssigmetalle können auch als elektrochemischer Energiespeicher eingesetzt werden. Solche Flüssigmetallbatterien sind seit den 1960er bekannt und werden stetig weiter erforscht 23. Im Inneren dieser Batterien befinden sich drei verschiedene Flüssigkeiten die aufgrund der unterschiedlichen Dichte übereinander liegen. Eine der bekanntesten Zellen ist die Natrium-Bismut-Batterie.

\subsection{Flüssigmetalle in Solarkraftwerken}

Auch in Solarkraftwerken wurden die guten Wärmeübertragungseigenschaften der Flüssigmetalle schon experimentell untersucht. Durch einen schweren Natriumbrand auf der PSA im Jahre 1986 wurden Flüssigmetalle als WTM in Solarkraftwerken jedoch über 25 Jahre nicht weiter betrachtet. Erst in jüngster Zeit wird die Forschung in diesem Bereich wieder aufgenommen. Im Folgenden werden die Erfahrungen mit Flüssigmetallen in Solarkraftwerken beschrieben. 


\subsubsection{Sandia CRTF (Central Receiver Test Facility)[24]}

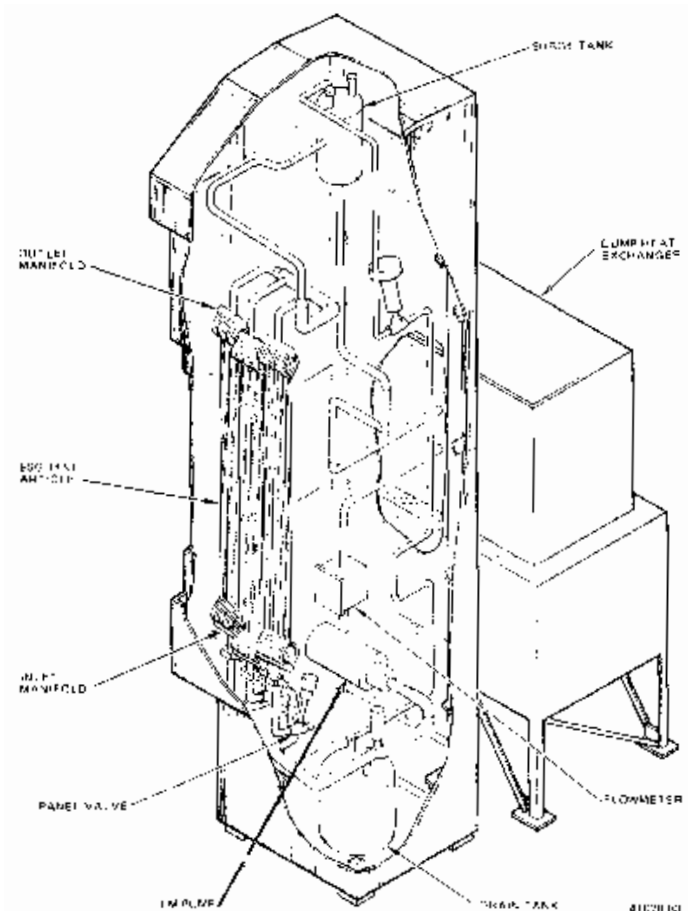

Abbildung 3.1.: Natrium Receivermodul auf der CRTF in Barstow

In der CRTF führte Sandia im Zeitraum zwischen 10. Oktober 1981 und 12. März 1982 erfolgreich Tests mit natriumgekühlten Receivermodulen durch. Die durchgeführten Tests dienten als Grundlagenuntersuchung des Verhaltens von Natrium unter solarer Einstrahlung. Des weiteren wollte man Erfahrung in der Fertigung und im Betrieb mit Natriumreceivern gewinnen.

Das getestete Receivermodul bestand aus drei parallel durchströmten Submodulen mit je 21 Absorberrohren (316 stainless steel, entspricht 1.4401 [X5CrNiMo17-12-2]). Jedes Rohr hatte einen Durchmesser von $19 \mathrm{~mm}$ mit einer Wandstärke von $1.2 \mathrm{~mm}$ und wurde stumpf in Verteiler/Sammler (Durchmesser: $102 \mathrm{~mm}$ ) eingeschweißt. Ein Untermodul hatte inklusive Verteiler und Sammler eine Gesamtlänge von $530 \mathrm{~cm}$ und eine Breite von $40 \mathrm{~cm}$. Die Rohre sind auf einem Kreisausschnitt mit $12 \mathrm{~m}$ Durchmesser angeordnet und repräsentieren somit einen Ausschnitt eines großen externen Receivers. Die Auslegung der Absorberrohre im Brennfleck basierte auf einer festgelegten Lebensdauer von 30 Jahren (ASME N-47). Die der Solarstrahlung ausgesetzte Apertur des Moduls war $3 \mathrm{~m}$ hoch und $1.2 \mathrm{~m}$ breit. Dieser Bereich wurde mit hochabsorbierender Farbe beschichtet (Pyromark).

Die Leistung am Auslegungszeitpunkt betrug 3x1 $\mathrm{MW}_{\text {th }}$ bei einer maximalen Wärmestromdichte von $1.5 \mathrm{MW} / \mathrm{m}^{2}$. Die Austrittstemperatur für den Auslegungszeitpunkt war $593 \pm 14^{\circ} \mathrm{C}$ bei einer Eintrittstemperatur von $288^{\circ} \mathrm{C}$.

Für die Zirkulation des Natriums im Kreislauf wurde eine elektromagnetische Pumpe EMP verwendet $\left(0.007 \mathrm{~m}^{3} / \mathrm{s}\right.$ am Auslegungszeitpunkt). Als Schutzgas diente Argon. Als Wärmesenke wurde ein Wärmeübertrager zur Umgebungsluft eingesetzt.

Bei Nacht wurde der Receiver entleert. Sämtliche Tanks und Rohre wurden 
dabei auf einer Temperatur von $204{ }^{\circ} \mathrm{C}$ gehalten. Die ungenaue Bestimmung der Wärmestromdichte auf der Apertur führte zu einer relativ großen Unsicherheit bei der Wirkungsgradberechnung. Die im Testbetrieb erreichten Receiverwirkungsgrade lagen zwischen 90-96 $\pm 10 \%$.

\subsubsection{CRS-Natriumreceiver auf der PSA}

Im Rahmen der IEA wurde 1981 das Projekt SSPS initiiert. Teil dieses Projekts war das CRS, bei dem zwei Natriumreceiver getestet wurden (Receiver in Kavität von Sulzer und externer Receiver von Franco-Tosi Industriale). Tabelle 3.1 zeigt die wichtigsten Anlagenparameter.

Tabelle 3.1.: Hauptanlagenparameter des CRS im Projekt SSPS auf der PSA 2527

\begin{tabular}{ll}
\hline Anlage & CRS im Projekt SSPS auf der PSA \\
\hline Heliostatenfeld & 93 Heliostate je $39.3 \mathrm{~m}^{2}=3656 \mathrm{~m}^{2}$ \\
Auslegungszeitpunkt & Tag $80,12 \mathrm{~h}$, Einstrahlung: $0.92 \mathrm{~kW} / \mathrm{m}^{2}$ \\
Temperaturbereich & $270{ }^{\circ} \mathrm{C}$ bis $530{ }^{\circ} \mathrm{C}$ \\
Schutzgas im Natrium-Kreislauf & Argon $(8 \mathrm{bar})$ \\
Kreiselpumpe Receiver-loop & $0.73-11.315 \mathrm{~kg} / \mathrm{s}(\mathrm{DP}=7.3 \mathrm{~kg} / \mathrm{s})$ \\
Kreiselpumpe Dampferzeuger-loop & $0.69-7.59 \mathrm{~kg} / \mathrm{s}(\mathrm{DP}=6.9 \mathrm{~kg} / \mathrm{s})$ \\
Solarleistung & $2880 \mathrm{~kW}$ th \\
Elektrische Leistung & $600 / 517 \mathrm{~kW}$ el $($ brutto $/$ netto $)$ \\
2-Tank-Speicher mit Natrium & je $70 \mathrm{~m}^{3}$, ca. $1 \mathrm{MWh}_{\mathrm{el}} / 5.5 \mathrm{MWh}$ th \\
Dampferzeuger & Wärmeübertragerfläche: $14.7 \mathrm{~m}^{2}$ \\
& $0.86 \mathrm{~kg} / \mathrm{s}$ Dampf bei $500{ }^{\circ} \mathrm{C} \mathrm{und} 105 \mathrm{bar}$ \\
\hline
\end{tabular}

\subsubsection{Sulzer-Receiver [25, 27, 28]}

Der erste Natriumreceiver wurde von Interatom (deutsch) ausgelegt und von Sulzer (Schweiz) gebaut. Der Receiver wurde in Kavitätenbauform mit achteckiger Apertur ausgeführt (siehe Abbildung 3.2).

Die Absorberfläche entsprach einer gekrümmten Wand mit einem Radius von $2.25 \mathrm{~m}$, einer Höhe von $3.57 \mathrm{~m}$ und einem Akzeptanzwinkel von $120^{\circ}$. Der Receiver bestand aus 6 parallelen, horizontal angeordneten Rohren, die in 14 Serpentinen von unten nach oben durchströmt wurden (Gesamtlänge: $87 \mathrm{~m}$ ). 


\section{Eigenschaften und Anwendungen von Flüssigmetallen}
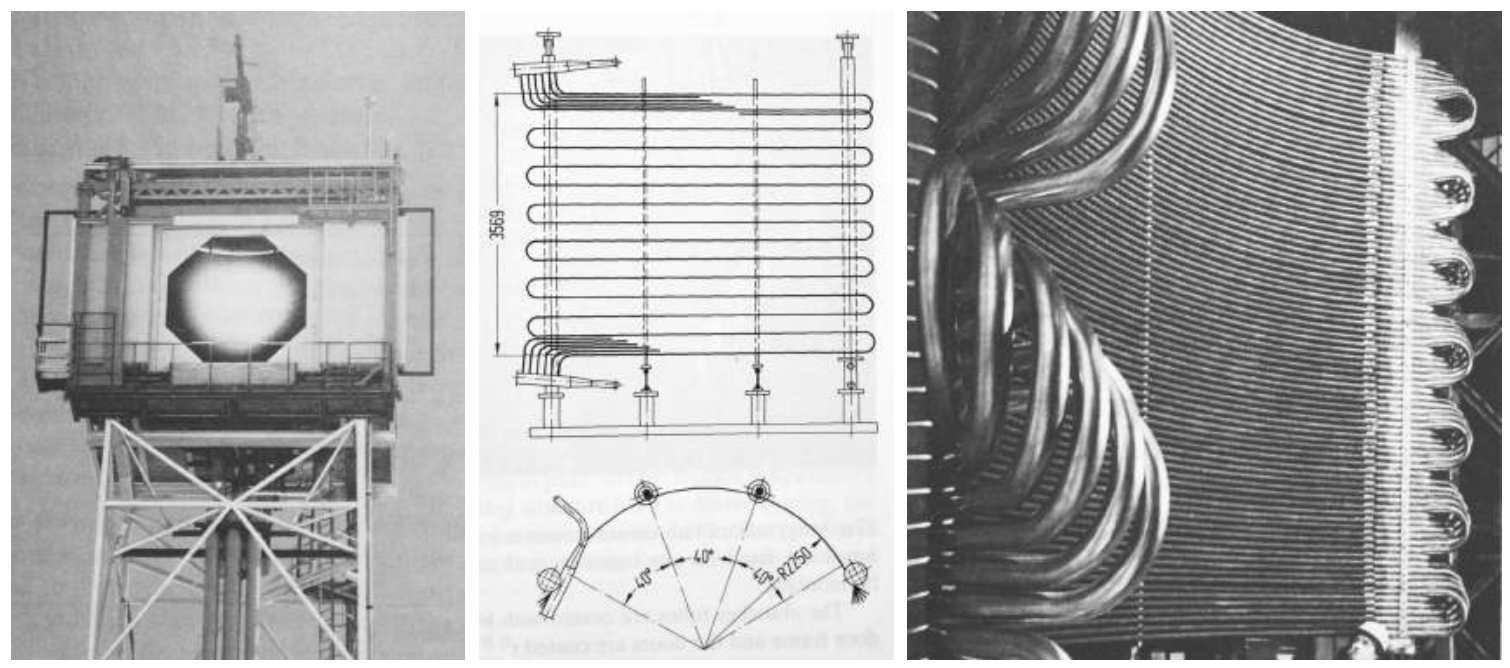

Abbildung 3.2.: Natriumreceiver in Kavitätenbauform von Interatom/Sulzer 28]

Die wichtigsten Receiverdaten sind in Tabelle 3.2 zusammengefasst. Die Absorberrohre wurden mit schwarzem Pyromark 2500 beschichtet. Die Kavität wurde mit $350 \mathrm{~mm}$ Isoliermaterial ausgelegt, um die thermischen Verluste auf $20 \mathrm{~kW}$ zu begrenzen.

Während der Testphase erreichte der Receiver lediglich einen Wirkungsgrad von $77 \%$. Grund hierfür war, dass aufgrund von Problemen des Dampfmotors nur $80 \%$ der Auslegungsleistung erreicht werden konnte.

Tabelle 3.2.: Hauptparameter des Natriumreceivers von Interatom/Sulzer $25,27,28$

\begin{tabular}{ll}
\hline Anlage & Natriumreceiver in Kavität auf der PSA \\
\hline Dimensionen außen & Höhe/Breite/Tiefe: 6 x 6 x $3.1 \mathrm{~m}$ \\
Aperturfläche (Achteck) & $9.7 \mathrm{~m}^{2}$ \\
Receiverfläche & $17 \mathrm{~m}^{2}$ (Rohre mit Spalte) \\
& $15 \mathrm{~m}^{2}$ (nur Rohre) \\
Wärmestromdichte & $0.6 \mathrm{MW} / \mathrm{m}^{2}$ (maximal) \\
& $0.16 \mathrm{MW} / \mathrm{m}^{2}$ (gemittelt) \\
Leistung am DP & $2.84 \mathrm{MW}$ th \\
Berechneter Wirkungsgrad & $88.3 \%$ \\
Rohrdurchmesser (außen) & $38 \mathrm{~mm}$ \\
Rohrwandstärke & $1.5 \mathrm{~mm}$ \\
Eintrittstemperatur & $270{ }^{\circ} \mathrm{C}$ \\
Austrittstemperatur & $530{ }^{\circ} \mathrm{C}$ \\
Druck & $4 \mathrm{bar}$ \\
Druckverlust & $0.45 \mathrm{bar}$ \\
Massenstrom & $7.34 \mathrm{~kg} / \mathrm{s}$ \\
\hline
\end{tabular}




\subsubsection{ASR - High-Flux-Experiment [28, 29]}

Der zweite Natriumreceiver (ASR = Advanced Sodium Receiver) auf der PSA wurde von Snamprogetti (Italien) ausgelegt und von Franco-Tosi Industriale (Italien) gebaut (siehe Abbildung 3.3).

Der Receiver bestand aus 5 Modulen mit je 39 parallelen Rohren. Alle Module wurden in einer Ebene angeordnet und seriell von unten nach oben durchströmt. Die Absorberrohre wurden mit schwarzem Pyromark beschichtet.
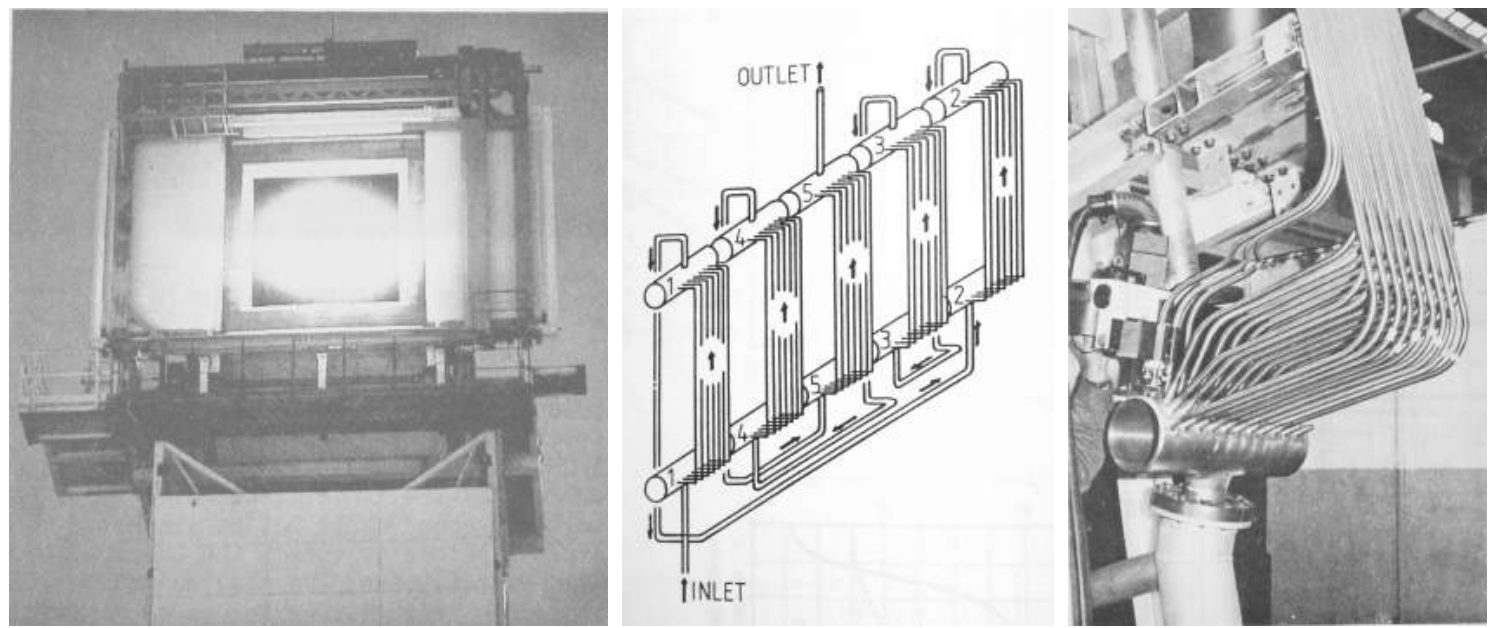

Abbildung 3.3.: Natriumreceiver von Snamprogetti/Franco-Tosi Industriale 30

Tabelle 3.3.: Hauptparameter des Natriumreceivers von Snamprogetti/Franco-Tosi Industriale 25, 27, 28.

\begin{tabular}{ll}
\hline Anlage & Externer Natriumreceiver (ASR) auf der PSA \\
\hline Dimensionen außen & Höhe/Breite: $2.85 \mathrm{~m} \mathrm{x} 2.73 \mathrm{~m}$ \\
Aperturfläche (Rechteck) & $7.94 \mathrm{~m}^{2}$ \\
Receiverfläche & $7.94 \mathrm{~m}^{2}$ \\
Wärmestromdichte & max. $1.38 \mathrm{MW} / \mathrm{m}^{2}$, gemittelt: $0.35 \mathrm{MW} / \mathrm{m}^{2}$ \\
Leistung am DP & $2.84 \mathrm{MW}_{\text {th }}$ \\
Berechneter Wirkungsgrad & $88 \%$ \\
Rohrdurchmesser & $14 \mathrm{~mm}$ \\
Rohrwandstärke & $1 \mathrm{~mm}$ \\
Eintrittstemperatur & $270{ }^{\circ} \mathrm{C}$ \\
Austrittstemperatur & $530{ }^{\circ} \mathrm{C}$ \\
Druck & $6 \mathrm{bar}$ \\
Druckverlust & $1.2 \mathrm{bar}$ \\
Massenstrom & $7.3 \mathrm{~kg} / \mathrm{s}$ \\
\hline
\end{tabular}


Nach etwa 500 Testtagen (ca. 900 h) und einer maximal erreichten Wärmestromdichte von $1.1 \mathrm{MW} / \mathrm{m}^{2}$ wurde das sogenannte High Flux Experiment gestartet. Dazu wurden zu den bisher verwendeten 93 Heliostaten (MCC) weitere $30 \mathrm{He}-$ liostate (MBB) hinzugezogen. Außerdem wurde die bisherige Zielpunktstrategie von 3 Zielpunkten auf einen Zielpunkt in der Mitte des Receivers reduziert. Damit wurde die Leistung auf 3.4 MW und die maximale Wärmestromdichte auf $2.51 \mathrm{MW} / \mathrm{m}^{2}$ erhöht. Außerdem wurde die Austrittstemperatur auf $560{ }^{\circ} \mathrm{C}$ angehoben. Der berechnete Wirkungsgrad lag damit bei $90.7 \%$ und die maximale Rohrtemperatur bei $635^{\circ} \mathrm{C}$.

Die erste Testphase im Zeitraum von August 1985 bis November 1985 lief erfolgreich ab. Dabei wurde ein großer Temperaturbereich und verschiedene Wärmestromdichten untersucht. Der Receiver zeigte stets hohe Wirkungsgrade über $90 \%$, obwohl dieser nicht für die hohen Wärmestromdichten ausgelegt wurde [29]. Im Frühjahr 1986 wurden weitere 10 Heliostate (ASINEL) installiert, um die Wärmestromdichte nochmals zu erhöhen. Diese zweite Testphase wurde jedoch durch den Natriumbrand im August 1986 abgebrochen.

\subsubsection{Natrium-Brand auf der PSA [26]}

Das Natriumfeuer brach durch austretendes Natrium während Wartungsarbeiten an einem defekten Ventil am 18. August 1986 aus. Während den Wartungsarbeiten stand die Anlage unter Druck. Das im Bereich des Ventils erstarrte Natrium hielt dem Leitungsdruck ( $\approx 8$ bar) nicht stand und geschätzte $10 \mathrm{t}$ Natrium traten aus, bevor der Druck genügend abfiel um weiteren Austritt zu verhindern. Im Folgenden wird der Unfallhergang genauer beschrieben:

11. Aug.: Tägliche Routineuntersuchung stellt erstarrtes Natrium (Natriumhydroxid) an einem Ventil zwischen Receiver und heißem Tank fest. Für die Reparatur wurde entschieden, den Bereich um das Ventil durch Einfrieren zu isolieren. Dazu wurden alle anderen Leitungen entleert. Die Begleitheizung wurde abgeschalten und die Dämmung im Bereich des Ventils entfernt.

18. Aug. 8:30: Die Temperatur in der Nähe des Ventils betrug noch $35^{\circ} \mathrm{C}$ und am Rohr in $3 \mathrm{~m}$ Entfernung noch $43^{\circ} \mathrm{C}$. Der Versuch, das Ventil zu bewegen schlug fehl. Die Temperatur des heißen Tanks war $270{ }^{\circ} \mathrm{C}$ und in den Leitungen in der Nähe $170^{\circ} \mathrm{C}$. 
18. Aug. 10:00: Beginn der Reparatur. Ventil wurde demontiert. Die Dichtschweißnaht wurde durch vorsichtiges Bohren entfernt.

18. Aug. 10:15: 2/3 der Umfangsnaht war bereits entfernt. Das Ventil öffnete plötzlich und Stücke von festem Natrium kamen aus der Leitung, gefolgt von einem Strom aus flüssigem Natrium.

18. Aug. 11:00: Das flüssige Natrium reagierte sofort mit der Umgebungsluft und füllte die Halle mit dichtem, weißen Rauch. Das Wartungspersonal flüchtete durch die Feuertüre in den Computerraum ohne ernsthafte Verletzungen. Der heiße Tank wurde sofort entlüftet, aber durch die große Menge an Argon vergangen etliche Minuten bis der Druck abfiel. Kurz darauf fiel die Stromversorgung aus. Die Notstrom-Dieselgeneratoren sprangen jedoch an, sodass die Entlüftung nicht unterbrochen wurde. Der Versuch, das Natrium vom heißen Tank in den kalten Tank zu transferieren schlug fehl, weil bereits die elektrischen Kabel durch das Feuer zerstört waren. 15 min lang strömte flüssiges Natrium aus $\left(\approx 12 \mathrm{~m}^{3}\right)$. Unglücklicherweise war der Natriumstrahl nach oben gerichtet und benetzte eine große Fläche, was zu einem großen Feuer führte. Durch das Belüftungssystem drang der Rauch auch in den Kontrollraum vor. Die Feuertüre war vom zuvor geflüchteten Wartungspersonal nicht geschlossen worden und so breitete sich das Feuer auch auf den Computerraum und anschließend auch auf den Kontrollraum aus. Schließlich zerstörte das Feuer Teile des Dachs und Fenster wodurch noch mehr Luft das Feuer anfachte.

18. Aug. 12:15: Das Feuer lies langsam nach. Ein Mann konnte die Halle betreten und stellte fest, dass kein Natrium mehr austrat. Das restliche noch brennende Natrium wurde mit Graphex-Pulver gelöscht. Die meisten Leitungen waren zerstört, jedoch waren beide Tanks unversehrt. Wahrscheinlich hat die große Wärmekapazität von $30 \mathrm{~m}^{3}$ Natrium im kalten Tank, der in unmittelbarer Nähe des Brandherdes stand, den Tank vor weiterem Schaden bewahrt.

\subsubsection{Jemalong Solar Thermal Station}

Seit 2011 werden in Australien (36 km westlich von Forbes) von VastSolar Receiverversuche mit Natrium durchgeführt. Im ersten Schritt wurden 200 einfach hergestellte Heliostate zur Demonstration eines $300 \mathrm{~kW}_{\text {th}}$-Receivers verwendet. 2012 wurde dieses Konzept hochskaliert auf 700 Heliostate für ein Receivermodul 


\section{Eigenschaften und Anwendungen von Flüssigmetallen}
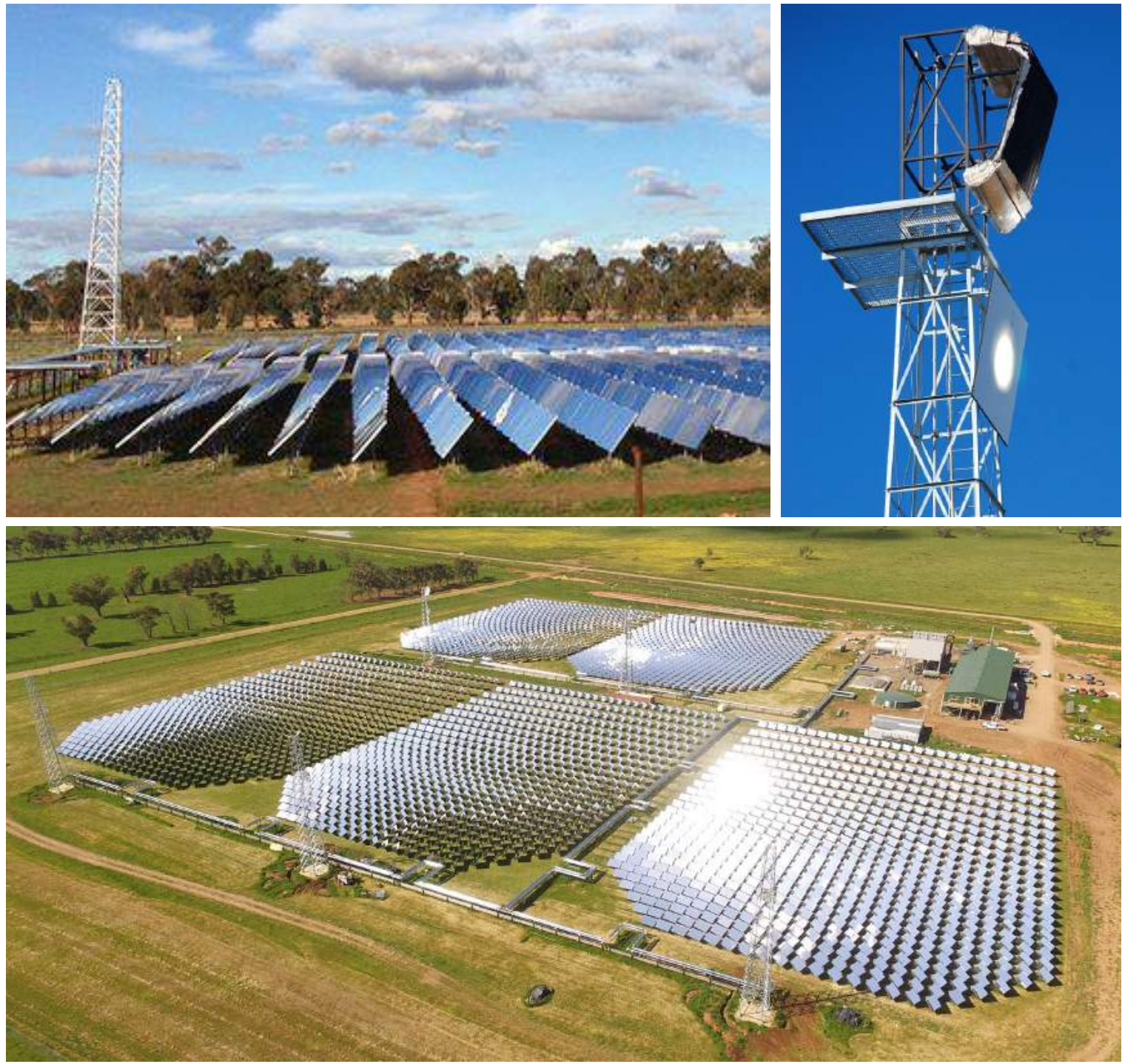

Abbildung 3.4.: Solarturmkraftwerk Jemalong Solar Thermal Station von VastSolar in Australien, Nähe Forbes 31

mit 1.2 $\mathrm{MW}_{\text {th }}$ auf einem Turm mit $27 \mathrm{~m}$ Höhe. Das Receivermodul wurde als planarer, externer Rohrreceiver mit $2 \mathrm{~m}^{2}$ ausgeführt (siehe Abbildung 3.4 rechts). Die Auslegung basiert auf einer maximal zulässigen Wärmestromdichte von 1.5 MW/m ${ }^{2}$ bei einem Temperaturhub von 270 auf $560{ }^{\circ} \mathrm{C} 31,32$.

Im September 2013 wurde entschieden, die Anlage als Mehrturmsystem mit 5 identischen Solartürmen (Gesamtleistung: $6 \mathrm{MW}_{\text {th }}$ mit 3500 Heliostaten) zu erweitern (siehe Abbildung 3.4 unten). Die Anlage beinhaltet einen thermischen Speicher mit Natrium und eine Dampfturbine mit $1.1 \mathrm{MW}_{\mathrm{el}}$, die ans Stromnetz angeschlossen werden soll 32 . 
Der Baubeginn war im April 2014, jedoch kam es am 14. Juni 2015 beim Test der drei bereits installierten Heliostatenfeldern zu einem Natriumleck und einem Natriumbrand [33]. Das Feuer wurde fachgerecht mit soda ash gelöscht und es gab keine Verletzten. Trotz des Brandes und der dadurch resultierenden Verzögerung konnte das Projekt im Frühjahr 2016 fertiggestellt werden. Für die nächste Skalierungsstufe plant VastSolar bereits ein $30 \mathrm{MW}_{\mathrm{el}}$-System mit 90 dieser Türme und einem Flüssigsalzspeicher $(4 \mathrm{~h})$. Der Baubeginn ist für Ende 2016 geplant.

\subsection{Potenzielle Flüssigmetalle in Solarkraftwerken}

In thermischen Solarkraftwerken wird die solare Strahlungsenergie zunächst in thermische Energie umgewandelt, bevor sie im Kraftwerksblock in elektrische Energie umgeformt wird. Bei Rohrreceivern wird die Solarstrahlung von den Rohren absorbiert und an ein Wärmeträgermedium (WTM) übertragen. Für die Auslegung und den Wirkungsgrad eines Receivers ist daher die Wahl des WTM entscheidend. Die Eigenschaften eines idealen WTM sind in Tabelle 3.4 zusammengefasst.

Tabelle 3.4.: Eigenschaften eines optimalen Wärmeträgermediums

\begin{tabular}{ll}
\hline Eigenschaft & Vorteil \\
\hline Niedriger Schmelzpunkt & Reduziert / Vermeidet Begleitheizung \\
Hoher Siedepunkt / chem. Stabilität & Großer Betriebsbereich in Flüssigphase \\
Hohe Wärmeleitfähigkeit & Guter Wärmeübergang \\
Hohe Wärmekapazität & Erlaubt direkte thermische Speicherung \\
& mit hoher Energiedichte \\
Geringer Dampfdruck & Betrieb bei Umgebungsdruck möglich \\
Geringe Viskosität & Geringer Druckverlust \\
Inertes Verhalten mit Strukturmaterial & Geringe Korrosion \\
Nicht toxisch / Nicht reaktiv & Verhindert Unfälle / Gesundheitsrisiken \\
Hohe Verfügbarkeit, einfache Herstellung & Geringe Kosten \\
\hline
\end{tabular}

Jedoch gibt es kein WTM, das alle diese Eigenschaften erfüllt. In Solarkraftwerken hat sich Luft, Wasser und in letzter Zeit besonders Flüssigsalz (vor 
allem auf Grund der geringen Speicherkosten) als WTM etabliert. Bezüglich der thermohydraulischen Eigenschaften im Receiver bieten die Flüssigmetalle jedoch ein großes Potenzial [6, 34, 35].

Die wichtigsten Betriebsparameter eines WTM sind die Temperaturgrenzen, in denen es betrieben werden kann. Der typische Temperaturbereich, in denen Kraftwerksprozesse betrieben werden ist: $250-600^{\circ} \mathrm{C}$. Mit der Beschränkung der Schmelz- und Siedetemperatur auf $T_{\text {schmelz }}<250{ }^{\circ} \mathrm{C}$ und $T_{\text {siede }}>700^{\circ} \mathrm{C}$ reduzieren sich die potenziellen Flüssigmetalle auf:

- Alkali-Metalle: Lithium, Natrium, Kalium

- Schwermetalle: Blei-Bismut, Cadmium

- Andere Metalle: Gallium, Indium, Zinn, Thallium

Eine weitere Einschränkung der Kosten auf $<20 € / \mathrm{kg}$ reduziert die Anzahl der potenziellen Metalle weiter. Tabelle 3.5 und Abbildung 3.5 zeigen die verbliebenen Metalle inklusive einiger Legierungen mit ihren Schmelz- und Siedepunkten sowie den Kostendaten im Vergleich zu verschiedenen Flüssigsalzen.

Tabelle 3.5.: Physikalische Eigenschaften und Kosten von Solar Salt, Natrium und Blei-Bismut nach [41], 42], [43], 37], 40]

\begin{tabular}{|c|c|c|c|c|c|}
\hline & & Solar Salt & Natrium & LBE & \\
\hline \multirow{6}{*}{$\begin{array}{l}0 \\
0 \\
\stackrel{10}{7} \\
\text { H } \\
11 \\
\text { E- }\end{array}$} & Dichte $\rho$ & 1820 & 852 & 9660 & {$\left[\mathrm{~kg} / \mathrm{m}^{3}\right]$} \\
\hline & Wärmekapazität $c_{p}$ & 1.52 & 1.28 & 0.15 & {$[\mathrm{~kJ} / \mathrm{kg} / \mathrm{K}]$} \\
\hline & Wärmeleitfähigkeit $\lambda$ & 0.52 & 68.10 & 13.35 & {$[\mathrm{~W} / \mathrm{m} / \mathrm{K}]$} \\
\hline & Dyn. Viskosität $\eta$ & 1.58 & 0.27 & 1.45 & {$[\mathrm{kPa} \cdot \mathrm{s}]$} \\
\hline & Kosten & 0.5 & 2.60 & 12.00 & {$[€ / \mathrm{kg}]$} \\
\hline & Kosten & 910 & 2215 & 115920 & {$\left[€ / \mathrm{m}^{3}\right]$} \\
\hline \multirow{4}{*}{ 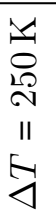 } & Energiedichte & 379 & 319 & 38 & {$[\mathrm{~kJ} / \mathrm{kg}]$} \\
\hline & Energiedichte & 690 & 272 & 362 & {$\left[\mathrm{MJ} / \mathrm{m}^{3}\right]$} \\
\hline & Kosten & 4.7 & 29.3 & 1152 & {$[€ / \mathrm{kWh}]$} \\
\hline & Speicherdichte & 191.6 & 75.6 & 100.6 & {$\left[\mathrm{kWh} / \mathrm{m}^{3}\right]$} \\
\hline \multirow{4}{*}{ 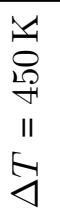 } & Energiedichte & 682 & 575 & 68 & {$[\mathrm{~kJ} / \mathrm{kg}]$} \\
\hline & Energiedichte & 1241 & 490 & 652 & {$\left[\mathrm{MJ} / \mathrm{m}^{3}\right]$} \\
\hline & Kosten & 2.6 & 16.3 & 640 & {$[€ / \mathrm{kWh}]$} \\
\hline & Speicherdichte & 344.9 & 136 & 181.1 & {$\left[\mathrm{kWh} / \mathrm{m}^{3}\right]$} \\
\hline
\end{tabular}




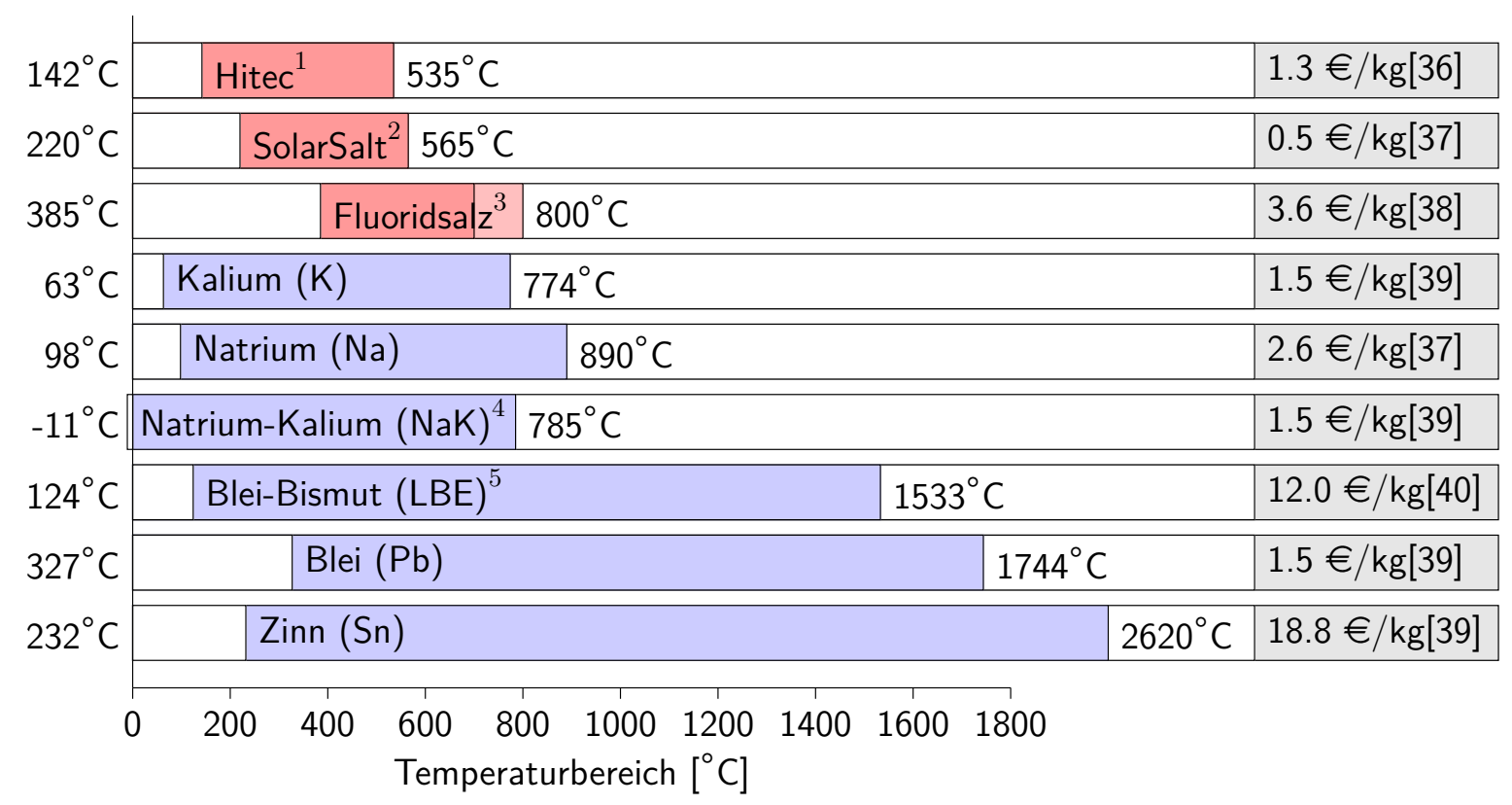

Abbildung 3.5.: Gegenüberstellung der Schmelz- und Siedepunkte sowie der spezifischen Kosten potenzieller Flüssigmetalle (blau) und Flüssigsalze (rot) für Solarkraftwerke. (1): Hitec $\left(53 \% \mathrm{KNO}_{3}+40 \% \mathrm{NaNO}_{2}+7 \% \mathrm{NaNO}_{3}\right)$, (2): Solar Salt $\left(60 \% \mathrm{NaNO}_{3}+40 \% \mathrm{KNO}_{3}\right), \quad(3)$ : Fluoridsalz $\left(8 \% \mathrm{NaF}+92 \% \mathrm{NaBF}_{4}\right)$ erfordert ab $700{ }^{\circ} \mathrm{C}$ Druckatmosphäre, (4): $\mathrm{NaK}(22 \% \mathrm{Na}+78 \% \mathrm{~K}),(5)$ : $\mathrm{Pb}-\mathrm{Bi}(55,5 \% \mathrm{Bi}+44,5 \% \mathrm{~Pb})$, alle Angaben in Massenprozent

Nach Tabelle 3.5 ergeben sich für Solar Salt die geringsten Anschaffungskosten. Wie im späteren Verlauf dieser Arbeit gezeigt wird, sind jedoch die Betriebskosten des Wärmeträgermediums von größerer Bedeutung. Für die thermische Speicherung hingegen sind die spezifischen Speicherkosten in $€ / \mathrm{kWh}$ entscheidend (siehe Abschnitt 4.2).

\subsection{Eigenschaften der Flüssigmetalle als WTM}

In diesem Kapitel werden alle für die Auslegung und den Betrieb notwendigen Eigenschaften der Flüssigmetalle analysiert und mit Solar Salt verglichen. Dazu gehören neben der Wärmeübertragung und damit dem Wirkungsgrad auch die Betriebsgrenzen bedingt durch die optische Güte des Heliostatenfeldes, der Druckverlust im WTM-Kreislauf, die Receivergeometrie, Betriebscharakteristik (Begleitheizung und das dynamische Verhalten bei fluktuierender solarer 
Einstrahlung), Pump- und Messtechnik sowie Korrosion und Lebensdauer. Des Weiteren wird auch auf die Toxizität und Reaktivität eingegangen und die Erfahrungen im Umgang mit Flüssigmetallen bzw. die daraus entwickelte Sicherheitstechnik beschrieben.

\subsubsection{Thermohydraulische Eigenschaften}

Da in dieser Arbeit ausschließlich Rohrreceiver untersucht werden, wird in diesem Abschnitt der Wärmeübergang in Rohren genauer untersucht. Die Wärmeübertragung in Rohren erfolgt maßgeblich durch erzwungene Konvektion auf der Rohrinnenseite. Es gilt:

$$
\dot{Q}_{\text {conv }}=\alpha \cdot A \cdot\left(T_{\mathrm{wi}}-T_{\text {bulk }}\right)
$$

Der Wärmeübergangskoeffizient $\alpha$ ist von den Stoffdaten des verwendeten WTM und der Strömung abhängig und kann mit Nusselt-Korrelationen abgeschätzt

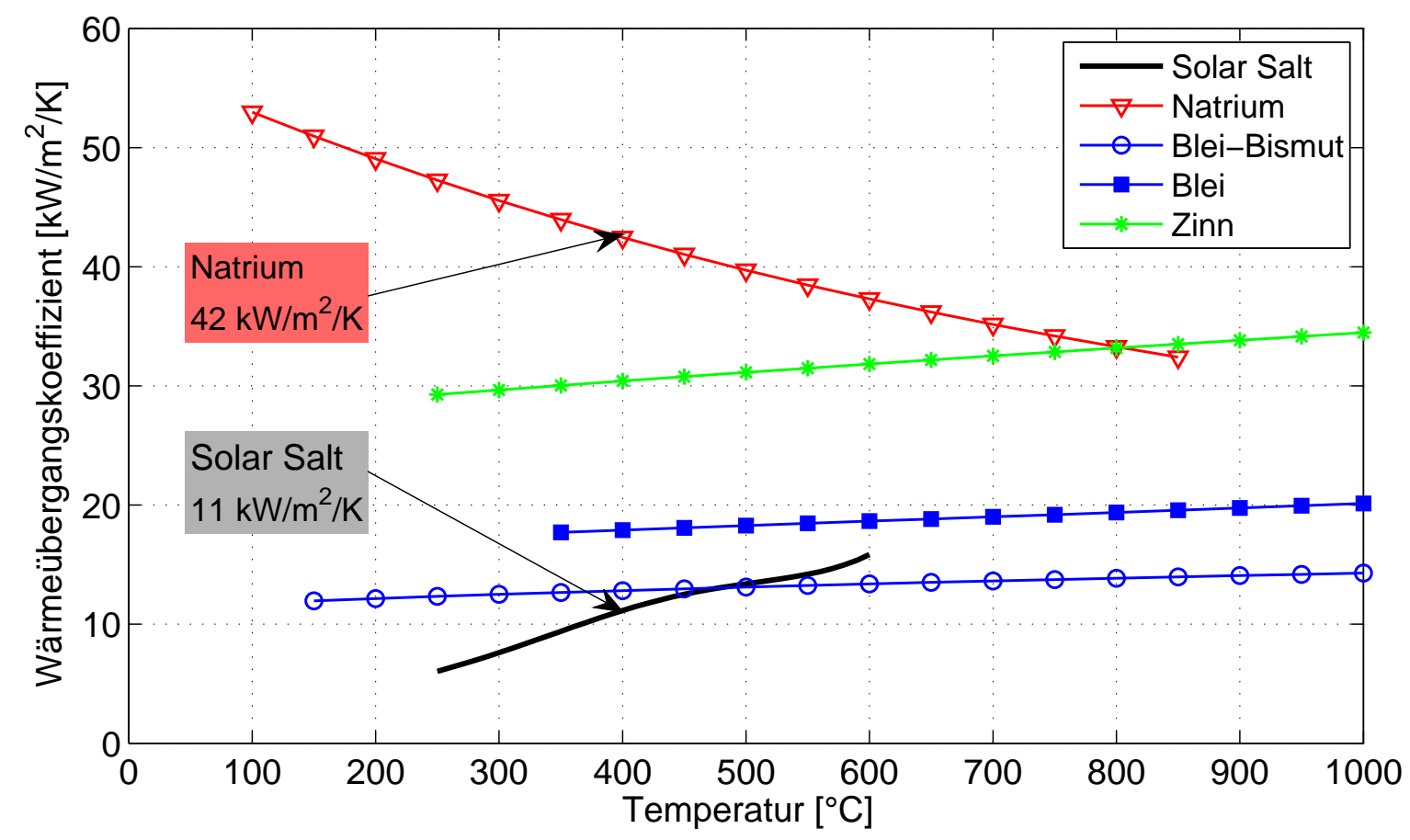

Abbildung 3.6.: Wärmeübergangskoeffizienten von Solar Salt und verschiedenen Flüssigmetallen in Abhängigkeit der Temperatur bei einer Rohrströmung mit $d_{\mathrm{i}}=15 \mathrm{~mm}, u=2.5 \mathrm{~m} / \mathrm{s}$ und $\mu=0.1 \mathrm{~mm}$. 
werden. Abbildung 3.6 zeigt die sich ergebenden Wärmeübergangskoeffizienten von Solar Salt und verschiedenen Flüssigmetallen bei einer Rohrströmung mit $d_{\mathrm{i}}=15 \mathrm{~mm}, u=2.5 \mathrm{~m} / \mathrm{s}$ und $\mu=0.1 \mathrm{~mm}$. Die verwendeten NusseltKorrelationen sind im Anhang E auf Seite 187 näher beschrieben.

Es zeigt sich, dass die Flüssigmetalle über den kompletten Temperaturbereich höhere Wärmeübergangskoeffizienten erreichen als Solar Salt. Durch die sehr hohe Wärmeleitfähigkeit von Natrium (100-200 mal höher als bei Solar Salt, siehe Anhang A auf Seite 177) werden hier die höchsten Wärmeübergangskoeffizient erreicht.

\subsubsection{Wärmestromdichtelimit}

Je nach WTM wird in der Literatur häufig eine maximal zulässige Wärmestromdichte angegeben. Nach [29] ergeben sich folgende Maximalwerte: Für Luft: $0.2 \mathrm{MW} / \mathrm{m}^{2}$, Wasserdampf: $0.4 \mathrm{MW} / \mathrm{m}^{2}$, Flüssigsalz: $0.8 \mathrm{MW} / \mathrm{m}^{2}$ und Natrium: $1.5 \mathrm{MW} / \mathrm{m}^{2}$. Tatsächlich ist aber die maximale Temperatur (des $\mathrm{WTM}^{2}{ }^{2}$ oder des Rohrmaterials $\}^{3}$ der beschränkende Faktor bei der Auslegung eines Receivers. Wird die Turbulenz in den Absorberrohren erhöht, so reduziert sich stets die Rohrtemperatur. Theoretisch kann daher jedes WTM für beliebige Wärmestromdichten ausgelegt werden, vorausgesetzt die entsprechende Turbulenz, bzw. die dafür nötige Pumpenleistung steht zur Verfügung. Tatsächlich gibt es folglich kein Wärmestromdichtelimit, sondern eine wirtschaftliche Begrenzung der Pumpenleistung und der maximalen Temperatur. Bei Solar Salt ist die maximale Temperatur durch die thermische Stabilität der Salzschmelze auf ca. $600{ }^{\circ} \mathrm{C}$ begrenzt 44. Beim konvektiven Wärmeübergang in einem Absorberrohr tritt die höchste Temperatur der Salzschmelze stets an der Rohrinnenoberfläche der bestrahlten Rohrseite auf. Wird diese Filmtemperatur auf $600{ }^{\circ} \mathrm{C}$ beschränkt, so kann damit bei einer gewissen Rohrgeometrie und thermischen Leistung die maximal zulässige Wärmestromdichte in Abhängigkeit der Pumpenleistung berechnet werden.

Die folgende Abschätzung gilt für ein einzelnes Absorberrohr, welches über die

\footnotetext{
${ }^{2}$ Siedetemperatur oder thermische Stabilität bei Mischungen.

${ }^{3}$ Schmelzpunkt des Rohrmaterials oder maximale Temperatur aus Lebensdauerberechnung.
} 
gesamte Rohrlänge und den halben Rohrumfang homogen bestrahlt wird. Die maximale Filmtemperatur ergibt sich somit am Rohrauslass, wo die mittlere Fluidtemperatur $T_{\text {bulk }}$ am höchsten ist. Mit der Vorgabe des Rohrdurchmessers, der thermischen Leistung und des Temperaturhubes im Rohr $\Delta T_{\text {bulk }}=(290-565)^{\circ} \mathrm{C}$ kann die Strömungsgeschwindigkeit $u$ unter Verwendung von mittleren Stoffwerten wie folgt berechnet werden.

$$
\begin{aligned}
& \dot{Q}_{\mathrm{th}}=\dot{V} \rho \cdot c_{p} \Delta T_{\mathrm{bulk}}=\frac{\pi d^{2}}{4} \cdot u \cdot \rho \cdot c_{p} \Delta T_{\mathrm{bulk}} \\
& \Rightarrow u=\frac{4 \cdot \dot{Q}_{\mathrm{th}}}{\pi d^{2} \cdot \rho \cdot c_{p} \Delta T_{\mathrm{bulk}}}
\end{aligned}
$$

Damit ergibt sich die Reynolds-Zahl zu

$$
\operatorname{Re}=\frac{\rho \cdot d \cdot u}{\eta}=\frac{4 \cdot \dot{Q}_{\mathrm{th}}}{\eta \cdot \pi d \cdot c_{p} \Delta T_{\mathrm{bulk}}}
$$

Durch die unterschiedlichen Prandtl-Zahlen müssen für Solar Salt und Natrium unterschiedliche Nusselt-Korrelationen verwendet werden:

$$
\begin{array}{ll}
N u_{\mathrm{SS}}=0.023 \cdot \operatorname{Re}^{4 / 5} \cdot \operatorname{Pr}^{0.4} & \text { nach Dittus-Boelter 45 } \\
N u_{\mathrm{Na}}=5.6+0.0165 \cdot \operatorname{Re}^{0.85} \cdot \operatorname{Pr}^{0.86} & \text { nach Chen \& Chiou 16 }
\end{array}
$$

Der Wärmeübergangskoeffizient ergibt sich aus

$$
\alpha=\frac{\lambda \cdot N u}{d}
$$

Mit Hilfe der bestrahlten Rohrfläche $\frac{\pi \cdot d}{2} L$ kann die Rohrlänge mit der thermischen Leistung und der vom Fluid absorbierten Wärmestromdichte beschrieben werden.

$$
\begin{gathered}
\dot{Q}_{\mathrm{th}}=\dot{q} \cdot \frac{\pi}{2} \cdot d \cdot L \\
\Rightarrow L=\frac{2 \cdot \dot{Q}_{\mathrm{th}}}{\pi \cdot \dot{q} \cdot d}
\end{gathered}
$$

Aus der fundamentalen Gleichung des konvektiven Wärmeübergangs lässt sich mit Gleichung (3.7) die Wärmestromdichte in Abhängigkeit des Wärmeüber- 
gangskoeffizienten berechnen:

$$
\begin{aligned}
& \dot{Q}_{\text {th }}=\alpha \cdot \frac{\pi d}{2} \cdot L \cdot\left(T_{\text {film }}-T_{\text {bulk }}\right) \\
& \Rightarrow \dot{q}=\alpha \cdot\left(T_{\text {film }}-T_{\text {bulk }}\right)
\end{aligned}
$$

Mit der Vorgabe der maximalen Filmtemperatur kann damit für jede Kombination aus Rohrdurchmesser und thermischer Leistung die maximale Wärmestromdichte berechnet werden.

Der Druckverlust ergibt sich mit Gleichung (3.2) und (3.7) zu

$$
\Delta p=\zeta \cdot \frac{L}{d} \cdot \rho \cdot \frac{u^{2}}{2}=\frac{\zeta \cdot 16 \cdot \dot{Q}_{\mathrm{th}}^{3}}{d^{6} \cdot \dot{q} \cdot \rho \cdot \pi^{3} \cdot\left(c_{p} \Delta T_{\text {bulk }}\right)^{2}}
$$

Die für den konvektiven Wärmeübergang bzw. für das Erreichen eines bestimmten Wärmeübergangskoeffizienten notwendige hydraulische Pumpenleistung, bei der die Einhaltung der maximalen Filmtemperatur gewährleistet ist, lässt sich folgendermaßen berechnen:

$$
P_{\mathrm{p}}=\dot{V} \cdot \Delta p=\frac{\dot{Q}_{\mathrm{th}}}{\left(\pi \cdot c_{p} \Delta T_{\mathrm{bulk}}\right)^{3}} \cdot \frac{\zeta \cdot 16 \cdot \dot{Q}_{\mathrm{th}}^{3}}{d^{6} \cdot \dot{q} \cdot \rho^{2}}
$$

Diese Pumpenleistung ist neben dem Temperaturhub im Absorberrohr $\Delta T_{\text {bulk }}$ noch von der ans Fluid übertragenen Leistung $\dot{Q}_{\text {th }}$ und dem Rohrdurchmesser $d$ abhängig. Die Wärmestromdichte $\dot{q}$ wird nach Gleichung (3.8) berechnet und ist ebenfalls von den drei Parametern $\Delta T_{\text {bulk }}, \dot{Q}_{\text {th }}$ und $d$ abhängig. Nach Entdimensionierung mit der thermischen Leistung und unter Berücksichtigung der Wirkungsgrade zur Wandlung in elektrische bzw. hydraulische Pumpenleistung ergibt sich die dimensionslose Pumpenleistung.

$$
P_{\mathrm{p}}^{*}=\frac{P_{\mathrm{p}}}{\dot{Q}_{\mathrm{th}}} \cdot \frac{1}{\eta_{\mathrm{PB}} \cdot \eta_{\mathrm{p}}}=\frac{\zeta \cdot 16 \cdot \dot{Q}_{\mathrm{th}}^{3}}{d^{6} \cdot \dot{q} \cdot \rho^{2} \cdot\left(\pi \cdot c_{p} \Delta T_{\mathrm{bulk}}\right)^{3}} \cdot \frac{1}{\eta_{\mathrm{PB}} \cdot \eta_{\mathrm{p}}}
$$

Für unterschiedliche Rohrdurchmesser und thermische Leistungen lässt sich Gleichung (3.11) für Solar Salt und Natrium jeweils als Kurvenschar darstellen (siehe Abbildung 3.7). Für die Abschätzung des Rohrreibungsbeiwerts $\zeta$ wird in diesem Vergleich die vereinfachte Gleichung nach Blasius mit $\zeta=0.3164 / R e^{0.25}$ verwendet [46]. 


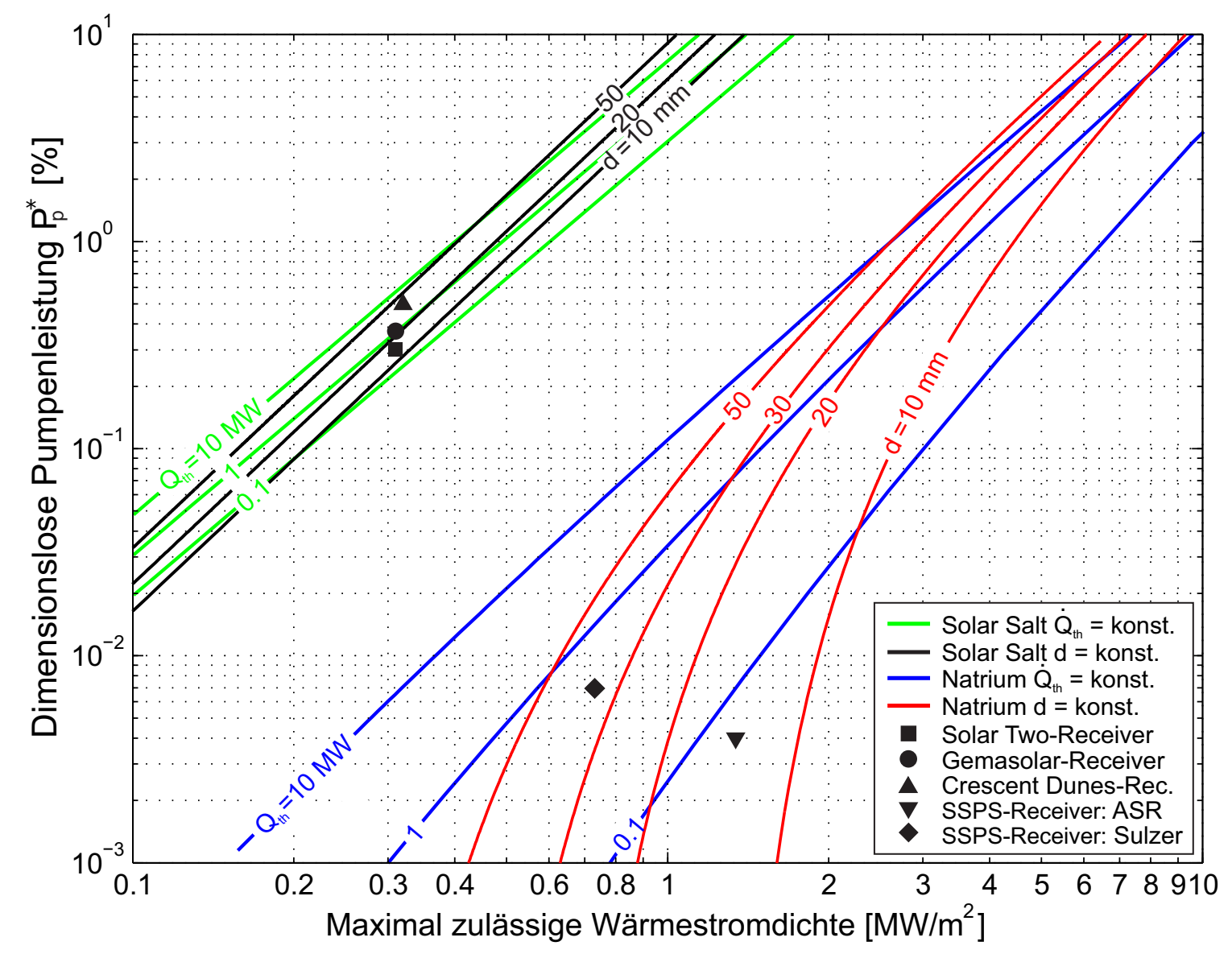

Abbildung 3.7.: Dimensionslose Pumpenleistung in Abhängigkeit der maximal zulässigen Wärmestromdichte (homogen auf halbem Rohrumfang) für verschiedene Durchmesser und thermische Leistungen. Rohrreibung nach Blasius: $\zeta=0.3164 / R e^{0.25}$ mit einem Kraftwerksblock- und Pumpenwirkungsgrad von $\eta_{\mathrm{PB}}=0.4$ und $\eta_{\mathrm{p}}=0.7$. Temperaturhub im Rohr: $\Delta T_{\text {bulk }}=290-565^{\circ} \mathrm{C}$. Maximale Filmtemperatur für Solar Salt und für Natrium: $T_{\text {film }}=600^{\circ} \mathrm{C}$.

Wenn die dimensionslose Pumpenleistung $P_{\mathrm{p}}^{*}=1$, dann wird die gesamte Brutto-Kraftwerksleistung für den Betrieb der Pumpe verwendet. Die dimensionslose Pumpenleistung bezieht sich dabei lediglich auf den Druckverlust im Absorberrohr ohne Verteiler, Sammler, Verbindungs- und Turmleitungen. Die dargestellten Ergebnisse beziehen sich auf eine über den halben Rohrumfang aufgebrachten, homogenen Wärmestromdichteverteilung. Im dargestellten Durchmesser- und Leistungsbereich ergibt sich für Solar Salt bei einer Beschränkung der Pumpenleistung auf 0.1 - 1\% der Brutto-Kraftwerksleistung eine Wärmestromdichte zwischen $0.1-0.6 \mathrm{MW} / \mathrm{m}^{2}$. Selbst bei einer um den Faktor 100 höheren Pumpenleistung ist die maximal zulässige Wärmestromdichte 
bei Solar Salt immernoch geringer als bei Natrium. Hinzu kommt, dass bei Natrium die hier dargestellte maximale Filmtemperatur von $T_{\text {film }}=600{ }^{\circ} \mathrm{C}$ nicht eingehalten werden muss, wodurch noch höhere Wärmestromdichten möglich sind. Die in Abbildung 3.7 angegebenen maximalen Wärmestromdichten gelten am Rohraustritt, wo die maximale Temperatur auftritt. An Orten mit $T_{\text {bulk }}<565^{\circ} \mathrm{C}$ können auch höhere Wärmestromdichten zugelassen werden. Daher ergibt sich eine Abweichung zwischen den hier angegebenen maximalen Wärmestromdichten und den Werten aus der Literatur, wo meistens die Maximalund Mittelwerte angegeben sind. Vant-Hull [47] berücksichtigt für Solar Salt zusätzlich noch die erhöhte Korrosion bei hohen Temperaturen, wodurch die Wärmestromdichte am Austritt auf $0.24 \mathrm{MW} / \mathrm{m}^{2}$ beschränkt wird.

Zusätzlich sind in Abbildung 3.7 für verschiedene realisierte Salz- und Natriumreceiver die sich ergebenden dimensionslosen Pumpenleistungen im Falle einer über den halben Rohrumfang aufgebrachten, homogenen Wärmestromdichteverteilung bei einer maximalen Filmtemperatur von $T_{\text {film }}=600{ }^{\circ} \mathrm{C}$ eingezeichnet $t^{\mathbb{}}$. Bei Solar Salt erhöht sich selbst bei einer Verdoppelung der Pumpenleistung die maximal zulässige Wärmestromdichte lediglich um ca. $0.1 \mathrm{MW} / \mathrm{m}^{2}$. In der Literatur wird daher häufig von einem Wärmestromdichtelimit bei Solar Salt gesprochen. Dieses Wärmestromdichtelimit ist allein durch die Stoffeigenschaften und die maximal zulässige Temperatur bestimmt und bei Solar Salt stets niedriger als die vom Heliostatenfeld verfügbare Wärmestromdichte [48. Daraus ergeben sich zwei Möglichkeiten für die Receiverauslegung: 1) Den Receiver zu vergrößern und die Zielpunkte der Heliostate auf der Receiverfläche verteilen, um dadurch die Wärmestromdichte zu reduzieren oder 2) Die Strömungsgeschwindigkeit (Turbulenz) zu erhöhen, um den Wärmeübergangskoeffizient zu steigern. Letztere Möglichkeit geht meist mit einem höheren Druckverlust einher, der eine höhere Pumpenleistung erfordert und damit den Systemwirkungsgrad senkt. Die erste Möglichkeit führt zu größeren thermischen Verlusten und erhöht das Receivergewicht und die thermische Masse. Bei der Auslegung von Salzreceivern muss daher ein Kompromiss zwischen Druckverlust und Absorberfläche gefunden werden. Im Projekt SolarTwo wurde der Receiver für eine

\footnotetext{
${ }^{4}$ Solar Two Einzelrohr: $d=18.6 \mathrm{~mm}, L=74.4 \mathrm{~m}, \dot{Q}_{\mathrm{th}}=0.66 \mathrm{MW}$, Gemasolar Einzelrohr: $d=21.7 \mathrm{~mm}, L=84.8 \mathrm{~m}, \dot{Q}_{\mathrm{th}}=0.94 \mathrm{MW}$, Crescent Dunes Einzelrohr: $d=38.7 \mathrm{~mm}, L=160 \mathrm{~m}, \dot{Q}_{\mathrm{th}}=3.55 \mathrm{MW}$, SSPS ASR Einzelrohr: $d=14 \mathrm{~mm}, L=14.3 \mathrm{~m}, \dot{Q}_{\mathrm{th}}=0.073 \mathrm{MW}$, SSPS Sulzer Einzelrohr: $d=35 \mathrm{~mm}, L=65.8 \mathrm{~m}, \dot{Q}_{\mathrm{th}}=0.47 \mathrm{MW}$.
} 
mittlere Wärmestromdichte von $0.43 \mathrm{MW} / \mathrm{m}^{2}$ ausgelegt mit einem Maximum von $0.83 \mathrm{MW} / \mathrm{m}^{2}$ [44, ,49]. Die Auslegung des Kraftwerks Gemasolar sieht eine leicht höhere Wärmestromdichte vor $\left(0.477 \mathrm{MW} / \mathrm{m}^{2}\right.$ mit einem Peak von: $1.0 \mathrm{MW} / \mathrm{m}^{2}$ ) 49. Wie Abbildung 3.7 zeigt, ist eine weitere Steigerung der Wärmestromdichte bei Solar Salt zwar theoretisch möglich, jedoch unwahrscheinlich, da die ohnehin schon hohe Pumpenleistung mit der Wärmestromdichte noch weiter ansteigt. Der geringe Wirkungsgradvorteil im Receiver durch die höhere Wärmestromdichte wird damit durch die dafür nötige, höhere Pumpenleistung vereitelt.

Bei der Auslegung von Salzreceivern wird stets versucht, die Absorberfläche zu minimieren, ohne die maximal zulässige Wärmestromdichte zu überschreiten. Bei Natrium ist diese Beschränkung durch die Stoffeigenschaften weit oberhalb der maximal vom Feld möglichen Wärmestromdichte und damit für die Auslegung nicht relevant. Grund hierfür ist die Wärmeleitfähigkeit, die im Vergleich zu Flüssigsalz um den Faktor 100-200 höher ist (siehe Stoffwerte Anhang A auf Seite 177), wodurch sehr hohe Wärmeübergangskoeffizienten erreicht werden. In Almeria wurde ein Natriumreceiver bereits experimentell mit Wärmestromdichten bis $2.5 \mathrm{MW} / \mathrm{m}^{2}$ getestet [29]. Die Spannungsuntersuchungen dieser Absorberrohre zeigen, dass maximale Wärmestromdichten bis $3 \mathrm{MW} / \mathrm{m}^{2}$ erreichbar sind. Folglich bietet Natrium die Möglichkeit für kleinere Receiver mit höheren Wirkungsgraden (geringere Absorberfläche führt zu geringeren Verlusten). Die Reduktion der Absorberfläche geht jedoch stets mit einer höheren Randstrahlung (engl. Spillage) einher. Die Verteilung der Zielpunkte der Heliostate (wie bei Salzreceivern notwendig) kann bei Natrium zwar auf einen Punkt reduziert werden, jedoch macht die Verkleinerung des Receivers nur unter gewissen Umständen Sinn. Aus thermodynamischer Sicht muss das Produkt aus Heliostatenfeld- und Receiverwirkungsgrad $\left(\eta_{\text {rec }} \cdot \eta_{\text {field }}\right)$ maximiert werden. Ökonomisch betrachtet müssen zusätzlich die Kosten von Heliostatenfeld und Receiver mit berücksichtigt werden. Kapitel 7 und 8 zeigen Parametervariationen der Absorberfläche zur Ermittlung der optimalen Receivergröße. 


\subsubsection{Receivergeometrie, Druckverlust und Wärmeübergang}

Die Stoffeigenschaften des verwendeten WTM haben einen maßgeblichen Einfluss auf die Receivergeometrie, den Druckverlust und den Wärmeübergang und damit auf den Wirkungsgrad, die Kosten und die Lebensdauer eines Receivers. In diesem Kapitel werden die Stoffwerte von Natrium und Blei-Bismut im Vergleich zu Solar Salt analysiert und ihre Auswirkungen auf die Receivergeometrie, den Druckverlust und den Wärmeübergang betrachtet. Für diese Abschätzung werden mittlere Stoffwerte verwendet und beim Vergleich zwischen Solar Salt und den Flüssigmetallen werden stets Receiver mit gleicher thermischen Leistung $\dot{Q}_{\text {th }}$ und gleicher Temperaturdifferenz $\Delta T_{\text {bulk }}$ zwischen Receiverein- und austritt betrachtet.

Eine wichtige Größe bei der Receiverauslegung ist der Volumenstrom. Aus der Energieerhaltung gilt:

$$
\dot{V}=n_{\mathrm{r}} \cdot n_{\mathrm{str}} \cdot \pi \cdot \frac{d_{\mathrm{r}}^{2}}{4} \cdot u_{\mathrm{r}}=\frac{\dot{Q}_{\mathrm{th}}}{\bar{\rho} \cdot \overline{c_{p}} \cdot \Delta T_{\mathrm{bulk}}}
$$

Die Größe $\left(n_{\mathrm{r}} \cdot n_{\mathrm{str}}\right)$ beschreibt dabei die Anzahl der parallel durchströmten Absorberrohre. Die volumetrische Wärmekapazität $\left(\rho \cdot c_{p}\right)$ von Solar Salt ist im Vergleich zu Natrium mehr als doppelt so hoch. Dadurch ergibt sich für Solar Salt ein verhältnismäßig niedriger Volumenstrom. Das Volumenstromverhältnis zwischen Solar Salt und Natrium bzw. Blei-Bismut (LBE) kann wie folgt abgeschätzt werden:

$$
\frac{\dot{V}_{\mathrm{Na}}}{\dot{V}_{\mathrm{SS}}}=\frac{\bar{\rho}_{\mathrm{SS}} \cdot \bar{c}_{p \mathrm{SS}}}{\bar{\rho}_{\mathrm{Na}} \cdot \bar{c}_{p \mathrm{Na}}} \approx 2.5 \quad \frac{\dot{V}_{\mathrm{LBE}}}{\dot{V}_{\mathrm{SS}}} \approx 1.9
$$

Um die kritische Reynolds-Zahl nicht zu unterschreiten um einen akzeptablen Wärmeübergang zu erhalten muss der verhältnismäßig niedrige Gesamtvolumenstrom bei Solar Salt durch einen kleinen Gesamtströmungsquerschnitt gepumpt werden, da $R e \sim \frac{\dot{V}}{d}$. Durch den geringeren Strömungsquerschnitt ergeben sich bei gleicher Absorberfläche stets längere Strömungswege im Receiver. Aus diesem Grund bestehen Receiver mit Solar Salt als WTM meist aus vielen seriell verschalteten Modulen mit jeweils wenig parallelen Rohren, in denen das Salz mäanderförmig strömt (2x12 Module bei Solar Two [50], 2x8 Module bei Gemasolar [51]). 
Bei externen, zylindrischen Rohrreceivern lässt sich die Absorberfläche nach Abbildung 3.8 berechnen.

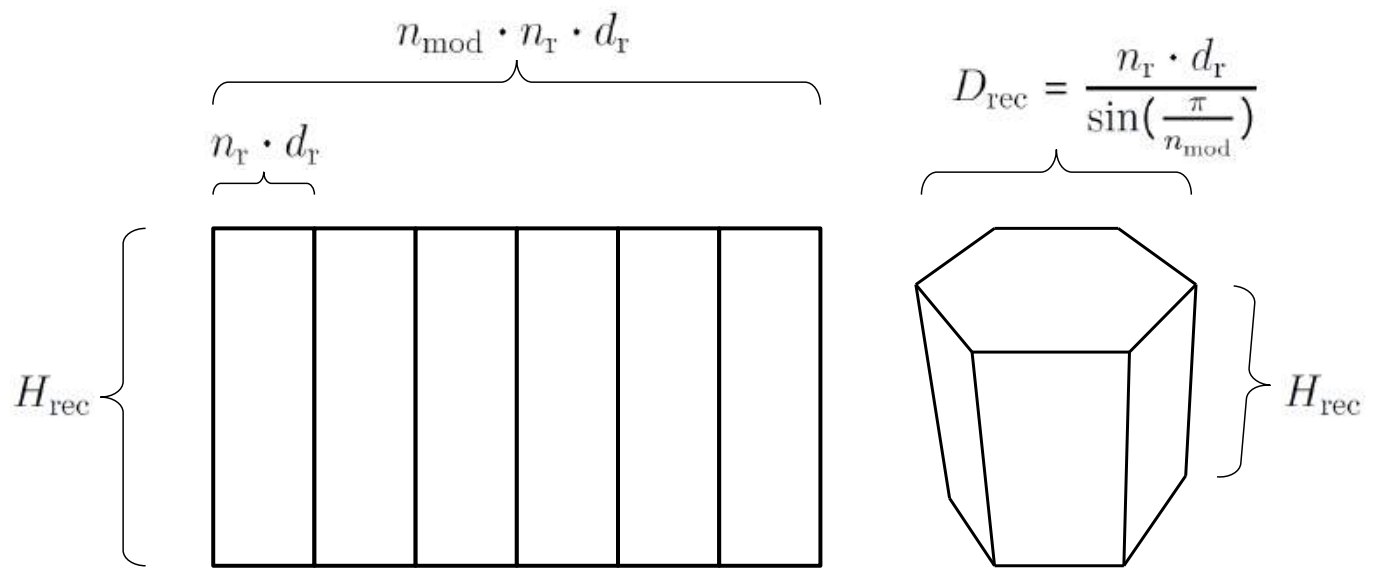

Abbildung 3.8.: Skizze eine zylindrischen Rohrreceivers

Mit der Anzahl und den Abmessungen der Absorberrohre sowie dem Verhältnis zwischen Receiverhöhe zu -durchmesser $H_{\text {rec }} / D_{\text {rec }}$ gilt für die projizierte Receiverfläche:

$$
A_{\text {rec }}=H_{\text {rec }} \cdot n_{\text {mod }} \cdot n_{\mathrm{r}} \cdot d_{\mathrm{r}}=\frac{H_{\mathrm{rec}}}{D_{\text {rec }}} \cdot \frac{n_{\mathrm{mod}} \cdot\left(n_{\mathrm{r}} \cdot d_{\mathrm{r}}\right)^{2}}{\sin \left(\frac{\pi}{n_{\mathrm{mod}}}\right)}=\frac{\dot{Q}_{\mathrm{th}}}{\dot{\dot{q}}}
$$

Damit lässt sich die Anzahl der mäanderförmig durchströmten Module folgendermaßen beschreiben:

$$
n_{\text {mod }}=\frac{\dot{Q}_{\mathrm{th}}}{H_{\mathrm{rec}} \cdot n_{\mathrm{r}} \cdot d_{\mathrm{r}} \cdot \dot{\dot{q}}}
$$

Gleichung (3.15) beschreibt die Gesamtanzahl an Modulen, unabhängig davon, ob diese parallel oder seriell durchströmt werden.

Die Strömungsgeschwindigkeit in den Absorberrohren wird mit der ReynoldsZahl und der dynamischen Viskosität $\eta$ wie folgt beschrieben:

$$
u_{\mathrm{r}}=\frac{R e \cdot \eta}{d_{\mathrm{r}} \cdot \rho}
$$

Wird Gleichung (3.12) nach $n_{\mathrm{r}} \cdot d_{\mathrm{r}}$ umgestellt und für die Strömungsgeschwindigkeit Gleichung (3.16) eingesetzt ergibt sich mit der Prandtl-Zahl $\operatorname{Pr}=\eta \cdot c_{p} / \lambda$ 
folgende Gleichung:

$$
\begin{aligned}
\frac{n_{\mathrm{r}, \mathrm{Na}} \cdot d_{\mathrm{r}, \mathrm{Na}}}{n_{\mathrm{r}, \mathrm{SS}} \cdot d_{\mathrm{r}, \mathrm{SS}}} & =\frac{c_{p \mathrm{SS}} \cdot \eta_{\mathrm{SS}}}{c_{p \mathrm{Na}} \cdot \eta_{\mathrm{Na}}} \cdot \frac{R e_{\mathrm{SS}}}{R e_{\mathrm{Na}}} \\
& =\frac{P r_{\mathrm{SS}} \cdot \lambda_{\mathrm{SS}}}{P r_{\mathrm{Na}} \cdot \lambda_{\mathrm{Na}}} \cdot \frac{R e_{\mathrm{SS}}}{R e_{\mathrm{Na}}} \approx 7,18 \cdot \frac{R e_{\mathrm{SS}}}{R e_{\mathrm{Na}}}
\end{aligned}
$$

Aufgrund der dynamischen Wärmestromdichteverteilung wird der Massenstrom im Receiver häufig in zwei Strömungswege unterteilt. Werden zwei Receiver mit gleicher Modulverschaltung miteinander verglichen (z.B. $n_{\text {str }}=2$ Strömungswege wie in Abbildung 5.10, Seite 84), kann die Gesamtlänge eines Strömungswegs mit Abbildung 3.8 berechnet werden: $L_{\mathrm{str}}=H_{\mathrm{rec}} \cdot \frac{n_{\mathrm{mod}}}{n_{\mathrm{str}}}$. Das Verhältnis der Gesamtströmungsweglängen ergibt sich damit aus Gleichung (3.15) und Einsetzen von Gleichung (3.17):

$$
\begin{aligned}
\frac{L_{\mathrm{str}, \mathrm{SS}}}{L_{\mathrm{str}, \mathrm{Na}}} & =\frac{H_{\mathrm{rec}, \mathrm{SS}} \cdot n_{\mathrm{mod}, \mathrm{SS}}}{H_{\mathrm{rec}, \mathrm{Na}} \cdot n_{\mathrm{mod}, \mathrm{Na}}}=\frac{n_{\mathrm{r}, \mathrm{Na}} \cdot d_{\mathrm{r}, \mathrm{Na}}}{n_{\mathrm{r}, \mathrm{SS}} \cdot d_{\mathrm{r}, \mathrm{SS}}} \cdot \frac{\overline{\dot{q}}_{\mathrm{Na}}}{\overline{\dot{q}}_{\mathrm{SS}}} \\
& =\frac{P r_{\mathrm{SS}} \cdot \lambda_{\mathrm{SS}}}{P r_{\mathrm{Na}} \cdot \lambda_{\mathrm{Na}}} \cdot \frac{\overline{\dot{q}}_{\mathrm{Na}}}{\overline{\dot{q}}_{\mathrm{SS}}} \cdot \frac{R e_{\mathrm{SS}}}{R e_{\mathrm{Na}}} \approx 7,18 \cdot \frac{\dot{\dot{q}}_{\mathrm{Na}}}{\dot{\bar{q}}_{\mathrm{SS}}} \cdot \frac{R e_{\mathrm{SS}}}{R e_{\mathrm{Na}}}
\end{aligned}
$$

Abbildung 3.9 zeigt dieses Verhältnis graphisch in Abhängigkeit der relativen mittleren Wärmestromdichte und dem Verhältnis der Reynoldszahlen.

Daraus wird deutlich, dass die Receivergeometrie sehr stark vom WTM abhängt. Die benötigte Strömungsweglänge im Receiver mit Solar Salt ist folglich bei gleicher Absorberfläche $\left(\overline{\dot{q}}_{\mathrm{Na}} / \overline{\dot{q}}_{\mathrm{SS}}=1\right)$ und gleicher Reynolds-Zahl 7,18 mal länger. Demnach wäre auch die Anzahl der Module bei Solar Salt entsprechend höher. Wird beispielsweise die Absorberfläche bei Natrium halbiert $\left(\overline{\dot{q}}_{\mathrm{Na}} / \overline{\dot{q}}_{\mathrm{SS}}=2\right)$ und die bestrahlte Rohrlänge ebenfalls halbiert $\left(\frac{L_{\mathrm{SS}}}{L_{\mathrm{Na}}}=2\right)$, so ergibt sich ein Reynolds-Zahl-Verhältnis von $R e_{\mathrm{Na}} / R e_{\mathrm{SS}} \approx 7$. Folglich bestehen Natriumreceiver tendenziell aus wenigen Modulen mit vielen parallel durchströmten Rohren, wohingegen Receiverkonstruktionen mit Solar Salt meist viele Module mit jeweils wenig parallelen Rohren aufweisen. Je nach Anforderung an den Receiver (Teillastverhalten und Druckverlust) ergibt sich ein Bereich an Modul- und Rohranzahlen für die Auslegung. Da aus optischen Gründen die Anzahl der seriell durchströmten Module (und damit die bestrahlte Strömungsweglänge) nicht beliebig reduziert werden kann, erreichen Natriumreceiver stets deutlich 


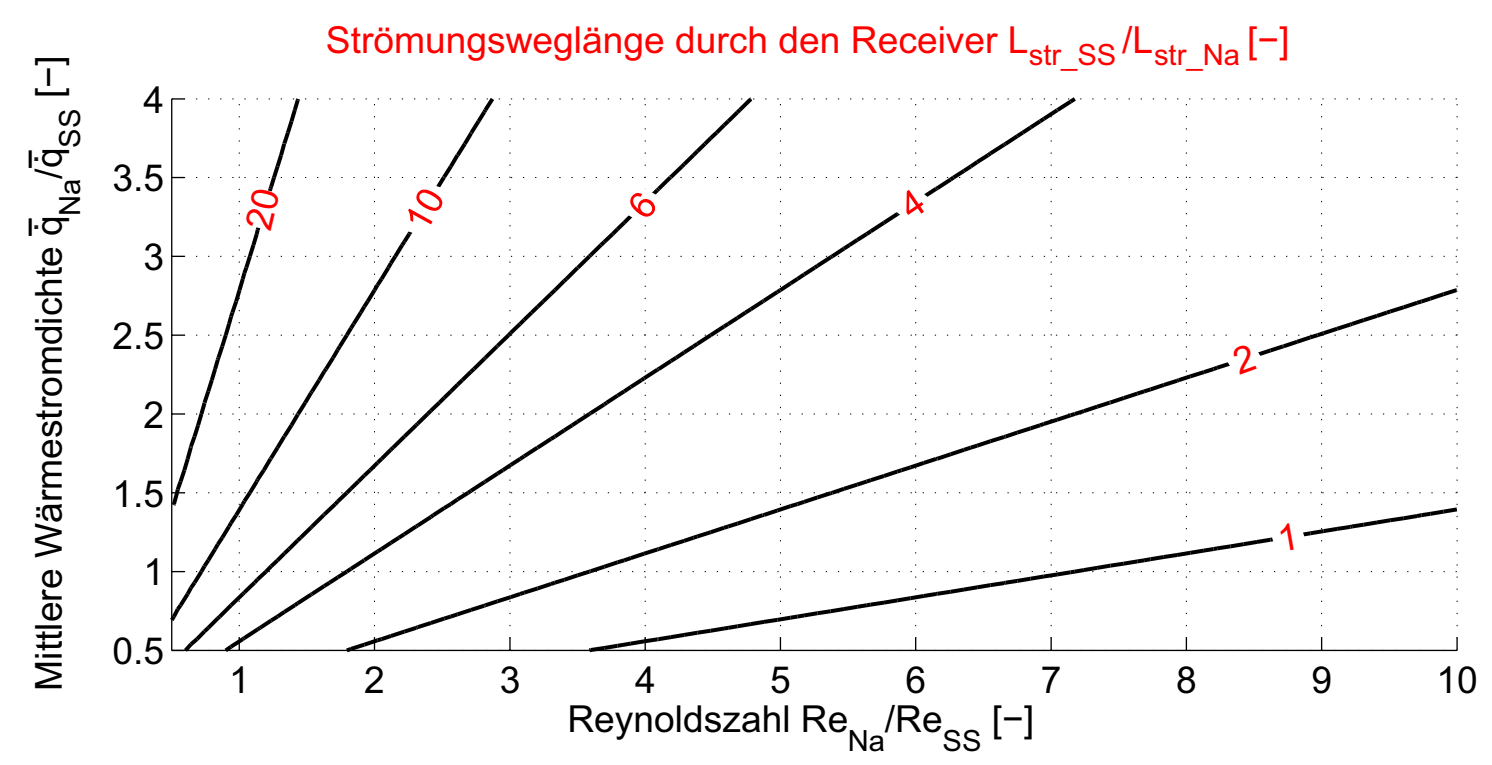

Abbildung 3.9.: Verhältnis der Strömungsweglängen zwischen Solar Salt und Natrium in Abhängigkeit der relativen mittleren Wärmestromdichte und dem Verhältnis der Reynoldszahlen bei gleicher thermischer Receiverleistung $\dot{Q}_{\text {th }}$, gleichem Temperaturunterschied $\Delta T_{\text {bulk }}$ zwischen Ein- und Auslass

höhere Reynolds-Zahlen. Die kritische Reynolds-Zahl wird somit im Teillastbetrieb mit Natrium erst bei deutlich geringerem Wärmeeintrag erreicht, wodurch ein größerer Betriebsbereich entsteht.

Bei der Receiverauslegung spielt neben dem Receiverwirkungsgrad auch der Druckverlust eine wichtige Rolle. Unter Vernachlässigung von Ein- und Auslaufeffekten gilt nach [46] für den Druckverlust eines Stromfadens durch den Receiver:

$$
\Delta p=\zeta \cdot \frac{L_{\mathrm{str}}}{d_{\mathrm{r}}} \cdot \rho \cdot \frac{u_{\mathrm{r}}^{2}}{2}=\zeta \cdot \frac{n_{\mathrm{mod}} \cdot H_{\mathrm{rec}}}{n_{\mathrm{str}} \cdot d_{\mathrm{r}}} \cdot \rho \cdot \frac{u_{\mathrm{r}}^{2}}{2}
$$

Mit den Gleichung (3.15) und (3.12) kann der Druckverlust wie folgt dargestellt werden:

$$
\begin{aligned}
\Delta p & =\zeta \cdot \frac{\dot{Q}_{\mathrm{th}}}{\overline{\dot{q}} \cdot n_{\mathrm{r}} \cdot n_{\mathrm{str}} \cdot d_{\mathrm{r}}^{2}} \cdot \rho \cdot \frac{u_{\mathrm{r}}^{2}}{2} \\
& =\zeta \cdot \frac{c_{p} \cdot \Delta T_{\mathrm{bulk}} \cdot \pi}{\overline{\dot{q}}} \cdot \rho^{2} \cdot \frac{u_{\mathrm{r}}^{3}}{8}
\end{aligned}
$$


Für den Vergleich zwischen Natrium und Solar Salt lässt sich damit das Verhältnis der Druckverluste folgendermaßen abschätzen:

$$
\begin{aligned}
\frac{\Delta p_{\mathrm{SS}}}{\Delta p_{\mathrm{Na}}} & =\frac{\zeta_{\mathrm{SS}}}{\zeta_{\mathrm{Na}}} \cdot \frac{c_{p \mathrm{SS}}}{c_{p \mathrm{Na}}} \cdot \frac{\overline{\dot{q}}_{\mathrm{Na}}}{\overline{\dot{q}}_{\mathrm{SS}}} \cdot\left(\frac{\rho_{\mathrm{SS}}}{\rho_{\mathrm{Na}}}\right)^{2} \cdot\left(\frac{u_{\mathrm{r}, \mathrm{SS}}}{u_{\mathrm{r}, \mathrm{Na}}}\right)^{3} \\
& \approx 5,45 \cdot \frac{\zeta_{\mathrm{SS}}}{\zeta_{\mathrm{Na}}} \cdot \frac{\dot{\dot{q}}_{\mathrm{Na}}}{\overline{\dot{q}}_{\mathrm{SS}}} \cdot\left(\frac{u_{\mathrm{r}, \mathrm{SS}}}{u_{\mathrm{r}, \mathrm{Na}}}\right)^{3}
\end{aligned}
$$

Dieser Zusammenhang ist für $\zeta_{\mathrm{SS}} / \zeta_{\mathrm{Na}}=1$ in Abbildung 3.10 dargestellt. Für übliche Rohrgeometrien und Strömungsgeschwindigkeiten ergeben sich leicht niedrigere Werte für $\zeta_{\mathrm{Na}}$ im Vergleich zu $\zeta_{\mathrm{SS}}$. Zur Abschätzung der Größenordnung ist diese Annahme jedoch zielführend.

Gleichung (3.21) berücksichtigt nur den Druckverlust der bestrahlten Rohrlänge ohne Umlenkungen, plötzlichen Querschnittsänderungen und Verbindungsrohre zwischen den Modulen. Durch die geringfügig höheren Druckverlustbeiwerte bei Solar Salt und der tendenziell höheren Anzahl an Verbindungsrohren zwischen den Modulen (siehe Gleichung (3.18)) stellt Gleichung (3.21) den Minimalwert für den Druckverlustquotienten dar.

Bei gleicher Absorberfläche und gleicher Strömungsgeschwindigkeit ergibt sich damit ein um den Faktor 5,45 höherer Druckverlust bei Solar Salt. Wird die Absorberfläche bei Natrium halbiert $\left(\overline{\dot{q}}_{\mathrm{Na}} / \overline{\dot{q}}_{\mathrm{SS}}=2\right)$ und zusätzlich die Strömungsgeschwindigkeit reduziert, erhöht sich dieser Faktor auf $10-100$.

In Abbildung 3.6 ist der Wärmeübergangskoeffizient in Abhängigkeit der Temperatur für verschiedene Flüssigmetalle dargestellt. Wie bereits erwähnt ist der Wärmeübergangskoeffizient zudem über die Nusselt-Zahl maßgeblich von der Strömung abhängig. Meist führen höhere Wärmeübergangskoeffizienten auch zu höheren Druckverlusten. Dies erhöht einerseits den thermischen Wirkungsgrad, führt aber andererseits zu einer höheren Pumpenleistung. Zur Bewertung eines WTMs hinsichtlich des Wärmeübergangs muss folglich stets der Druckverlust mitberücksichtigt werden.

Die hydraulische Pumpenleistung eines einzelnen Absorberrohres (komplette Strömungsweglänge durch einen Receiver) ergibt sich aus den Gleichungen (3.12) und (3.20). Mit $n_{\mathrm{r}}=1$ und $n_{\mathrm{str}}=1$ gilt:

$$
\dot{V} \cdot \Delta p=\dot{Q}_{\mathrm{th}} \cdot \zeta \cdot \frac{\pi \cdot \bar{\rho}}{\overline{\dot{q}}} \cdot \frac{u_{\mathrm{r}}^{3}}{8}
$$




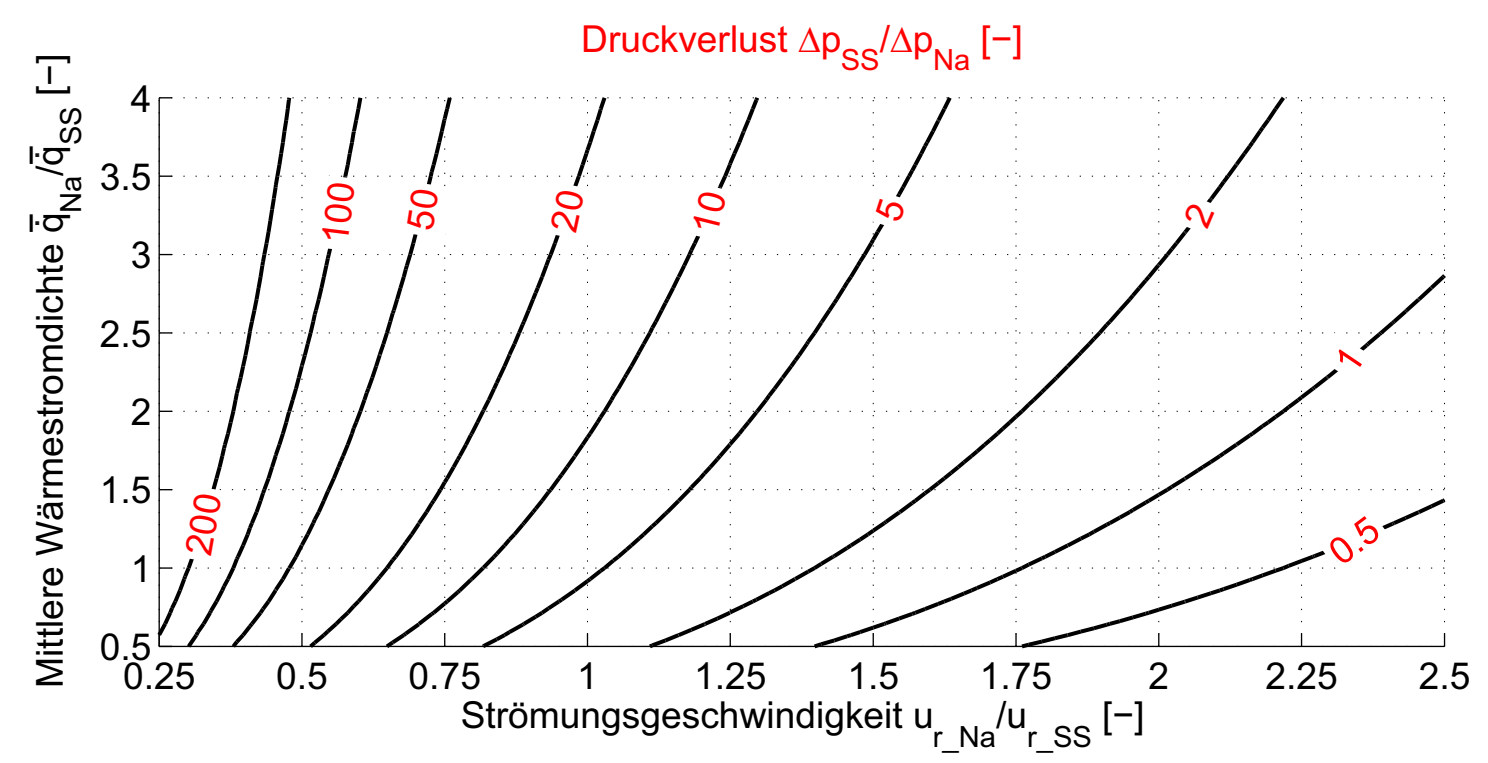

Abbildung 3.10.: Verhältnis der Druckverluste zwischen Solar Salt und Natrium in Abhängigkeit der relativen mittleren Wärmestromdichte und dem Strömungsgeschwindigkeitsverhältnis bei gleicher thermischer Receiverleistung $\dot{Q}_{\text {th }}$ und gleichem Temperaturunterschied $\Delta T_{\text {bulk }}$ zwischen Ein- und Auslass für $\zeta_{\mathrm{SS}} / \zeta_{\mathrm{Na}}=1$ ohne Berücksichtigung von Umlenkungen, plötzlichen Querschnittsänderungen und Verbindungsrohren zwischen den Modulen

Wird die Strömungsgeschwindigkeit durch Gleichung 3.12 ersetzt, ergibt sich

$$
\dot{V} \cdot \Delta p=\frac{\dot{Q}_{\mathrm{th}}^{4} \cdot \zeta \cdot 8}{\overline{\dot{q}} \cdot\left(\overline{c_{p}} \cdot \Delta T_{\mathrm{bulk}}\right)^{3} \cdot(\bar{\rho} \cdot \pi)^{2} \cdot d_{\mathrm{r}}^{6}}
$$

Damit lässt sich der Rohrdurchmesser in Abhängigkeit von der Pumpenleistung beschreiben:

$$
d_{\mathrm{r}}=\sqrt[6]{\frac{\dot{Q}_{\mathrm{th}}^{4} \cdot \zeta \cdot 8}{(\dot{V} \cdot \Delta p) \cdot \overline{\dot{q}} \cdot\left(\overline{c_{p}} \cdot \Delta T_{\mathrm{bulk}}\right)^{3} \cdot(\bar{\rho} \cdot \pi)^{2}}}
$$

Mit Gleichung (3.24) kann aus 3.12 die Strömungsgeschwindigkeit , die Reynolds-Zahl (3.16) und die Nusselt-Zahl (Korrelationen im Anhang E, Seite 187 berechnet werden. Da der Rohrreibungskoeffizient $\zeta$ vom Rohrdurchmesser und der Reynolds-Zahl abhängt, muss Gleichung 3.24 und damit auch 
$u_{\mathrm{r}}$, Re und $N u$ iterativ bestimmt werden. Nach [46] gilt für $\zeta$ :

$$
\frac{1}{\sqrt{\zeta}}=-2 \log _{10}\left[\frac{2.51}{R e \cdot \sqrt{\zeta}}+\frac{\mu}{3.71 \cdot d_{\mathrm{r}}}\right]
$$

Der Wärmeübergangskoeffizient ergibt sich schließlich aus:

$$
\alpha=\frac{\lambda \cdot N u}{d_{\mathrm{r}}}
$$

Abbildung 3.11 zeigt die Wärmeübergangskoeffizienten und die Reynolds-Zahlen in Abhängigkeit der hydraulischen Leistung für ein einzelnes Absorberrohr mit einer thermischen Leistung von $2.5 \mathrm{MW}_{\text {th }}$ mit $\Delta T_{\text {bulk }}=290-565^{\circ} \mathrm{C}$ für Solar Salt, Natrium und Blei-Bismut5

Je größer die hydraulische Leistung der Pumpe, desto höher der Wärmeübergangskoeffizient. Die linke Seite des Diagramms stellt den Extremfall ohne Massenstrom dar. Der Wärmeübergang beschränkt sich hier auf die Wärmeleitung. Natrium erreicht im Vergleich zu Solar Salt und Blei-Bismut schon bei geringer Pumpenleistung sehr hohe Wärmeübergangskoeffizienten. Außerdem ist der Gradient bei Natrium am größten, sodass eine geringe Steigerung der Pumpenleistung den Wärmeübergang im Vergleich zu Solar Salt und Blei-Bismut deutlich stärker erhöht. Des Weiteren führt die niedrige Viskosität von Natrium in Verbindung mit der niedrigen Dichte zu sehr hohen Reynolds-Zahlen und zu einem geringen Druckverlust. Bei Blei-Bismut führt die hohe Dichte einerseits zu sehr hohen Reynolds-Zahlen, andererseits aber auch zu hohen Druckverlusten. Bezogen auf die Pumpenleistung ergeben sich damit niedrigere Wärmeübergangskoeffizienten als bei Solar Salt.

Aus Abbildung 3.11 ergeben sich für $(\dot{V} \cdot \Delta p=$ konst. $)$ die folgenden Verhältnisse:

$$
\begin{array}{rlrl}
\alpha_{\mathrm{Na}} / \alpha_{\mathrm{SS}} & \approx 3.6 & R e_{\mathrm{Na}} / \operatorname{Re}_{\mathrm{SS}} & \approx 5.15 \\
\alpha_{\mathrm{LBE}} / \alpha_{\mathrm{SS}} & \approx 0.82 & R e_{\mathrm{LBE}} / \operatorname{Re}_{\mathrm{SS}} & \approx 7.19
\end{array}
$$

${ }^{5}$ Typischer Wert eines Absorberrohres eines $140 \mathrm{MW}_{\mathrm{th}}$-Receivers mit 56 parallelen Absorberrohren (2x28 Rohre) 


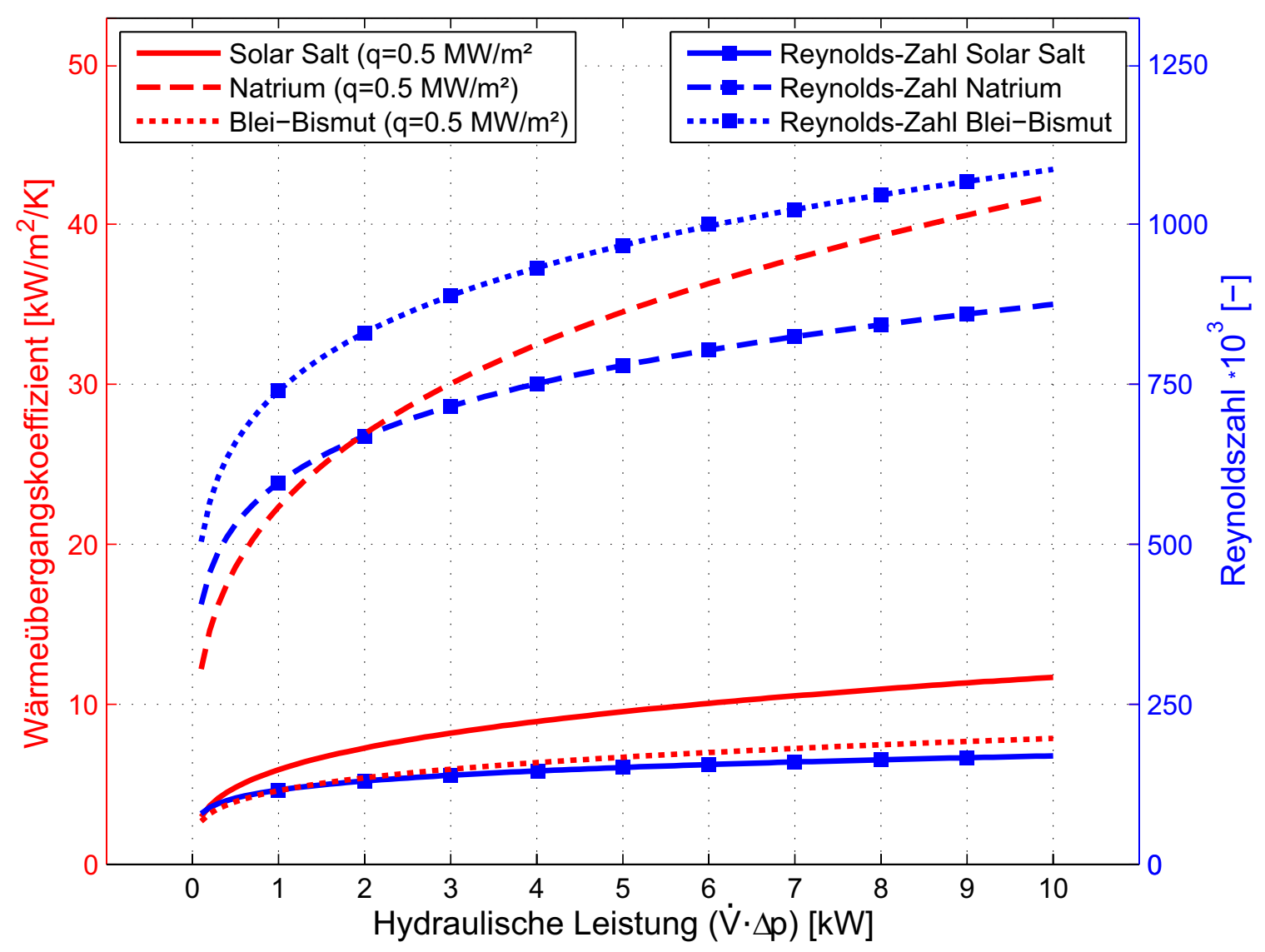

Abbildung 3.11.: Wärmeübergangskoeffizient und Reynolds-Zahl in Abhängigkeit der hydraulischen Leistung für ein Absorberrohr mit einer thermischen Leistung von $\dot{Q}_{\text {th }}=2.5 \mathrm{MW}_{\text {th }}$ für Solar Salt, Natrium und BleiBismut bei einer mittleren Wärmestromdichte von $\overline{\dot{q}}=0.5 \mathrm{MW} / \mathrm{m}^{2}$ und einem Temperaturunterschied zwischen Ein- und Austritt von $\Delta T_{\text {bulk }}=290-565^{\circ} \mathrm{C}$. Strömungsumlenkungen, Armaturen und Verbindungsrohre sind vernachlässigt.

Diese Verhältnisse bleiben nahezu konstant ${ }^{6}$, unabhängig von der Wahl von $\dot{Q}_{\mathrm{th}}$, $\overline{\dot{q}}$ und $\Delta T_{\text {bulk. }}$. Je nach Wahl dieser Parameter verschieben sich die Kurven in Abbildung 3.11 entsprechend den angegebenen Verhältnissen.

Die sich ergebenden Rohrdurchmesser aus Gleichung (3.24) sind für Solar Salt, Natrium und Blei-Bismut in Abbildung 3.12 dargestellt und stehen in direktem Zusammenhang mit den Wärmeübergangskoeffizienten und Reynolds-Zahlen aus Abbildung 3.11. Je größer die Pumpenleistung, desto kleiner wird der Rohrdurch-

\footnotetext{
${ }^{6}$ Die Sensitivitätsanalyse beinhaltet folgende Wertebereiche: $\Delta T_{\text {bulk }}=[175 \ldots 375]^{\circ} \mathrm{C}$, $\overline{\dot{q}}=[0.25 \ldots 1.0] \mathrm{MW} / \mathrm{m}^{2}$ und $\dot{Q}_{\mathrm{th}}=[1.5 \ldots 3.5] \mathrm{MW}$.

Innerhalb der angegebenen Intervalle ändert sich das Verhältnis der Wärmeübergangskoeffizienten um maximal $9.4 \%$ bezogen auf den Referenzwert. Den größten Einfluss auf das Verhältnis der Reynolds-Zahlen hat $\Delta T_{\text {bulk }}$ mit einer maximalen Abweichung von $14.3 \%$.
} 


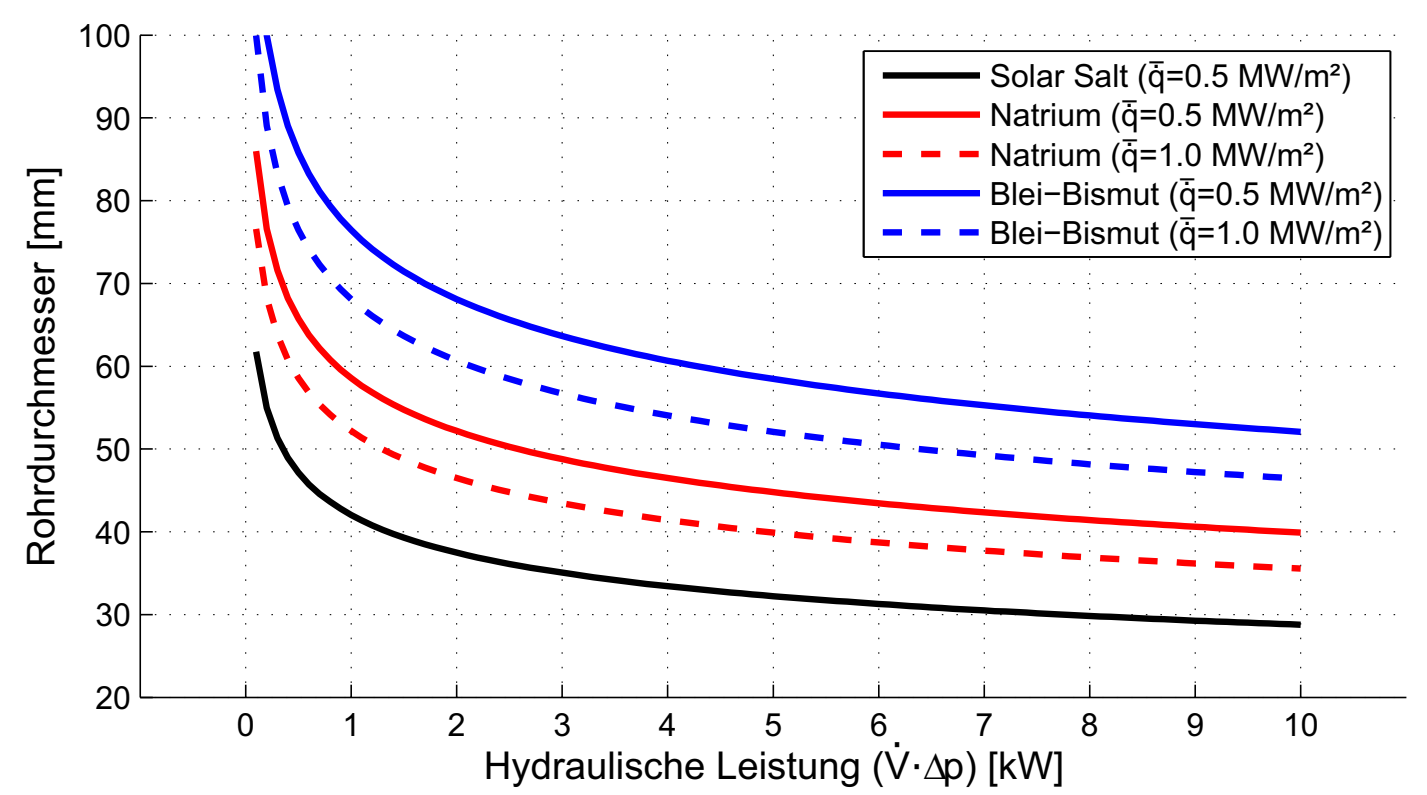

Abbildung 3.12.: Rohrdurchmesser in Abhängigkeit der hydraulischen Leistung für ein Absorberrohr mit einer thermischen Leistung von $\dot{Q}_{\text {th }}=$ $2.5 \mathrm{MW}_{\text {th }}$ für Solar Salt, Natrium und Blei-Bismut bei einem Temperaturunterschied zwischen Ein- und Austritt von $\Delta T_{\text {bulk }}=290-$ $565^{\circ} \mathrm{C}$ und einer mittleren Wärmestromdichte von $\overline{\dot{q}}=0.5 \mathrm{MW} / \mathrm{m}^{2}$ bzw. $\overline{\dot{q}}=1.0 \mathrm{MW} / \mathrm{m}^{2}$. Strömungsumlenkungen, Armaturen und Verbindungsrohre sind vernachlässigt.

messer. Der größere Volumenstrom der Flüssigmetalle (siehe Gleichung (3.13)) erfordert bei gleicher Pumpenleistung auch größere Rohrdurchmesser. Wird die mittlere Wärmestromdichte erhöht, reduziert sich damit bei gleicher thermischer Leistung die Absorberfläche und damit auch der Rohrdurchmesser (siehe Gleichung (3.24)).

Aus Abbildung 3.10 und 3.11 wird deutlich, dass die Receiverauslegung mit Natrium deutlich mehr Freiheiten bietet als mit Solar Salt, da in einem großen Parameterraum von Absorberfläche, Modulanzahl, Rohranzahl und -abmessungen sehr niedrige Druckverluste erreicht werden bei gleichzeitig hohen ReynoldZahlen und hohen Wärmeübergangskoeffizienten. Die Auslegung von Salzreceivern hingegen orientiert sich stets an der maximal zulässigen Wärmestromdichte (siehe Abschnitt 3.4.2) und muss hohe Druckverluste in Kauf nehmen, um einen akzeptablen Wärmeübergang zu erreichen.

Bei Blei-Bismut ist dieses Wärmestromdichtelimit zwar höher als bei Solar Salt, jedoch führt der niedrige Wärmeübergangskoeffizient zu hohen Rohrtemperaturen, die sich negativ auf die Lebensdauer auswirken können. 
Neben dem Einfluss auf die Receivergeometrie haben die Stoffeigenschaften des WTMs darüber hinaus ebenfalls Einfluss auf die Turmkonstruktion und das dafür notwendige Fundament. Die Receivergesamtmasse und damit die Dichte des WTMs ist hierfür maßgeblich. Ein Natriumreceiver ist nur etwa halb so schwer wie ein Salzreceiver gleicher Leistung. Ein Blei-Bismut-Receiver hingegen wiegt etwa das 5-fache. Da die vorhandenen Turmkostenkorrelationen das Receivergewicht nicht mitberücksichtigen, wird dieser Effekt in dieser Arbeit nicht weiter betrachtet.

\subsubsection{Begleitheizung, Korrosion und Lebensdauer}

In Solarkraftwerken mit Flüssigsalztechnologie ist stets darauf zu achten, dass das Salz in flüssigem Zustand bleibt. Ansonsten können Leitungen verstopfen, was zum Ausfall der gesamten Anlage führt. Daher werden sämtliche Rohrleitungen und Ventile mit Begleitheizungen ausgestattet, die Nachts und bei geringer Sonneneinstrahlung den Salzkreislauf auf Betriebstemperatur halten. Beim Solarkraftwerk Solar Two betrug die tägliche elektrische Energie für die Begleitheizung 6.83 $\mathrm{MWh}_{\mathrm{el}}$ (Online-Days) und 9.7 $\mathrm{MWh}_{\mathrm{el}}$ (Offline-Days) 44]. Selbst mit optimierter Beheizungsstrategie konnte die tägliche Heizenergie nicht unter $4 \mathrm{MWh}_{\mathrm{el}} / \mathrm{Tag}$ gesenkt werden, unabhängig davon, wieviel Energie vom Receiver täglich absorbiert wurde (zw. 0-250 $\mathrm{MWh}_{\text {th }}$ ).

Die hier betrachteten Flüssigmetalle besitzen alle eine geringere Energiedichte $\left(\rho \cdot c_{p}\right)$ als Solar Salt und die Alkalimetalle sowie Blei-Bismut haben zusätzlich niedrigere Schmelztemperaturen (siehe Abbildung 3.5). Daher fällt die zu erwartende Heizenergie bei Flüssigmetallen deutlich geringer aus. Bei Solar Salt werden die Heizdrähte gleichmäßig um den Rohrumfang verteilt, um ein gleichmäßiges Auftauen ohne Überhitzung zu gewährleisten. Bei den Alkalimetallen wird erwartet, dass ein einzelner Heizdraht entlang des Rohres genügt, da die hohe Wärmeleitfähigkeit die Wärme gleichmäßig auf den gesamten Rohrquerschnitt verteilt.

Im Betrieb kommen die WTM mit verschiedenen Strukturmaterialien (Rohre, Tanks, Dichtungen) in Kontakt. Besonders bei hohen Temperaturen treten vermehrt chemische Reaktionen (Korrosion) auf. Flüssigsalze können diese Korrosion erheblich beschleunigen. Im Betrieb eines Salzsystems kommt es dadurch 
zur Verunreinigung des WTMs und zu Ablagerungen an der Rohrinnenwand, die den Wärmeübergang reduzieren (Fouling genannt). Bei Flüssigsalzen gilt $R_{\mathrm{f}}=8.808 \times 10^{-5} \mathrm{~m}^{2} \mathrm{~K} / \mathrm{W} 52,53$, wodurch der Wärmeübergangskoeffizient um bis zu $50 \%$ reduziert wird. Dadurch steigen die Rohrtemperaturen auf der bestrahlten Rohrseite um ca. $70{ }^{\circ} \mathrm{C}[54]$.

Bei Alkalimetallen spielt Korrosion eine untergeordnete Rolle und Fouling kann vernachlässigt werden [54]. Die in Solarreceivern häufig verwendeten NickelBasislegierungen (Inconel) können bei Alkalimetallen daher vermutlich problemlos verwendet werden.

Die Verträglichkeit von Blei-Bismut mit Strukturmaterialien nimmt bei hohen Temperaturen immer weiter ab. Sowohl Korrosion als auch die hohe Löslichkeit von Legierungselementen der Stähle im Blei-Bismut erfordern eine aktive Sauerstoffkontrolle. Bis zu einer Strömungsgeschwindigkeit von etwa $2 \mathrm{~m} / \mathrm{s}$ kann mit einem kontrollierten Sauerstoffpotenzial eine schützende Oxidschicht aufrechterhalten werden [55]. Höhere Strömungsgeschwindigkeiten führen zu sehr schneller Erosion/Korrosion. Für höhere Temperaturen eignen sich Refraktärmetalle. Gegenstand der Forschung sind insbesondere auch schützende Keramikbeschichtungen [56]. Tabelle 3.6 zeigt eine Bewertung von Fachleuten bezüglich der Verträglichkeit von Flüssigmetallen mit verschiedenen Strukturmaterialien bei unterschiedlichen Temperaturen.

Solarturmreceiver sind hochbelastete Bauteile, die hohen Temperaturen und Temperaturgradienten ausgesetzt sind. Eine Dauerfestigkeit kann daher nicht erreicht werden, sondern lediglich eine Zeitstandsfestigkeit. Folgende Vorgänge müssen bei der Lebensdauerabschätzung berücksichtigt werden:

- Zyklische Ermüdung (Bauteilermüdung durch Risswachstum)

- Kriechen

- Reduktion der tragenden Wandstärke infolge Korrosion

- Wechselwirkung zwischen den Mechanismen

Bei Natriumreceivern ist die Belastung durch Innendruck stets geringer als bei Salzreceivern (siehe Abschnitt 3.4.3). Zudem ist die statische Last durch die geringere Dichte und die tendenziell kleineren Receiver ebenfalls geringer. Folglich sind bei Natriumreceivern geringere Primärspannungen zu erwarten. Auch die Korrosion ist bei Natrium weniger problematisch. Durch die hohe 


\begin{tabular}{|c|c|c|c|c|}
\hline 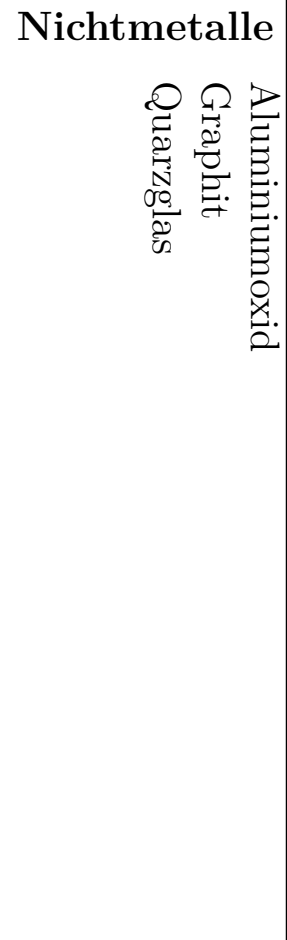 & 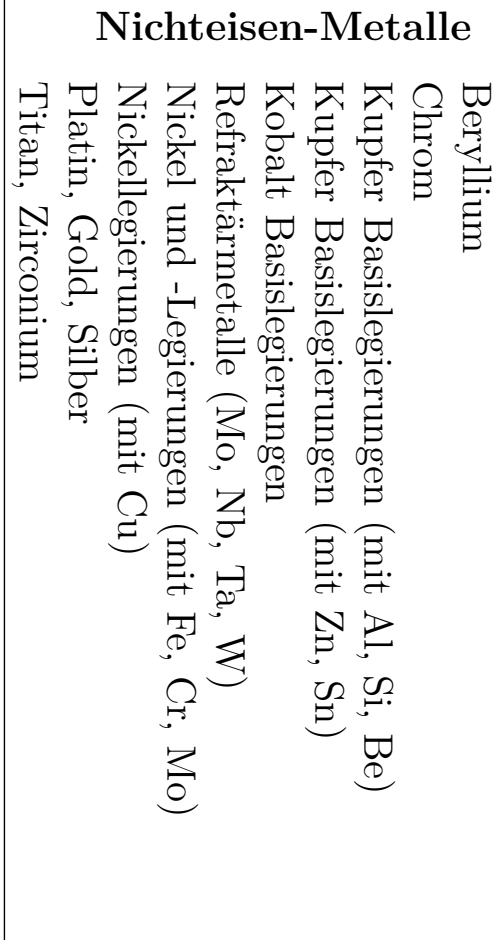 & & 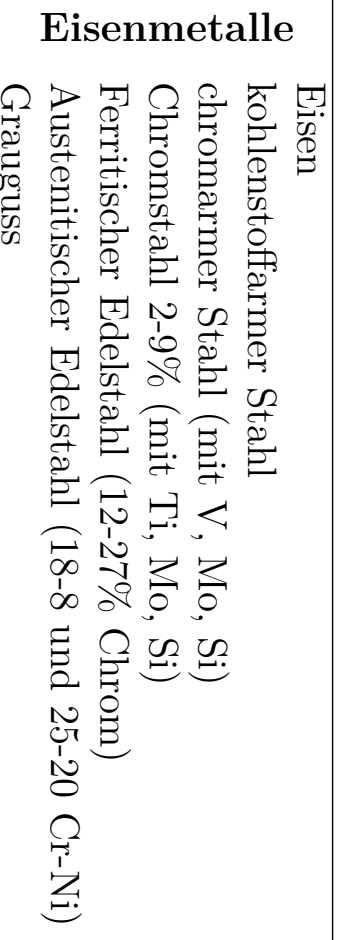 & 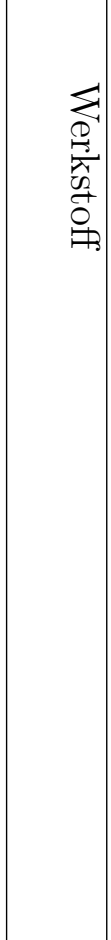 \\
\hline 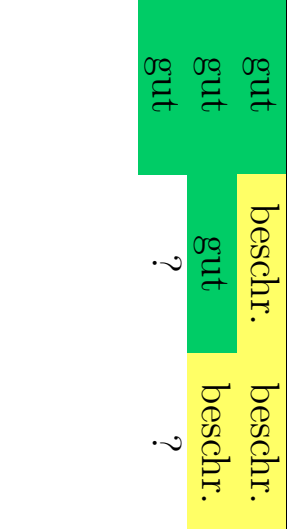 & 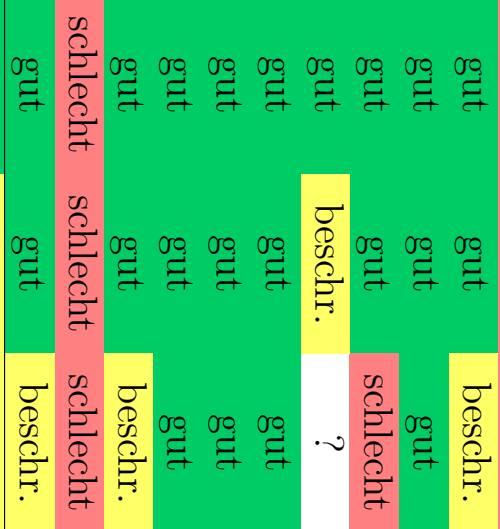 & & 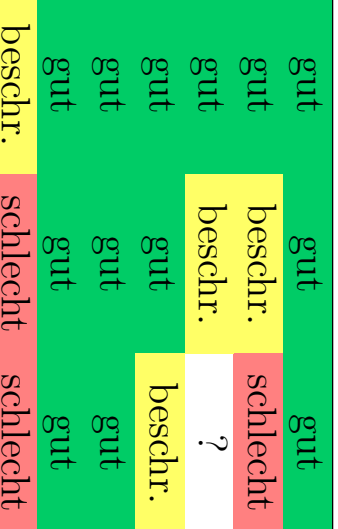 & 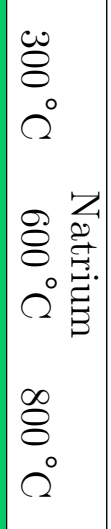 \\
\hline 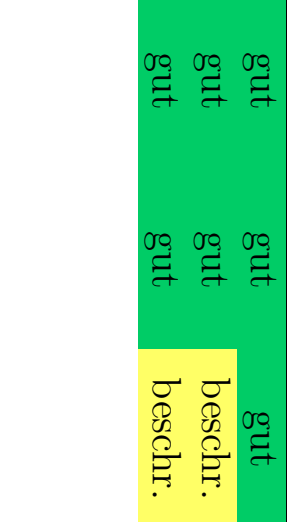 & 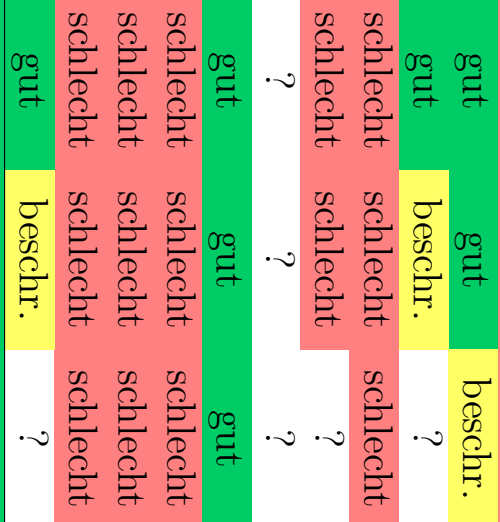 & & 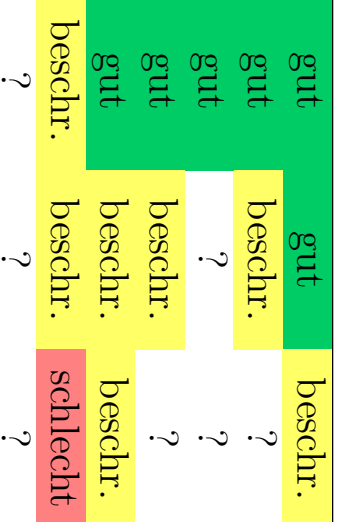 & 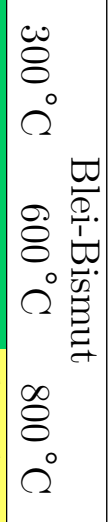 \\
\hline 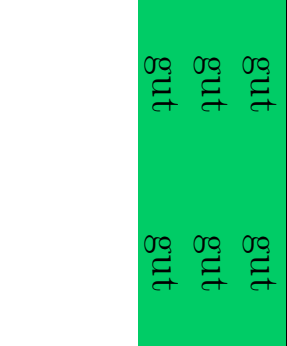 & 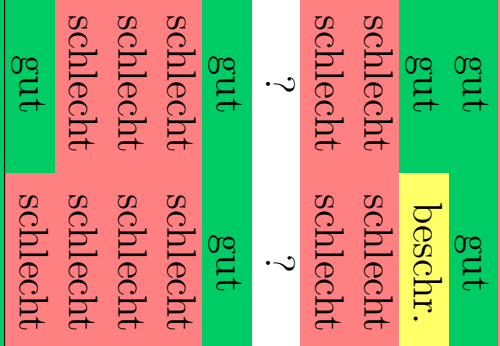 & & 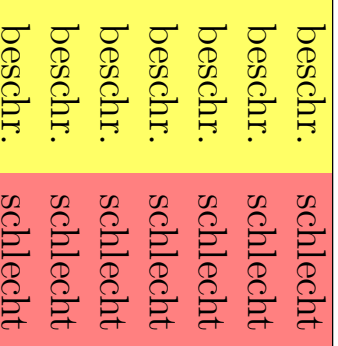 & 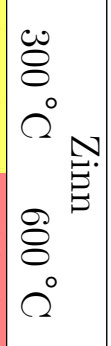 \\
\hline
\end{tabular}


Wärmeleitfähigkeit von Natrium entsteht prinzipiell auch eine homogenere Temperaturverteilung mit geringeren Temperaturspitzen. Die Reduktion der Zielpunkte auf die Receivermitte führt jedoch zu höheren Wärmestromdichten, die diesem Effekt entgegenwirken.

Die tatsächliche Spannungsverteilung und damit die Lebensdauer hängt maßgeblich von der Konstruktion des Receivers ab. Der Fokus dieser Arbeit liegt auf der thermischen und ökonomischen Auslegung ohne detaillierte Receiverkonstruktion.

\subsubsection{Einfrier- und Auftauvorgänge im Receiver}

Bei Receivern mit Flüssigsalz als WTM muss das Einfrieren der Salzschmelze stets verhindert werden, da sonst hohe Spannungen die Absorberrohre beschädigen können. Außerdem kommt es beim Auftauvorgang der Absorberrohre leicht zur Überhitzung. Die hohe Schmelztemperatur von Solar Salt $\left(\approx 220^{\circ} \mathrm{C}\right.$, siehe Abbildung 3.5) erschwert diese Aufgabe zusätzlich. Nachts und an Tagen schwacher Einstrahlung werden Salzreceiver daher entleert. Dennoch kann das Einfrieren einzelner Rohre - vor allem an windigen Tagen - nicht immer verhindert werden. Im Betrieb von Solar Two kam es im Zeitraum zwischen Juli und November 1998 über 30 mal zum unerwünschten Einfrieren der Salzschmelze in einzelnen Absorberrohren [44].

Die An- und Abfahrvorgänge (Entleeren, Befüllen und Vorheizen) reduzieren bei Salzreceivern die Verfügbarkeit und senken den Ertrag des Kraftwerks. Da die Gefriertemperatur von Natrium $\left(98^{\circ} \mathrm{C}\right)$ weit unterhalb der Betriebstemperatur liegt, ist ein unerwünschtes Einfrieren im Betrieb nicht zu erwarten. Um die Verfügbarkeit und den Ertrag von Natriumreceivern zu erhöhen, gibt es Überlegungen, auf die Entleerung komplett zu verzichten und den Receiver Nachts einfrieren zu lassen. Dieses Kapitel beinhaltet eine erste Abschätzung zur Realisierung dieser Überlegung. Die transienten, thermischen Simulationen vernachlässigen die Wärmeübertragung durch Konvektion, daher handelt es sich um eine konservative Abschätzung des Auftauvorgangs. 
Weitere Simulationsrandbedingungen sind:

- Rohrgeometrie: Inconel $601, d=50 \mathrm{~mm}, s=2 \mathrm{~mm}$

- Homogene Wärmestromdichte auf halber Rohraußenfläche: $100 \mathrm{~kW} / \mathrm{m}^{2}$

- Thermische Strahlung von Rohraußenfläche: $\epsilon=0.85, T_{\mathrm{amb}}=25^{\circ} \mathrm{C}$

- Adiabate Rohrenden

- Temperaturabhängige Stoffwerte (siehe Anhang A auf Seite 177)

- Keine Konvektion, nur Wärmeleitung

Abbildung 3.13 zeigt die Simulationsergebnisse für Solar Salt und Natrium. Bereits nach kurzer Zeit ist zu erkennen, dass die hohe Wärmeleitfähigkeit von Natrium (100-200 mal höher als bei Solar Salt) den Wärmeeintrag gleichmäßig auf den Rohrquerschnitt verteilt. Die Wärmeleitfähigkeit von Solar Salt (links im Bild) ist um den Faktor 40 niedriger als die des Rohrwerkstoffs, was dazu führt, dass die Rohrwand auf der strahlungsabgewandten Seite heißer wird als das Salz in der Rohrmitte. Dadurch kommt es zur Überhitzung von Absorberrohr und Flüssigsalz (siehe Abbildung 3.13 unten: Überhitzung des Salzes an der Rohrinnenwand der bestrahlten Rohrseite).

Mit der Simulation lassen sich die aus der Literatur bekannten Probleme beim Auftauen von Salzreceivern (siehe [58]) bestätigen. Ebenso treffen die Vermutungen zu, dass ein Auftauvorgang eines eingefrorenen Natriumreceivers dank der hohen Wärmeleitfähigkeit von Natrium von thermischer Seite her möglich erscheint. Das verwendete Modell vernachlässigt die Flüssigphase und die Konvektion, die den Auftauvorgang beschleunigt. In Realität ergeben sich somit bessere Wärmeübergänge. Aufgrund der kleineren Biot-Zahl von Solar Salt gegenüber Natrium spielt die Konvektion bei Solar Salt eine größere Rolle.

Pacheco führte 1995 Gefrier- und Schmelzexperimente mit Salzreceiverpanels durch [42]. Als größtes Problem wurde der Bereich zwischen Absorberrohr und Ofen detektiert, welcher weder mit Begleitheizung, noch mit Solarstrahlung aufgewärmt werden kann. Dennoch gelang das Auftauen innerhalb mehrerer Stunden durch vorsichtiges Fokussieren einzelner Heliostaten. Nach zwei Einfrierund Auftauzyklen wurde eine maximale bleibende Dehnung der Rohrdurchmesser von $4 \%$ gemessen. Der Bruch erfolgte nach 12 Zyklen [44].

Die Volumenänderung beim Phasenwechsel von Solar Salt beträgt ca. 10\% wohingegen bei Natrium lediglich ca. 2.5\% [59]. Somit ist davon auszugehen, dass die beim Auftauvorgang entstehenden Spannungen in der Rohrwand bei einem Natriumreceiver geringer sind als bei Solar Salt. Zudem besitzt Natrium ein sehr 

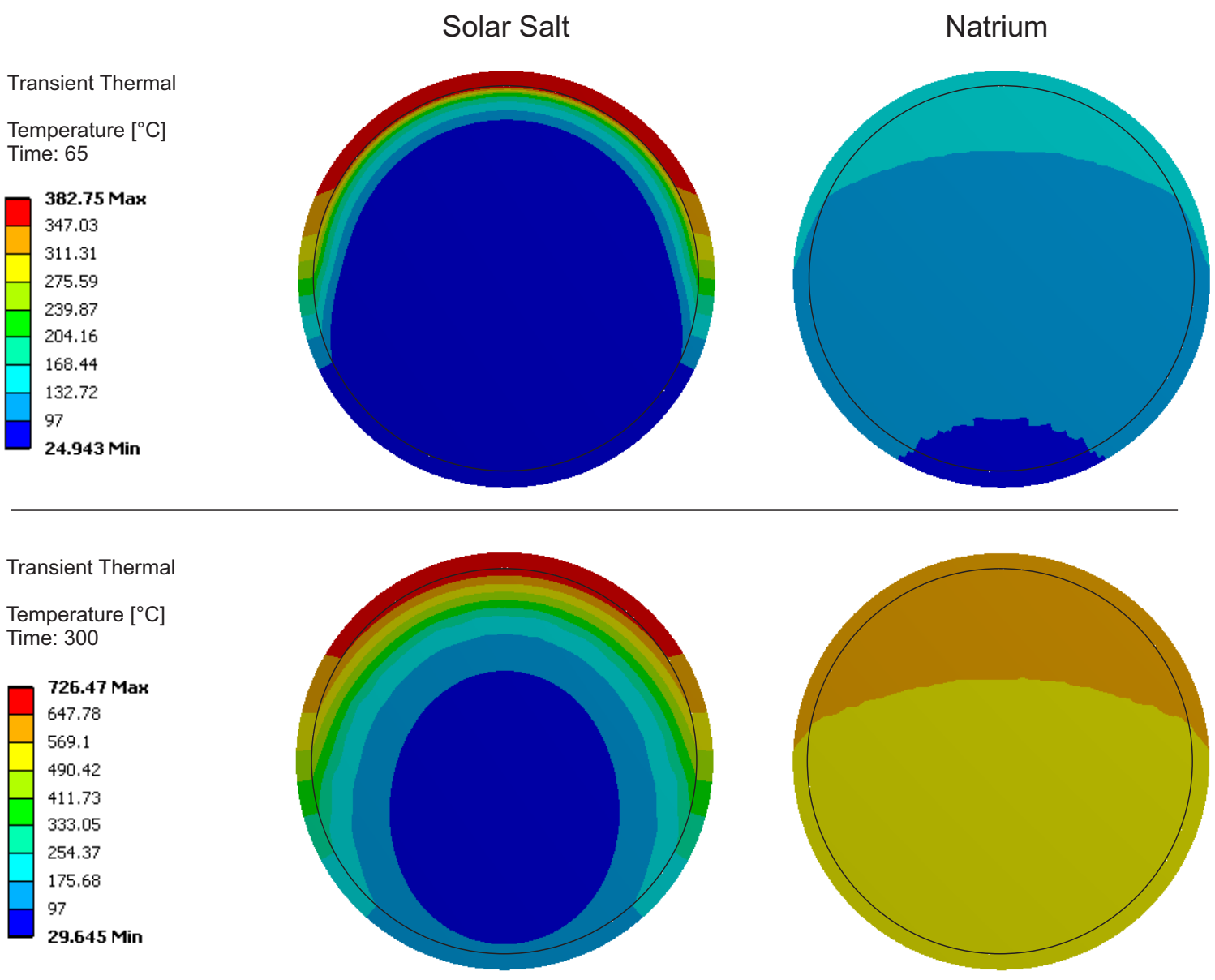

Abbildung 3.13.: Ergebnisse der transienten thermischen Simulation des Auftauvorgangs eingefrorener Absorberrohre: Temperaturverteilung der gefüllten Absorberrohre (links: Solar Salt, recht: Natrium). Oben: Zeitpunkt 1 (Natrium bereits weitgehend aufgetaut), Unten: Zeitpunkt 2 (Weite Teile von Solar Salt noch immer fest, Rohrtemperatur bereits über $700{ }^{\circ} \mathrm{C}$ )

niedriges Elastizitätsmodul von $10 \mathrm{GPa}$ bei $20{ }^{\circ} \mathrm{C}$ [60]. Bevor das Absorberrohr sich plastisch verformt, wird beim Auftauen zunächst die Natriumsäule komprimiert. In Anhang B auf Seite 181 ist eine Spannungsuntersuchung dokumentiert, nach der ein solares Aufschmelzen von eingefrorenen Natriumrohren möglich erscheint. Dieses Ergebnis muss noch durch Experimente bestätigt werden.

Mit Blei-Bismut können Absorberrohre voraussichtlich problemlos zyklisch eingefroren und aufgetaut werden, da die Volumenänderung beim Phasenwechsel $0 \%$ beträgt 43]. Die Wärmeleitfähigkeit ist zwar nicht so hoch wie bei Natrium, jedoch immer noch 20 bis 30 mal höher als bei Solar Salt (siehe Anhang A, Seite 177). Somit wird die Überhitzung der Rohre geringer sein als bei Solar Salt. 


\subsubsection{Förderung und Messtechnik}

Flüssigmetalle sind elektrisch leitfähige Fluide und können sich daher die Magnetohydrodynamik (MHD) zunutze machen. Zur Förderung von Flüssigmetallen können somit neben herkömmlichen, mechanischen Pumpen auch berührungslose, sogenannte elektromagnetische Pumpen (EMP) eingesetzt werden. Der große Vorteil dieser Pumpen ist das Fehlen sämtlicher beweglicher Teile und Dichtungen. Somit kann eine Leckage ausgeschlossen werden und bei einer potenziellen Sicherheitsentleerung des kompletten Kreislaufs in den Sumpftank stehen keine Strömungshindernisse wie Leitschaufeln einer mechanischen Pumpe im Weg. Einziger Nachteil dieser Pumpen ist der geringere Wirkungsgrad. In Abschnitt 4.3 werden diese Pumpen genauer beschrieben.

Bei durchsichtigen Medien wie Wasser und Luft gibt es eine Reihe an Messmethoden zur Strömungsmessung. Bei Flüssigmetallströmungen stellen die Undurchsichtigkeit, Korrosion und zum Teil auch die hohe Temperatur für die Messtechnik eine große Herausforderung dar. Über die MHD gibt es dennoch einige Messverfahren zur Bestimmung der Eigenschaften einer Flüssigmetallströmung:

Bei der Lorentzkraft-Anemometrie (LKA) wird die strömungsinduzierte Kraft auf einen Permanentmagneten in der Nähe eines Rohres mit Flüssigmetall gemessen. Aus dieser Kraftmessung lässt sich der Durchfluss und die mittlere Strömungsgeschwindigkeit bestimmen. Diese Technik steht kurz vor ihrem Einsatz in der Aluminium- und Stahlherstellung [61].

Die berührungslose, induktive Strömungstomographie (CIFT) ermöglicht die Rekonstruktion des Geschwindgkeitsfelds einer Flüssigmetallströmung durch die äußere Messung der stömungsinduzierten Verzerrung eines angelegten Magnetfelds. In einem Demonstrationsexperiment konnte gezeigt werden, dass das mittlere dreidimensionale Geschwindigkeitsfeld in einem mit Flüssigmetall gefüllten Zylinder rekonstruiert werden kann [62].

Zur Regelung des Massenstroms ist die Kenntnis der Füllstände in sämtlichen Tanks wichtig. In technischen Anlagen wird dies häufig mittels Drucksensoren realisiert. In druckbeaufschlagten Tanks ist dies jedoch nicht ohne weiteres möglich. Im Solar Two-Kraftwerk wurde der Füllstand im Receivereinlasstank mit 
radioaktiven Cäsium-137-Strahlern gemessen [44]. Dieses Verfahren ist nicht nur aufwändig und teuer, sondern oft auch fehleranfällig. Bei Flüssigmetallen wird auch hier die elektrische Leitfähigkeit genutzt. Mit mehreren über die Tankhöhe verteilten Elektroden (Zündkerzen) kann der Füllstand sehr zuverlässig und kostengünstig gemessen werden [63].

\subsubsection{Reaktivität und Toxizität}

Aufgrund der starken Korrosion von Zinn mit Strukturmaterialien (siehe Abschnitt 3.4.4 bleiben nach Abbildung 3.5 nur noch die Alkalimetalle und Blei bzw. Bleiverbindungen übrig.

Bleiverbindungen sind toxisch und bis auf Ausnahmen als fortpflanzungsgefährdend eingestuft [64]. Beim Betrieb eines Bleikreislaufes muss daher stets der Kontakt und die Aufnahme in den menschlichen Körper vermieden werden. Durch den geringen Dampfdruck $\left(4.21 \times 10^{-7} \mathrm{~Pa}\right.$ bei $\left.600 \mathrm{~K}\right)$ stellt die Gefahr durch Einatmen von Bleidämpfen kein Problem dar. Kommt es im Betrieb zu einer Leckage, erstarrt das ausgelaufene Blei und kann entfernt werden. Bei Kontakt von Blei oder Blei-Bismut mit Wasser oder Wasserdampf gibt es keine Reaktion.

Die Alkalimetalle sind als Reinstoff für den Menschen nicht toxisch. Jedoch kommt es beim Kontakt mit Sauerstoff zu teils heftigen exothermen Reaktionen. Unterhalb von $115{ }^{\circ} \mathrm{C}$ baut Natrium mit dem Sauerstoff aus der Umgebungsluft eine Oxidschicht auf, ohne weiter zu reagieren [65]. Bei Temperaturen zwischen 115 und $300{ }^{\circ} \mathrm{C}$ entsteht Natriumoxid $\left(\mathrm{Na}_{2} \mathrm{O}\right)$. Bei höheren Temperaturen entsteht Natriumperoxid $\left(\mathrm{Na}_{2} \mathrm{O}_{2}\right)$. Alle diese Reaktionen laufen unter großer Wärmeentwicklung ab. Ist zusätzlich Wasser bzw. feuchte Luft in der Umgebung entsteht daraus Natriumhydroxid $(\mathrm{NaOH})$ und Wasserstoff $\left(\mathrm{H}_{2}\right)$, welche wiederum mit dem Sauerstoff in der Luft zu Explosionen führen können. Im Gegensatz zu Feuern mit organischem Material ist der dichte weiße Rauch eines Natriumbrandes nicht brennbar. Die Flammen sind oft sehr klein und unscheinbar. Im Brandherd selbst entsteht ein grelles, hellgelbes Licht. Die Temperatur kann bis zum Siedepunkt auf ca. $880{ }^{\circ} \mathrm{C}$ ansteigen [65]. Brennende Alkalimetalle selbst können nicht explodieren, jedoch reagieren die oben genannten Komponenten 
des weißen Rauches mit der Feuchtigkeit der Luft und können Haut, Augen und die Lunge verätzen.

\subsubsection{Erfahrung und Sicherheit}

Im Laufe der letzten 50 Jahre wurde vor allem in der Nukleartechnik viel Erfahrung im Umgang mit Alkalimetallen gesammelt (siehe Abschnitt 3.1.1). Zahlreiche Leckagen und Brände führten dazu, Sicherheitsrichtlinien und Konstruktionsrichtlinien zu entwickeln [65].

Experimentelle Untersuchungen mit Natriumbränden zeigen einen großen Unterschied zwischen einer brennenden Natriumlache (pool fire) und einem brennenden Natriumfreistrahl (spray fire) hinsichtlich Ausbreitungsgeschwindigkeit und Temperatur [66]. Beträgt der Sauerstoffgehalt der Umgebung $<5 \%$ entsteht bei Natrium kein Brand [65]. Eine Möglichkeit zur Feuerbekämpfung ist, wie bei anderen Bränden auch, der Entzug von Sauerstoff.

Sämtliche wasserhaltigen Löschmittel (wie z.B. der Löschschaum in Feuerlöschern) eignen sich nicht zur Brandbekämpfung von Alkalimetallen. Auch Kohlenstoffdioxid $\left(\mathrm{CO}_{2}\right)$ eignet sich nicht zur Brandbekämpfung, da der Sauerstoff mit dem Alkalimetall weitere Oxide bildet. Die zur Brandlöschung ebenfalls eingesetzten Tetrachloride $\left(\mathrm{CCl}_{4}\right)$ bilden mit Alkalimetallen das Giftgas Kohlenoxiddichlorid (Phosgene). Als Löschmittel kommen daher lediglich chemisch getrocknetes Natriumhydrogencarbonat (sodium bicarbonate), Vermiculit und Graphex (Graphitpulver) in Frage. In Tests wurden damit Natriumfeuer innerhalb kürzester Zeit erfolgreich gelöscht. Als effektivstes aber auch teuerstes Löschmittel wurde Graphex identifiziert [67].

Als Schutzgas kommt bei Alkalimetallen Stickstoff sowie die Edelgase Helium, Neon, Argon, Krypton und Xenon in Betracht. Bei hohen Temperaturen kann Stickstoff mit den metallischen Strukturkomponenten reagieren, daher wird als Schutzgas in Alkalimetallkreisläufen meistens Helium oder Argon eingesetzt.

Die folgende Liste beschreibt die wichtigsten Vorkehrungen, die zum sicheren Betrieb eines Alkalimetallkreislaufes notwendig sind:

- Grundsätzlich werden unter allen Komponenten sogenannte Tropfschalen (engl.: driptrays) vorgesehen, die potenziell auslaufendes Natrium auffangen 
und die Kontaktoberfläche mit der Umgebung verringern und das Löschen vereinfachen.

- Der Kontakt von Alkalimetallen mit Beton ist unbedingt zu vermeiden, da ab $100{ }^{\circ} \mathrm{C}$ ein Teil des im Beton gebundenen Wassers verdampft und mit dem Natrium reagiert. Bei Kontakt von heißen Alkalimetallen mit Beton platzt dieser oberflächig ab und verteilt das Flüssigmetall weiter in der Umgebung. Daher werden alle Betonflächen, die potenziell in Kontakt mit dem Alkalimetall treten können, mit Schutzblechen verdeckt.

- Die Verwendung von Schutzgas im Kreislauf verhindert/reduziert das Eindringen von Luft im Falle einer Leckage und hält damit den Kreislauf rein.

- Die Verwendung einer Kaltfalle reinigt den Kreislauf im Betrieb von nicht erwünschten Oxiden, die Korrosion verursachen und dadurch weitere Sicherheitsrisiken darstellen.

- Einhausung des kompletten oder zumindest eines Teils des Kreislaufs. Im Falle eines Brandes kann die Einhausung mit Argon oder Stickstoff geflutet werden, wodurch die Reaktion zum Erliegen kommt.

- Drainagetank am tiefsten Punkt mit Fassungsvermögen des gesamten Kreislaufes. Im Falle einer Leckage kann möglichst viel Alkalimetall in den Drainagetank geleitet werden. Der Kreislauf muss so konstruiert sein, dass der komplette Inhalt allein durch die Schwerkraft entleert werden kann (möglichst viele senkrechte Rohre). Mit Hilfe eines Gegendrucks durch ein Schutzgas kann der Entleerungsprozess unter Umständen beschleunigt werden. Strömungsumlenkungen und -behinderungen (bspw. durch Ventile oder Leitbleche einer Pumpe) erschweren ebenfalls die Notentleerung. Elektromagnetischen Pumpen (EMP) sind diesbezüglich vorteilhaft.

- Konstruktive Maßnahmen, die jede Kontaktmöglichkeit - direkt oder indirekt - zwischen Luft-/Wasserleitungen und Flüssigmetallleitungen ausschließen.

\subsubsection{Verfügbarkeit und Kosten}

Natrium ist das sechsthäufigste Element auf der Erde. Aufgrund seiner Reaktivität kommt es aber nicht als Reinstoff, sondern immer in Natrium-Salz- 
Verbindungen vor. Natrium wird hauptsächlich aus Natriumchlorid gewonnen, zum Beispiel durch Schmelzflusselektrolyse in einer Downs-Zelle. Für die Herstellung von $1 \mathrm{~kg}$ Natrium werden etwa $10 \mathrm{kWh}$ benötigt [68]. Da Natrium in Verbindungen sehr reichlich vorhanden ist, hängt der Preis hauptsächlich von den Stromkosten und dem Preis vom ebenfalls bei der Elektrolyse entstehenden Chlor ab.

Die weltweite jährliche Förderung von Blei, Bismut und Zinn ist in Tabelle 3.7 dargestellt.

Tabelle 3.7.: Vorkommen und jährliche Förderung ausgewählter Metalle 69 71]

\begin{tabular}{lccc}
\hline Rohstoff & $\begin{array}{c}\text { Weltweite } \\
\text { Förderung }\end{array}$ & $\begin{array}{c}\text { Weltweite } \\
\text { Reserven }\end{array}$ & $\begin{array}{c}\text { Anteil } \\
\text { in Erdkruste }\end{array}$ \\
\hline Blei (2012) & $5.12 \mathrm{kt}$ & $89 \mathrm{kt}$ & $0.0018 \%$ \\
Bismut (2012) & $8.2 \mathrm{kt}$ & $320 \mathrm{kt}$ & $0.00002 \%$ \\
Zinn (2012) & $240 \mathrm{kt}$ & $4700 \mathrm{kt}$ & $0.0035 \%$ \\
Natrium & bedarfsabhängig & - & $2.64 \%$ \\
\hline
\end{tabular}




\section{Kraftwerkskonzepte und -komponenten für Flüssigmetallreceiver}

\subsection{Einteilung der Kraftwerkskonzepte für Flüssigmetallreceiver}

Es gibt eine Vielzahl von Kombinationsmöglichkeiten von Flüssigmetallreceivern, thermischen Energiespeichern und Kraftwerksblöcken. Dieses Kapitel gibt einen Überblick über die Kraftwerkskonzepte und Technologien zur solaren Stromerzeugung mit Solarturmkraftwerken, bei denen Flüssigmetalle involviert sind.

\subsubsection{Anbindung an eine Dampfturbine}

Da Dampfturbinen seit Ende des 19. Jahrhunderts Stand der Technik sind, wird diese Technologie auch in Solarkraftwerken eingesetzt. Übliche Dampfparameter von unterkritischen Turbinen mit Zwischenüberhitzung in der Leistungsklasse von $125 \mathrm{MW}_{\mathrm{el}}$ sind $162 \mathrm{bar} / 550{ }^{\circ} \mathrm{C} / 550{ }^{\circ} \mathrm{C} / 275^{\circ} \mathrm{C}$. Für überkritische Prozesse wird Dampf bei 250 bar $/ 620^{\circ} \mathrm{C} / 620^{\circ} \mathrm{C} / 275^{\circ} \mathrm{C}$ benötigt.

Die obere Prozesstemperatur ist durch die Überhitzung vorgegeben und liegt in der Regel zwischen 500-700 ${ }^{\circ} \mathrm{C}$. Da in konventionellen Dampfkraftwerken mit fossilen Energieträgern das Rauchgas nicht beliebig weit abgekühlt werden kann (Kondensation von Schwefelsäure), ist das untere Temperaturniveau der Wärmezufuhr auf ca. $250{ }^{\circ} \mathrm{C}$ beschränkt. Zur Wirkungsgradsteigerung durch 


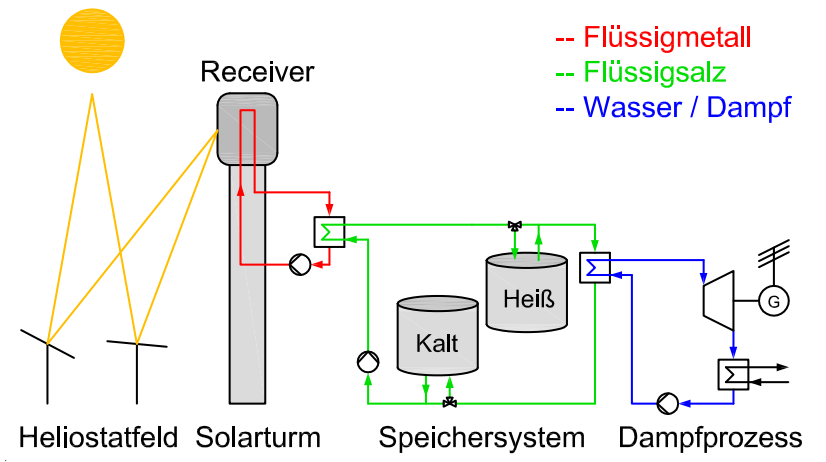

\author{
Dampfturbine (Rankine-Prozess) \\ Unter- und überkritischer Dampf \\ Untere Receivertemp.: $\quad 290^{\circ} \mathrm{C}$ \\ Obere Receivertemp.: $\quad 565^{\circ} \mathrm{C}$ (Flüssigsalz) \\ $640^{\circ} \mathrm{C}$ (LM-Speicher) \\ Mögliche Flüssigmetalle: $\mathrm{Na}, \mathrm{NaK}, \mathrm{K}$ \\ $\mathrm{Pb}, \mathrm{Pb}-\mathrm{Bi}, \mathrm{Sn}$
}

Abbildung 4.1.: Konzeptskizze zur Anbindung eines Flüssigmetallreceivers an einen Dampfprozess mit thermischem Energiespeicher

regenerative Speisewasservorwärmung] durchläuft ein Teilmassenstrom des thermodynamischen Kreisprozesses zwar niedrigere Temperaturen, jedoch bleibt das untere Temperaturniveau der Wärmezufuhr auf $250^{\circ} \mathrm{C}$.

In Solarkraftwerken gibt es diese Beschränkung durch das Rauchgas jedoch nicht, wodurch theoretisch tiefere untere Prozesstemperaturen möglich sind. Zur Wirkungsgradsteigerung des Dampfprozesses ist die regenerative Speisewasservorwärmung aber auch hier sinnvoll. Jedoch könnte der Anteil, der zusätzlich durch die Temperaturbeschränkung des Rauchgases notwendig ist, bei Solarkraftwerken vermieden werden. Des Weiteren muss für eine Prozessoptimierung das gesamte Kraftwerk betrachtet werden. Eine niedrigere untere Prozesstemperatur würde zwar in der Dampfturbine geringere Wirkungsgrade erzielen, jedoch gleichzeitig die Kosten des thermischen Speichers senken und höhere Receiverwirkungsgrade ermöglichen. Unter Umständen ist durch eine derartige Änderung der Prozessparameter ein höherer Gesamtwirkungsgrad erreichbar. Die aktuellen Dampfturbinen sind jedoch für fossile Energieträger optimiert und werden aller Voraussicht nach nicht für Solarkraftwerke weiter entwickelt. In Abbildung 4.1 wird Flüssigmetall nur im Receiver verwendet. Die Wärme wird anschließend über einen Sicherheitswärmeübertrager an das Speichersystem mit Flüssigsalz übertragen. Je nach Bedarf wird damit Frischdampf für die Turbine erzeugt. Insbesondere bei überkritischen Prozessen mit Temperaturen $>565{ }^{\circ} \mathrm{C}$

\footnotetext{
${ }^{1}$ Zur regenerativen Speisewasservorwärmung wird der Turbine stufenweise teilexpandierter Dampf entnommen, der in den mehreren Vorwärmern kondensiert. Die dabei abgegebene Kondensationswärme dient zur Aufheizung des Speisewassers in diesen Vorwärmstufen. Dieses Verfahren heißt innerer Wärmeaustausch. Das Kondensat, das in einem Vorwärmer anfällt, wird über ein Drosselventil auf den Druck im nächsten Vorwärmers gedrosselt, wobei wieder etwas Sattdampf entsteht, der sich dort verflüssigt.
} 
ist die thermische Speicherung mit Solar Salt kritisch. In diesem Fall können Flüssigmetallspeicher verwendet werden. Dadurch entfällt auch der sonst nötige Wärmeübertrager. In Abschnitt 4.2 werden mögliche Flüssigmetallspeicher diskutiert.

\title{
4.1.2. Anbindung an eine Gasturbine
}

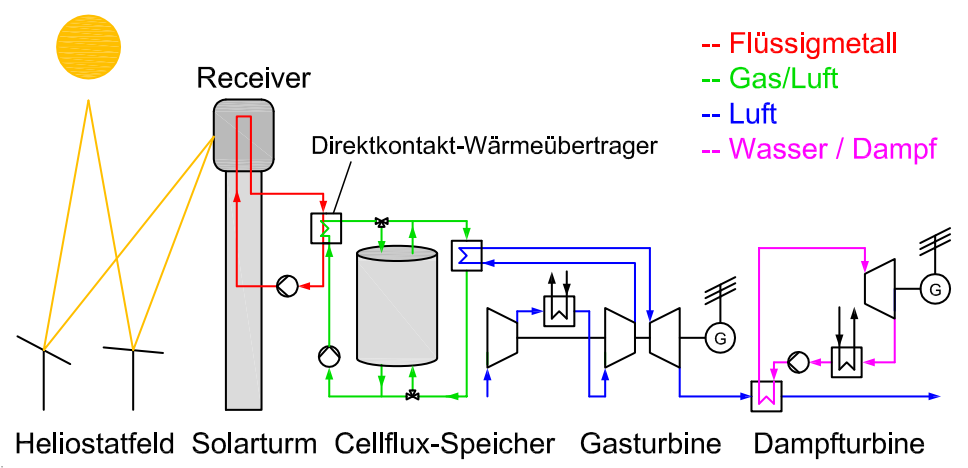

\author{
GuD-System: \\ Gasturbine (Brayton-Prozess) \\ Dampfturbine (Rankine-Prozess) \\ Als offener oder geschlossener Prozess \\ DHCX mit Cellflux-Speicherkonzept \\ Bei geschlossenem Prozess entfällt ein \\ Wärmeübertrager \\ Mögliche Flüssigmetalle: \\ Bis $850^{\circ} \mathrm{C}$ : $\quad \mathrm{Na}, \mathrm{Pb}, \mathrm{Pb}-\mathrm{Bi}, \mathrm{Sn}$ \\ Über $900^{\circ} \mathrm{C}$ : $\mathrm{Pb}, \mathrm{Pb}-\mathrm{Bi}, \mathrm{Sn}$
}

Abbildung 4.2.: Konzeptskizze zur Anbindung eines Flüssigmetallreceivers an eine Gasturbine mit nachgeschaltetem Dampfprozess

Zur Anbindung an eine Gasturbine sind höhere Temperaturen erforderlich, daher kann als Speicher kein Flüssigsalz verwendet werden. Eine Möglichkeit bietet ein Regeneratorspeicher (z.B. das Cellflux-Konzept [72]), wobei das Gas beispielsweise mit einem Direktkontaktwärmeübertrager (DCHX) durch das Flüssigmetall erhitzt wird. Mögliche Kombinationen zu Flüssigmetallen mit Nichtmetallen für DCHX finden sich in Abschnitt 4.3.3. Wenn die Gasturbine einen geschlossenen Kreisprozess durchläuft entfällt der in Abbildung 4.2 dargestellte Wärmeübertrager und das Gas aus dem Cellflux-Speicher kann direkt der Turbine zugeführt werden. Die Abwärme der Gasturbine kann noch für einen Dampfprozess genutzt werden. Aktuelle Kombikraftwerke $(\mathrm{GuD})$ erreichen Wirkungsgrade von bis zu $60 \%$ 73.

Natürlich sind auch sämtliche Abwandlungen des in Abbildung 4.2 dargestellten Konzepts möglich wie bspw. eine Gasturbine mit nachgeschaltetem Flüssigsalzspeicher und einer damit gespeisten Dampfturbine. In diesem Fall reduziert sich aber der Nutzungsgrad der Gasturbine erheblich, da diese nur bei Solarstrahlung betrieben werden kann. 


\title{
4.1.3. AMTEC - Alkali Metal Thermal to Electric Converter
}

Das Kernstück von AMTEC besteht aus einer speziellen Keramik namens BASE (beta-alumina solid electrolyte). Diese Keramik leitet positive Ionen aber wirkt gegenüber Elektronen als Isolator. Auf der heißen Seite verdampft Natrium und verursacht einen Überdruck. Durch die Druckdifferenz zwischen heißer und kalter Seite der BASE diffundieren die Natrium-Ionen durch die BASE zur Kathode während die Elektronen über einen Leiter außerhalb fließen und Arbeit verrichten bis sie an der Kathode mit den Ionen wieder zu neutralem Natriumdampf vereint werden. Auf der kalten Seite wird der Natriumdampf kondensiert und der Kreislauf beginnt von Neuem.

Der AMTEC-Kreislauf zeigt ein hohes Potenzial und besitzt außerdem keine beweglichen Teile. Die Wirkungsgrade liegen im Bereich zwischen 10-30 \% 74. Ein großes Problem ist die Degradation der BASE im Betrieb. Eine mögliche Anwendung der AMTEC-Zelle ist ein Hochtemperaturprozess mit einem Flüssigmetallspeicher (z.B. Thermocline mit Füllmaterial), der einen nachgeschalteten Dampfprozess speist (siehe Abbildung 4.3). Die AMTEC-Zelle arbeitet als Direktkonverter und ist somit wie eine PV-Zelle von der aktuellen Einstrahlung abhängig. Sofern die untere Temperatur der AMTEC-Zelle und die obere Prozesstemperatur des nachgeschalteten Dampfprozesses $565^{\circ} \mathrm{C}$ nicht übersteigt kann auch ein Salzspeicher wie in Abbildung 4.1 verwendet werden.

Das AMTEC-Konzept wurde 1966 erfunden und ist seither Gegenstand der Forschung [74, 75]. Aktuell gibt es noch keine kommerzielle AMTEC-Anlage.

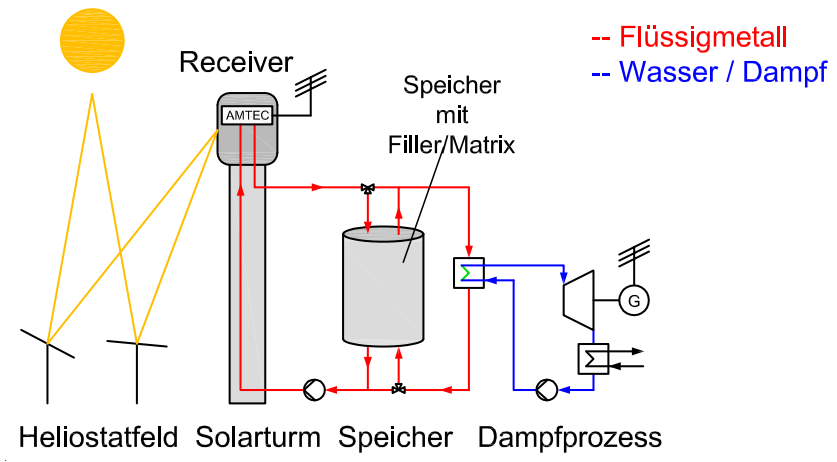

\author{
AMTEC-Zelle in Receiver \\ Nur für Alkalimetalle ( $\mathrm{Na}, \mathrm{NaK}, \mathrm{K})$ \\ Obere Temperatur: $600-1000^{\circ} \mathrm{C}$ \\ Thermocline-Speicher mit \\ geeignetem Füllmaterial / Matrix \\ Nachgeschalteter \\ Rankine-Prozess
}

Abbildung 4.3.: Konzeptskizze zur Anwendung einer AMTEC-Zelle als Hochtemperaturprozess in einem Solarturmkraftwerk 


\subsubsection{LM MHD Generator - Liquid Metal Magnetohydrodynamic Generator}

Eine weitere Möglichkeit der Stromerzeugung mit Flüssigmetallen bietet der MHD-Generator. Er nutzt die Eigenschaft der elektrischen Leitfähigkeit der Flüssigmetalle, die beschleunigt durch ein Magnetfeld eine Spannung induzieren. Zur Beschleunigung der Flüssigmetallströmung stehen verschiedene Möglichkeiten zur Verfügung. In der Ein-Komponenten-Strömung wird das Flüssigmetall im Receiver teilweise verdampft. Der damit einhergehende Dichteunterschied beschleunigt das Flüssigmetall. Die andere Möglichkeit ist eine Zwei-Komponenten-Strömung, bei der das Flüssigmetall mit einem thermodynamischen Arbeitsmedium (Dampf/Gas) gemischt wird. Mögliche Kombinationen aus Flüssigmetall und Gas sind in Abschnitt 3.4.7 und 4.3.3 beschrieben. Die Vermischung funktioniert wie ein Direktkontakt-Wärmeübertrager und fungiert gleichzeitig als Pumpe, wodurch der Kreislauf als Naturumlauf betrieben werden kann [76]. Die Ausdehnung des Gases treibt das Flüssigmetall durch das Magnetfeld. Anschließend werden die beiden Phasen wieder getrennt und der Kreislauf beginnt von neuem. Bei der Zwei-Komponenten-Strömung genügen bereits Temperaturen ab $300{ }^{\circ} \mathrm{C}$ für den Betrieb. Der MHD-Generator kann als Hochtemperaturprozess mit einem nachgeschalteten Brayton- oder Rankineprozess kombiniert werden, je nach Wahl des Flüssigmetalls und des thermodynamischen Arbeitsmediums (siehe Abbildung 4.4). Wie auch das AMTEC-Konzept hat der MHD-Generator keine beweglichen Komponenten. Der theoretische Wirkungsgrad des MHD-Generators übersteigt $60 \%$ 77 79 . Im Labor-Maßstab wurde bereits ein Wirkungsgrad von $48 \%$ bei einer Leistung von $10.8 \mathrm{~kW}$ gemessen [80].

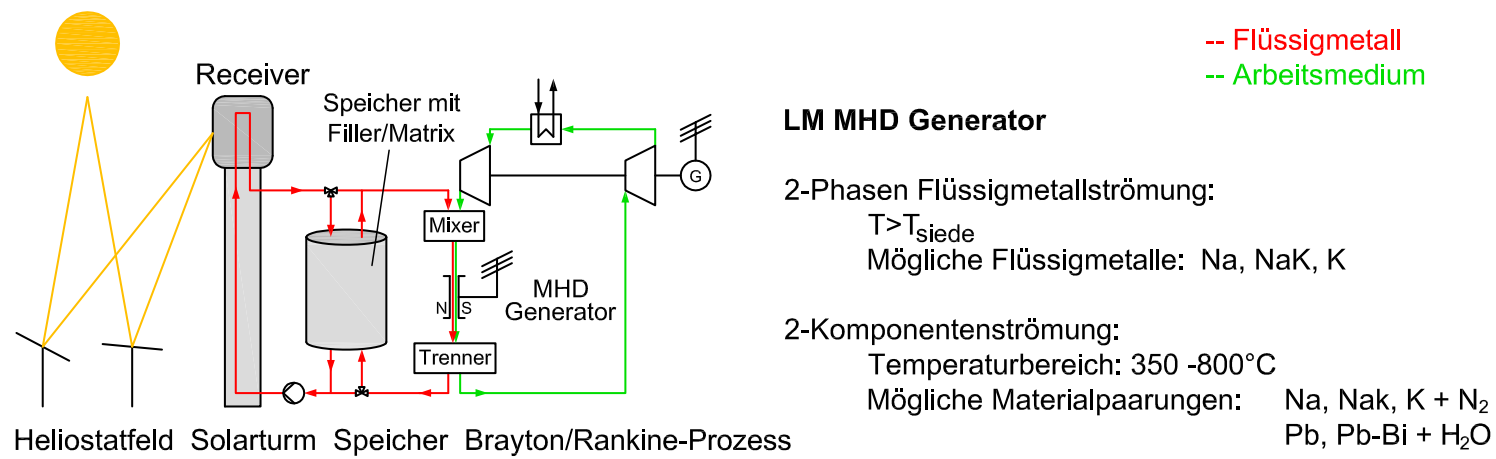

Abbildung 4.4.: Konzeptskizze zur Anwendung eines MHD-Generators als Hochtemperaturprozess in einem Solarturmkraftwerk 


\title{
4.1.5. Flüssigmetalldampfturbine
}

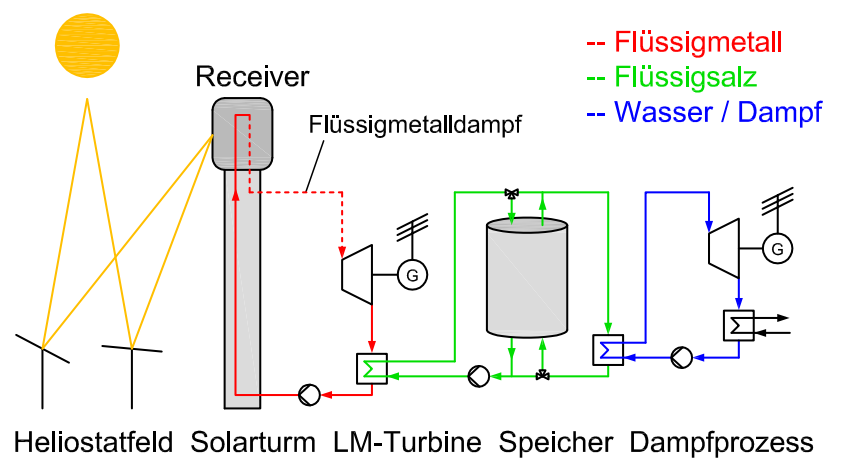

\author{
Flüssigmetalldampfturbine \\ Flüssigmetall wird im Receiver \\ vollständig verdampft \\ Ein Nachgeschalteter Speicher mit \\ Flüssigsalz und einer Dampfturbine \\ führt zur Grundlastfähigkeit des \\ Kraftwerks \\ Mögliche Flüssigmetalle: $\mathrm{Na}, \mathrm{NaK}, \mathrm{K}$
}

Abbildung 4.5.: Konzeptskizze einer Flüssigmetalldampfturbine mit nachgeschalteter Dampfturbine

Das am weitesten von der kommerziellen Nutzung entfernte Konzept ist die Flüssigmetalldampfturbine. Das flüssige Metall wird dabei im Receiver verdampft und anschließend in einer Turbine entspannt. Die verbleibende Restwärme kann in einem thermischen Speicher mit Flüssigsalz gespeichert und je nach Bedarf einem Dampfturbinenprozess zugeführt werden. Theoretische und experimentelle Untersuchungen mit Natriumdampf finden sich in der Solartechnik bisher nur bei Dish-Stirling-Maschinen 81, 82. Ein Nachteil dieses Konzepts ist der geringe Nutzungsgrad der Flüssigmetalldampfturbine, die nur tagsüber bei Solarstrahlung betrieben werden kann. Die Dampfturbine hingegen ist durch den Speicher von der Solarstrahlung entkoppelt und erreicht somit höhere Nutzungsgrade.

\subsection{Thermische Energiespeicher für Flüssigmetallsysteme}

Die Frage nach einer passenden Speichertechnologie kann nicht getrennt vom Kraftwerksprozess beantwortet werden. Neben der Speichertemperatur spielen auch die Speicherkosten eine wichtige Rolle. Die Kosten des 2-Tank Flüssigsalzspeichers des Solar Two Kraftwerks betrugen $23 € / \mathrm{kWh}$ [83]. Um die Kosten von Flüssigmetallspeichern abzuschätzen wurden im Rahmen dieser Arbeit verschiedene Speicherkonzepte untersucht. Die Modellierung basiert auf statio- 
nären Berechnungen mit konstanten, gemittelten Stoffwerten und erlaubt keine detaillierten Aussagen über das Speicherverhalten.

\subsubsection{Modellierung der thermischen Speicherkonzepte}

Latente und thermochemische Speicher werden bisher in der Industrie großtechnisch noch nicht eingesetzt, daher beschränkt sich die Betrachtung auf die folgenden, sensiblen Speicherkonzepte:

- 2-Tank-Speicher (direkt)

- Thermocline-Speicher mit schwimmender Trennwand (direkt)

- Thermocline-Speicher mit Matrix/Füllmaterial (indirekt)

- Segmentierter Thermocline-Speicher mit Matrix/Füllmaterial (indirekt)

Eine detailliertere Beschreibung der Konzepte befindet sich in [84]. Die benötigte thermische Energie im Speicher berechnet sich wie folgt:

$$
E_{\mathrm{th}}=\frac{P_{\mathrm{PB}} \cdot c_{\mathrm{st}}}{\eta_{\mathrm{PB}}}+E_{\mathrm{loss}}
$$

Der Faktor $E_{\text {loss }}$ beschreibt die thermischen Verluste (Wärmeverluste in Speicher, Leitungen und Kraftwerksblock).

Für einen Kraftwerksblock mit $P_{\mathrm{PB}}=125 \mathrm{MW}_{\text {el }}$ und einer Speicherkapazität von $c_{\mathrm{st}}=10 \mathrm{~h}$ Turbinenbetrieb ergibt sich mit einem angenommenen Wirkungsgrad von $\eta_{\mathrm{PB}}=0.4$ eine benötigte Speicherenergie von $E_{\mathrm{th}} \approx 3500 \mathrm{MWh}_{\mathrm{th}}$.

Der folgende Abschnitt zeigt eine Kostenabschätzung der oben genannten Speicherkonzepte mit Solar Salt, Natrium, Blei, Blei-Bismut und Zinn. Bei den indirekten Energiespeichern beträgt der Volumenanteil von Füllmaterial/Matrix im Speicher $75 \%$. Tabelle 4.1 zeigt die Speichertemperaturen sowie die verwendeten Stoff- und Kostendaten der Speicher- und Füllmaterialien. Die detaillierten konstruktiven Annahmen sowie Kostendaten für Struktur und Wärmedämmung finden sich in [84], [85] und [86]. Die indirekten Kosten werden mit $20 \%$ der Einzelkosten abgeschätzt. 
Tabelle 4.1.: Gemittelte Stoffwerte und Kostendaten von Solar Salt und verschiedenen Flüssigmetallen sowie der verwendeten Füllmaterialien nach 84

\begin{tabular}{|c|c|c|c|c|c|}
\hline Wärmeträgermedium & $\begin{array}{c}\text { Solar Salt } \\
\left(\mathrm{NaNO}_{3}+\mathrm{KNO}_{3}\right)\end{array}$ & $\begin{array}{l}\text { Natrium } \\
(\mathrm{Na})\end{array}$ & $\begin{array}{l}\mathrm{Blei})^{a} \\
(\mathrm{~Pb})^{-}\end{array}$ & $\begin{array}{l}\text { Blei-Bismut } \\
\quad(\mathrm{Pb}-\mathrm{Bi})\end{array}$ & $\begin{array}{l}\text { Zinn } \\
(\mathrm{Sn})\end{array}$ \\
\hline obere Temperatur $\left[{ }^{\circ} \mathrm{C}\right]$ & 565 & 565 & 625 & 565 & 565 \\
\hline untere Temperatur $\left[{ }^{\circ} \mathrm{C}\right]$ & 290 & 290 & 350 & 290 & 290 \\
\hline Dichte $\left[\mathrm{kg} / \mathrm{m}^{3}\right]$ & 1818 & 851 & 10305 & 9660 & 6330 \\
\hline Wärmekapazität [kJ/(kg K)] & 1.52 & 1.28 & 0.15 & 0.15 & 0.24 \\
\hline Kosten $[€ / \mathrm{kg}]$ & 1 & 2.6 & 1.6 & 12 & 18 \\
\hline Kosten $[€ / \mathrm{kWh}]$ & 8.6 & 26.7 & 140 & 1047 & 982 \\
\hline Energiedichte $[\mathrm{kJ} / \mathrm{kg}]$ & 417 & 351 & 41 & 41 & 66 \\
\hline Energiedichte $\left[\mathrm{MJ} / \mathrm{m}^{3}\right]$ & 758 & 299 & 425 & 398 & 418 \\
\hline Speicherdichte $\left[\mathrm{kWh} / \mathrm{m}^{3}\right]$ & 211 & 83 & 118 & 111 & 116 \\
\hline Füllmaterial & $\begin{array}{l}\text { Graphit } \\
\text { (C) }\end{array}$ & \multicolumn{2}{|c|}{$\begin{array}{c}\text { Quarzit } \\
\left(98 \% \mathrm{SiO}_{2}\right) \\
\end{array}$} & \multicolumn{2}{|c|}{$\begin{array}{l}\text { Aluminiumoxid } \\
\left(85 \% \mathrm{Al}_{2} \mathrm{O}_{3}\right)\end{array}$} \\
\hline Dichte $\left[\mathrm{kg} / \mathrm{m}^{3}\right]$ & 2500 & \multicolumn{2}{|c|}{2651} & \multicolumn{2}{|l|}{3560} \\
\hline Wärmekapazität [kJ/(kg K)] & 0.71 & \multicolumn{2}{|c|}{1.2} & \multicolumn{2}{|l|}{1.2} \\
\hline Kosten $[€ / \mathrm{kg}]$ & 1.5 & \multicolumn{2}{|c|}{0.5} & \multicolumn{2}{|l|}{1} \\
\hline
\end{tabular}

${ }^{a}$ Da der Schmelzpunkt von Blei bei $327^{\circ} \mathrm{C}$ liegt, wird die untere Temperatur auf $350{ }^{\circ} \mathrm{C}$ gesetzt und die gleiche Temperaturdifferenz wie bei den anderen Speichern genutzt.

\subsubsection{Ergebnisse und Vergleich mit Literaturdaten}

Abbildung 4.6 zeigt die Speicherkosten der direkten Speicherkonzepte. Daraus lässt sich ableiten, dass die direkte Speicherung von thermischer Energie in Flüssigmetallen nicht wirtschaftlich ist. Selbst bei Natrium sind die Speicherkosten mehr als doppelt so hoch wie beim 2-Tank-Speicher mit Flüssigsalz.

Abbildung 4.7 zeigt die Speicherkosten der indirekten Speicherkonzepte im Vergleich mit den direkten Speicherkosten von Solar Salt. Zur besseren Übersichtlichkeit sind nur Konzepte mit Speicherkosten $\leq 30 € / \mathrm{kWh}$ dargestellt. Die indirekte Speicherung in einem Thermocline mit Quarzit oder Aluminiumoxid als Füllmaterial und Flüssigmetall als WTM erreicht in diesem Modell kostenmäßig die gleiche Größenordnung wie die Speicherung mit Flüssigsalz. Bei den Schwermetallen kommt nur der segmentierte Speicher in Frage und selbst damit sind die Kosten höher als beim 2-Tank-Salzspeicher. Bei den Schwermetallen entstehen zudem zusätzliche Kosten für das Fundament, welches die größere 


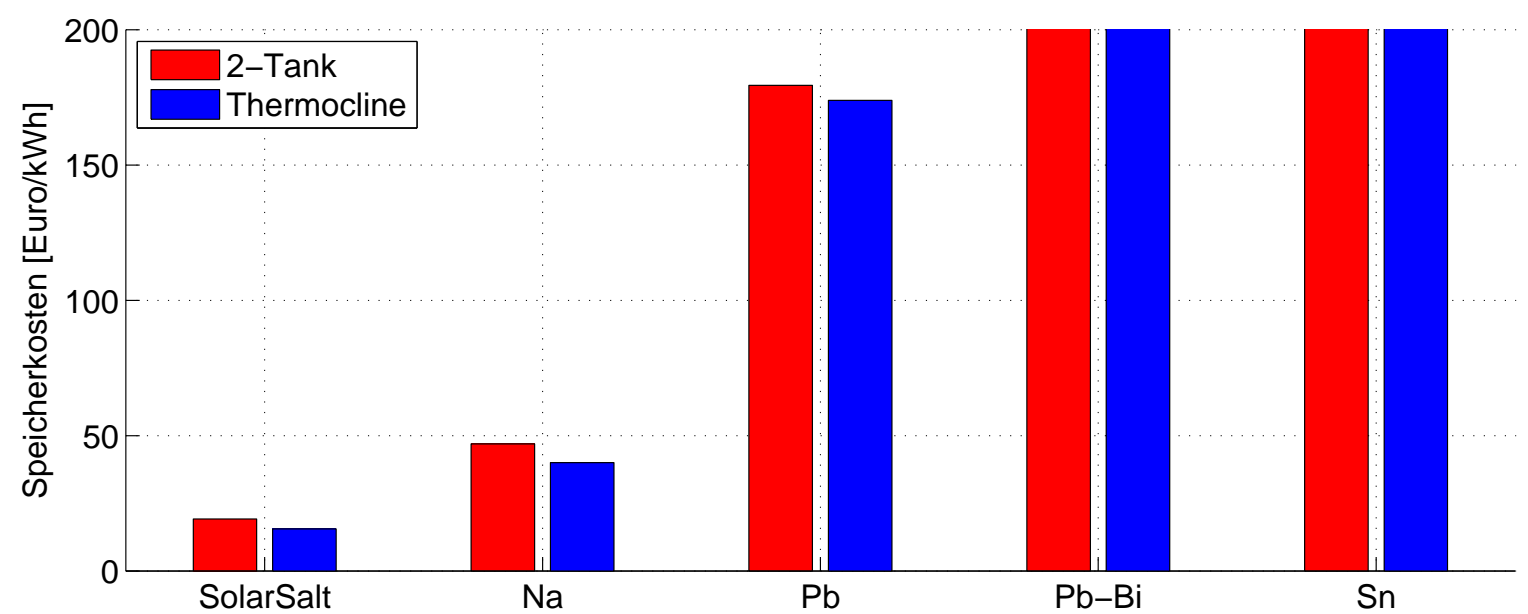

Abbildung 4.6.: Speicherkosten bei direkter thermischer Speicherung in 2-Tank und Thermocline-Speichern mit verschiedenen Wärmeträgermedien. Temperaturdifferenz: $\Delta T=275^{\circ} \mathrm{C}$, Speichergröße: $\approx 3500 \mathrm{MWh}_{\text {th }}$

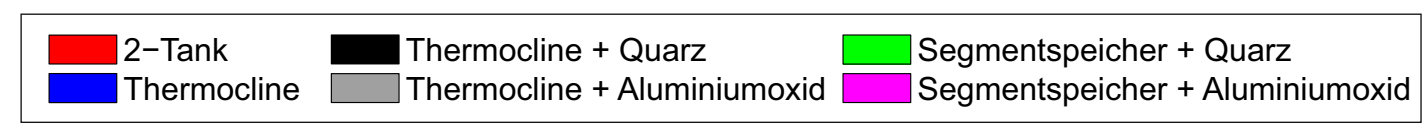

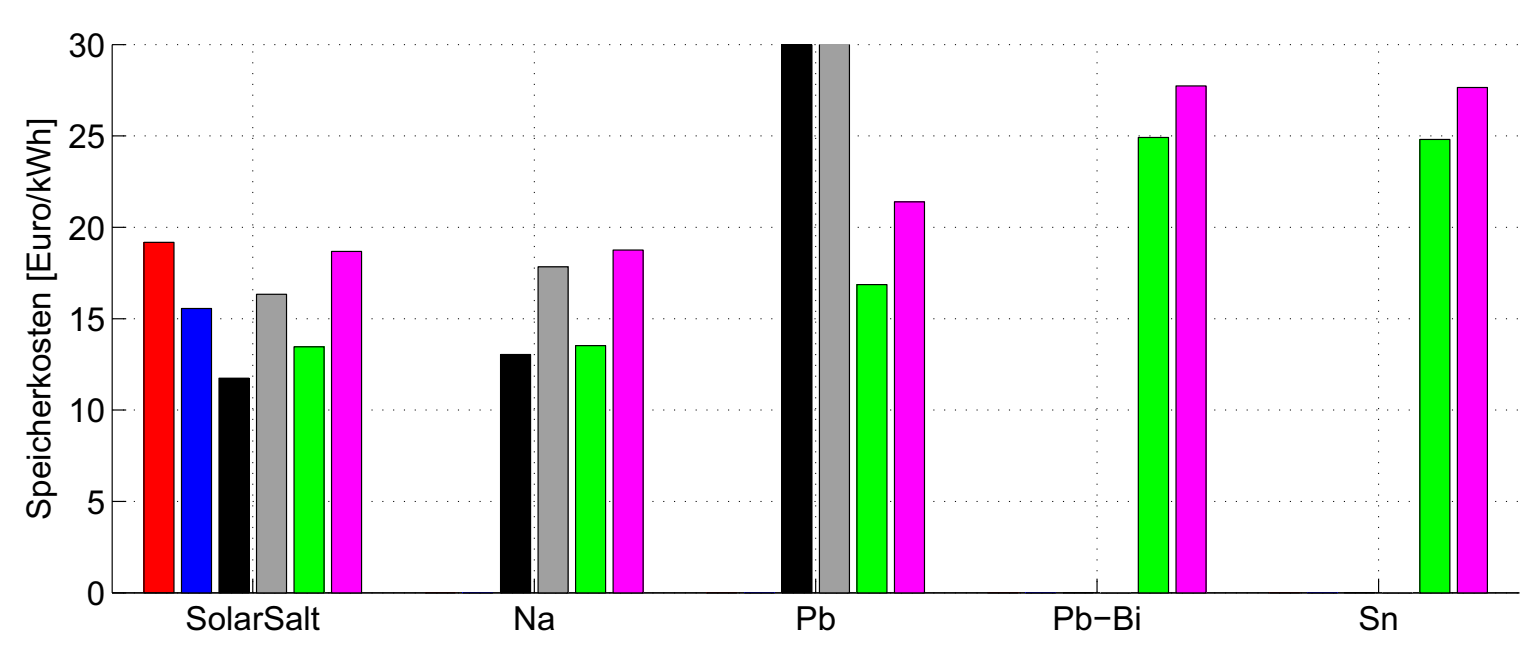

Abbildung 4.7.: Speicherkosten bei direkter und indirekter thermischer Speicherung in 2-Tank, Thermocline und Segmentierten Speichern mit verschiedenen Wärmeträger- und Füllmaterialien. Temperaturdifferenz: $\Delta T=275^{\circ} \mathrm{C}$, Speichergröße: $\approx 3500 \mathrm{MWh}_{\mathrm{th}}$

Masse durch die hohe Dichte aufnehmen muss. Diese Kosten sind im vorliegenden Modell jedoch nicht berücksichtigt. Auch die höheren Energieverluste beim Thermocline sowie die eventuellen Probleme bei der Materialverträglichkeit zwischen Flüssigmetall und Füllmaterial werden nicht berücksichtigt.

Aufgrund der geringeren Kosten und der bereits gesammelten Erfahrung mit 2-Tank-Speichern (z.B. in den SEGS-Kraftwerken [87]) wird im weiteren Verlauf 


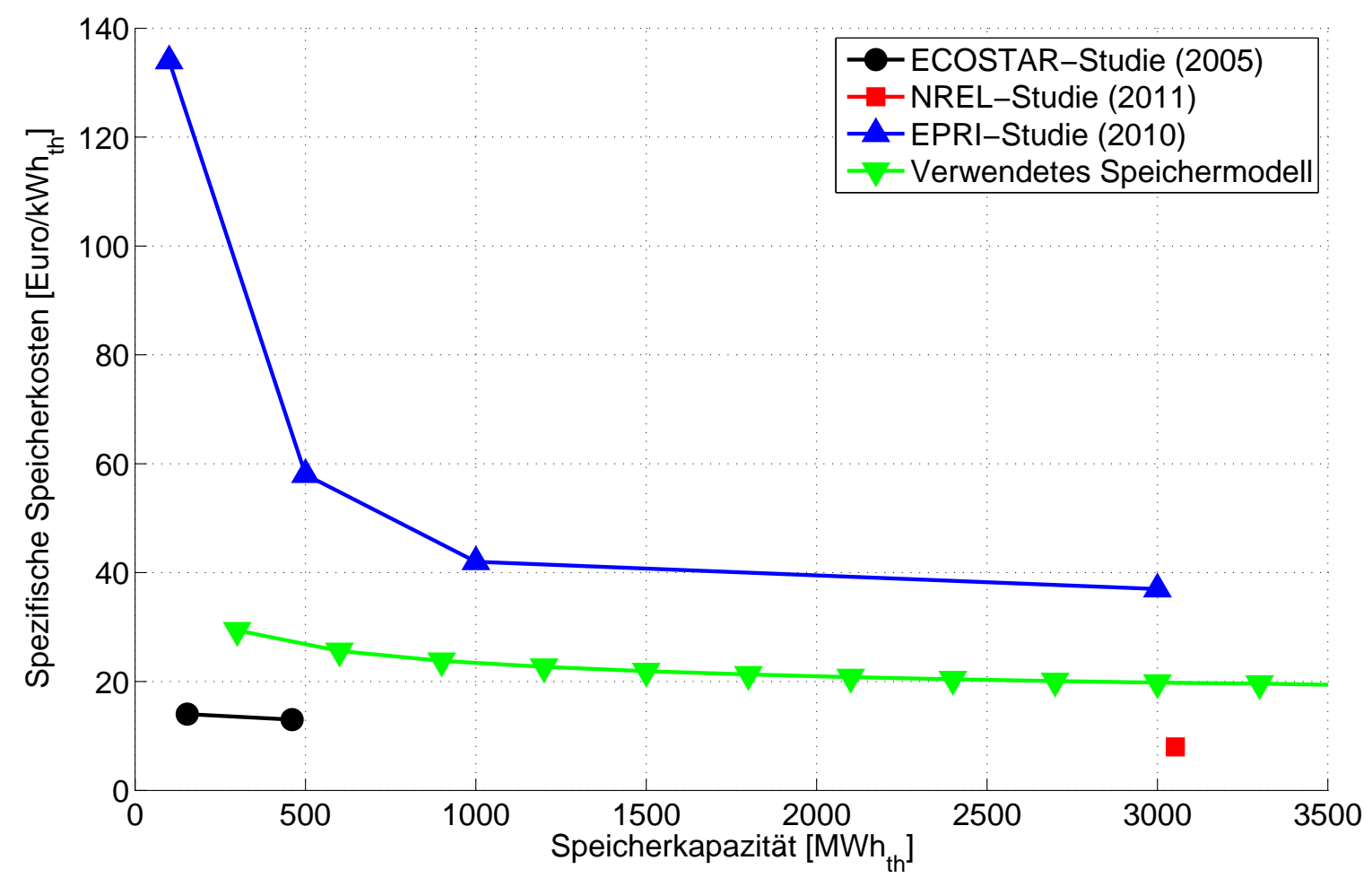

Abbildung 4.8.: Spezifische Speicherkosten für 2-Tank-Systeme mit SolarSalt als Speichermedium $\left(290-565^{\circ} \mathrm{C}\right)$ : ECOSTAR-Studie 88, NREL-Studie 85, EPRI-Studie [89]. Umrechnungskurs: $1 €=1.35$ US $\$$

dieser Arbeit das 2-Tank-Konzept mit Solar Salt verwendet. Abbildung 4.8 zeigt einen Vergleich der Speicherkosten des verwendeten Speichermodells mit verschiedenen Literaturwerten. Die starke Streuung der Kostendaten liegt vermutlich in unterschiedlichen Annahmen für die Salzkosten. Beim verwendeten Speichermodell ergibt sich die folgende Kostenaufteilung:

- Kosten Speichermedium: $45 \%$ (Solar Salt: $1 € / \mathrm{kg}$ )

- Heißer Tank: $20 \%$

- Kalter Tank: $18 \%$

- Indirekte Kosten: $17 \%$ 


\subsection{Elektromagnetische Pumpen (EMP) und Wärmeübertrager}

Zur Förderung von Flüssigmetallen kommen neben mechanischen Pumpen (Kreisel- oder Zahnradpumpen) und Mammutpumpen auch elektromagnetische Pumpen (EMP) zum Einsatz. Letztere nutzen die elektrische Leitfähigkeit der Flüssigmetalle, um damit durch ein Magnetfeld eine Strömung zu erzeugen. Alle EMP kommen ohne bewegliche Teile aus, sind wartungsfrei und daher langlebig. Das Fehlen beweglicher Dichtungen, die im Kreislauf offene und fehleranfällige Stellen bedeuten, sind ein entscheidender Vorteil. EMP erreichen bei Alkalimetallen Wirkungsgrade von $\approx 40 \%$ und bei Schwermetallen $\approx 10 \%$ 84. Jedoch werden die Verluste in Wärme umgewandelt, die in die Flüssigmetallströmung übergeht und somit dem System zugeführt wird und nicht direkt als Verlust gewertet werden kann. Nach Herstellerangaben lassen EMP den Betrieb bei sehr geringen Volumenströmen zu, wodurch geringe Teillastzustände $\leq 10 \%$ möglich sind. Des Weiteren können EMP bei eingefrorenem WTM angefahren werden. Grundlegend werden zwei Typen von EMP unterschieden: Konduktionspumpen und Induktionspumpen.

\subsubsection{Konduktionspumpe}

Hier wird ein unveränderliches Magnetfeld rechtwinklig zum stromdurchflossenen Leiter und zum Strömungskanal angelegt. Sobald eine Spannung an die Elektroden angelegt wird, wirkt die Lorentzkraft $(\vec{F}=I \cdot \vec{L} \times \vec{B})$ auf das Flüssigmetall. Der Vorteil dieses Typs ist der sehr einfache Aufbau und die hohe Förderhöhe. Dafür sind sie im Durchfluss limitiert [79]. Bei höheren Temperaturen ist der Kontakt zwischen Kupferleiter und Strömungskanal materialtechnisch schwierig. Bei Gleichstrompumpen (DC) sind mehrere 1000 A bei sehr niedrigen Spannungen nötig, daher wird dieser Pumpentyp nur bei kleinen Anlagen eingesetzt. Bei Wechselstrompumpen (AC) können die hohen Ströme über Transformatoren zwar kostengünstiger erzeugt werden, dafür entstehen aber zusätzliche Verluste. 


\subsubsection{Induktionspumpe}

Bei diesem Typ bewegt sich das Magnetfeld und induziert dabei den Strom in der Flüssigkeit selbst. Eine Stromquelle ist hier nur zur Erzeugung des wandernden Magnetfeldes notwendig. Es wird zwischen FLIP und ALIP unterschieden. Bei FLIP sind die Windungen auf beiden Seiten des Strömungskanals angeordnet. Bei ALIP werden auf der Außenseite des ringförmigen Strömungskanals Windungen angebracht während auf der Innenseite ein ferromagnetischer Kern sitzt. Durch die Wirbelstromverluste können Induktionspumpen ohne Begleitheizung anfahren. Außerdem sind sie auch bei hohen Temperaturen funktionsfähig. Induktionspumpen erreichen bei großen Durchflüssen höhere Wirkungsgrad als Konduktionspumpen, haben aber eine limitierte Förderhöhe [79].

Tabelle 4.2 zeigt verschiedene elektromagnetische Pumpen, die bereits gebaut und betrieben worden sind.

Tabelle 4.2.: Gebaute elektromagnetische Pumpen mit ihren Hauptparametern

\begin{tabular}{cccccccl}
\hline Typ & Fluid & $T\left[{ }^{\circ} \mathrm{C}\right]$ & $\Delta p[\mathrm{bar}]$ & $\dot{V}\left[\mathrm{~m}^{3} / \mathrm{h}\right]$ & $P[\mathrm{~kW}]$ & $\eta_{\mathrm{p}}[\%]$ & Anwendung \\
\hline FLIP & $\mathrm{Na}$ & 370 & 2.76 & 273 & 70 & 36 & Gebaut 1953 $[90$ \\
$\mathrm{DC}$ & $\mathrm{Na}$ & 410 & 5.17 & 1885 & 540 & 50 & EBR-II $(1955)[\overline{91}$ \\
$\mathrm{ALIP}$ & $\mathrm{Na}$ & 410 & 5.17 & 1885 & 600 & 45 & EBR-II $(1955) \sqrt{91}$ \\
$\mathrm{ALIP}$ & $\mathrm{Na}$ & 335 & 2.8 & 9600 & 1680 & 45 & Gebaut 1998 92 \\
ALIP & $\mathrm{Pb}-\mathrm{Bi}$ & 320 & 1.0 & 15 & 5.3 & 8.1 & LBE-Complex TC-1 93 \\
\hline
\end{tabular}

\subsubsection{Wärmeübertrager mit Flüssigmetallen}

In der chemischen Industrie kommen überall, wo ein Vermischen zweier Substanzen mit Sicherheit vermieden werden muss, sogenannte DoppelrohrWärmeübertrager (auch: Sicherheits-Wärmeübertrager) zum Einsatz. Da bei den in Solarkraftwerken angestrebten Temperaturen der direkte Kontakt von Natrium mit Solar Salt zur Bildung explosiver Gemische führen kann, werden in dieser Arbeit Doppelrohr-Wärmeübertrager betrachtet, obgleich in vergleichbaren Anwendungen zum Teil einfache Wärmeübertrager zum Einsatz kommen? Bei Doppelrohr-Wärmeübertragern (siehe Abbildung 4.9p wird ein Ringraum

\footnotetext{
${ }^{2}$ In natriumgekühlten Atomreaktoren werden teilweise im Dampferzeuger einfache Wärmeübertrager verwendet. Verschiedene Sicherheitssysteme sorgen dafür, dass im Falle
} 


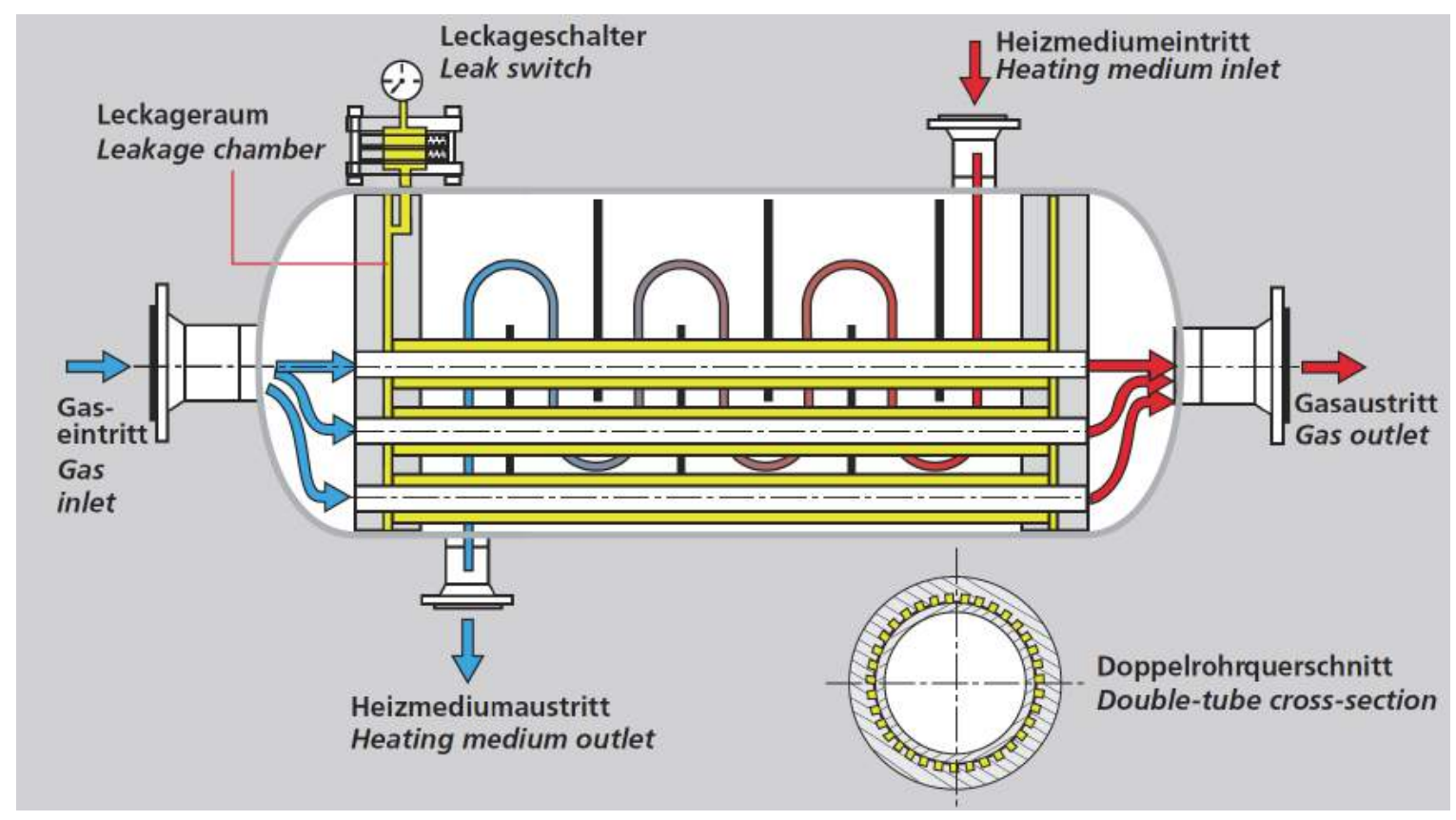

Abbildung 4.9.: Schaubild und Funktionsweise eines Doppelrohr-Wärmeübertragers mit innen strukturierten Rohren 96

durch innen strukturierte Rohre erzeugt. Zur Erhöhung der Wärmeübergangs werden die inneren Rohre hydraulisch aufgeweitet und an die Außenrohre gepresst, sodass Innenrohr und Außenrohr in direktem Kontakt sind und Wärme durch Leitung übertragen wird. Der durch die Struktur noch vorhandene Hohlraum zwischen beiden Rohren wird anschließend evakuiert und im Betrieb überwacht. Sobald eine Leckage auftritt erhöht sich dieser Druck, ohne dass beide Medien in Kontakt treten. Anschließend kann der Wärmeübertrager routinemäßig entleert und getauscht werden.

Da Blei- und Bleiverbindungen keine Reaktion mit Wasser/Dampf zeigen, können hier einfache Wärmeübertrager eingesetzt werden. Für höhere Wirkungsgrade sind auch Direktkontaktwärmeübertrager (DCHX) denkbar [95]. Allerdings gibt es beim Einleiten von unterkühltem Wasser in flüssiges Metall generell durch den plötzlichen Phasenwechsel und die damit verbundene starke Volumenausdehnung sogenannte „Dampfexplosionen“, die bei der Auslegung beachtet werden müssen.

eines Risses und dem Kontakt von Natrium mit Wasser/Dampf die Reaktion schnell zum Erliegen kommt (Notentleerung in Sumpftank) und der Schaden minimal ausfällt [94]. 



\section{Receivermodellierung ASTRID ${ }^{\odot}$}

Im Receiver wird die konzentrierte Solarstrahlung in Wärme umgewandelt. Für die Bewertung verschiedener Solarturmkonzepte ist die genaue Kenntnis über die Eigenschaften des Receiver erforderlich. Die allgemeinen Anforderungen an einen Receiver sind:

- Hoher Wirkungsgrad über großen Leistungsbereich (inklusive optischer, thermischer und hydraulischer Verluste)

- Geringe thermische Masse, um Aufwärmzeiten zu minimieren (Erhöhung der Betriebszeit und damit des Jahresertrags)

- Geringes Gewicht (Reduziert Tragstruktur, Fundament und damit Kosten des Turms und des Receivers)

- Hohe Lebensdauer (hohe Betriebssicherheit mit wenig Ausfallzeiten)

- Geringe Kosten

Diese Anforderungen hängen von vielen Faktoren ab und können nicht alle gleichzeitig maximiert werden. Während der Receiverauslegung muss daher eine Optimierung durchgeführt werden, um einen Kompromiss zwischen diesen verschiedenen Anforderungen zu erhalten.

Das Programm ASTRID ${ }^{\odot}$ (Advanced $\underline{\text { Solar }}$ Tubular ReceIver Design) zur Auslegung und Optimierung von Rohrreceivern wurde im Rahmen dieser Arbeit entwickelt. Die Auslegung eines Receivers erfolgt dabei in zwei Stufen. Im ersten Schritt (Vorauslegung) wird der Receiver analytisch berechnet (thermisch und hydraulisch). Innerhalb weniger Minuten kann ein ganzes Parameterfeld an Geometrievarianten berechnet und bewertet werden. Im zweiten Schritt erfolgt eine detaillierte thermische FEM-Berechnung. ASTRID ${ }^{\odot}$ erzeugt für die gewählte Variante automatisiert das FEM-Modell mit allen erforderlichen Daten. Durch die Kopplung mit dem Strahlverfolgungsprogramm FEMRAY/SPRAY kann 
für jeden Zeitpunkt im Jahr die durch das Heliostatenfeld entstehende Wärmestromdichteverteilung auf den Receiver aufgebracht werden. Des Weiteren können verschiedene Windsituationen automatisiert berechnet werden. Die daraus resultierenden mehrdimensionalen Receiverkennfelder können anschließend zur Optimierung und für die Jahresertragsrechnung genutzt werden.

Abschnitt 5.1 beschreibt die Vorauslegung, während Abschnitt 5.2 die thermische Modellierung mit FEM erläutert. Abschnitt 5.3 beschreibt die Validierung von ASTRID $^{\odot}$. Im ersten Schritt wurde die verwendete Vereinfachung mit eindimensionalen Fluidelementen und Nusselt-Korrelationen zur Modellierung des Wärmeübergangs mit einem detaillierten CFD-Modell verglichen und validiert. Anschließend wurde ein komplettes Modell des SOLUGAS Luftreceivers erstellt und mit den Messdaten verglichen (Abschnitt 5.3.2). Zuletzt sind drei Messpunkte des Solar Two Receiverexperiments mit ASTRID $^{\odot}$ nachmodelliert und ebenfalls mit den vorhandenen Messdaten abgeglichen worden (siehe Abschnitt 5.3.3.

\subsection{Analytische Vorauslegung von Rohrreceivern}

Ziel dieser Vorauslegung ist die Einschränkung der wesentlichen Receivergeometriedaten auf wenige Varianten, die anschließend mit genaueren Rechnungen untersucht werden können. Bei der Vorauslegung von Rohrreceivern werden die Verluste durch Konvektion und Strahlung sowie der Strahlungsaustausch zwischen benachbarten Komponenten bewusst vernachlässigt. Eine Wirkungsgradberechnung findet somit nicht statt. Als Software für die Vorauslegung wird MATLAB verwendet.

\subsubsection{Funktionsumfang und Programmstruktur}

Aus fertigungstechnischen Gründen werden Receiver häufig aus mehreren Modulen aufgebaut, die als Vieleck angeordnet sind. Jedes dieser Module ist identisch aufgebaut und ihre Verschaltung erfolgt entweder parallel oder seriell. Der Modellierungsansatz zur Vorauslegung von Rohrreceivern ist in Abbildung 5.1 dargestellt. Die geometrische Anordnung der Module spielt in der Vorauslegung 


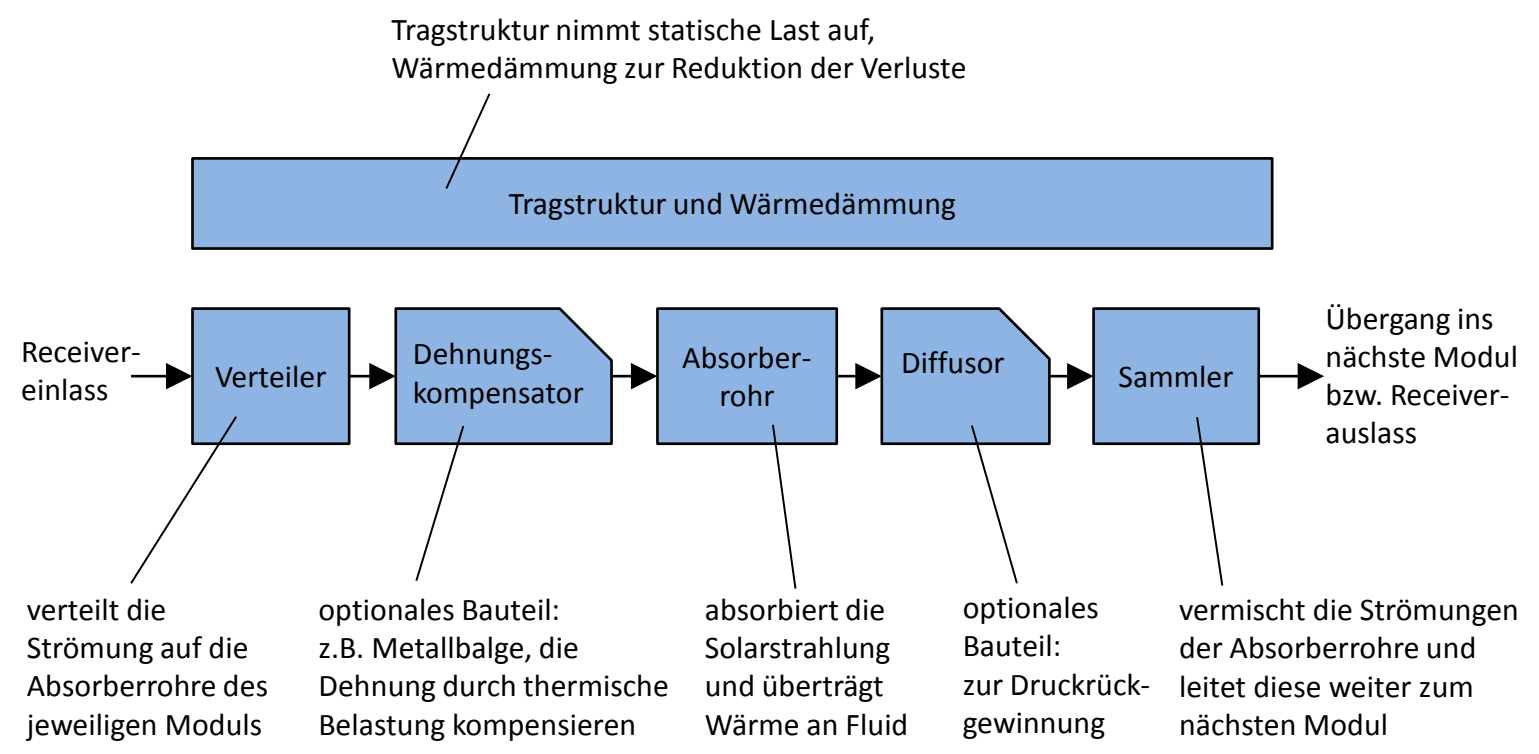

Abbildung 5.1.: Flussdiagramm zum Modellierungsansatz der Vorauslegung von Rohrreceivern mit ASTRID $^{\odot}$

keine Rolle, da die optischen Eigenschaften des Receivers nicht berücksichtigt werden. Das WTM gelangt jeweils über die Verteiler (V) in die Absorberrohre. Zur Kompensation der durch die thermische Belastung hervorgerufene Dehnung können optional Dehnungskompensatoren (z.B. Metallbalge) verwendet werden. Ebenfalls optional können Diffusoren zur Druckrückgewinnung eingesetzt werden. Im Sammler (S) wird das WTM zusammengeführt und verlässt den Receiver. In ASTRID $^{\odot}$ werden diese Komponenten für jedes Modul hydraulisch und thermisch ausgelegt. Zur thermischen Berechnung werden lediglich die Absorberrohre betrachtet. Alle anderen Komponenten werden als adiabat angenommen. Bei der hydraulischen Berechnung und der Kostenabschätzung werden jedoch sämtliche Komponenten aus Abbildung 5.1 berücksichtigt, inklusive der Verbindungsrohre zwischen den Modulen. Der Receiver kann als ebene Fläche, in zylindrischer Form oder als Vieleck modelliert werden. Die Absorberrohre können auf einer Linie oder versetzt angeordnet sein. Außerdem können sie offen liegen (externer Receiver) oder in einer Kavität. Damit können verschiedene Konfigurationen hinsichtlich den Kosten bewertet werden. Abbildung 5.2 zeigt schematisch die möglichen Receivermodellierungen.

Die Absorbereinheit selbst besteht aus parallel und/oder seriell verschalteten Modulen. Dies wird bereits in der Vorauslegung berücksichtigt. Zwei Modulverschaltungsalgorithmen sind im Programm hinterlegt. Beide bestehen aus zwei 
(a)

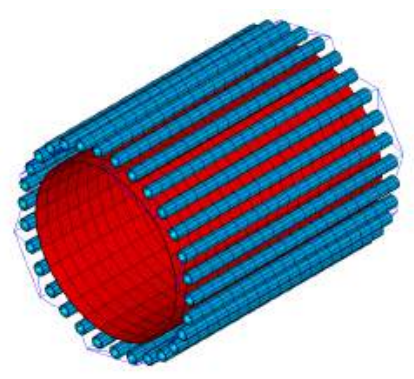

(b)

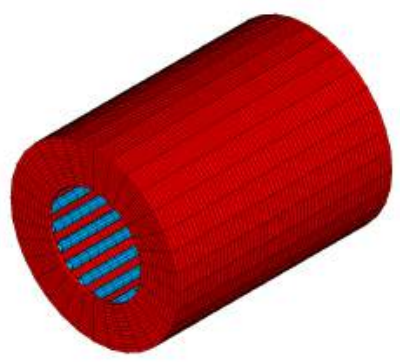

(c)

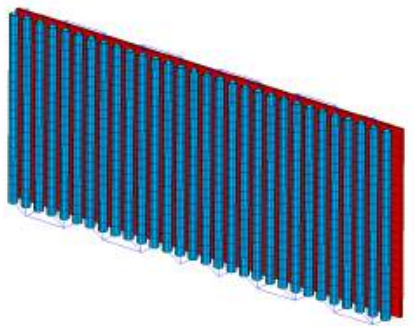

(d)

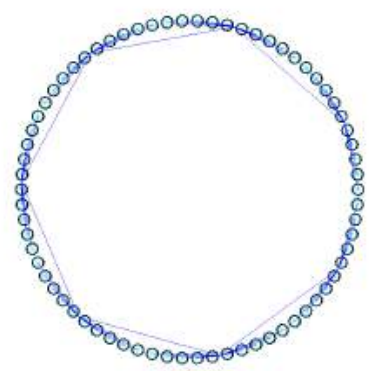

(e)

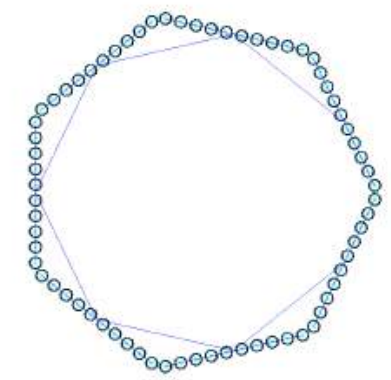

(f)

Abbildung 5.2.: Schematische Darstellung der möglichen Receivermodellierungen. Die roten Flächen stellen die thermische Dämmung dar: (a): Externer Receiver, (b): in Kavität, (c): planar, (d): kreisförmige Rohranordnung, (e): als regelmäßiges Polygon, (f): mit Versatz.

(a)

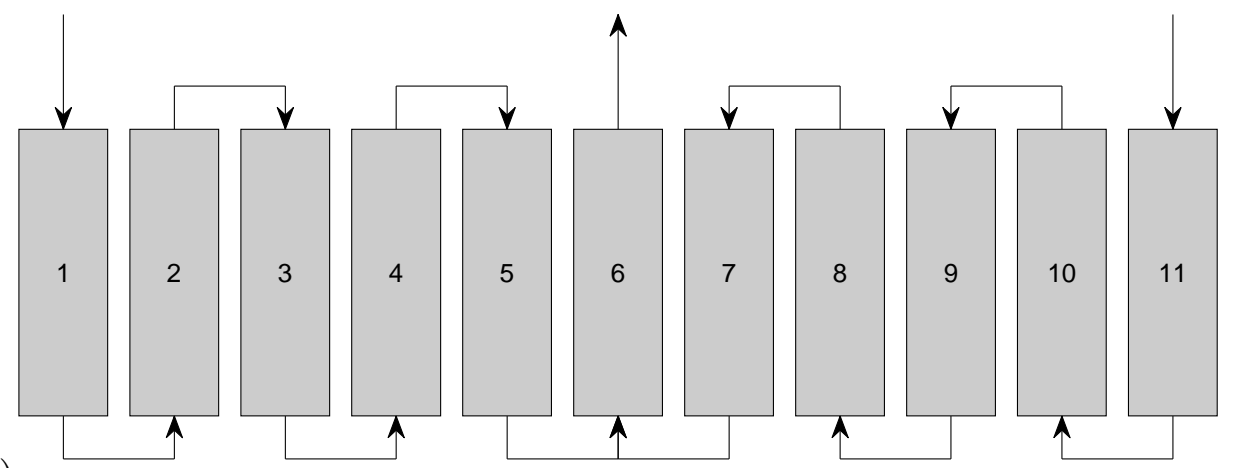

(b)

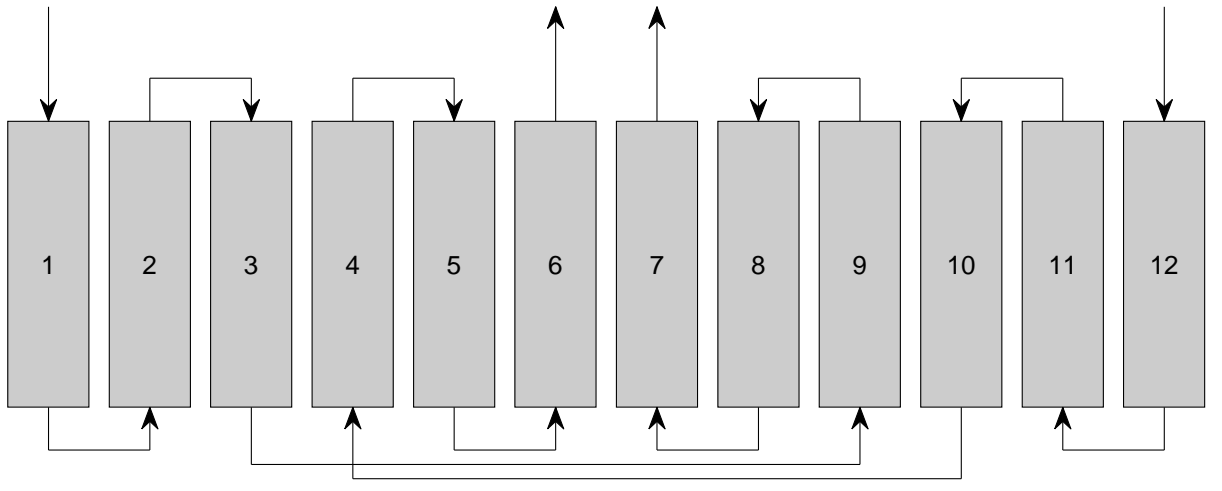

Abbildung 5.3.: Schematische Darstellung zweier Modulverschaltungsvarianten. 
Strömungswegen, die in Serpentinen durch die Receivermodule führen, bis sie den Receiver auf der gegenüberliegenden Seite verlassen. Bei einer Verschaltungsvariante findet zusätzlich eine Kreuzung der Strömungswege auf halber Strecke durch den Receiver statt. Abbildung 5.3 zeigt schematisch die beiden hinterlegten Modulverschaltungsvarianten.

Durch die direkte Eingabe der Modulverschaltungsmatrix sind auch komplexere Modulverschaltungen möglich. Mehr Details dazu befinden sich in Abschnitt 5.1.2.1.

Als WTM kann Luft, SolarSalt45 SolarSalt60 2 , Hitec, sowie die Flüssigmetalle Natrium, Blei, Blei-Bismut ${ }^{3}$, reines Zinn und Galinstan ${ }^{4}$ ausgewählt werden. Für Luft sind temperatur- und druckabhängige Stoffwerte im Programmcode hinterlegt. Bei den Flüssigsalzen und -metallen werden die Stoffwerte durch temperaturabhängige Korrelationen berechnet (siehe Anhang A Seite 177).

Bei der Berechnung werden alle Absorberrohre axial in beliebig feine Abschnitte unterteilt. Für jeden dieser Abschnitte werden die physikalischen Eigenschaften der Strömung (Dichte $\rho$, Strömungsgeschwindigkeit $u$, dynamische Viskosität $\eta$, Wärmekapazität $c_{p}$, Wärmeleitfähigkeit $\lambda$, Druckverlustbeiwert $\zeta$, Druckverlust $\Delta p$, Reynolds-Zahl Re, Prandtl-Zahl $\operatorname{Pr}$, Nusselt-Zahl $N u$, Wärmeübergangskoeffizient $\alpha$ ) in Abhängigkeit von lokal auftretendem Druck $p(i)$ und Temperatur T(i) berechnet. Für die Druckverlustberechnung werden nicht nur die Absorberrohre, sondern auch die Verteiler und Sammler mit Diffusoren sowie die Verbindungsrohre zwischen den Modulen berücksichtigt. Mehr Details dazu finden sich in Abschnitt 5.1.3.

In der Vorauslegung erfährt jedes Absorberrohr die gleiche Wärmestromdichteverteilung. Entlang der Absorberrohre kann zwischen einem homogenen, einem linear ansteigend/abfallenden und einem sinusförmigen Verlauf der Wärmestromdichte gewählt werden (siehe Abbildung 5.4 a). Des Weiteren kann der Wärmestromdichteverlauf über den Rohrumfang gewählt werden: Kompletter Rohrumfang bestrahlt, halber Rohrumfang oder lediglich die projizierte Rohrfläche. Die Verteilung über den Rohrumfang verändert lediglich die wärme-

\footnotetext{
${ }^{1}$ Salzmischung aus $45 \% \mathrm{NaNO}_{3}, 55 \% \mathrm{KNO}_{3}$ in Gewichtsprozent

${ }^{2}$ Salzmischung aus $60 \% \mathrm{NaNO}_{3}, 40 \% \mathrm{KNO}_{3}$ in Gewichtsprozent

${ }^{3}$ Eutektische Mischung aus Blei und Bismut: LBE mit 55.5\% Bi, 45.5\% Pb in Gewichtsprozent

${ }^{4}$ Bei Raumtemperatur flüssige Legierung aus $67 \%$ Gallium, 20.5\% Indium, $12.5 \%$ Zinn in Gewichtsprozent
} 
übertragende Fläche und damit den Wert der Wärmestromdichte. Wärmeleitung in Umfangsrichtung wird nicht berücksichtigt.

(a)

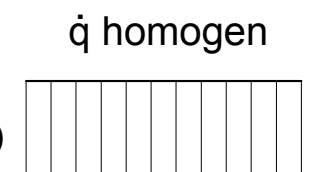

प11याय

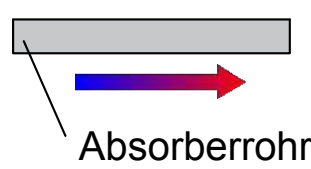

(e)

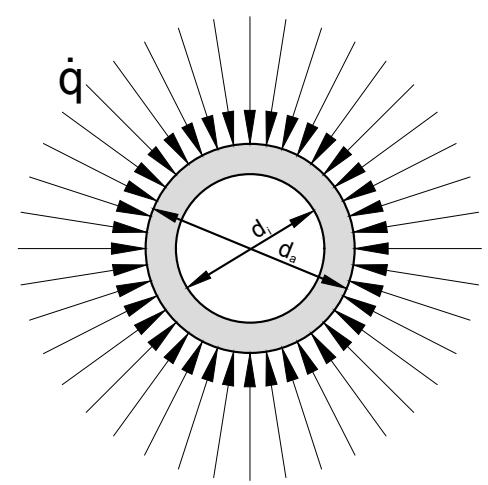

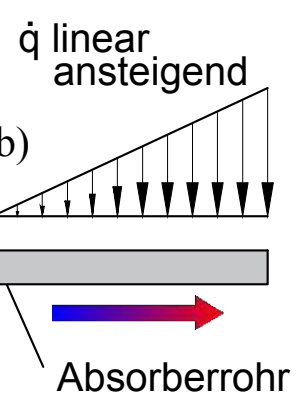

(c)
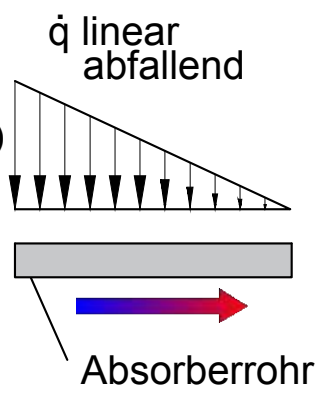

(f)

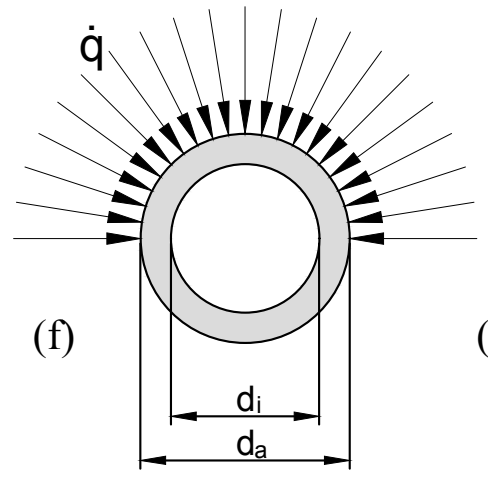

(d)
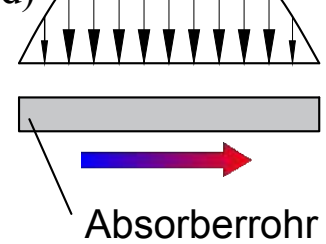

q sinusförmig

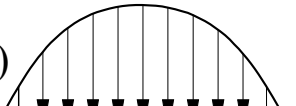

$\dot{q}$

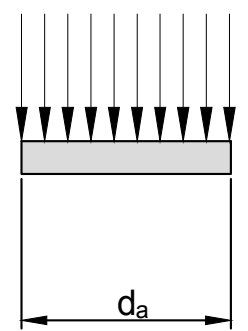

Abbildung 5.4.: Schematische Darstellung der möglichen Wärmestromdichteverteilung: (a-d): Wärmestromdichteverteilung entlang der Absorberrohre, (e-g): Wärmestromdichteverteilung über den Rohrumfang.

Mit der Vorgabe der thermischen Receiverleistung $\left(\dot{Q}_{\text {th }}\right)$, der Ein- und Auslasstemperatur ( $T_{\text {ein }}$ und $T_{\text {aus }}$ ) sowie der mittleren Strömungsgeschwindigkeit $(u)$ wird die Anzahl der Absorberrohre und damit die Receivergeometrie berechnet. Der Absolutdruck des WTM am Receivereinlass $p_{\max }$ dient zusammen mit den Material- und Festigkeitskennwerten zur Auslegung der Rohrwandstärke.

Für jede Variante werden die charakteristischen Receiverkenngrößen in eine Datei geschrieben, die anschließend zur Bewertung herangezogen werden kann. Diese beinhaltet auch die Druckverluste im Receiver bei verschiedenen Teillastzuständen sowie die modulweise gemittelten Werte aller thermohyraulischen Parameter. Zudem werden die Fluid- und Rohrtemperaturen, der Druckverlust, die Strömungsgeschwindigkeit sowie der Wärmeübergangskoeffizient entlang der Absorberrohre graphisch dargestellt. 
Abbildung 5.6 zeigt die graphische Ausgabe einer Beispielauslegung mit ASTRID. Die Modulverschaltung dieses Beispielreceivers ist in Abbildung 5.5 dargestellt. Als WTM wird Solar Salt verwendet. Beide parallel verlaufenden Strömungswege

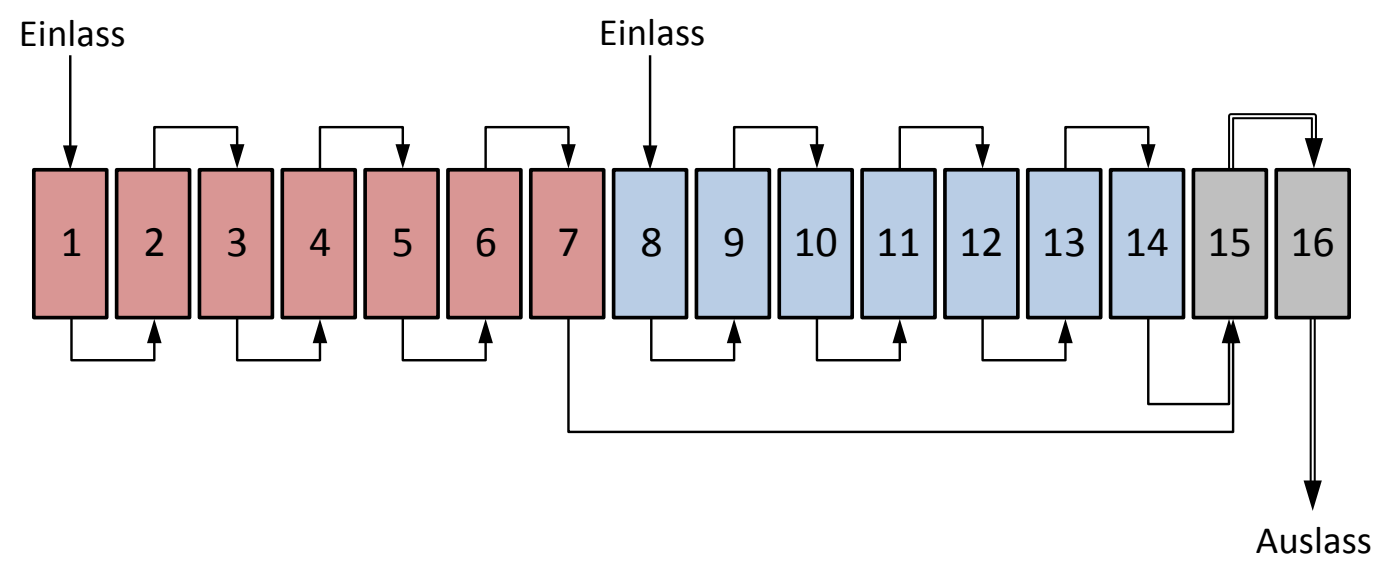

Abbildung 5.5.: Modulverschaltung einer Beispielauslegung mit ASTRID

(Modul 1 bis 7) und (Modul 8 bis 14) sind identisch aufgebaut und erfahren daher die gleichen Fluid- und Rohrtemperaturen. Diese beiden Strömungswege werden anschließend gemischt und durchströmen gemeinsam Modul 15 und 16 seriell. Der axial sinusförmige Verlauf der Wärmestromdichte in jedem Modul ist im oberen Teilbild in Abbildung 5.6 in grün dargestellt. Da in der Vorauslegung jedes Modul die gleiche Leistung erfährt, entspricht die Wärmestromdichte in jedem Modul der Absorberfläche. Modul 15 und 16 sind folglich etwa doppelt so breit wie die restlichen Module des Receivers.

Im mittleren Teilbild ist der Unterschied zwischen Rohrinnenwand- und Fluidtemperatur sowie der Druckverlust je Modul dargestellt. Die senkrechten Linien zu Beginn und am Ende jedes Moduls entsprechen den Druckverlusten in Verteiler und Sammler bzw. in den Übergängen zum nächsten Modul.

Im unteren Teilbild ist neben dem lokalen Wärmeübergangskoeffizient auch die Strömungsgeschwindigkeit dargestellt. Letztere hängt vom Massenstrom und der Anzahl der Rohre des Moduls ab. 

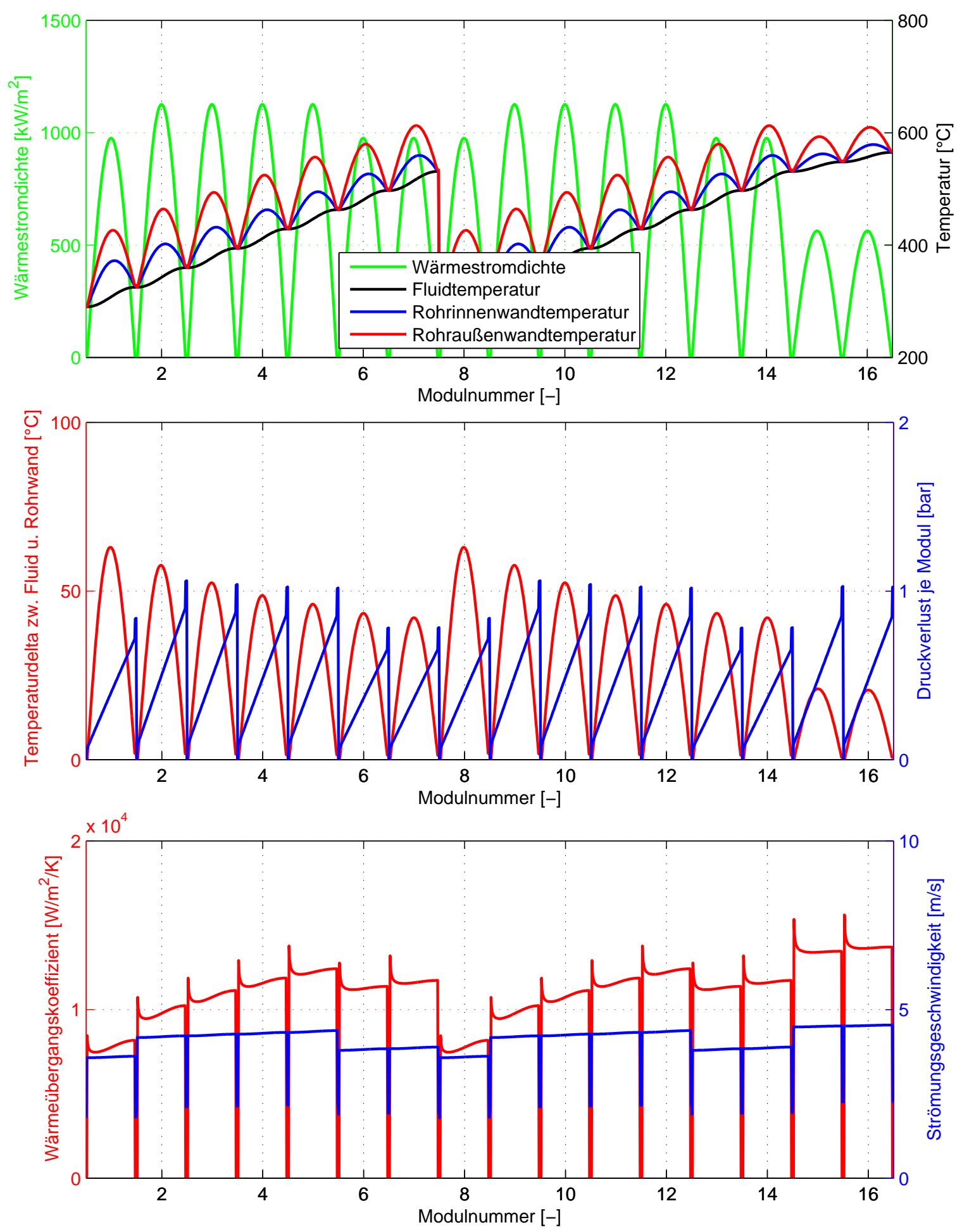

Abbildung 5.6.: Graphische Ausgabe der Receiverkenngrößen aus der Vorauslegung je Modul entlang eines Absorberrohres. Oben: Fluid- und Rohrtemperaturen sowie Wärmestromdichteverlauf, Mitte: Druckverlust und Temperaturdifferenz zwischen mittlerer Fluid- und Rohrinnenwandtemperatur, Unten: Wärmeübergangskoeffizient und Strömungsgeschwindigkeit. 
Abbildung 5.7 zeigt die Funktionsstruktur mit allen verwendeten MATLABFunktionen (*.m).

Receivermodellierung.m

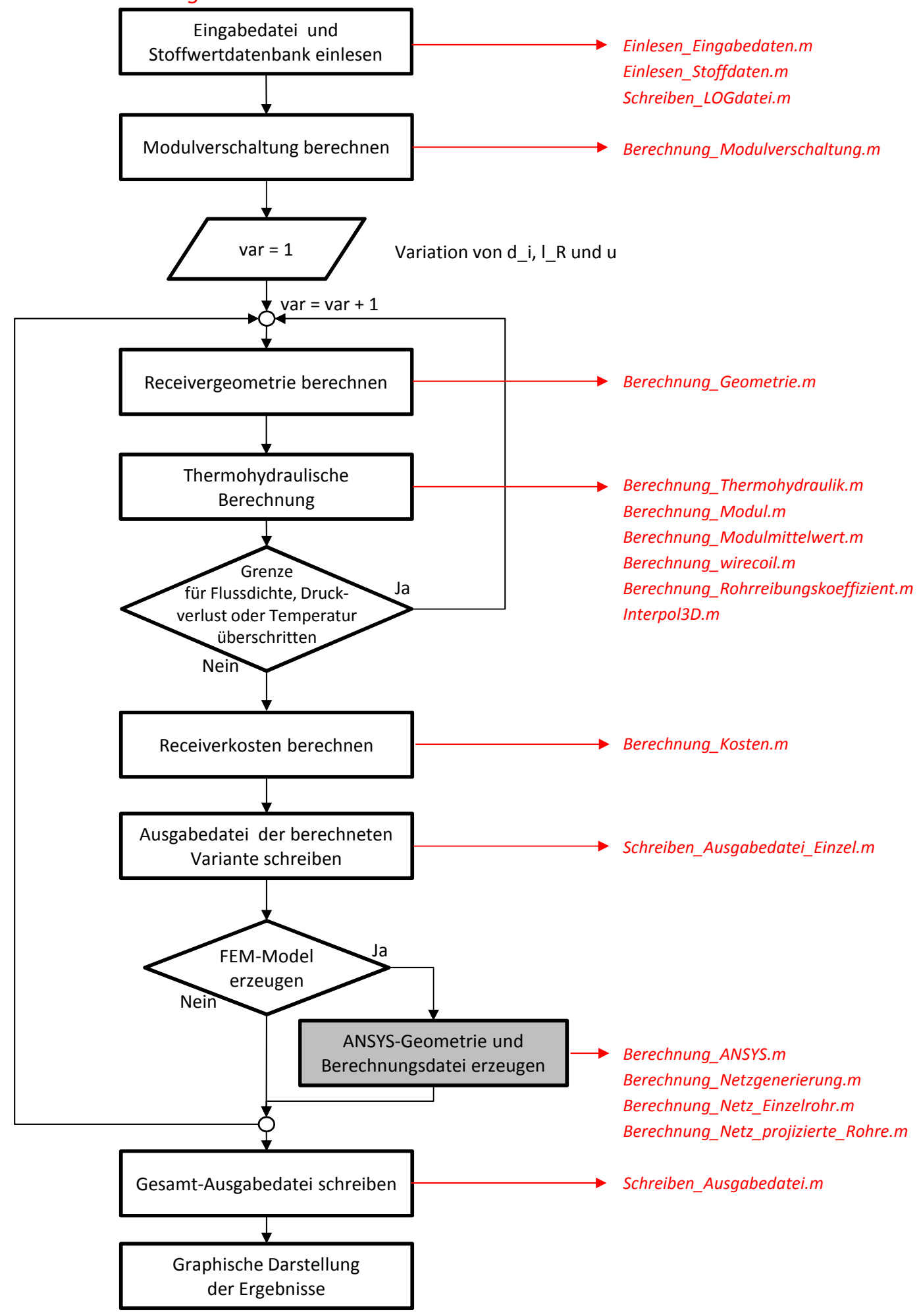

Abbildung 5.7.: Funktionsstruktur der Receivermodellierung (Vorauslegung und Erzeugung der FEM-Berechnungsdateien). 


\subsubsection{Berechnung der Receivergeometrie}

\subsubsection{Modulverschaltung}

Die Unterteilung des Receivers in mehrere gleich aufgebaute Module führt zu fertigungstechnischen Vorteilen und geringeren Kosten. Abbildung 5.8 zeigt die Anordung und Nummerierung der Module. Jedes Modul besitzt einen oberen und einen unteren Anschluss (einen Eingang und einen Ausgang). Die Module werden fortlaufend durchnummeriert, unabhängig der Modulverschaltung. Im manuellen Modus können die Module auch durch die Vorgabe der Koordinaten der Moduleckpunkte beliebig im Raum angeordnet werden.

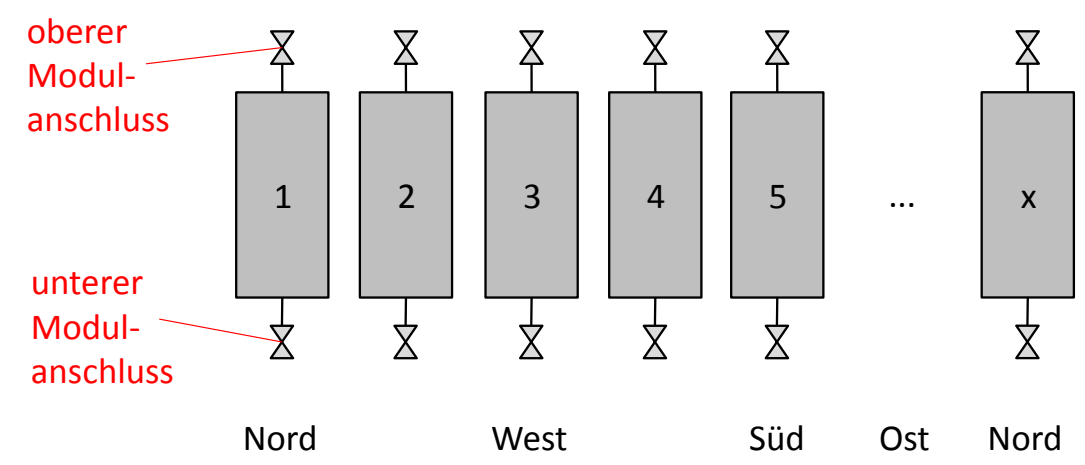

Abbildung 5.8.: Anordnung und Nummerierung der Module.

Programmtechnisch wird die Anzahl und die komplette Verschaltung der Module über folgende Modulverschaltungsmatrix definiert:

$$
M=\left[\begin{array}{c|c|c|c}
\text { Modul- } & \text { Folgemodul- } & \text { Einlass oben } & \text { Beidseitige } \\
\text { nummer } & \text { nummern } & \text { true/false } & \text { Bestrahlung } \\
& A|B| C \mid D & & \text { true/false }
\end{array}\right]
$$

Bei einer Parallelschaltung wird ein Anschluss mit mehreren Folgemodulen verbunden. Die Variablen $A, B, C, D$ stehen für bis zu vier möglichen Strömungsverzweigungen zu anderen Modulen. Ebenso sind Strömungsvereinigungen möglich. Bei den letzten Modulen (Receiverauslass) wird als Folgemodulnummer der Wert -1 gewählt. Bei speziellen Receiverkonfigurationen können die Module auch beidseitig bestrahlt werden (siehe letzte Spalte). Abbildung 5.9 zeigt ein Beispiel einer komplexen Modulverschaltung mit der zugehörigen Modulverschaltungsmatrix. In Modul 5 findet beispielsweise eine Strömungsvereinigung 
statt. Zudem ist die Fließrichtung von oben nach unten, daher ist der Eintrag in der vorletzten Spalte eine 1.

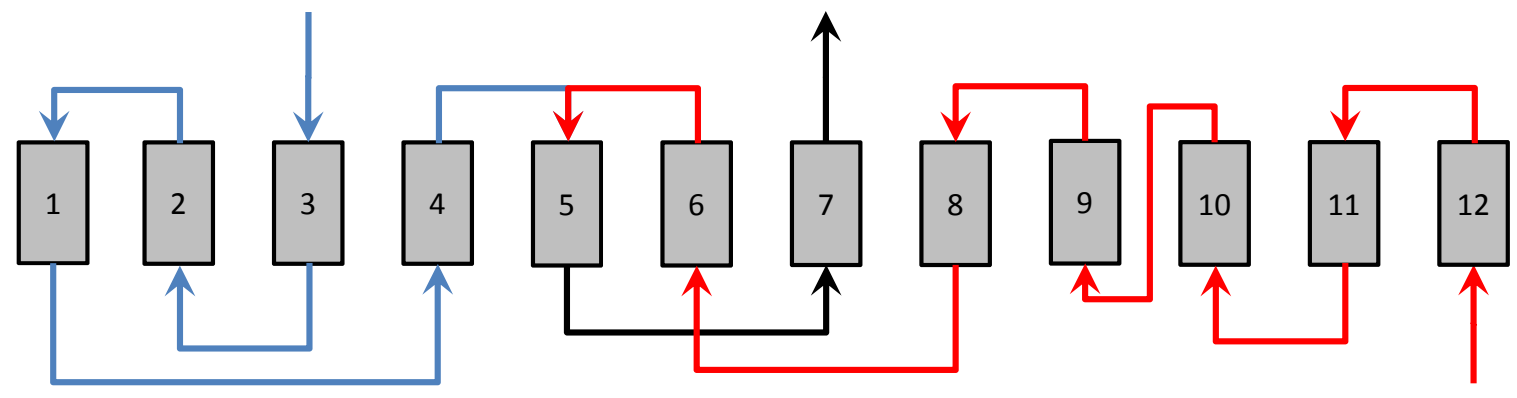

$$
M=\left[\begin{array}{c|cccc|c|c}
1 & 4 & 0 & 0 & 0 & 1 & 0 \\
2 & 1 & 0 & 0 & 0 & 0 & 0 \\
3 & 2 & 0 & 0 & 0 & 1 & 0 \\
4 & 5 & 0 & 0 & 0 & 0 & 0 \\
5 & 7 & 0 & 0 & 0 & 1 & 0 \\
6 & 5 & 0 & 0 & 0 & 0 & 0 \\
7 & -1 & 0 & 0 & 0 & 0 & 0 \\
8 & 6 & 0 & 0 & 0 & 1 & 0 \\
9 & 8 & 0 & 0 & 0 & 0 & 0 \\
10 & 9 & 0 & 0 & 0 & 0 & 0 \\
11 & 10 & 0 & 0 & 0 & 1 & 0 \\
12 & 11 & 0 & 0 & 0 & 0 & 0
\end{array}\right]
$$

Abbildung 5.9.: Beispiel einer komplexen Modulverschaltung mit zugehöriger Modulverschaltungsmatrix

Theoretisch ist eine beliebige Modulverschaltung mit Kombinationen aus Parallelund Reihenschaltung möglich. Im Programmcode sind zwei symmetrische Modulverschaltungen implementiert (siehe Abbildung 5.10). Beide haben jeweils zwei Strömungswege, welche die Module in Serpentinen durchlaufen. Der Einlass ist jeweils an den beiden Modulen auf der Nordseite und der Auslass auf der Südseite. Bei $t y p_{\text {mod }}=1$ (a) durchläuft ein Strang jeweils die Hälfte des Receiverumfangs. Bei typ $p_{\text {mod }}=2$ (b) wechselt jeder Strang nach einem Viertel des Receiverumfangs die Seit $5^{5}$. Bei ungerader Modulanzahl werden die Stränge im letzten Modul auf der Südseite zusammengeführt (siehe Abbildung 5.3a). Dadurch ergibt sich hier der doppelte Massenstrom, wie in den restlichen Modulen.

\footnotetext{
${ }^{5}$ Durch den veränderlichen Sonnenstand erscheint diese Modulverschaltung aus regelungstechnischer Sicht (konstante Receiveraustrittstemperatur) sinnvoll.
} 


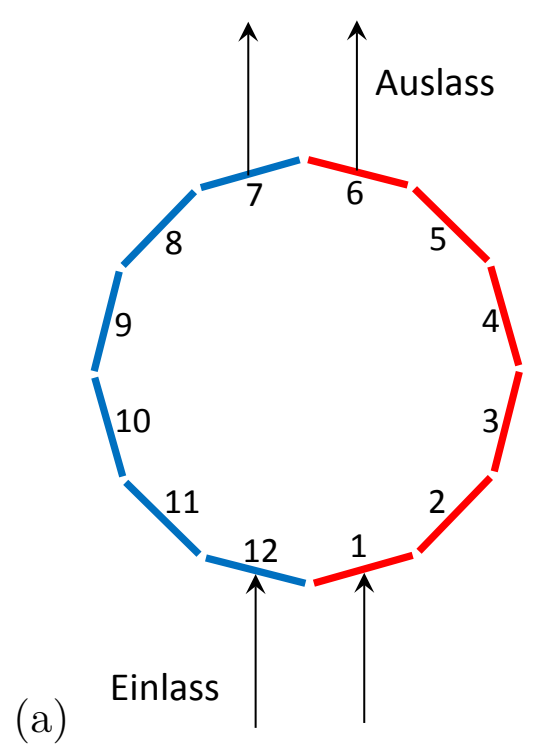

(a)

\begin{abstract}
Abbildung 5.10.: Prinzipskizze für symmetrische Modulverschaltungen. (a): ohne Kreuzung der Strömungswege, (b): Kreuzung der Strömungswege nach $1 / 4$ des Receiverumfangs.
\end{abstract}

\title{
5.1.2.2. Anzahl und Abmessungen der Absorberrohre
}

Der benötigte Massenstrom lässt sich aus der Receiverleistung und dem gewünschten Temperaturhub sowie der mittleren Wärmekapazität des WTM berechnen. Es gilt:

$$
\dot{m}_{\text {ges }}=\frac{\dot{Q}_{\text {th }}}{\overline{c_{p}} \cdot\left(T_{\text {aus }}-T_{\text {ein }}\right)}
$$

Mit der Kontinuitätsgleichung und der Strömungsgeschwindigkeit kann der Massenstrom durch ein Absorberrohr berechnet werden:

$$
\dot{m}_{\mathrm{r}}=\rho \cdot u_{\mathrm{r}} \cdot \pi \cdot \frac{d_{\mathrm{i}}}{4}
$$

Mit der Anzahl der Strömungswege im Receiver lässt sich damit die Anzahl der Absorberrohre pro Modul berechnen:

$$
n_{\mathrm{r}}=\frac{\dot{m}_{\mathrm{ges}}}{\dot{m}_{\mathrm{r}} \cdot n_{\mathrm{str}}}
$$


Der Wert für die Rohranzahl wird im Programm ganzzahlig gerundet und der tatsächliche Massenstrom durch ein Rohr neu berechnet. Alle weiteren Berechnungen verwenden dann ebenfalls die gerundete Rohranzahl.

Die Wandstärke der Absorberrohre und der Verteiler/Sammler wird mit der Druckgeräterichtlinie nach AD 2000-B1 [97] berechnet. Die genaue Berechnungsvorschrift ist im Anhang C, Seite 183 dokumentiert.

Sofern Diffusoren in der Berechnung berücksichtigt werden, ergibt sich der Innenduchmesser des Diffusorendes aus dem Diffusoröffnungswinkel und der Diffusorlänge (siehe Abbildung rechts)

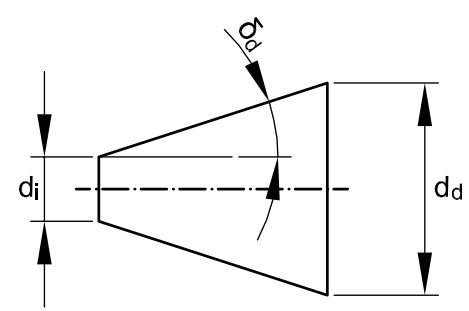

Es ergibt sich:

$$
\tan \delta_{\text {dif }}=\frac{d_{\text {dif }}-d_{\mathrm{i}}}{2 \cdot L_{\mathrm{dif}}} \quad \rightarrow \quad d_{\mathrm{dif}}=2 \cdot L_{\mathrm{dif}} \cdot \tan \delta_{\mathrm{dif}}+d_{\mathrm{i}}
$$

Der mittlere Receiverdurchmesser ergibt sich aus

$$
d_{\mathrm{rec}}=n_{\mathrm{r}} \cdot n_{\mathrm{mod}} \cdot \frac{\left(d_{\mathrm{a}}+z_{\mathrm{r}}\right)}{\pi}
$$

Bei der Berechnung der Modulbreite muss eine Fallunterscheidung getroffen werden. Für einen kreisrunden Receiver (siehe Abbildung $5.2 \mathrm{~d}$ ) gilt:

$$
b_{\mathrm{mod}}=\pi \cdot \frac{d_{\mathrm{rec}}}{n_{\mathrm{mod}}}
$$

Für prismenförmige Receiver, deren Grundfläche aus einem regelmäßigem Polygon besteht (siehe Abbildung 5.2 ) gilt für die Modulbreite:

$$
b_{\text {mod }}=n_{\mathrm{r}} \cdot\left(d_{\mathrm{a}}+z_{\mathrm{r}}\right)
$$

\subsubsection{Thermohydraulische Berechnung}

In der thermohydraulischen Berechnung werden die Druckverluste, Wärmeübergangskoeffizienten und Rohrtemperaturen berechnet. Für jede Variante (Kombi- 
nation aus $d_{\mathrm{i}}, u_{\mathrm{r}}$ und $\left.H_{\mathrm{rec}}\right)$ wird die Geometrie berechnet und anschließend die thermohydraulischen Parameter entlang eines Stromfadens durch den Receiver bestimmt. Dabei werden die Absorberrohre axial in kleine scheibenförmige Diskretisierungsabschnitte unterteilt. Eine Randbedingung in der Vorauslegung ist, dass jedes Modul die gleiche Leistung an das Fluid überträgt, unabhängig der Modulverschaltung.

Die Stoffwerte Dichte $\rho$, Wärmeleitfähigkeit $\lambda$, spezifische Wärmekapazität $c_{p}$, dynamische Viskosität $\eta$ und Prandtl-Zahl $\operatorname{Pr}$ werden für jeden Diskretisierungsabschnitt entsprechend der aktuell herrschenden Temperatur neu berechnet (Temperaturabhängige Stoffwerte siehe Anhang A, Seite 177).

Jedes Modul (bzw. jedes Rohr) wird in die folgenden Diskretisierungsabschnitte unterteilt:

- $\operatorname{Verteiler~}(i=1)$

- Übergang Verteiler - Absorberrohr $(i=2)$

- Unterteilung des Absorberrohres in $x$ Abschnitte mit einer Länge von je $l_{\text {sector }}$ (unabhängig von der Gesamtrohrlänge) $(i=3 \ldots x+2)$

- Diffusor $(i=x+3)$

- Übergang Diffusor - Sammler $(i=x+4)$

- Sammler $(i=x+5)$

Verteiler, Sammler, Diffusor und Übergänge werden als adiabat betrachtet und werden daher nur für die Berechnung des Druckverlustes berücksichtigt. Für jedes Modul werden die mittleren Temperaturen, Druckverluste und Wärmeübergangskoeffizienten in die Ergebnisdatei geschrieben. Des Weiteren enthält die Ergebnisdatei auch sämtliche thermohydraulischen Größen für jeden Diskretisierungsabschnitt.

\subsubsection{Rohrreibungskoeffizient und Druckverlust}

Zur Berechnung des Druckverlustes ist der Rohrreibungskoeffizient $\zeta$ entscheidend. Ihre Berechnung erfolgt nach dem VDI-Wärmeatlas 2006 46 und ist im Anhang für alle Komponenten und Übergänge im Anhang D, Seite 185 dokumentiert. 


\subsubsection{Strömungsgeschwindigkeit und Reynolds-Zahl}

Über die Kontinuitätsgleichung lässt sich die Strömungsgeschwindigkeit und damit die Reynolds-Zahl berechnen. Es gilt:

$$
\begin{aligned}
& \dot{m}_{\text {mod }}=\rho \cdot u_{\mathrm{r}}(i) \cdot n_{\mathrm{r}} \cdot A_{\mathrm{r}}(i) \quad \text { mit } \quad A_{\mathrm{r}}=\frac{\pi \cdot d_{\mathrm{i}}^{2}}{4} \\
& \rightarrow u_{\mathrm{r}}(i)=\frac{4 \cdot \dot{m}_{\mathrm{mod}}}{\rho(i) \cdot n_{\mathrm{r}} \cdot \pi \cdot d_{\mathrm{i}}^{2}}
\end{aligned}
$$

Mit der kinematische Viskosität lässt sich damit die Reynolds-Zahl sowie die Peclet-Zahl berechnen:

$$
\nu(i)=\frac{\eta(i)}{\rho(i)} \quad \rightarrow \operatorname{Re}(i)=\frac{u_{\mathrm{r}}(i) \cdot d_{\mathrm{i}}}{\nu(i)}, \quad \operatorname{Pe}(i)=\operatorname{Re}(i) \cdot \operatorname{Pr}(i)
$$

\subsubsection{Berechnung der Nusselt-Zahl}

Die Nusselt-Zahl beschreibt den konvektiven Wärmeübergang zwischen einer festen Oberfläche und einem strömenden Fluid. Je nach Geometrie und Strömungsart gibt es unterschiedliche Nusselt-Korrelationen mit verschiedenen Gültigkeitsbereichen. Für niedrige Prandtl-Zahlen, wie sie bei Flüssigmetallen vorkommen gibt es zudem gesonderte Korrelationen. Eine umfassende Übersicht zu verschiedenen Korrelationen für Flüssigmetalle findet sich in [16]. Im Programmcode für die Receivermodellierung sind folgende Nusselt-Korrelationen hinterlegt. Im Anhang E auf Seite 187 finden sich die dazugehörigen Formeln.

- $N u$ nach Gnielinski 45

- Nu nach Gnielinski unter Berücksichtigung der Einlaufstrecke 98

- Nu nach Hausen 99

- Nu für Alkalimetalle (Na, K, NaK) nach Chen \& Chiou [16

- $N u$ für Blei-Bismuth (Pb-Bi) nach Lubarsky and Kaufman 16

- $N u$ für Blei $(\mathrm{Pb})$ nach Kutateladze [16]

Der konvektive Wärmeübergangskoeffizient ergibt sich anschließend mit der Nusselt-Zahl:

$$
\alpha_{\mathrm{wtm}}(i)=\frac{N u(i) \cdot \lambda(i)}{d_{\mathrm{i}}}
$$




\subsubsection{Berechnung der Rohrwandtemperaturen}

Die Fluidtemperaturen in Verteiler, Diffusor und Sammler werden als konstant angenommen. Um die Fluid- und Rohrwandtemperaturen des Absorbers bestimmen zu können wird zunächst die Leistung bestimmt, die pro Diskretisierungsabschnitt an das WTM übertragen wird. Die Wärmestromdichte kann sowohl über den Rohrumfang als auch über die Absorberrohrlänge variieren. Die bestrahlte Länge des Rohrumfangs ergibt sich zu:

$$
\begin{aligned}
& L_{\mathrm{qth}}=\pi \cdot d_{\mathrm{i}} \quad \rightarrow \text { kompletter Umfang bestrahlt } \\
& L_{\mathrm{qth}}=\pi \cdot d_{\mathrm{i}} / 2 \quad \rightarrow \text { halber Umfang bestrahlt } \\
& L_{\mathrm{qth}}=d_{\mathrm{i}} \quad \rightarrow \text { projizierter Umfang bestrahlt }
\end{aligned}
$$

Mit der Vorgabe der Modulleistung und des Verlaufs der Wärmestromdichte entlang des Absorberrohrs sowie der Anzahl der Diskretisierungsabschnitte kann die Wärmestromdichte für jeden Absorberabschnitt berechnet werden:

$$
\begin{aligned}
& \dot{q}_{\mathrm{wtm}}(i)=\frac{\dot{Q}_{\text {mod_ges }}}{n_{\mathrm{r}} \cdot n_{\mathrm{bin}} \cdot L_{\mathrm{bin}} \cdot L_{\mathrm{qth}}} \quad \rightarrow \text { homogen entlang Absorber } \\
& \dot{q}_{\mathrm{wtm}}(i)=\frac{\dot{Q}_{\text {mod_ges }}}{n_{\mathrm{r}} \cdot n_{\text {bin }} \cdot L_{\text {bin }} \cdot L_{\mathrm{qth}}}\left[\frac{2 \cdot(i-1)}{n_{\text {bin }}}\right] \rightarrow \text { linear ansteigend } \\
& \dot{q}_{\mathrm{wtm}}(i)=\frac{\dot{Q}_{\text {mod_ges }}}{n_{\mathrm{r}} \cdot n_{\mathrm{bin}} \cdot L_{\mathrm{bin}} \cdot L_{\mathrm{qth}}}\left[\frac{2-2(i-1)}{n_{\mathrm{bin}}}\right] \rightarrow \text { linear abfallend } \\
& \dot{q}_{\mathrm{wtm}}(i)=\sin \left((i-3) \frac{\pi}{n_{\mathrm{bin}}}\right) \frac{\pi}{2} \frac{\dot{Q}_{\text {mod_ges }}}{n_{\mathrm{r}} \cdot n_{\text {bin }} \cdot L_{\text {bin }} \cdot L_{\mathrm{qth}}} \quad \rightarrow \text { sinusförmig }
\end{aligned}
$$

Daraus ergibt sich die ans Fluid übertragene Leistung für jeden Diskretisierungsabschnitt:

$$
\dot{Q}_{\mathrm{mod}}(i)=\dot{q}_{\mathrm{wtm}}(i) \cdot n_{\mathrm{r}} \cdot L_{\mathrm{bin}} \cdot L_{\mathrm{qth}}
$$

Die Fluidtemperatur entlang des Absorberrohrs lässt sich damit rekursiv für jeden Diskretisierungsabschnitt berechnen:

$$
T_{\mathrm{wtm}}(i)=T_{\mathrm{wtm}}(i-1)+\frac{\dot{Q}_{\mathrm{mod}}(i)}{\dot{m}_{\mathrm{mod}} \cdot c_{p}(i)}
$$


Für den konvektiven Wärmestrom im Absorberrohr gilt:

$$
\dot{Q}_{\text {mod }}(i)=\alpha_{\text {wtm }}(i) \cdot n_{\mathrm{r}} \cdot L_{\mathrm{bin}} \cdot d_{\mathrm{i}} \cdot \pi \cdot\left[T_{\mathrm{wi}}(i)-T_{\mathrm{wtm}}(i)\right]
$$

Durch Umformen lässt sich die Rohrinnenwandtemperatur ermitteln:

$$
T_{\mathrm{wi}}(i)=T_{\mathrm{wtm}}(i)+\frac{\dot{Q}_{\bmod }(i)}{\alpha_{\mathrm{wtm}}(i) \cdot n_{\mathrm{r}} \cdot L_{\mathrm{bin}} \cdot d_{\mathrm{i}} \cdot \pi}
$$

Für die Wärmeleitung in der Rohrwand gilt:

$$
\dot{Q}_{\text {mod }}(i)=\frac{2 \pi \cdot L_{\mathrm{bin}} \cdot n_{\mathrm{r}} \cdot \lambda_{\mathrm{r}} \cdot\left[T_{\mathrm{wa}}(i)-T_{\mathrm{wi}}(i)\right]}{\ln \left(d_{\mathrm{a}} / d_{\mathrm{i}}\right)}
$$

Durch Umformen ergibt sich die Rohraußenwandtemperatur:

$$
T_{\text {wa }}(i)=T_{\text {wi }}(i)+\frac{\dot{Q}_{\text {mod }}(i) \cdot \ln \left(d_{\mathrm{a}} / d_{\mathrm{i}}\right)}{2 \pi \cdot L_{\mathrm{bin}} \cdot n_{\mathrm{r}} \cdot \lambda_{\mathrm{r}}}
$$

Die aufzubringende Pumpenleistung eines Moduls ergibt sich aus:

$$
P_{\mathrm{p}}(i)=\frac{\dot{m}_{\mathrm{mod}} \cdot \Delta p(i)}{\rho(i) \cdot \eta_{\mathrm{p}}}
$$

\subsubsection{Berechnung der Receiverkosten}

Zur überschlägigen Abschätzung der Receiverkosten wird für jede Variante eine Kostenberechnung anhand von spezifischen Kostendaten durchgeführt. Tabelle 5.1 zeigt die zur Berechnung verwendeten Kostenparameter.

Für die Berechnung der Materialkosten muss zunächst die Masse des Receivers (Absorberrohre, Verteiler und Sammler) berechnet werden:

$$
\begin{aligned}
& m_{\mathrm{r}}=n_{\mathrm{mod}} \cdot n_{\mathrm{r}} \cdot \rho_{\mathrm{r}} \cdot \frac{\pi}{4} \cdot\left(d_{\mathrm{a}}{ }^{2}-d_{\mathrm{i}}{ }^{2}\right) \cdot L_{\mathrm{r}} \\
& m_{\mathrm{v}}=n_{\mathrm{mod}} \cdot \rho_{\mathrm{v}} \cdot \frac{\pi}{4} \cdot\left({d_{\mathrm{av}}}^{2}-{d_{\mathrm{v}}}^{2}\right) \cdot b_{\mathrm{mod}} \\
& m_{\mathrm{s}}=n_{\mathrm{mod}} \cdot \rho_{\mathrm{s}} \cdot \frac{\pi}{4} \cdot\left({d_{\mathrm{as}}}^{2}-{d_{\mathrm{s}}}^{2}\right) \cdot b_{\mathrm{mod}}
\end{aligned}
$$


Damit ergeben sich die Materialkosten für die Absorberrohre, den Verteiler und den Sammler.

$$
K_{\mathrm{r}, \mathrm{v}, \mathrm{s}}=k_{\mathrm{r}, \mathrm{v}, \mathrm{s}} \cdot m_{\mathrm{r}, \mathrm{v}, \mathrm{s}}
$$

Die Materialkosten der Isolierung sind vom Receiveraufbau abhängig. Bei Rundumfeldreceivern gilt als Bezugsfläche für die spezifischen Kosten der Zylindermantel, der von den Absorberrohren umschlossen wird. Bei Verteiler und Sammler wird als Bezugsfläche jeweils die Rohraußenfläche verwendet. Für Receiver in einer Kavität wird als Bezugsfläche entsprechend die Zylindermantelfläche, welche die Absorberrohre umschließt verwendet. Zudem gibt es hier noch eine kreisringförmige Dämmfläche, die die Apertur formt sowie eine Kreisfläche am hinteren Ende der Kavität. Abbildung 5.11 veranschaulicht die Bezugsflächen für die Berechnung der Isolationskosten.

Die Materialkosten für die Dämmung ergeben sich damit wie folgt:

$$
K_{\mathrm{r}, \mathrm{v}, \mathrm{s}}=k_{\mathrm{iso}} \cdot A_{\mathrm{iso} \_\mathrm{r}, \mathrm{v}, \mathrm{s}}
$$

Tabelle 5.1.: Kostenparameter für die überschlägige Kostenabschätzung in der Receivervorauslegung 100

\begin{tabular}{lll}
\hline Parameter & Variable & Einheit \\
\hline \hline Materialkosten Absorberrohre & $k_{\mathrm{r}}$ & $€ / \mathrm{kg}$ \\
Materialkosten Verteiler & $k_{\mathrm{v}}$ & $€ / \mathrm{kg}$ \\
Materialkosten Sammler & $k_{\mathrm{s}}$ & $€ / \mathrm{kg}$ \\
Materialkosten Dämmung & $k_{\text {iso }}$ & $€ / \mathrm{m}^{2}$ \\
Stückkosten Metallbälge & $k_{\text {comp }}$ & $€ / \mathrm{St}$ \\
Stückkosten Diffusoren & $k_{\text {dif }}$ & $€ / \mathrm{St}$ \\
Material/Fertigung Tragstruktur & $k_{\text {struct }}$ & $€ / \mathrm{m}^{2}$ \\
Fertigungskosten Bohrungen in S/V & $k_{\text {drill }}$ & $€ / \mathrm{St}$ \\
Fertigungskosten Schweißnähte Umfang Absorber & $k_{\text {weld_r }}$ & $€ / \mathrm{St}$ \\
Fertigungskosten Schweißnähte Umfang V/S & $k_{\text {weld_v,s }} € / \mathrm{m}$ \\
Fertigungskosten allg. pro Modul & $k_{\text {mod }}$ & $€ / \mathrm{St}$ \\
Montagekosten Dämmung je für V,Absorber,S & $k_{\text {assy_r,v,s }} € / \mathrm{St}$ \\
\hline
\end{tabular}


Receiver mit außen liegenden Rohren

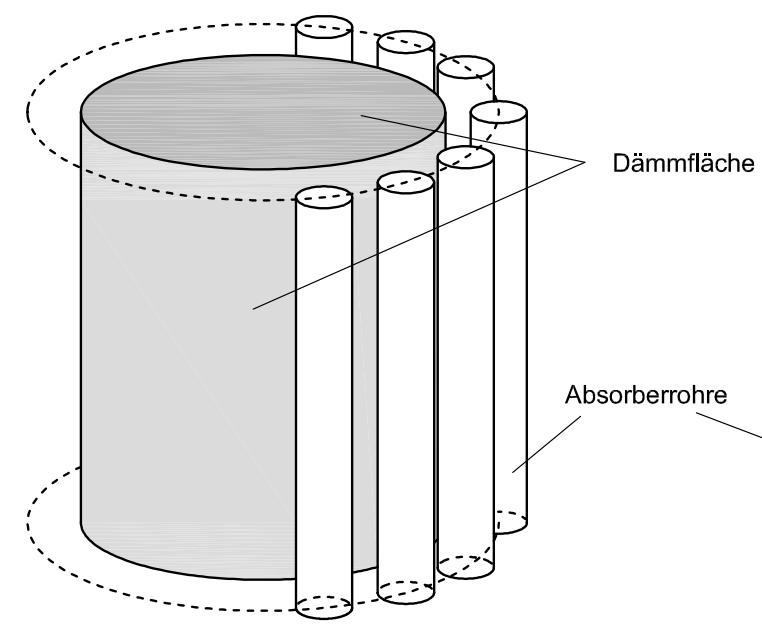

Receiver mit Rohre in Cavity

Abbildung 5.11.: Definition der Bezugsflächen für die Kostenabschätzung der thermischen Isolation von Rundumfeld- und Cavityreceivern.

Die Metallbälge und die Diffusoren werden über Stückkosten berechnet:

$$
\begin{aligned}
K_{\mathrm{comp}} & =k_{\mathrm{comp}} \cdot n_{\mathrm{r}} \cdot n_{\mathrm{mod}} \\
K_{\mathrm{dif}} & =k_{\mathrm{dif}} \cdot n_{\mathrm{r}} \cdot n_{\mathrm{mod}}
\end{aligned}
$$

Die Fertigungskosten für Bohrungen in Verteiler/Sammler sowie die Erstellung der Tragstruktur ergeben:

$$
\begin{aligned}
K_{\text {drill_v }, \mathrm{s}} & =k_{\text {drill }} \cdot 2 \cdot n_{\mathrm{r}} \cdot n_{\mathrm{mod}} \\
K_{\text {struct }} & =k_{\text {struct }} \cdot A_{\text {iso }}
\end{aligned}
$$

Die Schweißnähte zw. Absorber/Diffusor, Absorber/Metallbalg, Verteiler/Metallbalg und Diffusor/Sammler:

$$
\begin{aligned}
K_{\text {weld_r,comp,dif,s }} & =k_{\text {weld_r }} \cdot 4 \cdot n_{\text {mod }} \cdot n_{\mathrm{r}} \\
K_{\text {weld_mod }} & =\left[k_{\text {weld__v }}+k_{\text {weld_s }}\right] \cdot n_{\text {mod }} \cdot \pi \cdot\left(d_{\text {av }}+d_{\text {as }}\right)
\end{aligned}
$$

Die Montagekosten für die Isolation wird pauschal für Verteiler, Sammler und Absorber berechnet:

$$
K_{\text {assy_r,v,s }}=3 \cdot k_{\text {assy_r,v, }, \mathrm{s}}
$$




\subsection{Thermische Receivermodellierung mit FEM}

In diesem Kapitel wird die thermische Receivermodellierung mit FEM detailliert beschrieben. In der Receivervorauslegung mit ASTRID werden die Verluste durch Konvektion und Strahlung sowie der Strahlungsaustausch zwischen benachbarten Komponenten vernachlässigt. Für eine detailliertere Betrachtung insbesondere einer Wirkungsgradberechnung können diese Einflüsse nicht mehr ignoriert werden. Daher wird der Receiver mit FEM modelliert und simuliert. Als Simulationsprogramm wird ANSYS verwendet. Über die fortranähnliche, programmeigene Skriptsprache APDL, kann die Berechnung komplett automatisiert werden. Basierend auf den Ergebnissen der Vorauslegung werden in ASTRID alle für die Berechnung notwendigen Parameter in APDL-Makros geschrieben, sodass für den Nutzer keine weiteren Schritte notwendig sind.

ASTRID verfügt zudem über eine Schnittstelle zu einem Strahlverfolgungsprogramm (SPRAY). Dadurch kann die reale Wärmestromdichteverteilung, wie sie zu einem bestimmten Zeitpunkt vom Heliostatenfeld auf dem Receiver erzeugt wird, berücksichtigt werden.

Die Geometriedaten des Receivers werden aus der Vorauslegung übernommen. Alternativ kann die Receivergeometrie auch manuell vorgegeben werden. In diesem Fall können die Module auch unterschiedliche Rohranzahlen und -geometrien aufweisen und beliebig im Raum angeordnet sein. Die Erstellung der Rechennetze geschieht ebenfalls über ASTRID.

Die generierten APDL-Makros beinhalten einen Algorithmus zur Regelung der Receiveraustrittstemperatur. Es besteht die Wahl zwischen einer iterativen Skalierung der Wärmestromdichteverteilung auf dem Receiver oder einer Regelung des Massenstroms, um die gewünschte Receiveraustrittstemperatur zu erhalten. Letztere berücksichtigt auch die Modulverschaltung und die Anzahl der Strömungswege, deren Massenströme getrennt voneinander entsprechend auf Austrittstemperatur geregelt werden. Dieser Algorithmus läuft ohne weitere Intervention des Benutzers automatisiert ab.

Abbildung 5.12 zeigt schematisch die in ASTRID angewendete Vorgehensweise der thermischen Receivermodellierung mittels FEM. Die einzelnen Schritte werden im Folgenden detailliert beschrieben. 


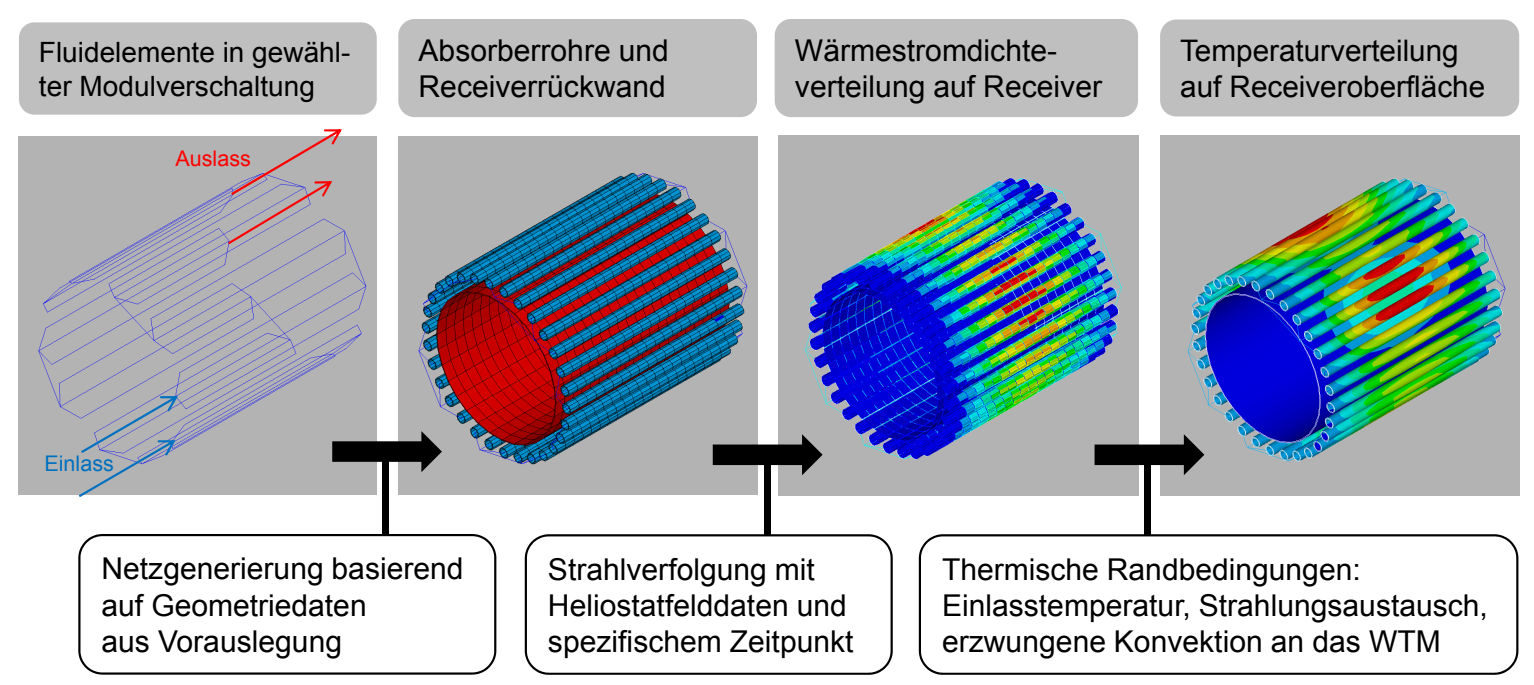

Abbildung 5.12.: Schematischer Aufbau und Vorgehensweise der in ASTRID implementierten thermischen Receivermodellierung mit FEM.

\subsubsection{Geometrieerstellung und Netzgenerierung}

Sämtliche Geometriedaten sowie die Anzahl und Verschaltung der Module werden aus der Vorauslegung übernommen. Die Rohrdiskretisierung kann beliebig erfolgen. Das gröbste FEM-Modell besteht aus lediglich projizierten Rohrflächen. In diesem Fall wird um den Receiverumfang für jedes Rohr ein Element erzeugt. Werden die Absorberrohre komplett abgebildet muss die Elementanzahl über Rohrumfang und -länge angegeben werden. Die Dämmfläche (Receiverrückwand oder Kavität) kann als externes Modell eingebunden werden oder bei einfachen Geometrien (z.B. Zylinder) direkt im Code erzeugt werden.

Als Grundlage zur Netzgenerierung dienen Knoten. Entsprechend der Diskretisierung werden durch einen Algorithmus aus den Geometriedaten Knoten im Raum erstellt und daraus Elemente definiert. Der große Vorteil dieser Herangehensweise ist, dass die Knotennummern frei gewählt werden können und die Zuordnung von Knotennummer und Position im Raum stets möglich ist, wodurch die Auswertung einfacher wird. Außerdem vereinfacht sich die Kopplung von Fluid- und Oberflächenelementen, wodurch die Rechenzeit zur Netzgenerierung reduziert wird6.

${ }^{6}$ Für die Konvektion auf der Rohrinnenseite müssen die Oberflächenelemente surf152 mit den Fluidelementen fluid116 gekoppelt werden. Der sonst notwendige und zeitintensive Suchalgorithmus mit dem Befehl mstole entfällt hier, da die Elemente direkt aus den bekannten Knotennummern generiert werden. 


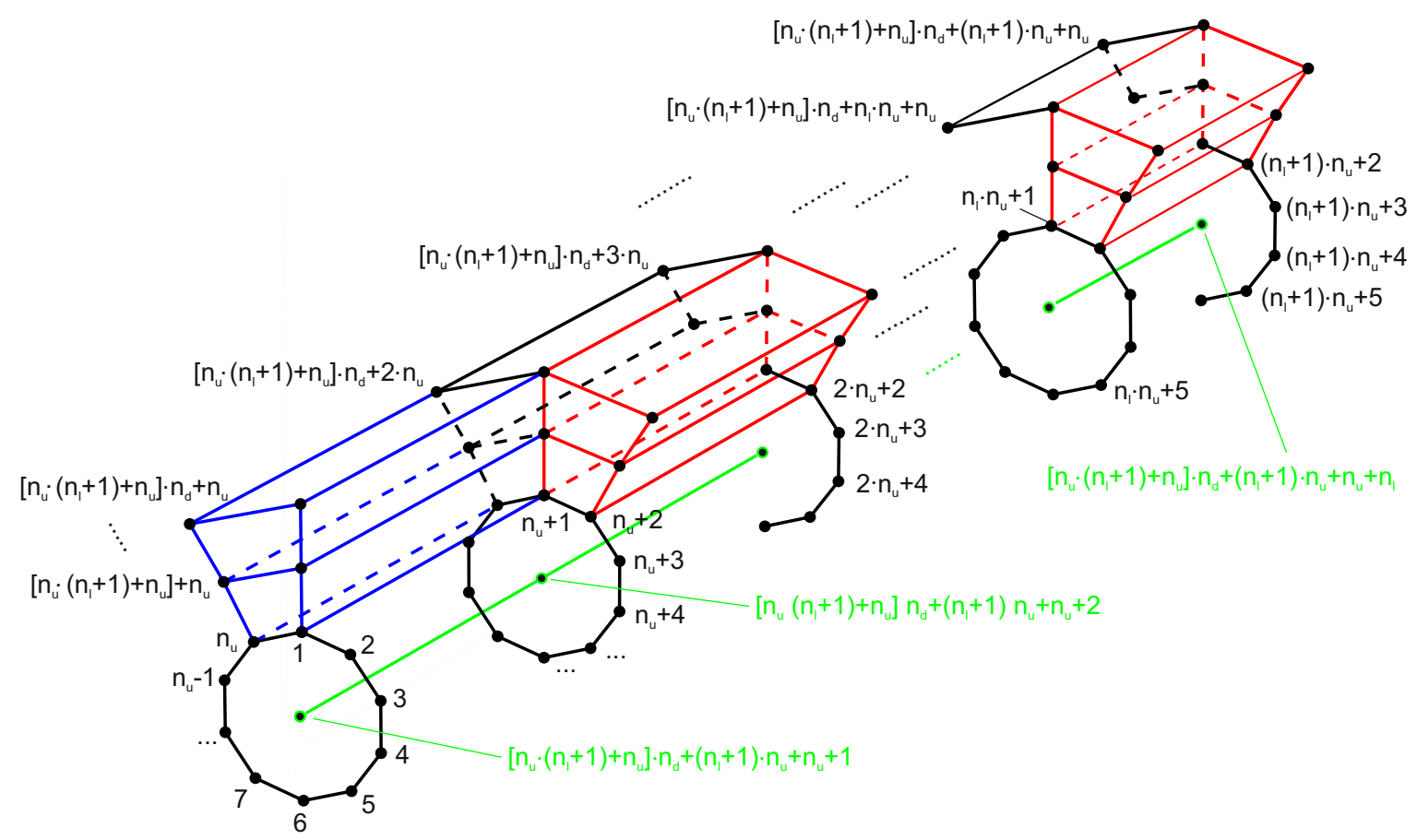

Abbildung 5.13.: Knotennummerierung der Absorberrohre in ASTRID mit $n_{\mathrm{u}}$ : Anzahl der Elemente in Rohrumfangsrichtung, $n_{\mathrm{l}}$ : Anzahl der Elemente in Rohrlängsrichtung, $n_{\mathrm{d}}$ : Anzahl der Elemente über die Rohrdicke. Grüne Linie: Fluidelemente.

\subsubsection{Volumenelemente}

Sowohl die Absorberrohre, als auch die Rückwand bzw. Kavität werden mit dem thermischen 3D-Element solid70 ohne Zwischenknoten modelliert. Jedes Element (Hexaeder) besitzt somit acht Knoten. Abbildung 5.13 zeigt beispielhaft die Knotenpositionierung und -nummerierung anhand eines Absorberrohres. Zur Elementgenerierung müssen diese in einer speziellen Reihenfolge angegeben werden.

\subsubsection{Fluidelemente}

Als Fluidelemente werden Elemente des Typs fluid116 verwendet. Diese 1DFluidelemente berücksichtigen den Massen- und Wärmetransport. Die Knoten dieser Elemente befinden sich alle auf dem gedachten Strömungsfaden in der Rohrmitte und haben die gleiche Diskretisierung wie die Rohrelemente selbst. Jedes Fluidelement besteht aus zwei Knoten. Der hydraulische Durchmesser der 
Rohrströmung wird über die real constants des Elementtyps angegeben. Die Reihenfolge in der die Knotennummern den Elementen zugewiesen werden ergibt die Strömungsrichtung bzw. den Massentransport (siehe grüne Linie in Abbildung 5.13). Ein Algorithmus generiert gemäß der Modulverschaltungsmatrix aus Abschnitt 5.1.2.1 automatisch die Fluidelemente in der richtigen Reihenfolge. Auch die Fluidelemente für Verteiler und Sammler werden automatisch entsprechend der Modulverschaltung erzeugt.

\subsubsection{Oberflächenelemente}

Zur einfacheren Auswertung werden die Konvektionsrandbedingungen nicht direkt auf die Seitenflächen der Volumenelemente aufgebracht. Sowohl auf der Rohrinnenseite, als auch auf der Rohraußenseite werden daher mit den vorhandenen Knoten zusätzliche thermische Flächenelemente des Typs surf152 erzeugt. Auf der Rohraußenseite teilen sie sich jeweils die vier Knoten der Seitenfläche des Volumenelements. Auf der Rohrinnenseite werden die Elemente zusätzlich mit den beiden Knoten des jeweiligen Fluidelements verbunden. Daher bestehen die Flächenelemente auf der Rohrinnenseite aus (4+2) Knoten. Auch diese Elemente werden automatisiert durch einen Algorithmus erzeugt.

Zur Auswertung des infraroten Strahlungsaustausches wird zusätzlich noch ein weiteres Element des Typs surf252 auf sämtliche im Strahlungsaustausch stehenden Komponenten (Rohraußenoberfläche, Receiverrückwand bzw. Kavität) definiert.

\subsubsection{Randbedingungen}

Dieses Kapitel beschreibt, welche Randbedingungen berücksichtigt und wie diese modelliert werden. Des Weiteren wird die Vorgehensweise zur Regelung der Receiveraustrittstemperatur beschrieben. 


\subsubsection{Materialdaten in ANSYS}

In ASTRID werden die Stoffwerte der verschiedenen WTM durch druck- bzw. temperaturabhängige Polynome berechnet (siehe Anhang A, Seite 177). In ANSYS können die Materialdaten aber nicht temperatur- und wertkontinuierlich als Polynom angegeben werden. Daher erstellt ASTRID stattdessen aus diesen Polynomen Stoffwerttabellen, in denen ANSYS linear interpoliert.

\subsubsection{Massenstrom- und Temperaturrandbedingung}

Die Massenströme werden entsprechend der Anzahl an Strömungswegen und parallel verschalteten Absorberrohren auf die Fluidelemente aufgebracht. An den ersten Knoten der Fluidelemente am Einlass wird eine feste Temperaturrandbedingung gesetzt (Receivereintrittstemperatur). Zudem wird bei der Dämmung an sämtliche Knoten der absorberabgewandten Seite ebenfalls eine feste Temperaturrandbedingung gesetzt. Bei externen Receivern entspricht diese Fläche der Innenseite des Hohlzylinders der Rückwand. Bei Receivern in einer Kavität entsprechend der Außenseite der umhüllenden Dämmung. Diese Temperaturrandbedingung erleichtert die Konvergenz der nichtlinearen Rechnung, da die Temperatur dieser Knoten unveränderlich ist und sich die Wärmeströme entsprechend anpassen müssen.

\subsubsection{Erzwungene Konvektion auf der Rohrinnenseite}

Für die erzwungene Konvektion auf der Rohrinnenseite wird der Wärmeübergangskoeffizient benötigt. Dieser wird zunächst aus der Vorauslegung übernommen (Gleichung (5.11)). Die Größe der Wärmeübertragungsflächen (Rohrinnenwand) hängt von der Diskretisierung der Rohre ab. Je mehr Elemente verwendet werden, desto besser wird der kreisförmige Querschnitt angenähert. Um auch bei geringer Elementanzahl vergleichbare Ergebnisse zu erhalten, wird der Wärmeübergangskoeffizient entsprechend dem Verhältnis aus realer, kreisförmiger 
Fläche und diskretisierter Fläche umgerechnet. Es gilt:

$$
\alpha_{\mathrm{wtm}, \mathrm{FEM}}=\alpha_{\mathrm{wtm}, \mathrm{real}} \cdot \frac{\pi \cdot d_{\mathrm{i}}}{n_{u} \cdot d_{\mathrm{i}} \cdot \sin \left(\frac{\pi}{n_{u}}\right)}
$$

\subsubsection{Konvektionsverluste}

Die Konvektionsverluste an die Umgebung werden ebenfalls über einen Wärmeübergangskoeffizienten berechnet. Bei außen liegenden Rohren (Rundumfeldreceiver) sind die an der Konvektion beteiligten Flächen jeweils der zur Umgebung offen liegende Teil der Absorberrohre (Hälfte des Rohrumfangs). Auch hier wird der Wärmeübergangskoeffizient entsprechend der Rohrdiskretisierung mit Gleichung (5.38) angepasst. Bei Kavitäten werden die Konvektionsverluste an der Innenwand der Kavität berechnet.

\subsubsection{Infraroter Strahlungsaustausch}

Der gegenseitige Strahlungsaustausch der Rohre untereinander und mit der Receiverrückwand wird ebenfalls berücksichtigt. Dazu wird die jeweilige Emissivität des Absorbers und der Rückwand definiert. Jedes Element, das einen Sichtfaktor bezüglich eines anderen Elements hat steht im Strahlungsaustausch. Zur schnelleren Berechnung können verschiedene Elementgruppierungen in separaten Strahlungsräumen zusammengefasst werden, in denen die Sichtfaktorberechnung durchgeführt wird. Elemente, die nicht Teil dieser Gruppierung sind werden dann bei der Sichtfaktorberechnung ignoriert. Zur Auswertung der Wärmeströme durch Strahlungsaustausch dient das in Abschnitt 5.2.1.3 beschriebene Oberflächenelement surf252

\subsubsection{Wärmestromdichteverteilung}

Für die Wärmestromdichteverteilung auf dem Receiver stehen verschiedene Optionen zur Verfügung. Zum einen besteht die Möglichkeit, die Wärmestromdichte analog zur Vorauslegung aufzubringen (siehe hierzu Abbildung 5.4). In diesem Fall erfährt jedes Rohr die gleiche Wärmestromdichte und auf die Rückwand 
bzw. die Kavität wird keine Wärmestromdichte aufgebracht.

Eine weitere Möglichkeit zur Aufbringung der Wärmestromdichte bietet das Einlesen einer Wärmestromdichteverteilung über eine externe Datei, die beispielsweise mit HFLCAL erzeugt werden kann. Die über den Receiverumfang und der Höhe verteilten Werte werden entsprechend der Rohrdiskretisierung interpoliert und auf die offen liegende Hälfte der Absorberrohre aufgebracht. Auch hier ist die Rückwand bzw. Kavität ohne Wärmestromdichte.

Die dritte Möglichkeit bietet der FEMRAY-Ansatz. Hierzu wird ein zuvor definiertes Heliostatenfeld (z.B. in HFLCAL) mit dem Strahlverfolgungsprogramm SPRAY simuliert. Dabei wird die reale Receivergeometrie verwendet. Die Ausgabe von SPRAY beinhaltet eine detaillierte Wärmestromdichteverteilung auf den Absorberrohren und der Receiverrückwand bzw. der Kavität. Je nach Tag und Uhrzeit ergibt sich bei diesem Ansatz eine realistische, lokal aufgelöste Wärmestromdichteverteilung auf dem Receiver. Über ein zuvor definiertes Makro können automatisch verschiedene Zeitpunkte nacheinander berechnet und daraus ein Receiverkennfeld erzeugt werden.

\subsubsection{Temperaturregelung und Teillastverhalten}

In aller Regel wird bei Solarreceivern eine konstante Austrittstemperatur angestrebt (Temperatur des heißen Speichers). Entsprechend der solaren Einstrahlung muss daher der Massenstrom geregelt werden, um diese Temperatur zu erreichen. Selbst für den Auslegungszeitpunkt, der in der Vorauslegung berechnet wird, ergeben sich in ANSYS andere Austrittstemperaturen, da hier zusätzlich der Receiverwirkungsgrad berücksichtigt wird. Daher stehen in ASTRID zwei Optionen zur Temperaturregelung zur Verfügung.

Eine Möglichkeit ist den in der Vorauslegung berechneten Massenstrom zum Auslegungszeitpunkt konstant zu halten und die auf den Receiver eintreffende Leistung $\dot{Q}_{\text {inc }}$ zu skalieren, bis die gewünschte Austrittstemperatur $T_{\text {aus,soll }}$ erreicht ist. Die verwendete Ansatzfunktion des Receiverwirkungsgrades über dem Lastbereich lautet:

$$
\eta_{\mathrm{rec}}=\eta_{\mathrm{opt}}-\frac{\dot{q}_{\mathrm{loss}} \cdot A_{\mathrm{ap}}}{\dot{Q}_{\mathrm{inc}}}
$$


Über die Abschätzung von $\eta_{\text {opt }}$ und $\dot{q}_{\text {loss }}$ kann der zu erwartende Wirkungsgrad und damit die folgende Gleichung gelöst werden.

$$
\frac{\dot{Q}_{\mathrm{inc}} \cdot \eta_{\mathrm{rec}}}{\dot{m} \cdot c_{p}}+T_{\text {ein }}-T_{\text {aus }, \text { soll }}=0
$$

Mit Hilfe des Newton-Verfahrens wird die Leistung der ersten Iteration (i=2) berechnet:

$$
\dot{Q}_{\mathrm{inc}, \mathrm{i}+1}=\dot{Q}_{\mathrm{inc}, \mathrm{i}}-\frac{\left(T_{\mathrm{aus}}-T_{\mathrm{aus}, \mathrm{soll}}\right) \cdot \dot{m} \cdot c_{p}}{\eta_{\mathrm{opt}}}
$$

Alle weiteren Iterationen verwenden das Sekanten-Verfahren, bis die Receiveraustrittstemperatur ausreichend genau (nach Vorgabe) erreicht ist:

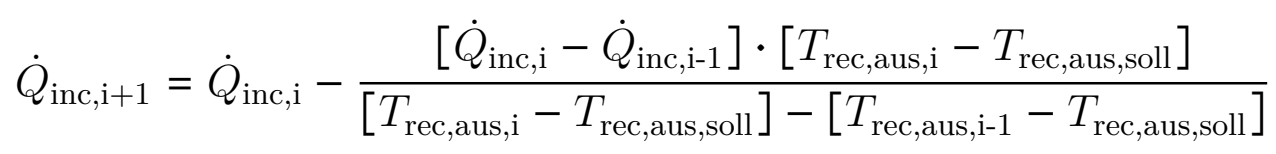

In der Funktionsstruktur (siehe Abbildung 5.14) ist dieser Programmabschnitt grün markiert.

Eine andere Möglichkeit ist die Regelung des Massenstroms, ohne Skalierung der Wärmestromdichteverteilung. Insbesondere für die Berechnung von Teillastzuständen und inhomogener bzw. unsymmetrischer Bestrahlung des Receivers (in Morgen- und Abendstunden) wird der Massenstrom für jeden Strömungsweg separat auf die gewünschte Austrittstemperatur geregelt. Im Fall einer Strömungsvereinigung werden die Massenströme aller Strömungswege vor der Vereinigung so geregelt, dass bei der Vereinigung die gleichen Fluidtemperaturen herrschen. Bei jeder Iteration werden sämtliche für die Strömung relevanten Größen neu berechnet.

Für die erste Berechnung in ANSYS (i=1) wird anhand der Wärmestromdichteverteilung auf dem Receiver ein erster Schätzwert für die Massenströme ermittelt. Für die erste Iteration (i=2) wird das Newton-Verfahren für jeden Strömungsweg (j) separat angewandt:

$$
\dot{m}_{\mathrm{j}, \mathrm{i}+1}=\dot{m}_{\mathrm{j}, \mathrm{i}}+\frac{\left[T_{\mathrm{aus}, \mathrm{j}, \mathrm{i}}-T_{\mathrm{aus}, \mathrm{soll}}\right] \cdot\left[\overline{c_{p}} \cdot \dot{m}_{\mathrm{j}, \mathrm{i}}^{2}\right]}{\dot{Q}_{\mathrm{inc}, \mathrm{i}}-\dot{Q}_{\mathrm{loss}, \mathrm{i}}}
$$


Alle weiteren Iterationen verwenden das Sekanten-Verfahren:

$$
\dot{m}_{\mathrm{j}, \mathrm{i}+1}=\dot{m}_{\mathrm{j}, \mathrm{i}}-\frac{\left[\dot{m}_{\mathrm{j}, \mathrm{i}}-\dot{m}_{\mathrm{j}, \mathrm{i}-1}\right] \cdot\left[T_{\mathrm{aus}, \mathrm{j}, \mathrm{i}}-T_{\mathrm{aus}, \mathrm{soll}}\right]}{\left[T_{\mathrm{aus}, \mathrm{j}, \mathrm{i}}-T_{\mathrm{aus}, \mathrm{soll}}\right]-\left[T_{\mathrm{aus}, \mathrm{j}, \mathrm{i}-1}-T_{\mathrm{aus}, \mathrm{soll}}\right]}
$$

Für jedes Absorberrohr wird anhand des neu berechneten Massenstromes die Strömungsgeschwindigkeit, Reynolds-Zahl, Nusselt-Zahl und damit die Wärmeübergangskoeffizienten neu berechnet. Der Massenstrom wird solange iteriert, bis die vorgegebene Receiveraustrittstemperatur ausreichend genau (nach Vorgabe) erreicht ist. In Abbildung 5.14) ist dieser Programmabschnitt blau hinterlegt.

\subsubsection{Netzstudie}

Bei den in dieser Arbeit durchgeführten Netzstudien zeigt sich eine starke Abhängigkeit des WTMs. Je nach Konfiguration und Medium sind unterschiedlich feine Diskretisierungen nötig. Bei Solar Salt als WTM wird der Receiverwirkungsgrad tendenziell schon mit groben Rechennetzen (Absorberrohre mit $n_{\mathrm{u}}=6, n_{\mathrm{l}}=10$ ) ausreichend genau berechnet. Für die Berechnung der maximalen Absorbertemperatur sind jedoch feinere Netze mit mindestens $n_{\mathrm{u}}=10$ und $n_{\mathrm{l}}=20$ nötig. Bei Natrium verhält es sich umgekehrt. Mit groben Netzen wird die Absorbertemperatur bereits gut abgeschätzt. Für die genaue Berechnung des thermischen Wirkungsgrades bedarf es jedoch sehr feiner Netze. Die Ursache dieser Abhängigkeiten liegt vermutlich in den temperaturabhängigen Stoffeigenschaften begründet.

Um die Netzunabhängigkeit der Ergebnisse sicherzustellen muss bei jeder Receiverauslegung stets eine Netzstudie durchgeführt werden. 


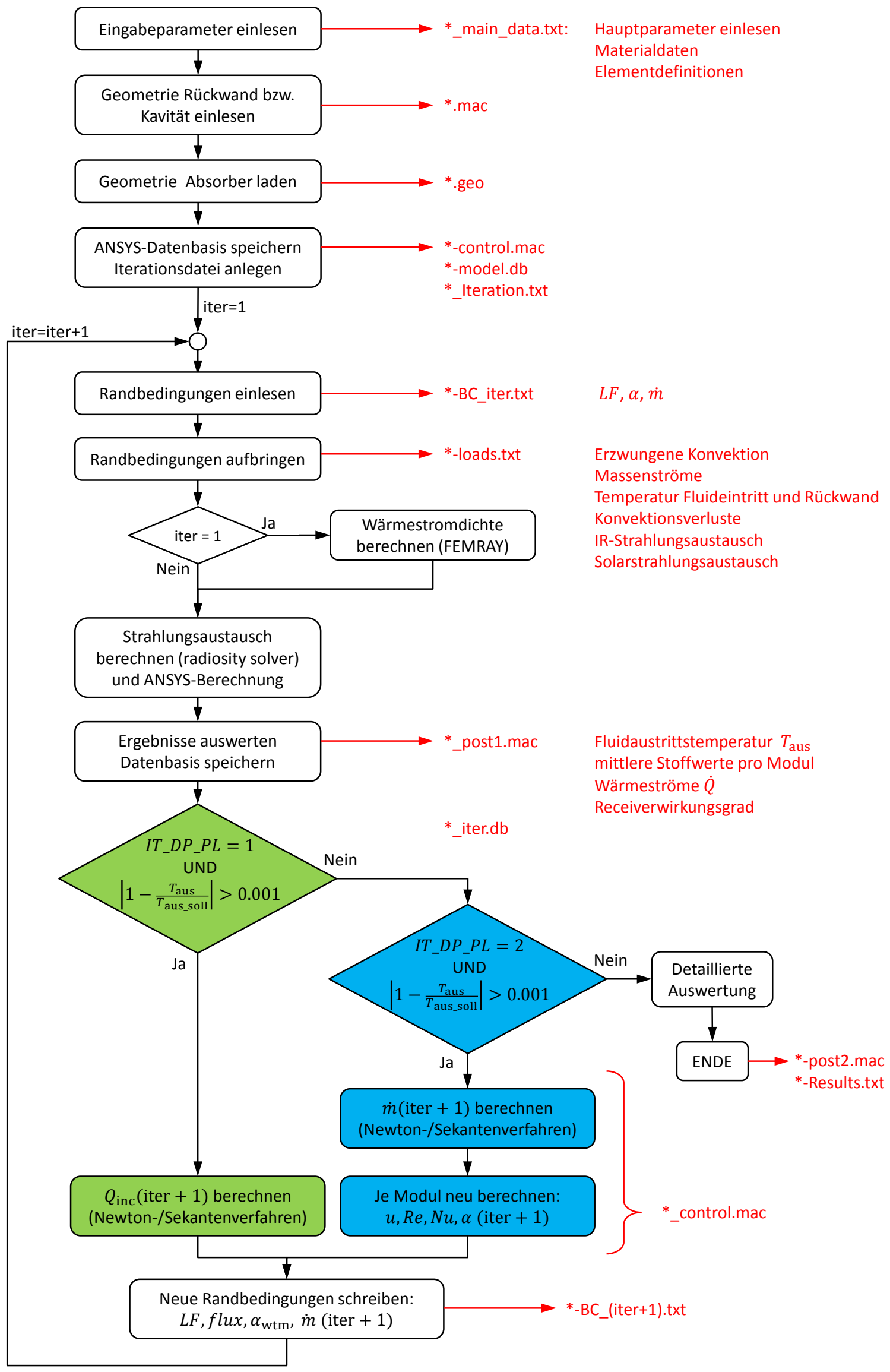

Abbildung 5.14.: Flussdiagramm der thermischen Receivermodellierung mit FEM. Wärmestromdichte- und Massenstromiteration für Teillastfälle. 


\subsection{Validierung des ASTRID-Receivermodells}

Das ASTRID-Receivermodell wurde im Zuge dieser Arbeit anhand verschiedener Projekte und Messdaten validiert. Im ersten Schritt wurde die in ASTRID verwendete Modellierung mit eindimensionalen Fluidelementen und Nusselt-Korrelationen mit einem detaillierten CFD-Modell validiert (siehe Abschnitt 5.3.1). Anschließend wurde mit ASTRID der SOLUGAS-Receiver simuliert und mit den Messergebnissen verglichen (Abschnitt 5.3.2). Zum Abschluss wurde noch der Solar Two-Receiver an drei Messpunkten simuliert und die Wirkungsgrade mit den Ergebnissen der veröffentlichten Messdaten verglichen (Abschnitt 5.3.3).

\subsubsection{Validierung mit CFD-Modell}

Da das Wärmeübertragungsverhalten von Flüssigmetallen (niedrige PrandtlZahl) sehr stark von anderen Flüssigkeiten (mit hohen Prandtl-Zahlen) abweicht, werden die Simulationen zur Validierung sowohl für Blei-Bismut $(\operatorname{Pr} \approx 0.02)$ als auch für Solar Salt $(P r \approx 6.7)$ durchgeführt 101 .

Der Vergleich umfasst verschiedene Konfiguration (einzelnes Absorberrohr, Receivermodul, ganzer Receiver). Die Simulationen beinhalten homogene und inhomogene Wärmestromdichteverteilungen bei unterschiedlichen Temperaturniveaus. Neben stationären Rechnungen wird auch das instationäre Modellverhalten untersucht. Bei der Modellierung mit ASTRID wird das WTM durch eindimensionale Fluidelemente vereinfacht und der Wärmeübergangskoeffizient mit Nusselt-Korrelationen für Rohrströmungen berechnet. Das detaillierte CFDModell berücksichtigt den gesamten Strömungsquerschnitt und berechnet lokale Wärmeübergangskoeffizienten. Je nach Wärmeträgermedium kommen unterschiedliche Turbulenzmodelle und turbulente Prandtl-Zahlen zur Anwendung. Die detaillierte Modellbeschreibung ist in [101] dokumentiert.

Abbildung 5.15 zeigt den Vergleich eines inhomogen bestrahlten Absorberrohres mit LBE als Wärmeträgermedium. 

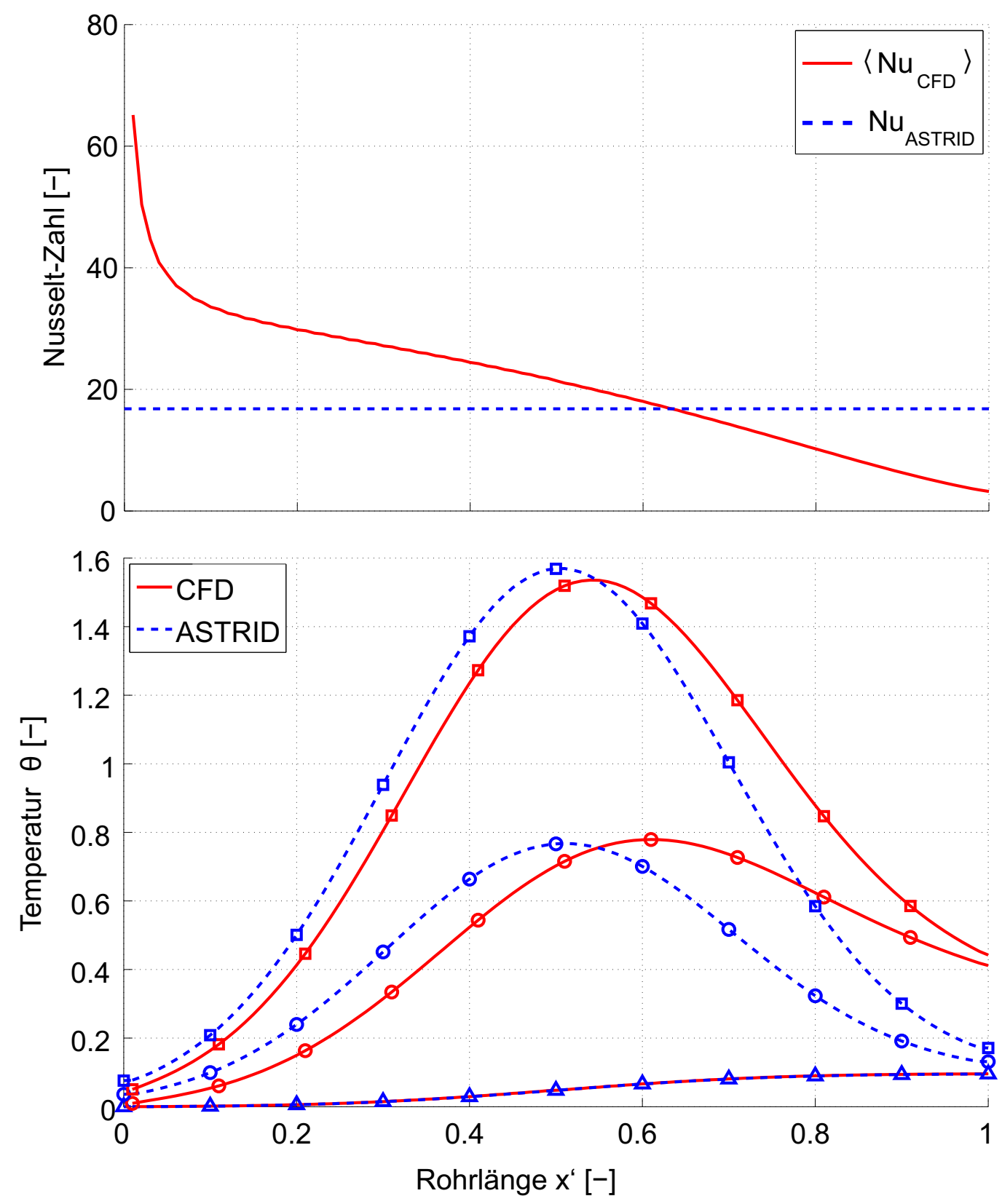

Abbildung 5.15.: Vergleich der Simulation eines inhomogen bestrahlten Rohres mit CFD und mit ASTRID in Anlehnung an [101]. Oben: Verlauf der Nusselt-Zahl entlang der Rohrlänge. Durchgezogene Linie: aus CFD-Simulation (gemittelt über Rohrumfang), Gestrichelte Linie: In ASTRID verwendete Korrelation für LBE. Unten: Dimensionslose Temperaturverteilung entlang der Rohrlänge. Durchgezogene Linie für CFD und gestrichelte Linie für ASTRID, mittlere Fluidtemperatur $(\triangle)$, Rohrinnenwandtemperatur auf bestrahlter Seite ( O), Rohraußenwandtemperatur auf bestrahlter Seite ( $\square)$. 
Die entdimensionierte Temperatur und Rohrlänge ist dabei:

$$
\theta=\frac{\left(T-T_{\mathrm{b} 0}\right) \cdot \lambda_{\mathrm{f}}}{\left\langle q_{\mathrm{wi}}\right\rangle_{\mathrm{L}} \cdot R_{\mathrm{i}}} \quad ; \quad x^{\prime}=\frac{x}{L}
$$

mit $\quad T_{\mathrm{b} 0}=$ Mittlere Fluidtemperatur am Eintritt

$\lambda_{\mathrm{f}}=$ Wärmeleitfähigkeit des Fluids

$\left\langle q_{\mathrm{wi}}\right\rangle_{\mathrm{L}}=$ Über den Umfang und die Rohrlänge gemittelte Wärmestromdichte auf der Rohrinnenseite

$x=$ Koordinate in Rohrlängsrichtung $[0 \ldots L]$

Aufgrund der Vernachlässigung der Konvektions- und Strahlungsverluste sind die resultierenden Fluidtemperaturen beider Modelle identisch (Energieerhaltung). Die maximale Rohrtemperatur stimmt in beiden Modellen gut überein, jedoch gibt es eine Abweichung des Ortes, wo diese Maximalwerte erreicht werden. Bei ASTRID stimmt der Ort der maximalen Temperatur mit dem Ort der maximalen Wärmestromdichte überein, während beim CFD-Modell dieser Peak weiter hinten in Richtung Rohraustritt verschoben ist. Auch die Temperatur am Rohraustritt ist beim CFD-Modell deutlich höher. Grund für diese Abweichung ist die lokal unterschiedliche Nusselt-Zahl beider Modelle (siehe Abbildung 5.15 oben). Im CFD-Modell ist zunächst die Nusselt-Zahl höher, fällt aber gegen Ende deutlich ab unter den konstanten Wert, welcher im ASTRID-Code verwendet wird. Dies verschlechtert den Wärmeübergang und führt zu höheren Rohrtemperaturen. Dieser Vergleich zeigt die Grenzen des ASTRID-Modells. Um die maximale Rohrtemperatur exakt bestimmen zu können, ist die Kenntnis der lokalen Nusselt-Zahl erforderlich. Wird im ASTRID-Modell die Nusselt-Zahl im Bereich der maximalen Wärmestromdichte überschätzt, so ergeben sich zu niedrige Rohrtemperaturen.

Es zeigt sich, dass für Rohrströmungen die verwendeten Nusselt-Korrelationen ausreichend genau sind 101. 


\subsubsection{SOLUGAS-Projekt}

Im Zeitraum zwischen 2008 bis 2014 wurde unter der Leitung von ABENGOA das Projekt SOLUGAS durchgeführt. Innerhalb dieses Projekts wurde die erste solarhybride Gasturbine zusammen mit einem metallischen Rohrreceiver im Megawatt-Maßstab gebaut und über $1000 \mathrm{~h}$ getestet. Dabei wurden Receiveraustrittstemperaturen bis $800{ }^{\circ} \mathrm{C}$ erreicht [102]. Abbildung 5.16 zeigt die Anlage und den Receiveraufbau. Die wichtigsten technischen Daten der Anlage sind in Tabelle 5.2 zusammengefasst.

Tabelle 5.2.: Technische Daten des SOLUGAS-Projekts 102,103

\begin{tabular}{ll}
\hline Receivertyp & in Kavität, metallische Absorberrohre \\
\hline Apertur & $5.94 \mathrm{~m}^{2}$ \\
Receiverleistung & $3.2 \mathrm{MW}_{\text {th }}$ \\
Wärmeträgermedium & Luft bei 9 bar, $\Delta p_{\text {rec }}=250 \mathrm{mbar}$ \\
Einlasstemperatur & $300-350{ }^{\circ} \mathrm{C}$ \\
Auslasstemperatur & $\operatorname{max.~} 800{ }^{\circ} \mathrm{C}$ \\
Rohrtemperatur & $\max .1000{ }^{\circ} \mathrm{C},\left(\right.$ Dämmung max. $\left.1200{ }^{\circ} \mathrm{C}\right)$ \\
Absorber & 10 parallel verschaltete Module als Polyeder angeordnet \\
& Je Modul 17 Absorberrohre, mit Versatz angeordnet \\
& $d_{\mathrm{i}}=19.6 \mathrm{~mm}, s=1.4$ mm, $L=6$ m $(5$ m bestrahlt) \\
Brennkammer & Werkstoff: Nickel-Basis-Legierung (Inconel 617$)$ \\
Turbine & Aufheizung auf $1150{ }^{\circ} \mathrm{C}$ durch fossiles zufeuern \\
\hline
\end{tabular}

Aus fertigungstechnischen Gründen sind die Rohre nicht in einer Ebene, sondern versetzt zueinander angeordnet (siehe Abbildung 5.2. Teilbild f bzw. Abbildung 5.17). Außerdem ist jedes Absorberrohr über einen Metallbalg mit dem Verteiler verbunden. Dieser vorgespannte Metallbalg gleicht die im Betrieb auftretende thermische Dehnung (bis zu $40 \mathrm{~mm}$ ) aus. Zur Druckrückgewinnung ist am Ende jedes Absorberrohrs vor dem Übergang zum Sammler ein Diffusor angebracht. Die Außenseite des Receivers ist thermisch und optisch gedämmt, sodass die Haltestruktur im Betrieb $150^{\circ} \mathrm{C}$ nicht überschreitet.

Durch die Anordnung der Heliostate und des Receivers kommt es im Betrieb zu einer sehr inhomogenen Wärmestromdichteverteilung. Um dennoch ein homogenes Temperaturfeld zu erhalten wurden die Massenströme der einzelnen Module angepasst. Dazu wurden an den Modulen mit geringerer Wärmestromdichte jeweils an den Eingängen der Absorberrohre Blenden installiert, um den 

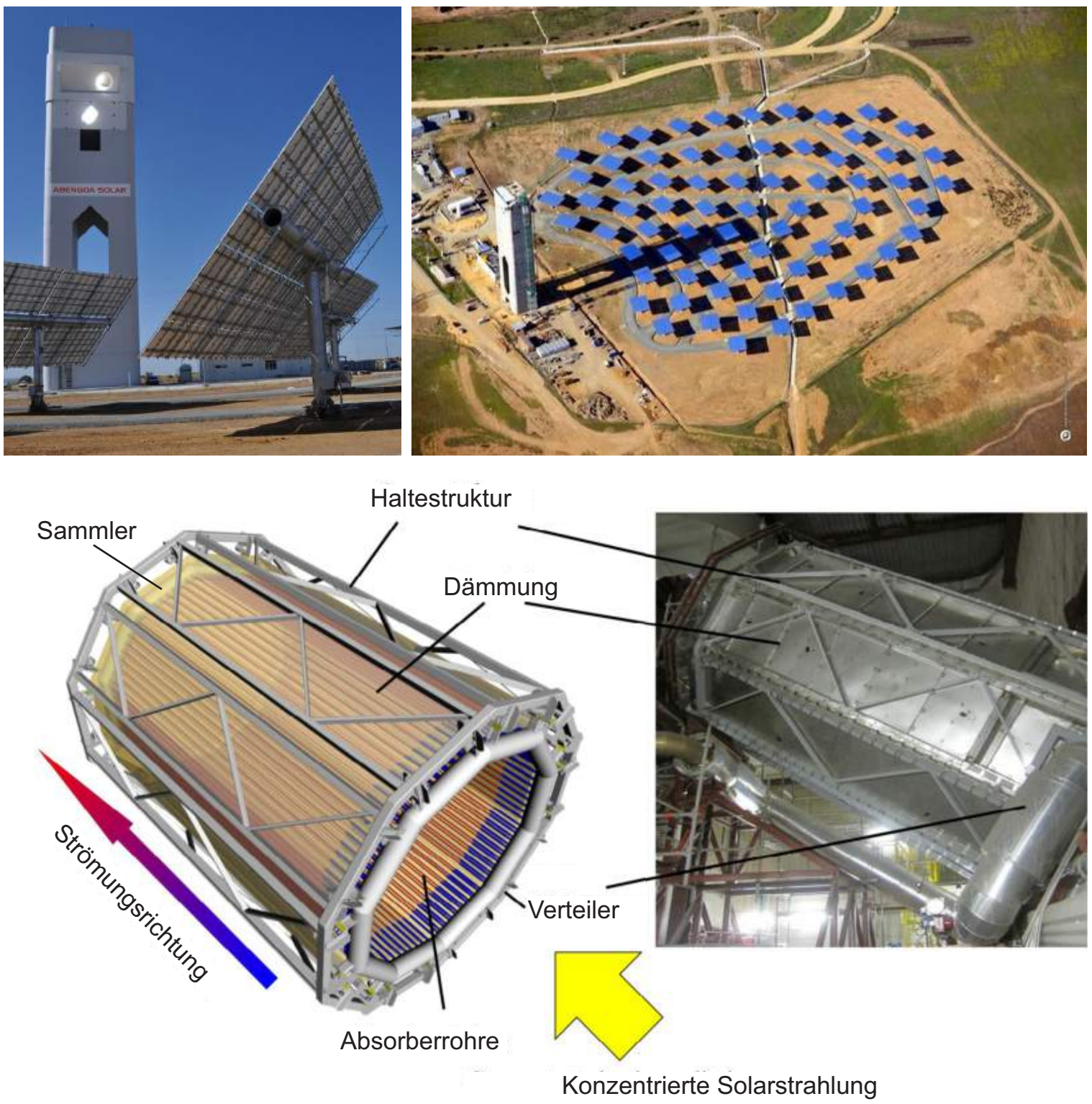

Abbildung 5.16.: Oben: Heliostatenfeld und Turm des SOLUGAS-Projekts in der Ebene von Sanlúcar la Mayor in der Nähe von Sevilla in Andalusien (2012). Unten: Prinzipbild des Receivers und ausgeführte Installation im Turm 102, 103].

Massenstrom zu reduzieren bzw. an den Modulen mit hoher Wärmestromdichte zu erhöhen. Damit ergeben sich folgende Massenströme (Hier beispielhaft für den 8. Juni um $13 \mathrm{~h})$ :

\begin{tabular}{lll}
\hline Modulnummer & Massenstrom $\dot{m}_{\text {mod }}$ & Wärmeübergangskoeffizient $\alpha$ \\
\hline $2,3,4,7,8,9(\dot{q}$ niedrig $)$ & $0.492 \mathrm{~kg} / \mathrm{s}$ & $430 \mathrm{~W} / \mathrm{m}^{2} / \mathrm{K}$ \\
$5,6,1,10(\dot{q}$ hoch $)$ & $0.7 \mathrm{~kg} / \mathrm{s}$ & $580 \mathrm{~W} / \mathrm{m}^{2} / \mathrm{K}$ \\
\hline
\end{tabular}




\subsubsection{Modellierung}

Die thermische Modellierung des SOLUGAS-Receivers mit FEM ist mit ASTRID entsprechend nach Abschnitt 5.2 durchgeführt worden. Die Komponenten wie Verteiler, Sammler, Faltenbalge und Diffusoren wurden in der thermischen Rechnung vernachlässigt, da sie thermisch gedämmt sind und folglich an der Energiewandlung nicht teilnehmen. In der hydraulischen Berechnung der Vorauslegung werden diese Komponenten berücksichtigt, da sie dort eine relevante Rolle für den Druckverlust spielen. Sämtliche Randbedingungen (wie die Massenstromverteilung in den Absorberrohren, Druck, Eintritts- und Umgebungstemperatur) sind im FEM-Modell berücksichtigt. Die Diskretisierung der Absorberrohre beträgt 50 Elemente über die Rohrlänge und 10 Elemente in Umfangsrichtung. Die Geometrie der Kavität wird als externe Datei eingelesen. Die Absorptivität der Absorberrohre bzw. der gedämmten Kavität beträgt $\epsilon_{\mathrm{r}}=0.9$ bzw. $\epsilon_{\mathrm{cav}}=0.3$. Die Wärmestromdichteverteilung auf den Absorberrohren und der Kavität wird mit dem Strahlverfolgungsprogramm FEMRAY/SPRAY berechnet, welches neben den Heliostatpositionen auch die optische Güte und die Nachführungsfehler sowie die für jeden Heliostat individuelle, deflektometrisch vermessene Spiegelkrümmung (canting) berücksichtigt [100].

Abbildung 5.17 zeigt das FEM-Modell mit Absorberrohren in der Kavität und die Wärmestromdichteverteilung auf den Absorberrohren am 8. Juni um $13 \mathrm{~h}$.
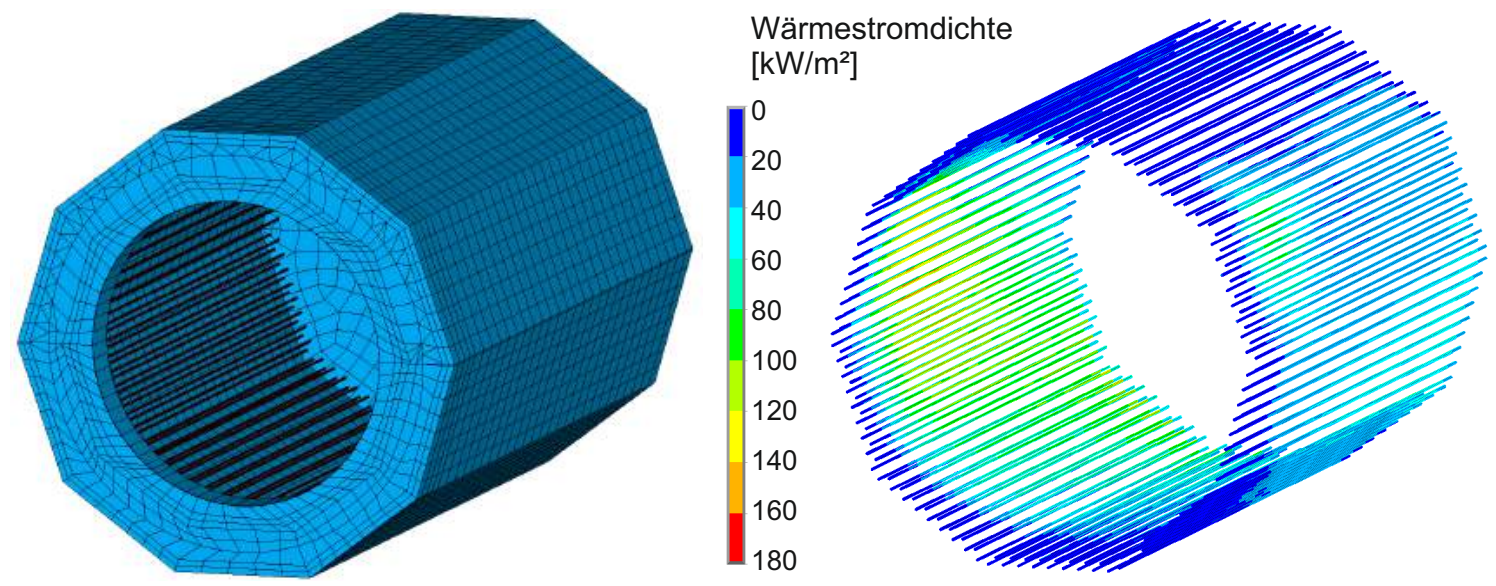

Abbildung 5.17.: Links: FEM-Modell (Absorberrohre in Kavität). Rechts: Mit FEMRAY/SPRAY berechnete Wärmestromdichteverteilung auf Absorberrohren am 8. Juni um $13 \mathrm{~h}$. 


\subsubsection{Ergebnisse und Vergleich mit Messdaten}

Ein kritischer Punkt bei der Simulation des Receivers ist der Verlust durch Konvektion, da für große Kavitätreceiver bisher keine genauen Korrelationen existieren. Der Wärmeübergangskoeffizient für die Konvektionsverluste an die Umgebung wurde daher über eine Parametervariation mit ANSYS und dem Vergleich der Gesamt-Austrittstemperatur ermittelt. Mit diesem berechneten Wärmeübergangskoeffizient stimmen nicht nur die Gesamt-Austrittstemperaturen, sondern auch die Austrittstemperaturen der einzelnen Absorberrohre sehr gut überein (siehe Abbildung 5.18).

Im Verlauf des SOLUGAS-Projekts wurden 16 Messpunkte zwischen März und August 2013 mit dem gleichen FEM-Modell berechnet und ausführlich dokumentiert (siehe [102]). Daher beschränkt sich der Vergleich hier auf den einen Messpunkt am 8. Juni um $13 \mathrm{~h}$ (siehe Abbildung 5.18).

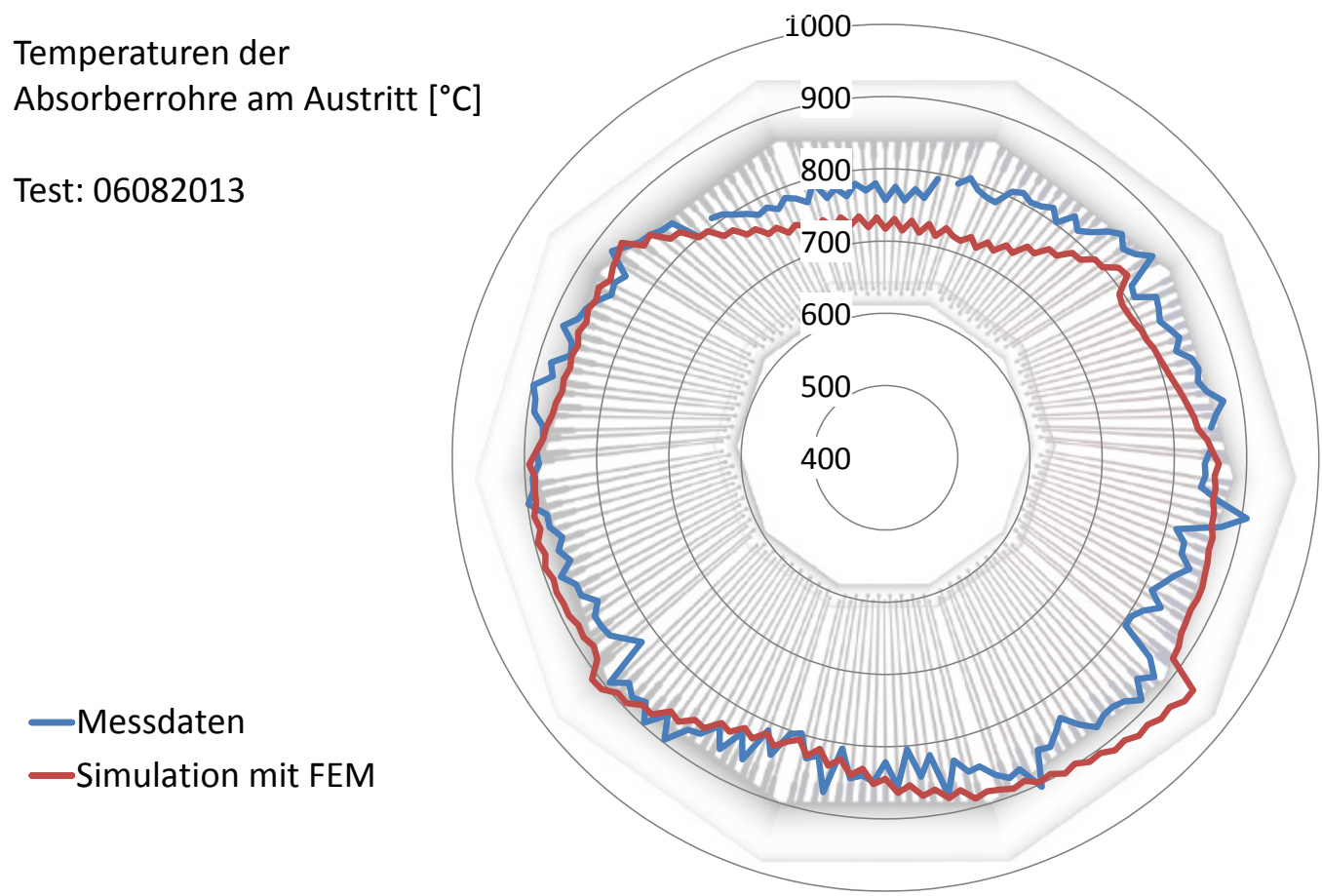

Abbildung 5.18.: Vergleich der Luft-Austrittstemperaturen aus Messung und Simulation für den 8. Juni um $13 \mathrm{~h}$ über den kompletten Receiverumfang für alle Absorberrohre. Gemessen wurde jeweils über ein Thermoelement am Absorberrohrauslass im unbestrahlten Bereich hinter der Dämmung. 


\subsubsection{SolarTwo-Projekt}

Dieser Abschnitt beschreibt die Simulation des Solar Two-Receivers mit ASTRID und den Vergleich der Ergebnisse mit Literaturdaten. Außerdem wird das Receiververhalten beim fiktiven Tausch des WTMs von Solar Salt auf Natrium untersucht.

\subsubsection{Anlagedaten von SolarTwo [52], [44], [50]}

Im Jahre 1995 wurde die Pilotanlage Solar One zu Solar Two umgewandelt. Zu den existierenden 1818 Heliostaten mit je $39.1 \mathrm{~m}^{2}$ wurden weitere 108 Heliostate mit je $95 \mathrm{~m}^{2}$ hinzugefügt. Damit besaß die Anlage insgesamt 1926 Heliostate mit einer reflektierenden Gesamtfläche von $82750 \mathrm{~m}^{2}$. Als Wärmeträgerund Speichermedium wurde Solar Salt verwendet. Die Speicherkapazität reichte für $3 \mathrm{~h}$ Turbinenbetrieb

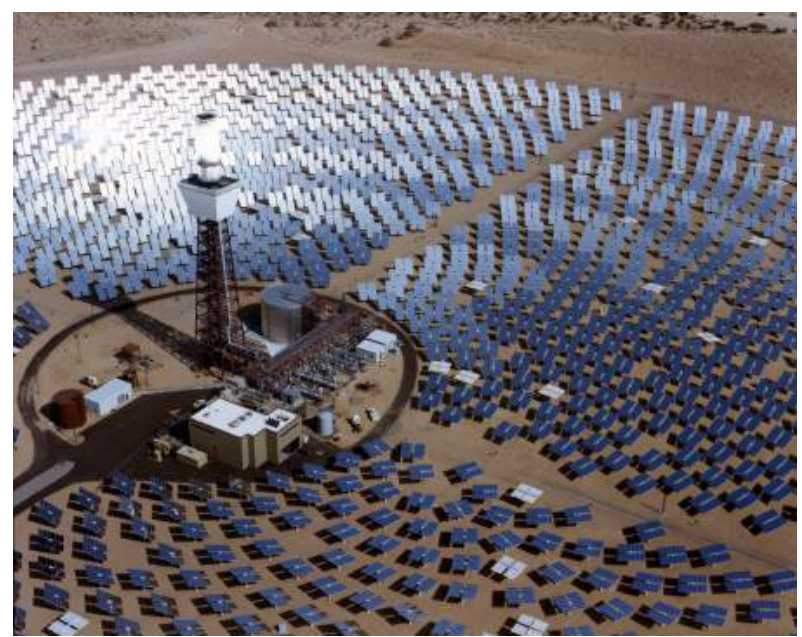

Abbildung 5.19.: Das Solarkraftwerk Solar Two in der Mojave-Wüste in Kalifornien, USA 104 $\left(10 \mathrm{MW}_{\mathrm{el}}\right)$. Abbildung 5.20 zeigt das Anlagenschema von Solar Two. Nach erfolgreichem Betrieb wurde die Anlage im Jahre 1999 außer Betrieb genommen.

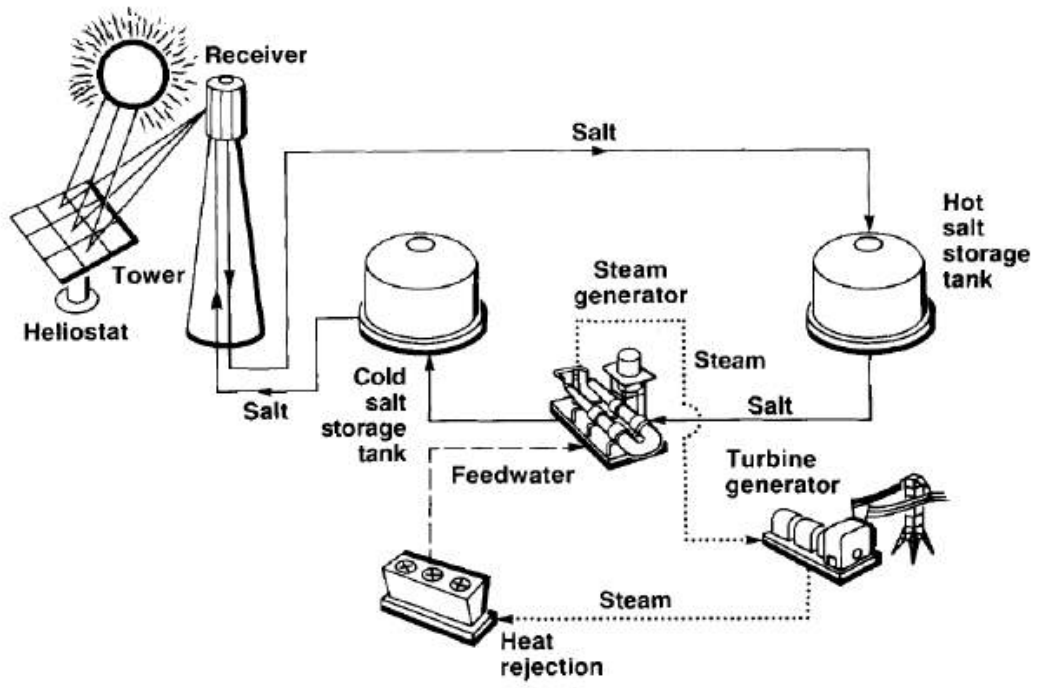

Abbildung 5.20.: Anlagenschema von Solar Two nach 105. 
Tabelle 5.3.: Technische Daten der SolarTwo-Anlage 44

\begin{tabular}{ll}
\hline Anlage & Solar Two \\
\hline Standort & Daggett, Kalifornien \\
Betrieb & $1995 / 99$, Receiverbetrieb: $1800 \mathrm{~h}$ \\
Anlagenkonfiguration & Externer Receiver mit $360^{\circ}$-Feld \\
Wärmeträgermedium & Solar Salt \\
Thermische Receiverleistung & $42.2 \mathrm{MW}$ \\
Einlasstemperatur & $290^{\circ} \mathrm{C}$ \\
Auslasstemperatur & $565^{\circ} \mathrm{C}$ \\
Wärmestromdichte & max.: $850 \mathrm{~kW} / \mathrm{m}^{2}$, gemittelt: $430 \mathrm{~kW} / \mathrm{m}^{2}$ \\
Anzahl Module & 24 \\
Anzahl Strömungswege & 2 \\
Anzahl Rohre pro Modul & 32 \\
Rohraußendurchmesser & $21 \mathrm{~mm}$ \\
Rohrwandstärke & $1.2 \mathrm{~mm}$ \\
Receiverhöhe & $6.2 \mathrm{~m}$ \\
Receiverdurchmesser & $5.1 \mathrm{~m}$ \\
Turmhöhe & $76.2 \mathrm{~m}$ (Receiver Mittellinie über Grund) \\
Receiverfläche & $99.3 \mathrm{~m}^{2}$ (Durchmesser: $5.1 \mathrm{~m}$, Höhe: $6.2 \mathrm{~m}$ ) \\
\hline
\end{tabular}
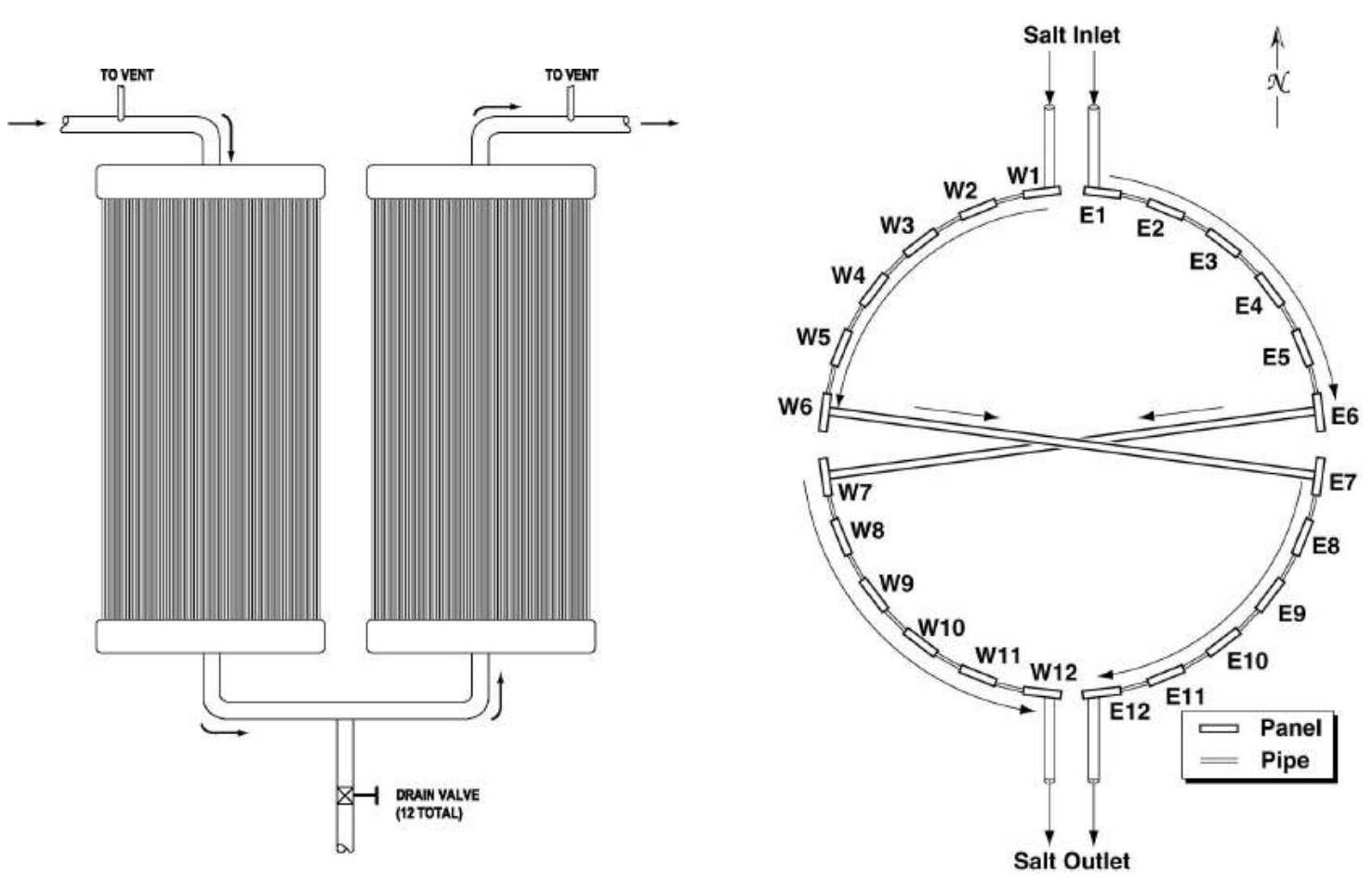

Abbildung 5.21.: Modulverschaltung im SolarTwo-Receiver 83. 


\subsubsection{Modellierung SolarTwo Referenzkonzept}

Um eine bessere Vergleichbarkeit der verschiedenen Varianten (Solar Salt und Natrium) zu erhalten wurde nicht das tatsächliche Heliostatenfeld der Solar TwoAnlage verwendet, sondern mit HFLCAL $]^{7}$ ein neues Feld erstellt und optimiert, welches eine ähnliche Aufstellung aufweist.

Feldauslegung: Mit zunehmender Entfernung vom Äquator ergeben sich in HFLCAL Heliostatenfelder mit sehr hohem Anteil an Heliostaten im Norden (bei Standorten auf der Nordhalbkugel). Dadurch ergibt sich eine höhere Wärmestromdichte auf der Receivernordseite, die auch mit Zielpunktoptimierung nicht wesentlich reduziert werden kann. Bei Salzreceivern kann durch diesen Effekt das Wärmestromdichtelimit überschritten werden, was in HFLCAL nicht berücksichtigt wird. Um dem entgegen zu wirken, werden Heliostaten aus dem Norden entfernt und entsprechend mehr Heliostate (mit schlechterem Wirkungsgrad) in den Süden gestellt. Durch diese Aufstellung ergibt sich eine homogenere Wärmestromdichteverteilung auf dem Receiver, jedoch erhöhen sich die Kosten des Heliostatenfelds. In HFLCAL kann der selbe Effekt erreicht werden, indem die geographische Breite reduziert wird. Bei der Feldauslegung in HFLCAL ergibt sich ein dem SolarTwo-Kraftwerk ähnliches Heliostatenfeld, wenn die geographische Breite auf $18^{\circ} \mathrm{N}$ statt wie real $34.867^{\circ} \mathrm{N}$ gesetzt wird.

Tabelle 5.4 zeigt die Parameter der Heliostatenfeldoptimierung für SolarTwo aus HFLCAL ohne Zielpunktverteilung.

Das daraus resultierende Heliostatenfeld ist in Abbildung 5.22 dargestellt. Die Farbskala beschreibt den jeweiligen Heliostatwirkungsgrad am Auslegungszeitpunkt.

Für die Ermittlung der optimalen Zielpunktverteilung mit SPRAY gelten die folgenden Annahmen:

Spiegelwinkelfehler (mirror slope error DEVSLO): $1.3 \mathrm{mrad}$

Winkelfehler in Rotationsachsen:

$0.65 \mathrm{mrad}$

(error in rotation of tracking axes)

Anzahl an Strahlen (Gesamt/pro Element): $\quad 9 \times 10^{8} / 324$

\footnotetext{
${ }^{7}$ HFLCAL: Siehe Abkürzungsverzeichnis und 106
} 
Tabelle 5.4.: Eingabedaten in HFLCAL für die Modellierung von Solar Two

\section{Heliostat}

Heliostatfläche

Spiegelreflektivität

$9,57 \cdot 12,93\left(=121 \mathrm{~m}^{2}\right)$

Strahlfehler (beam error)

$89.34 \%$

Feldauslegung (Field layout)

Feldaufstellung

$3.664 \mathrm{mrad}$

Minimaler Abstand zwischen Heliostaten (amin)

„slip planes“

Parameter für Heliostatabstand (ar)

$17.62 \mathrm{~m}$

$5.936 \mathrm{~m}$

Parameter für Heliostatabstand (br)

0.0481

Parameter für Heliostatabstand (ustart)

$21.715 \mathrm{~m}$

Parameter für Felddichte (fpack)

0.766

Abstand zwischen Turm und erster Heliostatenreihe $65.0 \mathrm{~m}$

\section{Feldwirkungsgrade (Field efficiencies)}

Berechnung der DNI

Höhe über NN

nach Hottel 107

Breitengrad

Receivermittelpunkt über Grund (ath)

$603 \mathrm{~m}$

$18.0^{\circ}$ statt $\left(34.867^{\circ}\right)$

$84.36 \mathrm{~m}$

Turmradius für Verschattung (thor)

$6.0 \mathrm{~m}$

\section{Systemleistung (System performance)}

Receiver: Zylinderradius

$2.55 \mathrm{~m}$

Receiver: Zylinderhöhe

$6.2 \mathrm{~m}$

Receivermodellierung

predefined $(\mathrm{icav}=4)$

Model-Nr.2 (Receiver mit konstanter Oberflächentemperatur)

Parameter $1=\eta_{\mathrm{opt}}$

0.942

Parameter $2=\dot{q}_{\text {loss }}$

$0.025 \mathrm{MW}$

Auslegungszeitpunkt

21. März, $12 \mathrm{~h}$

Einfallende Strahlung auf Receiver zum DP

$48 \mathrm{MW}$

\section{Kostenberechnung (Cost calculation)}

Kostenmodell

Heliostatenfeld

Turmkostenkorrelation

K1

H1

$\alpha$

Spezifische Receiverkosten

Landkosten

O\&M Kosten

Anuitätenfaktor (ANU)
16031

$130 € / \mathrm{m}^{2}$

$K 1+H 1 \cdot \operatorname{ath}^{\alpha} \quad$ [€]

250000

14.78

2.39

$115000 € / \mathrm{MW}$

$3 € / \mathrm{m}^{2}$

$3 \%$ von Systemkosten

0.0937 


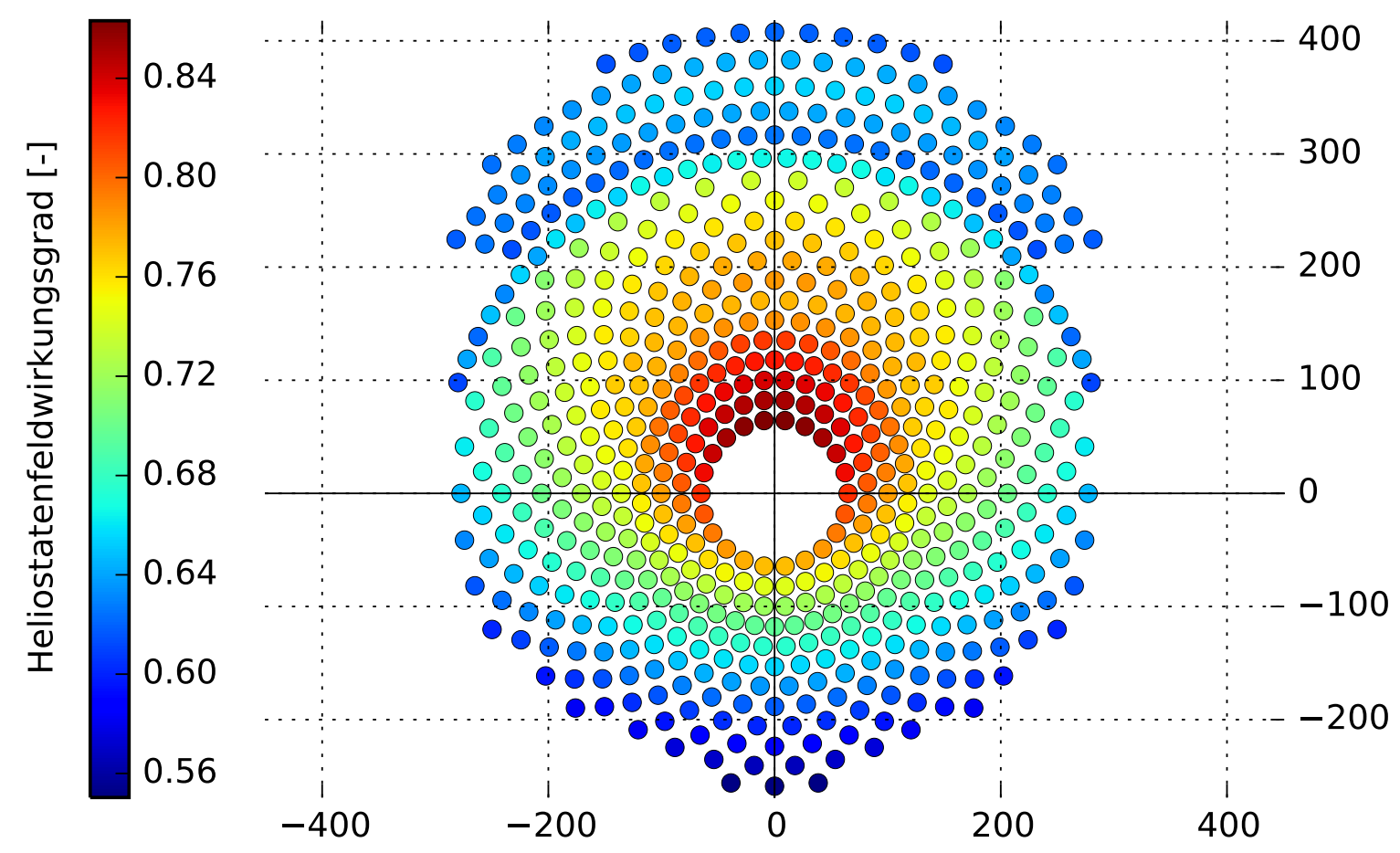

Abbildung 5.22.: Ergebnisse der Heliostatfeldoptimierung mit HFLCAL für Solar Two bei einem Breitengrad von $18^{\circ} \mathrm{N}$ : Heliostatenfeldaufstellung (insgesamt 621 Heliostate) und Heliostatenfeldwirkungsgrad am Auslegungszeitpunkt (21. März, 12h)

Die Zielpunktverteilung wird so gewählt, dass eine möglichst homogene Wärmestromdichteverteilung entsteht. Dabei findet nur eine Verschiebung der Zielpunkte in vertikaler Richtung statt. Entsprechend dem projizierten Bild jedes Heliostaten auf dem Receiver wird dessen Zielpunkt so weit wie möglich vertikal verschoben.

Die Wärmestromdichteverteilung der angewendeten Zielpunktverteilung ist in Abbildung 5.23 dargestellt. Zum Auslegungszeitpunkt ergibt sich eine maximale Wärmestromdichte von $\dot{q}_{\max }=1.55 \mathrm{MW} / \mathrm{m}^{2}$ bei folgenden Heliostatenfeldwirkungsgraden:

$\begin{array}{llll}\text { Blockierung und Abschattung: } & 99.09 \% & \text { Kosinus: } & 84.87 \% \\ \text { Atmosphärische Abschwächung: } & 97.45 \% & \text { Intercept: } & 93.38 \% \\ \text { Gesamtfeld mit Reflektivität: } & 68.37 \% & (50.06 \% \text { im Jahresmittel })\end{array}$




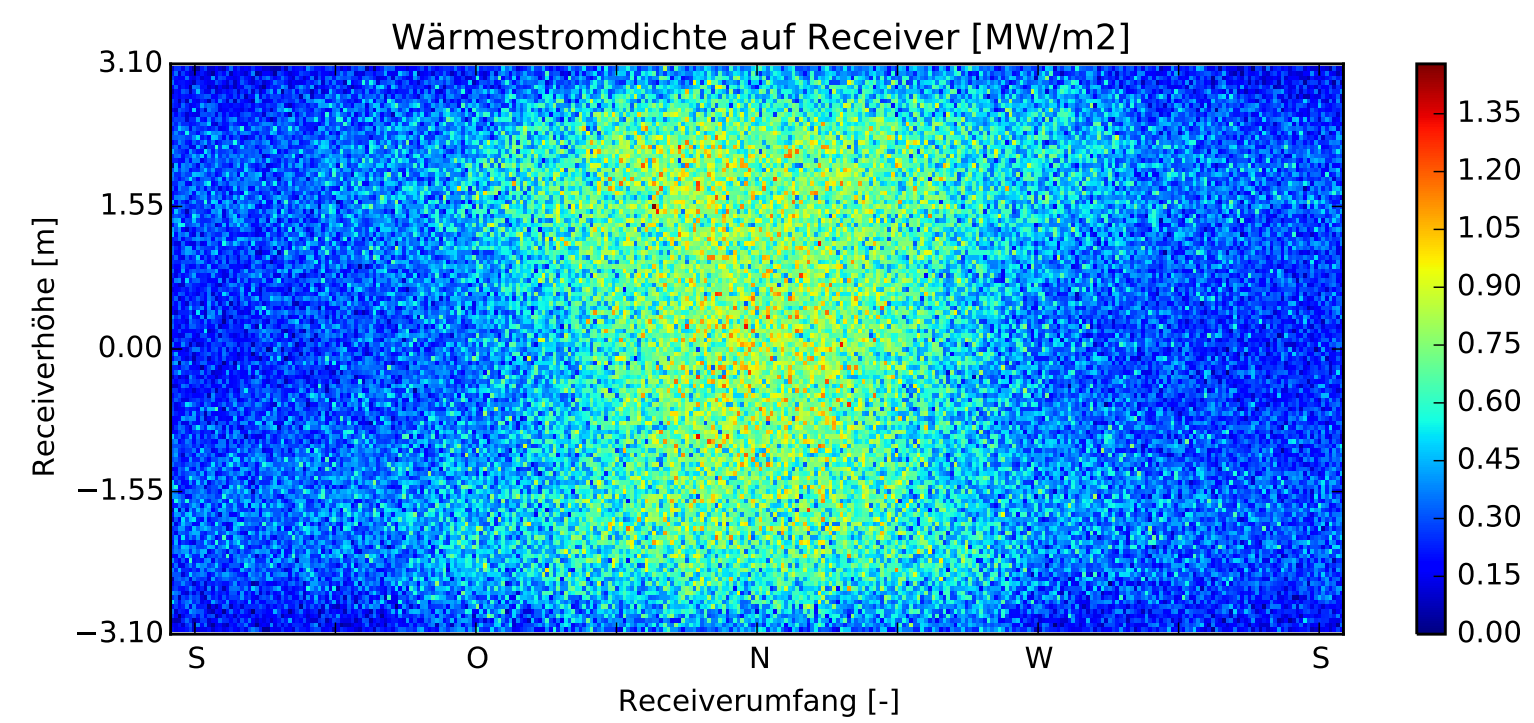

Abbildung 5.23.: Wärmestromdichteverteilung auf der Receiveroberfläche mit Zielpunktverteilung am Auslegungszeitpunkt (21. März, 12h). Maximale Wärmestromdichte: $\dot{q}_{\max }=1.55 \mathrm{MW} / \mathrm{m}^{2}$, Mittlere Wärmestromdichte: $\overline{\dot{q}}=0.44 \mathrm{MW} / \mathrm{m}^{2}$, Heliostatenfeldwirkungsgrad: $\eta_{\text {feld }}=$ $68.4 \%$.

Receivermodellierung: Der Receiver wird wie in Kapitel 5 beschrieben modelliert. Dabei werden die geometrischen Randbedingungen der Solar Two-Anlage aus Tabelle 5.3 mit der Modulverschaltung nach Abbildung 5.24 verwendet. Die Absorptions- bzw. Emissionskoeffizienten für die Solarstrahlung sowie die Strahlung im infraroten Bereich werden für die Absorberrohre und die Rückwand in Anlehnung an 52 wie folgt gewählt:

$$
\begin{aligned}
\epsilon_{\text {sol_r }}=0.95 & \begin{array}{l}
\text { Absorptionsgrad Absorberrohre Solarstrahlung } \\
\text { (entspricht optischem Wirkungsgrad) }
\end{array} \\
\epsilon_{\text {sol_cav }}=0.20 & \begin{array}{l}
\text { Absorptionsgrad Receiverrückwand Solarstrahlung } \\
\epsilon_{\text {ir_r }}=0.83
\end{array} \\
& \begin{array}{l}
\text { Absorptionsgrad Absorberrohre im infraroten Bereich } \\
\text { (Strahlungsaustausch in FEM-Simulationen) }
\end{array} \\
\epsilon_{\text {ir_cav }}=0.84 & \text { Absorptionsgrad Receiverrückwand im infraroten Bereich }
\end{aligned}
$$

Die Konvektionsverluste an die Umgebung werden über einen konstanten Wärmeübergangskoeffizient mit den zur Umgebung gerichteten Rohrflächen berechnet. Der Strahlungsaustausch wird für jedes Modul (Absorberrohre und entsprechender Teil der Rückwand) separat berechnet. 


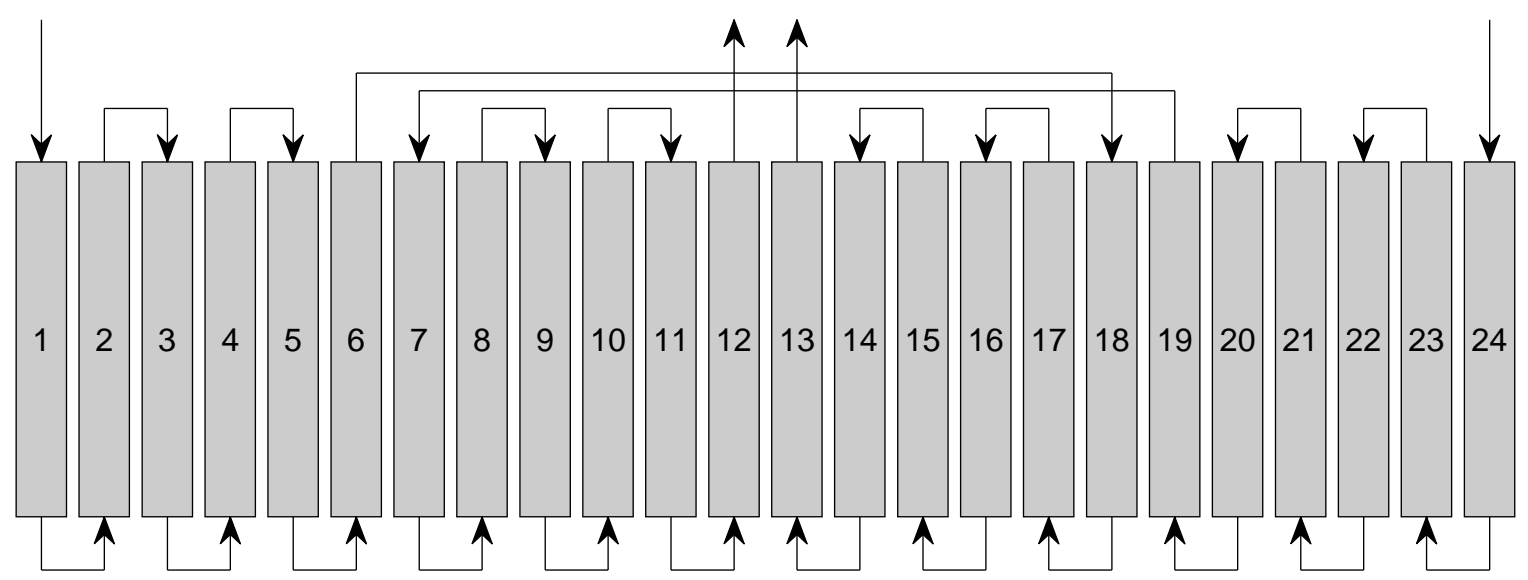

Abbildung 5.24.: Modulverschaltung von Solar Two mit Modulnummerierung

\subsubsection{Ergebnisse und Vergleich mit Messergebnissen}

Zur Validierung des ASTRID-Codes wurden verschiedene Testpunkte des Solar Two-Receivers simuliert und die Ergebnisse miteinander verglichen 101 (siehe Tabelle 5.5). Da externe Receiver wie in Solar Two dem Wind direkt ausgesetzt sind, ist eine genaue Bestimmung der konvektiven Verluste sehr wichtig. Daher beinhaltet der hier vorgestellte Vergleich die mit CFD berechneten

Tabelle 5.5.: Vergleich der Messdaten von drei Testtagen des Solar Two-Receivers 44 mit den Simulationsergebnissen aus ASTRID (mit CFD berechnete Konvektionsverluste)

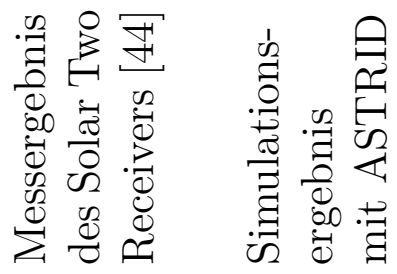

\begin{tabular}{lll}
\hline Testtag: $29.9 .97\left(u_{\text {wind }}=0.67 \mathrm{~m} / \mathrm{s}\right)$ & & \\
Thermische Verluste $[\mathrm{MW}]$ & 2.27 & 2.18 \\
Wirkungsgrad [\%] & 88.8 & 89.0 \\
\hline Testtag: $\mathbf{5 . 3 . 9 9}\left(u_{\text {wind }}=3.4 \mathrm{~m} / \mathrm{s}\right)$ & & \\
Thermische Verluste $[\mathrm{MW}]$ & 3.04 & 2.44 \\
Wirkungsgrad [\%] & 86.7 & 88.4 \\
\hline Testtag: 23.3.99 $\left(u_{\text {wind }}=8.9 \mathrm{~m} / \mathrm{s}\right)$ & & \\
Thermische Verluste $[\mathrm{MW}]$ & 2.75 & 2.77 \\
Wirkungsgrad [\%] & 85.6 & 85.8 \\
\hline
\end{tabular}


Wärmeübergangskoeffizienten unter Berücksichtigung der Absorbergeometrie sowie der am Testtag gemessenen Windgeschwindigkeit und -richtung (Details siehe 108]).

Bis auf den Testtag mit $u_{\text {wind }}=3.4 \mathrm{~m} / \mathrm{s}$ stimmen die Simulationsergebnisse gut mit den veröffentlichten Messergebnissen überein. Im Allgemeinen stehen die Verluste von externen Receivern im direkten Verhältnis zur Absorbertemperatur und der Windgeschwindigkeit. Da die Receiveraustrittstemperatur Absorbertemperaturen in allen drei Testfällen nahezu gleich ist, müssen auch die Absorbertemperaturen in der gleichen Größenordnung sein. Folglich hängen die Konvektionsverluste nur noch von der Windgeschwindigkeit ab. Die Messergebnisse zeigen einen höheren thermischen Verlust bei $u_{\text {wind }}=3.4 \mathrm{~m} / \mathrm{s}$ als bei $u_{\text {wind }}=8.9 \mathrm{~m} / \mathrm{s}$, daher kann angenommen werden, dass diese Abweichung durch Messfehler zustande kommt. 


\section{Konzeptauswahl, Bewertungsmethodik und Spezifikation}

In diesem Kapitel werden die verwendeten Kraftwerkskonzepte detailliert beschrieben. Darüber hinaus wird die Methodik vorgestellt, mit der sie bewertet werden. Für alle wichtigen Komponenten wird die Vorgehensweise zur Ermittlung der Wirkungsgradkennlinien und Kostenfunktionen beschrieben und wie diese in die Jahresertrags- und LCOE-Berechnung einfließen. In Anhang G auf Seite 191ff sind die detaillierten Spezifikationen der einzelnen Konzepte dokumentiert.

\section{1. Übergeordnete Annahmen}

Die Absorberfläche von Salzreceivern wird durch das Wärmestromdichtelimit bestimmt und muss größer gewählt werden, als die durch das Heliostatenfeld mögliche Strahlungskonzentration (siehe Abschnitt 3.4.2). Bei Flüssigmetallreceivern wird die Absorberfläche nicht mehr durch Beschränkungen des Wärmeträgermediums, sondern durch den optischen Wirkungsgrad des Heliostatenfeldes bestimmt (Randstrahlung (engl. spillage) steigt je kleiner die Absorberfläche). Aus diesem Grund muss bei Flüssigmetallreceivern zusätzlich eine ReceivergröBenstudie durchgeführt werden, um die optimale Konfiguration zu identifizieren. Seit dem Bau des ersten kommerziellen Solarturmkraftwerks mit Flüssigsalz als Wärmeträger- und Speichermedium (Gemasolar in 2011) hat sich diese Technologie etabliert und die Skalierung zu größeren Leistungen erfolgte bereits in 2015 
Tabelle 6.1.: Auswahl an Solarturmkraftwerken mit Flüssigsalz als Wärmeträger- und Speichermedium.

\begin{tabular}{lcccc}
\hline Name & Land & Leistung & Status & Netzsynchronisation \\
\hline Gemasolar & Spanien & $19.9 \mathrm{MW}_{\text {el }}$ & Im Betrieb & 2011 \\
Crescent Dunes & USA & $110 \mathrm{MW}_{\text {el }}$ & Im Betrieb & 2015 \\
Supcon & China & $50 \mathrm{MW}_{\text {el }}$ & Im Bau & - \\
Noor III & Marokko & $150 \mathrm{MW}_{\text {el }}$ & Im Bau & 2017 (geplant) \\
Atacama-1 & Chile & $110 \mathrm{MW}_{\text {el }}$ & Im Bau & 2018 (geplant) \\
Golmud & China & $200 \mathrm{MW}_{\text {el }}$ & Im Bau & 2018 (geplant) \\
Rice (RSEP) & USA & $150 \mathrm{MW}_{\text {el }}$ & In Entwicklung & 2016 (geplant) \\
Redstone & Südafrika & $100 \mathrm{MW}_{\text {el }}$ & In Entwicklung & 2018 (geplant) \\
Copiapó & Chile & $260 \mathrm{MW}_{\text {el }}$ & In Entwicklung & 2019 (geplant) \\
\hline
\end{tabular}

mit Crescent Dunes. Tabelle 6.1 zeigt eine Auswahl an Solarturmkraftwerken mit Flüssigsalz als Wärmeträger- und Speichermedium.

Aufgrund des derzeitigen Erfolgs von Flüssigsalz als WTM werden in dieser Arbeit alle Flüssigmetallkonzepte mit einem Referenz-Salzturmkonzept verglichen. Als Vergleichskriterium werden die Stromgestehungskosten LCOE herangezogen.

\subsubsection{Basiskonzept und Leistungsklasse}

Bei der Bewertung und dem Vergleich von Kraftwerken spielt der Kraftwerksblock und die Leistungsklasse eine wesentliche Rolle. Bei kleinen Anlagen ergeben sich tendenziell höhere Stromgestehungskosten, daher geht der Trend hin zu größeren Kraftwerksblöcken. Außerdem entscheidet die Leistungsklasse auch über die verwendete Speichertechnologie. Die in Tabelle 6.1 aufgeführten Kraftwerke verwenden alle eine Dampfturbine (Rankine-Kreisprozess) mit einer Leistung von 100-150 $\mathrm{MW}_{\text {el }}$ pro Turbine (mit Ausnahme von Gemasolar und Supcon). Um diesem Trend und der Verfügbarkeit von Dampfturbinen gerecht zu werden, werden sämtliche Konzepte in dieser Arbeit mit einer Leistungsklasse von $125 \mathrm{MW}_{\text {el }}$ ausgelegt und bewertet. Um den Nutzungsgrad der Turbine zu erhöhen und die Grundlastfähigkeit zu gewährleisten, muss das Heliostatenfeld mit dem Solarvielfachen $(S M)$ größer dimensioniert werden. Aus Voruntersuchungen und dem Vergleich des $S M$ von existierenden Anlangen ergibt sich damit eine thermische Kraftwerksleistung von ca. $700 \mathrm{MW}_{\text {th }}$. 
Für diese Leistung sind etwa 115 ha reflektierende Spiegelfäche nötig. Bei einem Einturmsystem ergibt sich damit ein relativ großes Heliostatenfeld mit geringerem Wirkungsgrad Aus diesem Grund können Mehrturmsysteme mit mehreren kleineren Feldern und einem zentralen Kraftwerksblock höhere Wirkungsgrade erzielen. Jedoch kommen hier die zusätzlichen Leitungen als weiterer Kostenfaktor hinzu. Um diesen Effekt abzuschätzen wird in dieser Arbeit jedes Konzept sowohl als Einturm- als auch als Mehrturmsystem (5x140 $\left.\mathrm{MW}_{\text {th }}\right)$ ausgelegt. Abbildung 6.1 zeigt das Referenzkonzept mit Flüssigsalz. Wie auch bei Gemasolar und Crescent Dunes beträgt die Salztemperatur im Betrieb 290-565 ${ }^{\circ} \mathrm{C}$. Durch den drucklosen Speicher handelt es sich bei diesem Konzept um einen offenen Salzkreislauf. Die Höhendifferenz zwischen Grund und Receiver (Turmhöhe) muss daher von der Salzpumpe überwunden werden und wird nicht rekuperiert.

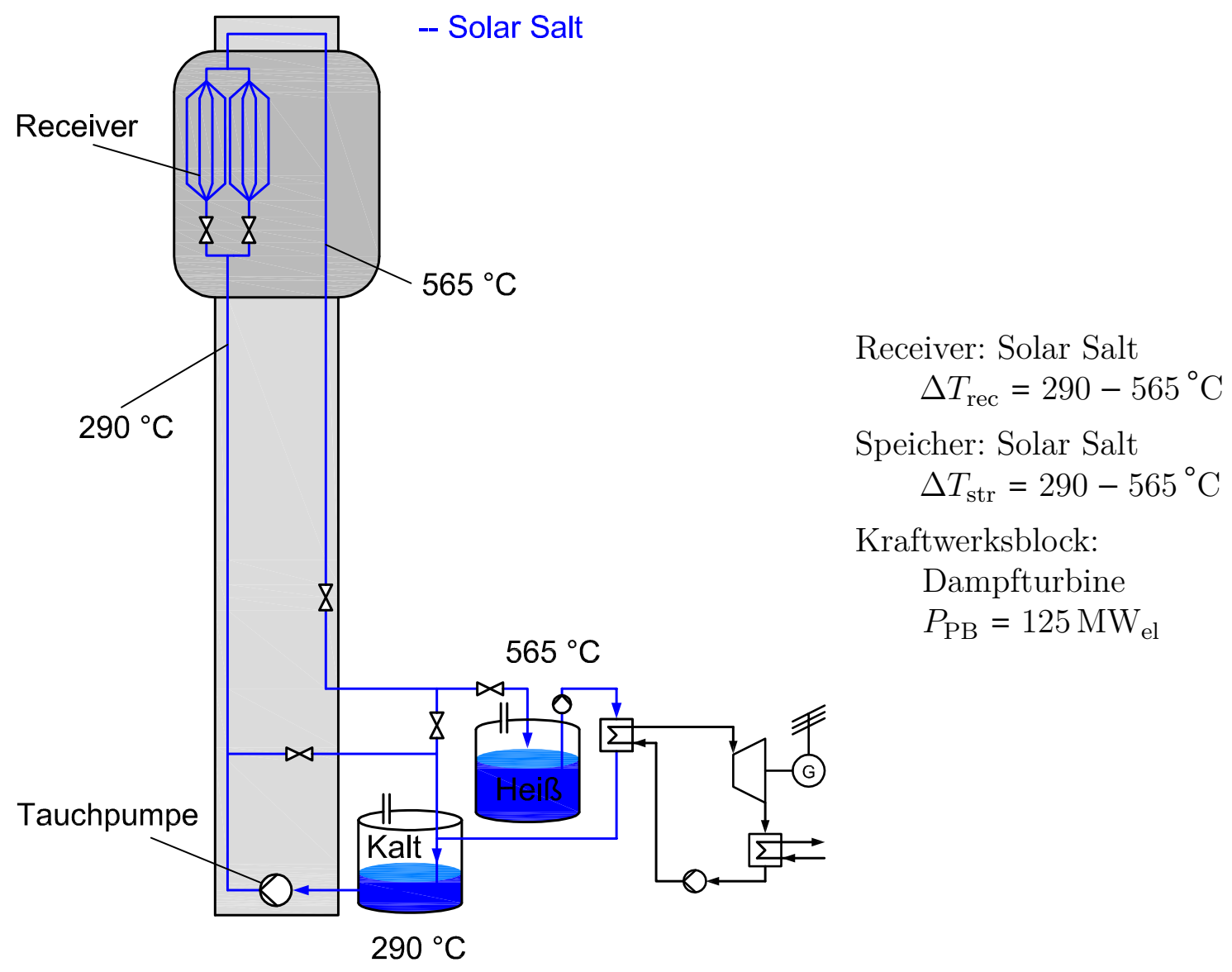

Abbildung 6.1.: Referenzkonzept mit Solar Salt als Wärmeträger- und Speichermedium und Dampfturbine mit Luftkühlung.

\footnotetext{
${ }^{1}$ Atmosphärische Auslöschung nimmt bei großen Abständen zwischen Heliostat und Receiver $\mathrm{zu}$, ebenso die Randstrahlung (Spillage) durch Strahlaufweitung.
} 
Alle Flüssigmetallkonzepte werden mit diesem Referenzkonzept verglichen. Für jedes Konzept wird in der Jahresertragsrechnung die kostenoptimale Speichergröße ermittelt. Voruntersuchungen zeigen, dass sich bei der gegebenen Leistungsklasse kostenoptimale Speichergrößen bei einer Kapazität von etwa $3400 \mathrm{MWh}_{\text {th }}$ ergeben. Da die direkte thermische Speicherung in Flüssigmetallen in dieser Größenordnung und dem angegebenen Temperaturbereich sehr teuer ist (siehe Abschnitt 4.2.2 werden die hier untersuchten Flüssigmetallkonzepte alle mit einem thermischen Speicher mit Flüssigsalz sowie einem zusätzlichen Wärmeübertrager ausgelegt.

Abbildung 6.2 zeigt das Konzept: Receiver-only. Im Vergleich zum Referenzkonzept mit Flüssigsalz wird lediglich der Salzreceiver durch einen (kleineren) Flüssigmetallreceiver ersetzt. Thermischer Speicher und Kraftwerksblock bleiben

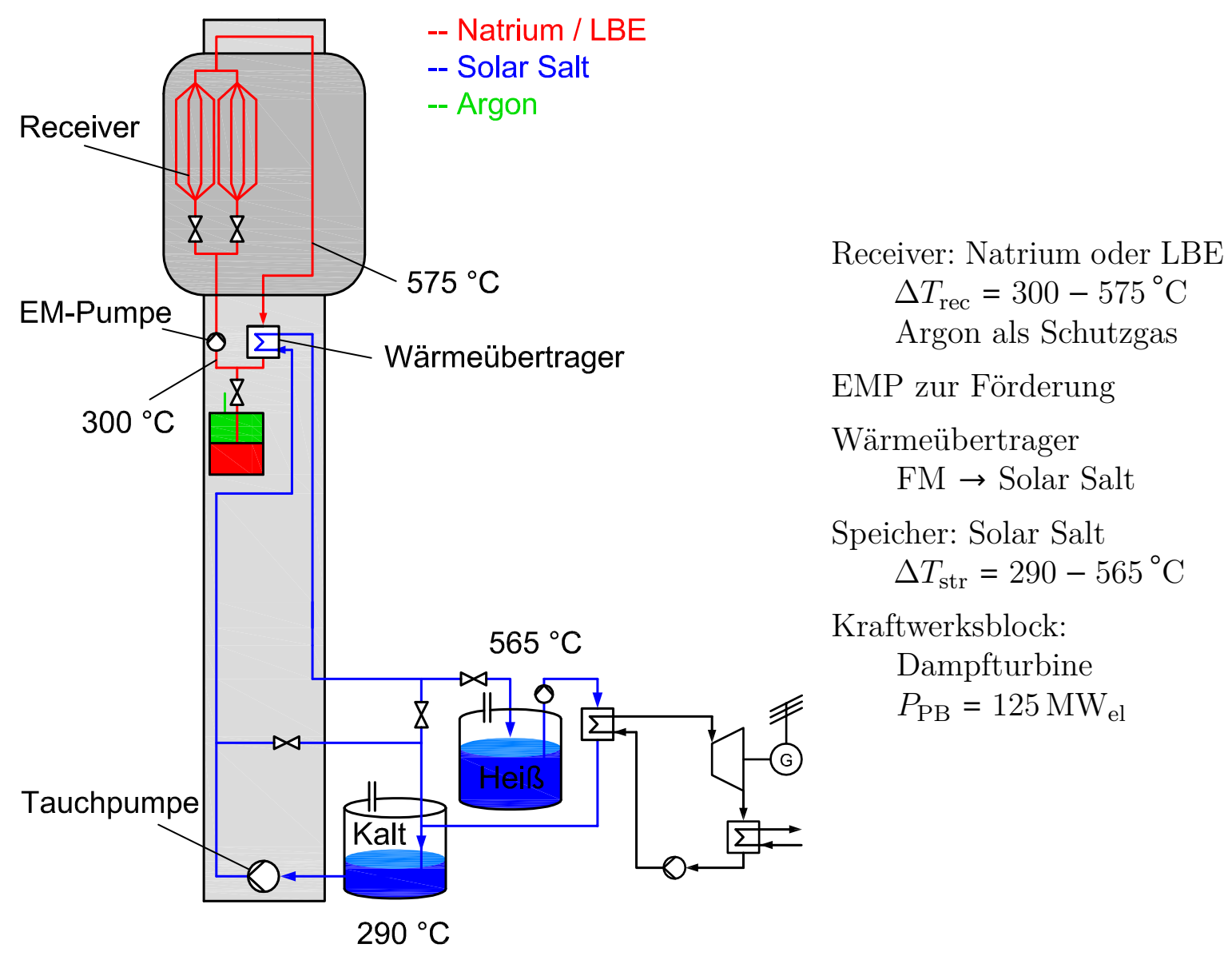

Abbildung 6.2.: Konzept: Receiver-only. Flüssigmetall im Receiver mit Wärmeübertrager auf Solar Salt als Speichermedium und Dampfturbine mit Luftkühlung. 
unverändert ${ }^{2}$. Durch die höheren Wärmestromdichten sind im Flüssigmetallreceiver höhere Wirkungsgrade erreichbar. Zudem kann ein kleiner und leichterer und somit kostengünstigerer Receiver verwendet werden. Bei Crescent Dunes wiegt allein das Flüssigsalz im Receiver $317 \mathrm{t}$ 15. Durch die geringe Dichte von Natrium und das Potenzial für kleinere Receiver kann die Receivermasse deutlich reduziert werden. Diese Gewichtsreduktion senkt auch die Turmkosten, was aber in dieser Arbeit nicht weiter untersucht wird. Zur Förderung von Natrium wird eine elektromagnetische Pumpe (EMP) verwendet (Vorteile siehe Abschnitt 4.3). Durch den deutlich geringeren Druckverlust bei Flüssigmetallen (siehe Abschnitt 3.4.3) wird außerdem die benötigte Pumpenergie im Betrieb wesentlich reduziert. Durch den höheren Wirkungsgrad kann der Receiver auch im Teillastbetrieb bei geringerer Strahlungsintensität (Morgenund Abendstunden) betrieben werden. Dies legt die Vermutung nahe, dass mit Flüssigmetallen ein höherer Jahresertrag möglich ist.

Der Nachteil dieses Konzeptes ist der zusätzlich notwendige Wärmeübertrager. Die Grädigkeit des Wärmeübertragers wird mit $10 \mathrm{~K}$ abgeschätzt, wodurch die Temperatur des Flüssigmetalls im Receiver im Betrieb $300-575{ }^{\circ} \mathrm{C}$ beträgt. Die Limitierung der Salztemperatur auf $T_{\mathrm{SS}, \max }=565^{\circ} \mathrm{C}$ bleibt damit weiterhin bestehen. Als WTM werden die beiden aussichtsreichsten Flüssigmetalle (Natrium und LBE, siehe Abschnitt 3.3 und 3.4 untersucht.

Ein weiteres in dieser Arbeit untersuchtes Konzept ist das Tower-loop-Konzept (siehe Abbildung 6.3). Der Unterschied zum Receiver-only-Konzept besteht darin, dass der geschlossene Flüssigmetallkreislauf mit Steig- und Fallleitung bis zum Grund geführt wird. Der hohe Druckverlust bei offenen Salzsystemen von etwa $0.2 \mathrm{bar} / \mathrm{m}$ Turmhöhe wird damit vermieden und die benötigte Pumpenergie weiter reduziert. Auch der statische Druck in Steig- und Fallleitung wird im Falle von Natrium um mehr als die Hälfte reduziert $t^{3}$, wodurch geringere Wandstärken und geringere Kosten für die Steig- und Fallleitung entstehen. Für LBE ist dieses Konzept aufgrund der hohen Dichte und die damit verbundenen hohen statischen Drücke nicht geeignet $t^{3}$.

\footnotetext{
${ }^{2}$ Die Größe des thermischen Speichers hängt auch vom Wirkungsgrad des Receivers ab, da beispielsweise bei einem höheren Ertrag evtl. ein größerer Speicher zu geringeren LCOE führt. Bei der Optimierung wird jeweils die Speichergröße ermittelt, die zu den niedrigsten LCOE führt. Daher ergeben sich je nach Konzept leicht unterschiedliche Speichergrößen.

${ }^{3}$ Bei einer Turmhöhe von $200 \mathrm{~m}$ ergeben sich folgende statische Drücke in der Steigleitung am Grund: Solar Salt: 37 bar $+\Delta p_{\text {rec }}$, Natrium: 17 bar $+\Delta p_{\text {rec }}$, LBE: 203 bar $+\Delta p_{\text {rec }}$.
} 


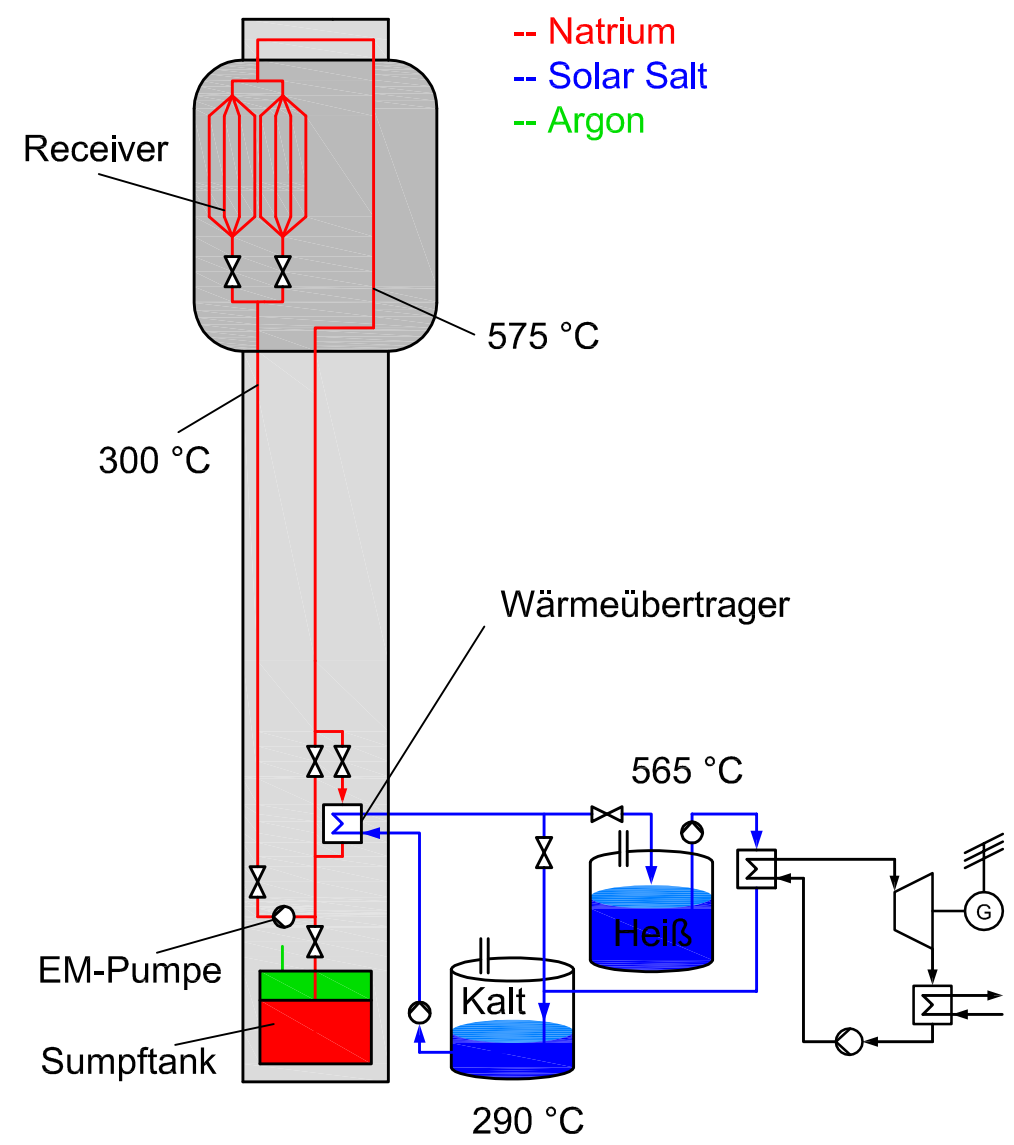

Receiver: Natrium

$\Delta T_{\text {rec }}=300-575{ }^{\circ} \mathrm{C}$

Argon als Schutzgas

EMP zur Förderung

Wärmeübertrager

$\mathrm{FM} \rightarrow$ Solar Salt

Speicher: Solar Salt

$\Delta T_{\text {str }}=290-565^{\circ} \mathrm{C}$

Kraftwerksblock:

Dampfturbine

$P_{\mathrm{PB}}=125 \mathrm{MW}_{\mathrm{el}}$

Abbildung 6.3.: Konzept: Tower-loop. Flüssigmetall in Receiver und Turmleitung mit Wärmeübertrager auf Solar Salt als Speichermedium und Dampfturbine mit Luftkühlung. Der Sumpftank hat das Fassungsvermögen des gesamten Natriums im Kreislauf. Im Falle einer Leckage oder bei Wartungen wird sämtliches Natrium in den Sumpftank entleert.

\subsubsection{Kraftwerksblock}

Nach [109] gibt es eine starke Abhängigkeit zwischen der Leistungsklasse und dem Wirkungsgrad von Dampfturbinen. Wie in Abschnitt 6.1.1 beschrieben, wird der Systemvergleich in dieser Arbeit in der Leistungsklasse von $125 \mathrm{MW}_{\mathrm{el}}$ durchgeführt. Die verwendete Dampfturbine besitzt einen Kondensator mit Luftkühlung. Tabelle 6.2 zeigt die wichtigsten Parameter des Kraftwerksblocks und Abbildung 6.4 das für alle Varianten verwendete Wirkungsgradkennfeld der Dampfturbine in Abhängigkeit vom Lastfall und der Umgebungstemperatur. Der Kraftwerksblock wird maximal bis $125 \mathrm{MW}_{\mathrm{el}}$ ohne Überlast betrieben. 


\section{1. Übergeordnete Annahmen}

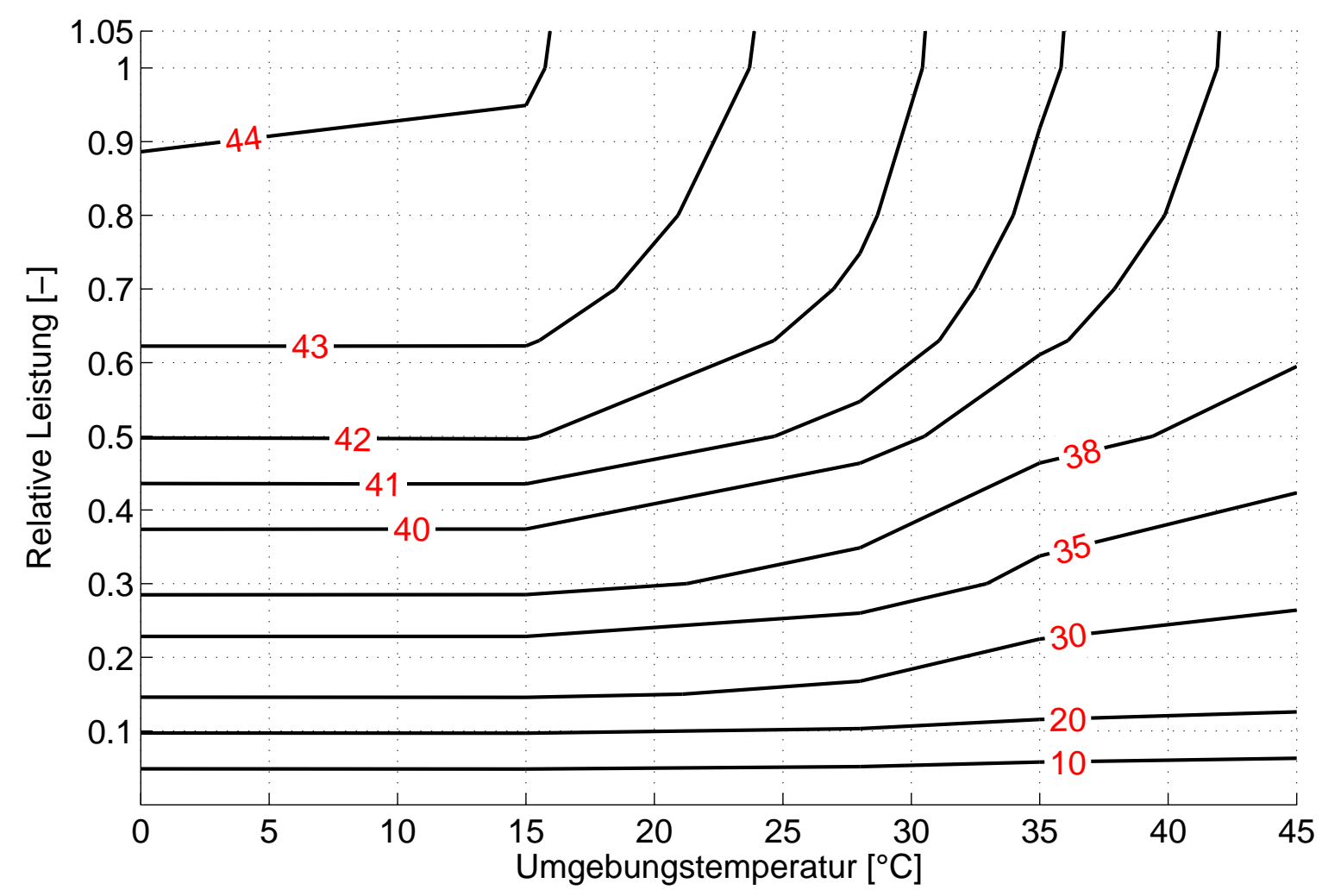

Abbildung 6.4.: Wirkungsgradkennfeld der Dampfturbine (125 $\mathrm{MW}_{\text {el }}$, Werte in \%) in Abhängigkeit vom Lastfall und der Umgebungstemperatur 100

Tabelle 6.2.: Eigenschaften des verwendeten Kraftwerksblocks nach 100

\begin{tabular}{ll}
\hline Dampfturbinentyp & ZÜ-Kondensation-Maschine \\
\hline Nennleistung brutto & $125 \mathrm{MW}_{\text {el }}$ \\
Dampfmassenstrom (HD/ND) ${ }^{a}$ & $100.8 / 89.5 \mathrm{~kg} / \mathrm{s}$ \\
Dampfdruck (FD/ZÜ) & $155 / 34 \mathrm{bar}$ \\
Dampftemperatur (FD/ZÜ) & $552 / 552^{\circ} \mathrm{C}$ \\
Druckverlust (FD/ZÜ) & $10 / 2 \mathrm{bar}$ \\
Parasitäre Verluste (online/offline) & $5000 / 1250 \mathrm{~kW}$ el \\
Abdampfdruck & $125 \mathrm{mbar}$ \\
Abdampftemperatur & $50^{\circ} \mathrm{C}$ \\
Abdampfmassenstrom & $71.8 \mathrm{~kg} / \mathrm{s}$ \\
Speisewassertemperatur & $239^{\circ} \mathrm{C}$ \\
Leistung Luftkondensator & $161 \mathrm{MW}$ th \\
\hline
\end{tabular}

\footnotetext{
${ }^{a} \mathrm{HD}=$ Hochdruck, ND $=$ Niederdruck.

${ }^{b} \mathrm{FD}=$ Frischdampf, ZÜ $=$ Zwischenüberhitzung.
} 


\subsubsection{Standort und Auslegungszeitpunkt}

Die Vergleichsstudie wurde für den Standort Postmasburg in Südafrika durch-

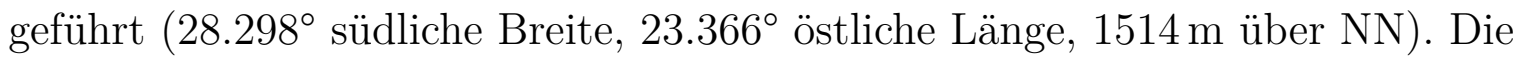
maximale DNI beträgt: $1091 \mathrm{~W} / \mathrm{m}^{2}$. Neben den stündlich aufgelösten DNI-Daten sind auch die Messdaten der Windgeschwindigkeit in $10 \mathrm{~m}$ über Grund (max. $13.1 \mathrm{~m} / \mathrm{s})$, die Umgebungstemperatur $\left(-4.8\right.$ bis $\left.35.9^{\circ} \mathrm{C}\right)$, der Umgebungsdruck sowie die relative Luftfeuchtigkeit gegeben, mit deren Hilfe die Wirkungsgrade von Receiver und Kraftwerksblock stündlich aufgelöst berechnet werden können.

\subsubsection{Heliostatenfeldauslegung}

Sämtliche Heliostatenfelder wurden mit HFLCAL ausgelegt. Da diese Auslegung vom Receiverwirkungsgrad abhängt, sind mehrere Iterationen zwischen der Receiverberechnung mit ASTRID und der Heliostatenfeldauslegung nötig. Sofern nicht anders angegeben wurde in HFLCAL das Receivermodell 101 (user defined, icav=3) nach [106 verwendet. Für die erste Auslegung wurden zunächst Schätzbzw. Erfahrungswerte für die vier Parameter $\eta_{\mathrm{opt}}, \epsilon, d T_{\mathrm{w}}$ und $\alpha$ angenommen. Für den Receiverwirkungsgrad gilt dabei die folgende Gleichung nach [106:

$$
\begin{aligned}
\eta_{\text {rec }} & =\eta_{\text {opt }}-\left[\left(\epsilon \cdot \sigma_{\mathrm{SB}} \cdot \bar{T}^{4}\right)+(\alpha \cdot(\bar{T}-300))\right] \cdot \frac{A_{\text {rec }}}{P_{\text {inc }}} \\
\bar{T} & =\frac{T_{\text {aus }}+T_{\text {ein }}}{2}+d T_{\mathrm{w}}
\end{aligned}
$$

mit $\quad \eta_{\text {opt }}$ : Optischer Receiverwirkungsgrad

$\epsilon$ : Emissivität der Receiveroberfläche

$d T_{\mathrm{w}}$ : Temperaturdifferenz der Receiverwand

$\alpha$ : Konvektiver Wärmeübergangskoeffizient

Nach der Heliostatenfeldoptimierung wurde anschließend mit ASTRID die thermische FEM-Berechnung durchgeführt, um die tatsächliche Wirkungsgradkennlinie des Receivers zu erhalten. Mit dieser neuen Kennlinie können die zuvor geschätzten vier Parameter in Gleichung (6.1) über einen Kurvenfit neu bestimmt und die Heliostatenfeldoptimierung erneut durchgeführt werden. Dieser 
iterative Vorgang wurde mehrmals wiederholt, um den Einfluss des Receiverwirkungsgrades auf die Feldauslegung mitzuberücksichtigen.

Die bei Salzreceivern notwendige Zielpunktverteilung wurde bei der Heliostatenfeldoptimierung nicht mitberücksichtigt, sondern erst im Nachgang durchgeführt. Dabei wurden für ausgewählte Zeitpunkte für jeden Heliostat die Zielpunkte berechnet und anschließend mit SPRAY der Feldwirkungsgrad bestimmt. In Abbildung 6.5 ist die Differenz der Wirkungsgrade des Heliostatenfeldes des Referenzsystems Solar Salt mit und ohne Zielpunktverteilung dargestellt.
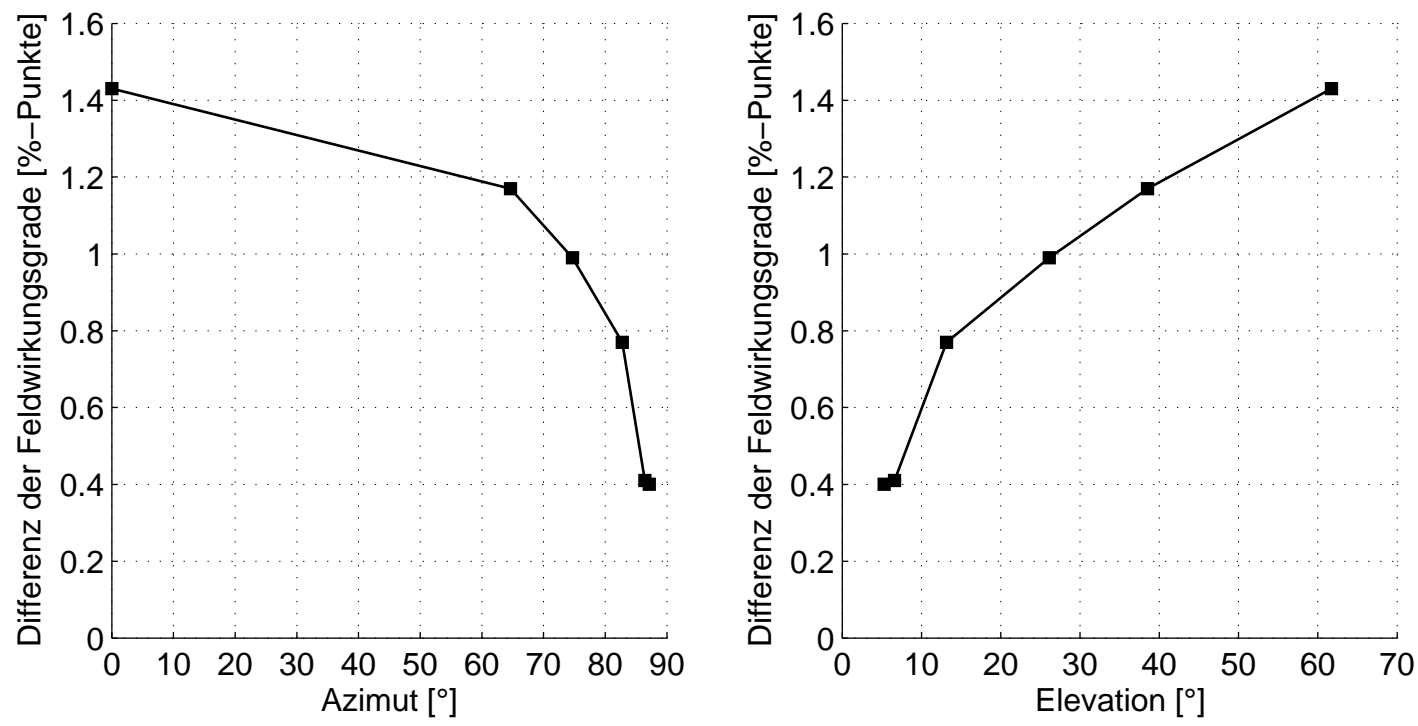

Abbildung 6.5.: Differenz der Feldwirkungsgrade in Abhängigkeit von Azimutwinkel und Elevation für verschiedene Zeitpunkte am Standort Postmasburg (Wirkungsgrad ohne Zielpunktverteilung abzüglich des Wirkungsgrades mit Zielpunktverteilung).

In den Morgenstunden (geringe Elevation und hohe Azimutwinkel) sind die Zielpunkte nahe der Receivermitte angeordnet und gegenüber der Berechnung ohne Zielpunktverteilung vermindert sich der Feldwirkungsgrad um lediglich $0.4 \%$. Bei hohen Leistungen und Wärmestromdichten um die Mittagszeit (Azimut $=0^{\circ}$ und hoher Elevation) müssen die Zielpunkte weiter verteilt werden, um das Wärmestromdichtelimit nicht zu überschreiten. Dabei verringert sich der Feldwirkungsgrad durch eine höhere Randstrahlung um ca. 1.4\%-Punkte.

In der vorliegenden Arbeit wurde für jedes Heliostatenfeld ein Wirkungsgradkennfeld mit SPRAY ohne Zielpunktverteilung erzeugt (in Abhängigkeit von 
Azimut und Elevation). In diesem Kennfeld kann zur Jahresertragsberechnung je nach Zeitpunkt/Sonnenstand der Wirkungsgrad des Heliostatenfeldes interpoliert werden. Bei den Varianten mit Zielpunktverteilung (Solar Salt-Varianten) muss dieses Kennfeld entsprechend Abbildung 6.5 angepasst werden.

\subsubsection{Receivermodellierung und Wärmeträgermedium}

Die Receiverauslegung wurde wie in Kapitel 5 beschrieben mit ASTRID und ANSYS durchgeführt. Mit der Wärmestromdichteverteilung wurden in der Vorauslegung eine Vielzahl an Varianten mit verschiedenen Modulanzahlen, verschaltungen und Rohrdurchmessern berechnet und hinsichtlich Kosten, Druckverlust und Teillastverhalten bewertet. Anschließend wurden in der detaillierten FEM-Berechnung die mehrdimensionalen Receiverwirkungsgradkennfelder für verschiedene Zeitpunkte und Windfälle berechnet. Abbildung 6.6 zeigt beispielhaft eine Wirkungsgradkennlinie mit den resultierenden Absorbertemperaturen. Der Feldwirkungsgrad nimmt ab den frühen Morgenstunden bis $12 \mathrm{~h}$ stetig zu. Grund hierfür sind hauptsächlich die geringeren Kosinusverluste bei höheren Sonnenständen. Der Receiverwirkungsgrad steigt zunächst stark an, da die Leistungszunahme auch mit einer Zunahme des Massenstromes und damit der Strömungsgeschwindigkeit (Turbulenz) einhergeht, wodurch der Wärmeübergang verbessert wird. Zwischen 8-12 h beträgt die Zunahme nur noch wenige Prozentpunkte.

Die maximale Absorbertemperatur hängt stark von der Wärmestromdichteverteilung und vom verwendeten WTM ab. In dieser Arbeit werden die drei WTM Solar Salt, Natrium und Blei-Bismut untersucht.

Die höchsten Absorbertemperaturen enstehen gegen $9 \mathrm{~h}$, da der Receiver zu dieser Uhrzeit einseitig bestrahlt wird. Im weiteren Verlauf sinkt bis $12 \mathrm{~h}$ die maximale Absorbertemperatur trotz höherer Receiverleistung.

Für die in dieser Arbeit durchgeführte Jahresertragsrechnung wurden die am Auslegungstag (21. März) berechneten Wirkungsgradkennfelder verwendet. Für jede Stunde im Jahr wurde daraus je nach Wind- und Lastzustand der Receiverwirkungsgrad interpoliert. 


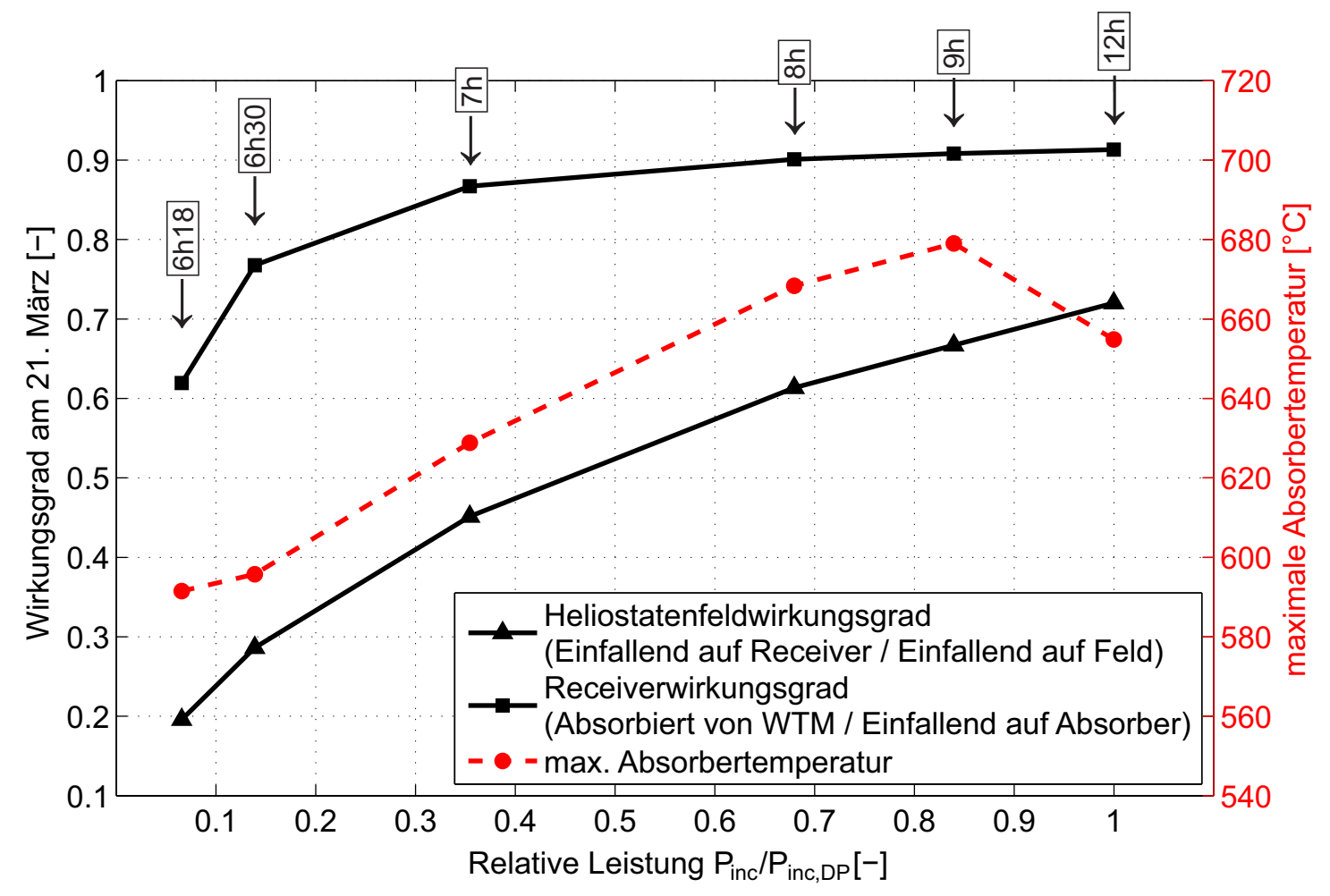

Abbildung 6.6.: Beispielergebnis eines mit ASTRID berechneten Receivers an verschiedenen Uhrzeiten am 21.März ohne Windeinfluss (Wirkungsgrade und maximale Absorbertemperaturen).

\subsubsection{Leitungssystem und thermischer Energiespeicher}

Für jede berechnete Variante wurde die Steig- und Fallleitung mit der Druckgeräterichtlinie ausgelegt (siehe Anhang, Gleichung (C.1), Seite 183). Für die Wandstärke ist dabei der statische Druck am Grund maßgebend. Bei Systemen mit offenen Flüssigsalzkreisläufen kommen zur Förderung mechanische Pumpen zum Einsatz. Durch die hohe Förderhöhe (> Turmhöhe) sind hohe Leistungen erforderlich, wodurch im Teillastbetrieb der minimale Volumenstrom nicht beliebig reduziert werden kann. In dieser Arbeit wurde daher die Leistung der Salzreceiver auf $\dot{Q}_{\text {th }} \geq 20 \%$ begrenzt. Für sämtliche hier durchgeführten Jahresertragsberechnungen wurden für alle Salzpumpen die in steag EBSILON hinterlegte Pumpenkennlinie verwendet (siehe Abbildung 6.7 links).

Für die Förderung von Natrium wurden in dieser Arbeit ausschließlich elektromagnetischen Pumpen angenommen (Vorteile siehe Abschnitt 4.3). Für die Anwendung in Solarturmkraftwerken der untersuchten Leistungsklasse sind

\footnotetext{
${ }^{4}$ EBSILON Professional Version 12.01 von STEAG Energy Services
} 

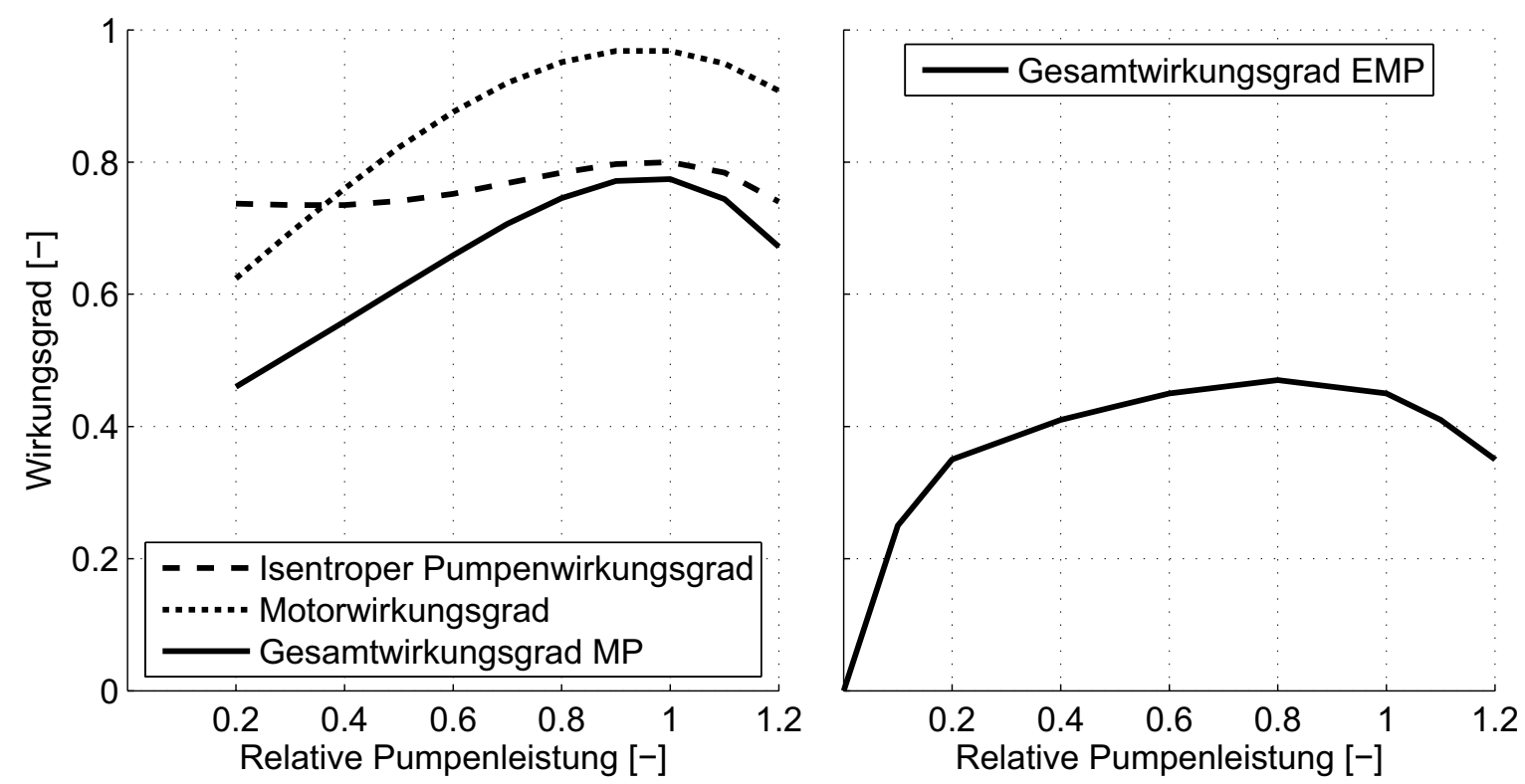

Abbildung 6.7.: Verwendete Wirkungsgradkennlinien. Links: Mechanische Pumpen (MP) für Flüssigsalz und Blei-Bismut. Rechts: Elektromagnetische Pumpen (EMP) für Natrium 92 .

große Pumpen mit hohen Durchflussmengen bis $8500 \mathrm{~m}^{3} / \mathrm{h}$ und Druckverlusten bis 4 bar notwendig. Eine EMP in dieser Größenordnung $\left(9600 \mathrm{~m}^{3} / \mathrm{h}\right.$ bei 2.8 bar $)$ wurde bereits gebaut und ausgiebig getestet [92]. Die dort dokumentierte Wirkungsgradkennlinie wurde für die hier durchgeführten Jahresertragsrechnungen verwendet (siehe Abbildung 6.7 rechts).

Die geringen Wirkungsgrade der EMP spiegeln sich in einer Temperaturerhöhung des Flüssigmetalls wieder. Dieser Einfluss wurde in den Berechnungen dieser Arbeit nicht berücksichtigt. Auch Blei-Bismut kann mit EMP gefördert werden, jedoch ergeben sich noch geringere Wirkungsgrade als bei Natrium. Daher wurden hier die LBE-Systeme wie die Salzsysteme mit mechanischen Pumpen ausgelegt.

Unabhängig vom jeweiligen Konzept wurde für diese Arbeit die Annahme getroffen, dass sämtliche Pumpen des Kraftwerks nach den in Abbildung 6.7 dargestellten spezifischen Wirkungsgradkennlinien arbeiten.

Aus den Voruntersuchungen zu thermischen Speichersystemen (siehe Abschnitt 4.2.2) kommen für die direkte Speicherung beim verwendeten Kraftwerksblock (Dampfturbine mit $125 \mathrm{MW}_{\mathrm{el}}$ ) und dem Temperaturniveau (290$565^{\circ} \mathrm{C}$ ) aus Kostengründen lediglich die 2-Tank-Systeme mit Solar Salt als 
Speichermedium in Frage. Die kostengünstigeren Varianten mit Thermoclinen und Füllmaterialien sind derzeit noch nicht Stand der Technik und wurden daher hier nicht weiter betrachtet. Sämtliche hier berechneten Konzepte wurden daher mit einem 2-Tank-System mit Solar Salt berechnet. 


\subsection{Bewertungsmethodik und LCOE-Berechnung}

Als Grundlage zur Berechnung der Stromgestehungskosten (LCOE) dient das ECOSTAR-Berechnungsmodell 88 in welchem das von der IEA vorgeschlagene Kostenmodell [110] zur Berechnung und Bewertung von erneuerbaren Energien hinterlegt ist. Ausgehend von den stündlichen DNI- und Wetterdaten wird dabei der Jahresenergieertrag des Kraftwerks berechnet und mit den jährlichen Anlagenkosten ins Verhältnis gesetzt. Die Gesamtleistung des Solarturmkraftwerkes ergibt sich für jeden Zeitpunkt aus:

$$
P_{\text {sys }}(t)=D N I(t) \cdot A_{\text {feld }} \cdot \eta_{\text {feld }}(t) \cdot \eta_{\text {rec }}(t) \cdot \eta_{\text {stor }}(t) \cdot \eta_{\mathrm{pb}}(t)
$$

Die jährlichen Kosten ergeben sich aus:

$$
K_{\mathrm{jahr}}=K_{\mathrm{inv}} \cdot F C R+K_{\mathrm{O} \& \mathrm{M}} \quad ; \quad F C R=\frac{i_{\mathrm{r}} \cdot\left(1+i_{\mathrm{r}}\right)^{n_{\text {jahre }}}}{\left(1+i_{\mathrm{r}}\right)^{n_{\text {jahre }}}-1}
$$

mit $\quad K_{\text {inv }}$ : Investitionskosten (Land, Heliostatenfeld, Turm, Receiversystem, WTM, Rohrleitungen, Wärmeübertrager, Speichersystem, Kraftwerksblock) + indirekte Kosten (Engineering, Risikozuschlag, Management)

FCR: Annuitätenfaktor (Fixed Charge Rate)

$K_{\mathrm{O \& M}}$ : Jährliche Lohn-, Wasser-, Betriebs- und Wartungskosten

+ Versicherung

$i_{\mathrm{r}}$ : Zinssatz

$n_{\text {jahre: }}$ Laufzeit des Darlehens in Jahren

Die LCOE ergeben sich damit zu:

$$
L C O E=\frac{K_{\text {jahr }}}{\int_{t_{0}}^{t_{\text {end }}} P_{\text {sys,el }}(t)-P_{\text {loss }, \text { par }}(t) d t} \quad\left[€ / \mathrm{kWh}_{\text {el }}\right]
$$

Die Verluste $P_{\text {loss,par }}$ in Gleichung 6.4 beinhalten den Stromverbrauch zum Betrieb der gesamten Anlage (Heliostatenbewegung, Steuerungssysteme, sämtliche Pumpen und Begleitheizung) im Online- und Offlinebetrieb. 
Bei der Betriebsstrategie ist es möglich, den Kraftwerksblock bedarfsabhängig mit einer vorgegebenen Lastkurve zu betreiben. In dieser Arbeit wurde bei allen Systemen die Annahme getroffen, dass der Energiebedarf im Stromnetz stets vorhanden ist und somit der Kraftwerksblock möglichst immer unter Vollast betrieben wird, sofern es die Einstrahlung bzw. der Speicherzustand zulässt. Die Betriebszustände eines Solarturmkraftwerkes sind in Abbildung 6.8 dargestellt. Folgende Optionen sind möglich:

1. Keine Solarstrahlung, Speicher leer

$\rightarrow$ Kraftwerksblock offline

2. Solarstrahlung zu gering für Betrieb des Kraftwerksblocks, Speicher leer $\rightarrow$ Speicher wird beladen

3. Solarstrahlung ausreichend für Betrieb des Kraftwerksblocks, Speicher leer $\rightarrow$ Kraftwerksblock online und Speicher wird beladen

4. Solarstrahlung ausreichend für Betrieb des Kraftwerksblocks, Speicher voll $\rightarrow$ Kraftwerksblock online, Heliostaten werden defokussiert (Dumping)

5. Solarstrahlung zu gering für Betrieb des Kraftwerksblocks, Speicher voll $\rightarrow$ Speicher wird entladen, Kraftwerksblock online

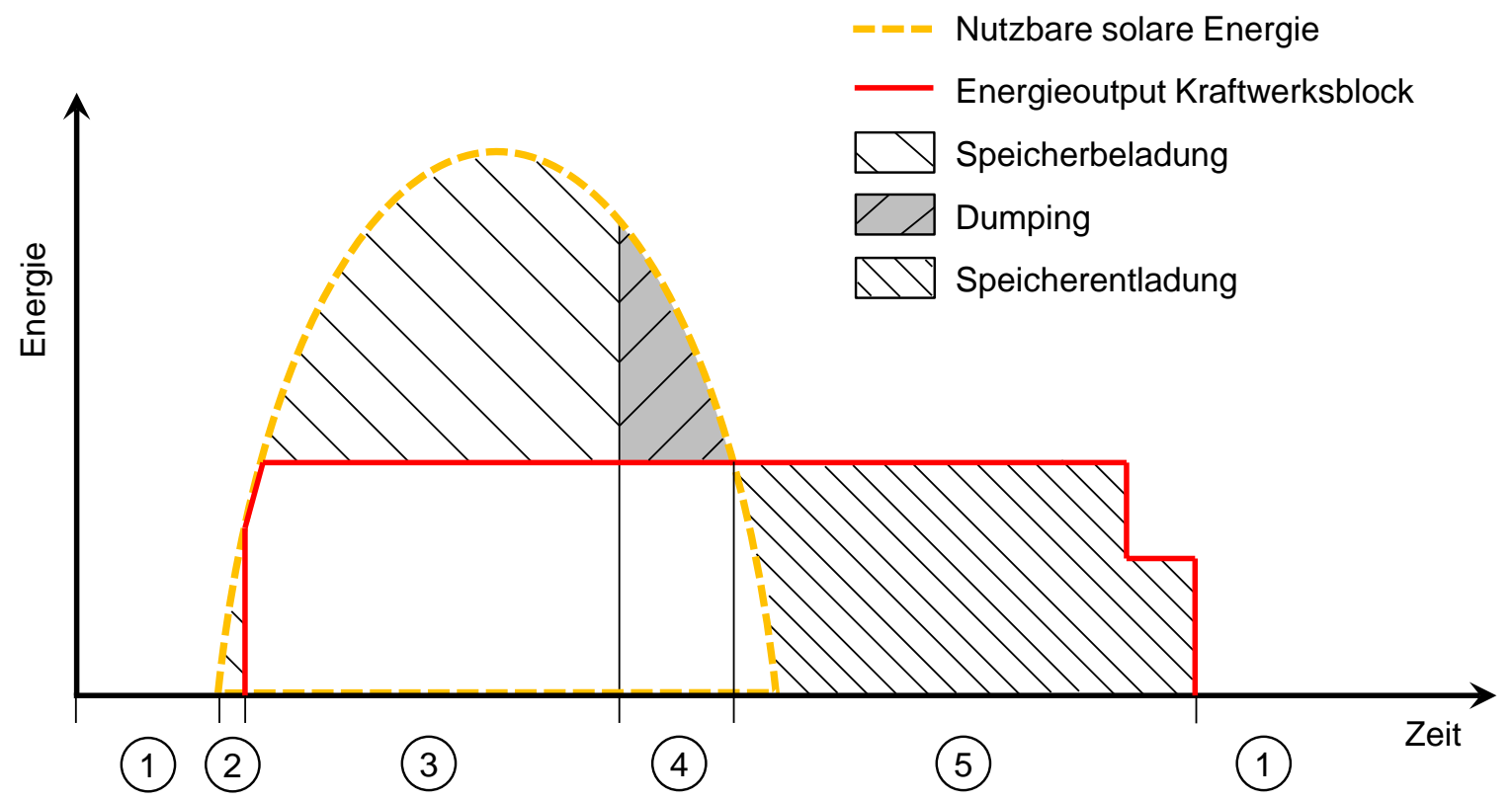

Abbildung 6.8.: Mögliche Betriebszustände eines Solarturmkraftwerkes 


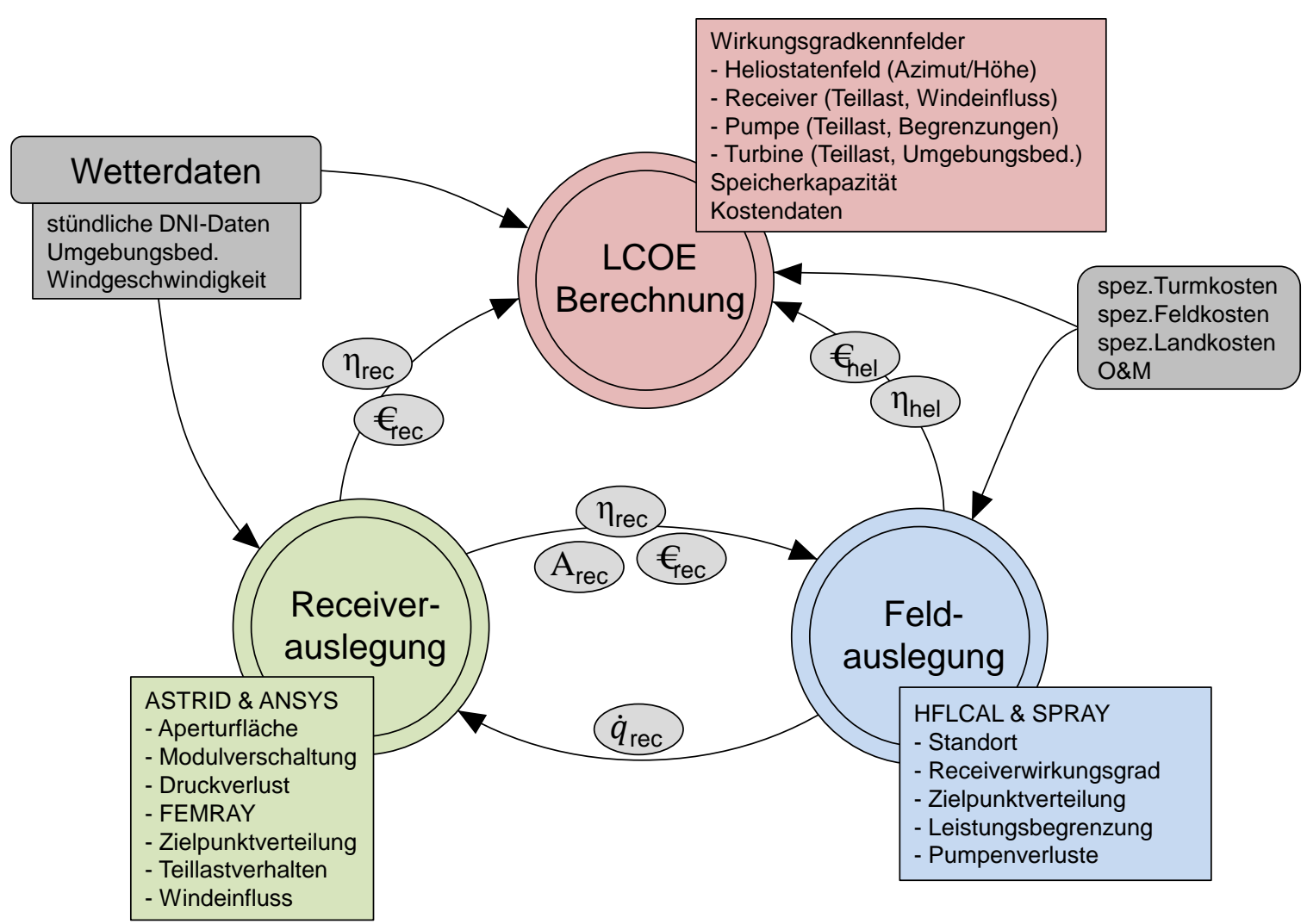

Abbildung 6.9.: Vorgehensweise bei der Systemauslegung und LCOE-Berechnung

Vor der eigentlichen Jahresertrags- und LCOE-Berechnung wurden alle Einzelkomponenten der jeweiligen Konfigurationen in mehreren Schleifen optimiert. Abbildung 6.9 zeigt die Vorgehensweise bei der Systemauslegung und -optimierung. Im ersten Schritt wird mit einem geschätzten Receiverwirkungsgrad und den Hauptanlagenparametern (Standort, Leistung, Aperturfläche) ein Heliostatenfeld mit HFLCAL erzeugt und mit Hilfe von spezifischen Kostendaten (Heliostatenfeld, Turm, Receiver, O\&M, parasitäre Verluste) optimiert. Der zweite Schritt beinhaltet die detaillierte Receiverauslegung mit ASTRID (siehe Kapitel 5). Aus den Daten der Feldauslegung (Anordnung der Heliostate und Turmhöhe) wird mittels Strahlverfolgung die Wärmestromdichteverteilung auf dem Receiver ermittelt. In ASTRID wird der Receiver hydraulisch und thermisch unter Berücksichtigung der Kosten optimiert. Mit den berechneten Daten kann die Feldauslegung verfeinert werden.

In der LCOE-Berechnung wird schließlich zur Ermittlung des Energieertrags ein komplettes Jahr in stündlicher Aufösung simuliert. Um das kostenoptimale 
Verhältnis zwischen Speicherkapazität und Dumping zu ermitteln wird bei jeder Variante eine Parametervariation der Speicherkapazität durchgeführt.

\subsubsection{Systemmodellierung und Jahresertragsberechnung}

Zur Ertragsberechnung wurde für jede Stunde im Jahr anhand des Sonnenstandes der Wirkungsgrad des Heliostatenfeldes aus dem mit SPRAY erzeugten Kennfeld interpoliert und damit die auf den Receiver auftreffenden Strahlung $P_{\text {inc }}$ berechnet. Mit der Windgeschwindigkeit auf Receiverhöhe und $P_{\text {inc }}$ wurde anschließend die thermische Receiverleistung sowie die benötigte Pumpenleistung aus den zuvor erzeugten Kennfeldern interpoliert. Der Speicherwirkungsgrad wurde als konstant angenommen mit $\eta_{\mathrm{st}}=0.99$. Je nach Zustand des Kraftwerksblocks (Anfahrvorgang oder Betrieb) $)^{5}$ und des thermischen Speichers sowie der angewendeten Betriebsstrategie und der vorgegebenen Lastkurve (Strombedarf) wurde das Kraftwerk stündlich geregelt (siehe auch Abschnitt 6.2). Mit den Umgebungsbedingungen der Luft wurde schließlich für jede Stunde die Brutto-Kraftwerksleistung (siehe Abbildung 6.4) und abzüglich der parasitären Verluste durch Pumpen, Begleitheizung und Nachführen der Heliostate die Netto-Kraftwerksleistung berechnet, die integriert über das Jahr den Jahresenergieertrag ergibt.

\subsubsection{Kostenmodelle}

\subsubsection{Heliostatenfeldkosten}

Nach [11] liegen die Kosten für Heliostatenfelder zwischen $98-170 € / \mathrm{m}^{2}$ reflektierende Spiegelfäch ${ }^{6}$. Der Mittelwert aus den in 111 angegebenen Kostendaten wird in dieser Arbeit verwendet. Er beträgt:

$$
K_{\text {Feld }}=130 € / \mathrm{m}^{2} \text { Spiegelfläche }+3 € / \mathrm{m}^{2} \text { Land }
$$

\footnotetext{
${ }^{5}$ Für jeden Anfahrvorgang der Turbine werden ca. $150 \mathrm{MWh}_{\text {th }}$ benötigt. Die thermische Masse und die thermischen Verluste des Receivers sowie des Leitungssystems werden ebenfalls mitberücksichtigt.

${ }^{6}$ Werte in Euro Jahr 2010, Dollarwechselkurs: $1.4 \$=1 €$.
} 


\subsubsection{Turmkosten}

Die Turmhöhe hat einen großen Einfluss sowohl auf den Wirkungsgrad des Heliostatenfeldes (Kosinuswirkungsgrad), als auch auf die parasitären Verluste im WTM-Kreislauf (Pumpenleistung). Dies macht sich vor allem bei offenen Systemen (wie in Abbildung 6.1) bemerkbar. Kommerzielle Anlagen haben Turmhöhen $]^{7}$ zwischen 100 und $200 \mathrm{~m}$. Abbildung 6.10 zeigt verschiedene Turmkostenkorrelationen in Abhängigkeit von der Turmhöhe. In dieser Arbeit wird die im Projekt SalzturmPLUS [113] entwickelte Korrelation verwendet:

$$
K_{\text {turm }}=250000+14.77 \cdot H_{\text {turm }}^{2.392} \quad[€]
$$

Neben der Turmhöhe werden die Turmkosten auch vom Receivergewicht beeinflusst (vergleiche Abschnitt 3.4.2). Dieser Einfluss wird in dieser Arbeit nicht mitberücksichtigt.

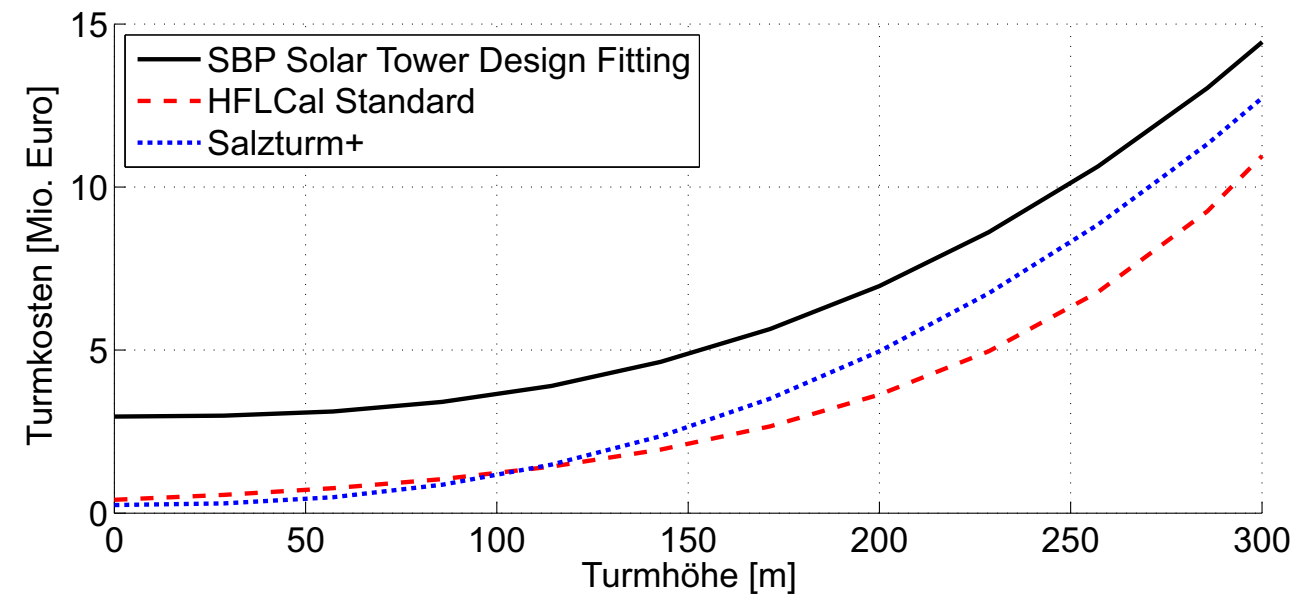

Abbildung 6.10.: Vergleich verschiedener Turmkostenkorrelationen [114, 113

\subsubsection{Receiverkosten}

Nach Umrechnung der Receiverkosten für Mehraugenkavitäten von Babcock und Wilcox 115 auf einen externen Receiver mit einer mittleren Wärmestromdichte von $0.5 \mathrm{MW} / \mathrm{m}^{2}$ ergibt sich die in Abbildung 6.11 dargestellte Kostenaufteilung.

${ }^{7}$ Gemasolar: $140 \mathrm{~m}$ [11, Cerescent Dunes: $200 \mathrm{~m}$ 112. 

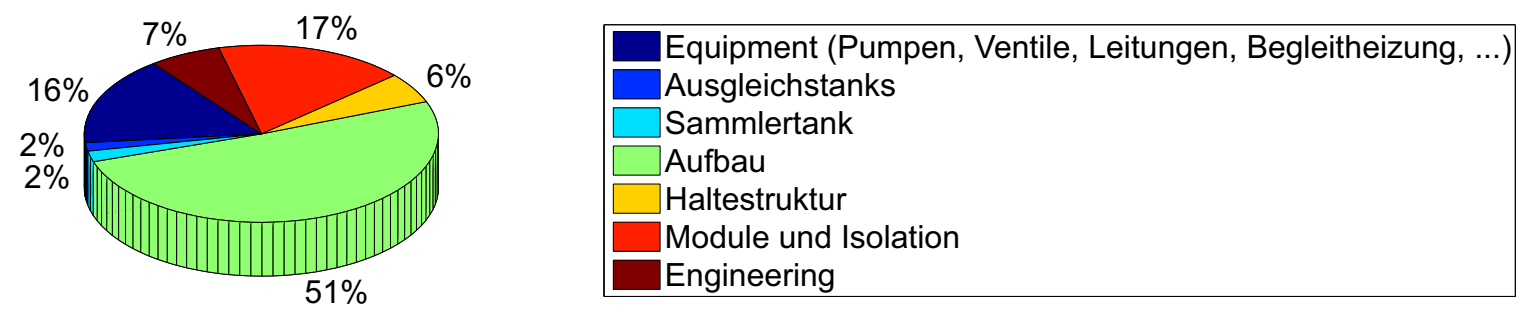

Abbildung 6.11.: Kostenaufteilung für externe Receiver in Anlehnung an 115

Der Aufbau des Receivers nimmt mit 51\% den größten Kostenanteil ein. Bei gleicher Receiverleistung bleiben die Kosten für das Equipment und die Tanks konstant. Wird die Wärmestromdichte erhöht bzw. die Absorberfläche verkleinert, so verringern sich auch die Kosten von Aufbau, Struktur, Module, Isolation und Engineering 8 . In der Receivergrößenstudie (siehe Abschnitt 7.1.2) wurden Natriumreceiver der gleichen Leistung mit verschiedenen Wärmestromdichten ausgelegt. Mit der Receiver- und Rohrgeometrie sowie den Materialkosten wurde eine Receiverkostenfunktion in Abhängigkeit der Absorberfläche bzw. der Wärmestromdichte hergeleitet. Die Receiverkosten setzen sich dabei aus einem Sockelbetrag (Equipment und Tanks) und einem über die Absorberfläche skalierbaren Betrag (Absorberrohre, Haltestruktur, Aufbau) zusammen. Für das Referenzsystem mit Solar Salt betragen die Receiverkosten in dieser Arbeit $115 € / \mathrm{kW}_{\text {th }}$ bei $\overline{\dot{q}}=0.5 \mathrm{MW} / \mathrm{m}^{2}$. Bei Natriumreceivern ergeben sich durch die geringere Absorberfläche sowie der geringeren Anzahl an Absorberrohren und Modulverbindungen auch geringere Receiverkosten. Um die Sensitivität der Receiverkosten beurteilen zu können werden in dieser Arbeit für Natriumreceiver zwei verschiedene Kostenfunktionen (Kostensenkung um 25\% bzw. um $40 \%$ bei halber Absorberfläche) untersucht:

$$
\begin{array}{lll}
K_{\text {rec }, 1}=\frac{28.476}{\overline{\dot{q}}}+57.381 & {[€ / \mathrm{kW}]} & \text { mit } \overline{\dot{q}} \text { in }\left[\mathrm{MW} / \mathrm{m}^{2}\right] \\
K_{\mathrm{rec}, 2}=\frac{46.438}{\dot{\dot{q}}}+21.899 & {[€ / \mathrm{kW}]} & \text { mit } \dot{\dot{q}} \text { in }\left[\mathrm{MW} / \mathrm{m}^{2}\right]
\end{array}
$$

Beide Kostenfunktionen (Gleichungen (6.7) bis (6.8)) skalieren die Receiverkosten linear mit der Absorberfläche basierend auf $115 € / \mathrm{kW}_{\text {th }}$ bei $\overline{\dot{q}}=0.5 \mathrm{MW} / \mathrm{m}^{2}$.

\footnotetext{
${ }^{8}$ Es wird angenommen, dass die Kosten für das Engineering linear mit den absoluten Receiverkosten skalieren. Je größer der Receiver, desto höher sind auch die Kosten für das Engineering.
} 


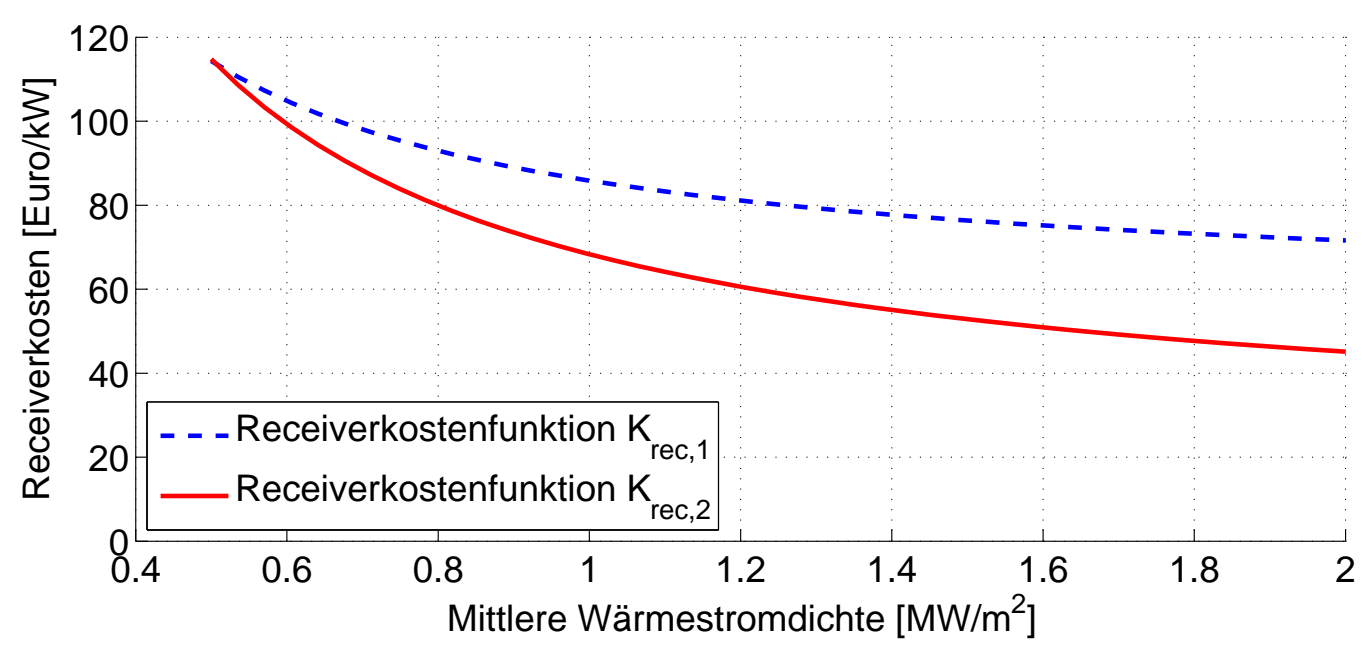

Abbildung 6.12.: Spezifische Receiverkostenfunktionen für Natriumreceiver in Abhängigkeit der mittleren Wärmestromdichte

Die Kostenfunktion $K_{\text {rec,1 }}$ entspricht einer Kostensenkung um $25 \%$ bei einer Halbierung der Absorberfläche $\left(86 € / \mathrm{kW}_{\text {th }}\right.$ bei $\left.\overline{\dot{q}}=1.0 \mathrm{MW} / \mathrm{m}^{2}\right)$. Bei $K_{\text {rec }, 2}$ beträgt die Kostensenkung $40 \%$ bei Halbierung der Absorberfläche $\left(68 € / \mathrm{kW}_{\text {th }}\right.$ bei $\overline{\dot{q}}=1.0 \mathrm{MW} / \mathrm{m}^{2}$ ). Abbildung 6.12 zeigt beide Receiverkostenfunktionen.

\subsubsection{Wärmeübertrager Natrium / Solar Salt}

In dieser Arbeit werden die Wärmeübertrager mit einer Grädigkeit von $10 \mathrm{~K}$ ausgeführt (siehe Abbildung 6.13). Auf Basis von [116] wurden die beiden folgenden Kostenfunktionen ermittelt, die den Kostenbereich für den vorliegenden Anwendungsfall abdecken. In erster Näherung ergeben sich durch den erhöhten Materialaufwand etwa die doppelten Kosten im Vergleich zu einem einfachen Wärmeübertrager ohne Doppelrohrtechnik.

$$
\begin{aligned}
K_{\mathrm{HX}, \text { min }} & =10.7140[€ / \mathrm{kW}] \\
K_{\mathrm{HX}, \text { max }} & =28.5710[€ / \mathrm{kW}]
\end{aligned}
$$



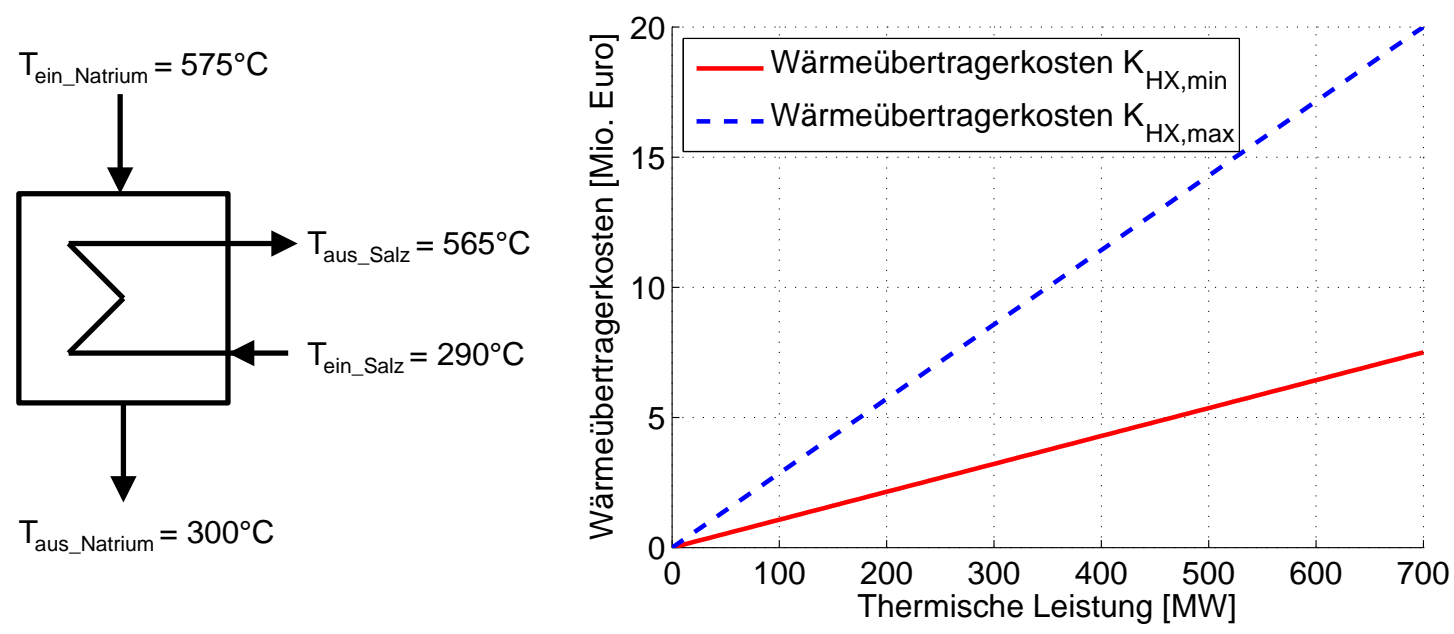

Abbildung 6.13.: Daten und Kostenfunktionen der Doppelrohr-Wärmeübertrager mit Natrium / Solar Salt.

\subsubsection{Wärmeträgermedium und Leitungen}

Die Menge des WTMs im Receiverkreislauf wurde mit dem Volumen des Receiversystems (Absorberrohre, Verteiler, Sammler und Verbindungsleitungen) sowie den Steig- und Fallleitungen ${ }^{9}$ abgeschätzt und mit dem Faktor 1.1 multipliziert. Für die Anschaffungskosten der WTM wurden die folgenden Werte (entsprechend Abbildung 3.5) angenommen:

- Solar Salt: $1.0 € / \mathrm{kg}$

- Natrium: $2.6 € / \mathrm{kg}$

- Blei-Bismut: $12.0 € / \mathrm{kg}$

Sowohl bei Solar Salt als auch bei den Flüssigmetallen fallen im Betrieb Kosten durch die Degradation bzw. Erneuerung/Reinigung der WTM an. Aufgrund mangelnder Daten wurden diese Kosten in dieser Arbeit vernachlässigt.

Zur Berechnung der Leitungskosten (ohne Dämmung) wurden folgende Annahmen getroffen: Kalte Steigleitung: $3 € / \mathrm{kg}$, heiße Fallleitung $10 € / \mathrm{kg}$. Der Rohrdurchmesser von Steig- und Fallleitung wurde für jede Variante so ausgelegt, dass der Druckverlust im Auslegungszeitpunkt $\approx 1$ bar beträgt. Zur Ermittlung der Rohrwandstärke wurde die Druckgeräterichtline AD 2000 [97] herangezogen.

${ }^{9}$ Beim Konzept Receiver-only befindet sich in den Steig- und Falleitungen Solar Salt, daher bleiben diese unberücksichtigt. Beim Konzept Tower-loop sind die Steig- und Fallleitungen mit Natrium gefüllt und gehen daher in die Berechnung mit ein. 
Bei Mehrturmsystemen sind die Kosten der wärmegedämmten Verbindungsleitungen zwischen den Türmen und dem zentralen Speicher/Kraftwerksblock signifikant für die LCOE. Da diese Kosten schwer abschätzbar sind und keine belastbare Quelle dafür gefunden werden konnte, wurden stattdessen die Leitungskosten berechnet, bei denen das Mehrturmsystem bezüglich des Einturmsystems die gleichen LCOE erreicht. Damit kann eine Aussage getroffen werden, wie hoch die Leitungskosten des Mehrturmsystems maximal sein dürfen, damit die gleichen LCOE zu erwarten sind.

\subsubsection{Speicherkosten}

Auf Basis der Ergebnisse der Speichermodellierung (Abschnitt 4.2) wird in dieser Arbeit der 2-Tank Speicher mit Solar Salt mit den in Abschnitt 4.2.2 angegebenen Speicherkosten verwendet. Eine Ausgleichsrechnung mit den Ergebnissen der Speichermodellierung liefert die folgende Kostenfunktion in Abhängigkeit der Speicherkapazität:

$$
K_{\mathrm{st}}=a \cdot E_{\mathrm{st}}^{b}+c \quad \text { in }\left[€ / \mathrm{kW} \mathrm{h}_{\mathrm{th}}\right] \quad \text { mit }\left\{\begin{array}{l}
a=155.6 \\
b=-0.3549 \\
c=10.55 \\
E_{\mathrm{st}} \text { in } \mathrm{MWh}_{\mathrm{th}}
\end{array}\right.
$$

\subsubsection{Kosten des Kraftwerksblocks}

Nach einer Studie von Black \& Veatch für NREL liegen die Kosten für den Kraftwerksblock zwischen $900-975 € / \mathrm{kW}_{\text {el }}$ installierter Leistung [117]. In dieser Arbeit wurde für die Kosten des gesamten Kraftwerksblocks folgende Annahme getroffen:

$$
K_{\mathrm{pb}}=950 € / \mathrm{kW}_{\mathrm{el}}
$$

Für die verwendete Dampfturbine mit einer Brutto-Nennleistung von $125 \mathrm{MW}_{\mathrm{el}}$ (Details siehe Abschnitt 6.1.2) ergeben sich damit die Kosten des Kraftwerksblocks: $K_{\mathrm{pb}}=118.75 \mathrm{Mio} €$. 


\subsubsection{Betriebs- und Wartungskosten}

Die in dieser Arbeit verwendeten Annahmen für die Finanzierungs-, Betriebsund Wartungskosten sind in Tabelle 6.3 zusammengefasst.

Damit ergibt sich der Annuitätenfaktor aus Gleichung (6.3) zu:

$$
F C R=\frac{0.08 \cdot 1.08^{25}}{1.08^{25}-1}=0.0937
$$

Tabelle 6.3.: Weitere Kostenannahmen für Finanzierung, Betrieb und Wartung nach 100.

Lohnkosten

Angestelle (ohne Feldwartung)

Angestellte für Feldwartung

Wasserkosten

O\&M des Equipments

Versicherung

Anlagenlaufzeit / Laufzeit des Darlehens

Kreditzinsrate $i_{\mathrm{r}}$

Engineering, Risikozuschlag und Management

Stromverbrauch der Heliostate

Stromkosten der Anlage während Offline-Zeiten
$48000 € /$ Angestellter/Jahr

25 Personen

0.03 Personen $/ \mathrm{m}^{2}$ Spiegelfläche

$200000 € / \mathrm{Jahr}$

$3 \%$ der Investitionskosten

$0.7 \%$ des EPC

25 Jahre

$8 \%$

$35 \%$ der Investitionskosten

$55 \mathrm{~W}_{\mathrm{el}}$

$0.074 € / \mathrm{kW}_{\text {th }}$

\subsubsection{Kosten für Sicherheitstechnik}

Sowohl bei Solar Salt als auch bei den Flüssigmetallen müssen gewisse Sicherheitsvorkehrungen berücksichtigt werden, um einen unfallfreien Betrieb zu gewährleisten. Neben der Massenstrom- und Temperaturüberwachung ist auch die Detektion der Füllstandshöhen in Behältern wichtig. Aufgrund der elektrischen Leitfähigkeit der Flüssigmetalle ergeben sich hier zusätzliche Möglichkeiten für die Messtechnik (siehe Abschnitt 3.4.6). Die nötigen Sicherheitsvorkehrungen für den Betrieb eines Natriumkreislaufes werden in Abschnitt 3.4 .8 beschrieben. Diese beinhalten hauptsächlich konstruktive Maßnahmen, die bei der Konstruktion des Kreislaufes mitberücksichtigt werden müssen und nicht zwangsläufig zu höheren Kosten führen. Daher werden in dieser Arbeit die Kosten für die Sicherheitstechnik nicht gesondert aufgeschlüsselt, sondern sind Teil der Receiverkosten (Equipment). 



\section{Vergleichsstudie Mehrturmsystem (5x140 MW)}

In diesem Kapitel werden die Ergebnisse des Systemvergleichs von Salzreceivern mit Flüssigmetallreceivern als Mehrturmkonfiguration mit $5 \times 140 \mathrm{MW}_{\text {th }}$ vorgestellt. Mehrturmsysteme weisen im Vergleich zu Einturmsystemen folgende charakteristische Merkmale auf: Es sind höhere optische Wirkungsgrade des Heliostatenfeldes erzielbar (Abstand vom Turm zur ersten Heliostatenreihe kann geringer gewählt werden, da kein Platz für Speicher und Kraftwerksblock vorgesehen werden muss). Zudem reduziert sich bei Mehrturmsystemen die atmosphärische Abschwächung (Extinktion), da die Heliostatenfelder insgesamt kleiner sind. Im Betrieb können einzelne Felder/Türme für Wartungsarbeiten abgeschaltet werden, ohne dass das gesamte Kraftwerk still steht. Somit ergibt sich ein höherer Nutzungsgrad. Im Gegenzug fallen bei Mehrturmsystemen zusätzliche Kosten der Leitungen von jedem Turm zum zentralen Kraftwerksblock an. Die übergeordneten Annahmen sowie die detaillierten Konzeptbeschreibungen sind in Kapitel 6 beschrieben bzw. im Anhang G auf Seite 191 dokumentiert.

\subsection{Ergebnisse der Komponentenauslegung}

\subsubsection{Heliostatenfeldauslegung}

Bei der Auslegung der Heliostatenfelder für Salzsysteme wurde zunächst eine Auslegung und Optimierung mit HFLCAL ohne Zielpunktverteilung durchgeführt. Anschließend wurde eine Parameterstudie mit verschiedenen Zielpunkten erstellt und mit SPRAY berechnet. Für den 21. März ergeben sich die Wirkungsgrade nach Tabelle 7.1. Für die Jahresertragsrechnung wurden für alle Zeitpunkte die Wirkungsgrade ohne Zielpunktvorgabe entsprechend Abbildung 6.5 reduziert. 


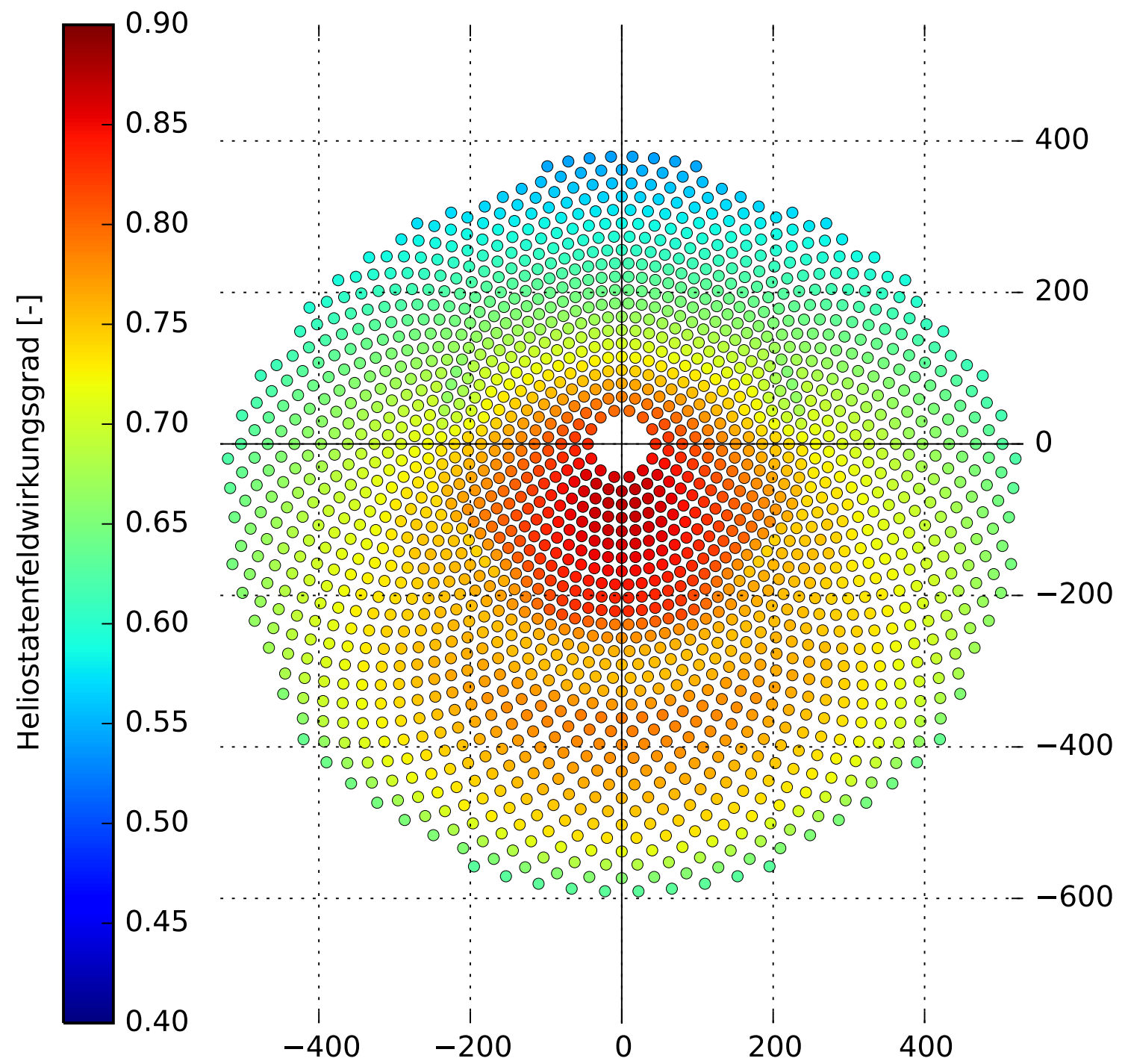

Abbildung 7.1.: Heliostatenfeld für Na-Receiver $\left(A_{\text {rec }}=274 \mathrm{~m}^{2}\right)$ mit optischen Wirkungsgraden am 21. März um $12 \mathrm{~h}: \dot{Q}_{\text {th }}=140 \mathrm{MW}_{\mathrm{th}}, 1771$ Heliostate.

Tabelle 7.1.: Optische Wirkungsgrade des Heliostatenfeldes (Referenz-Salzsystem mit $140 \mathrm{MW}_{\mathrm{th}}$-Receiver), berechnet mit SPRAY

\begin{tabular}{ccccc}
\hline Zeit & $\begin{array}{c}\text { Azimut } \\
\text { in }\left[^{\circ}\right]\end{array}$ & $\begin{array}{c}\text { Elevation } \\
\text { in }\left[^{\circ}\right.\end{array}$ & $\begin{array}{c}\eta_{\text {field }}(\text { mit SPRAY) } \\
\text { ohne Zielpunktverteilung }\end{array}$ & $\begin{array}{c}\eta_{\text {field }} \text { (mit SPRAY) } \\
\text { mit Zielpunktverteilung }\end{array}$ \\
\hline $6 \mathrm{~h} 24$ & 87.15 & 5.28 & 0.2515 & 0.2475 \\
$6 \mathrm{~h} 30$ & 86.43 & 6.60 & 0.2937 & 0.2896 \\
$7 \mathrm{~h} 00$ & 82.76 & 13.17 & 0.4537 & 0.4460 \\
$8 \mathrm{~h} 00$ & 74.70 & 26.12 & 0.6106 & 0.6007 \\
9h00 & 64.64 & 38.51 & 0.6634 & 0.6517 \\
12h00 & 0 & 61.71 & 0.7169 & 0.7026 \\
\hline
\end{tabular}




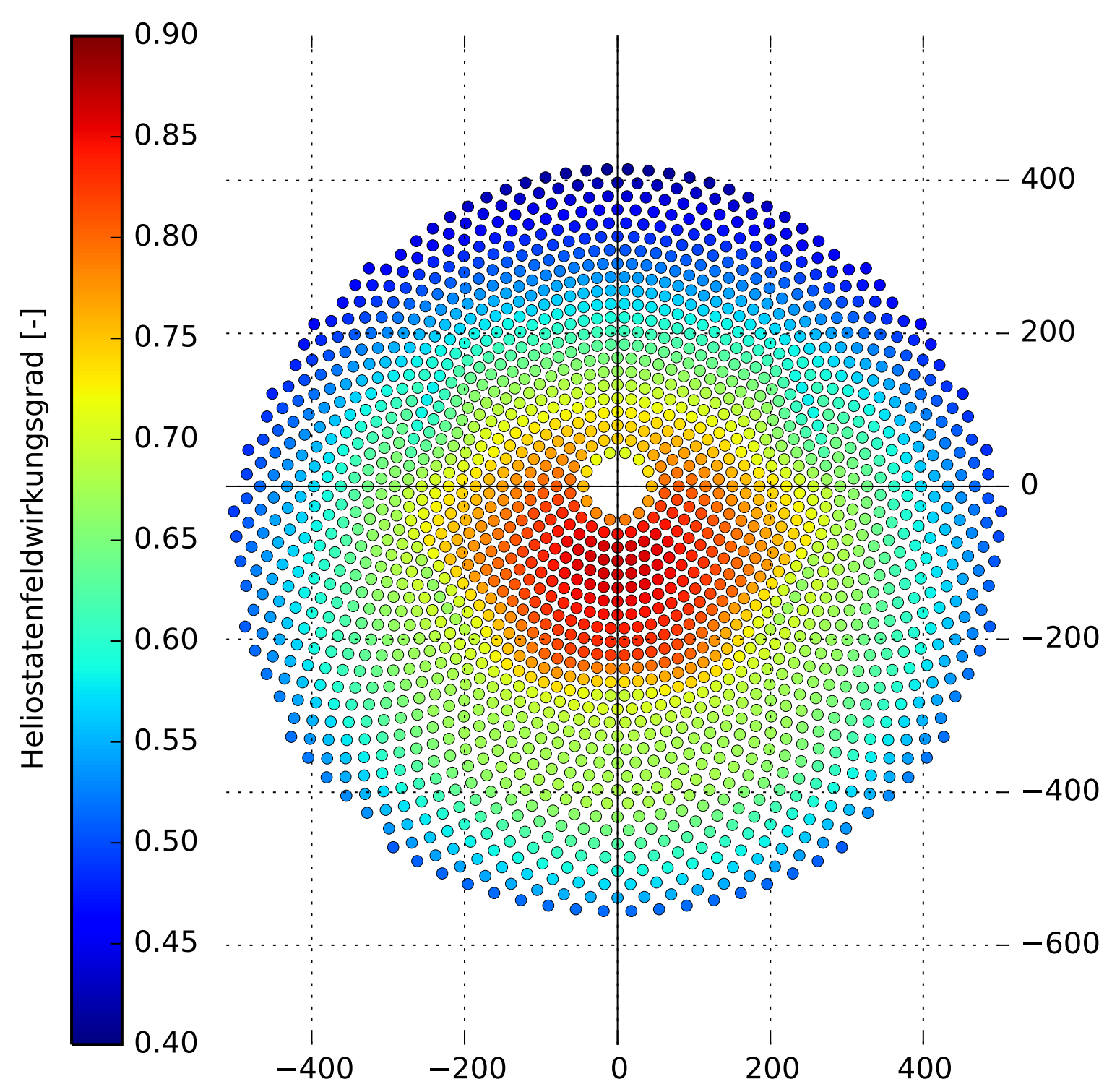

Abbildung 7.2.: Heliostatenfeld für Na-Receiver $\left(A_{\text {rec }}=113 \mathrm{~m}^{2}\right)$ mit optischen Wirkungsgraden am 21. März um $12 \mathrm{~h}: \dot{Q}_{\mathrm{th}}=140 \mathrm{MW}_{\mathrm{th}}, 1924$ Heliostate.

Bei der Auslegung von Flüssigmetallreceivern wurde komplett auf eine Zielpunktverteilung verzichtet. Wie in Abschnitt 3.4 .2 beschrieben, muss dabei stets eine Receivergrößenstudie durchgeführt werden, um das ideale Verhältnis zwischen Heliostatenfeld- und Receiverkosten zu erhalten. Da das Heliostatenfeld stets in Zusammenhang mit dem Receiver betrachtet werden muss, wurde für jede Receivergröße eine neue Heliostatenfeldauslegung durchgeführt. Abbildung 7.1 und Abbildung 7.2 zeigen die mit HFLCAL optimierten Heliostatenfelder für zwei unterschiedlich große Natriumreceiver gleicher Leistung.

Sowohl die Anzahl und Anordnung der Heliostate als auch die optischen Wirkungsgrade unterscheiden sich erheblich. Bei kleinen Receivern (Abbildung 7.2) 


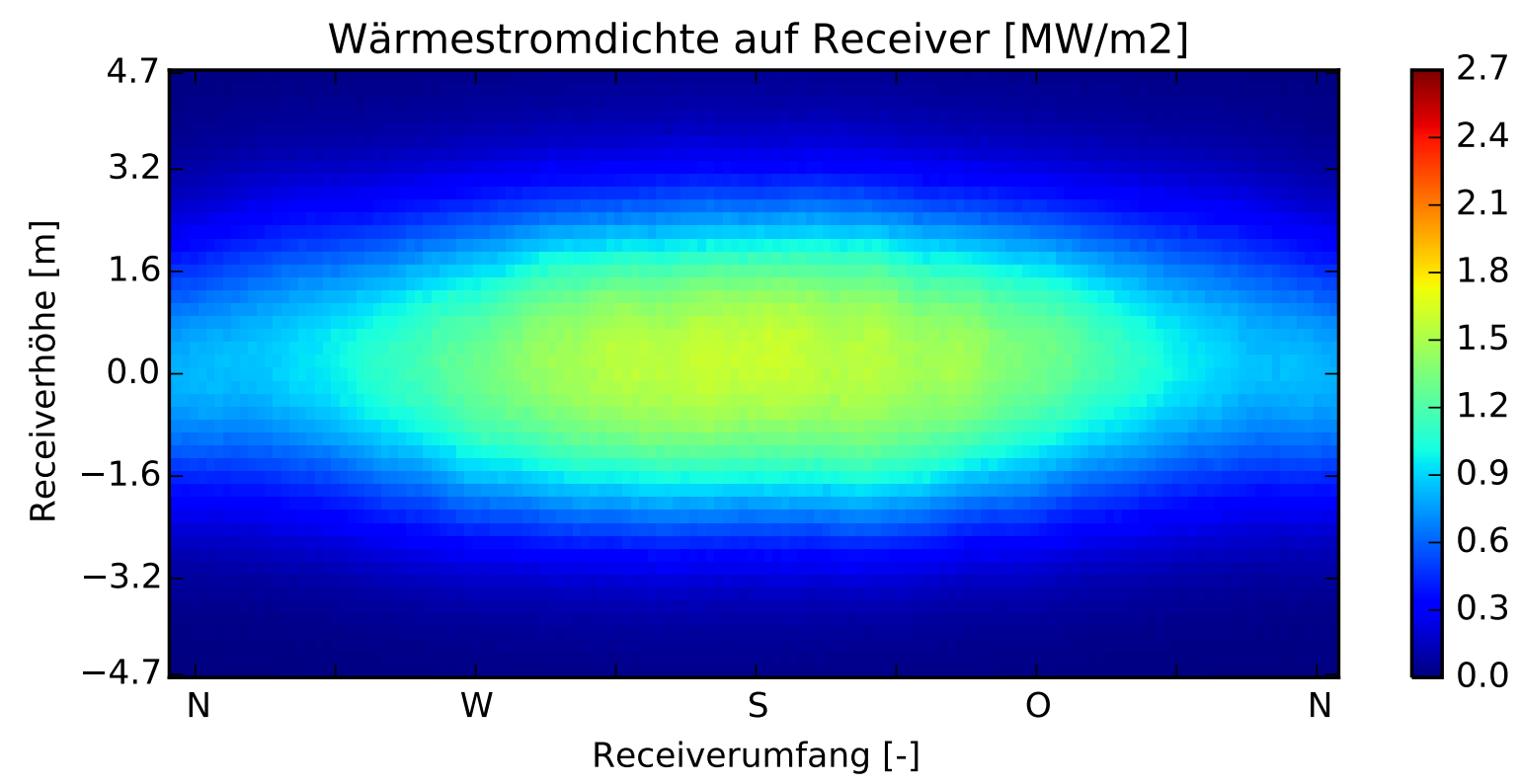

Abbildung 7.3.: Wärmestromdichteverteilung auf Na-Receiver $\left(A_{\text {rec }}=274 \mathrm{~m}^{2}\right)$ ohne Zielpunktverteilung in $\mathrm{MW} / \mathrm{m}^{2}$ am 21 . März um $12 \mathrm{~h}: \dot{Q}_{\mathrm{th}}=$ $140 \mathrm{MW}_{\text {th }}, \dot{q}_{\text {max }} \approx 1.6 \mathrm{MW} / \mathrm{m}^{2}$.

entsteht deutlich mehr Randstrahlung. Vor allem bei weit entfernten Heliostaten nimmt der Wirkungsgrad stark ab, da ihr Bild durch die Strahlaufweitung mit zunehmender Entfernung vom Receiver größer wird. Der Optimierungsalgorithmus in HFLCAL erzeugt daher ein dichter gestelltes und im Verhältnis zu Abbildung 7.1 kreisrunderes Heliostatenfeld. Trotz dieser Optimierung ergeben sich bei kleineren Receivern stets geringere Feldwirkungsgrade infolge der erhöhten Randstrahlung und der zunehmenden Verschattung und Blockierung der Heliostate.

Tabelle 7.2 zeigt die sich ergebenden Wirkungsgrade für beide Receivergrößen. Die Frage, welche Receivergröße optimal ist, hängt von weiteren Faktoren ab und wird in den nächsten Abschnitten weiter behandelt.

Abbildung 7.3 und 7.4 zeigen jeweils die sich durch das Heliostatenfeld ergebende Wärmestromdichteverteilung auf der Receiveroberfläche (abgewickelte Zylinderfläche) ohne Zielpunktverteilung für zwei verschiedene Receivergrößen gleicher Leistung. Beim größeren Receiver (Abbildung 7.3) ensteht eine maximale Wärmestromdichte von $\dot{q}_{\max } \approx 1.6 \mathrm{MW} / \mathrm{m}^{2}$. Da sich diese maximale Wärmestromdichte lediglich auf die Receivermitte konzentriert, besteht die Möglichkeit einer Zielpunktverteilung, ohne die Randstrahlung signifikant zu erhöhen (Siehe Auslegung von Salzreceivern mit $\dot{q}_{\max } \approx 1.0 \mathrm{MW} / \mathrm{m}^{2}$ ). 


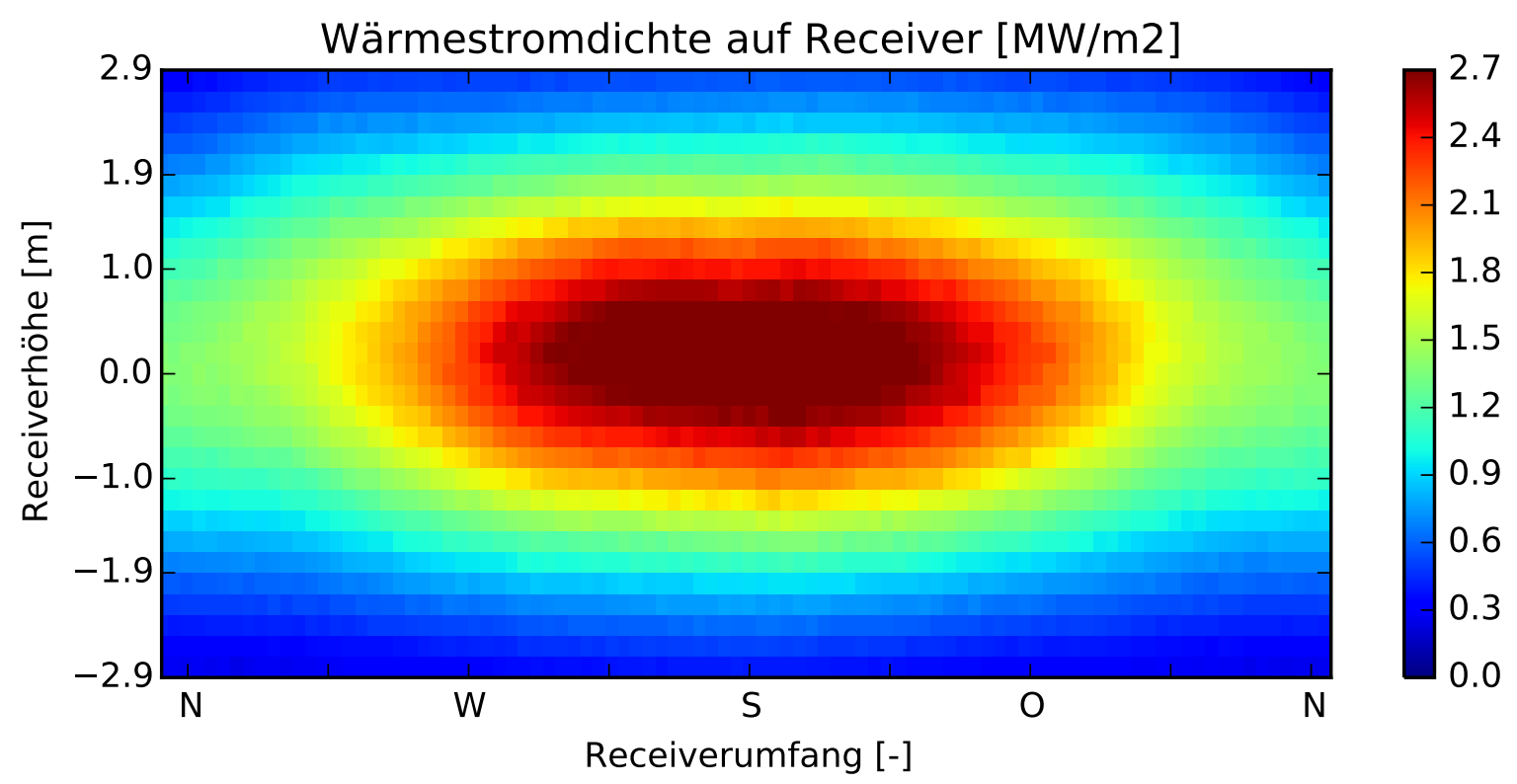

Abbildung 7.4.: Wärmestromdichteverteilung auf Na-Receiver $\left(A_{\text {rec }}=113 \mathrm{~m}^{2}\right)$ ohne Zielpunktverteilung in $\mathrm{MW} / \mathrm{m}^{2}$ am 21 . März um $12 \mathrm{~h}: \dot{Q}_{\mathrm{th}}=$ $140 \mathrm{MW}_{\text {th }}, \dot{q}_{\text {max }} \approx 2.9 \mathrm{MW} / \mathrm{m}^{2}$.

Wird die Absorberfläche reduziert, so erhöht sich bei gleicher Leistung auch die Wärmestromdichte. Beim kleinen Receiver resultiert hier eine maximale Wärmestromdichte von $\dot{q}_{\max } \approx 2.9 \mathrm{MW} / \mathrm{m}^{2}$ (siehe Abbildung 7.4). Selbst ohne Zielpunktverteilung ergibt sich im Verhältnis zum großen Receiver (Abbildung 7.3 ) eine Reduktion des Intercept-Wirkungsgrades um $\approx 6 \%$-Punkte (im

Tabelle 7.2.: Ergebnisse aus der Feldauslegung: Vergleich der Heliostatenfelder für verschieden große Receiver gleicher Leistung. Berechnung am 21.3./12 h mit SPRAY, Jahresmittelwert mit HFLCAL

\begin{tabular}{|c|c|c|c|c|}
\hline Receivergröße & \multicolumn{2}{|c|}{$274 \mathrm{~m}^{2}$} & \multicolumn{2}{|c|}{$113 \mathrm{~m}^{2}$} \\
\hline Leistung am 21.3. um $12 \mathrm{~h}$ & \multicolumn{2}{|c|}{$140 \mathrm{MW}_{\text {th }}$} & \multicolumn{2}{|c|}{$140 \mathrm{MW}_{\mathrm{th}}$} \\
\hline Anzahl Heliostate & \multicolumn{2}{|c|}{1771} & \multicolumn{2}{|c|}{1924} \\
\hline Reflektivität der Spiegel & \multicolumn{2}{|c|}{$89.3 \%$} & \multicolumn{2}{|c|}{$89.3 \%$} \\
\hline Feldwirkungsgrade & $21.3 . / 12 \mathrm{~h}$ & Jahresmittel $^{a}$ & $21.3 . / 12 \mathrm{~h}$ & Jahresmittel $^{a}$ \\
\hline Kosinuswirkungsgrad & $87.1 \%$ & $79.3 \%$ & $86.3 \%$ & $78.9 \%$ \\
\hline Blockierung \& Verschattung & $97.0 \%$ & $92.9 \%$ & $96.4 \%$ & $91.8 \%$ \\
\hline Extinktion & $95.8 \%$ & $95.6 \%$ & $93.3 \%$ & $95.6 \%$ \\
\hline Intercept-Wirkungsgrad & $99.6 \%$ & $98.4 \%$ & $94.0 \%$ & $88.0 \%$ \\
\hline Gesamtwirkungsgrad & $72.0 \%$ & $61.9 \%$ & $65.2 \%$ & $54.3 \%$ \\
\hline
\end{tabular}

${ }^{a}$ Werte aus HFLCAL ohne aiming 
Jahresmittel sogar über $8 \%$-Punkte). Der gesamte Feldwirkungsgrad, bestehend aus Kosinusverlusten, Blockierung und Verschattung, Absorption der Spiegel, Atmosphärische Abschwächung (Extinktion) und Randstrahlung (InterceptWirkungsgrad) reduziert sich um $\approx 7 \%$-Punkte im Vergleich zum großen Receiver (Sowohl Auslegungszeitpunkt als auch Jahresmittel).

Je kleiner der Receiver, desto geringer der Wirkungsgrad des Heliostatenfeldes. Gleichzeitig nimmt der Wirkungsgrad des Receivers zu, da die Verluste durch die geringere Absorberfläche abnehmen.

\subsubsection{Receivermodellierung}

Wie bereits in Abschnitt 3.4 .2 beschrieben, muss für die Flüssigmetallreceiver stets eine Receivergrößenstudie durchgeführt werden. Abbildung 7.5 zeigt die Ergebnisse der Receivergrößenstudie der Natriumreceiver graphisch. Auf der Abszisse ist die Absorberfläche dargestellt, über die die mittlere Wärmestromdichte direkt mit der Beziehung $\overline{\dot{q}}=\dot{Q}_{\text {th }} / A_{\text {rec }}$ abgeleitet werden kann. Zusätzlich ist noch die maximale solare Wärmestromdichte (berechnet mit SPRAY) für die jeweilige Auslegung aufgetragen. Für den Receiver gilt: Je kleiner der Receiver, desto höher der Receiverwirkungsgrad. Außerdem nimmt der Windeinfluß auf den Receiverwirkungsgrad zu, je größer die Absorberfläche ist $\left(\Delta \eta_{\mathrm{R}}=2.27 \%\right.$ Punkte bei $A_{\text {rec }}=274 \mathrm{~m}^{2}$ zwischen Windstille und einer Windgeschwindigkeit von $20 \mathrm{~m} / \mathrm{s}$ auf Receiverhöhe).

Der Gesamtwirkungsgrad $\left(\eta_{\text {field }} \cdot \eta_{\text {rec }}\right)$ zeigt, dass eine Reduktion der Absorberfläche aus thermodynamischer Sicht keinen signifikanten Vorteil bringt. Bis zu einer mittleren Wärmestromdichte von ca. $0.7 \mathrm{MW} / \mathrm{m}^{2}$ (Reduktion der Absorberfläche auf ca. $78 \%$ des Referenz-Salzsystems) steigt der Gesamtwirkungsgrad leicht an. Der Wirkungsgradvorteil bei weiterer Reduktion der Absorberfläche wird durch den geringeren Feldwirkungsgrad wieder aufgehoben.

Tabelle G.1 auf Seite 214 zeigt alle Parameter und Ergebnisse der Receivergrößenstudie. Durch die Anwendung einer Zielpunktstrategie im Falle des Referenzsystems mit Solar Salt kann die maximale Wärmestromdichte von $2.5 \mathrm{MW} / \mathrm{m}^{2}$ auf $1.2 \mathrm{MW} / \mathrm{m}^{2}$ reduziert werden. Gleichzeitig reduziert sich der Feldwirkungsgrad um $1.4 \%$ durch höhere Randstrahlung. Des Weiteren ergibt sich ein um 


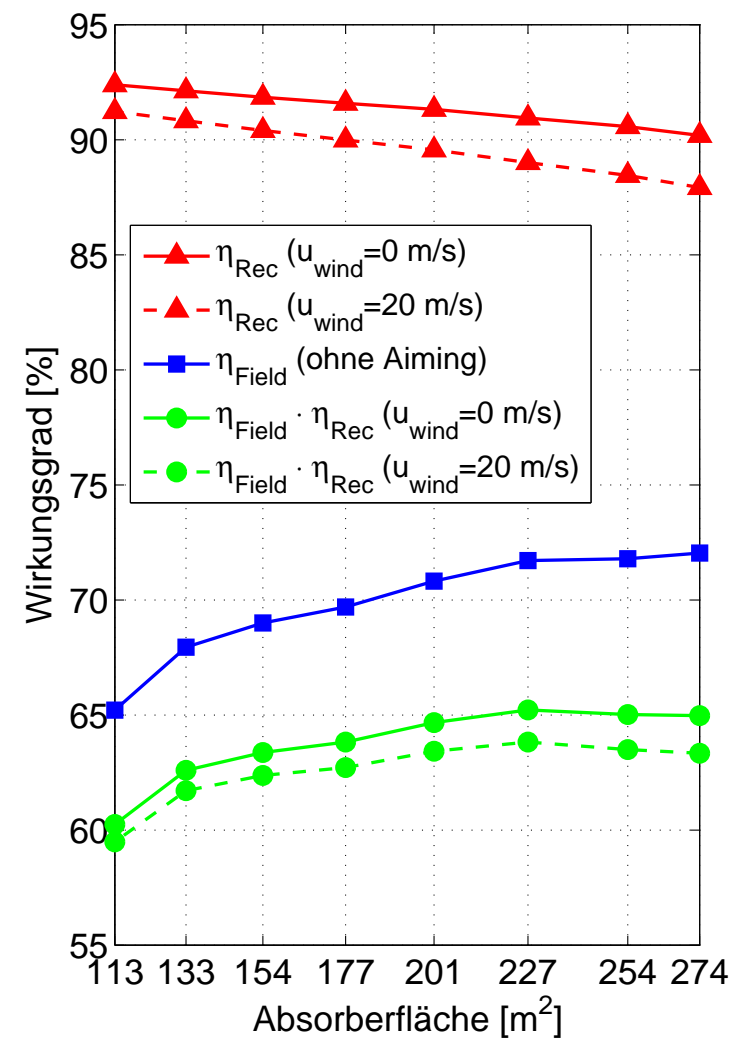

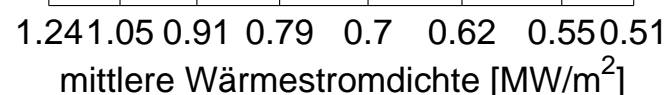

$2.992 .712 .492 .3 \quad 2.21 \quad 2.08 \quad 1.981 .66$ maximale Wärmestromdichte $\left[\mathrm{MW} / \mathrm{m}^{2}\right]$

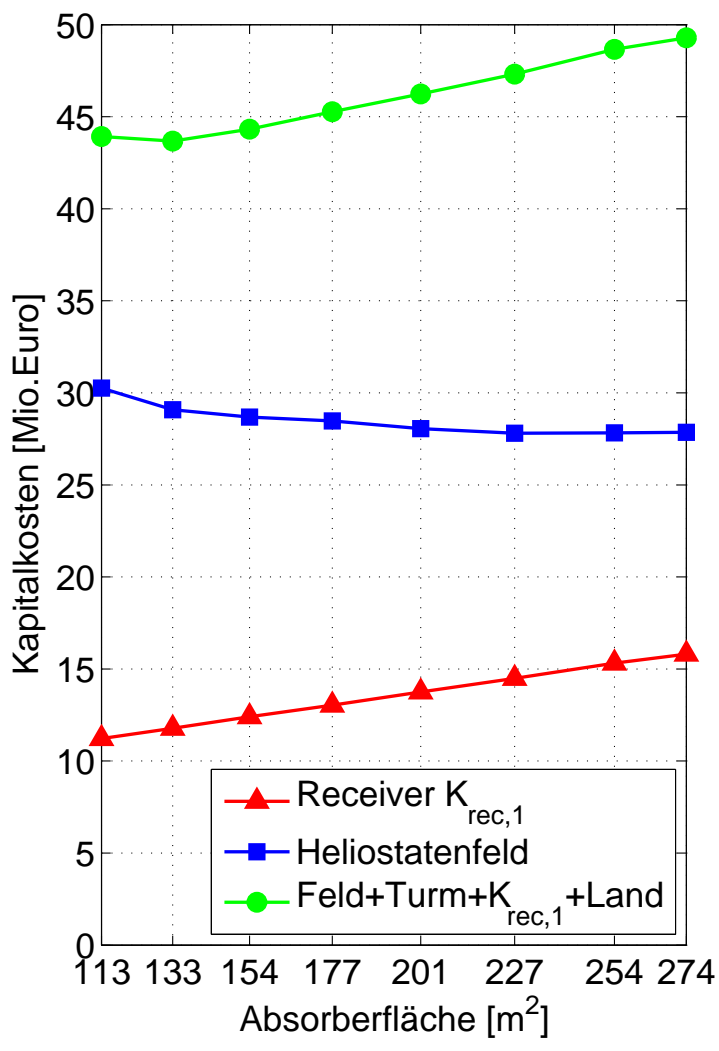

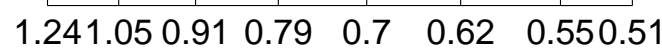
mittlere Wärmestromdichte $\left[\mathrm{MW} / \mathrm{m}^{2}\right]$

$2.992 .712 .492 .3 \quad 2.21 \quad 2.08 \quad 1.981 .66$ maximale Wärmestromdichte $\left[\mathrm{MW} / \mathrm{m}^{2}\right]$

Abbildung 7.5.: Links: Receiver- und Feldwirkungsgrade von Natriumreceivern am $21.3 \mathrm{um} 12 \mathrm{~h}$ in Abhängigkeit der Absorberfläche sowie der Windgeschwindigkeit bei einer Leistung von $140 \mathrm{MW}_{\text {th }}$. Rechts: Kapitalkosten von Receiver, Heliostatenfeld und Gesamtkapitalkosten (Feld+Turm+Receiver).

den Faktor 15 höherer Druckverlust beim Referenzkonzept. Im Teillastbetrieb (um 6 h30) sinkt die Reynolds-Zahl beim Salzreceiver auf 10000 ab, während die Natriumreceiver noch hohe Reynolds-Zahlen (Faktor 5-7 höher) aufweisen und durch die damit verbundenen höheren Wärmeübergangskoeffizienten auch deutlich höhere Wirkungsgrade (1-12\%-Punkte) erreichen.

Eine ökonomische Betrachtung der Receivergrößenstudie ist auf der rechten Seite in Abbildung 7.5 dargestellt. Die Kosten für das Heliostatenfeld sind linear abhängig von der Anzahl an Heliostaten bzw. vom Gesamtwirkungsgrad $\left(\eta_{\text {field }} \cdot \eta_{\text {rec }}\right)$. Je geringer der Gesamtwirkungsgrad, desto mehr Heliostate werden 
für die gleiche Leistung benötigt. Im vorliegenden Fall werden beim höchsten Gesamtwirkungsgrad 1768 Heliostate und beim niedrigsten Gesamtwirkungsgrad 1924 Heliostate verwendet. Das entspricht einem Zuwachs um $8.8 \%$ und damit einer Steigerung der Investitionskosten um ebenfalls $8.8 \%$ (

Die Receiverkosten hängen linear von der Absorberfläche ab (siehe Abschnitt 6.2.2.3). Selbst bei der konservativen Abschätzung der Receiverkosten $\left(K_{\text {rec }, 1}\right)$ reduzieren sich die Kosten um $29 \%$ ( $=3.2$ Mio.€) bei einem Absorberflächenverhältnis von 227/113 $=2.0$. Bei einer Verringerung der Absorberfläche sinken die Receiverkosten folglich mehr als die dadurch entstehende Kostensteigerung des Heliostatenfeldes. Die Summe der Kosten aus Heliostatenfeld, Turm, Receiver und Land zeigt, dass die niedrigsten Anlagenkosten trotz höherer Feldkosten bei sehr kleinen Receivern liegen.

Die Stromgestehungskosten ergeben sich aus den jährlichen Anlagenkosten (Investition und Betrieb) und der Netto-Jahresenergie. Abbildung 7.5 zeigt lediglich die Wirkungsgrade zu einem spezifischen Zeitpunkt (21.3 um 12 h). Die Berechnung der Jahresenergie erfordert aber die Kenntnis des Teillastverhaltens. Abbildung 7.6 zeigt die Wirkungsgrade zweier unterschiedlich großer Natriumreceiver in Abhängigkeit vom Lastfall. Es fällt auf, dass bei Teillast der Wind einen sehr viel größeren Einfluss auf den Receiverwirkungsgrad hat. Die grün hinterlegten Balken stellen die jährliche Betriebszeit des Receivers für den Standort Postmasburg in Südafrika dar (aus DNI-Messdaten berechnet). Ein Großteil befindet sich im Lastfallbereich zwischen [0.7 - 1], jedoch wird der Receiver auch häufig bei $P_{\text {inc }} / P_{\text {inc,DP }}=0.2$ betrieben.

Damit in der Jahresertragsrechnung für jeden beliebigen Zeitpunkt der Receiverwirkungsgrad in Abhängigkeit vom Lastfall und der Windgeschwindigkeit berechnet werden kann, wird die mit FEM berechnete Receiverwirkungsgradkennlinie für jeden Windfall mit folgender Gleichung gefittet.

$$
\eta_{\mathrm{rec}}=\eta_{0}-\dot{q}_{\mathrm{loss}} \cdot \frac{A_{\mathrm{rec}}}{P_{\mathrm{inc}, \mathrm{DP}}} \cdot\left(\frac{P_{\mathrm{inc}, \mathrm{DP}}}{P_{\mathrm{inc}}}\right)^{\gamma}
$$

Die vier Parameter $\eta_{0}, \dot{q}_{\text {loss }}, P_{\text {inc,DP }}$ und $\gamma$ sind für jede Variante in der jeweiligen Spezifikation im Anhang G auf Seite 191ff dokumentiert. 


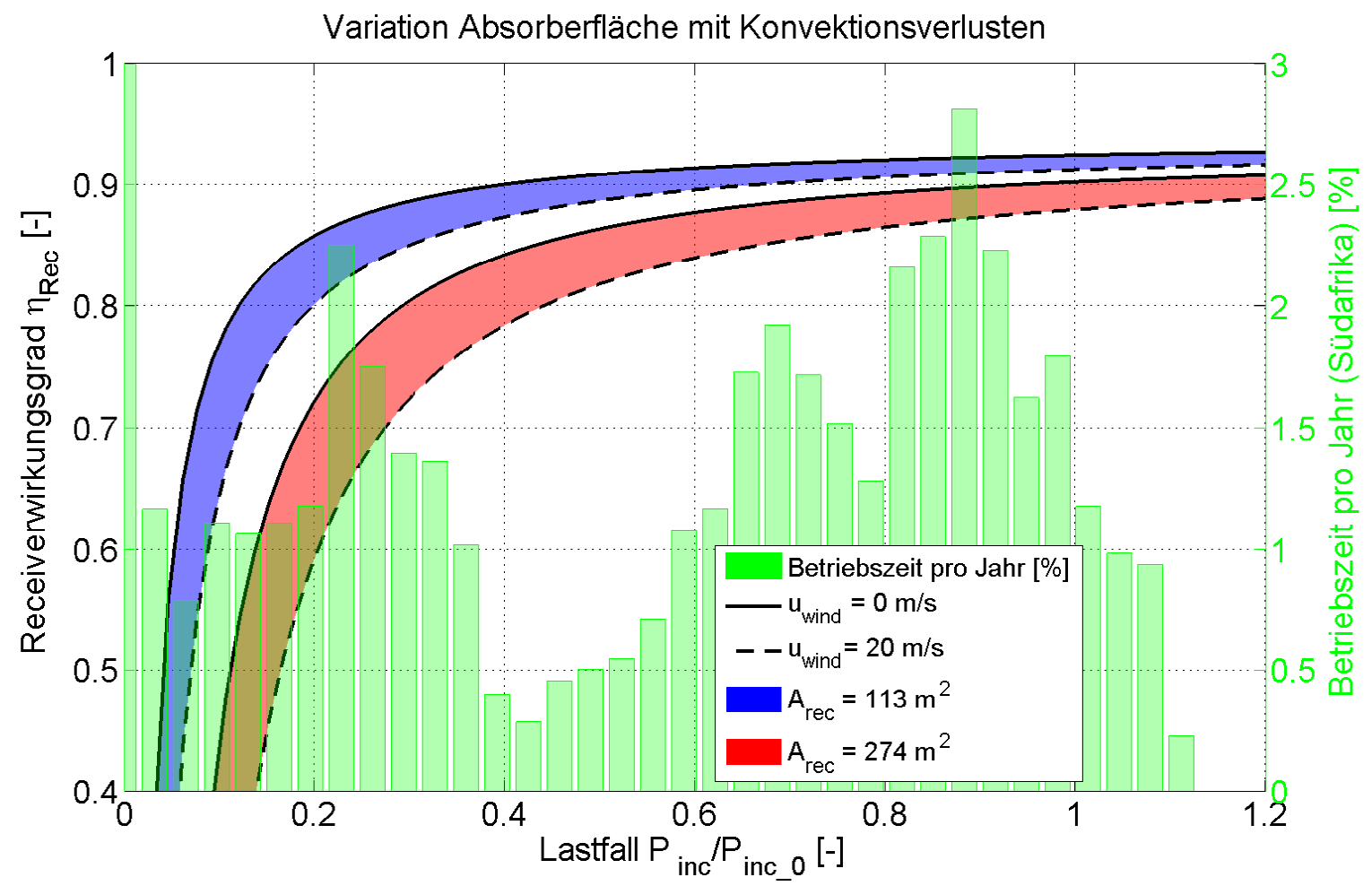

Abbildung 7.6.: Receiverwirkungsgrade von Natriumreceivern in Abhängigkeit der Last sowie der Windgeschwindigkeit für zwei verschiedene Receivergrößen bei einer Leistung von $P_{\text {inc,DP }}=140 \mathrm{MW}_{\text {th }}$. In Grün: Betriebszeit pro Jahr für den Standort Postmasburg, Südafrika

\subsection{Ergebnisse der LCOE-Berechnung}

\subsubsection{Solar Salt im Vergleich zu Natrium}

Abbildung 7.7 zeigt die Änderung der LCOE der Natriumsysteme (in Receiveronly und Tower-loop-Variante) relativ zum Referenzsystem mit Solar Salt mit den in Kapitel 6 definierten Randbedingungen. Neben den beiden untersuchten Receiverkostenfunktionen sind auch die Grenzen der Wärmeübertragerkosten dargestellt. Selbst mit der konservativen Receiverkostenfunktion $K_{\text {rec, } 1}$ beträgt die Reduktion der LCOE bereits bis zu 8\%, bezogen auf das Referenzsystem mit Solar Salt. Mit der Receiverkostenfunktion $K_{\text {rec,2 }}$ ergeben sich sogar bis zu $11 \%$. Der Unterschied zwischen den Varianten Receiver-only und Tower-loop besteht lediglich im zusätzlichen Druckverlust in der Steigleitung und der damit verbundenen höheren Pumpenergie für den Salzkreislauf. Allein dieser Effekt ändert die LCOE um 2-2.5\%. 


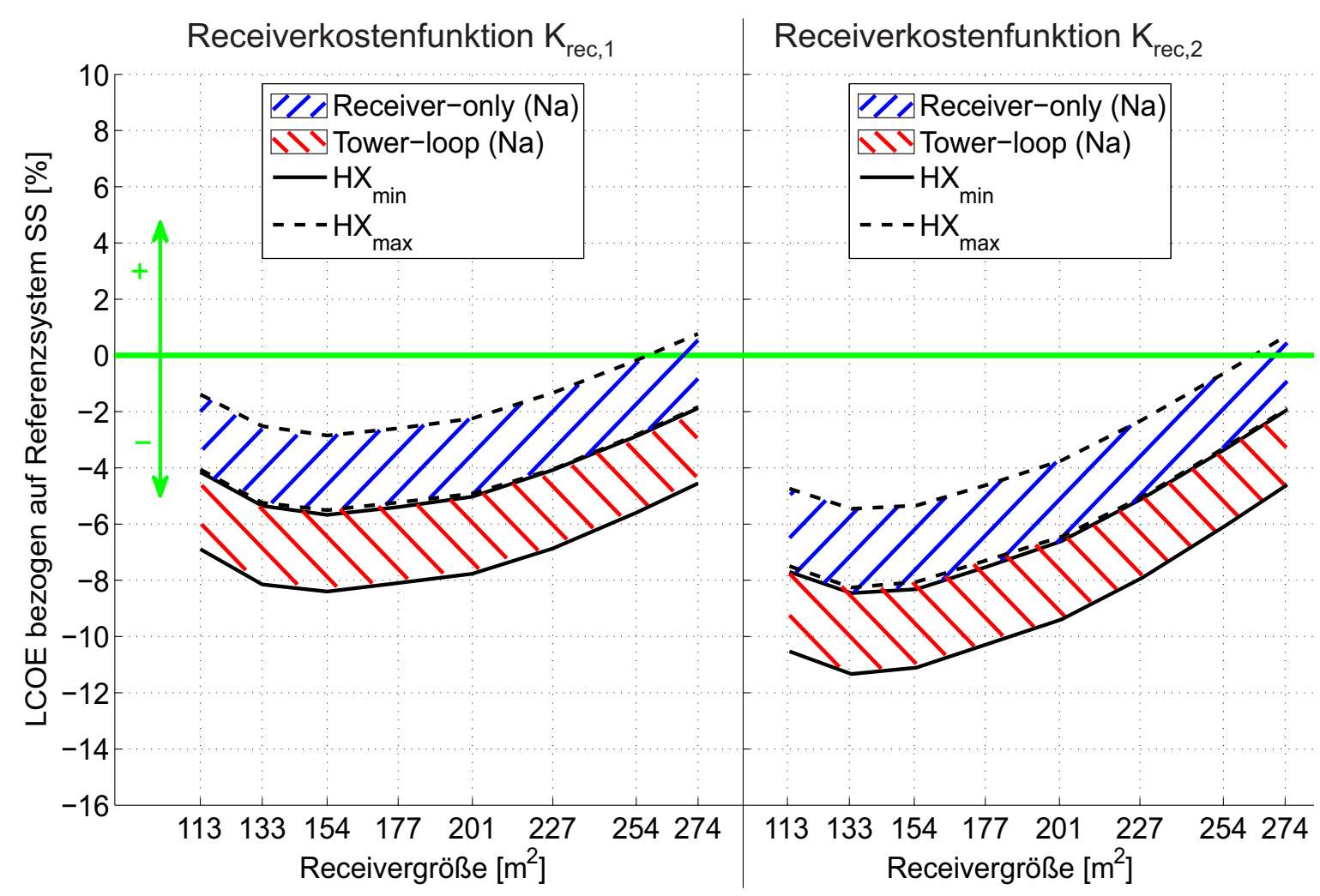

Abbildung 7.7.: Relative Änderung der LCOE von Natriumsystemen bezogen auf das Referenz-Salzsystem jeweils als Mehrturmkonfiguration $\left(5 \times 140 \mathrm{MW}_{\mathrm{th}}\right)$. Links: Receiverkostenfunktion $K_{\text {rec, } 1}$, Rechts: Receiverkostenfunktion $K_{\mathrm{rec}, 2}$. Jeweils als Receiver-only und Tower-loopVarianten mit Variation der Wärmeübertragerkosten $K_{\mathrm{HX} \text {,min }}$ und $K_{\mathrm{HX} \text { max }}$ bei jeweils kostenoptimaler Speichergröße.

Abbildung 7.8 zeigt die Ergebnisse des Referenzsystems und des Natriumsystems mit den geringsten LCOE (Receiverkostenfunktion $K_{\mathrm{rec}, 2}, A_{\mathrm{rec}}=133 \mathrm{~m}^{2}$ in Tower-loop-Variante mit $\left.K_{\mathrm{HX} \text {,min }}\right)$ im Vergleich. Das beim Referenzsystem leicht größere Heliostatenfeld kann über das Jahr mehr thermische Energie auf den Receiver konzentrieren. Der höhere Wirkungsgrad des Natriumreceivers führt zwar relativ zu einem höheren Wärmeertrag. Im absoluten Vergleich ist jedoch die vom Referenzsystem umgewandelte, elektrische Brutto-Energie immer noch höher als die entsprechende Brutto-Energie des Natriumsystems. Erst die höhere Betriebsenergie (durch Pumpen und Begleitheizung) des Salzsystems führt dazu, dass beide Systeme in Summe auf nahezu den selben Netto-Energieertrag kommen.

Im rechten Diagrammteil sind die Investitionskosten der einzelnen Komponenten beider Konfigurationen sowie deren relativer Beitrag zu den Gesamtinvestitions- 

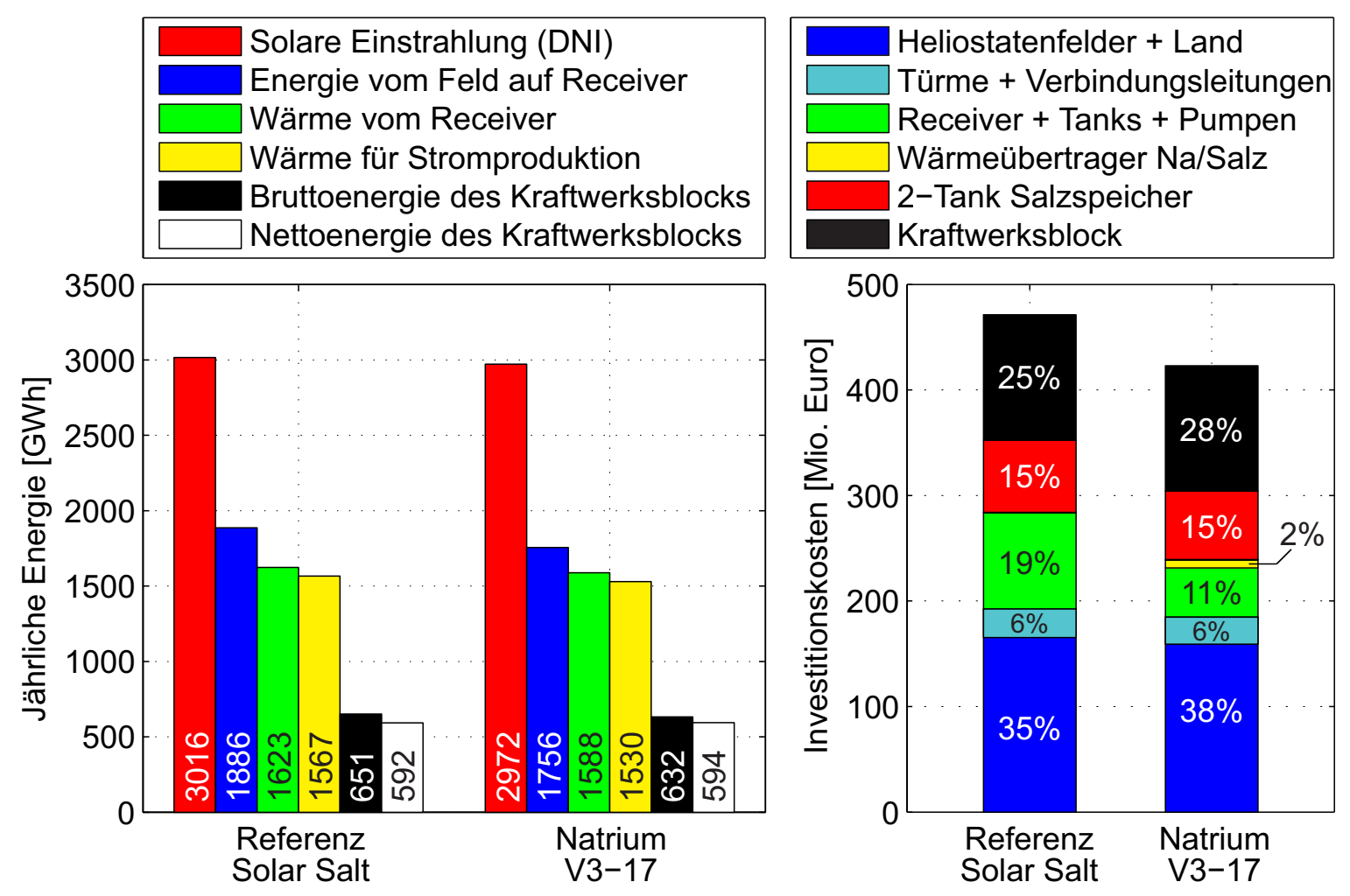

Abbildung 7.8.: Vergleich zwischen Referenzsystem Solar Salt und der Natriumvariante mit den geringsten $\operatorname{LCOE}\left(A_{\mathrm{rec}}=133 \mathrm{~m}^{2}\right.$ in Tower-loopKonfiguration mit Receiverkostenfunktion $\left.K_{\text {rec, } 2}\right)$. Links: Jährliche Energieumsätze in einzelnen Komponenten, Rechts: Investitionskosten der Einzelkomponenten.

kosten dargestellt.

Die wichtigsten Daten beider Systeme sind in Tabelle 7.3 zusammengefasst. Beide Systeme resultieren in ähnlichen Jahreswirkungsgraden von $20 \%$. Der niedrigere optische Wirkungsgrad beim Natriumsystem wird durch den höheren Receiverwirkungsgrad und die geringeren parasitären Verluste kompensiert. Den größten Kostenanteil nehmen jeweils die Heliostatenfelder ein. Der deutlich kostengünstigere Natriumreceiver (Absorberfläche $54 \%$ geringer) ist der ausschlaggebende Grund für die Reduktion der LCOE.

Die Kosten des Wärmeträgermediums im Receiverkreislauf liegen im Verhältnis zu den Anlagenkosten im \%o-Bereich und sind daher in Abbildung 7.8 und Tabelle 7.3 nicht einzeln aufgeführt, sondern in den Receiverkosten enthalten. 
Tabelle 7.3.: Vergleich zwischen Referenzsystems Solar Salt und dem Natriumsystems mit den geringsten LCOE $\left(A_{\text {rec }}=133 \mathrm{~m}^{2}\right.$ in Tower-loop-Variante mit Receiverkostenfunktion $\left.K_{\text {rec }, 2}\right)$

\begin{tabular}{lcc}
\hline Variante & Referenz & Natrium \\
& Solar Salt & V3-17 \\
\hline Absorberfläche $\left[\mathrm{m}^{2}\right.$ ] & 291 & 133 \\
Druckverlust $(21.3 . / 12 \mathrm{~h}$ ) [bar] & 14.82 & 0.81 \\
Gesamtmasse Absorberrohre leer [t] & 19.3 & 10.0 \\
Gesamtmasse des WTMs im Receiver [t] & 24.1 & 9.4 \\
\hline \hline Heliostatenfeldwirkungsgrad [\%] & 62.5 & 59.1 \\
Receiverwirkungsgrad [\%] & 86.1 & 90.4 \\
Energieverluste (Speicher, Dumping, ... ) [\%] & 96.5 & 96.3 \\
Turbinenwirkungsgrad [\%] & 41.5 & 41.3 \\
Parasitäre Verluste (Pumpen, Begleitheizung) [\%] & 90.9 & 94.0 \\
Gesamtwirkungsgrad [\%] & 19.6 & 20.0 \\
\hline \hline Kosten Heliostatenfeld [Mio.€] & 165 & 159 \\
Kosten Land [Mio.€] & 27 & 26 \\
Kosten Receiversystem [Mio.€] & 92 & 46 \\
Kosten Wärmeübertrager [Mio.€] & 0 & 8 \\
Kosten Thermischer Speicher [Mio.€] & 68 & 65 \\
Kosten Kraftwerksblock [Mio.€] & 119 & 119 \\
Gesamtkosten [Mio.€] & 471 & 422 \\
\hline
\end{tabular}

\subsubsection{Sensitivitätsanalyse des Feldwirkungsgrades}

Die Genauigkeit des Heliostatenfeldes ist maßgeblich für den optischen Wirkungsgrad verantwortlich. Heliostate mit einer präzisen Nachführung erzeugen weniger Randstrahlung als ungenauere Heliostate und führen daher zu einem höheren optischen Wirkungsgrad. Je geringer die Absorberfläche, desto entscheidender ist eine präzise Nachführung. Durch die geringere Absorberfläche der Natriumreceiver ergibt sich hier eine stärkere Abhängigkeit von der Genauigkeit des Heliostatenfeldes, als bei den (größeren) Salzreceivern. In diesem Abschnitt wird daher die Sensitivität der Nachführgenauigkeit auf die LCOE untersucht. Für die zuvor ausgelegten Heliostatenfelder wurden die Winkelfehler $\sigma_{\text {slope }}$ und $\sigma_{\text {tracking }}$ (in beiden Achsen) ausgehend von den Standardwerten $\left(\sigma_{\text {slope }}=1.3 \mathrm{mrad}\right.$ und $\left.\sigma_{\text {tracking }}=0.65 \mathrm{mrad}\right)$ um $25 \%$ verringert (auf $\sigma_{\text {slope }}=0.975 \mathrm{mrad}$ und $\sigma_{\text {tracking }}=0.4875 \mathrm{mrad}$ ) bzw um $50 \%$ erhöht (auf $\sigma_{\text {slope }}=1.95 \mathrm{mrad}$ und $\sigma_{\text {tracking }}=0.975 \mathrm{mrad}$ ). 


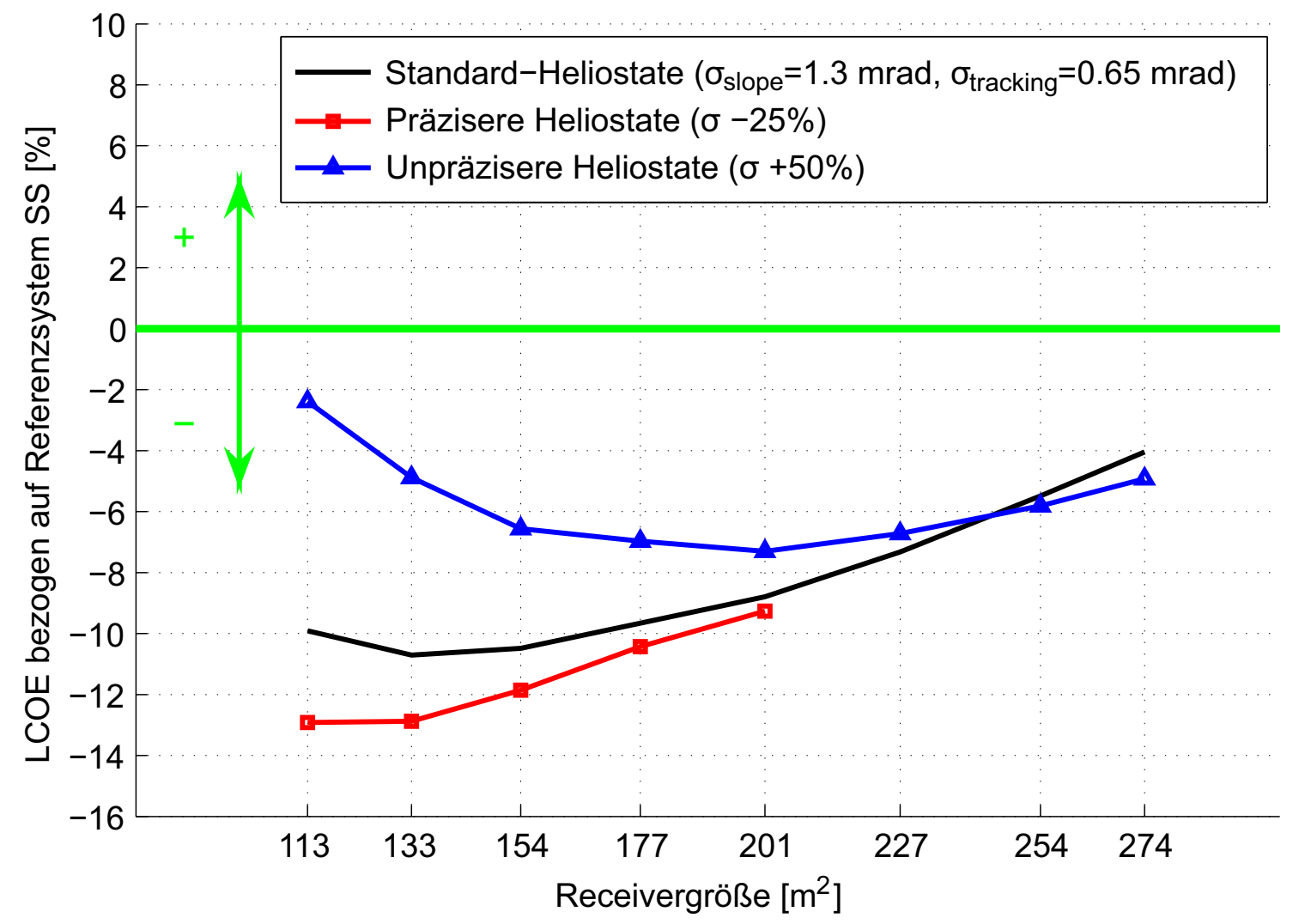

Abbildung 7.9.: Ergebnisse der Sensitivitätsanalyse über die Nachführgenauigkeit der Heliostate: Für jede Genauigkeitsklasse ist jeweils die relative Änderung der LCOE der Natriumsysteme bezogen auf die mit der selben Genauigkeitsklasse berechneten Referenz-Salzsysteme dargestellt. Alle Natriumvarianten als Tower-loop-Konfiguration mit Receiverkostenfunktion $K_{\mathrm{rec}, 2}$ und Wärmeübertragerkosten $K_{\mathrm{HX} \text {,min }}$.

Abbildung 7.9 zeigt die Ergebnisse dieser Sensitivitätsanalyse für die Towerloop-Varianten mit der Receiverkostenfunktion $K_{\text {rec,2 }}$ und den Wärmeübertragerkosten $K_{\mathrm{HX} \text {,min }}$. Die Sensitivitätsanalyse zeigt, dass sich sowohl die optimale Receivergröße als auch die relativen Werte der LCOE verändern. Je unpräziser die Heliostaten, desto größer der optimale Receiver und desto höher die LCOE. Dennoch sind bei Natriumsystemen selbst mit sehr unpräzisen Heliostaten die LCOE immernoch um ca. $7 \%$ geringer als beim Referenzsystem mit Solar Salt und derselben Heliostatengenauigkeit. 


\subsubsection{Solar Salt im Vergleich zu Blei-Bismut}

Wie auch bei Natrium ergibt sich bei der Auslegung von LBE-Receivern bei gleicher Leistung ein deutlich höherer Volumenstrom als bei Solar Salt (siehe Gleichung (3.13), Seite 39), was sich tendenziell in weniger und dafür breiteren Modulen äußert. Die hohe Dichte von LBE (siehe Stoffwerte Anhang A, Seite 177 ff) führt in Kombination mit der verhältnismäßig geringen Viskosität zwar einerseits zu sehr hohen Reynolds-Zahlen und verbessert damit den Wärmeübergang, andererseits resultiert dadurch aber auch eine hohe Gesamtmasse, sodass die Tower-loop-Konfiguration zu extrem hohen statischen Drücken ( $>300$ bar) führen würde (Receiver + Steig- und Fallleitung mit LBE gefüllt). Theoretisch können diese Anforderungen technisch erfüllt werden, jedoch erscheint aus Kostengründen die Tower-loop-Konfiguration mit LBE aufgrund der hohen Rohrwandstärken in Steig- und Fallleitung, dem für die hohe Gesamtmasse entsprechenden Turmfundament und der hohe Menge an LBE als ungünstiges Konzept. Daher wurde mit LBE lediglich die Receiver-only-Konfiguration untersucht.

Abbildung 7.10 zeigt die Änderung der LCOE aller berechneten LBE-Systeme relativ zum Referenzsystem mit Solar Salt (Spezifikation der LBE-Systeme siehe Anhang G.5). Es ergibt sich ein Potenzial von bis zu $6 \%$ geringeren LCOE bezogen auf das Referenzsystem mit Solar Salt. Wie auch bei den Natriumsystemen liegt der Grund für diese Reduktion weniger im höheren Ertrag, sondern hauptsächlich in den geringeren Receiverkosten, die durch die kleineren Absorberflächen mit höherer Wärmestromdichte zurückzuführen sind.

Tabelle 7.4 zeigt die wichtigsten technischen Daten des LBE-Receivers mit $A_{\text {rec }}=154 \mathrm{~m}^{2}$ im Vergleich zum Referenzreceiver mit Solar Salt. Ein weiterer Nachteil der hohen Dichte von LBE ist der hohe statische Druck im Receiver, der eine höhere Wandstärke fordert. Dies führt zu hohen Rohrtemperaturen. Im Gegensatz zu Solar Salt gibt es keine durch das Medium limitierte maximale Wärmestromdichte, jedoch führen die hohen Absorbertemperaturen (bzw. die hohen Temperaturgradienten zwischen Vorder- und Rückseite) zu hohen thermischen Spannungen und zu einer geringeren Lebensdauer.

Weitere Untersuchungen zur Lebensdauer von mit LBE gekühlten Absorberroh- 


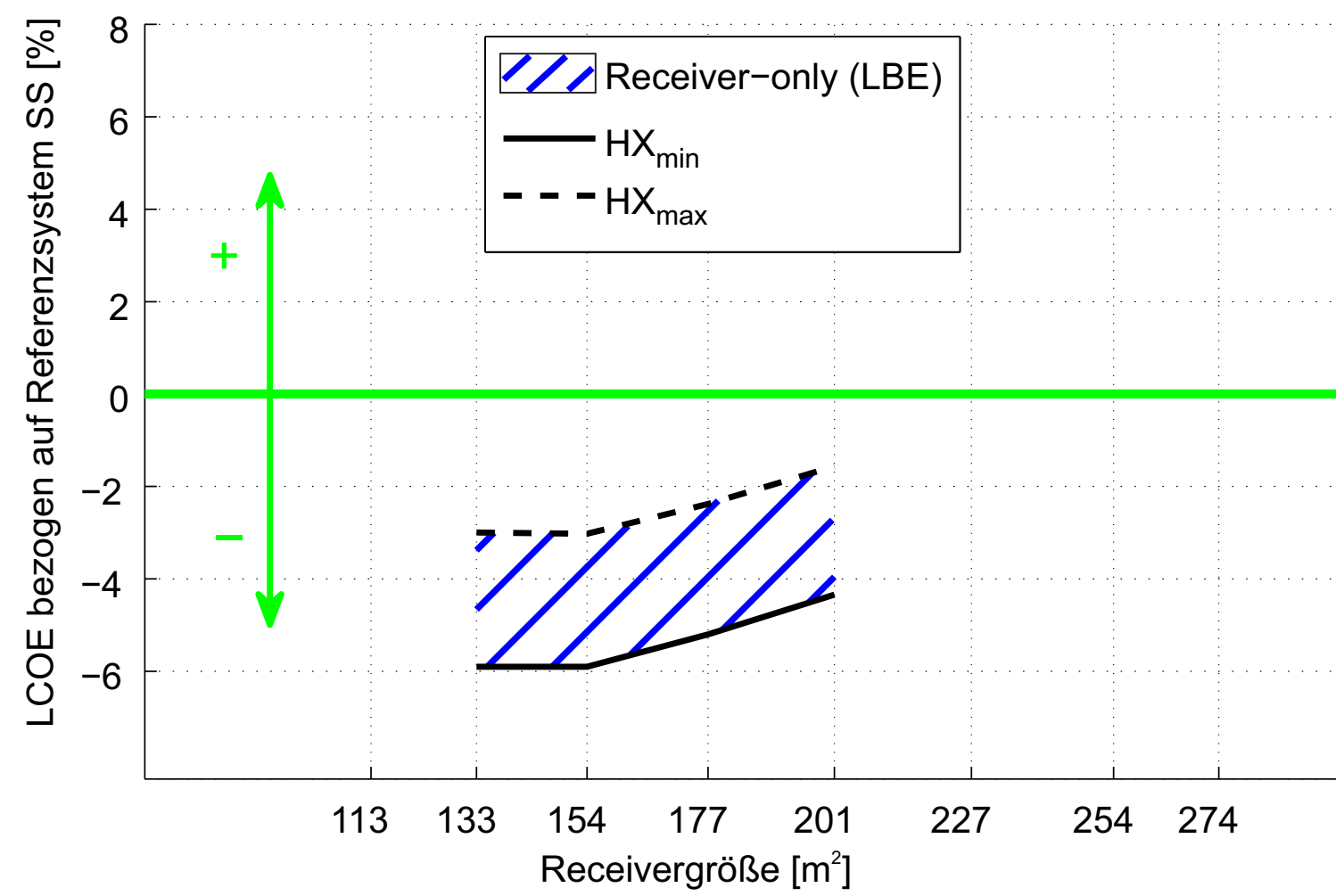

Abbildung 7.10.: Relative Änderung der LCOE von LBE-Systemen bezogen auf das Referenz-Salzsystem als Mehrturmkonfiguration (5x140 $\left.\mathrm{MW}_{\text {th }}\right)$. Receivergrößenstudie in Konfiguration Receiver-only und Receiverkostenfunktion $K_{\text {rec }, 2}$ (siehe Abschnitt 6.1.1) mit minimalen und maximalen Wärmeübertragerkosten $K_{\mathrm{HX} \text {,min }}$ und $K_{\mathrm{HX} \text {,max }}$.

ren unter hohen Wärmestromdichten sind notwendig für eine aussagekräftige Bewertung. Aktuell wird ein derartiger Receiver am KIT untersucht [118].

Die hohen Absorbertemperaturen und die Auswirkungen des höheren Receivergewichtes bei LBE sind in den dargestellten Ergebnissen nicht detailliert abgebildet. Um das Kostenreduktionspotenzial von LBE-Systemen zu bestätigen müssen diese Punkte mitberücksichtigt werden.

\subsubsection{Kostenanalyse der Wärmeträgermedien}

Für die Bewertung von WTM werden in der Literatur oft die Investitionskosten in $€ / \mathrm{kg}$ als Bewertungskriterium herangezogen und die Betriebskosten des Mediums vernachlässigt. Für einen aussagekräftigen Vergleich sind aber neben den thermischen Eigenschaften vor allem die Betriebskosten entscheidend. Die 
elektrische Energie zum Betrieb von Förderpumpe und Begleitheizung senkt den Nettoertrag des Kraftwerks und damit die LCOE.

Aus Gründen der Übersichtlichkeit beschränkt sich die folgende einfache Abschätzung auf die Pumpenleistung zur Überwindung des Druckverlustes im Receiver. Die Pumpenergie für den restlichen WTM-Kreislauf und die Energiekosten der Begleitheizung sowie die Kosten für Reinigung bzw. Erneuerung des WTMs werden vernachlässigt.

Als Grundlage für diesen Vergleich dienen die hier ausgelegten Receiver für Solar Salt, Natrium und Blei-Bismut. Die einmaligen Investitionskosten der Menge an WTM im Receiver wurden mit einem Annuitätenfaktor $F C R=0.0937$ (siehe Gleichung (6.13) auf Seite 139) und einem Zuschlag für die indirekten Kosten von $35 \%$ (siehe Tabelle 6.3) berechnet und verteilen sich gleichmäßig auf die Abschreibungszeit der Anlage (hier: 25 Jahre). Die Werte der jährlichen Pumpenergie (Nur Anteil durch Druckverlust im Receiver) wurden aus der Jahresertragsrechnung der jeweiligen Variante übernommen. Obwohl die elektrische Energie meist zu höheren Preisen auf dem Strommarkt verkauft wird, wurden die Stromkosten für die Pumpen hier mit den LCOE des jeweiligen Systems berechnet.

Abbildung 7.11 zeigt die jährlichen Finanzierungs- und Betriebskosten bei der Verwendung von Solar Salt, Natrium und LBE. Sowohl die jährlichen Kosten der Abschreibung als auch die jährlichen Betriebskosten sind mit den jährlichen Ge-

Tabelle 7.4.: Vergleich zwischen dem Receiver des Referenzsystems Solar Salt und dem des LBE-Systems mit den geringsten $\operatorname{LCOE}\left(A_{\mathrm{rec}}=154 \mathrm{~m}^{2}\right.$ in Receiver-only-Konfiguration mit Receiverkostenfunktion $\left.K_{\mathrm{rec}, 2}\right)$

\begin{tabular}{lcc}
\hline & Referenz Solar Salt & LBE V2-26 \\
\hline Absorberfläche $\left[\mathrm{m}^{2}\right.$ ] & 291 & 154 \\
Anzahl seriell durchströmter Module [-] & $2 \times 12$ & $2 \times 3$ \\
Druckverlust $(21.3 . / 12 \mathrm{~h}$ ) [bar] & 14.82 & 3.29 \\
Anzahl Rohre pro Modul [-] & 29 & 89 \\
Rohrinnendurchmesser [mm] & 32.8 & 35.4 \\
Rohrwandstärke [mm] & 1.4 & 2.5 \\
Gesamtmasse Absorberrohre leer [t] & 19.3 & 27.1 \\
Gesamtmasse WTM im Receiver [t] & 24.1 & 94.3 \\
Maximale Rohrtemperatur $(21.3 . / 9 \mathrm{~h})\left[{ }^{\circ} \mathrm{C}\right]$ & 609 & 896 \\
\hline
\end{tabular}




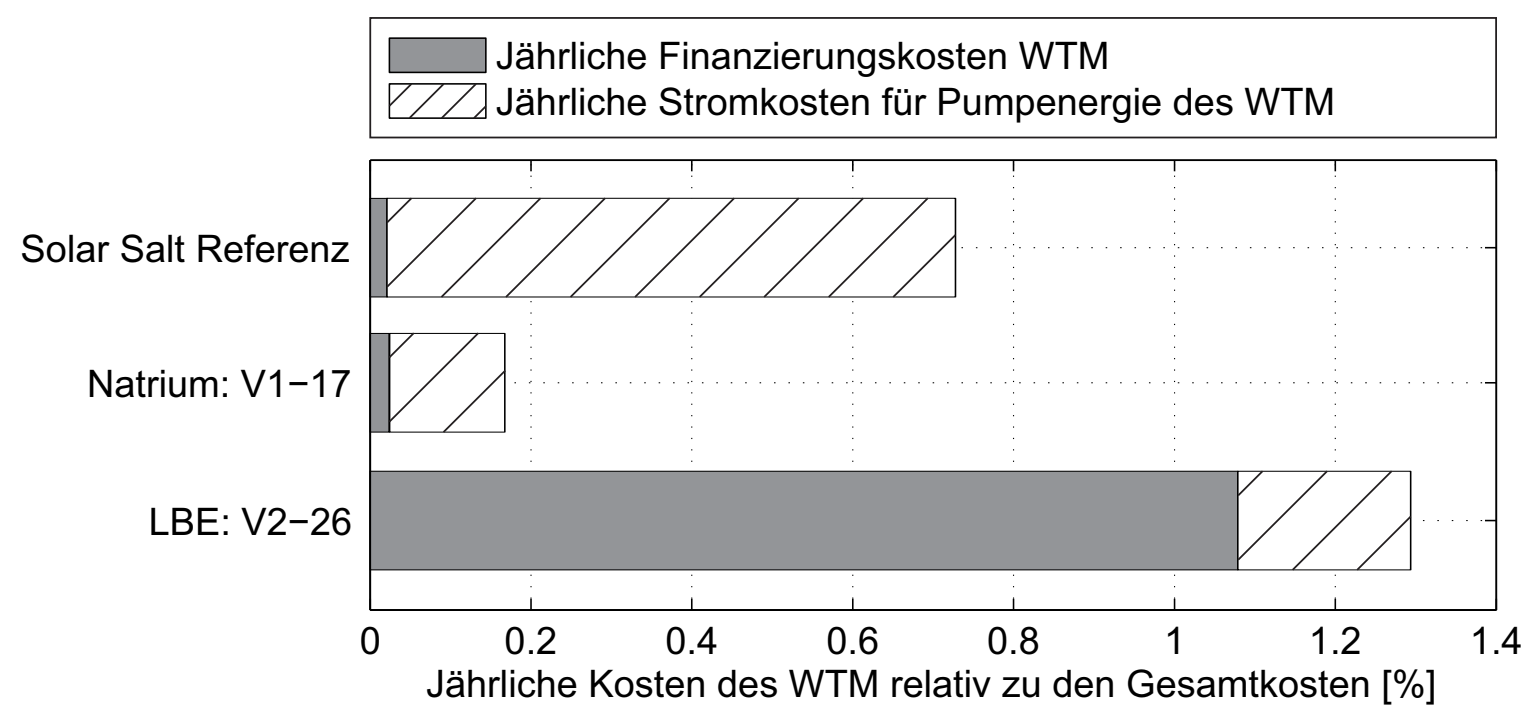

Abbildung 7.11.: Vergleich der jährlichen Kosten des WTM im Receiver (Investitionund Betriebskosten) für Solar Salt und Natrium bei einer Abschreibungslaufzeit von 25 Jahren. Zur Berechnung der Stromkosten wird lediglich die benötigte Pumpenergie zur Überwindung des Druckverlustes im Receiver herangezogen. Bei Solar Salt und LBE wird eine mechanische Pumpe eingesetzt und bei Natrium eine EMP.

samtkosten entdimensioniert dargestellt. Damit lässt sich neben dem Vergleich zwischen den Medien gleichzeitig der Kostenanteil am Gesamtkraftwerk bewerten. Durch die geringe Dichte von Natrium und die sehr kompakte Bauweise des Natriumreceivers führt dazu, dass trotz der höheren spezifischen Kosten von $K_{\mathrm{Na}}=2.6 € / \mathrm{kg}$ sich für Solar Salt $K_{\mathrm{SS}}=1 € / \mathrm{kg}$ und Natrium sehr ähnliche jährliche Finanzierungskosten für das WTM ergeben. Bei LBE hingegen führt die hohe Dichte zusätzlich zu den hohen spezifischen Kosten $K_{\mathrm{LBE}}=12 € / \mathrm{kg}$ zu sehr hohen Finanzierungskosten.

Auffällig bei Solar Salt und Natrium ist der sehr große Unterschied in den jährlichen Betriebskosten der Pumpe. Der hohe Druckverlust bei Salzreceivern ${ }^{1]}$ führt zu einer hohen Pumpenergie damit zu hohen jährlichen Stromkosten. Trotz der deutlich geringeren Wirkungsgrade der bei Natrium eingesetzten EMP (siehe Abbildung 6.7, Seite 128) ergeben sich für Solar Salt ca. 4-fach höhere jährliche Kosten. Werden zudem die Betriebskosten der Begleitheizung und der Pumpenergie für den restlichen WTM-Kreislauf berücksichtigt steigen die jährlichen Stromkosten weiter an. Aus diesem Blickwinkel betrachtet erscheint eine Bewertung der WTM nach ihren spezifischen Kosten in $€ / \mathrm{kg}$ wenig sinnvoll.

\footnotetext{
${ }^{1}$ Um einen technisch sinnvollen Wärmeübergang zu erhalten, entstehen bei Salzsystemen hohe Druckverluste (siehe Abschnitt 3.4.3 bzw. Auslegung in Tabelle 7.3).
} 



\section{Vergleichsstudie Einturmsystem (700 MW-Receiver)}

Dieses Kapitel beinhaltet die Vergleichsstudie von Einturmsystemen mit Solar Salt (Referenzsystem) und Natrium. Wie beim Vergleich der Mehrturmsysteme wurde für Natrium sowohl die Receiver-only als auch die Tower-loopVarianten berücksichtigt. Ebenso wurde eine Receivergrößenstudie durchgeführt. Die übergeordneten Annahmen sowie die Methodik zur Jahresertrags- und LCOE-Berechnung sind in Kapitel 6 beschrieben. Die detaillierte Spezifikation aller berechneten Varianten ist im Anhang $\mathrm{H}$ auf Seite 215ff dokumentiert. Im Gegensatz zu Mehrturmsystemen sind bei Einturmsystemen die thermischen Speicher sowie der Kraftwerksblock direkt neben dem Turm angeordnet. Dadurch können die Heliostate nicht so dicht am Turm stehen wie bei Mehrturmsystemen, was geringere Feldwirkungsgrade zur Folge hat. Ein wesentlicher Unterschied der Einturmsysteme ist das Fehlen der Verbindungsleitungen zum zentralen Kraftwerksblock, die bei Mehrturmsystemen notwendig sind.

\subsection{Ergebnisse der Komponentenauslegung}

\subsubsection{Heliostatenfeldauslegung}

Die Auslegung und Optimierung der Heliostatenfelder wurde mit HFLCAL durchgeführt. Beim Referenz-Salzsystem wurde das Receiver-model: 502 verwendet, um schon bei der Feldauslegung die parasitären Verluste mitzuberücksichtigen (Details siehe Anhang F). Dabei wurde für die Feldauslegung der Betriebsbereich des Receivers auf [10-114\%] der Auslegungsleistung beschränkt. 


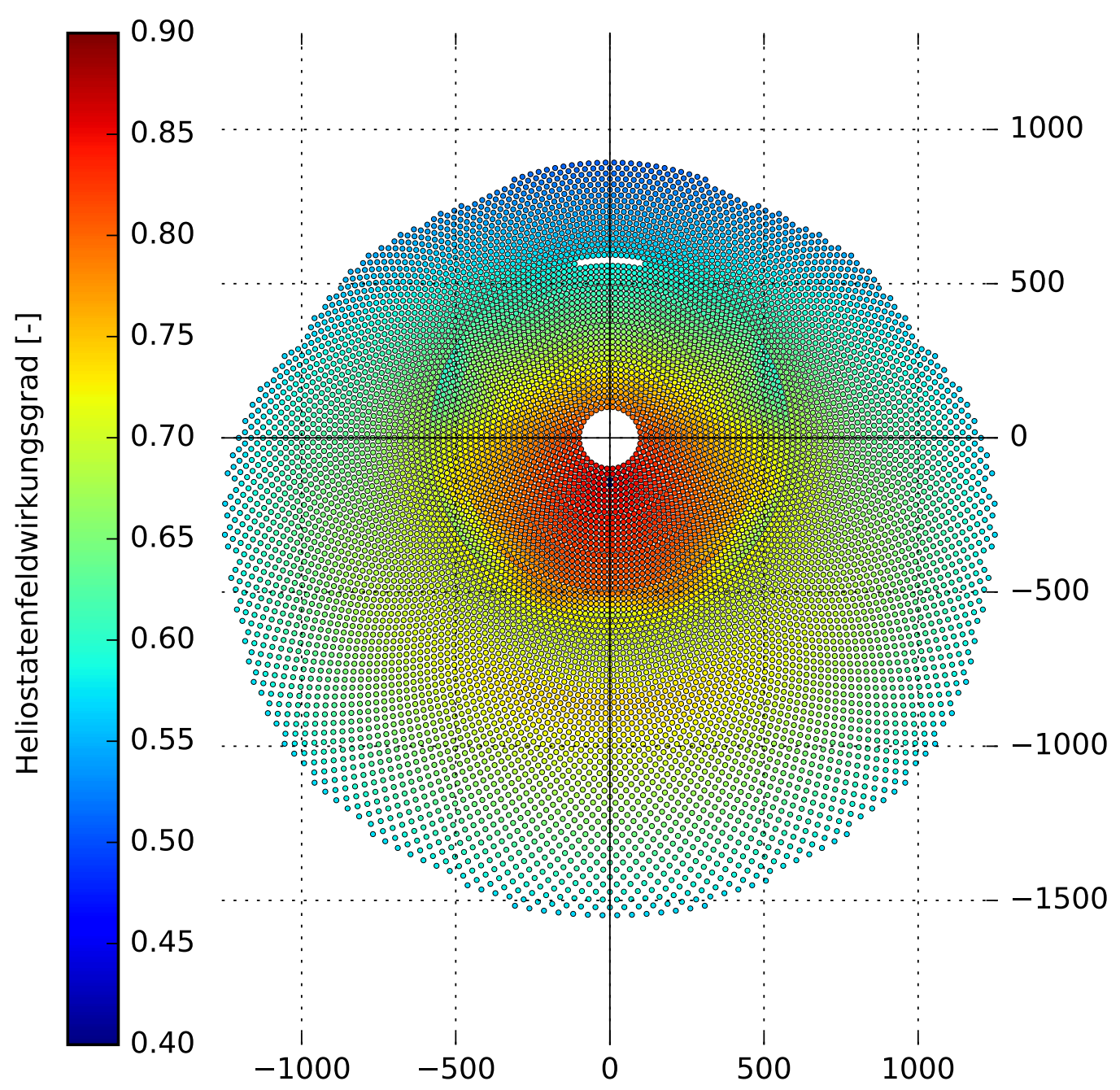

Abbildung 8.1.: Heliostatenfeld des Referenz-Salzsystems $\left(A_{\mathrm{rec}}=1376 \mathrm{~m}^{2}\right)$ mit optischen Wirkungsgraden am 21. März um $12 \mathrm{~h}: \dot{Q}_{\mathrm{th}}=700 \mathrm{MW}_{\mathrm{th}}$, 10137 Heliostate.

Tabelle 8.1.: Optische Wirkungsgrade und Wärmestromdichten auf Receiver (ReferenzSalzsystem mit $700 \mathrm{MW}_{\text {th }}$-Receiver), berechnet mit SPRAY

\begin{tabular}{|c|c|c|c|c|}
\hline Zeit & $\begin{array}{l}\text { Azimut } \\
\text { in }\left[^{\circ}\right]\end{array}$ & $\begin{array}{l}\text { Elevation } \\
\text { in [] }\end{array}$ & $\begin{array}{c}\eta_{\text {field }}(\text { mit SPRAY) } \\
\text { ohne Zielpunktverteilung }\end{array}$ & $\begin{array}{l}\eta_{\text {field }}(\text { mit SPRAY) } \\
\text { mit Zielpunktverteilung }\end{array}$ \\
\hline 6h30 & 86.43 & 6.60 & 0.2755 & 0.2756 \\
\hline 7h00 & 82.76 & 13.17 & 0.4331 & 0.4267 \\
\hline $8 \mathrm{~h} 00$ & 74.70 & 26.12 & 0.5818 & 0.5640 \\
\hline $9 \mathrm{~h} 00$ & 64.64 & 38.51 & 0.6292 & 0.6067 \\
\hline $12 \mathrm{~h} 00$ & 0 & 61.71 & 0.6760 & 0.6474 \\
\hline \multicolumn{3}{|c|}{ Max. Wärmestromdichte um 12h00: } & $2.23 \mathrm{MW} / \mathrm{m}^{2}$ & $0.93 \mathrm{MW} / \mathrm{m}^{2}$ \\
\hline
\end{tabular}




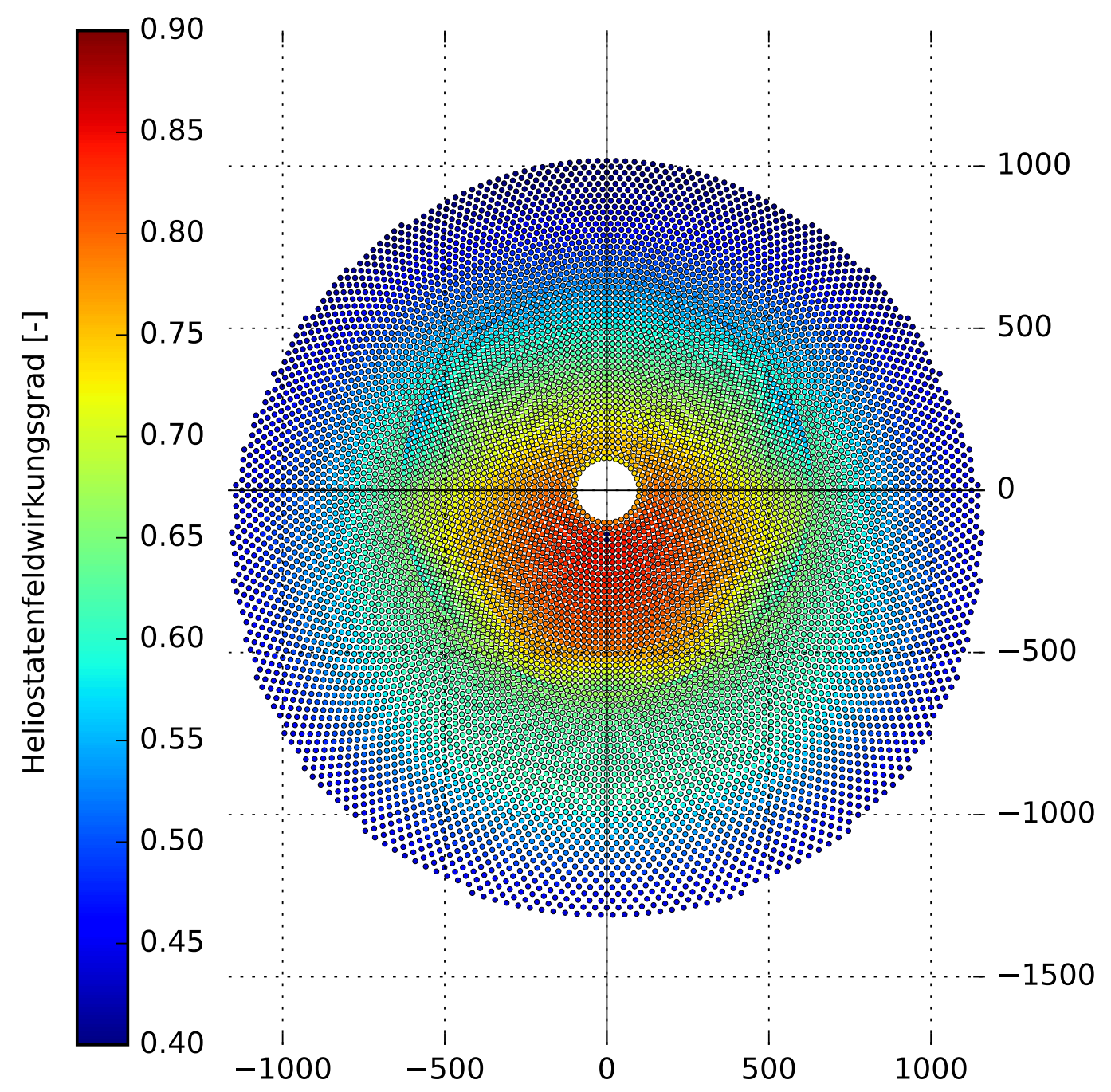

Abbildung 8.2.: Heliostatenfeld für Natriumreceiver $\left(A_{\text {rec }}=490 \mathrm{~m}^{2}\right)$ mit optischen Wirkungsgraden am 21. März um $12 \mathrm{~h}: \dot{Q}_{\text {th }}=700 \mathrm{MW}_{\text {th }}, 10471 \mathrm{He}-$ liostate.

Abbildung 8.1 zeigt die Feldaufstellung des Referenz-Salzsystems mit den einzelnen Heliostatwirkungsgraden am Auslegungszeitpunkt und Tabelle 8.1 die optischen Wirkungsgrade bei Teillast. Das in Abbildung 8.2 dargestellte Heliostatenfeld für einen sehr kleinen Natriumreceiver ( $\approx 35 \%$ der Absorberfläche des Referenz-Salzreceivers aus Abbildung 8.1) zeigt deutlich die Abhängigkeit des optischen Wirkungsgrades von der Feld- und Receivergröße. Die Randstrahlung nimmt mit größeren Heliostatenfeldern und kleineren Receivern zu. Auch die atmosphärische Abschwächung nimmt mit größeren Heliostatenfeldern zu. Die äußeren Heliostaten erreichen daher bei Sonnenhöchststand einen Wirkungsgrad von lediglich $40 \%$ (siehe Abbildung 8.2). Die Strahlaufweitung nimmt 
Tabelle 8.2.: Ergebnisse aus der Feldauslegung: Vergleich der Heliostatenfelder für verschieden große Receiver gleicher Leistung. Berechnung am 21.3./12 h mit SPRAY, Jahresmittelwert mit HFLCAL

\begin{tabular}{|c|c|c|c|c|}
\hline Receivergröße & \multicolumn{2}{|c|}{$1376 \mathrm{~m}^{2}$} & \multicolumn{2}{|c|}{$490 \mathrm{~m}^{2}$} \\
\hline Leistung am 21.3. um $12 \mathrm{~h}$ & \multicolumn{2}{|c|}{$700 \mathrm{MW}_{\mathrm{th}}$} & \multicolumn{2}{|c|}{$700 \mathrm{MW}_{\text {th }}$} \\
\hline Anzahl Heliostate & \multicolumn{2}{|c|}{10137} & \multicolumn{2}{|c|}{10471} \\
\hline Reflektivität der Spiegel & \multicolumn{2}{|c|}{$89.3 \%$} & \multicolumn{2}{|c|}{$89.3 \%$} \\
\hline Feldwirkungsgrade & $21.3 . / 12 \mathrm{~h}$ & Jahresmittel $^{a}$ & $21.3 . / 12 \mathrm{~h}$ & Jahresmittel $^{a}$ \\
\hline Kosinuswirkungsgrad & $86.6 \%$ & $78.9 \%$ & $85.3 \%$ & $78.1 \%$ \\
\hline Blockierung \& Verschattung & $96.8 \%$ & $93.3 \%$ & $95.9 \%$ & $91.8 \%$ \\
\hline Extinktion & $90.4 \%$ & $91.1 \%$ & $88.2 \%$ & $91.4 \%$ \\
\hline Intercept-Wirkungsgrad & $95.6 \%$ & $98.1 \%$ & $94.9 \%$ & $88.8 \%$ \\
\hline Gesamtwirkungsgrad & $64.7 \%$ & $58.7 \%$ & $61.1 \%$ & $52.0 \%$ \\
\hline
\end{tabular}

${ }^{a}$ Werte aus HFLCAL ohne aiming

mit dem Abstand zwischen Heliostat und Receiver zu. Dadurch erhöht sich die Randstrahlung, was dazu führt, dass in der Feldoptimierung die Heliostaten dichter gestellt werden und sich insgesamt ein kreisrunderes Heliostatenfeld ergibt. Beim (größeren) Referenz-Salzreceiver (Abbildung 8.1) sinkt der optische Wirkungsgrad der Heliostaten zwar ebenfalls mit zunehmendem Abstand vom Receiver, jedoch in viel geringerem Umfang. Bei der Feldoptimierung können daher die Abstände zwischen den Heliostaten vergrößert und somit die Verluste durch Verschattung und Blockierung minimiert werden. Tabelle 8.2 zeigt die einzelnen Wirkungsgrade beider Heliostatenfelder. Der Unterschied im optischen Wirkungsgrad zwischen diesen beiden Heliostatenfeldern für sehr unterschiedliche Receivergrößen $\left[1376 \mathrm{~m}^{2}\right.$ und $\left.490 \mathrm{~m}^{2}\right]$ beträgt zum Auslegungszeitpunkt $3.6 \%$ - Punkte und im Jahresmittel etwa $6.7 \%$ - Punkte ${ }^{a}$.

\subsubsection{Receivermodellierung}

Wie in Abschnitt 3.4 .2 beschrieben, muss für die Natriumreceiver stets eine Receivergrößenstudie gemacht werden, da zunächst nicht bekannt ist, welche Receivergröße zu den geringsten LCOE führt. Im vorhergehenden Abschnitt wurden bereits die Eigenschaften eines Heliostatenfeldes für einen sehr kleinen Natriumreceiver beschrieben. Abbildung 8.3 zeigt die Ergebnisse der Receivergrößenstudie 


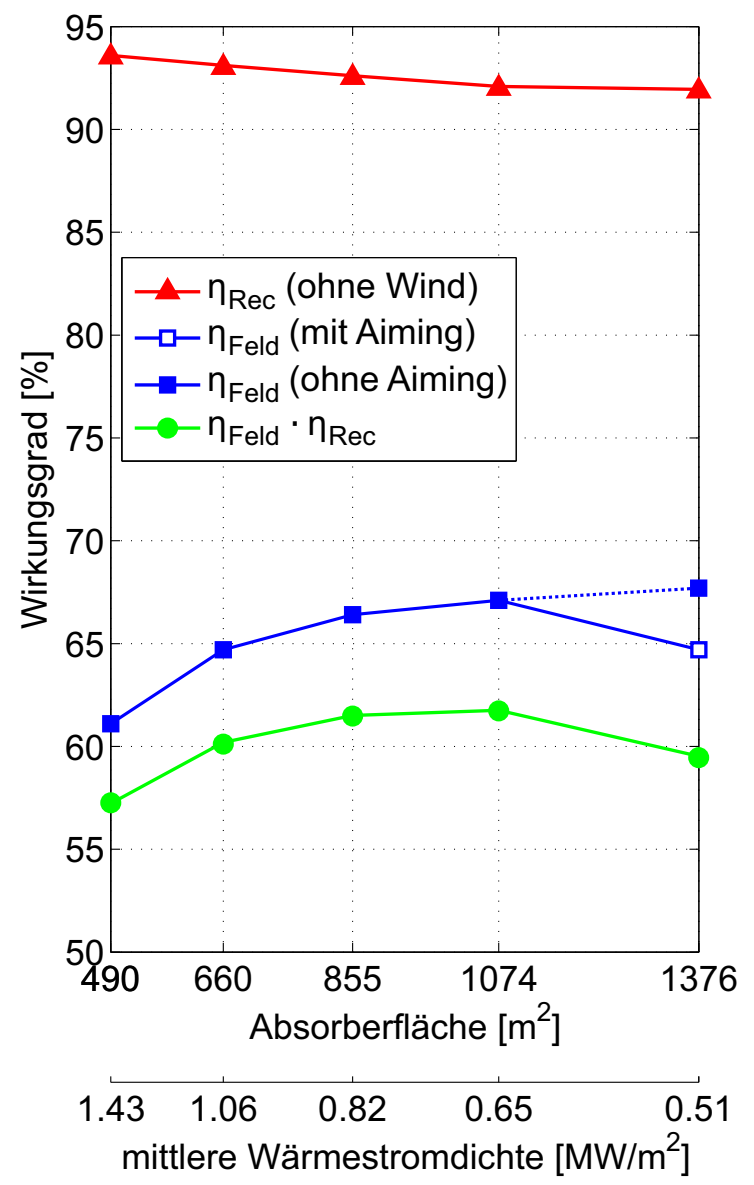

$\begin{array}{lllll}3.43 & 3.00 & 2.60 & 2.42 & \mathbf{0} 2.23 \\ & 0.93\end{array}$

maximale Wärmestromdichte $\left[\mathrm{MW} / \mathrm{m}^{2}\right]$

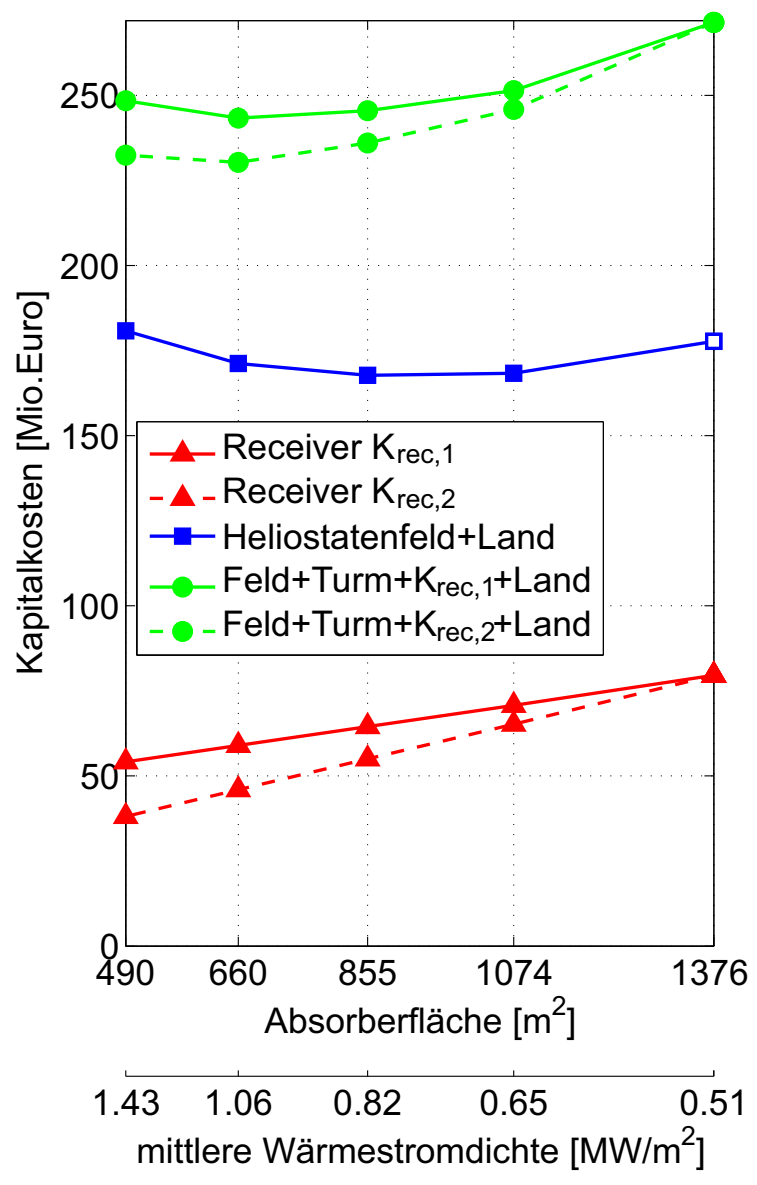

$\begin{array}{lllll}3.43 & 3.00 & 2.60 & 2.42 & 2.23 \\ & 0.93\end{array}$ maximale Wärmestromdichte $\left[\mathrm{MW} / \mathrm{m}^{2}\right]$

Abbildung 8.3.: Ergebnisse der Receivergrößenstudie. Links: Receiver- und Feldwirkungsgrade von Referenz-Salzsystem $\left(A_{\text {rec }}=1376 \mathrm{~m}^{2}\right)$ und Natriumsystemen $\left(A_{\text {rec }}=490-1074 \mathrm{~m}^{2}\right)$ am 21.3 um $12 \mathrm{~h}$ in Abhängigkeit der Absorberfläche bei einer Leistung von $700 \mathrm{MW}_{\text {th }}$. Rechts: Kapitalkosten von Receiver, Heliostatenfeld und dem Gesamtkapital (Feld+Land+Turm+Receiver).

graphisch. Für jede Receivergröße wurde eine Receiver- und Feldoptimierung durchgeführt. Die Ergebnisse zeigen, dass je geringer die Absorberfläche, desto geringer der optische Wirkungsgrad (erhöhte Randstrahlung). Jedoch führt die bei Salzreceivern notwendige Zielpunktverteilung zur Einhaltung des Wärmestromdichtelimits (siehe Abschnitt 3.4.2) ebenfalls zu einer Reduktion des optischen Wirkungsgrades. Am Auslegungszeitpunkt (21.3 um $12 \mathrm{~h}$ ) senkt sich dadurch im vorliegenden Fall der optische Wirkungsgrad um $2.6 \%$ - Punkte, wodurch die maximale Wärmestromdichte von 2.23 auf $0.93 \mathrm{MW} / \mathrm{m}^{2}$ reduziert 
wird. Bei Natrium gibt es kein zu beachtendes Wärmestromdichtelimit, daher werden jene Varianten ohne Zielpunktverteilung ausgelegt. Durch diese Maßnahme ergibt sich zusammen mit den höheren Receiverwirkungsgraden der Natriumreceiver ein um ca. 2\% - Punkte höherer Gesamtwirkungsgrad, wenn die Absorberfläche um bis zu $50 \%$ bezüglich des Referenz-Salzreceivers reduziert wird. Bei weiterer Reduktion der Absorberfläche fällt der Gesamtwirkungsgrad trotz steigendem Receiverwirkungsgrad stark ab. Die in Abbildung 8.3 dargestellten Wirkungsgrade gelten für Windstille am Auslegungszeitpunkt (21.3 um 12 h). Tabelle H.1 auf Seite 232 zeigt eine Übersicht dieser Receivergrößenstudie mit weiteren Auslegungsparametern und Ergebnissen von Heliostatenfeld und Receiver für den Auslegungszeitpunkt und den Teillastfall am 21.3 um 6 h30. Die Optimierung des Heliostatenfeldes ergibt für alle Varianten ähnliche Turmhöhen [298-316 m]. Deutlich unterscheiden sich die Receiver hinsichtlich des Druckverlustes, welcher beim Referenz-Salzreceiver um den Faktor fünf höher ist, als bei den Natriumvarianten.

Abbildung 8.3 zeigt rechts die Kapitalkosten von Heliostatenfeld, Turm und Receiver. Je höher der Gesamtwirkungsgrad (siehe Abbildung 8.3 links), desto weniger Heliostate werden für die gleiche Receiverleistung benötigt und desto günstiger wird das Heliostatenfeld. Die Receiverkosten sinken linear mit geringerer Absorberfläche entsprechend den Receiverkostenfunktionen nach Gleichung (6.7) bzw. 6.8 ab (siehe Seite 135). Die Summe der Kapitalkosten von Heliostatenfeld, Turm und Receiver ergeben damit ein Kostenminimum für das Natriumsystem mit einer Receivergröße von $A_{\text {rec }}=660 \mathrm{~m}^{2}$. Der höchste Wirkungsgrad wird jedoch beim Natriumsystem mit einem größeren Receiver $\left(A_{\text {rec }}=1074 \mathrm{~m}^{2}\right)$ erreicht.

Über die LCOE kann mit Abbildung 8.3 noch keine Aussage gemacht werden, da hier nur die Wirkungsgrade am Auslegungszeitpunkt bei Windstille dargestellt sind. Mit der Jahresertragsrechnung auf Basis von stündlichen DNI-Daten und unter Berücksichtigung der jeweiligen Zustände von Heliostatenfeld, Receiver, Speicher und Kraftwerksblock kann für jede Variante der Jahresenergieertrag und damit die LCOE berechnet werden (Ergebnisse siehe Abschnitt 8.2).

Trotz der hohen Wärmeübergangskoeffizienten führt vor allem bei den kleinen Natriumreceivern die hohe Wärmestromdichte zu hohen Rohrtemperaturen. Abbildung 8.4 und 8.5 (folgende Doppelseite) zeigen beispielhaft die 
Wärmestromdichte- und Temperaturverteilungen des jeweils am höchsten belasteten Rohres des ersten Moduls für den Referenz-Salzreceiver und den Natriumreceiver mit $A_{\text {rec }}=855 \mathrm{~m}^{2}$. Es lässt sich sehr gut erkennen, wie beim Referenz-Salzreceiver die Wärmestromdichte durch eine Zielpunktverteilung auf die gesamte Receiverhöhe $[-12.7 \ldots+12.7] \mathrm{m}$ verteilt wird mit einem Maximum von ca. $1.0 \mathrm{MW} / \mathrm{m}^{2}$ in der Receivermitte bei $L=0$. Das Salz wird in diesem Rohr von 290 auf $370{ }^{\circ} \mathrm{C}$ erwärmt. Die maximale Rohrinnen- und Rohraußenwandtemperatur beträgt $450^{\circ} \mathrm{C}$ bzw. $525^{\circ} \mathrm{C}$. Abbildung 8.4 unten zeigt die Temperaturverteilung über den Rohrumfang in der Receivermitte $(L=0)$, wo die höchste Wärmestromdichte und Rohrtemperatur auftritt.

Beim Natriumreceiver fällt zunächst die geringere Rohrlänge [-8.5 . + 8.5] $\mathrm{m}$ auf. Aufgrund der Auslegung mit lediglich drei seriell verschalteten Modulen, beträgt der Temperaturhub des Natriums im ersten Modul 300 bis $389^{\circ} \mathrm{C}$. Durch den Verzicht der Zielpunktverteilung und den geringeren Receiverdurchmesser ergeben sich beim Natriumreceiver sehr hohe maximale Wärmestromdichten von $\approx 2.5 \mathrm{MW} / \mathrm{m}^{2}$. Durch diese hohe Wärmestromdichte ergeben sich entsprechend auch hohe Rohraußenwandtemperaturen von bis zu $625^{\circ} \mathrm{C}$. Durch die hohen Wärmeübergangskoeffizienten erreicht der Natriumreceiver trotz der höheren Fluidtemperatur geringere Rohrinnenwandtemperaturen als der Referenz-Salzreceiver. Der sehr hohe Temperaturgradient in der Rohrwand kommt durch die verhältnismäßig geringe Wärmeleitfähigkeit des Rohrmaterials (13-25 W/m² /K [119]) zustande. Der in Almería getestete Natriumreceiver zeigte im Betrieb sehr ähnliche Wärmestromdichten und Temperaturgradienten ${ }^{1}$. Die damals durchgeführten Spannungsuntersuchungen zeigen, dass Wärmestromdichten bis $3 \mathrm{MW} / \mathrm{m}^{2}$ erreichbar sind 29], obwohl der Receiver ursprünglich nur für maximal $1.4 \mathrm{MW} / \mathrm{m}^{2}$ ausgelegt wurde $\mathrm{f}^{2}$.

\footnotetext{
${ }^{1}$ Beim Natriumreceiver (ASR) in Almería betrug der gemessene, maximale Temperaturgradient zwischen Rohraußen- und innenwand $\left(680-525=155^{\circ} \mathrm{C}\right)$ bei einer maximalen Wärmestromdichte von $\approx 2.5 \mathrm{MW} / \mathrm{m}^{2}$. Durch die Beschichtung mit Pyromark wurden sogar Temperaturen um $\left.775{ }^{\circ} \mathrm{C} 29\right]$ erreicht.

${ }^{2} \mathrm{Im}$ Anschluß an die High Flux Experiment-Testreihe mit $2.5 \mathrm{MW} / \mathrm{m}^{2}$ wurden die am höchsten bestrahlten Rohre zudem metallurgisch untersucht, um eine etwaige Zeitstandsermüdung festzustellen. Der Vergleich mit baugleichen, unbestrahlten Rohren zeigte keinen Unterschied 29].
} 

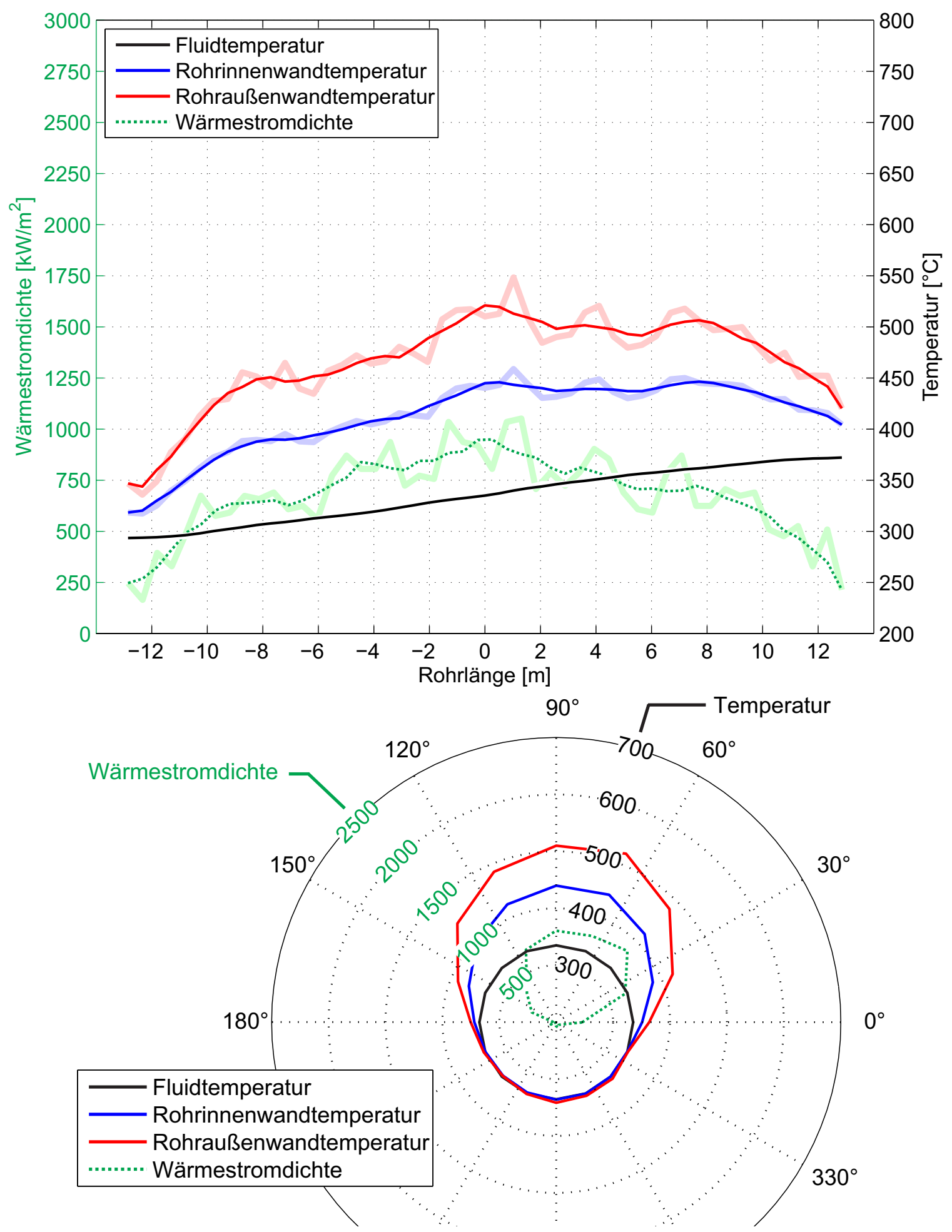

Abbildung 8.4.: Ergebnis der Receiversimulation des Referenz-Salzreceivers mit $A_{\text {rec }}=1376 \mathrm{~m}^{2}$. Oben: Wärmestromdichte- und Temperaturverteilung entlang des höchstbelasteten Absorberrohres des ersten Moduls am 21. $3 \mathrm{um} 12 \mathrm{~h}$ mit $\dot{Q}_{\text {th }}=700 \mathrm{MW}_{\mathrm{th}}$. Lokale Werte aus FEMBerechnung auf der feldzugewandten Rohrseite als blasse Linien dargestellt. Geglättete Kurve mit gleitendem Durchschnitt in Farbe darüber. Unten: Wärmestromdichte- und Temperaturverteilung desselben Rohres in Umfangsrichtung, ausgewertet bei $L=0$ (Receivermitte). Feldzugewandte Richtung bei $90^{\circ}$. 

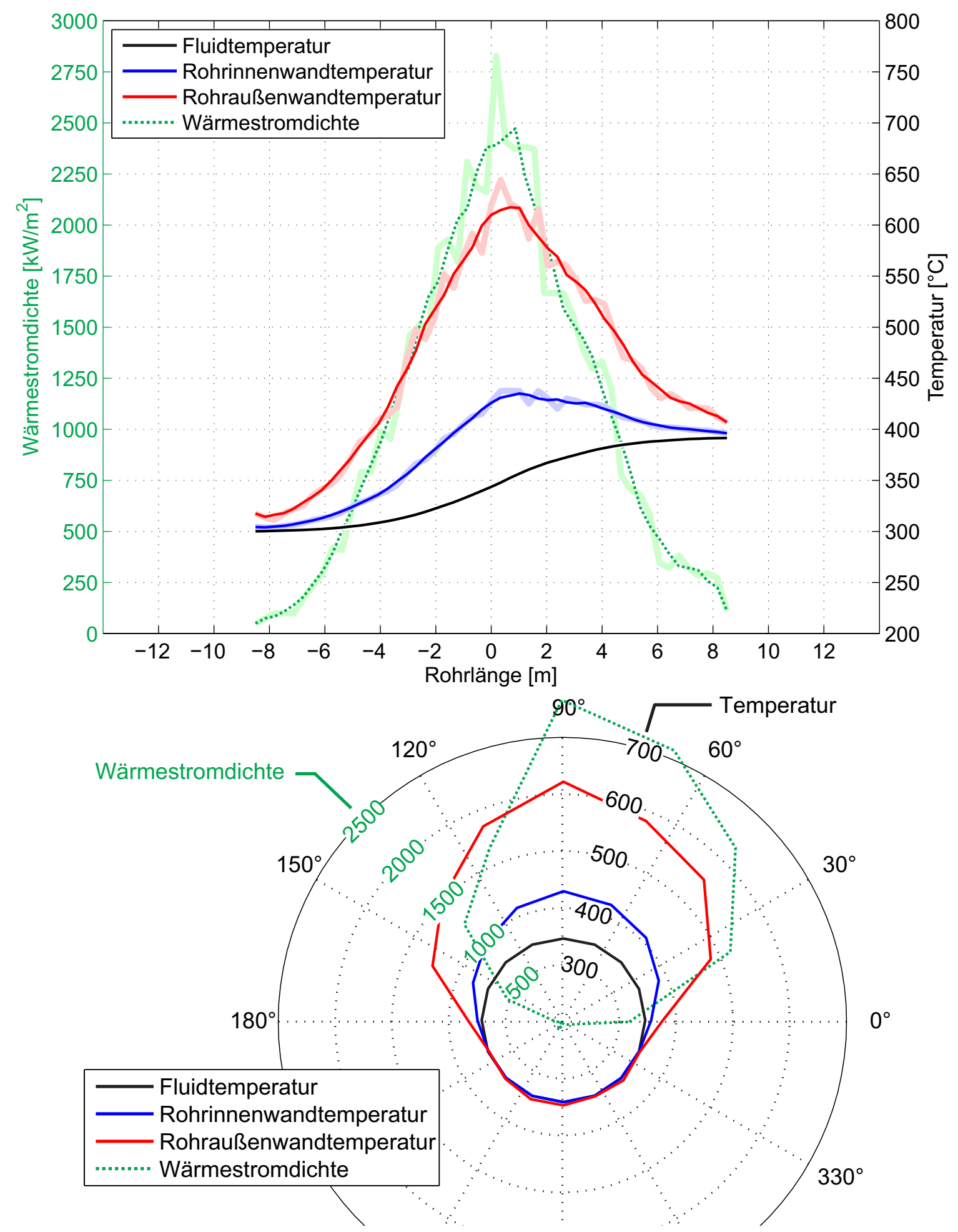

Abbildung 8.5.: Ergebnis der Receiversimulation der Natriumreceiver V2-32 bzw. V3-52 mit $A_{\text {rec }}=855 \mathrm{~m}^{2}$. Oben: Wärmestromdichte- und Temperaturverteilung entlang des höchstbelasteten Absorberrohres des ersten Moduls am 21. 3 um $12 \mathrm{~h}$ mit $\dot{Q}_{\text {th }}=700 \mathrm{MW}_{\text {th }}$. Lokale Werte aus FEM-Berechnung auf der feldzugewandten Rohrseite als blasse Linien dargestellt. Geglättete Kurve mit gleitendem Durchschnitt in Farbe darüber. Unten: Wärmestromdichte- und Temperaturverteilung desselben Rohres in Umfangsrichtung, ausgewertet $L=0$ (Receivermitte). Feldzugewandte Richtung bei $90^{\circ}$. 


\subsection{Ergebnisse der LCOE-Berechnung}

Die Jahresertragsrechnung wurde für alle Receivergrößen sowohl als Receiveronly als auch in Tower-loop-Konfiguration mit jeweils minimalen und maximalen Wärmeübertragerkosten durchgeführt und jeweils die Speichergröße mit den geringsten LCOE iterativ ermittelt. Für die Konfiguration Receiver-only können die LCOE bezogen auf das Referenz-Salzsystem um bis zu $13 \%$ reduziert werden. Bei den Tower-loop-Varianten sind es sogar fast 16\%. Die Natriumsysteme mit den geringsten LCOE weisen gegenüber dem Referenz-Salzreceiver eine um bis zu $65 \%$ geringere Absorberfläche auf. Aber selbst mit geringfügig reduzierter Absorberfläche (um 20\%) sinken die LCOE bereits um 8-11\%.

Der Grund für die geringeren Stromgestehungskosten der Natriumsysteme

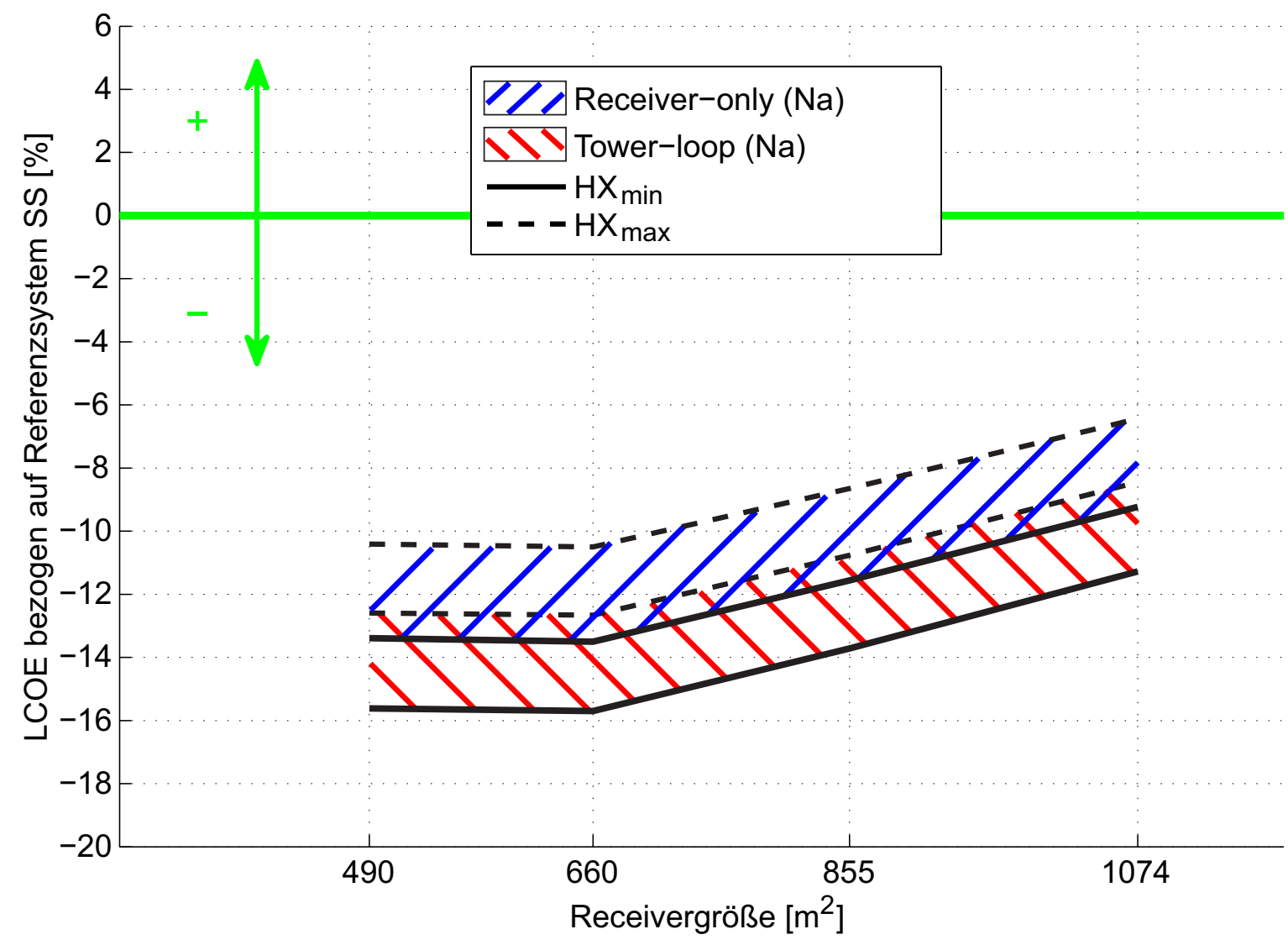

Abbildung 8.6.: Relative Änderung der LCOE von Natriumsystemen bezogen auf das Referenz-Salzsystem als Einturmkonfiguration $\left(700 \mathrm{MW}_{\mathrm{th}}\right)$. mit Receiverkostenfunktion $K_{\mathrm{rec}, 2}$. Receiver-only und Tower-loop-Varianten (siehe Abschnitt 6.1.1 mit Variation der Wärmeübertragerkosten $K_{\mathrm{HX} \text {,min }}$ und $K_{\mathrm{HX} \text {,max }}$ bei jeweils kostenoptimaler Speichergröße. 

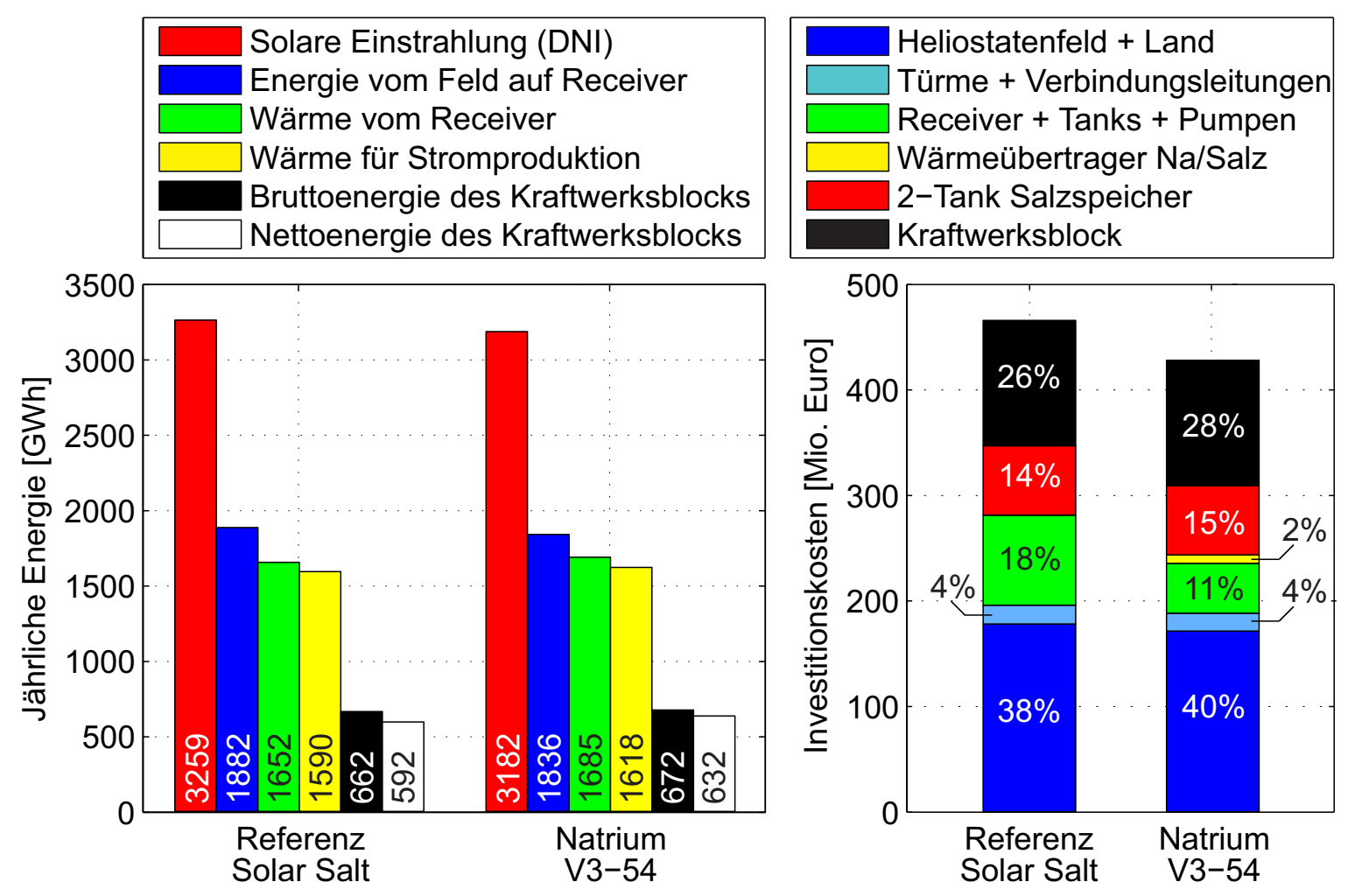

Abbildung 8.7.: Vergleich zwischen Referenzsystem Solar Salt und der Natriumvariante V3.54 $\left(A_{\text {rec }}=660 \mathrm{~m}^{2}\right.$ in Tower-loop-Konfiguration mit Receiverkostenfunktion $\left.K_{\mathrm{rec}, 2}\right)$. Links: Jährliche Energieumsätze in einzelnen Komponenten, Rechts: Investitionskosten.

liegt zum Einen am höheren Jahresenergieertrag und zum Anderen an den geringeren Anlagenkosten. Abbildung 8.7 und Tabelle 8.3 zeigen die jährlichen Energieumsätze und die Investitionskosten für das Referenz-Salzsystem und das Natriumsystem V3-54 mit $A_{\text {rec }}=660 \mathrm{~m}^{2}$ in Tower-loop-Konfiguration. Der höhere Jahresertrag des Natriumsystems liegt zu einem kleinen Anteil am höheren Receiverwirkungsgrad. Hauptsächlich schlagen jedoch die geringeren parasitären Verluste (Pumpenergie und Begleitheizung) zu Buche. Beim relativen Vergleich beider Systeme zeigt sich ein um $4 \%$ höherer Brutto-Jahresenergieertrag beim Natriumsystem gegenüber dem Referenz-Salzsystem. Abzüglich der parasitären Verluste ergibt sich beim Natriumsystem ein um $9 \%$ höherer NettoJahresenergieertrag. Der Gesamtwirkungsgrad des Natriumsystems ist damit um $2 \%$-Punkte höher als der des Referenz-Salzsystems. Die geringeren Kosten des Natriumsystems liegen zu einem kleinen Anteil in den geringeren Kosten des Heliostatenfeldes. Die ausschlaggebende Kostenreduktion wird jedoch durch den kostengünstigen Natriumreceiver erreicht. Insgesamt reduzieren sich damit 
Tabelle 8.3.: Vergleich der Jahreswirkungsgrade und Anlagenkosten des Referenzsystems Solar Salt und des Natriumsystem V3-54 mit $A_{\text {rec }}=660 \mathrm{~m}^{2}$

\begin{tabular}{lccc}
\hline Variante & Referenz & Natrium & Verhältnis \\
& Solar Salt & V3-54 & \\
\hline Heliostatenfeldwirkungsgrad [\%] & 57.75 & 57.70 & 1.00 \\
Receiverwirkungsgrad [\%] & 87.78 & 91.79 & 1.05 \\
Energieverluste (Speicher, Dumping, ... $)[\%]$ & 96.23 & 96.02 & 1.00 \\
Turbinenwirkungsgrad [\%] & 41.62 & 41.54 & 1.00 \\
Parasitäre Verluste (Pumpen, Begleitheizung) [\%] & 89.47 & 93.99 & 1.05 \\
\hline Pumpe Receiver-Kreislauf + HX [GWh] & 21.97 & 2.32 & 0.11 \\
Pumpe Salz HX + Dampferzeuger-Loop [GWh] & 0.90 & 1.59 & 1.77 \\
$\quad$ Parasitäre Verluste im Kraftwerksblock [GWh] & 28.43 & 29.27 & 1.03 \\
$\quad$ Begleitheizung [GWh] & 18.31 & 6.38 & 0.35 \\
\hline Gesamtwirkungsgrad [\%] & 18.16 & 19.86 & 1.09 \\
\hline \hline Kosten Heliostatenfeld + Turm [Mio.€] & 177.3 & 172.5 & 0.97 \\
Kosten Land [Mio.€] & 18.3 & 15.5 & 0.85 \\
Kosten Receiversystem + WTM [Mio.€] & 85.4 & 46.0 & 0.54 \\
Kosten Wärmeübertrager [Mio.€] & 0 & 8.0 & - \\
Kosten Thermischer Speicher [Mio.€] & 65.6 & 65.6 & 1.00 \\
Kosten Kraftwerksblock [Mio.€] & 118.8 & 118.8 & 1.00 \\
Indirekte Kosten [Mio.€] & 162.9 & 149.7 & 0.92 \\
\hline Gesamtkosten [Mio.€] & 447.2 & 412.2 & 0.92 \\
\hline
\end{tabular}

die Anlagenkosten um ca. $8 \%$.

Die für dieses Ergebnis ausschlaggebende Komponente ist der Receiver. Tabelle 8.4 zeigt den Receiver des Referenz-Salzsystem und den des Natriumsystems mit $A_{\text {rec }}=660 \mathrm{~m}^{2}$ im Vergleich. Die Receiver unterscheiden sich deutlich in Größe, Gewicht und Wirkungsgrad. Das Gewicht der befüllten Absorberrohre (ohne Tanks, Dämmung und Tragstruktur) ist beim Referenz-Salzreceiver um den Faktor 2.75 höher. Trotz der höheren maximalen Rohrtemperatur beim Natriumsystem sind die Wärmeverluste aufgrund der reduzierten Absorberfläche um über $35 \%$ geringer, was sich in einem höheren Receiverwirkungsgrad äußert. Die Reynolds-Zahlen im Natriumreceiver sind am Auslegungspunkt gegenüber dem Salzreceiver um den Faktor $\approx 10$ höher. Die Reynolds-Zahl sinkt im Teillastbetrieb nahezu linear mit der Einstrahlung, daher wird die kritische Reynolds-Zahl (laminare Rohrströmung) beim Natriumreceiver erst bei viel 
Tabelle 8.4.: Vergleich zwischen dem Receiver des Referenzsystems Solar Salt und dem Natriumreceiver V3-54 mit $A_{\text {rec }}=660 \mathrm{~m}^{2}$.

\begin{tabular}{|c|c|c|c|}
\hline Variante & $\begin{array}{c}\text { Referenz } \\
\text { Solar Salt }\end{array}$ & $\begin{array}{l}\text { Natrium } \\
\text { V3-54 }\end{array}$ & Einheit \\
\hline Absorberfläche & 1376 & 660 & {$\left[\mathrm{~m}^{2}\right]$} \\
\hline Anzahl seriell durchströmter Module & $2 \times 5$ & $2 \times 3$ & {$[-]$} \\
\hline Druckverlust (21.3./12 h) & 13.16 & 1.87 & [bar] \\
\hline Anzahl Rohre pro Modul & 142 & 131 & {$[-]$} \\
\hline Rohrinnendurchmesser & 32.8 & 50 & {$[\mathrm{~mm}]$} \\
\hline Rohrwandstärke & 1.4 & 1.4 & {$[\mathrm{~mm}]$} \\
\hline Gesamtmasse Absorberrohre leer & 65 & 24.1 & {$[t]$} \\
\hline Masse des WTMs im Receiver & 82.3 & 29.4 & {$[\mathrm{t}]$} \\
\hline Min./Max. Strömungsgeschwindigkeit ${ }^{a}$ & $3.79 / 4.18$ & $4.41 / 4.63$ & {$[\mathrm{~m} / \mathrm{s}]$} \\
\hline Min. Reynolds-Zahl ${ }^{a}$ & 68000 & 569000 & {$[-]$} \\
\hline Min./Max. Wärmeübergangskoeffizient ${ }^{a}$ & $7.9 / 12.7$ & $29.2 / 32.0$ & {$\left[\mathrm{~kW} / \mathrm{m}^{2} / \mathrm{K}\right]$} \\
\hline Verweilzeit des WTMs im Receiver ${ }^{a}$ & 55 & 16 & {$[\mathrm{~s}]$} \\
\hline Receivereintrittstemperatur & 290 & 300 & $\left.{ }^{\circ} \mathrm{C}\right]$ \\
\hline Receiveraustrittstemperatur & 565 & 575 & $\left.{ }^{\circ} \mathrm{C}\right]$ \\
\hline Maximale Rohrtemperatur ${ }^{b}$ & 628 & 689 & {$\left[{ }^{\circ} \mathrm{C}\right]$} \\
\hline Maximale Wärmestromdichte $^{a}$ & 1.1 & 3.11 & {$\left[\mathrm{MW} / \mathrm{m}^{2}\right]$} \\
\hline Absoluter Absorptionsgrad ${ }^{a, d}$ & 94.8 & 94.7 & {$[\%]$} \\
\hline Von Rohren absorbierte Leistung $^{a}$ & 721.4 & 711.7 & {$\left[\mathrm{MW}_{\mathrm{th}}\right]$} \\
\hline Ans Fluid übertragene Leistung ${ }^{a}$ & 699.7 & 699.9 & {$\left[\mathrm{MW}_{\mathrm{th}}\right]$} \\
\hline Wärmeverluste gesamt ${ }^{a}$ & 42.5 & 27.4 & {$\left[\mathrm{MW}_{\mathrm{th}}\right]$} \\
\hline Receiverwirkungsgrad ${ }^{a, c}$ & 91.9 & 93.1 & {$[\%]$} \\
\hline
\end{tabular}

${ }^{a}$ Am 21.3. um $12 \mathrm{~h}$

${ }^{b}$ Am 21.3. um $9 \mathrm{~h}$

${ }^{c}$ Von Natrium absorbiert / Real auf Rohre einfallend

${ }^{d}$ Absorptionsgrad des Rohrmaterials bzw. der Beschichtung: 0.93

geringerer Einstrahlung erreicht, wodurch der Betriebsbereich und damit der Ertrag gesteigert wird.

Ein weiterer interessanter Aspekt beim Betriebsverhalten ist die Verweilzeit des WTM im Receiver. Die in dieser Arbeit durchgeführte Ertragsrechnung ignoriert das dynamische Receiververhalten und geht davon aus, dass der Receiver stets exakt auf die Austrittstemperatur von $565{ }^{\circ} \mathrm{C}$ bei Salz und $575{ }^{\circ} \mathrm{C}$ bei Natrium geregelt wird. Die deutlich kürzere Verweilzeit im Natriumreceiver von $16 \mathrm{~s}$ gegenüber den $55 \mathrm{~s}$ beim Salzreceiver führt beim dynamischen Verhalten und der Regelung zu Vorteilen. So kann bei Gradienten in der Einstrahlung (z.B. infolge 
eines Wolkendurchgangs) der Massenstrom und damit die Austrittstemperatur deutlich schneller angeglichen werden. Insbesondere im Teillastbereich bei noch längeren Verweilzeiten erhöht sich die Trägheit des Regelkreises beim Salzsystem weiter. Durch die ungenaue Vorhersage der Wolkenbewegung und damit der Wärmestromdichteverteilung werden Salzreceiver bei Bewölkung zur Vermeidung von zu hohen Salztemperaturen im sogenannten cloud standby betrieben, bei welchem der Massenstrom derart eingestellt wird, dass sich ohne Wolken eine Salztemperatur von $510{ }^{\circ} \mathrm{C}$ einstellen würde [52]. Diese Sicherheitseinstellung vermindert den Jahresertrag bei Salzreceivern. Beim Natriumreceiver existiert einerseits diese Temperaturbeschränkung nicht und andererseits kann durch die kürzere Verweilzeit im Receiver auch die Austrittstemperatur genauer geregelt werden. Dieser zusätzliche Vorteil von Natrium ist in dieser Arbeit nicht berücksichtigt.

\subsection{Vergleich von Einturm- u. Mehrturmsystemen}

In der vorliegenden Arbeit wurden sowohl Einturm- als auch Mehrturmsysteme untersucht. Beim relativen Vergleich der Konzepte (Einturmsystem Salz zu Einturmsystem Natrium sowie Mehrturmsystem Salz zu Mehrturmsystem Natrium) spielt die Genauigkeit der verwendeten Kostenkorrelationen eine untergeordnete Rolle, wodurch die Unsicherheit der Ergebnisse sehr gering ist. Im absoluten Vergleich (Mehrturm- zu Einturmsysteme) spielen die absoluten Kosten (vor allem der Verbindungsleitungen) eine wichtige Rolle. Die vorliegende Arbeit kann daher keine detaillierte Aussage dazu geben, ob Einturmsysteme oder Mehrturmsysteme geringere LCOE aufweisen.

Unter der Voraussetzung, dass sämtliche Kostenkorrelationen Gültigkeit haben können die Kosten der Verbindungsleitungen ermittelt werden, bei denen das Mehrturmsystem in den gleichen LCOE resultiert wie das Einturmsystem. Diese Kostengleichheit wird im vorliegenden Fall bei spezifischen Leitungskosten von durchschnittlich $1730 € / \mathrm{m}$ erreicht. In diesem Fall bleibt für das Mehrturmsystem der Vorteil in der Betriebsweise durch die Modularität. Für höhere Leitungskosten ist das Einturmsystem kostengünstiger. 


\section{Zusammenfassung und Ausblick}

Diese Arbeit befasst sich mit der solarthermischen Stromerzeugung durch Solarturmkraftwerke mit Flüssigmetallen als Wärmeträgermedium. Neben Historie und aktueller Nutzung von Flüssigmetallen werden auch die Stoffeigenschaften und weitere wichtige Betriebseigenschaften und Komponenten im Detail beschrieben und diskutiert und mit dem Wärmeträgermedium Solar Salt verglichen. Die Verwendung von Flüssigmetallen wirkt sich nicht nur auf den Receiver, sondern auf das gesamte Kraftwerk aus. So ergeben sich beispielsweise durch die reduzierte Absorberfläche von Flüssigmetallreceivern andere Feldaufstellungen. Der Schwerpunkt dieser Arbeit liegt auf einem Vergleich zwischen der aktuell vorherrschenden Flüssigsalztechnologie und der Verwendung von Flüssigmetallen als Wärmeträgermedium. Durch Unsicherheiten in Kosten und Betriebscharakteristiken verschiedener Komponenten wird dieser Vergleich immer schwieriger, je unterschiedlicher die Kraftwerke aufgebaut sind. Um diese Unsicherheiten so weit wie möglich zu eliminieren beschränkt sich die hier durchgeführte Potenzialstudie auf das optische System und den Receiverkreislauf. Der thermische Energiespeicher mit Flüssigsalz und der Kraftwerksblock bleiben bei allen Konzepten und Varianten unverändert ${ }^{3}$, wohl wissend, dass mit höherwertigen Kraftwerksprozessen eine weitere Kostensenkung möglich ist. Somit stellt diese Arbeit die untere Grenze des Kostenreduktionspotenzials dar. Zusätzliche Maßnahmen wie höherwertige Dampfprozesse, Gasturbinen und Hochtemperaturenergiespeicher können die Stromgestehungskosten weiter senken.

Selbst unter dieser Prämisse (ohne Veränderung des Kraftwerksblocks) zeigen die Jahresertrags- und LCOE-Berechnungen für Flüssigmetalle bereits ein großes Potenzial zur Reduktion der Stromgestehungskosten. Bezüglich des Referenzkonzepts mit externem Salzreceiver können mit externen Natriumreceivern

\footnotetext{
${ }^{3}$ Beim thermischen Energiespeicher wird lediglich die Speicherkapazität variiert, um die geringsten Stromgestehungskosten der jeweiligen Variante zu ermitteln.
} 
die LCOE um bis zu $11 \%$ bei Mehrturmsystemen (5x140 $\mathrm{MW}_{\text {th}}$-Receiver) und um bis zu $16 \%$ bei Einturmsystemen $\left(1 \times 700 \mathrm{MW}_{\text {th }}\right.$-Receiver $)$ reduziert werden. Das Kostenreduktionspotenzial ist bei Einturmsystemen höher, da die höheren Türme beim Salzsystem relativ gesehen zu höheren Pumpenleistungen führen. Die geringeren LCOE der Flüssigmetallsysteme resultieren zu einem Teil aus dem höheren Jahresertrag von $\approx 2 \%$ gegenüber dem Referenz-Salzsystem. Hauptverantwortlich für die Ertragssteigerung sind die geringen Druckverluste des Natriumreceivers (Faktor 7-18 geringer gegenüber Salzreceiver) und die Verwendung eines geschlossenen Flüssigmetallkreislaufs ${ }^{4}$. Der Hauptbeitrag zur Senkung der LCOE ensteht durch die geringeren Receiverkosten. Im Vergleich zum Referenz-Salzreceiver kann beim Natriumreceiver gleicher Leistung die Absorberfläche um etwa die Hälfte reduziert werden bei gleichzeitiger Reduktion des Receivergewichts um ca. $60 \%$. Mit der verwendeten Receiverkostenfunktion $K_{\text {rec }, 2}$ reduzieren sich die Receiverkosten des Natriumsystems um $48 \%$ gegenüber dem Salzreceiver. Trotz des notwendigen Wärmeübertragers auf Solar Salt sind dadurch die Gesamtkosten der Anlage um ca. $8 \%$ geringer als beim Referenz-Salzsystem.

In der vorliegenden Arbeit wurde das dynamisches Verhalten des Receivers nicht berücksichtigt. Mit Einbeziehung der An- und Abfahrvorgänge kann die Ertragsrechnung weiter detailliert werden. Dazu gehört die Simulation von Befüll- und Entleerungsvorgängen, die Massenstromregelung bei fluktuierender Einstrahlung sowie die Optimierung der Betriebsgrenzen. Durch die niedrige Schmelztemeratur von $98^{\circ} \mathrm{C}$ und die kürzere Verweilzeit von Natrium im Receiver ergibt sich vermutlich gegenüber Solar Salt ein weiterer Vorteil. Im Falle von Natrium ist insbesondere die Option einer Zirkulation des WTM bis zum Einfrieren mit solarem Auftauen der Absorberrohre interessant. Mit dieser Betriebsweise könnte die Anfahrzeit bis zur Betriebsbereitschaft reduziert und der Ertrag weiter gesteigert werden.

Die exzellenten Wärmeübertragungseigenschaften von Natrium sind unbestritten. In dieser Arbeit konnte außerdem ein großes Potenzial zur Senkung der Stromgestehungskosten identifiziert werden. Der einzige Nachteil und Hauptgrund für die bisher eingeschränkte Nutzung von Natrium ist die hohe Reaktivität mit

\footnotetext{
${ }^{4}$ Salzkreisläufe werden aufgrund des thermischen Speichers als offene Kreisläufe betrieben. Die Förderpumpe muss daher die Turmhöhe überwinden, was eine hohe Leistung erfordert.
} 
Wasser und Sauerstoff. Im Laufe der letzten 30 Jahre wurden zahlreiche Sicherheitsrichtlinien für den Betrieb von Alkalimetallkreisläufen entwickelt. Dennoch können Unfälle beim Umgang mit Alkalimetallen nicht ausgeschlossen werden. Eine weitere Steigerung der Sicherheit könnten hermetisch geschlossene Kreisläufe bieten. Elektromagnetische Pumpen werden bereits zur berührungslosen Förderung von Natrium eingesetzt. Dasselbe Prinzip könnte auch für Ventile zur Massenstromregelung angewendet werden. Damit könnten herkömmliche Ventile, die im Betrieb stets eine gewisse Leckage aufweisen und ein Sicherheitsrisiko darstellen! vermieden werden. Eine weitere Möglichkeit für einen hermetischen Natriumkreislauf könnten Wärmerohre (engl. heat pipes) bieten, die sogar ohne Förderpumpe im Naturumlauf betrieben werden können (siehe auch [81]).

Nach [120 ergeben sich bei Solarturmkraftwerken mit einem Konzentrationsfaktor von $C=1000$ optimale Systemwirkungsgrade bei einer Receivertemperatur von $\approx 850^{\circ} \mathrm{C}$. Höhere Prozesstemperaturen stellen hohe Materialanforderungen an die Turbinen und sind daher bisher oft nicht wirtschaftlich 121. Gleichzeitig können höhere Prozesstemperaturen auf Seiten der Wärmegestehung, insbesondere bei der thermohydraulischen Auslegung von Flüssigmetallreceivern von Vorteil sein, sodass sich auch hier geringere LCOE ergeben könnten. Im Kapitel 4 sind zudem verschiedene innovative Kraftwerkskonzepte mit Flüssigmetallreceivern dargestellt, deren weitere Erforschung ebenfalls zu einer Kostensenkung führen könnte. Da Stickstoff und Natrium chemisch inert sind, könnte die Kombination mit einer $\mathrm{N}_{2}$-Gasturbine insbesondere aus sicherheitstechnischen Gründen eine interessante Lösung bieten.

\footnotetext{
${ }^{5}$ Der Grund für den Natriumbrand in Almería war ein undichtes Ventil (siehe Abschnitt 3.2.2.3). Außerdem stellen Ventile ein Strömungshindernis dar und verzögern den Abfluss in den Drainagetank im Falle einer Notentleerung.
} 



\section{A. Stoffwerte}

Verwendete Korrelationen zur Berechnung der Stoffwerte der verschiedenen WTM.

Solar Salt mit $T$ in ${ }^{\circ} \mathrm{C}$ nach Pacheco 42 :

$$
\begin{aligned}
& \rho=-0.636 \cdot T+2090 \quad\left[\mathrm{~kg} / \mathrm{m}^{3}\right] \\
& c p=1443+0.172 \cdot T \quad[\mathrm{~J} / \mathrm{kg} / \mathrm{K}] \\
& \lambda=0.443+1.9 \cdot 10^{-4} \cdot T \quad[\mathrm{~W} / \mathrm{m} / \mathrm{K}] \\
& \eta=22.714-0.12 \cdot T+2.28 \cdot 10^{-4} \cdot T^{2}-1.474 \cdot 10^{-7} \cdot T^{3} \quad[\mathrm{kPa} \mathrm{s}]=[\mathrm{t} / \mathrm{s} / \mathrm{m}]
\end{aligned}
$$

Hitec mit $T$ in ${ }^{\circ} \mathrm{C}$ nach Boerema $[6]$ :

$$
\begin{aligned}
& \rho=-0.733 \cdot T+2080 \quad\left[\mathrm{~kg} / \mathrm{m}^{3}\right] \\
& c p=1000 \cdot \frac{1.56-T}{1000} \quad[\mathrm{~J} / \mathrm{kg} / \mathrm{K}] \\
& \lambda=0.78-1.25 \cdot 10^{-3} \cdot(T-273.15)+1.6 \cdot 10^{-6} \cdot(T-273.15)^{2} \quad[\mathrm{~W} / \mathrm{m} / \mathrm{K}] \\
& b=5.9 \cdot \frac{(T-273.15)-9.638}{990.362} \\
& \eta=\frac{e^{b}+e^{-b}}{e^{b}-e^{-b}}-0.999 \quad[\mathrm{~Pa} \mathrm{~s}]=[\mathrm{kg} / \mathrm{s} / \mathrm{m}]
\end{aligned}
$$

Natrium mit $T$ in K nach Fink und Leibowitz [41:

$$
\begin{array}{ll}
\rho=219+275.32 \cdot\left(1-\frac{T}{2503.7}+511.58 \cdot\left(1-\frac{T}{2503.7}\right)^{0.5} \quad\left[\mathrm{~kg} / \mathrm{m}^{3}\right]\right. \\
c p=1.6582-8.479 \cdot 10^{-4} \cdot T+4.4541 \cdot 10^{-7} \cdot T^{2}-2992.6 \cdot T^{-2} & {[\mathrm{~kJ} / \mathrm{kg} / \mathrm{K}]} \\
\lambda=124.67-0.11381 \cdot T+5.5226 \cdot 10^{-5} \cdot T^{2}-1.1842 \cdot 10^{-8} \cdot T^{3} & {[\mathrm{~W} / \mathrm{m} / \mathrm{K}]} \\
\eta=e^{6.4406-0.3958 \cdot \ln (T)+\frac{556.835}{T}} \quad[\mathrm{~Pa} \mathrm{~s}]=[\mathrm{kg} / \mathrm{s} / \mathrm{m}] &
\end{array}
$$


Blei mit $T$ in $\mathrm{K}$ nach dem LBE-Handbuch [43]:

$$
\begin{aligned}
& \rho=11367-1.1944 \cdot T \quad\left[\mathrm{~kg} / \mathrm{m}^{3}\right] \\
& c p=175.1-4.961 \cdot 10^{-2} \cdot T+1.985 \cdot 10^{-5} \cdot T^{2}-2.099 \cdot 10^{-9} \cdot T^{3} \\
& -1.524 \cdot 10^{6} \cdot T^{-2} \quad[\mathrm{~J} / \mathrm{kg} / \mathrm{K}] \\
& \lambda=9.2+0.011 \cdot T \quad[\mathrm{~W} / \mathrm{m} / \mathrm{K}] \\
& \eta=4.55 \cdot 10^{-4} \cdot e^{\frac{1069}{T}} \quad[\mathrm{~Pa} \mathrm{~s}]=[\mathrm{kg} / \mathrm{s} / \mathrm{m}]
\end{aligned}
$$

Blei-Bismut (LBE) mit $T$ in $\mathrm{K}$ nach dem LBE-Handbuch [43]:

$$
\begin{array}{lrr}
\rho=11096-1.3236 \cdot T \quad\left[\mathrm{~kg} / \mathrm{m}^{3}\right] & \\
c p=159-2.72 \cdot 10^{-2} \cdot T+7.12 \cdot 10^{-6} \cdot T[\mathrm{~K}]^{2} & {[\mathrm{~J} / \mathrm{kg} / \mathrm{K}]} \\
\lambda=3.61+1.517 \cdot 10^{-2} \cdot T-1.741 \cdot 10^{-6} \cdot T^{2} & {[\mathrm{~W} / \mathrm{m} / \mathrm{K}]} \\
\eta=4.94 \cdot 10^{-4} \cdot e^{\frac{754.1}{T}} \quad[\mathrm{~Pa} \mathrm{~s}]=[\mathrm{kg} / \mathrm{s} / \mathrm{m}] &
\end{array}
$$

Zinn mit $T$ in K nach Sharafat [Sharafat2000] und Assael [Assael2010]:

$$
\begin{aligned}
& \rho=7374.7-676.5 \cdot 10^{-3} \cdot T \quad\left[\mathrm{~kg} / \mathrm{m}^{3}\right] \\
& c p=9.97-9.15 \cdot 10^{-3} \cdot T+6.5 \cdot 10^{-6} \cdot T^{2} \quad[\mathrm{cal} / \mathrm{mol} / \mathrm{K}] \\
& \lambda=13.8+0.0133 \cdot(T-273.15) \quad[\mathrm{W} / \mathrm{m} / \mathrm{K}] \\
& \eta=6.547 \cdot 10^{5} \cdot T^{-2.107}+0.549 \quad[\mathrm{kPa} \mathrm{s}]=[\mathrm{t} / \mathrm{s} / \mathrm{m}]
\end{aligned}
$$

Für den Rohrwerkstoff wird Inconel ALLOY617 (2.4663) verwendet 119 

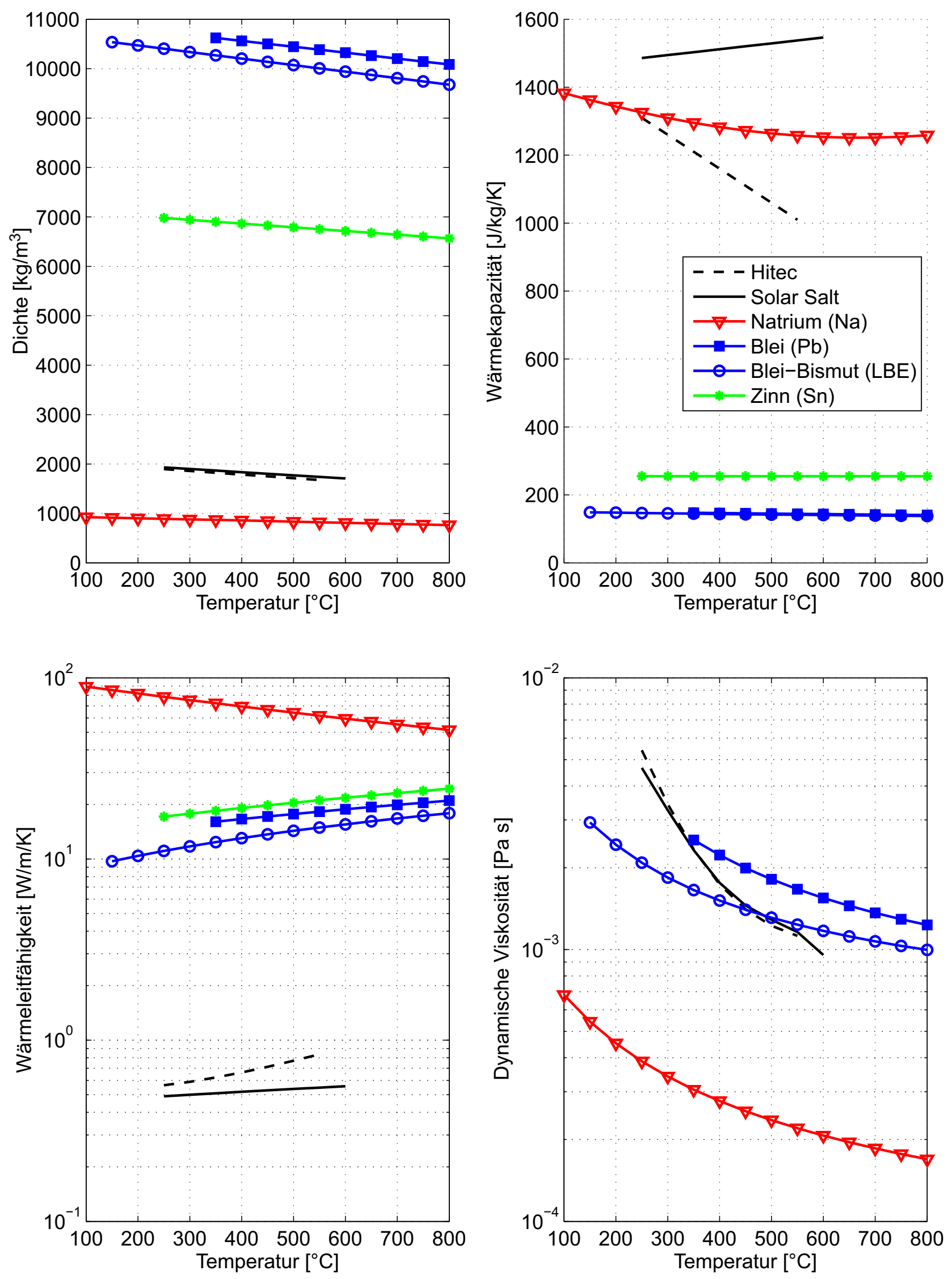



\section{B. Spannungsuntersuchung des Auftauvorgangs eines mit Natrium befüllten Rohres}

(von Ralf Uhlig, Dez. 2016)

Ziel: Analyse der Spannungen in einem Absorberohr, welche durch die Volumenänderung beim Schmelzen von Natrium hervorgerufen werden.

Vorgehensweise/FE-Modell: Modellierung eines Rohrabschnittes mit einem Außendurchmesser von $46 \mathrm{~mm}$, einer Wandstärke von $1 \mathrm{~mm}$ und einer Länge von $0.5 \mathrm{~m}$. Das Natrium sowie das Rohr werden als Festkörper simuliert. Es wird ein Bereich von $0.1 \mathrm{~m}$ Länge als „flüssiges“ Natrium betrachtet während der restliche Teil als fest betrachtet wird.

Die Berücksichtigung der Pressung infolge der Volumenänderung beim Phasenübergang erfolgt durch eine Anpassung des Wärmeausdehnungskoeffizienten. Dieser wurde iterativ so angepasst, dass bei einer Temperatur von $100{ }^{\circ} \mathrm{C}$ eine Volumenzunahme von $2.5 \%$ des „flüssigen“ Natriums resultiert. Die nachfolgenden Plots zeigen die radiale Dehnung (UX) und die Längsdehnung (UZ) des flüssigen Natriums unter Verwendung des angepassten Wärmeausdehnungskoeffizienten.

Adapted thermal expansion Type: Directional Deformation (X Axis) Unit: $\mathrm{mm}$

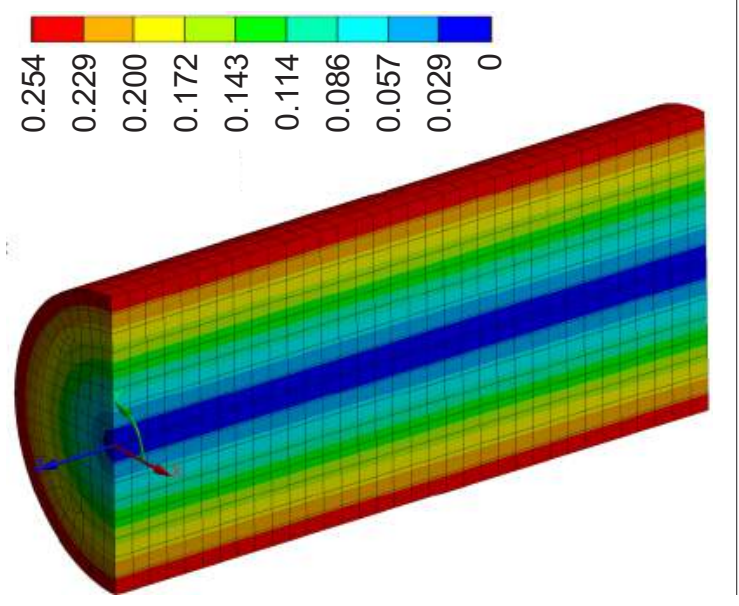

\section{Adapted thermal expansion}

Type: Directional Deformation (Z Axis)

Unit: $\mathrm{mm}$
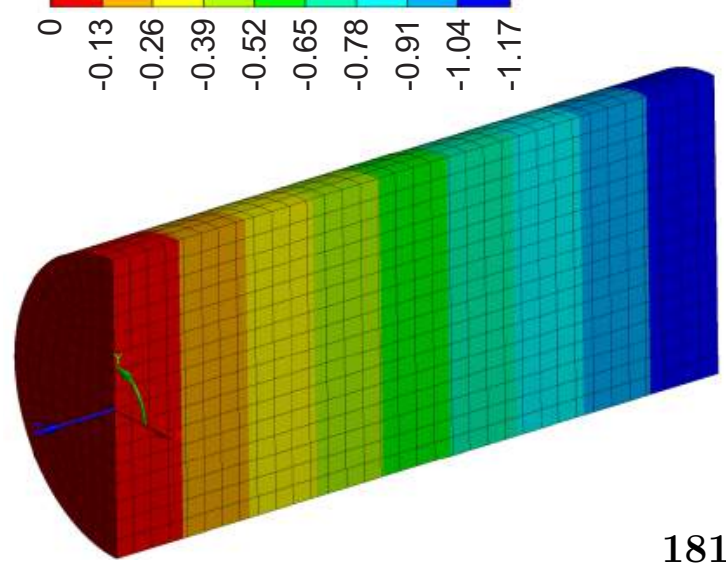


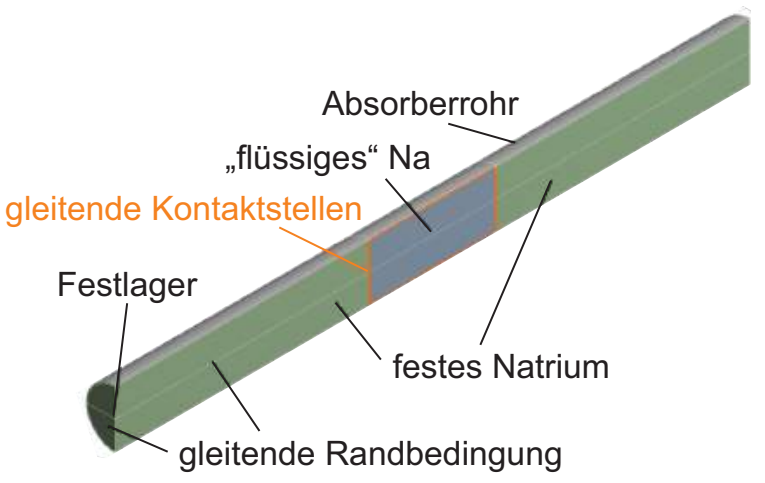

In einem zweiten Schritt wurden für das gesamte Modell (Rohr, festes und flüssiges Natrium) die thermischen Dehnungen und somit die Spannungen im Rohr berechnet. Dazu wurde das gesamte Natrium mit temperaturabhängigen Stoffwerten nach [41] modelliert. Für den flüssigen Teil des Natriums wurde die Wärmedehnung durch den angepassten fiktiven Wärmeausdehnungskoeffizienten $(0.000151 / \mathrm{K})$ ersetzt. Für das Rohr wurden die temperaturabhängigen Stoffwerten für den Werkstoff ALLOY617 [119] verwendet. An den Berührungsflächen zwischen flüssigem und festem Natrium sowie zwischen flüssigem Natrium und Rohrwand wurde eine Kontaktbedingung definiert, die eine Verschiebung der Körper zueinander (aber keine Ablösung) ermöglicht. (Vereinfachung zur sicheren Seite, da durch Volumenzunahme nur Druck zwischen den Körpern auftritt). An der Symmetriefläche und an der Stirnfläche wurde eine Randbedingung modelliert die eine Verschiebung innerhalb dieser Ebene ermöglicht. An einem Punkt wurde zusätzlich eine Festlagerung definiert.

Die Auswertung der Spannungen ergibt relativ geringe Spannungen von ca. $5 \mathrm{MPa}$. Trotz der starken konservativen Vereinfachung des Modells (harte Grenze zwischen flüssig und fest, keine Aufschmelzung der Randbereiche) liegen die Spannungen deutlich unter der Fließgrenze des Rohrmaterials. Eine Aufschmelzung des Natriums beim Anfahren des Receivers durch Solarstrahlung scheint somit möglich.

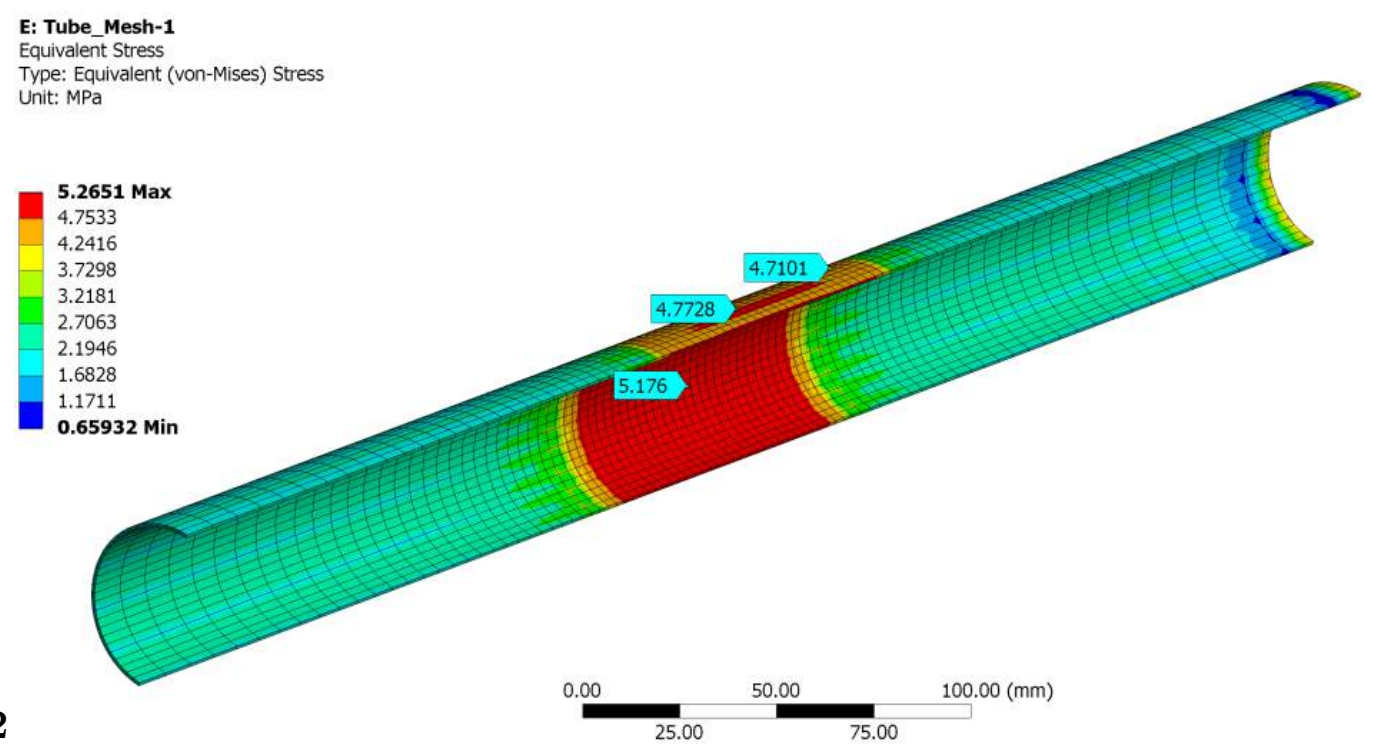




\section{Berechnung der Rohrwandstärken}

Die Wandstärke der Absorberrohre wird mit der Druckgeräterichtlinie nach AD 2000-B1 97 berechnet:

$$
s=\frac{p_{\max } \cdot d_{\mathrm{i}}}{20 \cdot w_{\mathrm{v}} \cdot \frac{\sigma_{\mathrm{zul} \_}}{S_{\mathrm{F}}}+p_{\max }} \quad \text { mit } p_{\max } \text { in }[\mathrm{bar}]
$$

Der Außendurchmesser der Absorberrohre ergibt sich damit zu

$$
d_{\mathrm{a}}=d_{\mathrm{i}}+2 \cdot s
$$

Die Innendurchmesser von Verteiler (V) und Sammler (S) ergeben sich aus der Definition, dass ihre Querschnittsflächen jeweils die Summe aller parallel durchströmten Absorberrohrquerschnittsflächen multipliziert mit einem Skalierungsfaktor sind:

$$
A_{\mathrm{v}, \mathrm{s}}=f_{\mathrm{v}, \mathrm{s}} \cdot \Sigma A_{\mathrm{r}} \quad \rightarrow \quad d_{\mathrm{v}, \mathrm{s}}{ }^{2}=f_{\mathrm{v}, \mathrm{s}} \cdot n_{\mathrm{r}} \cdot d_{\mathrm{i}}^{2}
$$

Die Durchmesser von Verteiler und Sammler ergeben sich daraus zu

$$
d_{\mathrm{v}, \mathrm{s}}=d_{\mathrm{i}} \cdot \sqrt{f_{\mathrm{v}, \mathrm{s}} \cdot n_{\mathrm{r}}}
$$

Für die Wandstärken von Verteiler und Sammler wird ebenfalls die Kesselformel angewendet. Die Durchmesser ergeben sich damit zu:

$$
d_{\mathrm{av}, \mathrm{as}}=d_{\mathrm{v}, \mathrm{s}}+2 \cdot \frac{p_{\max } \cdot d_{\mathrm{v}, \mathrm{s}}}{20 \cdot w_{\mathrm{v}} \cdot \frac{\sigma_{\mathrm{zu} \_\_}}{S_{\mathrm{F}}}+p_{\max }} \quad \text { mit } p_{\max } \text { in }[\mathrm{bar}]
$$





\section{Druckverlustberechnung im Receiver}

Zur Berechnung des Druckverlustes ist der Rohrreibungskoeffizient $\zeta$ entscheidend. Innerhalb des Verteilers, Sammlers und in den Absorberrohren selbst wird der Rohrreibungskoeffizient iterativ über folgende Formel nach VDIWärmeatlas 2006, Seite Lab2, Gl.10 bestimmt:

$$
\frac{1}{\sqrt{\zeta(i)}}=-2 \cdot \log _{10}\left[\frac{2,51}{\operatorname{Re}(i) \cdot \sqrt{\zeta(i)}}+\frac{t}{3,71 \cdot d_{\mathrm{i}}}\right]
$$

Der Übergang vom Verteiler in das Absorberrohr kommt einer plötzlichen Querschnittsverengung gleich. Nach VDI-Wärmeatlas 2006, Seite Lac1, Bild 2 ergibt sich durch lineare Regression nährungsweise (für $R e=10^{4}$ ):

$$
\zeta=-0,4 \cdot\left(\frac{A_{\mathrm{i}}}{A_{\mathrm{v}}}\right)+0,5=-0,4 \cdot\left(\frac{d_{\mathrm{i}}}{d_{\mathrm{v}}}\right)^{2}+0,5
$$

Der Diffusor entspricht einer stetigen Querschnittsverengung. Nach VDIWärmeatlas 2006, Seite Lac3, Bild 8 ergibt sich durch eine Polynomregression 4ter Ordnung:

$$
\zeta=\left[1-\left(\frac{d_{\mathrm{i}}}{d_{d}}\right)^{2}\right]^{2} \cdot\left[2 \cdot 10^{-6} \cdot \delta^{4}-0,0002 \cdot \delta^{3}+0,0065 \cdot \delta^{2}-0,0255 \cdot \delta+0,182\right]
$$

Der Übergang vom Diffusor in den Sammler entspricht einer plötzlichen Querschnittserweiterung. Nach VDI-Wärmeatlas 2006, Seite Lac2, Gl.7 gilt hierfür:

$$
\zeta=\left[1-\left(\frac{A_{d}}{A_{\mathrm{s}}}\right)\right]^{2}=\left[1-\left(\frac{d_{d}}{d_{\mathrm{s}}}\right)^{2}\right]^{2}
$$


Der Druckverlust im Absorberrohr sowie in Verteiler und Sammler wird nach VDI-Wärmeatlas 2006, Seite Lab1, Gl.1 berechnet:

$$
\Delta p(i)=\zeta(i) \cdot \frac{\Delta L}{d_{\mathrm{i}, \mathrm{v}, \mathrm{s}}} \cdot \rho(i) \cdot \frac{u(i)^{2}}{2}
$$

Im Absorberrohr entspricht die charakteristische Länge einem Diskretisierungsabschnitt $\Delta L=L_{\mathrm{bin}}$ mit dem dazugehörigen Durchmesser $d_{\mathrm{i}}$. Im Verteiler und Sammler wird hierfür die halbe Modulbreite $b_{\text {mod }} / 2$ verwendet mit den entsprechenden Innenduchmessern $d_{\mathrm{v}}$ und $d_{\mathrm{s}}$.

Der Übergang zwischen Verteiler und Absorberrohr kommt einer plötzlichen Querschnittsverengung gleich. Der Druckverlust hierfür errechnet sich nach VDI-Wärmeatlas 2006, Seite Lac1, Gl.2:

$$
\Delta p(i)=\zeta(i) \cdot \rho(i) \cdot \frac{u(i+1)^{2}}{2}
$$

Der Übergang zwischen Absorberrohr und Sammler bzw. zwischen Diffusor und Sammler kommt einer plötzlichen Querschnittserweiterung gleich. Der Diffusor selbst hingegen entspricht einer stetigen Querschnittserweiterung. Der Druckverlust dieser beiden Fälle errechnet sich nach VDI-Wärmeatlas 2006, Seite Lac2, Gl.7 und Gl.8 zu:

$$
\Delta p(i)=\zeta(i) \cdot \rho(i) \cdot \frac{u(i)^{2}}{2}
$$




\section{E. Verwendete Nusselt-Korrelationen}

$\mathrm{Nu}$ nach Gnielinski 45 :

Für $10^{4}<\operatorname{Re}(i)<10^{6}$ und $0.1<\operatorname{Pr}(i)<1000$

$$
N u(i)=\frac{\frac{\zeta(i)}{8} \cdot(\operatorname{Re}(i)-1000) \cdot \operatorname{Pr}(i)}{1+12.7 \cdot \sqrt{\frac{\zeta(i)}{8}} \cdot\left(\operatorname{Pr}(i)^{\frac{2}{3}}-1\right)}
$$

Nu nach Gnielinski unter Berücksichtigung der Einlaufstrecke [98]: Für $2300<\operatorname{Re}(i)<10^{4}$ und $0.6<\operatorname{Pr}(i)<1000$

$$
\begin{aligned}
& N u_{2}=1.615 \cdot\left[2300 \cdot \operatorname{Pr}(i) \frac{d_{\mathrm{i}}}{L_{\mathrm{r}}}\right]^{1 / 3} \\
& N u_{3}=\left(\frac{2}{1+22 * \operatorname{Pr}(i)}\right)^{1 / 6} \cdot \sqrt{2300 \cdot \operatorname{Pr}(i) \frac{d_{\mathrm{i}}}{L_{\mathrm{r}}}} \\
& N u_{L}=\left[49.371+\left(N u_{2}-0.7\right)^{3}+N u_{3}^{3}\right]^{1 / 3} \\
& N u_{T}=\frac{(0.0308 / 8) \cdot 10^{4} \operatorname{Pr}(i)}{1+12.7 \cdot \sqrt{(0.0308 / 8)}\left(\operatorname{Pr}(i)^{2 / 3}-1\right)} \cdot\left[1+\left(\frac{d_{\mathrm{i}}}{L_{\mathrm{r}}}\right)^{2 / 3}\right] \\
& \gamma=\frac{\operatorname{Re}(i)-2300}{10^{4}-2300} \\
& N u(i)=(1-\gamma) N u_{L}+\gamma N u_{T}
\end{aligned}
$$


Für $10^{4}<\operatorname{Re}(i)<10^{6}$ und $0.1<\operatorname{Pr}(i)<1000$

$$
\begin{gathered}
\xi=\frac{1}{\left(1.8 \cdot \log _{10} \cdot \operatorname{Re}(i)-1.5\right)^{2}} \\
N u(i)=\frac{(\xi / 8) \cdot \operatorname{Re}(i) \cdot \operatorname{Pr}(i)}{1+12.7 \cdot \sqrt{(\xi / 8)} \cdot\left(\operatorname{Pr}(i)^{2 / 3}-1\right)}\left[1+\frac{1}{3}\left(\frac{d_{\mathrm{i}}}{x}\right)^{2 / 3}\right] \quad \text { mit } x=\left[0 ; L_{\mathrm{r}}\right]
\end{gathered}
$$

Nu nach Hausen 99:

Für $3000<\operatorname{Re}(i)<10^{6}$ und $0.6<\operatorname{Pr}(i)<500$

$$
N u(i)=0.0235 *\left(\operatorname{Re}(i)^{0.8}-230\right) \cdot\left(1.8 \operatorname{Pr}(i)^{0.3}-0.8\right) \cdot\left[1+\left(\frac{d_{\mathrm{i}}}{L_{\mathrm{r}}}\right)^{2 / 3}\right]
$$

Nu für Alkailimetalle ( $\mathrm{Na}, \mathrm{K}, \mathrm{NaK}$ ) nach Chen and Chiou [16]:

$$
N u(i)=5.6+\left(0.0165 * P e(i)^{0.85}\right) \cdot\left(\operatorname{Pr}(i)^{0.01}\right)
$$

Nu für Blei-Bismut (Pb-Bi) nach Lubarsky and Kaufman [16]:

$$
N u(i)=0.625 \cdot \operatorname{Pe}(i)^{0.4}
$$

Nu für Blei $(\mathrm{Pb})$ nach Kutateladze [16]:

$$
N u(i)=5.9+0.015 \cdot P e(i)^{0.8}
$$




\section{F. Receivermodell 502 in HFLCAL}

Das Receivermodell 502 basiert auf dem in HFLCAL hinterlegten StandardModell 101 [106]. Ergänzend dazu kann eine Leistungsbegrenzung im Receiver (minimale und maximale Leistung) sowie einer Reduktion des ReceiverWirkungsgrades auf Grund von parasitären Verlusten berücksichtigt werden. Die entsprechenden Parameter werden im File RecEffAdd.dat definiert:

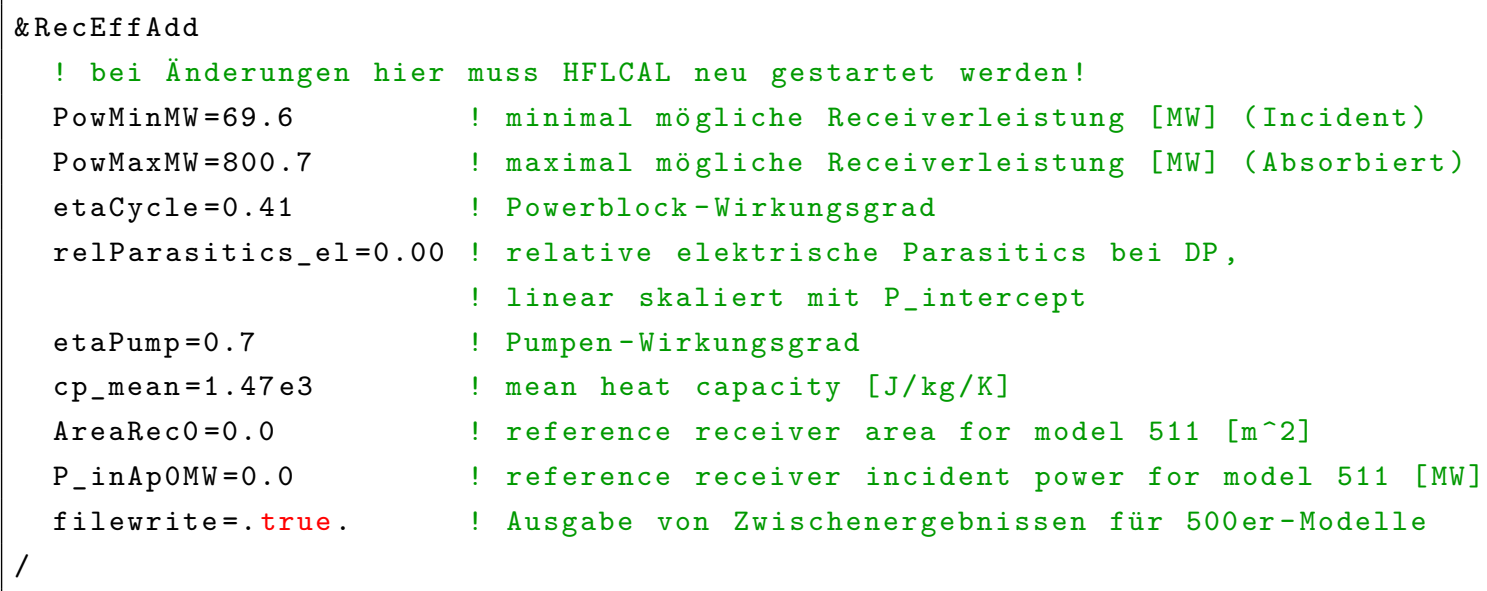

Die Berücksichtigung der turmhöhenabhängigen Pumpenverluste sind im Code folgendermaßen abgebildet:

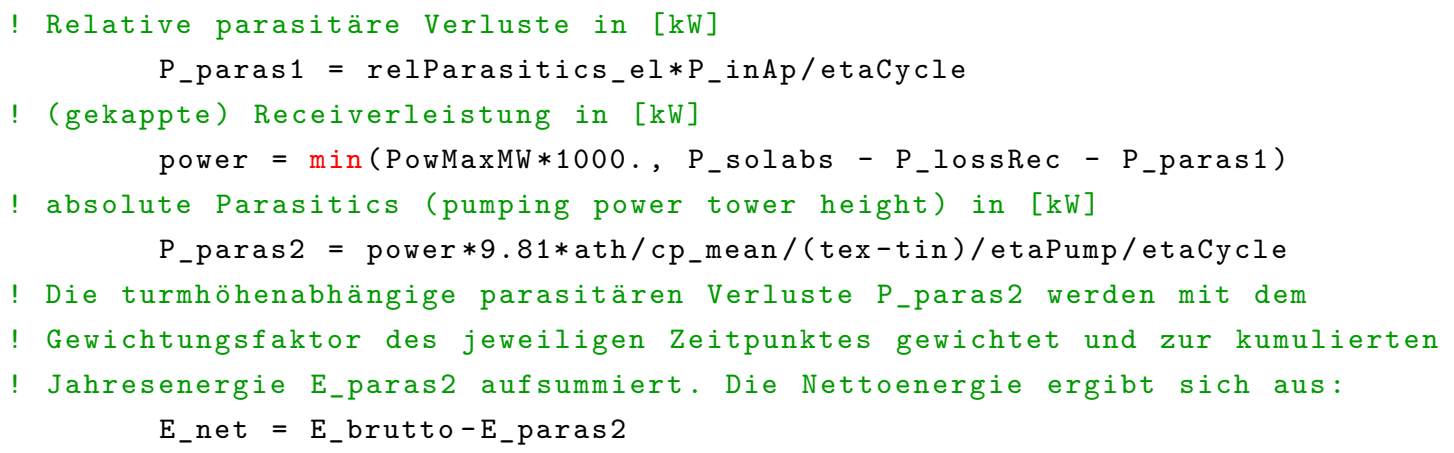





\section{G. Spezifikationstabellen der Mehrturmsysteme}

\section{G.1. Übersicht der Konfigurationen}

\begin{tabular}{|c|c|c|c|c|c|c|}
\hline 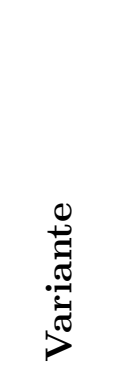 & 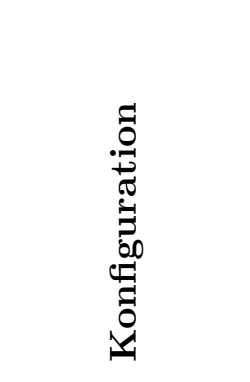 & 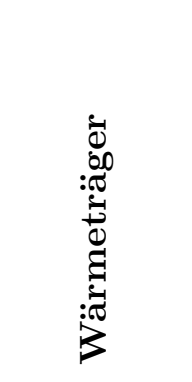 & 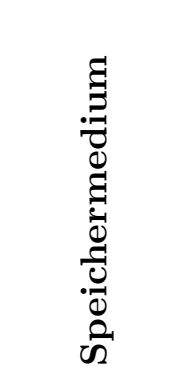 & 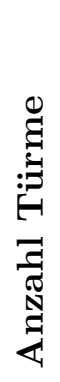 & 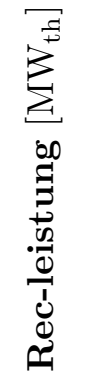 & 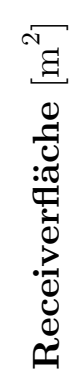 \\
\hline V 1.1 & Referenz Salz & Solar Salt & Solar Salt & 5 & 140 & 275 \\
\hline V 2.11 & Receiver-only & Natrium & Solar Salt & 5 & 140 & 274 \\
\hline V 2.12 & Receiver-only & Natrium & Solar Salt & 5 & 140 & 254 \\
\hline V 2.13 & Receiver-only & Natrium & Solar Salt & 5 & 140 & 227 \\
\hline V 2.14 & Receiver-only & Natrium & Solar Salt & 5 & 140 & 201 \\
\hline V 2.15 & Receiver-only & Natrium & Solar Salt & 5 & 140 & 177 \\
\hline V 2.16 & Receiver-only & Natrium & Solar Salt & 5 & 140 & 154 \\
\hline V 2.17 & Receiver-only & Natrium & Solar Salt & 5 & 140 & 133 \\
\hline V 2.18 & Receiver-only & Natrium & Solar Salt & 5 & 140 & 113 \\
\hline V 2.24 & Receiver-only & LBE & Solar Salt & 5 & 140 & 201 \\
\hline V 2.25 & Receiver-only & $\mathrm{LBE}$ & Solar Salt & 5 & 140 & 177 \\
\hline V 2.26 & Receiver-only & LBE & Solar Salt & 5 & 140 & 154 \\
\hline V 2.27 & Receiver-only & $\mathrm{LBE}$ & Solar Salt & 5 & 140 & 133 \\
\hline V 3.11 & Tower-loop & Natrium & Solar Salt & 5 & 140 & 274 \\
\hline V 3.12 & Tower-loop & Natrium & Solar Salt & 5 & 140 & 254 \\
\hline V 3.13 & Tower-loop & Natrium & Solar Salt & 5 & 140 & 227 \\
\hline V 3.14 & Tower-loop & Natrium & Solar Salt & 5 & 140 & 201 \\
\hline V 3.15 & Tower-loop & Natrium & Solar Salt & 5 & 140 & 177 \\
\hline V 3.16 & Tower-loop & Natrium & Solar Salt & 5 & 140 & 154 \\
\hline V 3.17 & Tower-loop & Natrium & Solar Salt & 5 & 140 & 133 \\
\hline V 3.18 & Tower-loop & Natrium & Solar Salt & 5 & 140 & 113 \\
\hline
\end{tabular}

Betriebsbereiche der Medien: Solar Salt $\left(290-565^{\circ} \mathrm{C}\right)$, Natrium und LBE $\left(300-575{ }^{\circ} \mathrm{C}\right)$ 


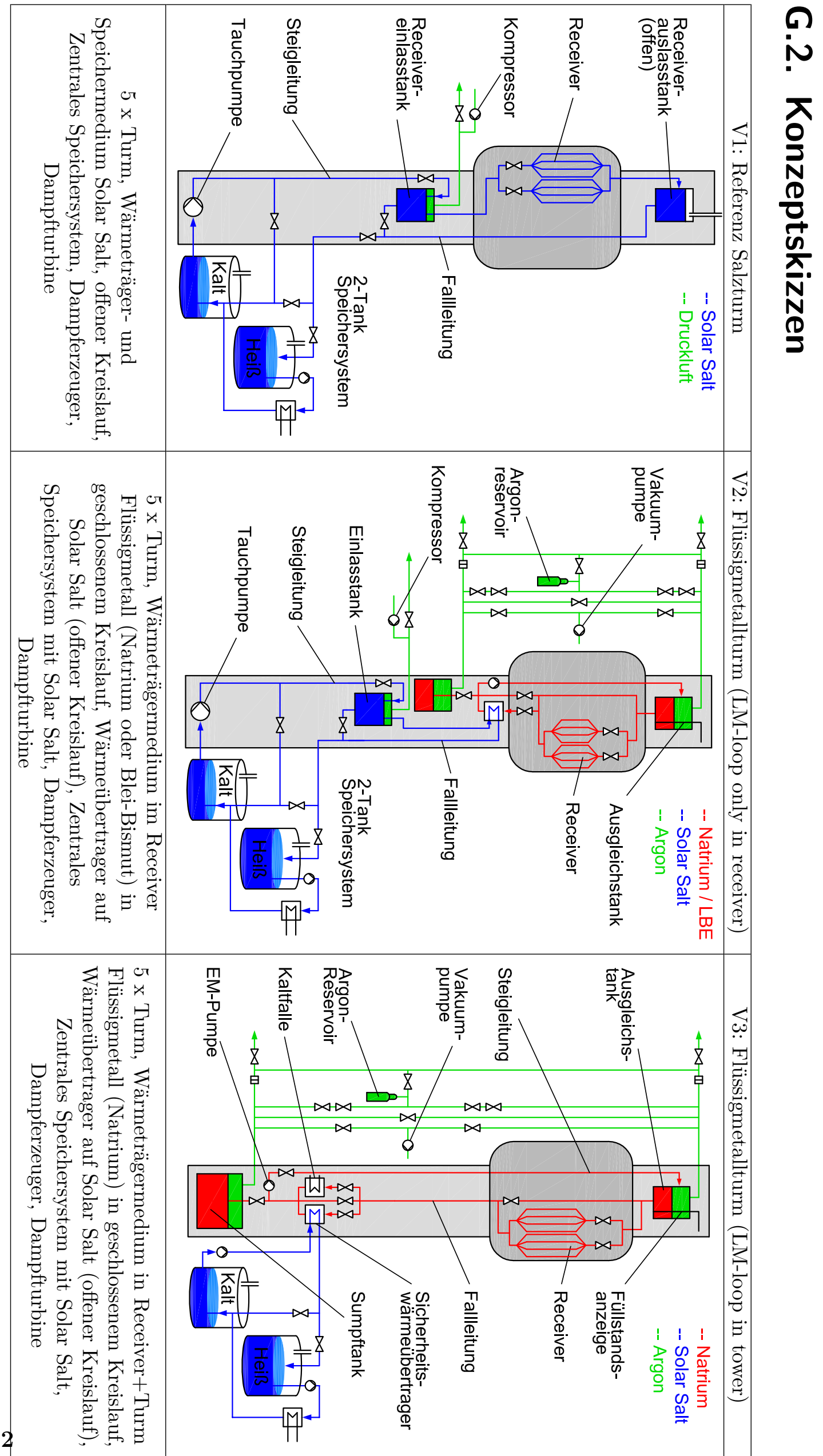




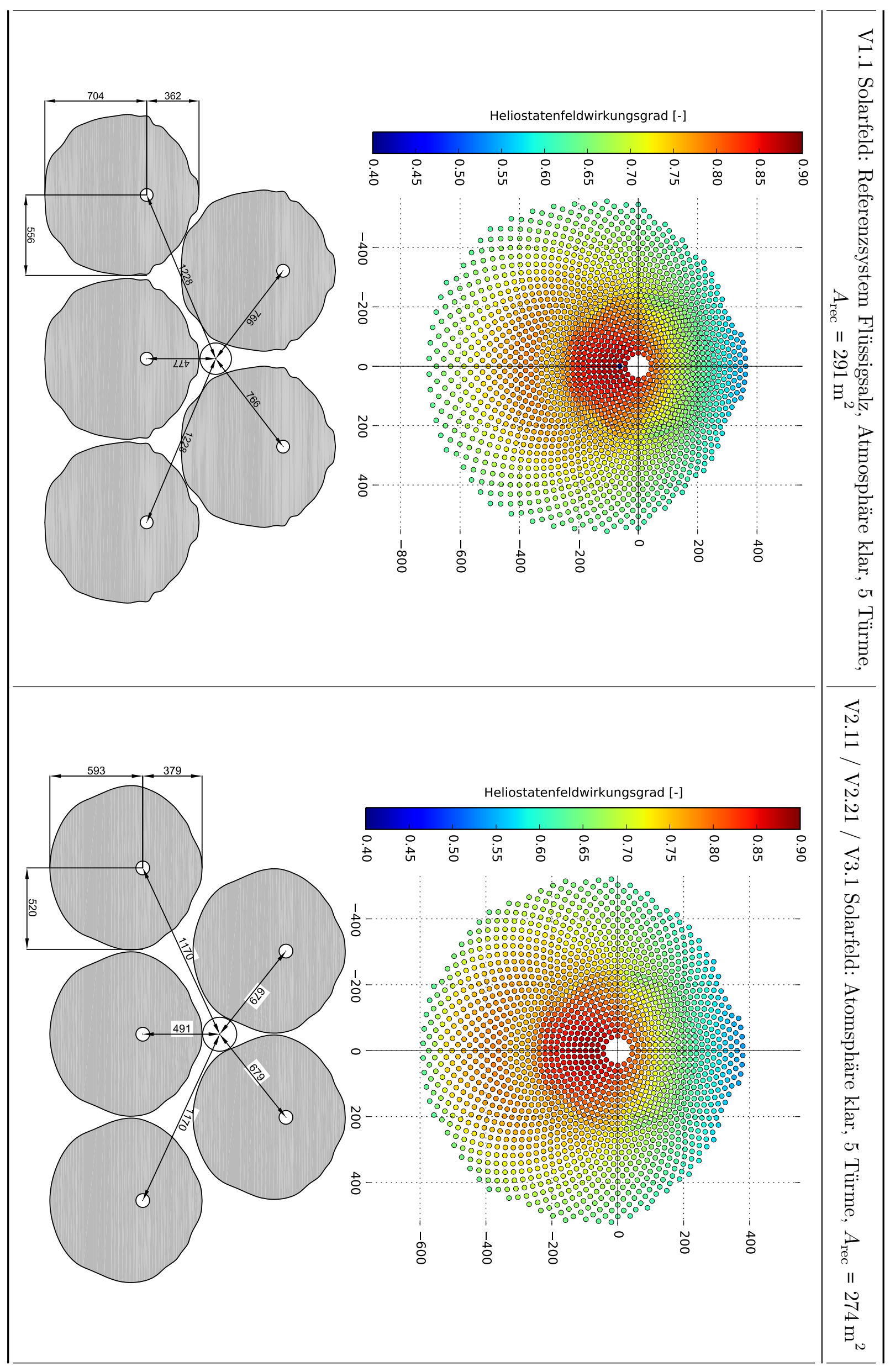




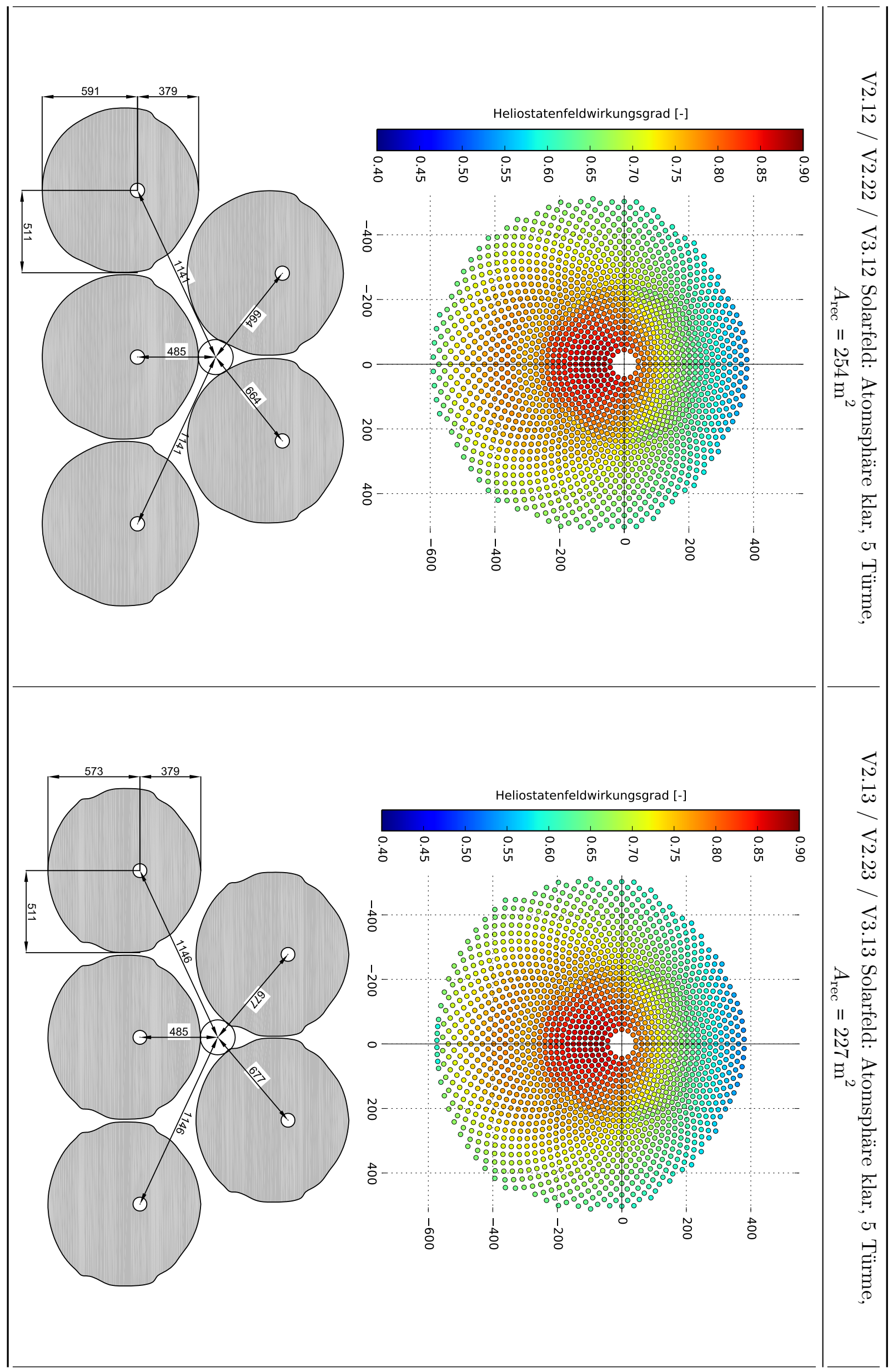




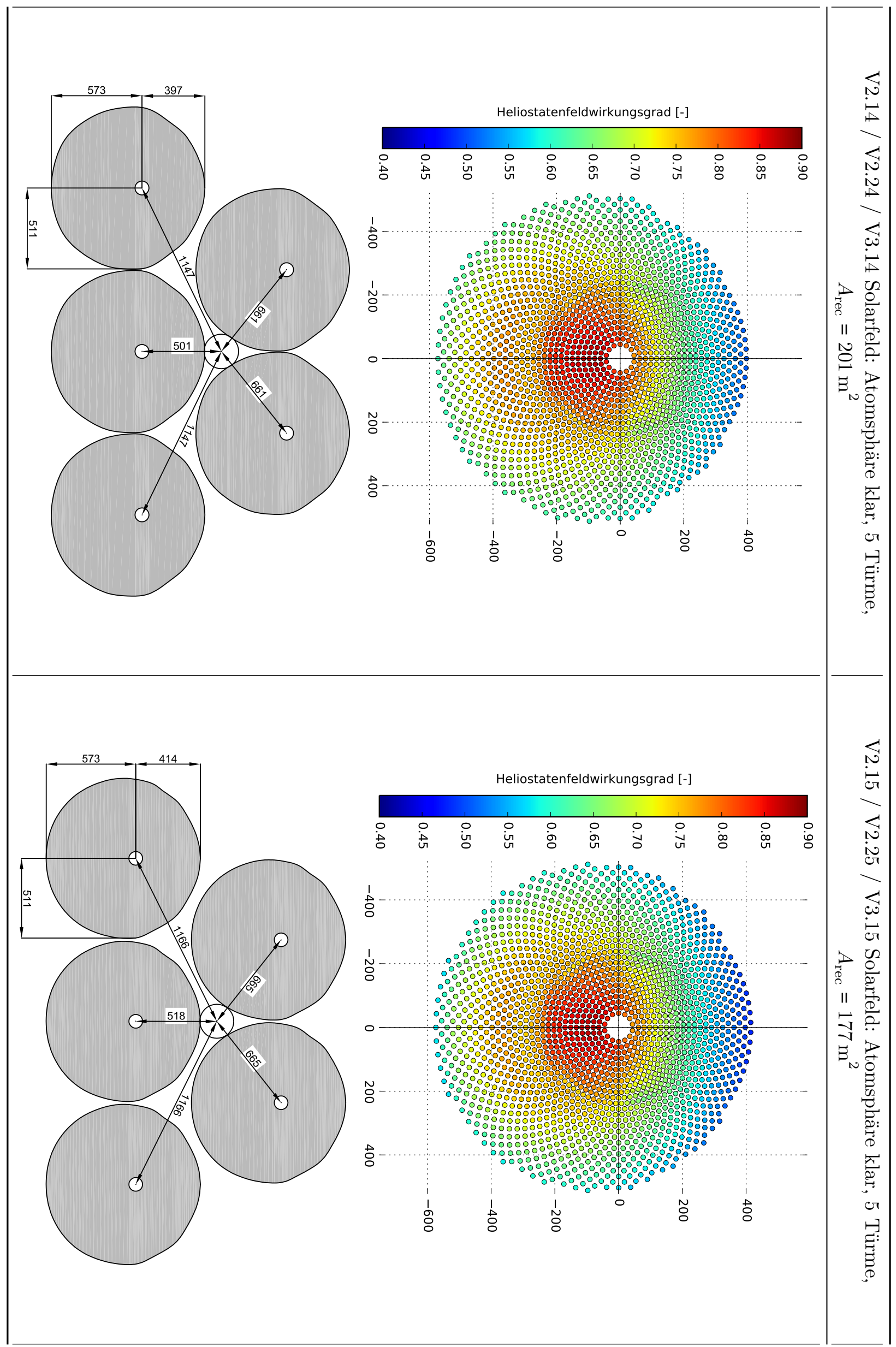




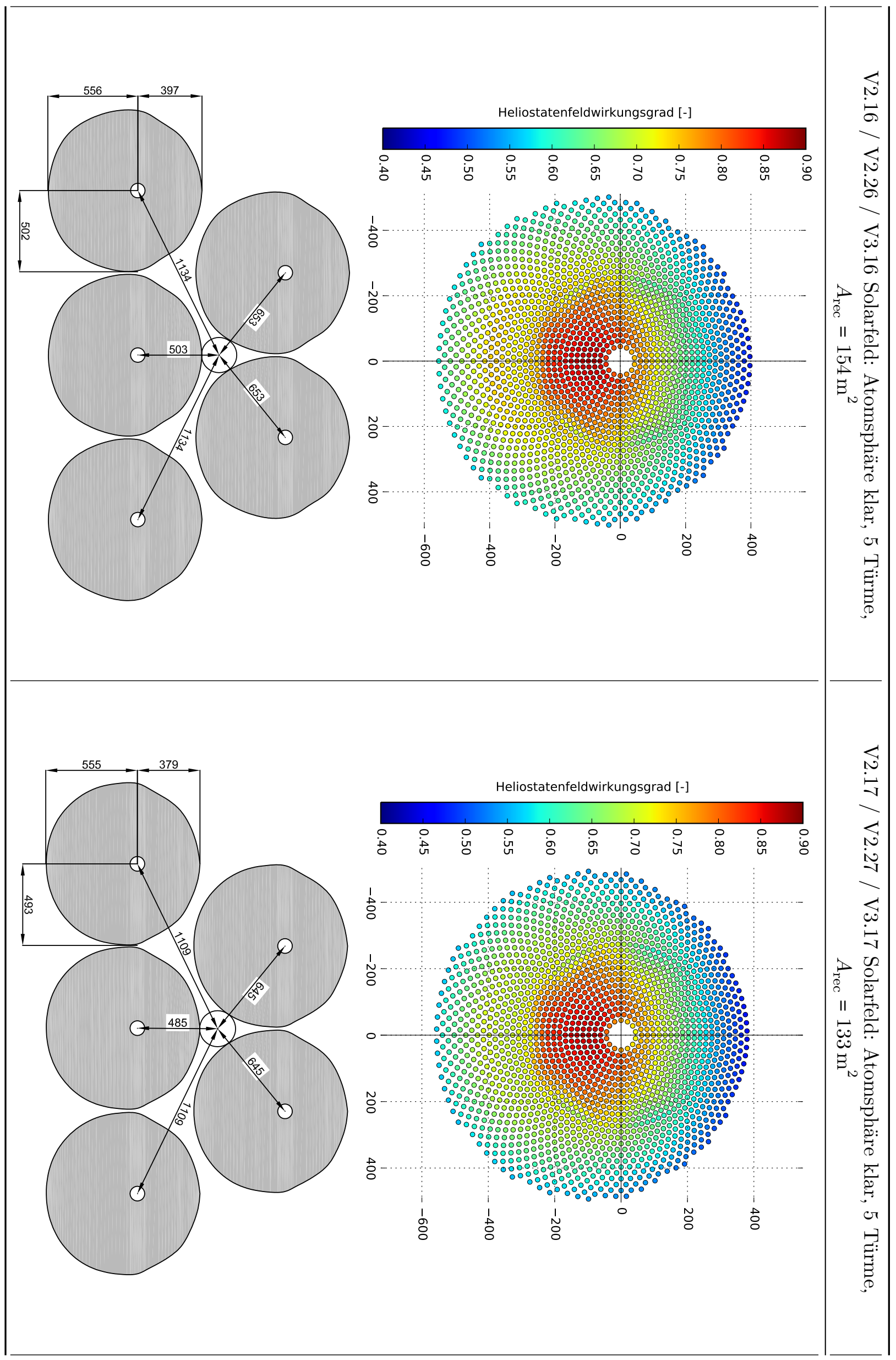




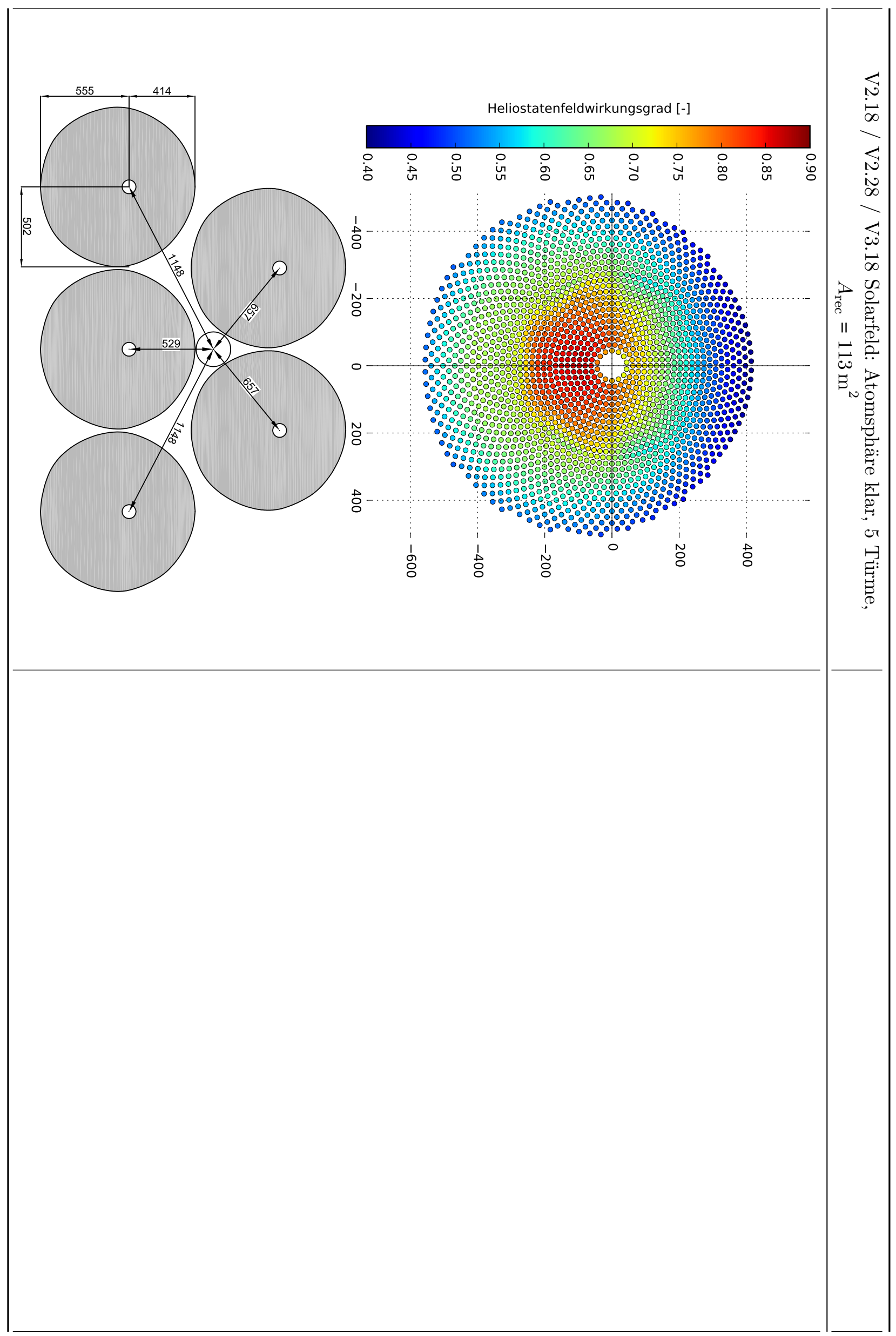




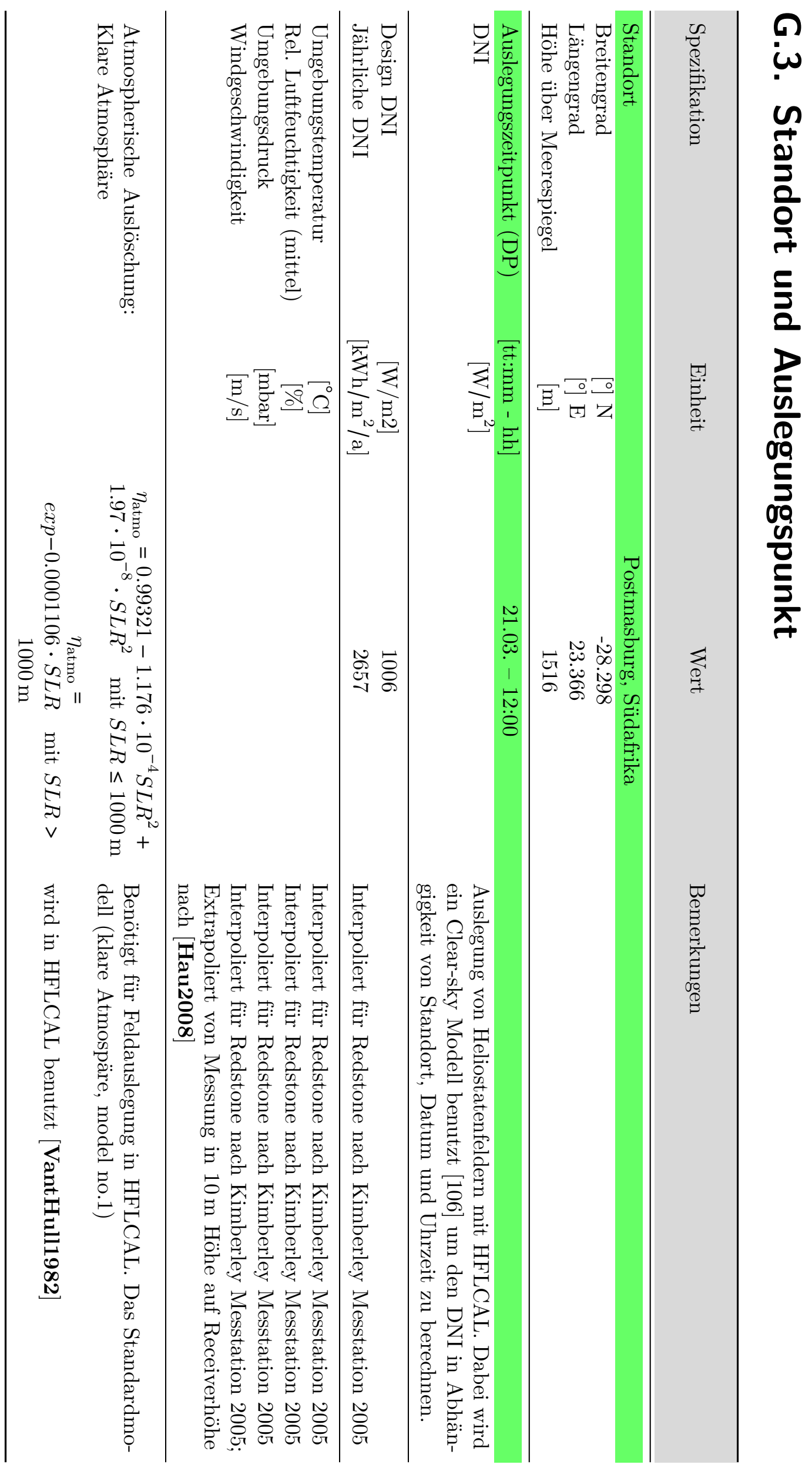




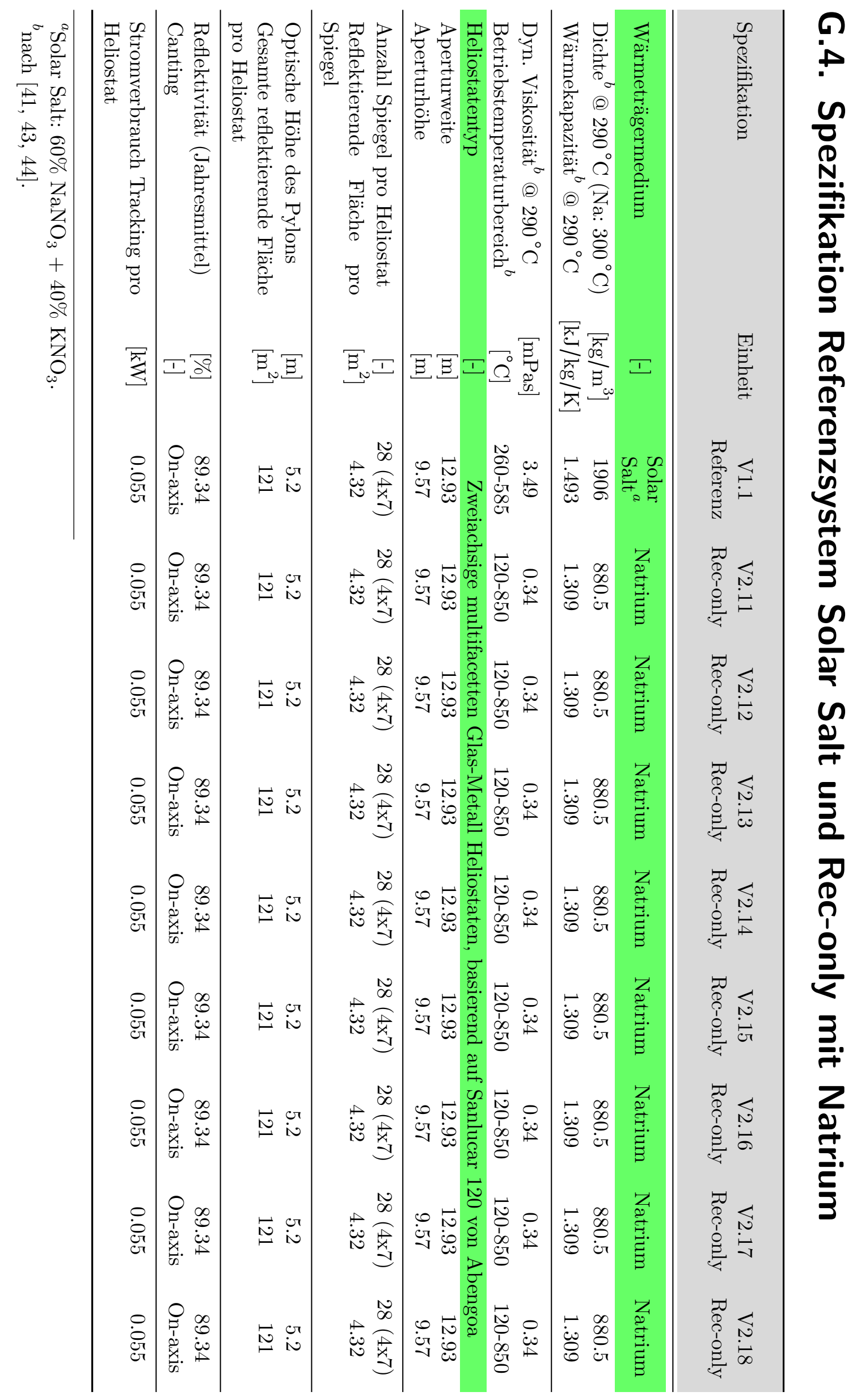




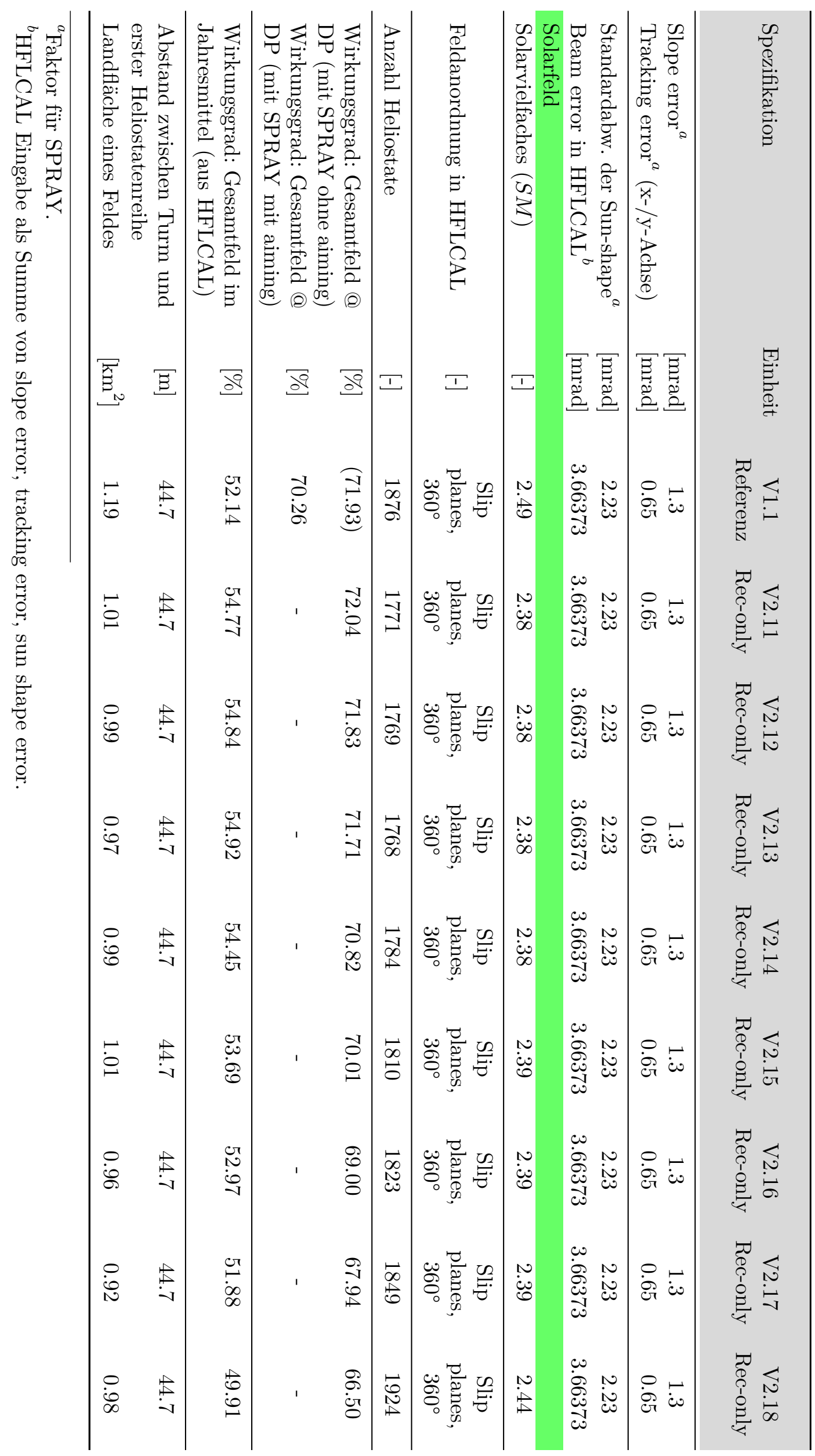




\begin{tabular}{|c|c|c|c|c|c|c|c|c|c|}
\hline 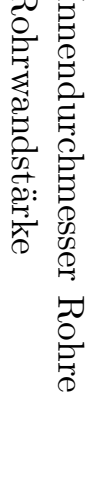 & 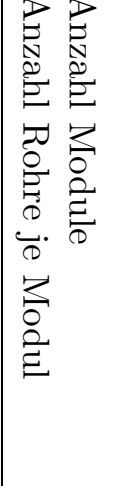 & 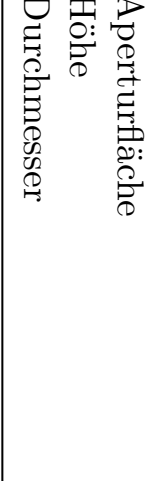 & 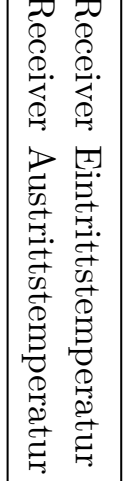 & 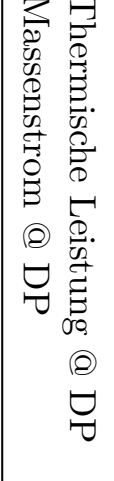 & 曷 & 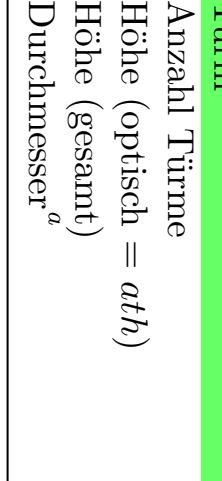 & 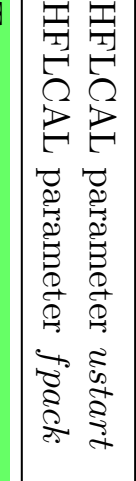 & 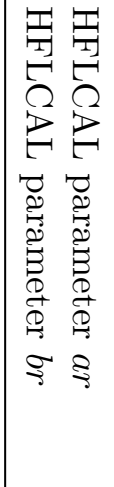 & 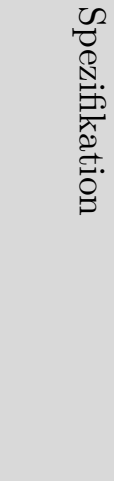 \\
\hline 宦宦 & $I I$ & 百互画 & $\vec{\Omega} \Omega$ & 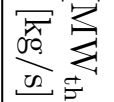 & $I$ & EEI & $I I$ & $I I$ & 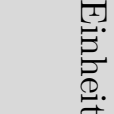 \\
\hline 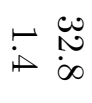 & $\stackrel{0}{0}$ & 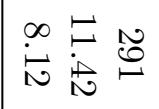 & 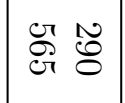 & 总若 & & $\infty$ & 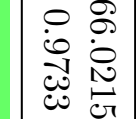 & 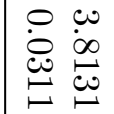 & 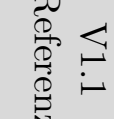 \\
\hline 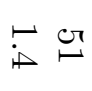 & 屯芲 & $0: 0$ & 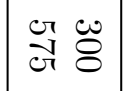 & 兑 & & $\infty \underset{\infty}{\infty}$ & 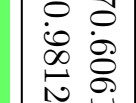 & 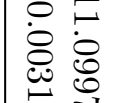 & 总 \\
\hline 芦留 & 忠萼 & $0 \begin{array}{lll}0 & 0 & 0 \\
\end{array}$ & 烝 & 造苦 & & & 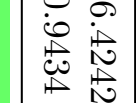 & 总尊 & 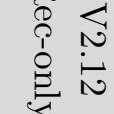 \\
\hline 芦 & 幽䔲 & 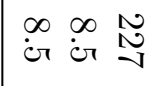 & 㳫 & 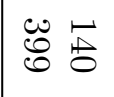 & & & 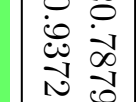 & 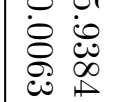 & 惫 \\
\hline 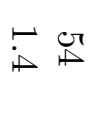 & $\ddot{\sim}$ & $\infty \infty \stackrel{0}{\stackrel{0}{g}}$ & 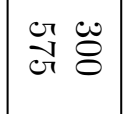 & 递 & & $\infty$ & 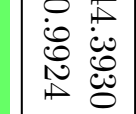 & 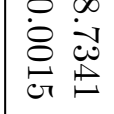 & 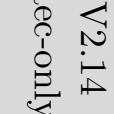 \\
\hline$\vec{\square}$ & 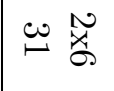 & 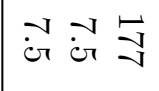 & 穿 & 若范 & & & 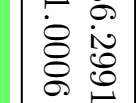 & 它 & 焉 \\
\hline$\ddot{\oplus}$ & 菦蔡 & フレ壱 & 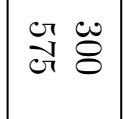 & 造范 & & & 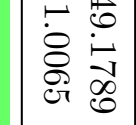 & 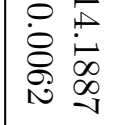 & 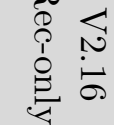 \\
\hline$\vec{\oplus} \stackrel{\circ}{\circ}$ & $\tilde{\approx}$ & 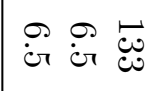 & 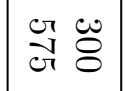 & 若若 & 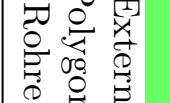 & $b_{\infty}$ & 它客 & $\begin{array}{l}0 \\
0 \\
0 \\
0 \\
0 \\
0 \\
0 \\
0\end{array}$ & 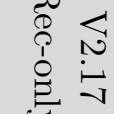 \\
\hline 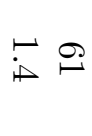 & 唗尊 & $\theta$ & $\stackrel{9}{\mathcal{G}} \stackrel{8}{8}$ & 造崌 & & & 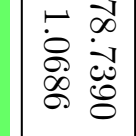 & 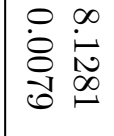 & 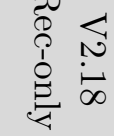 \\
\hline
\end{tabular}




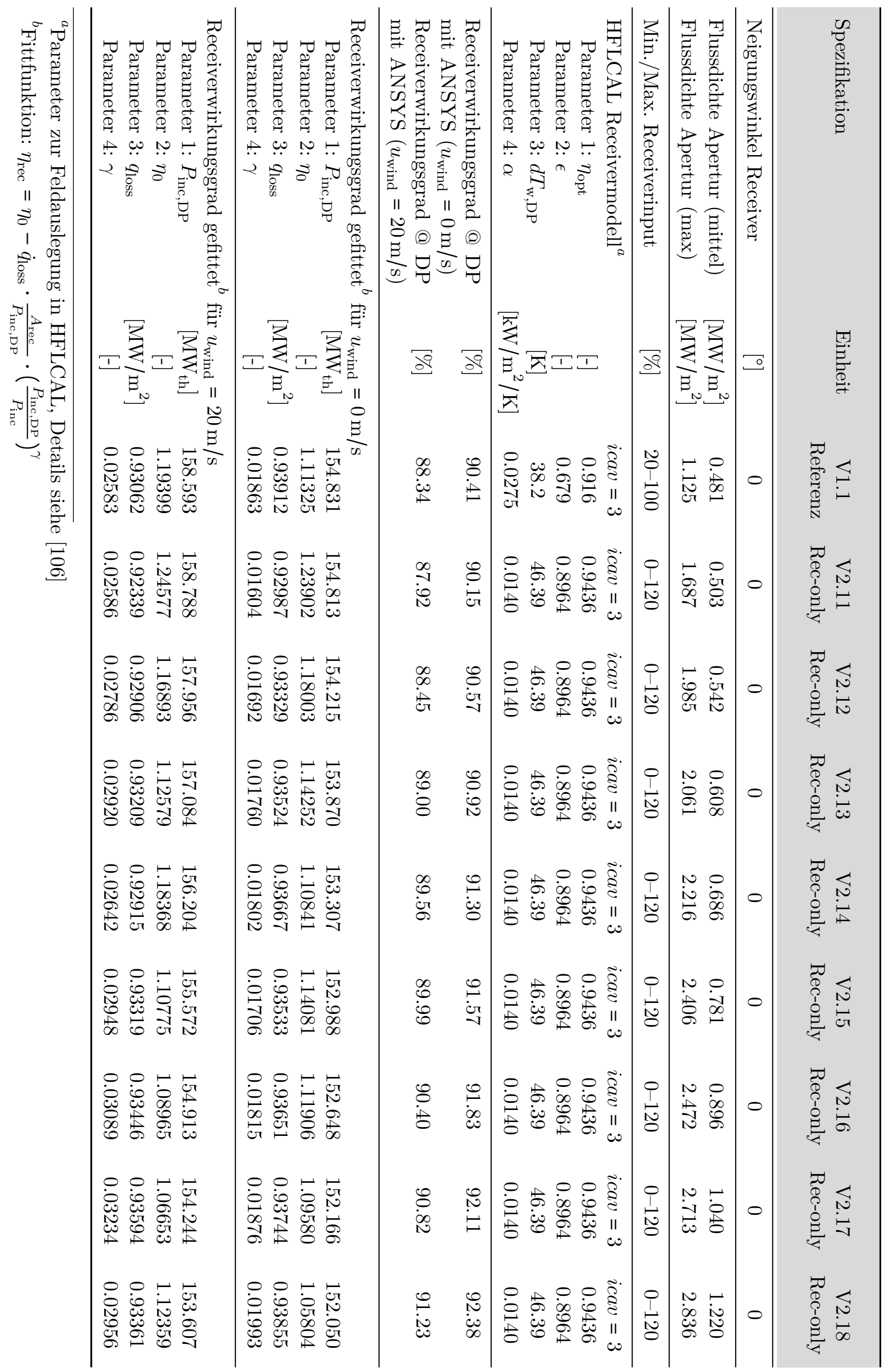




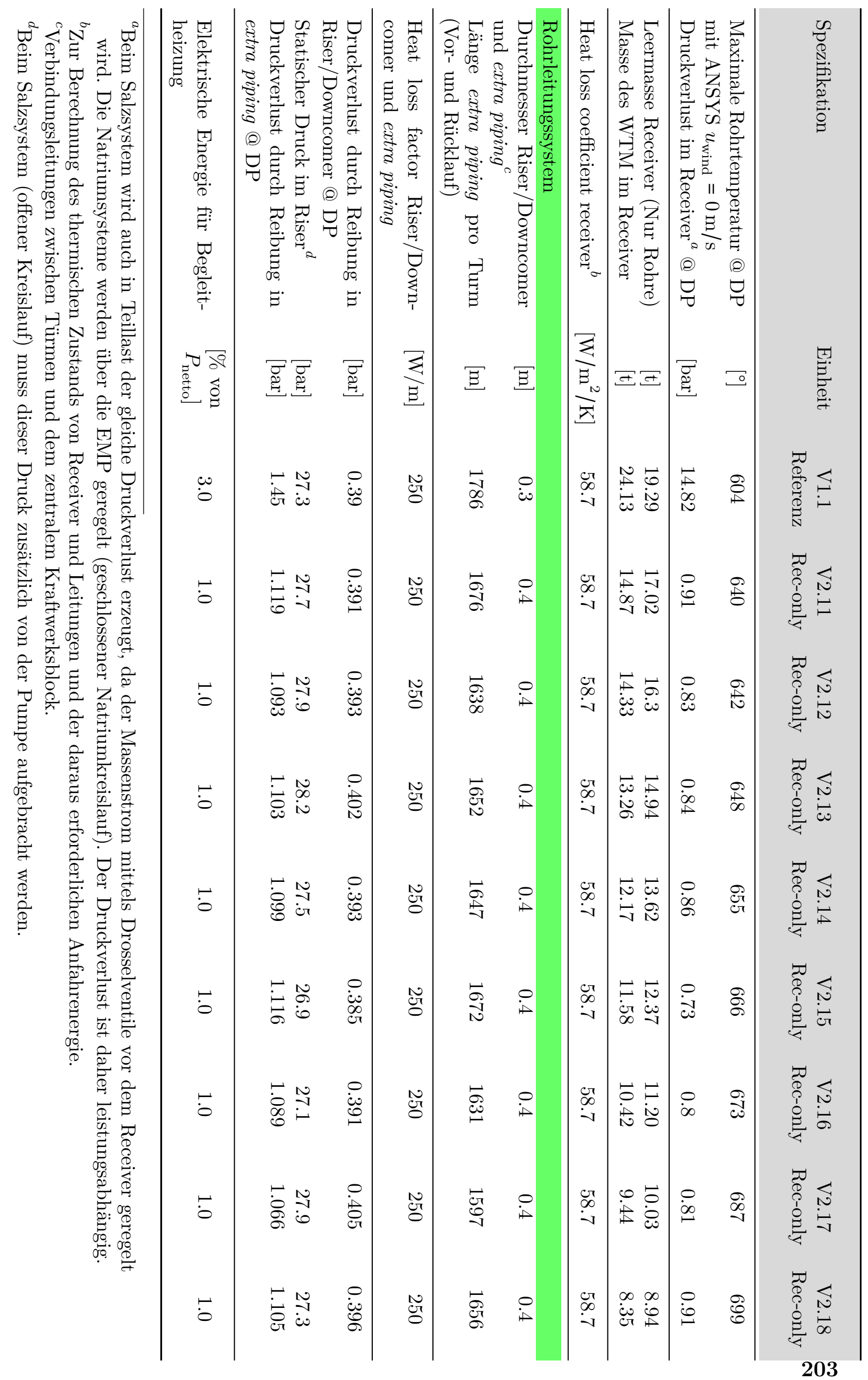




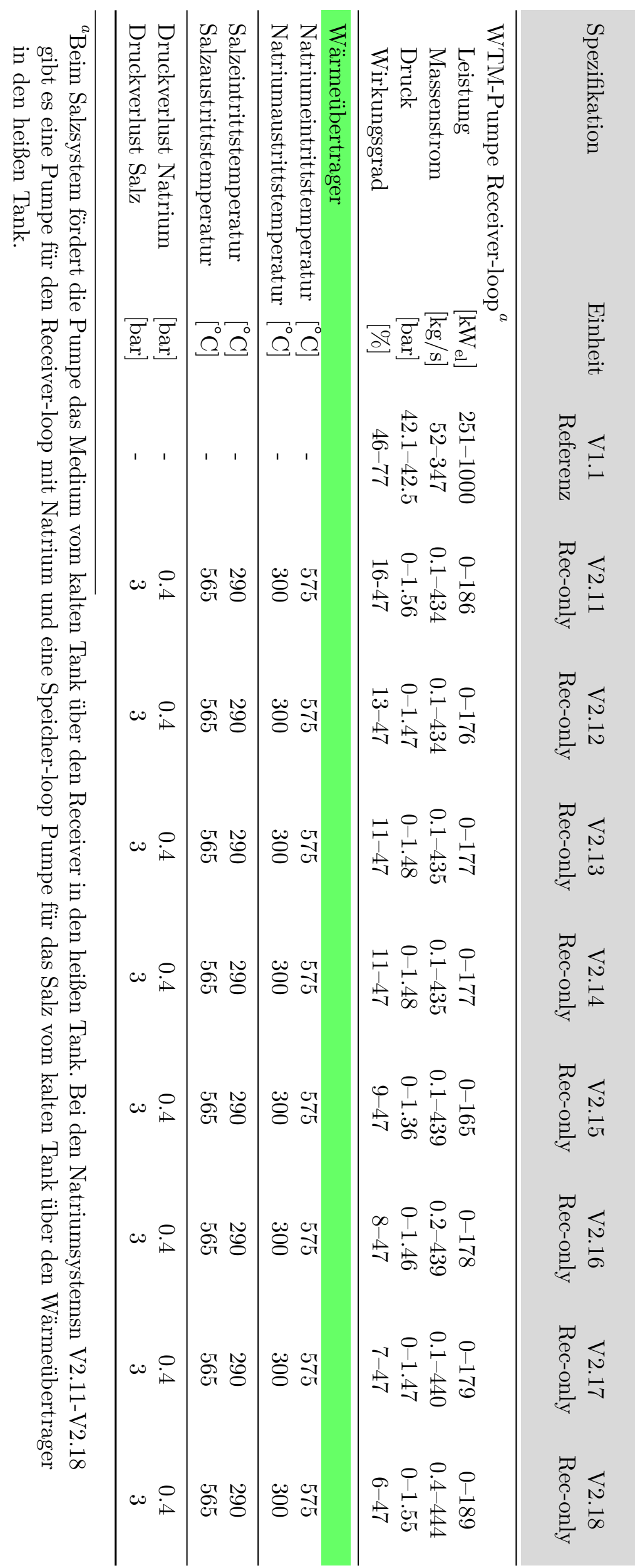




\begin{tabular}{|c|c|c|c|c|c|c|c|c|}
\hline 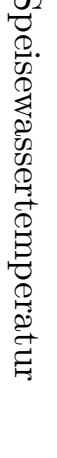 & 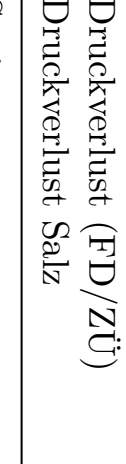 & 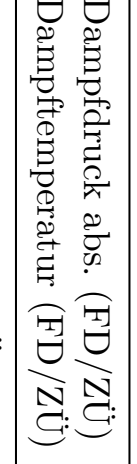 & 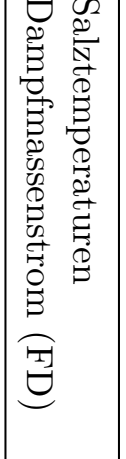 & 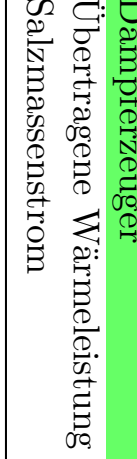 & 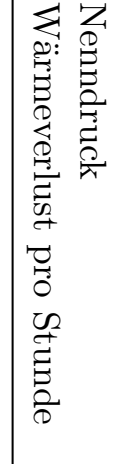 & 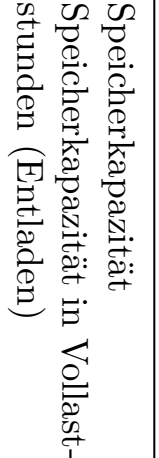 & 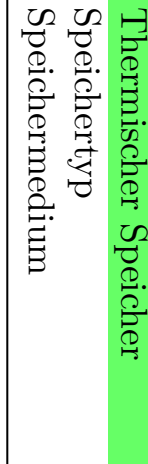 & 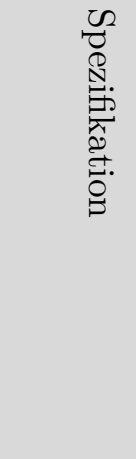 \\
\hline & 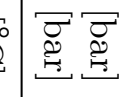 & 它 & $\frac{F}{\Xi}$ & 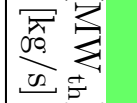 & 军莺 & $\Xi 3$ & $I I$ & 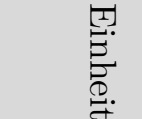 \\
\hline 芯 & $-\frac{8}{N}$ & 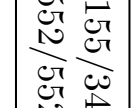 & 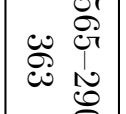 & 送 & 葱- & 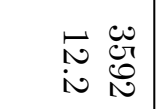 & & \\
\hline 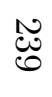 & $+\frac{5}{N}$ & 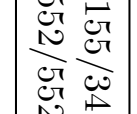 & 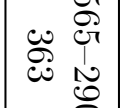 & $\mathscr{8}$ & 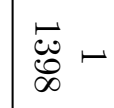 & $\mid$ & & \\
\hline 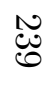 & $+\frac{5}{10}$ & 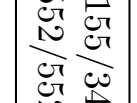 & 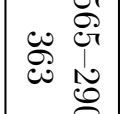 & & - & 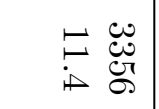 & & \\
\hline 胥 & $+\frac{5}{N}$ & 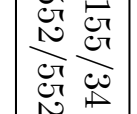 & 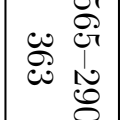 & & 蛋 - & 岕 & & 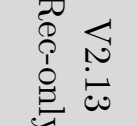 \\
\hline 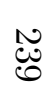 & $-\frac{5}{1}$ & 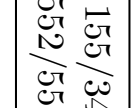 & 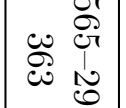 & 惫 & 䓌 & 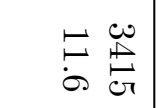 & & 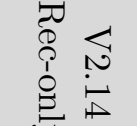 \\
\hline 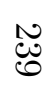 & $-\frac{5}{N}$ & 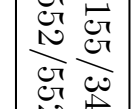 & 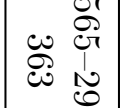 & $\ddot{\mathscr{\omega}}$ & 志 - & 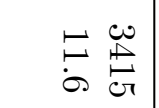 & & $\stackrel{\rho}{B c}$ \\
\hline 离 & $+\frac{5}{10}$ & 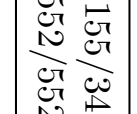 & 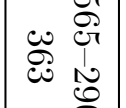 & 0 & $\mid$ & 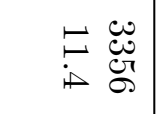 & & 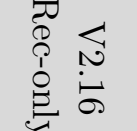 \\
\hline 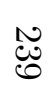 & $+\frac{\vec{b}}{v}$ & 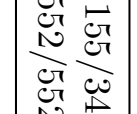 & 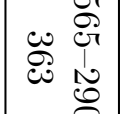 & & $\infty_{\infty}^{-}$ & 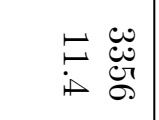 & & $8 \vec{g}$ \\
\hline 源 & $+\frac{0}{10}$ & 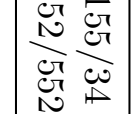 & 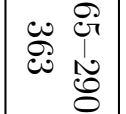 & & 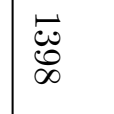 & $\begin{array}{l}5 \\
\end{array}$ & & 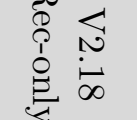 \\
\hline
\end{tabular}




\begin{tabular}{|c|c|c|c|c|c|c|c|c|}
\hline 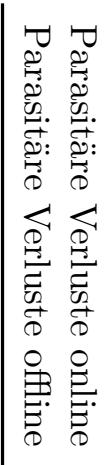 & 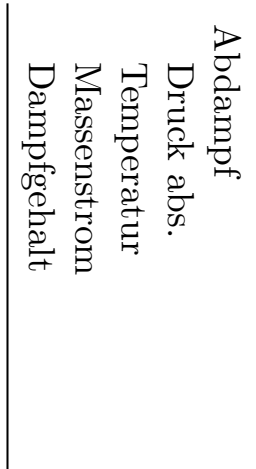 & 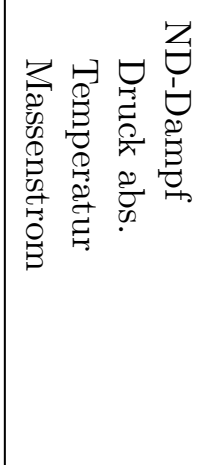 & 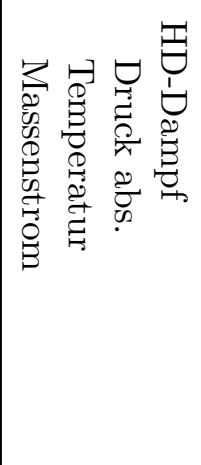 & 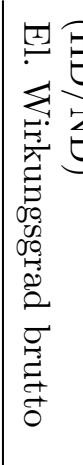 & 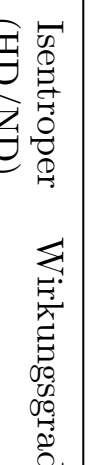 & 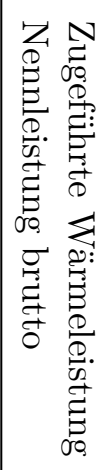 & 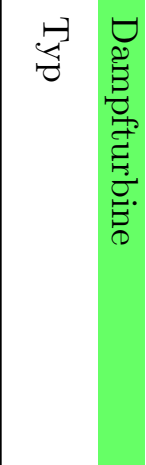 & 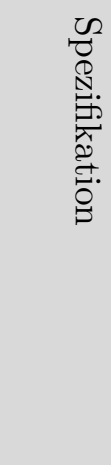 \\
\hline 军军 & 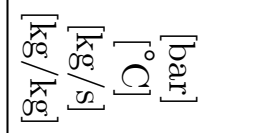 & 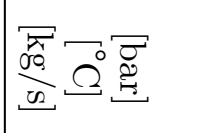 & 要 & 39 & $\bar{a}$ & 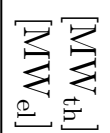 & I & 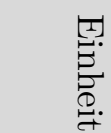 \\
\hline \begin{tabular}{|l}
$\vec{N}$ \\
di g \\
d
\end{tabular} & 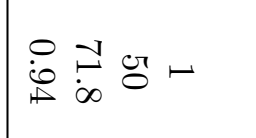 & 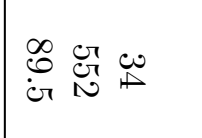 & 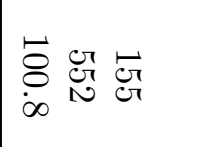 & 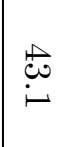 & $\underset{\substack{\infty \\
\infty \\
\infty}}{\stackrel{\infty}{0}}$ & 蝔 & 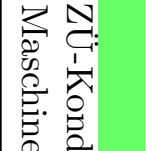 & 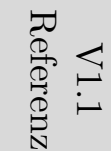 \\
\hline 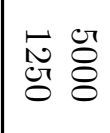 & 尼 & 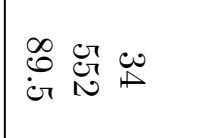 & 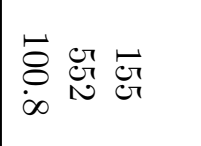 & 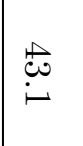 & $\underset{\substack{\infty \\
\infty}}{\stackrel{\infty}{0}}$ & 忥芯 & 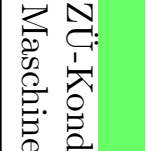 & 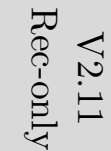 \\
\hline 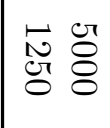 & 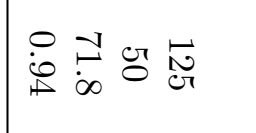 & 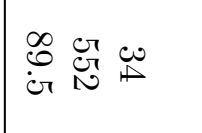 & 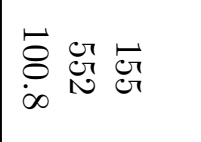 & 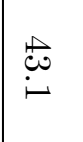 & $\begin{array}{l}\stackrel{\infty}{v} \\
\underset{0}{0}\end{array}$ & 芯芯 & 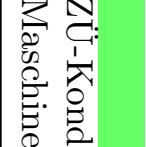 & 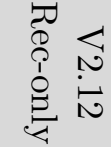 \\
\hline \begin{tabular}{|l}
$\vec{N}$ \\
di g \\
d
\end{tabular} & 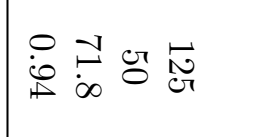 & 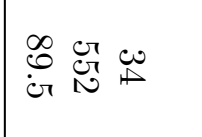 & 离空 & $\stackrel{\substack{0 \\
i}}{i}$ & $\begin{array}{l}\stackrel{\infty}{\sim} \\
\underset{\infty}{0}\end{array}$ & 芯密 & 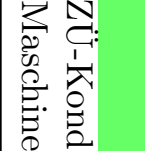 & 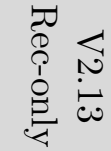 \\
\hline 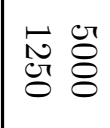 & 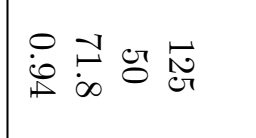 & 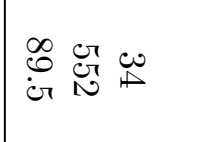 & 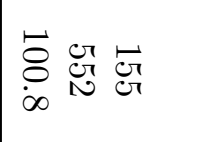 & $\stackrel{\substack{0.0 \\
i \sim}}{i}$ & $\underset{\substack{\infty \\
\infty \\
\infty}}{0}$ & 芯楞 & 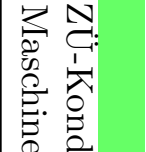 & 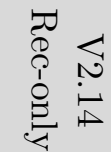 \\
\hline 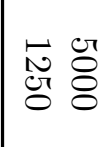 & 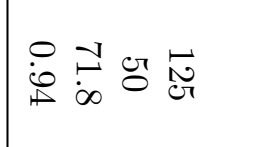 & 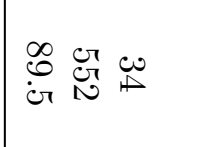 & 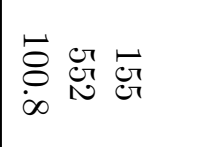 & 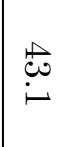 & 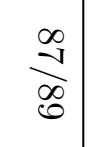 & 芯芯 & 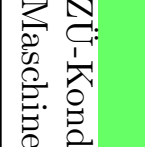 & 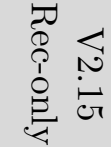 \\
\hline 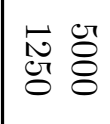 & 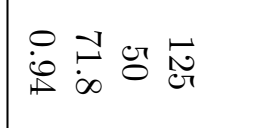 & 象然 & 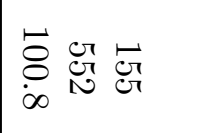 & 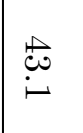 & $\underset{\substack{\infty \\
\infty \\
0 \\
0}}{\mid}$ & 芯芯 & 常 & 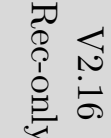 \\
\hline cr & 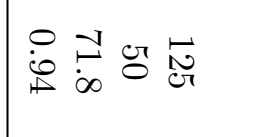 & 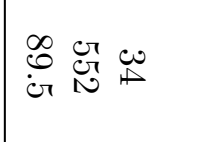 & 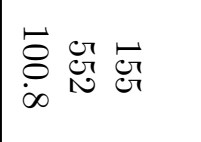 & $\stackrel{\substack{0 \\
i}}{i}$ & $\begin{array}{l}\stackrel{\infty}{\sim} \\
\stackrel{\infty}{0}\end{array}$ & 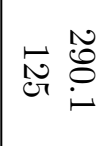 & 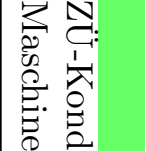 & 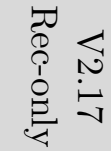 \\
\hline c్ & 光 & 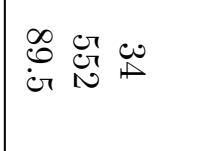 & 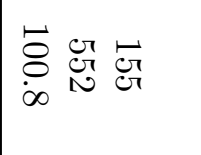 & $\stackrel{\substack{u \\
i}}{i}$ & $\underset{\substack{\infty \\
\infty}}{\stackrel{\infty}{0}}$ & 芯芯 & 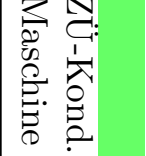 & 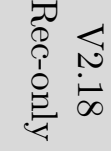 \\
\hline
\end{tabular}




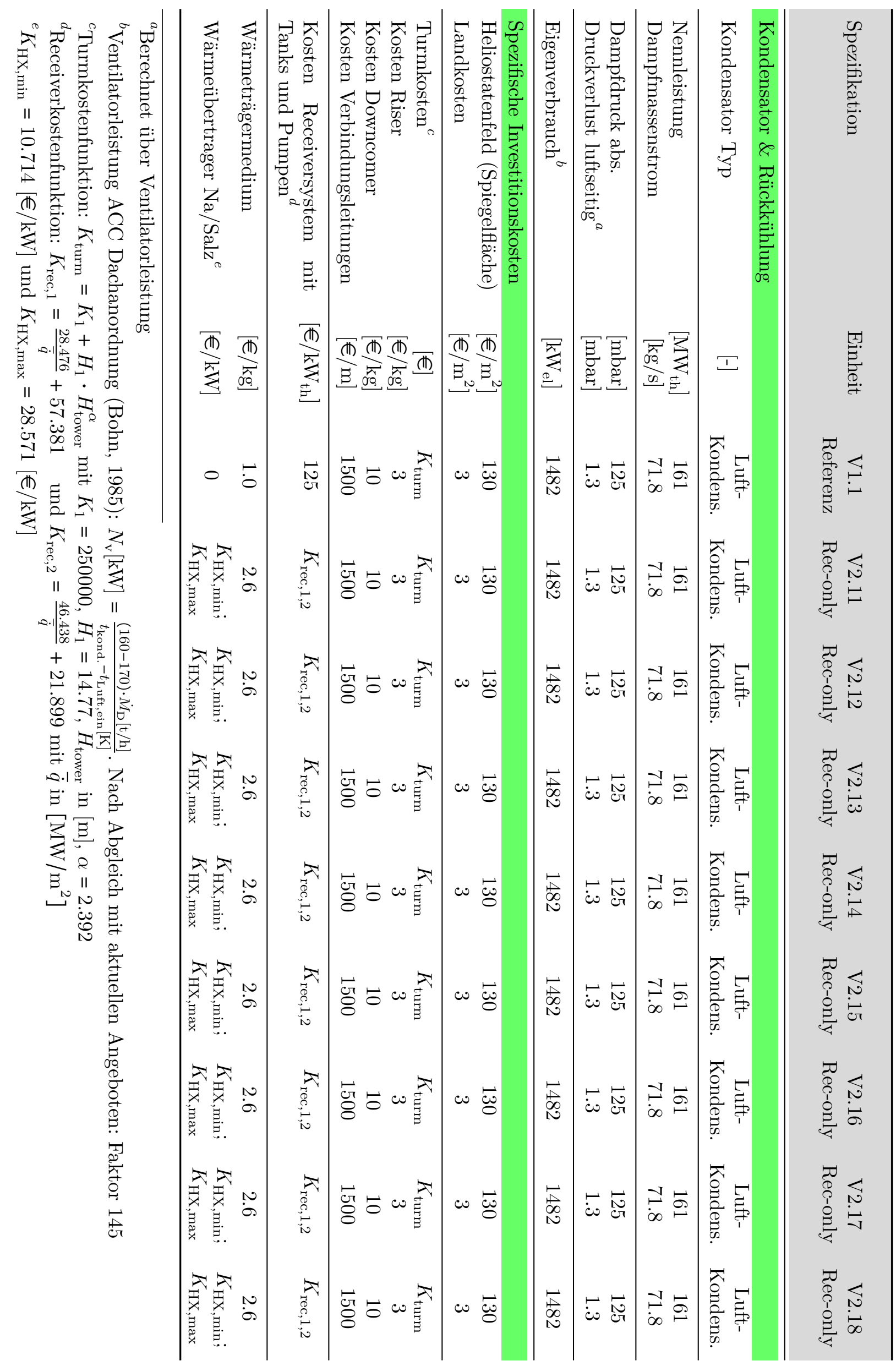




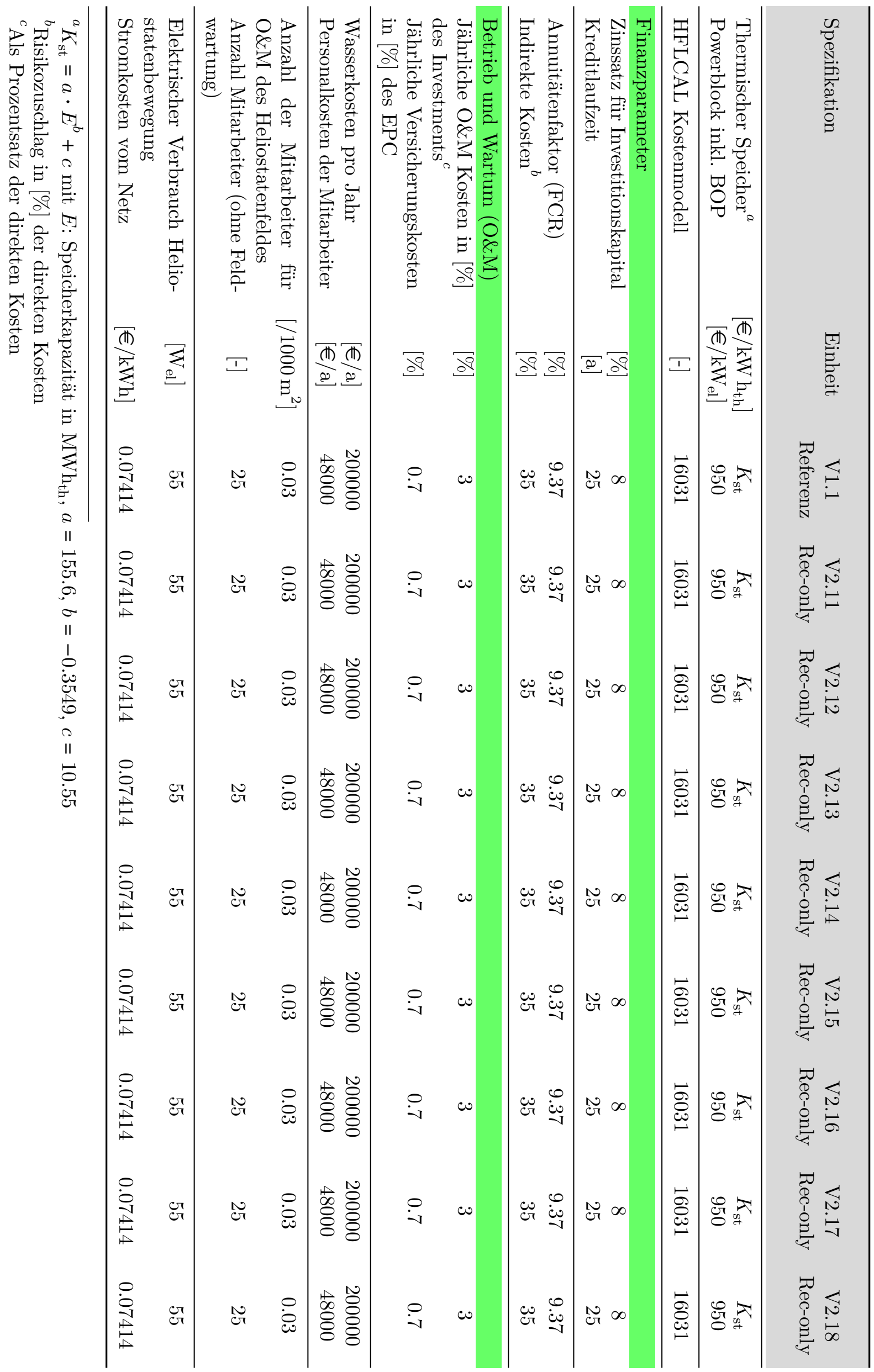




\begin{tabular}{|c|c|c|c|c|c|c|c|c|c|}
\hline 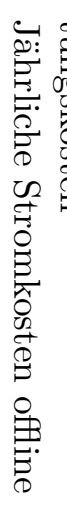 & 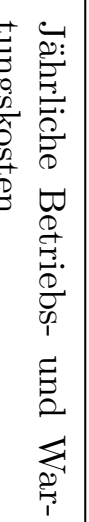 & 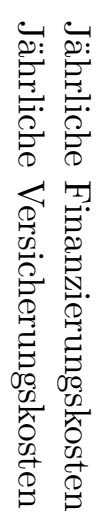 & 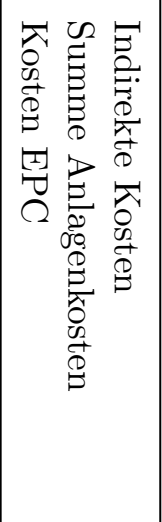 & 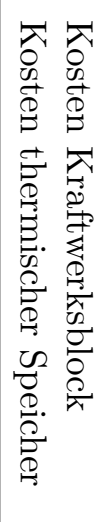 & 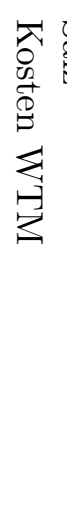 & 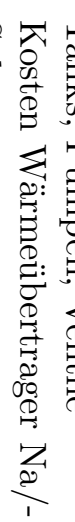 & 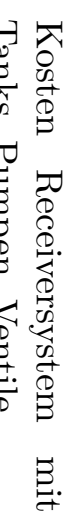 & 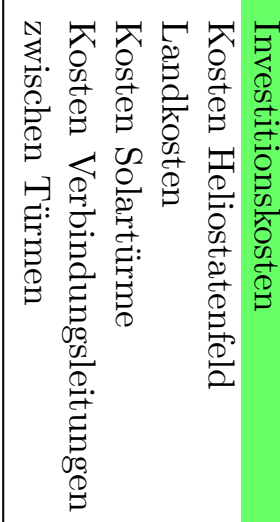 & 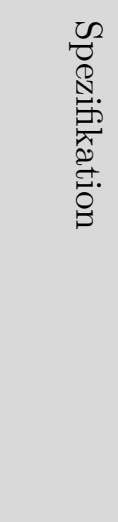 \\
\hline 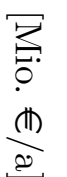 & 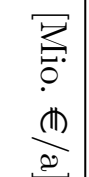 & 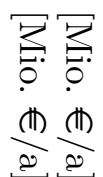 & 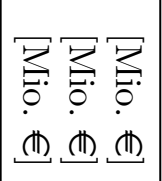 & 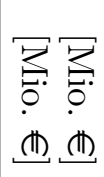 & $\begin{array}{l}\text { 帯 } \\
: \\
\text { (i) }\end{array}$ & $\begin{array}{l}\text { ?: } \\
0 \\
\text { (1) }\end{array}$ & $\begin{array}{l}\text { ?: } \\
?: \\
\text { (1) }\end{array}$ & 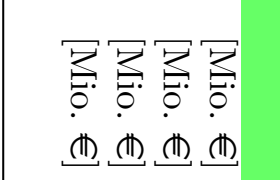 & 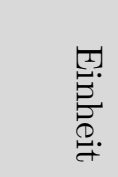 \\
\hline$\underset{i}{i}$ & $\begin{array}{l}\vec{o} \\
\dot{\sigma}\end{array}$ & 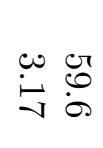 & 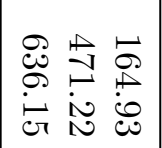 & 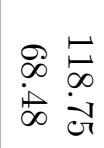 & $\stackrel{P}{\dot{C}_{0}}$ & $\stackrel{\circ}{8}$ & $\stackrel{0}{\stackrel{\bullet}{\sigma}}$ & 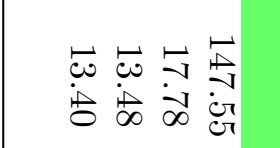 & 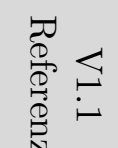 \\
\hline ن & $\begin{array}{l}\vec{O} \\
\dot{0} \\
\stackrel{1}{\sigma} \\
\dot{c}\end{array}$ & 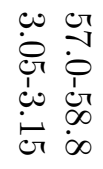 & 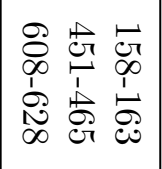 & 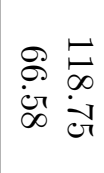 & 完 & $\begin{array}{l}-1 \\
\dot{1} \\
1 \\
0 \\
0 \\
0\end{array}$ & $\begin{array}{l}0 \\
\infty \\
0 \\
1 \\
0 \\
0 \\
i v\end{array}$ & 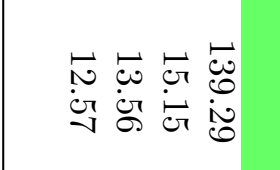 & 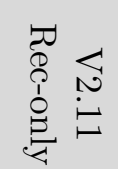 \\
\hline ن & $\begin{array}{l}\overrightarrow{c r} \\
\dot{0} \\
\dot{1} \\
\dot{0} \\
\dot{c}\end{array}$ & 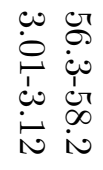 & 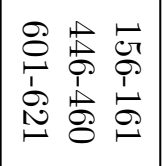 & 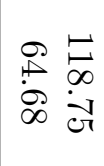 & $\stackrel{0}{\dot{0}}$ & $\begin{array}{l}-1 \\
\dot{1} \\
1 \\
0 \\
0 \\
0\end{array}$ & 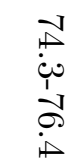 & 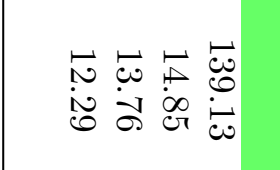 & 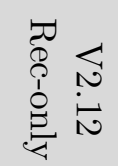 \\
\hline$\underset{i}{i}$ & $\begin{array}{l}\overrightarrow{c r} \\
\infty \\
1 \\
\stackrel{1}{\sigma} \\
i v\end{array}$ & 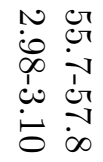 & 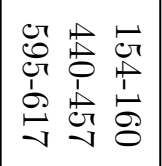 & 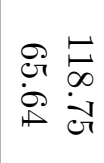 & $\stackrel{0}{6}$ & $\begin{array}{l}-1 \\
\text { ît } \\
1 \\
0 \\
0 \\
0\end{array}$ & $\begin{array}{l}\infty \\
\infty \\
i 1 \\
1 \\
N \\
\stackrel{1}{N} \\
-v\end{array}$ & 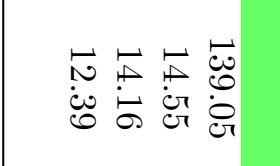 & 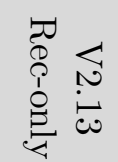 \\
\hline$\underset{\substack{i \\
\infty}}{0}$ & $\begin{array}{l}\overrightarrow{c r} \\
\infty \\
1 \\
\stackrel{1}{\sigma} \\
i v\end{array}$ & 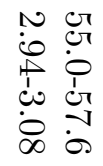 & 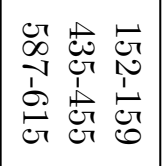 & 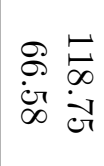 & $\stackrel{0}{=}$ & 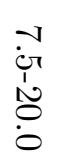 & $\begin{array}{l}9 \\
1 \\
i \\
1 \\
0 \\
0 \\
0\end{array}$ & 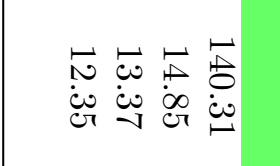 & 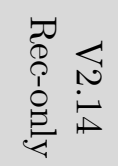 \\
\hline$\underset{i}{i}$ & $\begin{array}{c}\vec{c} \\
\dot{\infty} \\
\dot{1} \\
\sigma \\
\dot{i}\end{array}$ & 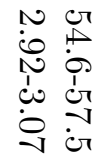 & 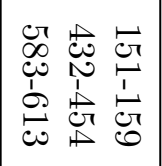 & 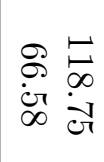 & $\stackrel{0}{v}$ & $\begin{array}{l}-1 \\
01 \\
1 \\
0 \\
0 \\
0\end{array}$ & $\begin{array}{l}\text { r } \\
0 \\
0 \\
0 \\
0 \\
0 \\
0\end{array}$ & 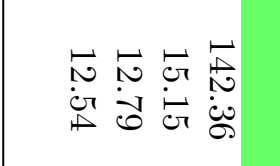 & 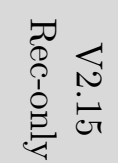 \\
\hline$\stackrel{0}{i}$ & 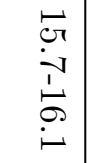 & $\begin{array}{ll}1 & 0 \\
\infty & 0 \\
\infty & 0 \\
1 & 0 \\
0 & 0 \\
0 & 0 \\
8 & 0\end{array}$ & 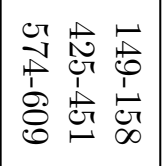 & 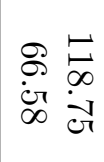 & $\stackrel{P}{\dot{c} r}$ & 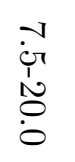 & $\begin{array}{l}c r \\
\stackrel{c}{c} \\
1 \\
1 \\
\stackrel{0}{0} \\
i\end{array}$ & 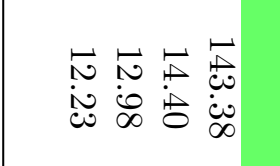 & 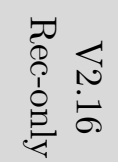 \\
\hline $\begin{array}{l}0 \\
\text { î. }\end{array}$ & $\begin{array}{l}\vec{c} \\
\dot{1} \\
\stackrel{\vec{\sigma}}{0} \\
0\end{array}$ & 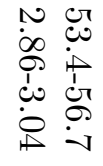 & 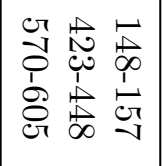 & $\begin{array}{l}\infty \\
\stackrel{\vec{\sigma}}{\infty} \\
\dot{\infty} \vec{v}\end{array}$ & $\stackrel{P}{\dot{e}}_{0}$ & $\begin{array}{l}-1 \\
01 \\
1 \\
0 \\
0 \\
0\end{array}$ & 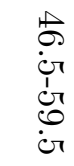 & 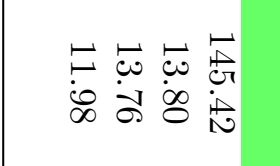 & 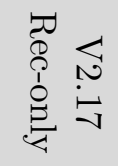 \\
\hline $\begin{array}{l}\text { Oे } \\
\text { i }\end{array}$ & $\begin{array}{l}\overrightarrow{e r} \\
0 \\
0 \\
0 \\
0 \\
0\end{array}$ & $\begin{array}{ll}N & 0 \\
\infty & 0 \\
\infty & 0 \\
1 & 0 \\
1 & 0 \\
0 & 0 \\
0 & 0 \\
\infty & 0\end{array}$ & 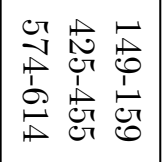 & 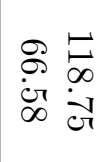 & $\stackrel{0}{i}$ & $\begin{array}{l}\overrightarrow{1} \\
\dot{c} \\
1 \\
0 \\
0 \\
0\end{array}$ & 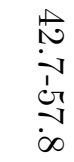 & 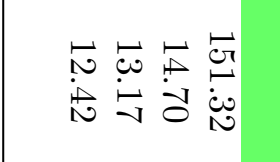 & 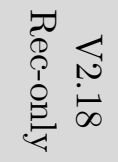 \\
\hline
\end{tabular}




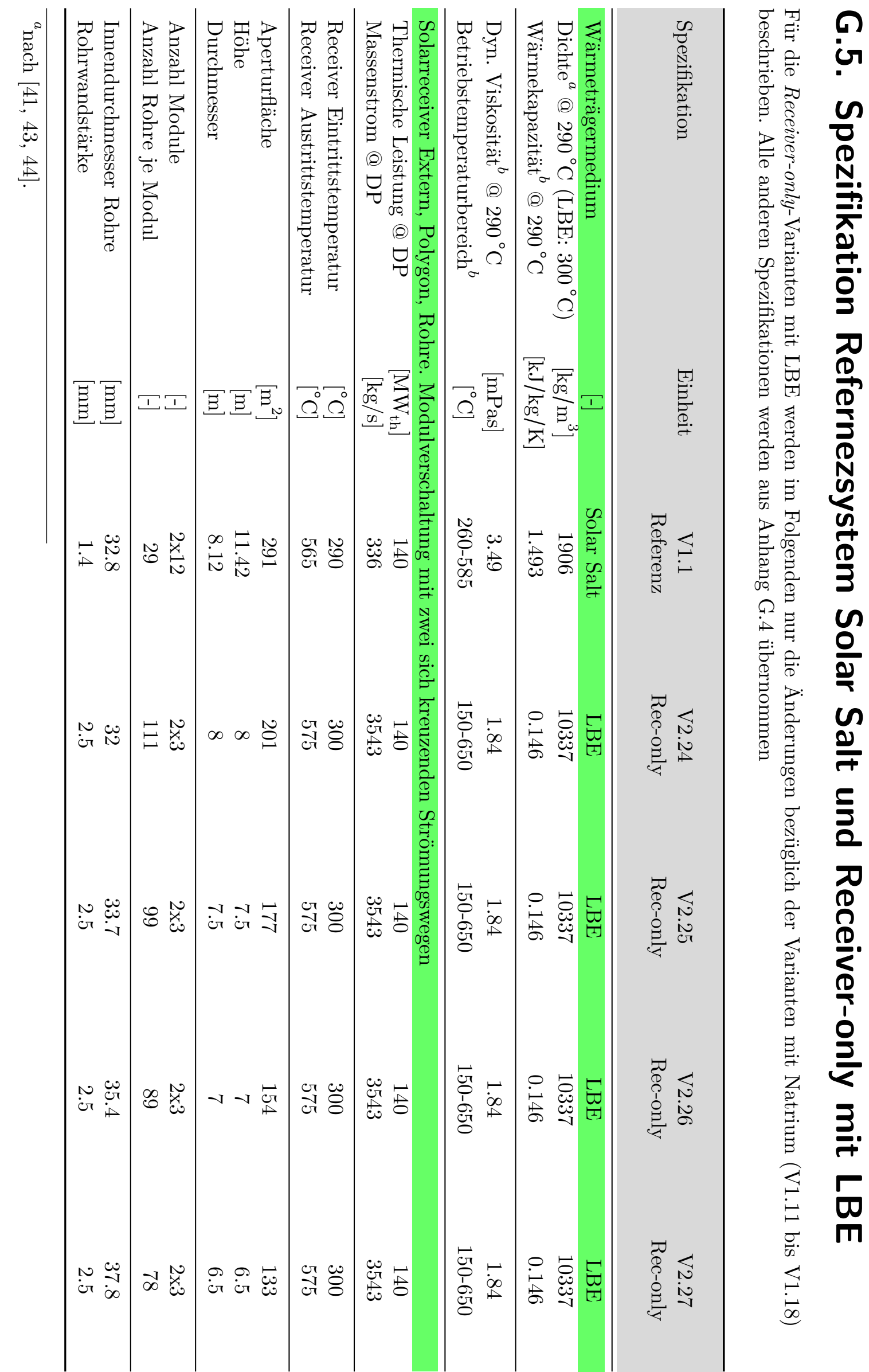




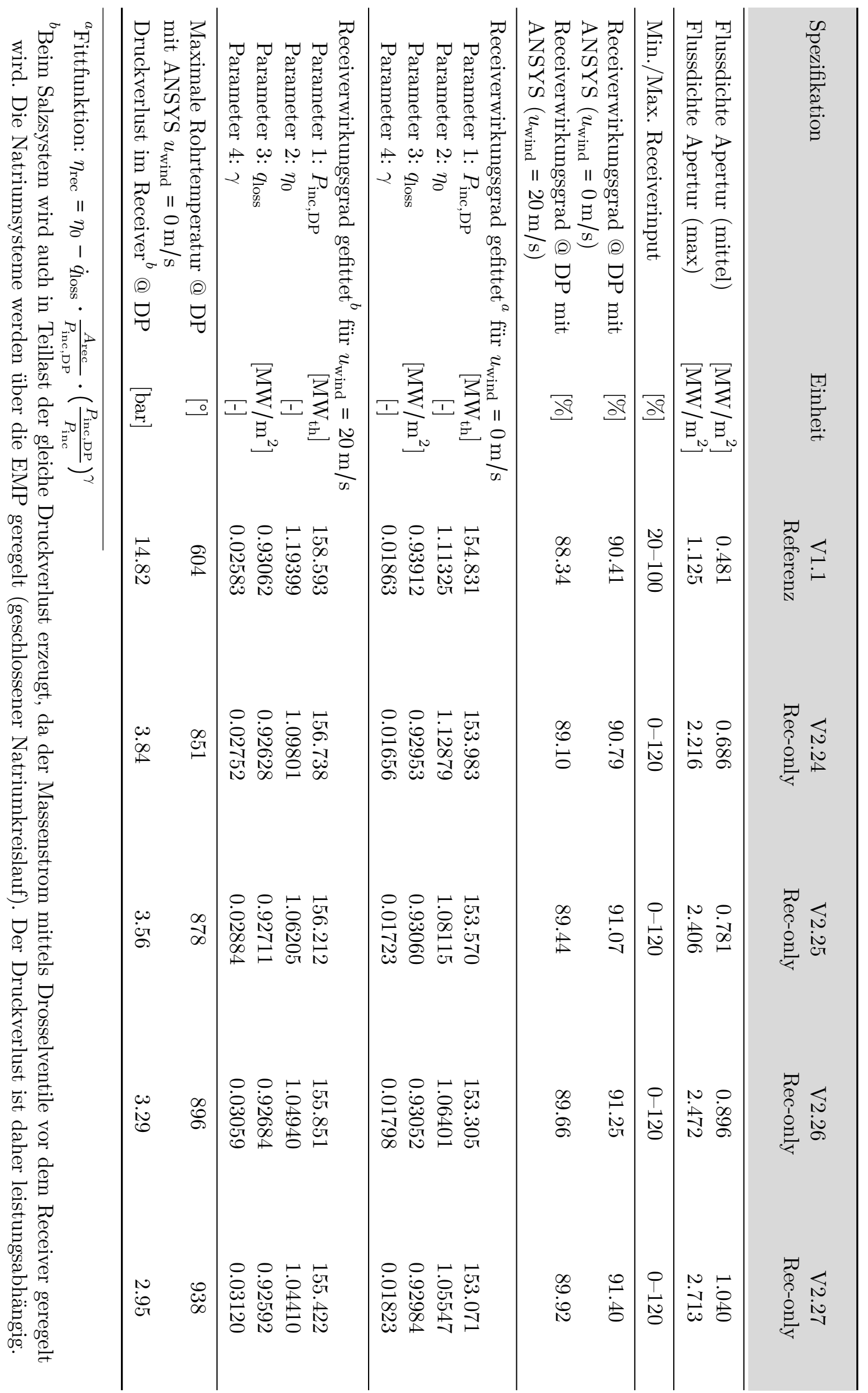




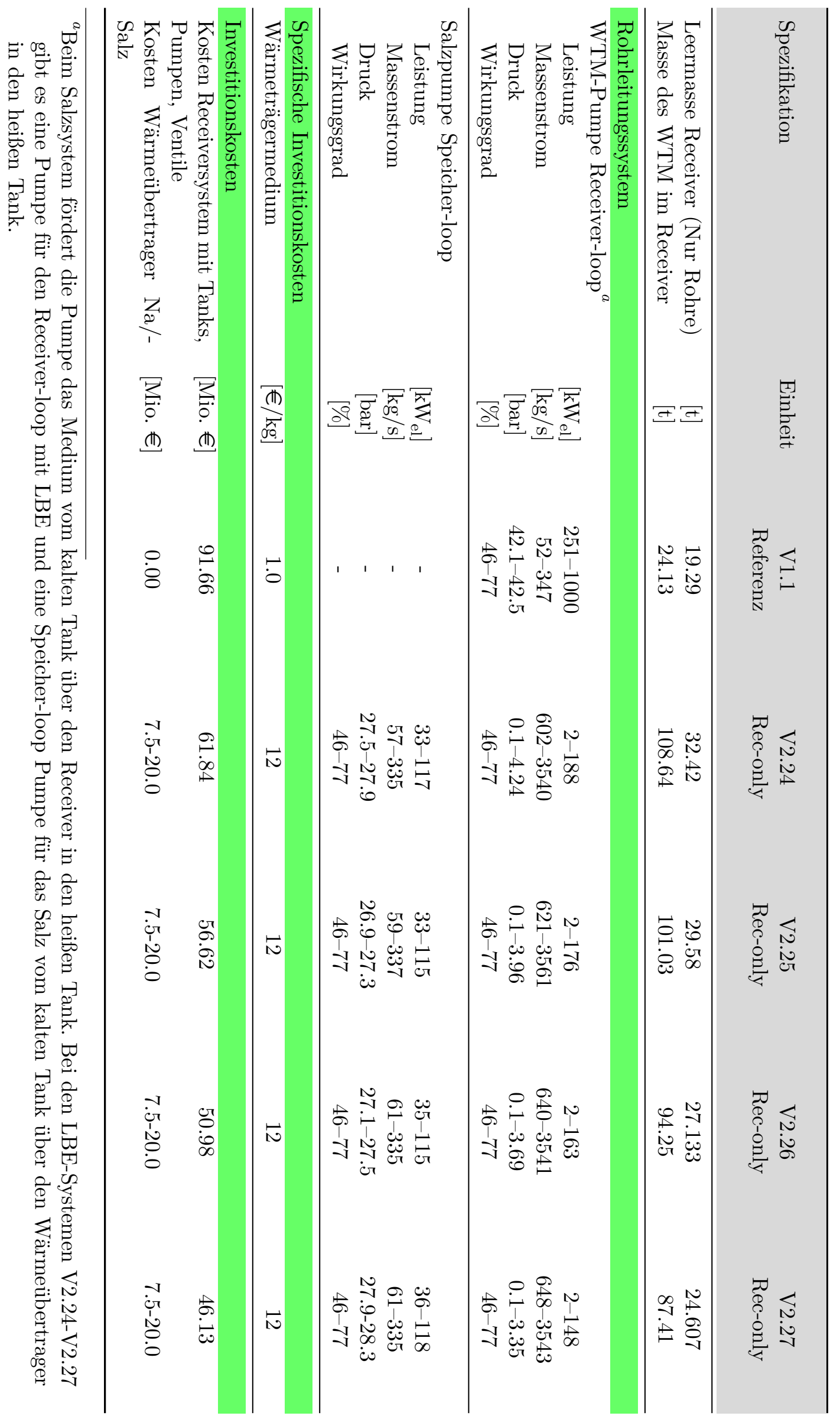




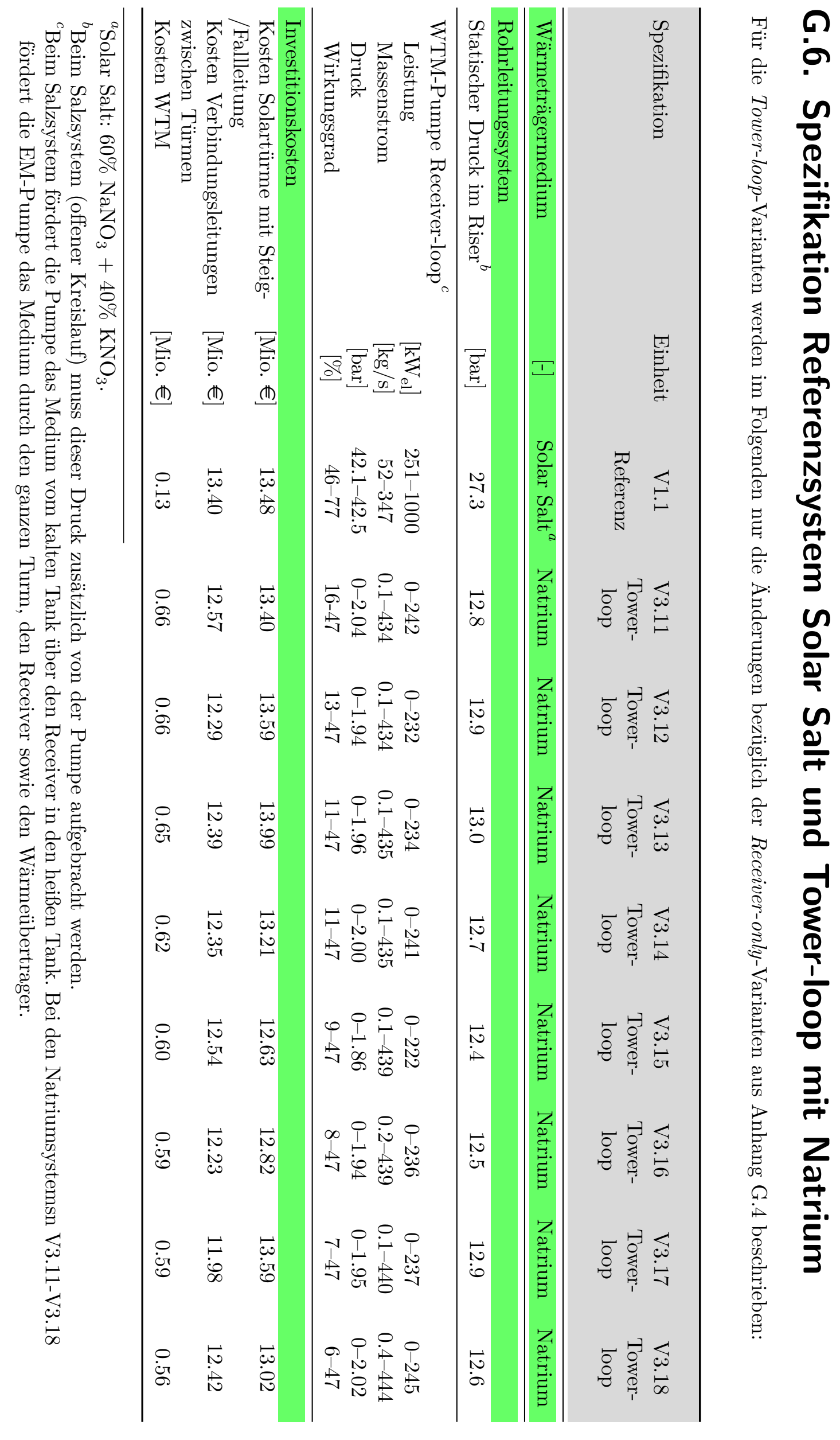




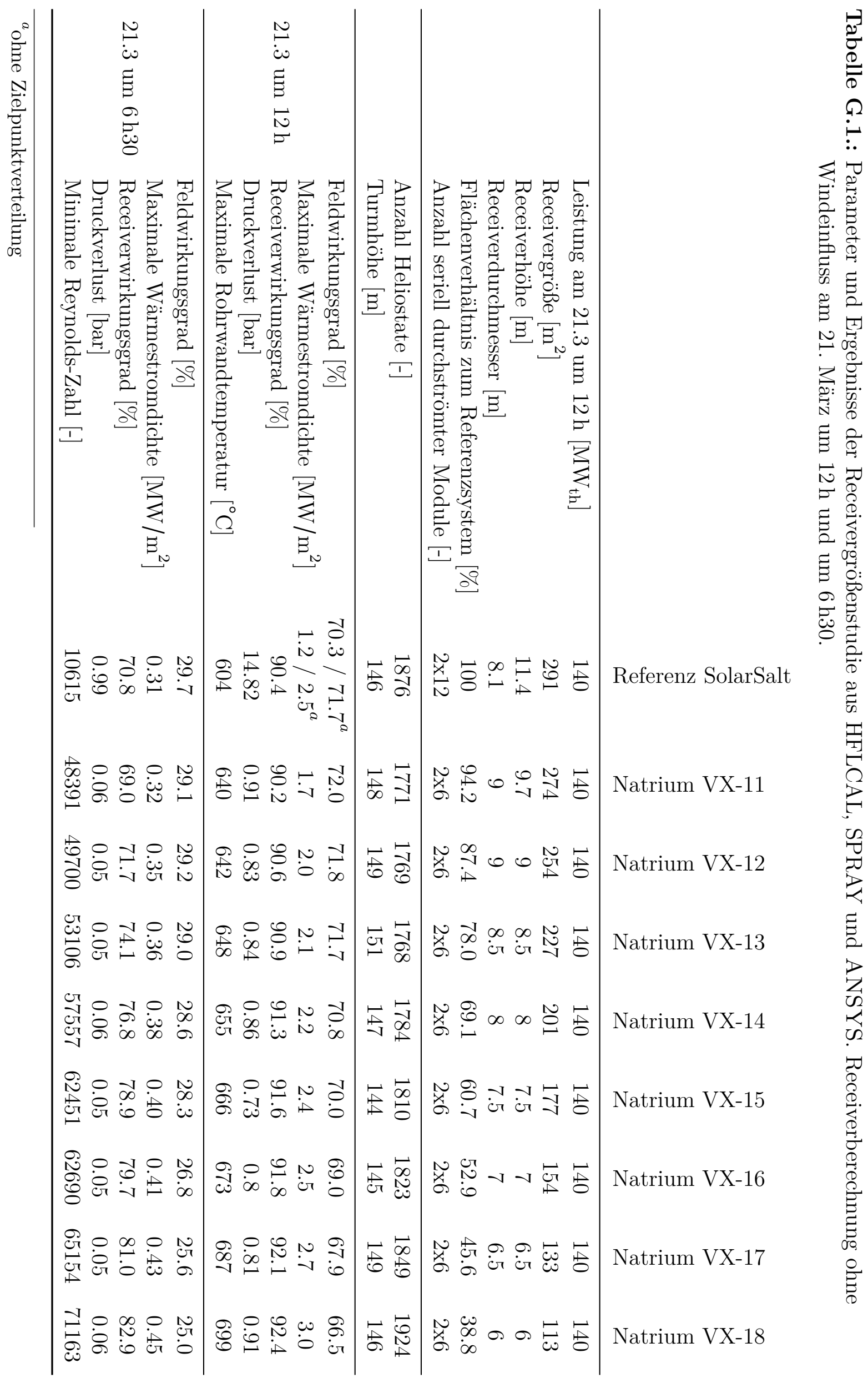




\section{H. Spezifikationstabellen der Einturmsysteme}

\section{H.1. Übersicht der Konfigurationen}

\begin{tabular}{|c|c|c|c|c|c|c|}
\hline 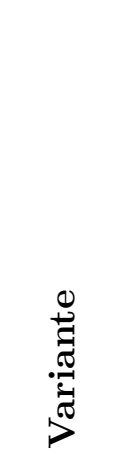 & 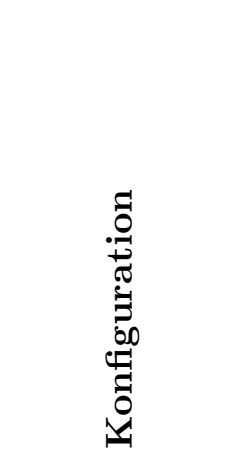 & 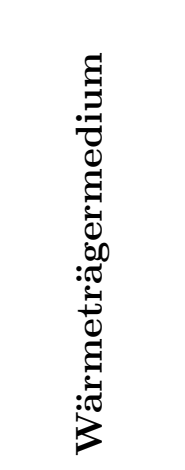 & 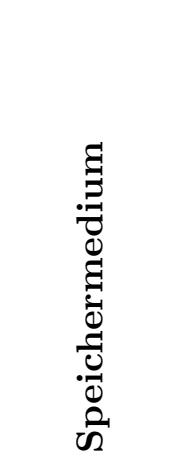 & 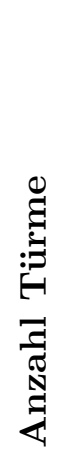 & 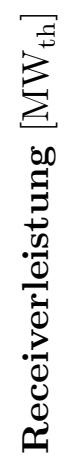 & 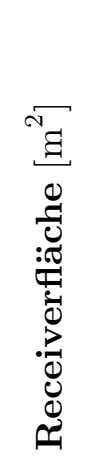 \\
\hline V 1.2 & $\begin{array}{l}\text { Referenz } \\
\text { Salzturm }\end{array}$ & Solar Salt & Solar Salt & 1 & 700 & 1376 \\
\hline V 2.31 & Receiver-only & Natrium & Solar Salt & 1 & 700 & 1074 \\
\hline V 2.32 & Receiver-only & Natrium & Solar Salt & 1 & 700 & 855 \\
\hline V 2.33 & Receiver-only & Natrium & Solar Salt & 1 & 700 & 704 \\
\hline V 2.34 & Receiver-only & Natrium & Solar Salt & 1 & 700 & 660 \\
\hline V 2.35 & Receiver-only & Natrium & Solar Salt & 1 & 700 & 490 \\
\hline V 3.51 & Tower-loop & Natrium & Solar Salt & 1 & 700 & 1074 \\
\hline V 3.52 & Tower-loop & Natrium & Solar Salt & 1 & 700 & 855 \\
\hline V 3.53 & Tower-loop & Natrium & Solar Salt & 1 & 700 & 704 \\
\hline V 3.54 & Tower-loop & Natrium & Solar Salt & 1 & 700 & 660 \\
\hline V 3.55 & Tower-loop & Natrium & Solar Salt & 1 & 700 & 490 \\
\hline
\end{tabular}




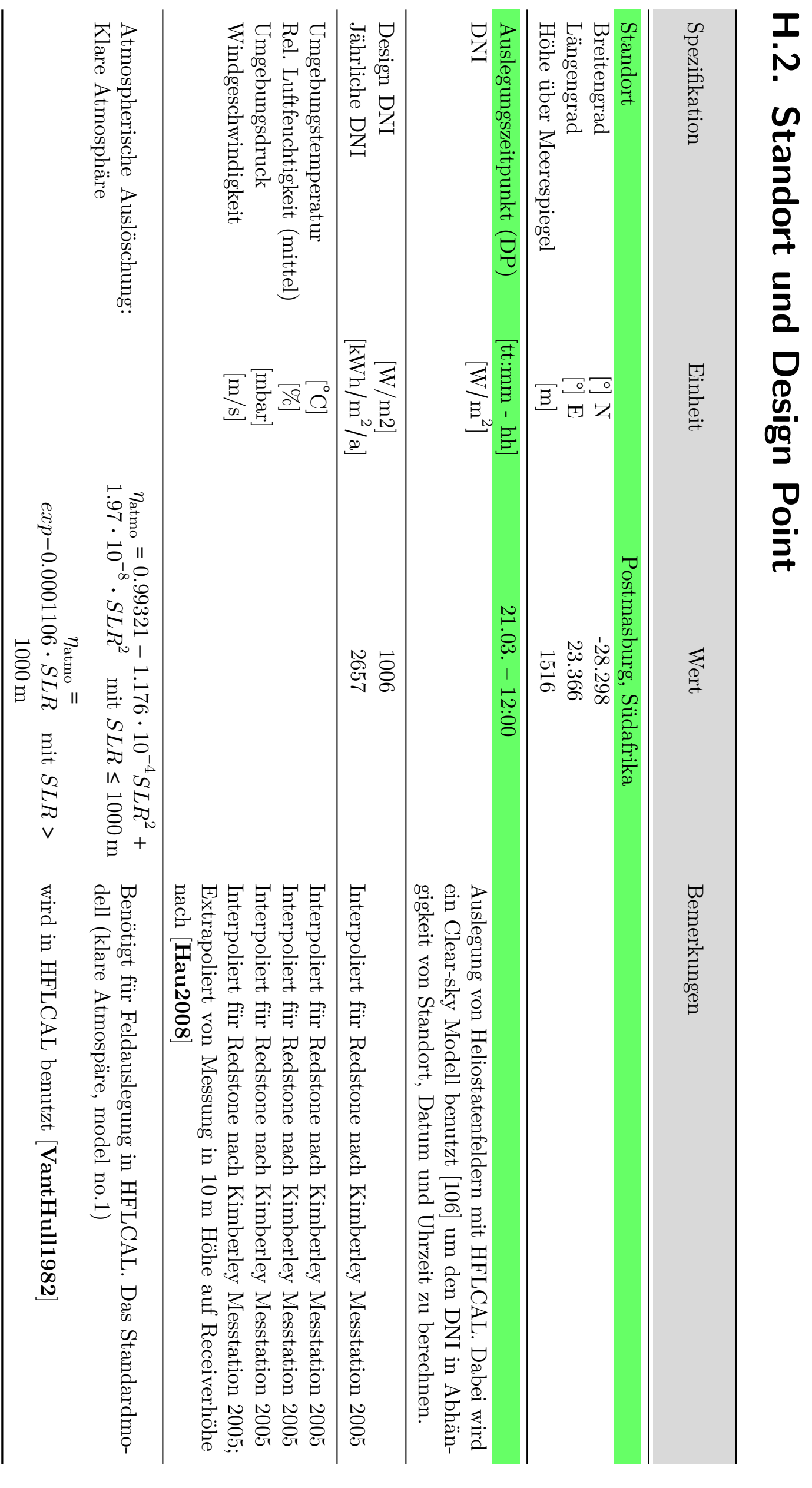




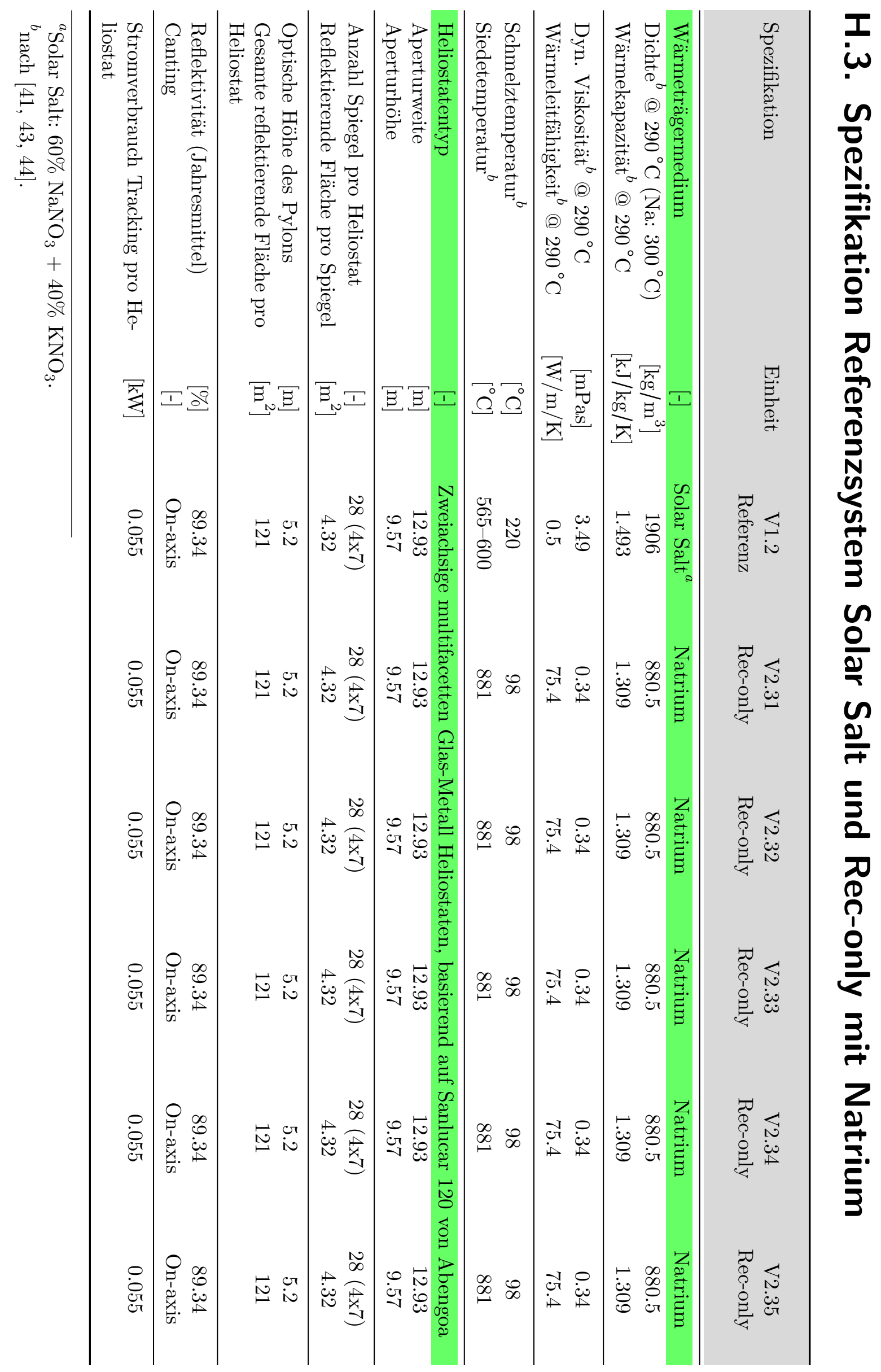




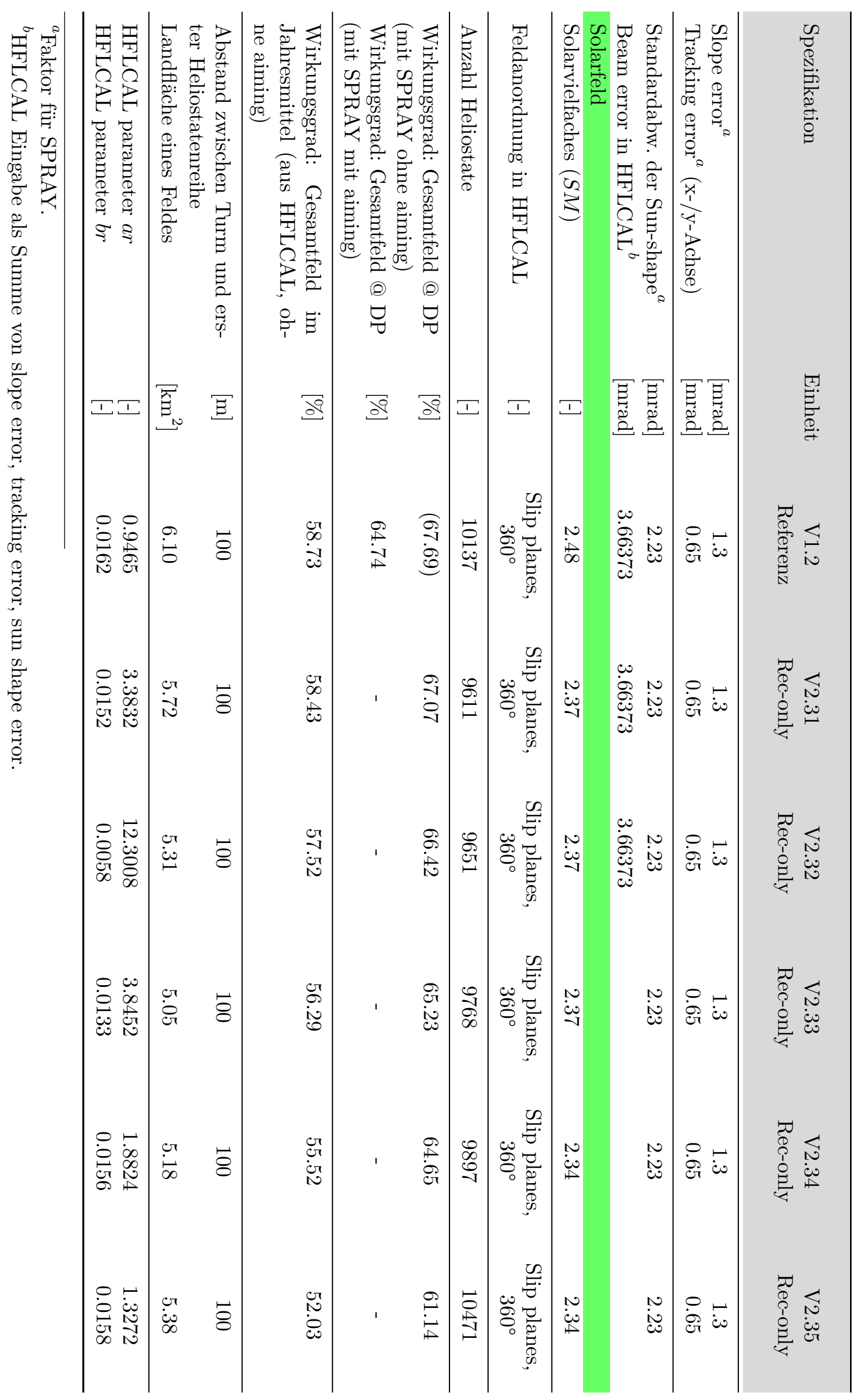




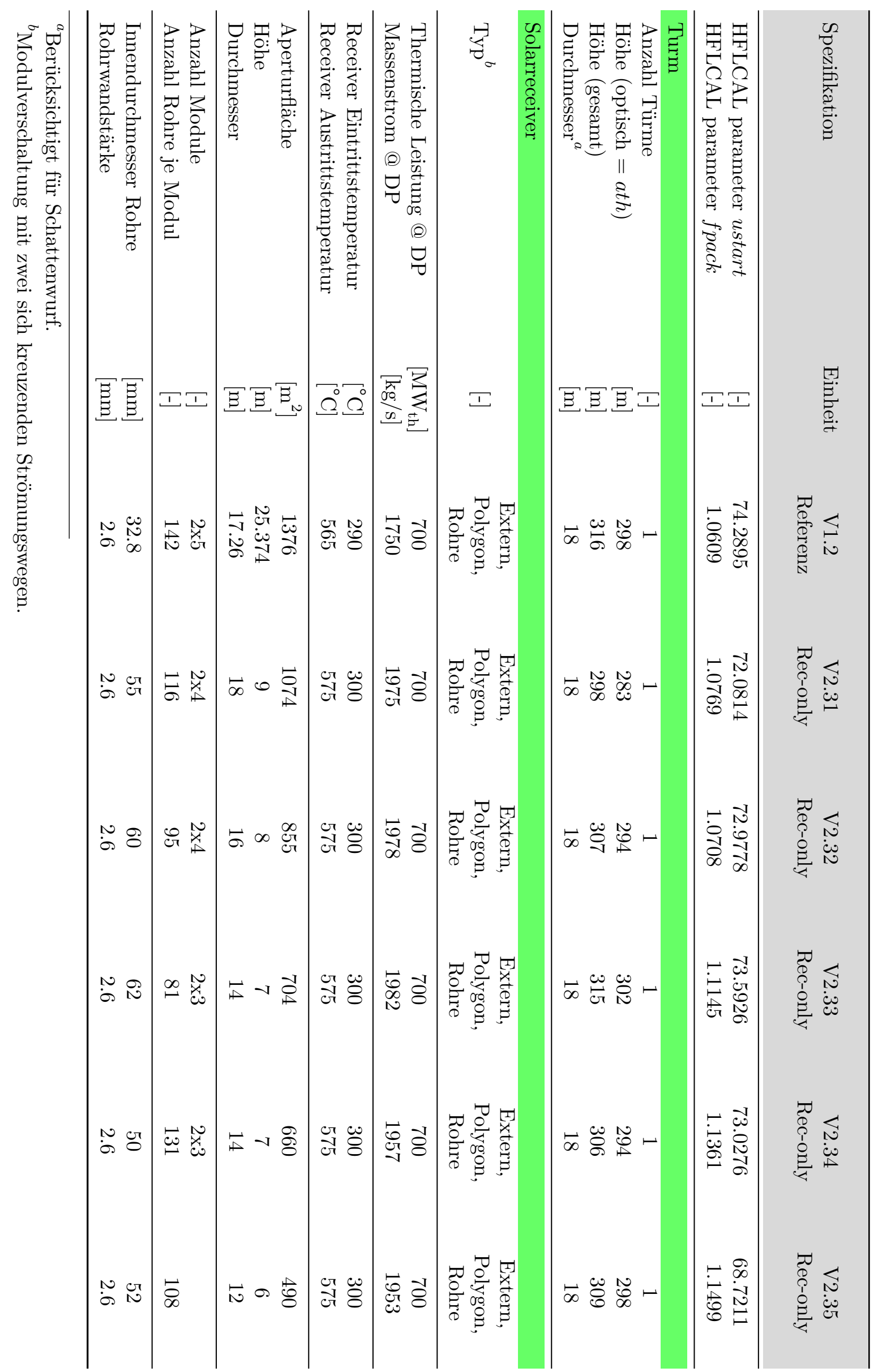




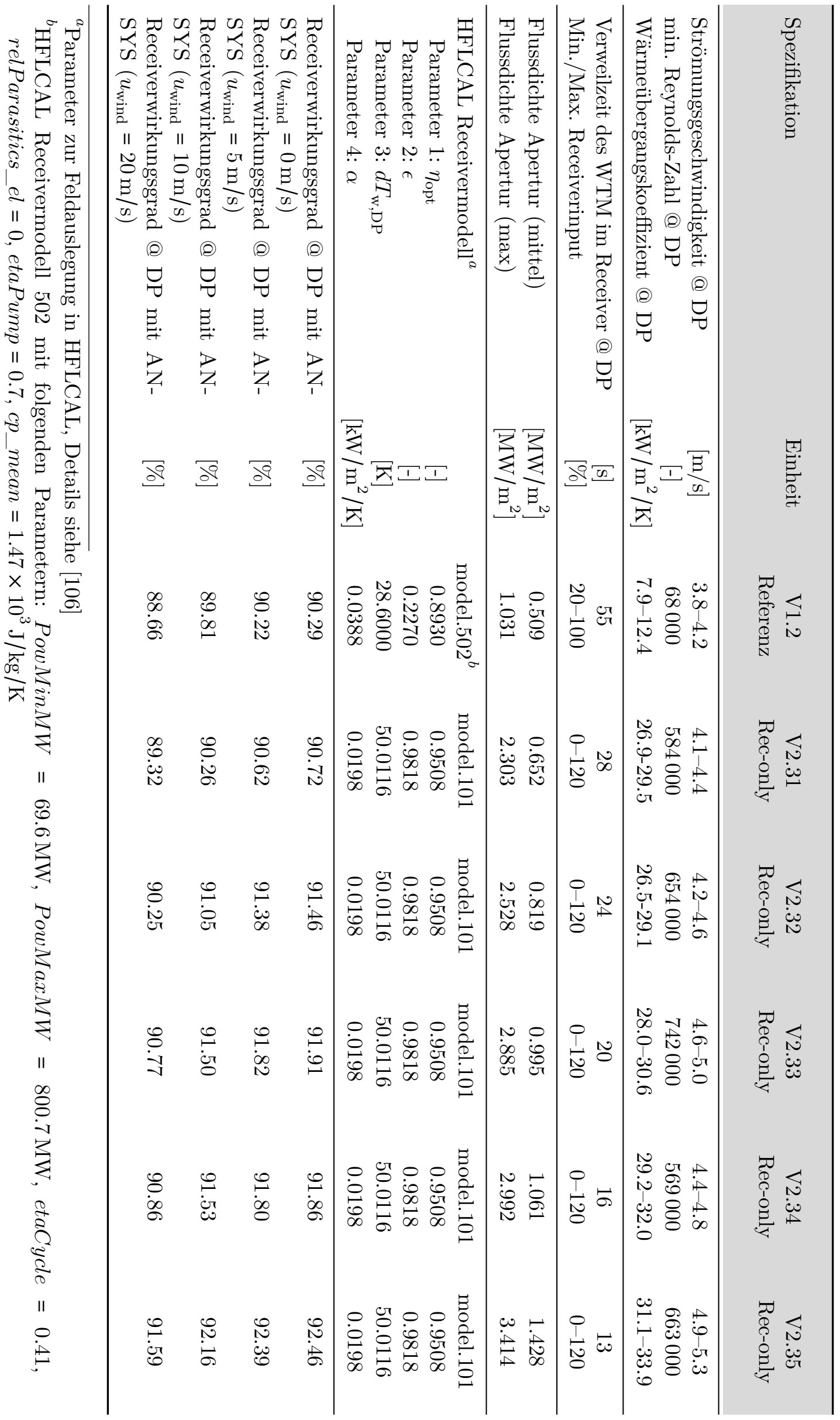




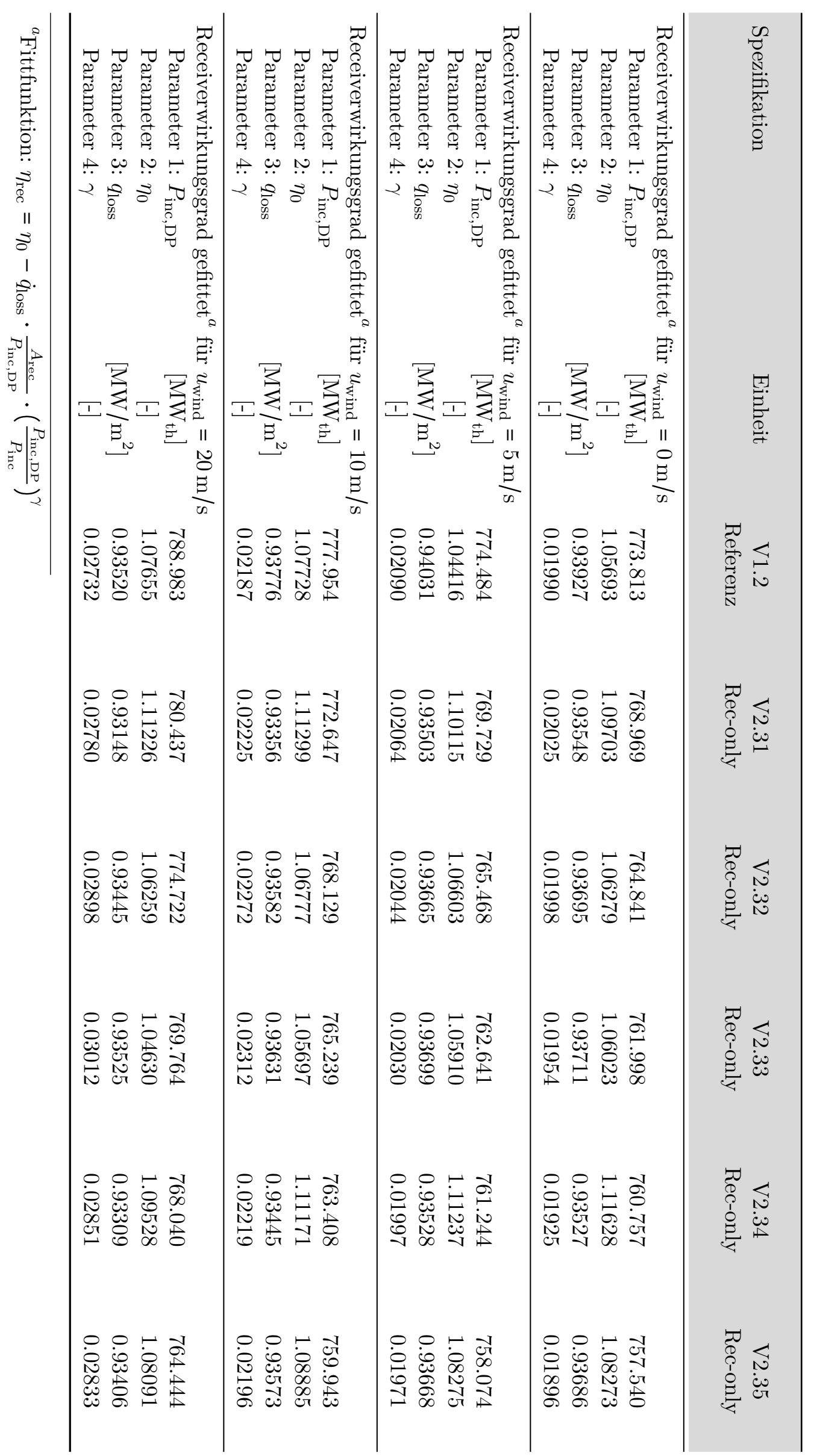




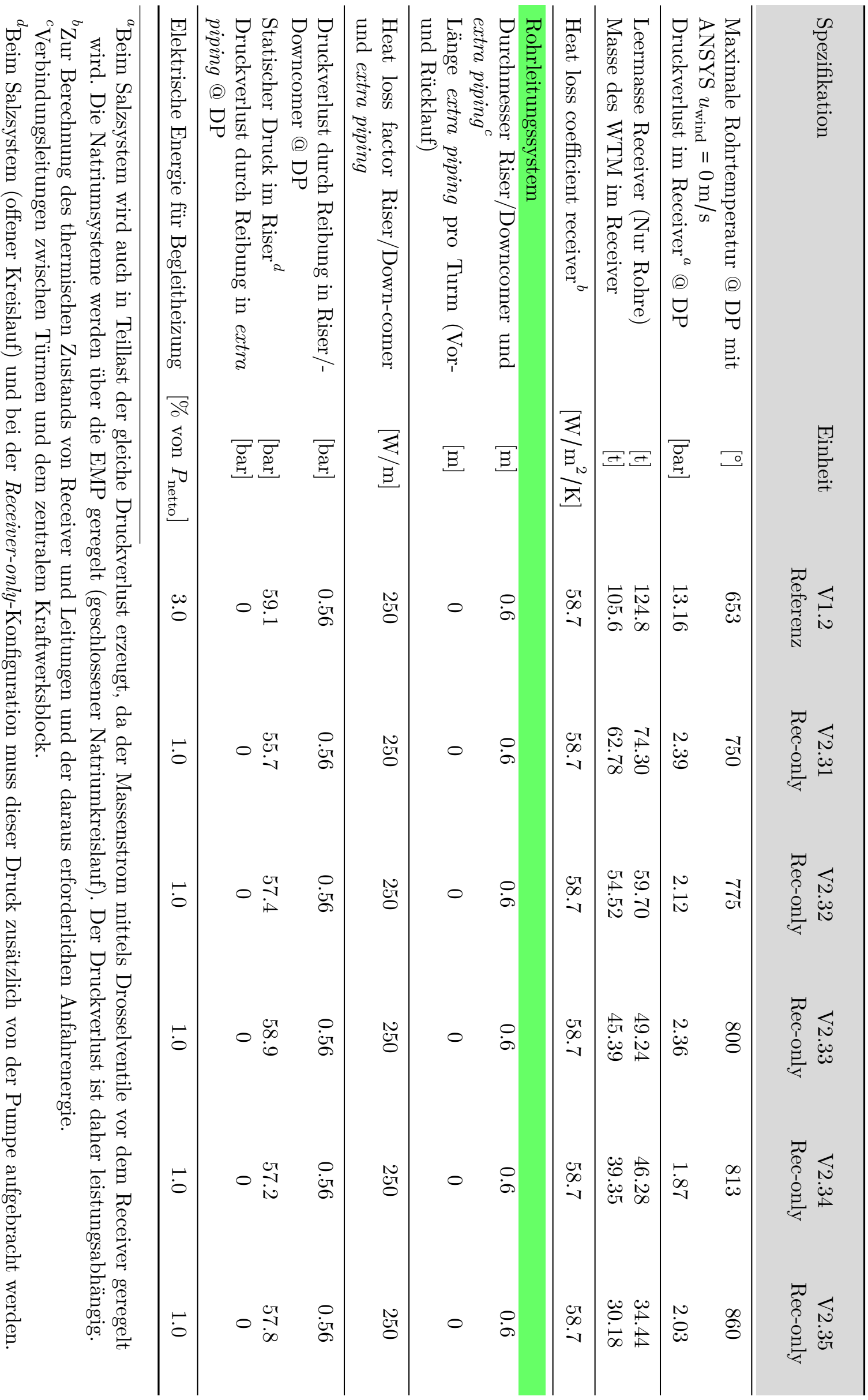




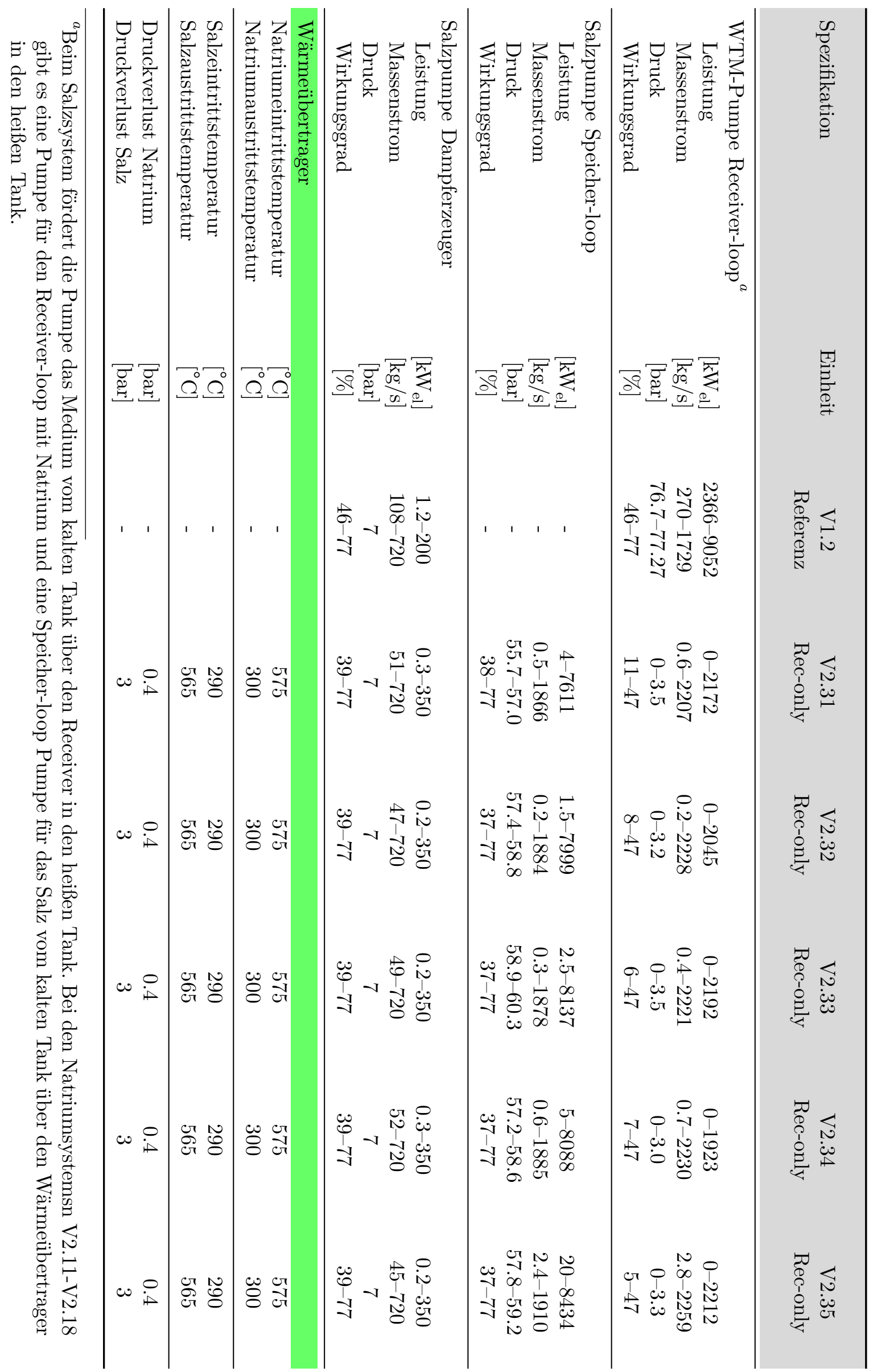




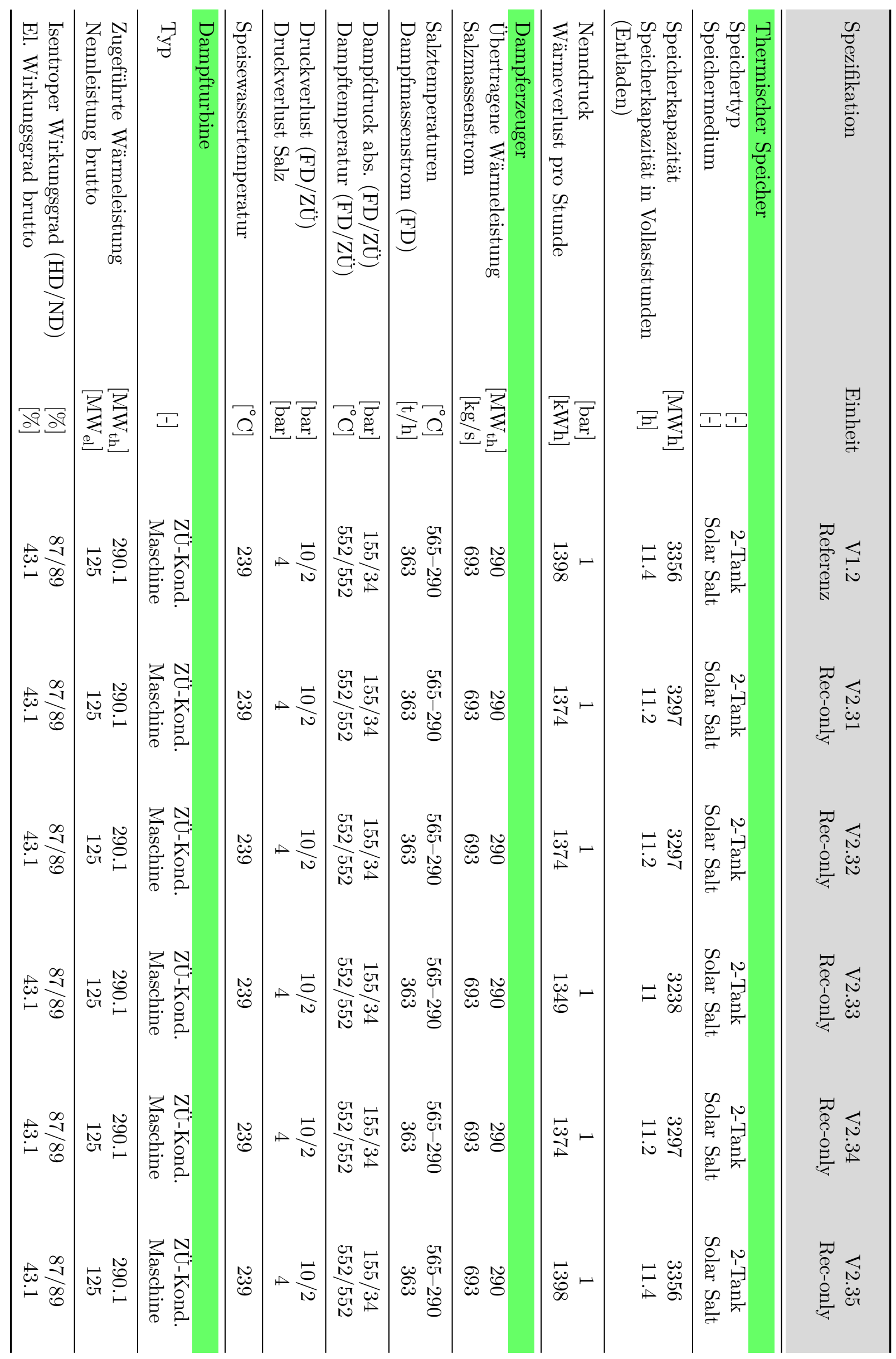




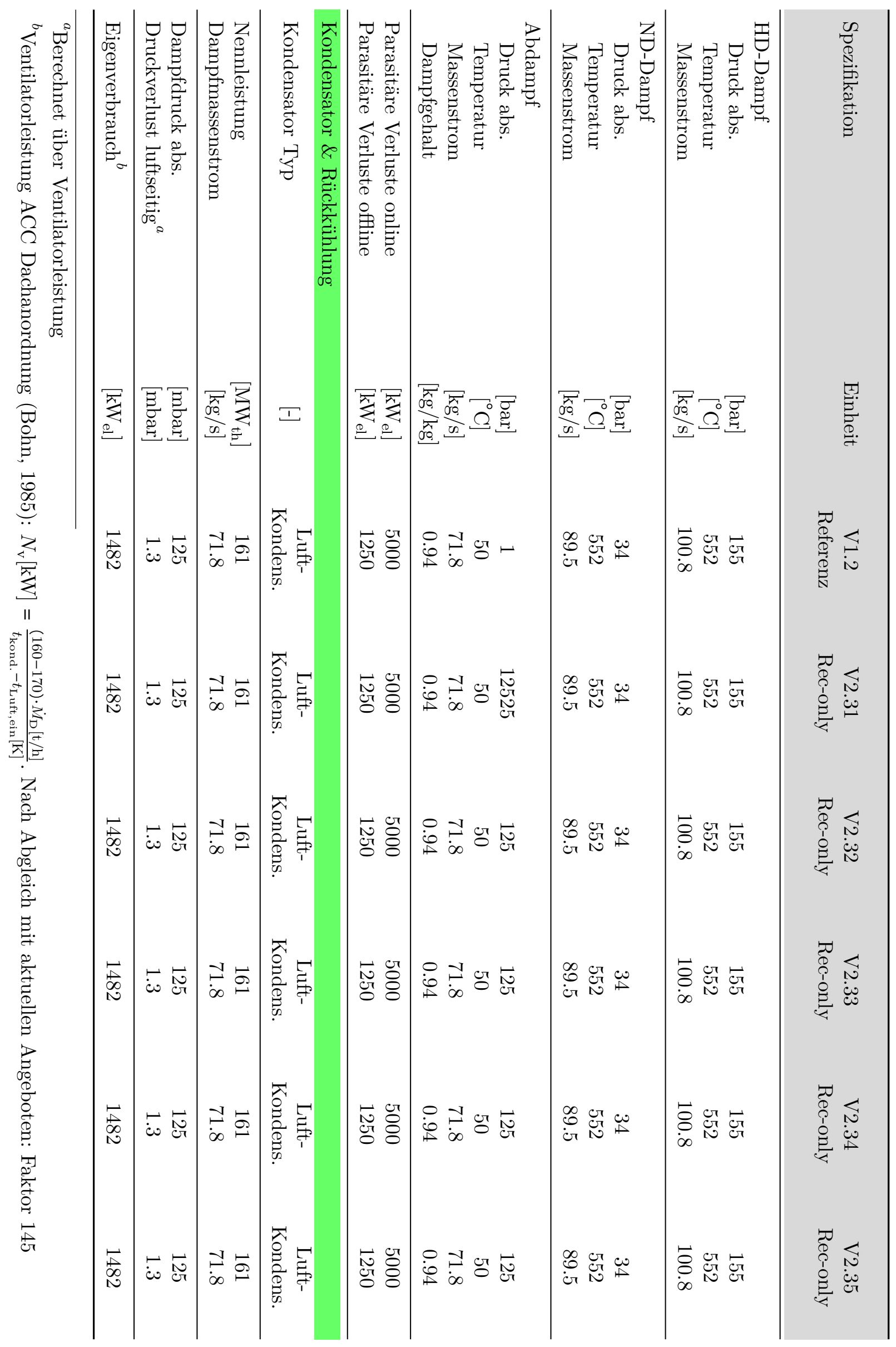




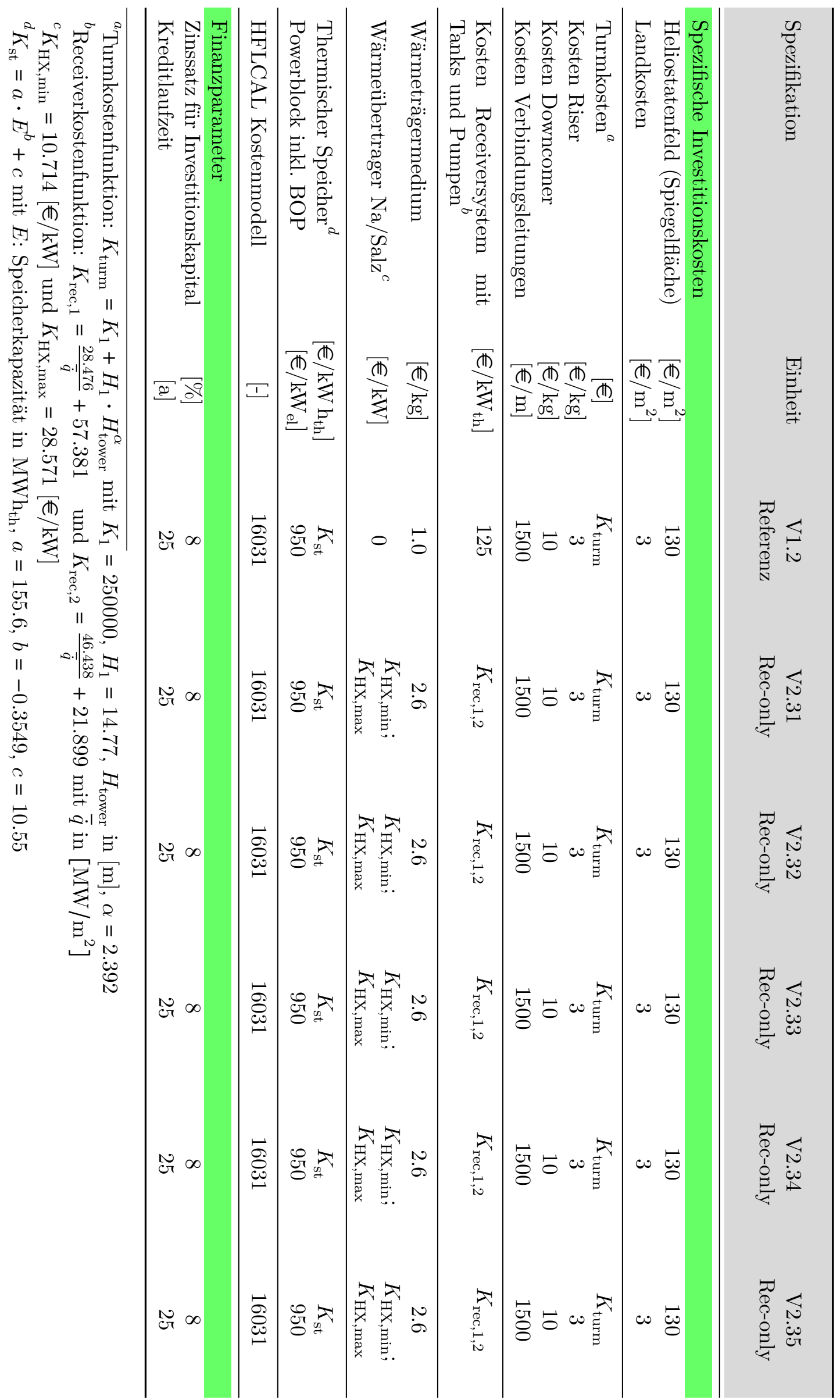




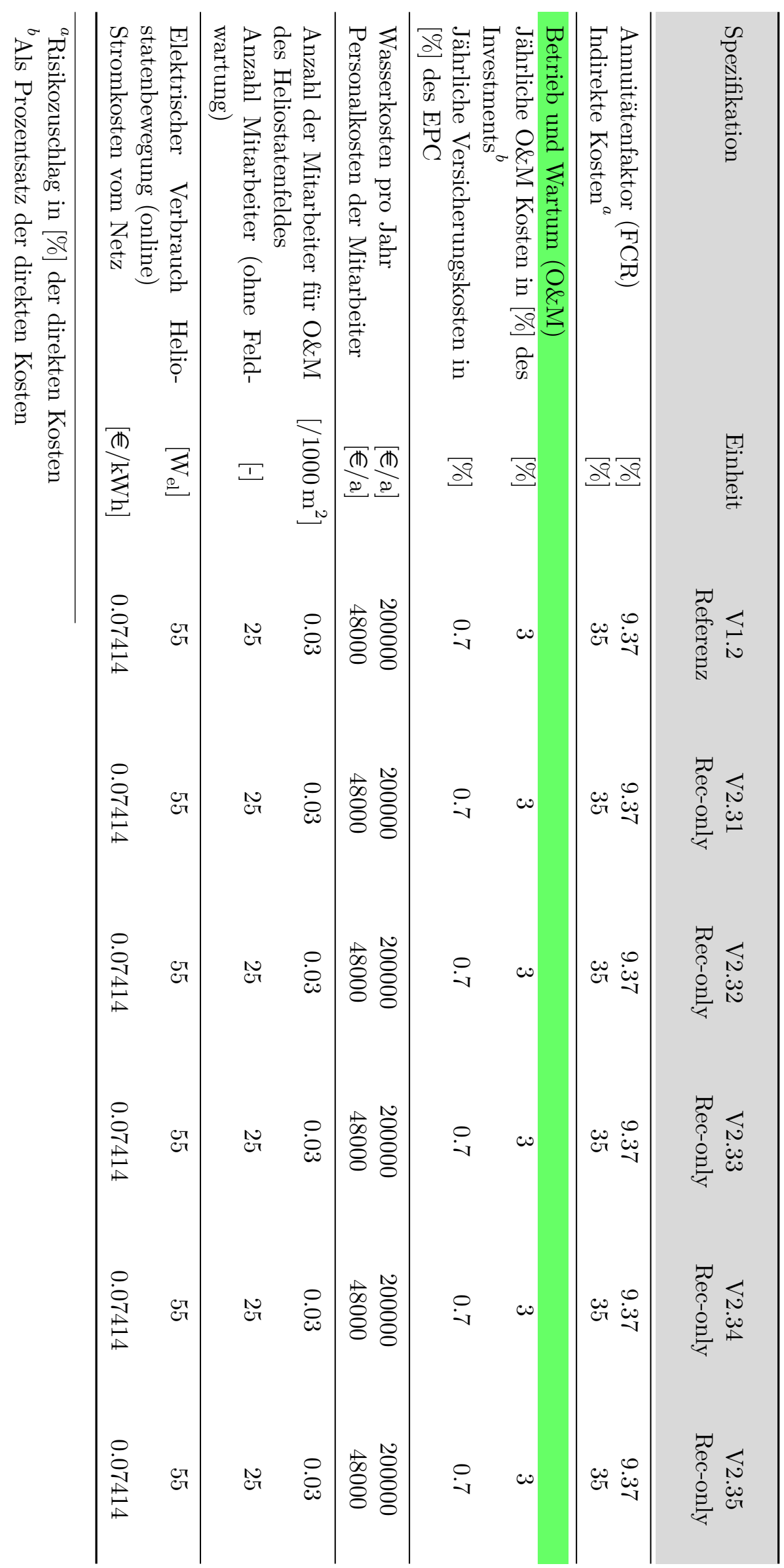




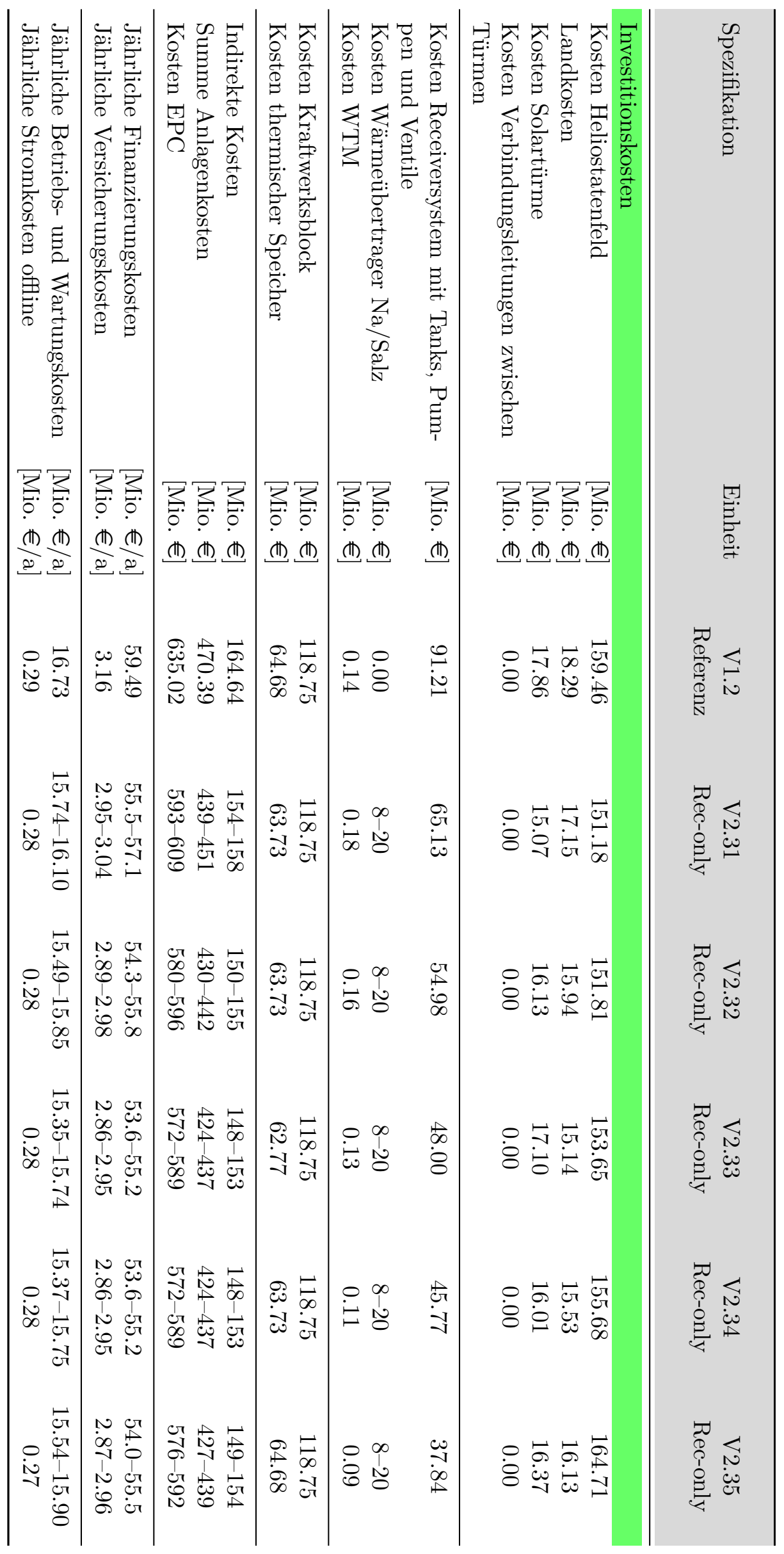




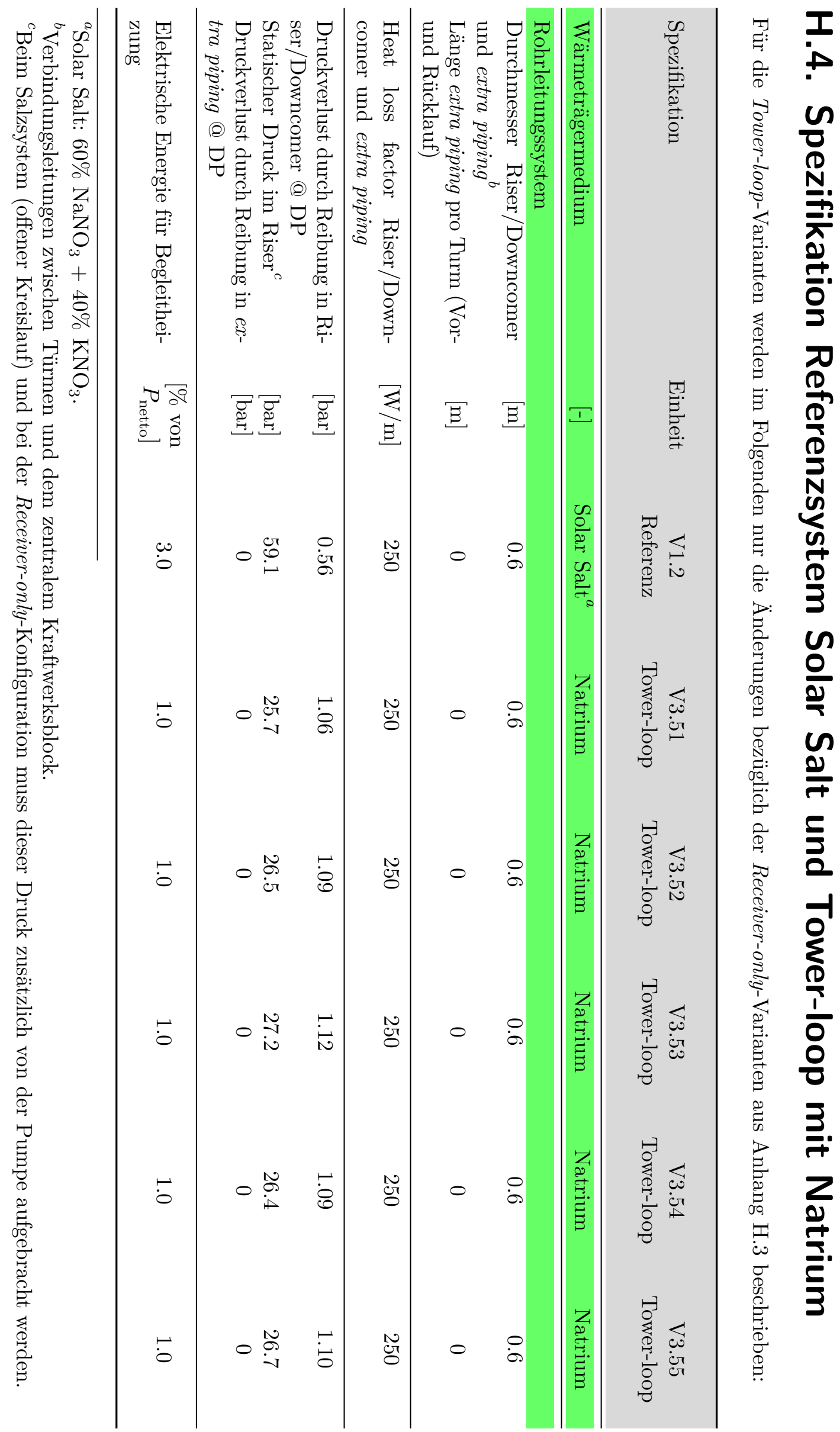




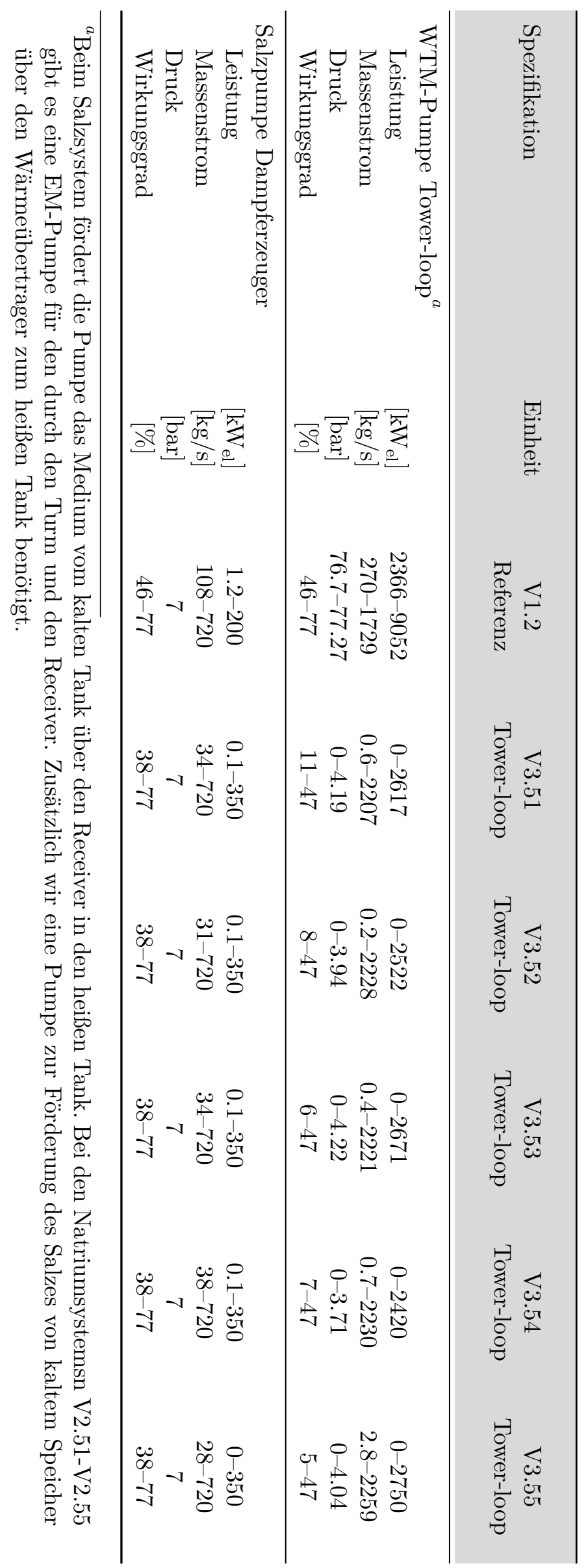




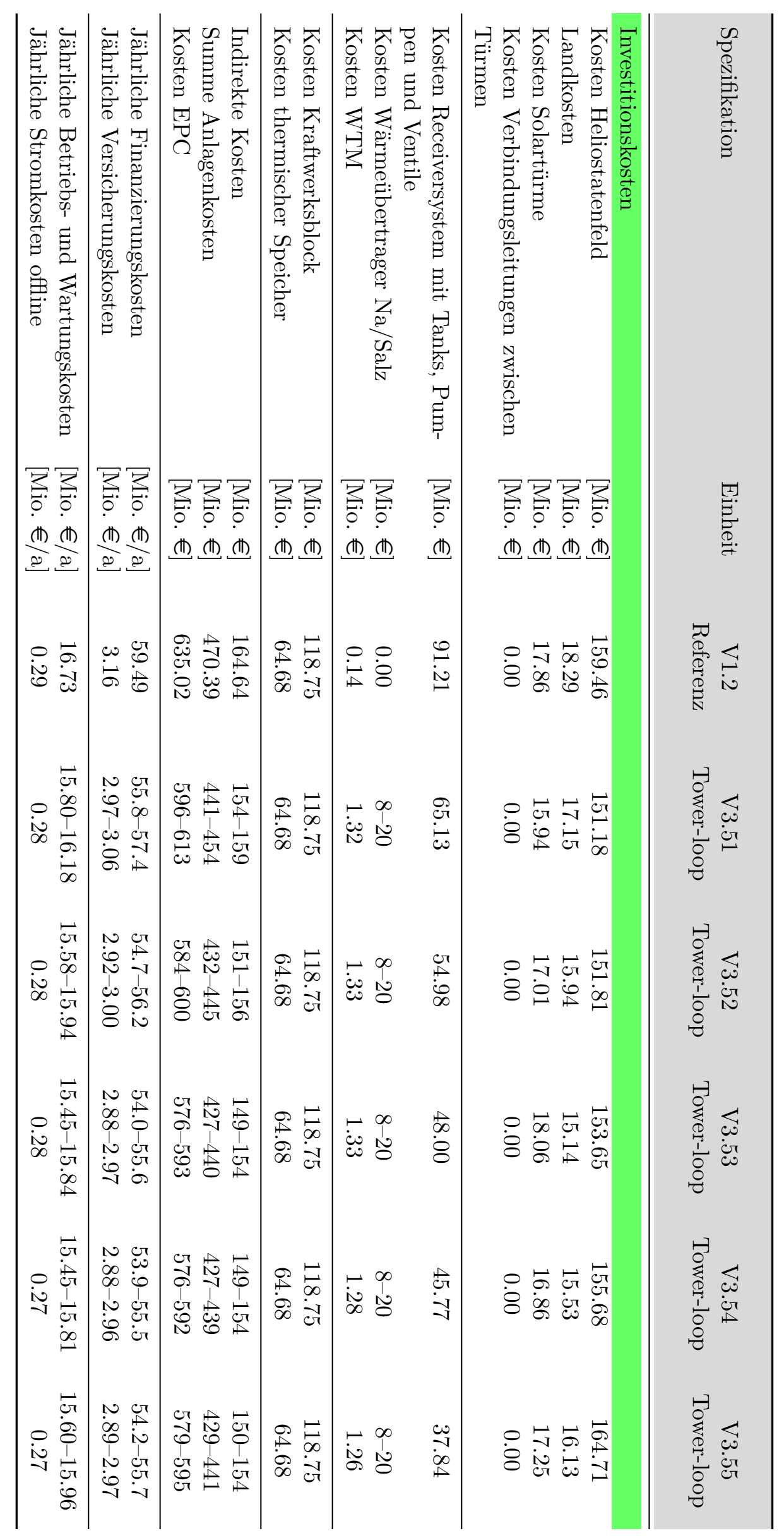




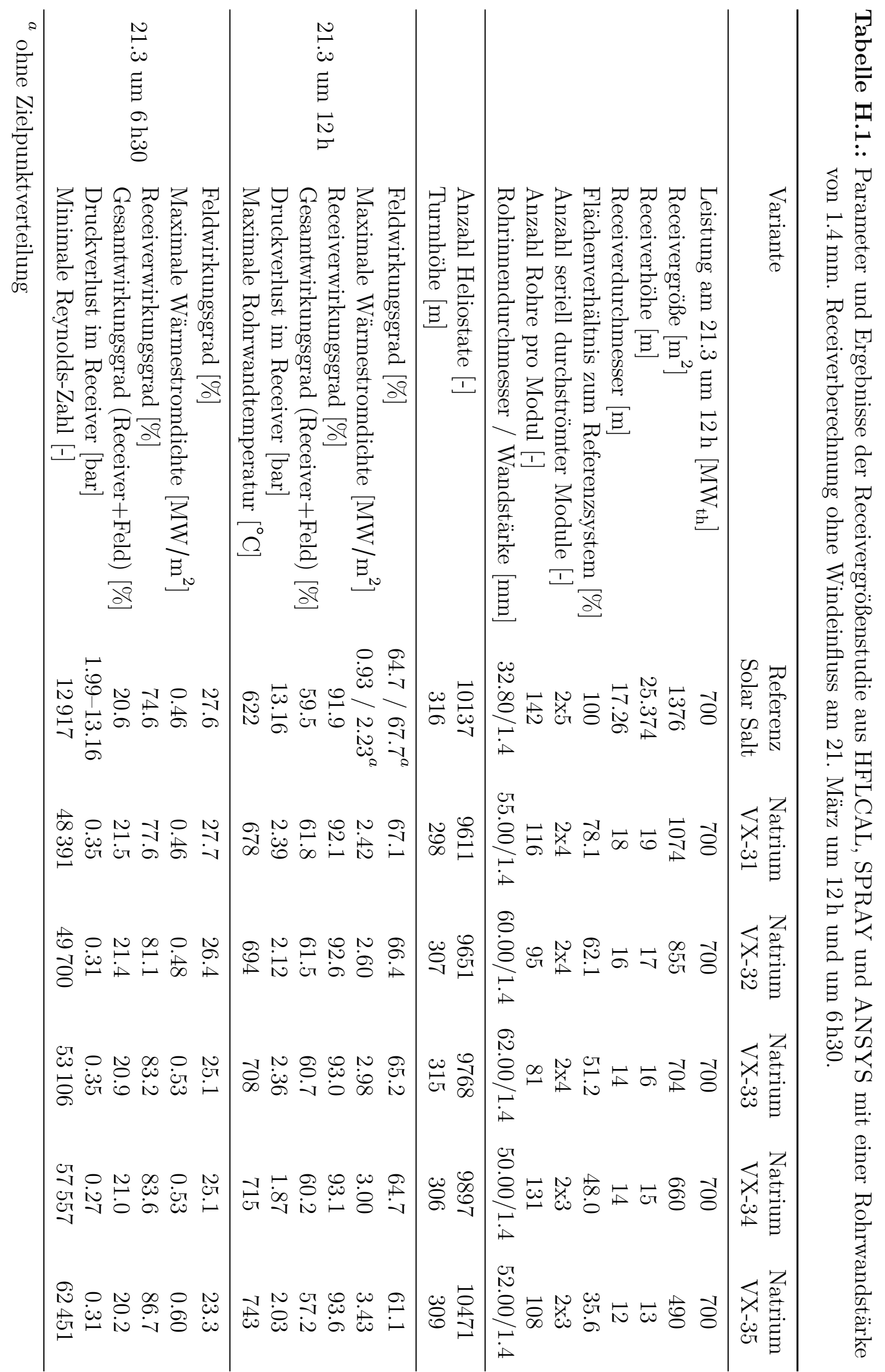




\section{Literatur}

[1] WorldBank. Breakdown of Electricity Generation by Energy Source. http://www.tsp-data-portal.org/. Abgerufen im April 2015.

[2] BMWI. Bruttostromerzeugung nach Energieträgern - in TWh. Arbeitsgruppe Erneuerbare Energien-Statistik (AGEE-Stat), Arbeitsgemeinschaft Energiebilanzen. http://www.bmwi.de/DE/Themen/Energie/ Energiedaten-und-analysen/Energiedaten/energietraeger.html, 15.10.2014.

[3] Europarat. Schlußfolgerungen der Vorsitzes des des europäischen Rates (Brüssel). Tagung vom 8./9. März 2007 in Brüssel, 7224/1/07, REV 1, CONCL 1. http://energy.iep-berlin.de/pdf/Schlussfolgerungen.pdf, 2.Mai 2007.

[4] Fraunhofer. Stromgestehungskosten erneuerbare Energien. Institut für solare Energiesysteme ISE. https://www.ise.fraunhofer.de/de/veroeffentlichungen/veroeffentlichungen-pdf-dateien/studien-und-konzeptpapiere/ studie-stromgestehungskosten-erneuerbare-energien.pdf, November 2013.

[5] Burgaleta, J. I., Arias, S. und Ramirez, D. „Gemasolar, the first tower thermosolar commercial plant with molten salt storage". In: SolarPaces International Conference (2011).

[6] Boerema, N., Morrison, G., Taylor, R. und Rosengarten, G. „Liquid sodium versus Hitec as a heat transfer fluid in solar thermal central receiver systems“. In: Solar Energy 86 (2012), S. 2293-2305.

[7] Phillips, J. N. Economic Analysis of Advanced Ultra-Supercritical Pulverized Coal Power Plants: A Cost-Effective CO2 Emission Reduction Option? Advances in Materials Technology for Fossil Power Plants, Proceedings from the Sicth International Conference 2010. Electric Power Research Institute (EPRI). 2011. 
[8] Sizmann, R. Solarchemisches Potential der Sonnenstrahlung. Becker und Funken, Ludwig-Maximilians-Universität München, 1989.

[9] IEA. Technology Roadmap Concentrating Solar Power. International Energy Agency (OECD/IEA. 2010.

[10] Kearney, D. „Status of the SEGS plants“. In: Solar World Congress 1992 (1991), S. 545-550.

[11] Torresol. Gemasolar, Connected to the Sun. Booklet Torresol Energy. http://www.torresolenergy.com, 4.Oktober 2011.

[12] Azcarraga, G. Evaluating the effectiveness of molten salt storage with solar plants. presentation (Torresol Energy). 2012.

[13] Burgaleta, J. I. „Operative advantages of a central tower solar plant with thermal storage system“. In: SolarPaces International Conference (2009).

[14] SolarReserve. http://www.solarreserve.com/en/global-projects/csp/ crescent-dunes. Abgerufen im Oktober 2016.

[15] SolarReserve. Crescent Dunes Solar Energy Project. project overview, fact sheet. www.SolarReserve.com. 9.2013.

[16] Pacio, J., Marocco, L. und Wetzel, T. „Review of data and correlations for turbulent forced convective heat transfer of liquid metals in pipes". In: Heat ans Mass Transfer 51.2 (2015), S. 153-164.

[17] IAEA. Status of Fast Reactor Research and Technology Development. International Atomic Energy Agency, IAEA-TECDOC-1691. Vienna, 2012.

[18] WNN. Russia connects BN-800 fast reactor to grid. WNN - World Nuclear News, 11. Dezember 2015. http://www.world-nuclear-news.org/NNRussia-connects-BN800-fast-reactor-to-grid-11121501.html, 11.12.2015.

[19] AtomInfo.Ru. BN-800 wurde in den kommerziellen Betrieb genommen. AtomInfo.Ru, 1. November 2015. http://www.atominfo.ru/newso/v0477.htm, Aberufen am 2.1.2016.

[20] Kolbenschmidt. Hohlventile. Motorservice, Technical Market Support, 4.Auflage, August 2014. http://download.ms-motor-service.de/ximages/ ks_50003991-01_web.pdf, 2.Mai 2007. 
[21] Mercedes. Mercedes-Benz 540 K Cabriolet B. Abgerufen am 2. Januar 2016. https://www.mercedes-benz.com/de/mercedes-benz/classic/ museum/mercedes-benz-540-k-cabriolet-b/.

[22] PC-Games-Hardware. Flüssigmetall-CPU-Kühler Danamics LMX Superleggera im Test. http://www.pcgameshardware.de/LuftkuehlungHardware-217993/Tests/Danamics-LMX-Superleggera-mit-Fluessigmetalltechnik-zum-Erfolg-706765/, 13. August 2010. Abgerufen am 13.08.2020.

[23] Bradwell, D. „Magnesium-antimony liquid metal battery for stationary energy storage“. In: Am. Chem. Soc 134 (2012), S. 1895-1897.

[24] Sodium Solar Receiver Experiment, Final Report. SAND-82-8192. Rockwell International Corp. 1.12.1983.

[25] Wettermark, G. „Performance of the SSPS Solar Power Plants at Almeria“. In: Journal of Solar Energy Engineering 110 (Nov. 1988), S. 235247.

[26] Lessons from the SSPS-CRS sodium fire incident. Motor Columbus. Deutsche Forschungs- und Versuchsanstalt für Luft- und Raumfahrt DFVLR. März 1987.

[27] Kesselring, P. The IEA/SSPS Solar Thermal Power Plants - Facts and Figures - Final Report of the International Test and Evaluation Team (ITET): Volume 4: Book of Summaries. Springer Verlag, 1986.

[28] Casal, F. Solar Thermal Power Plants, Achievements and Lessons Learned. Springer-Verlag, 1987.

[29] Schiel, W. The IEA/SSPS High Flux Experiment, Testing the Advanced Sodium Receiver at Heat Fluxes up to $2.5 \mathrm{MW} / \mathrm{m}^{2}$. Springer Verlag, 1987.

[30] Kesselring, P. The IEA/SSPS Solar Thermal Power Plants - Facts and Figures - Final Report of the International Test and Evaluation Team (ITET): Volume 1: Central Receiver System (CRS). Springer Verlag, 1986.

[31] VastSolar. Commercially Sustainable CSP Scalable, High Efficiency, Low Cost. ASTRI Symposium. 11. Februar 2015. 
[32] Coventry, J., Pye, J., Andraka, C., Blanco, M. und Fisher, J. „Sodium receivers for solar power towers: a review". In: Energy Procedia, SolarPACES 201469 (2015).

[33] VastSolar. Chemical leak successfully managed at solar research 85 development facility outside Forbes NSW. Pressemitteilung. 14.6.2015.

[34] Singer, C., Buck, R., Pitz-Paal, R. und Müller-Steinhagen, H. „Assessment of solar power tower driven ultra supercritical steam cycles applying tubular central receivers with varied heat transfer media". In: Proceedings of Energy Sustainability 2009 ().

[35] Pacio, J., Fritsch, A., Singer, C. und Uhlig, R. „Liquid metals as efficient coolants for high-intensity point-focus receivers: implications to the design and performance of next-generation CSP systems“. In: Energy Procedia 49 (2014), S. 647-655.

[36] Pacheco, J. E., Showalter, S. K. und Kolb, W. J. „Development of a Molten-Salt Thermocline Thermal Storage System for Parabolic Trough Plants“. In: Solar Energy Engineering 124 (2002), S. 153-159.

[37] Herrmann, U. und Kearney, D. W. „Survey of Thermal Energy Storage for Parabolic Trough Power Plants“. In: Solar Energy Engineering 124 (2002), S. 145-152.

[38] Mantz, R. Molten Salts 15: In Memory of Robert Osteryoung. The Electrochemical Society, ecs transactions, Vol. 3 No. 35, 2007.

[39] Pacio, J. und Wetzel, T. „Assessment of liquid metal technology status and research paths for their use as efficient heat transfer fluids in solar central receiver systems“. In: Solar Energy 93 (2013), S. 11-22.

[40] Pacio, J., Singer, C., Wetzel, T. und Uhlig, R. „Thermodynamic evaluation of liquid metals as heat transfer fluids in concentrated solar power plants“. In: Applied Thermal Engineering 60 (2013), S. 295-302.

[41] Fink, J. K. und Leibowitz, L. Thermodynamic and transport properties of sodium liquid and vapour. ANL/RE-95/2. Argonne National Laboratory, Reactor Engineering Division. January 1995. 
[42] Pacheco, J. E., Ralph, M. E., Chavez, J. M., Dunkin, S. R., Rush, E. E., Ghanbari, C. M. und Matthews, M. W. Results of Molten Salt Panel and Component Experiments for Solar Central Receivers. SAND1994-2525. Department of Energy, Sandia National Laboratories, Albuquerque, New Mexico 87185 und Livermore, California 94550. 1995.

[43] OECD/NEA. Handbook on Lead-bismuth Eutectic Alloy and Lead Properties, Materials Compatibility, Thermal-hydraulics and Technologies. NEA No. 6195, ISBN 978-92-64-99002-9. Nuclear Energy Agency. 2007.

[44] Pacheco, J. E. Final Test and Evaluation Results from the Solar Two Project. SAND2002-0120. Department of Energy, Sandia National Laboratories, Albuquerque, New Mexico 87185 und Livermore, California 94550. 2002.

[45] Incropera, F. P. Fundamentals of Heat and Mass Transfer, Hoboken: Wiley. pp. 490 and 515. John Wiley und Sons; 6. Auflage (1. September 2006), 2006.

[46] VDI. VDI-Wärmeatlas. Springer Vieweg, 2006 (10.Auflage).

[47] Vant-Hull, L. „The Role of Allowable Flux Density in the Design and Operation of Molten-Salt Solar Central Receivers“. In: Journal of Solar Energy Engineering 124.ASME (2002), S. 165-169.

[48] Smith, D. C. „Design and optimization of tube-type receiver panels for molten salt application“. In: Solar Engineering ASME 2 (1992), S. 10291036 .

[49] Lata, J. M., Rodríguez, M. und Lara, M. Á. de. „High Flux Central Receivers of Molten Salts for the New Generation of Commercial StandAlone Solar Power Plants". In: Solar Energy Engineering 130 (2008), S. $1-5$.

[50] Litwin, R. Z. Receiver System: Lessons Learned from Solar Two. SAND2002-0084. Department of Energy, Sandia National Laboratories, Albuquerque, New Mexico 87185 und Livermore, California 94550. 2002.

[51] NREL. System Advisor Model (SAM) Case Study Gemasolar. Fuentes De Andalucia, Spain. 2013. 
[52] Zavoico, A. B. Solar Power Tower Design Basis Document. SAND20012100. Department of Energy, Sandia National Laboratories, Albuquerque, New Mexico 87185 und Livermore, California 94550. 2001.

[53] Rodríguez-Sánchez, M. R., Soria-Verdugo, A. und Almendros-Ibánez, J. A. „Thermal design guidelines of solar power towers". In: Applied Thermal Engineering 63 (2014), S. 428-438.

[54] Logie, W., Asselineau, C., Pye, J. und Coventry, J. „Temperature and Heat Flux Distributions in Sodium Receiver Tubes“. In: Solar Research conference Aisa-Pacific (2015), S. 1-10.

[55] Ilincev, G. „Research results on the corrosion effects of liquid heavy metals $\mathrm{Pb}, \mathrm{Bi}$ and $\mathrm{Pb}-\mathrm{Bi}$ on structural materials with and without corrosion inhibitors". In: Nuclear Engineering and Design 217 (2002), S. 167-177.

[56] Lorenzin, N. und Abánades, A. „A review on the application of liquid metals as heat transfer fluid in Concentrated Solar Power technologies“. In: International Journal of Hydrogen energy 41 (2016), S. 6990-6995.

[57] LIMTECH. Interne Bewertung von Fachleuten im Rahmen der HelmholtzAllianz LIMTECH (2012-2017).

[58] Pacheco, J. E. und Dunkin, S. R. Assessment of molten-salt solar central receiver freeze-up and recovery events. Department of Energy, Sandia National Laboratories, Albuquerque, New Mexico 87185 and Livermore, California 94550. 1996, SAND96-0331C.

[59] Boerema, N., Taylor, R. A., Morrison, G. und Rosengarten, G. „Solidliquid phase change modelling of metallic sodium for application in solar thermal power plants“. In: Solar Energy 119 (2015), S. 151-158.

[60] Samsonov, G. V. Handbook of the Physicochemical Properties of the Elements. Springer US, 1968. DOI: 10.1007/978-1-4684-6066-7.

[61] Kolesnikov, Y., Karcher, C. und Thess, A. „Lorentz Force Flowmeter for Liquid Aluminum: Laboratory Experiments and Plant Tests". In: Metallurgical and materials transactions 42.B (2011), S. 441-450.

[62] Ratajczak, M., Wondrak, T. und Stefani, F. „A gradiometric version of contactless inductive flow tomography: theory and first applications". In: Philosophical Transactions of the royal society A 374.2070 (2016). 
[63] M. Daubner F. Fellmoser, L. S. Technische Beschreibung der Versuchsanlage ALINA zur Untersuchung eines Natrium-Freistrahls. KIT Scientific reports 7570. Karlsruher Institut für Technologie (KIT), Institut für Kern- und Energietechnik (IKET). 2011.

[64] Umweltbundesamt. Leitfaden zur Anwendung umweltverträglicher Stoffe. http://www.umweltbundesamt.de/sites/default/files/medien/publikation/long/2325.pdf. Abgerufen am 09.2015. Februar 2003.

[65] Anderson, F. A. A primer for the safe use of liquid alkali metals. ORNLTM-1740. Oak ridge national laboratory, U.S. Atomic energy commission. January 1967.

[66] Bae, J. H., Ahn, D. H., Kim, Y. C. und Cho, M. „An Experimental Study on the Characteristics of Sodium Fires". In: Journal of Korean Nuclear Society 26.4 (1994), S. 471-483.

[67] Chander, R., Sundararajan, A. und R.D.Kale. Status of studies on sodium fires and protection systems in indian LMFBR programme. XA0201166. Indira Gandhi Centre for Atomic Research, Department of Atomic Energy.

[68] Wietelmann, U. Winnacker/Küchler. Chemische Technik: Prozesse und Produkte, Alkali- und Erdalkalimetalle. Roland Dittmeyer, Wilhelm Keim, Gerhard Kreysa, Alfred Oberholz, Band 6 b: Metalle., 2006.

[69] USGS. United States Geological Survey. Commodity Statistics and Information of minerals. http://minerals.usgs.gov/minerals/pubs/commodity, 11.Sept 2014.

[70] Wedepohl, K. H. „The composition of the continental crust". In: Geochimica et Cosmochimica Acta 59.7 (1995), S. 1217-1232.

[71] Binder, H. H. Lexikon der chemischen Elemente. S. Hirzel Verlag, Stuttgart, 1999.

[72] Steinmann, W.-D., Laing, D. und Odenthal, C. „Development of the CellFlux Storage Concept for Sensible Heat". In: Journal of Solar Energy Engineering 136.011011 (Februar 2014), S. 1-8. 
[73] BDW. Das leistungsfähigste Kraftwerk der Welt. Bild der Wissenschaft online, 20. Mai 2011. http://www.wissenschaft.de/home/-/journal_content/ 56/12054/938790/, Aufgerufen im November 2016.

[74] Wu, S. Y., Xiao, L. und Cao, Y.-D. „A review on advances in alkali metal thermal to electric converters (AMTECs)“. In: International Journal of energy research 33 (3. Juli 2009), S. 868-892.

[75] Díez, N., Onea, A., Scherrer, S., Weisenburger, A. und Hering, W. Direct Energy Conversion of Heat to Electricity Using AMTEC. 978-1-46737172-8/15/ European Union. Karlsruher Institut for Technology (KIT). 2015.

[76] Saito, M., Nakamura, M., Nezu, A., Kamiyama, K. und Fujiie, Y. „Basic study on liquid-metal MHD power generation system using two-phase natural circulation“. In: MHD-International Conference 1.Chapter 19 (1996), S. 165-175.

[77] Branover, H. und El-Boher, A. „Promising applications of the liquid metal MHD energy conversion technology“. In: Energy Conversion Engineering Conference 2.IECEC-89., Proceedings of the 24th Intersociety (1989), S. 1051-1058.

[78] Petrick, M. „Liquid-metal magnetohydrodynamics“. In: IEEE Spectrum 2.Issue 3 (1965), S. 137-151.

[79] Baker, R. S. Handbook of electromagnetic pump technology. ISBN: 0444012745, 9780444012746. University of Michigan. 1987.

[80] Elliott, D. G. „Direct Current Liquid-Metal Magnetohydrodynamic Power Generation“. In: AIAA Journal 4.4 (1966), S. 627-634.

[81] Adkins, D., Andraka, C. E., Moreno, J. B., Rawlinson, K. S., Showalter, S. K. und Moss, T. A. „Heat Pipe Solar Receiver Development Activities at Sandia National Laboratories". In: Submitted to the Renewable and Advanced Energy Conference for the 21st Century Conference (April 1999).

[82] Laing, D. und Palsson, M. „Hybrid Dish/Stirling Systems: Combustor and Heat Pipe Receiver Development". In: Journal of Solar Energy Engineering 124.DOI: 10.1115/1.1465433 (Mai 2002), S. 176-181. 
[83] Reilly, H. E. und Kolb, G. J. An Evaluation of Molten-Salt Power Towers Including Results of the Solar Two Project. SAND2001-3674. Department of Energy, Sandia National Laboratories, Albuquerque, New Mexico 87185 und Livermore, California 94550. 2001.

[84] Fritsch, A., Flesch, J., Geza, V., Singer, C., Uhlig, R. und Hoffschmidt, B. „Conceptual study of central receiver systems with liquid metals as efficient heat transfer fluids". In: Energy Procedia 69 (2015), S. 644-653.

[85] Jonemann, M. Advanced Thermal Storage System with Novel Molten Salt. NREL/SR-5200-58595, DE-AC36-08GO28308. NREL, National renewable energy laboratory. May 2013.

[86] Kelly, B. und Kearney, D. Thermal Storage Commercial Plant Design Study for a 2-Tank Indirect Molten Salt System. NREL/SR-550-40166, DE-AC36-99-GO10337. NREL, National renewable energy laboratory. July 2006.

[87] Kearney, D. und Price, H. „Assessment of thermal energy storage for parabolic trough solar power plants". In: Proceedings of 2004 Solar Conference (ASME) (2004), S. 1-7.

[88] Pitz-Paal, R. European Concentrated Solar Thermal Road-Mapping (ECOSTAR). SES6-CT-2003-502578. Deutsches Zentrum für Luft- und Raumfahrt e.V. 2004.

[89] Libby, C. Solar Thermocline Storage Systems - Preliminary Design Study. EPRI, Palo Alto, CA: 2010. 1019581. Electric Power Research Institute (EPRI). 2010.

[90] Polzin, K. „Liquid metal pump technologies for nuclear surface power“. In: In Proceedings of Space Nuclear Conference (2007).

[91] Blake, L. „Conduction and induction pumps for liquid metals“. In: Proceedings of the IEE - Part A: Power Engineering 104 (1957), S. 4967.

[92] Ota, H., Katsuki, K., Funato, M., Taguchi, J., Fanning, A. W., Doi, Y., Nibe, N., Ueta, M. und Inagaki, T. „Development of 160m3/min large capacity sodium-immersed selfcooled electromagnetic pump“. In: Nuclear Science and Technology 41 (2004), S. 511-523. 
[93] Gromov, B. Development of molten lead-bismuth target complex tc-1 for the lansce-accelerator. State Scientific Center of Russian Federation, Institute for Physics and Power Engineering. Technical Report ORNLTM-1993, 2001.

[94] Ahn, S. J. „Evaluation of a Sodium water Reaction Event Caused by Steam Generator Tubes Break in the Prototype Generation IV Sodiumcooled Fast Reactor". In: Nuclear Engineering and Technology 48.4 (2016), S. 952-964.

[95] Abdulla, S. H. „Liquid-Metal/Water Direct Contact Heat Exchange: Flow Visualization, Flow Stability, and Heat Transfer Using Real-Time X-Ray Imaging“. In: Nuclear Science and Engineering 150.2 (Juni 2005), S. $182-220$.

[96] GEA. GEA Heat Exchangers auf der EP Shanghai in China, 30.10. bis 1.11.2013, Shanghai World Expo Exhibition \& Convention Center, Stand 1N15. Pressemitteilung. 11. Sep. 2013.

[97] TÜV. AD2000 Regelwerk. Beuth, Berlin, April 2015 (9.Auflage).

[98] VDI. VDI-Wärmeatlas, Ga5, Gl.28. Springer Vieweg, 2006.

[99] Baehr, H. D. Wärme- und Stoffübertragung 7, 222-225. Springer Verlag, 1974.

[100] DLR. Interner Datensatz der Abteilung PFS (Punktfokusierende Systeme) des Instituts für Solarforschung.

[101] Fritsch, A., Uhlig, R., Marocco, L., Frantz, C., Flesch, R. und Hoffschmidt, B. „A comparison between transient CFD and FEM simulations of solar central receiver tubes using molten salt and liquid metals". In: Solar Energy Not jet published (2016).

[102] Korzynietz, R., Brioso, J. A., Río, A. del, Quero, M., Gallas, M., Uhlig, R., Ebert, M., Buck, R. und Teraji, D. „Solugas - Comprehensive analysis of the solar hybrid Brayton plant". In: Solar Energy 135 (2016), S. 578-589.

[103] Uhlig, R. SOLUGAS Receiver handbook. Confidential Report. Deutsches Zentrum für Luft- und Raumfahrt, Institut für Solarforschung, Punktfokussierende Systeme, Receiverentwicklung. 1.5.2012. 
[104] DoE. Solar Two experimental solar power plant. Work of the United States Department of Energy. http://earthobservatory.nasa.gov/Features/ RenewableEnergy/Images/solar_two.jpg, Abgerufen im Juli 2015.

[105] Falcone, P. K. A handbook for central receiver design. Unlimited Release, SAND 86-8009. Sandia National Laboratories Livermore. December 1986.

[106] Schwarzbözl, P. The User's Guide to HFLCAL. A Software Program for Heliostat Field Layout Calculation. Software Release Visual HFLCAL VH12, Cologne 2009.

[107] Hottel, H. C. „A simple model for estimating the transmittance of direct solar radiation through clear atmospheres“. In: Solar Energy 18.2 (1976), S. $129-134$.

[108] Uhlig, R., Frantz, C. und Fritsch, A. „Effects of Vertically Ribbed Surface Roughness on the Forced Convective Heat Losses in Central Receiver Systems“. In: AIP Conference Proceedings (Solar Paces 2015) 1734 (2016).

[109] Milles, U. und Horenburg, P. Basisenergy 17 - Strom aus Gas und Kohle. ISSN 1438-3802, www.bine.info. FIZ Karlsruhe GmbH · Leibniz-Institut für Informationsinfrastruktur. Februar 2011.

[110] IEA. Guidelines for the Economic Analysis of Renewable Energy Technology Applications. ISBN: 92-64-13481-6. International Energy Agency, Workshop on the Economics of Renewable Energy Technologies. 1991.

[111] Kolb, G. J., Ho, C. K., Mancini, T. R. und Gary, J. A. Power Tower Technology Roadmap and Cost Reduction Plan. SAND2011-2419. Department of Energy, Sandia National Laboratories, Albuquerque, New Mexico 87185 und Livermore, California 94550. 2011.

[112] SolarReserve. Crescent Dunes Solar Energy Project. Fact Sheet. Sept. 2013.

[113] Singer, C. „Assessment of Improved Molten Salt Solar Tower Plants“. In: SolarPaces 2013, Energy Procedia 49 (2014), S. 1553-1562.

[114] Weinrebe, G., vonReeken, F., Wöhrbach, M., Plaz, T., Göcke, V. und Balz, M. "Towards Holistic Power Tower System Optimization“. In: SolarPaces 2013, Energy Procedia 49 (2014), S. 1573-1581. 
[115] Babcock und Wilcox. Molten Salt Receiver Subsystem Research Experiment - Executive Summary. SAND84-8178. Department of Energy, Sandia National Laboratories, Albuquerque, New Mexico 87185 und Livermore, California 94550. 1984.

[116] Persönliches Gespräch mit Firma Deller und überschlägige Auslegung zur Kostenabschätzung eines Doppelrohrwärmeübertragers für Natrium/Solar Salt mit einer Grädigkeit von $10 \mathrm{~K}$ bei einer Leistung von $140 \mathrm{MW}$. Wilhelm Deller Wärmeübertrager, Siegen, im Sommer 2015.

[117] BV. Cost and performance data for power generation technologies. Black \& Veatch, prepared for the National Renewable Energy Laboratory NREL. February 2012.

[118] Flesch, J., Fritsch, A., Cammi, G., Marocco, L., Fellmoser, F., Pacio, J. und Wetzel, T. „Construction of a test facility for demonstration of a liquid lead-bismuth-cooled 10kW thermal receiver in a solar furnace arrangement - SOMMER“. In: Energy Procedia, SolarPACES 201469 (2015), S. 1259-1268.

[119] SpecialMetals. Werkstoffdatenblatt für INCONEL alloy 617 (UNS N06617/W.Nr. 2.4663a). Special Metals Corporation, 2005 (Mar 05), Publication Number SMC-029. http://www.specialmetals.com/assets/documents/alloys/inconel/inconel-alloy-617.pdf, 01.09.2015.

[120] Lovegrove, K. Concentrating solar power technology - Principles, developments and applications. Woodhead Publishing Limited, ISBN: 978-184569-769-3, 2012.

[121] Singer, C. Solarturmreceiver für überkritische Dampfprozesse und ihre technische und ökonomische Bewertung. Springer Verlag, 2013. 

In einem Solarturmkraftwerk werden hunderte bis tausende Spiegel (sogenannte Heliostate) einzeln der Sonne nachgeführt, sodass das Sonnenlicht auf die Spitze eines Turmes konzentriert wird. Dort befindet sich der Receiver, der die Solarstrahlung absorbiert und an das Wärmeträgermedium weitergibt. Große thermische Energiespeicher erlauben die ganztägige Stromproduktion (auch Nachts). In aktuellen Solarturmkraftwerken kommen als Wärmeträger- und Speichermedium häufig flüssige Nitratsalze wie Solar Salt zum Einsatz. Aufgrund ihrer hohen Wärmekapazität und den geringen Kosten eignen sie sich sehr gut zur thermischen Energiespeicherung, jedoch bringen sie auch einige Nachteile mit sich. Flüssigmetalle weisen in vielen Punkten Vorteile gegenüber den Flüssigsalzen auf. In der vorliegenden Arbeit werden die Eigenschaften von Flüssigmetallen detailliert analysiert und mit Solar Salt verglichen. Zur Bewertung werden die Jahreserträge bzw. die Stromgestehungskosten (LCOE) herangezogen.

Die Ergebnisse zeigen ein Potenzial zur Reduktion der Stromgestehungskosten mit Flüssigmetallen um bis zu $16 \%$ gegenüber dem Referenzsystem mit Solar Salt, ohne dabei den Kraftwerksblock und den thermischen Speicher zu verändern.

\section{Logos Verlag Berlin}

